Andreas Siemes

\title{
Marktorientierte Kreditrisikobewertung
}

Eine empirische Untersuchung mittels Künstlicher Neuronaler Netze 


\section{Andreas Siemes}

\section{Marktorientierte Kreditrisikobewertung}

Kreditinstitute werden heute vor die Herausforderung gestellt, einen effektiven und effizienten Risikomanagementprozess zu etablieren. Insbesondere bei der Bewertung von Unternehmensanleihen und Krediten wird diese Notwendigkeit deutlich. Die Arbeit greift diese Problemstellung auf, indem ein Bewertungskonzept entwickelt und mittels eines geeigneten Instrumentes implementiert wird. Hierzu zieht der Autor das Instrument der Künstlichen Neuronalen Netze heran und weist mit Hilfe einer breit angelegten empirischen Untersuchung die beachtenswerte Leistungsfähigkeit des dargestellten Ansatzes nach. Unternehmensbezogene Daten, makroökonomische Kennzahlen und kreditspezifische Ausstattungsmerkmale werden mit Hilfe von Neuronalen Netzen analysiert und die individuelle Kreditrisikoprämie treffsicher abgeleitet.

Andreas Siemes absolvierte nach einer Ausbildung zum Bankkaufmann das Studium der Wirtschaftswissenschaften an der Universität Düsseldorf und der Michigan State University, USA. Anschließend arbeitete er als Consultant bei einer Unternehmensberatung im Bereich Bankenberatung und als Dozent der zeb-Akademie. Von 1999-2001 war er am Lehrstuhl für Betriebswirtschaftslehre, insbesondere Controlling der Westfälischen Wilhelms-Universität Münster tätig. Im Jahr 2001 erfolgte die Promotion. Heute ist der Autor Geschäftsführer der BHS Consulting GmbH in Düsseldorf. 
Marktorientierte Kreditrisikobewertung 


\section{Beiträge zum Controlling}

Herausgegeben von Wolfgang Berens

Band 2

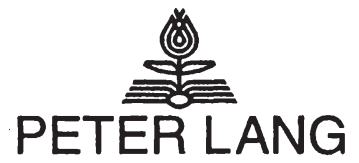

Frankfurt am Main - Berlin - Bern · Bruxelles - New York · Oxford · Wien 


\section{Andreas Siemes}

\section{Marktorientierte Kreditrisikobewertung}

Eine empirische Untersuchung mittels

Künstlicher Neuronaler Netze

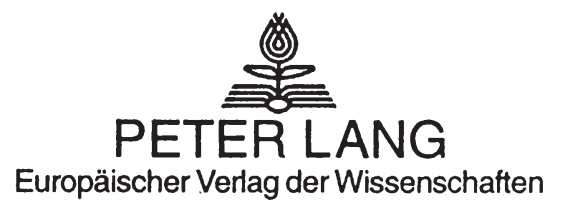


Die Deutsche Bibliothek - CIP-Einheitsaufnahme

Siemes, Andreas:

Marktorientierte Kreditrisikobewertung : eine empirische

Untersuchung mittels Künstlicher Neuronaler Netze / Andreas

Siemes. - Frankfurt am Main ; Berlin ; Bern ; Bruxelles ; New

York; Oxford ; Wien : Lang, 2002

(Beiträge zum Controlling; Bd. 2)

Zugl.: Münster (Westfalen), Univ., Diss., 2001

ISBN3-631-39217-6

Open Access: The online version of this publication is published on www.peterlang.com and www.econstor.eu under the international Creative Commons License CC-BY 4.0. Learn more on how you can use and share this work: http://creativecommons. org/licenses/by/4.0.

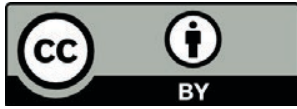

This book is available Open Access thanks to the kind support of ZBW - Leibniz-Informationszentrum Wirtschaft.

Gedruckt auf alterungsbeständigem, săurefreiem Papier.

\author{
D6 \\ ISSN 1618-825X \\ ISBN3-631-39217-6 \\ ISBN 978-3-631-75322-4 (eBook) \\ CC Peter Lang $\mathrm{GmbH}$ \\ Europäischer Verlag der Wissenschaften \\ Frankfurt am Main 2002 \\ Alle Rechte vorbehalten.
}

Das Werk einschließlich aller seiner Teile ist urheberrechtlich geschützt. Jede Verwertung außerhalb der engen Grenzen des Urheberrechtsgesetzes ist ohne Zustimmung des Verlages unzulässig und strafbar. Das gilt insbesondere für Vervielfältigungen, Übersetzungen, Mikroverfilmungen und die Einspeicherung und Verarbeitung in elektronischen Systemen.

Printed in Germany 123457

www.peterlang.de 


\section{Für Anne}

Andreas Siemes - 978-3-631-75322-4

Downloaded from PubFactory at 01/11/2019 06:12:16AM

via free access 
Andreas Siemes - 978-3-631-75322-4

Downloaded from PubFactory at 01/11/2019 06:12:16AM

via free access 
"Prediction is very difficult, especially if it's about the future" (Nils Bohr, Nobel Laureate in Physics) 
Andreas Siemes - 978-3-631-75322-4

Downloaded from PubFactory at 01/11/2019 06:12:16AM

via free access 


\section{GELEITWORT}

Kreditinstitute stehen heute aufgrund von aufsichtsrechtlichen und marktwirtschaftlichen Entwicklungen vor der Herausforderung, einen Risikomanagementprozess für die Identifikation, Bewertung, Analyse und Steuerung von Kreditrisiken zu etablieren. Grundlage für diesen Prozess und die hiermit korrespondierenden operativen Kreditentscheidungen ist eine risikoadjustierte Bepreisung des einzelnen Kreditgeschäfts. Nur auf Basis eines effizienten und effektiven Kreditzinskalkulationsverfahrens kann ein langfristig ertragreiches Kreditgeschäft sichergestellt werden. Alljährlich erscheinen neue, wissenschaftliche oder praktische Beiträge, die sich mit dem Problem der Herleitung eines Kreditzinskalkulationsverfahrens auseinandersetzen, jedoch ist ein allgemein akzeptiertes Konzept bisher nicht vorgestellt worden.

Das vorliegende Werk greift die Problemstellung der Kreditrisikobewertung auf und stellt ein Konzept vor, das den grundlegenden Anforderungen der Richtigkeit und Einzelbewertung gerecht werden kann. Das entwickelte Konzept wird mittels eines geeigneten Instrumentes implementiert und anhand von ausgewählten Daten validiert. Hierzu zieht der Autor das Instrument der Künstlichen Neuronalen Netze heran und dokumentiert mit Hilfe einer breit angelegten Untersuchung die Prognosegüte des in der Arbeit vorgestellten Ansatzes.

Die Arbeit überzeugt durch ihre theoretische Fundierung, eine innovative Konzeptentwicklung, die Konkretisierung als Modell bis hin zur Instrumentierung und durch die arbeitsreiche empirische Validierung. Darüber hinaus sind die weitreichenden Einsatzmöglichkeiten des Prognosemodells zu nennen, welche die erhebliche praktische Relevanz der Arbeit erklären. Das Prognosemodell zeigt eine beachtenswerte Leistungsfähigkeit im Vergleich zu alternativen Verfahren.

Neben ihrer praktischen Verwertbarkeit bietet die Untersuchung eine breite Grundlage für weitere wissenschaftliche Arbeiten. Ich wünsche ihr daher eine weite Verbreitung in Wissenschaft und Praxis.

Münster im Januar 2002

Prof. Dr. Wolfgang Berens 
Andreas Siemes - 978-3-631-75322-4

Downloaded from PubFactory at 01/11/2019 06:12:16AM

via free access 


\section{VORWORT}

Die vorliegende Arbeit entstand während meiner Tätigkeit als wissenschaftlicher Mitarbeiter am Lehrstuhl für Betriebswirtschaftslehre, insbesondere Controlling an der Wirtschaftswissenschaftlichen Fakultät der Westfälischen Wilhelms-Universität Münster und wurde unter dem Titel „Marktorientierte Kreditrisikobewertung - eine empirische Untersuchung mittels Künstlicher Neuronaler Netze“ im November 2001 als Dissertationsschrift angenommen. Meinem akademischen Lehrer, Herm Prof. Dr. Wolfgang Berens, danke ich für die wissenschaftliche und persönliche Betreuung der Arbeit. Insbesondere durch die von ihm gewährten akademischen Freiheiten konnte diese Arbeit entstehen. Herrn Prof. Dr. Ulrich W. Thonemann danke ich für die Übernahme des Zweitgutachtens.

Maßgeblich für das Gelingen meiner Arbeit waren die exzellenten Arbeitsbedingungen am Lehrstuhl. Insbesondere in arbeitsintensiveren Phasen wurde ich durch meine Kollegen entlastet. Für sorgfältiges Korrekturlesen und hilfreiche Diskussionen möchte ich Herrn Dr. Dieter Ernst, Herm Dipl.-Kfm. Jan Oliver Ernst, Herm Dipl.-Kfm. Sascha Henk, Herrn Dr. Andreas Hoffjan, Herrn Dipl.-Kfm. Klaus Jakobs, Frau Dipl.Kffr. Anke Kreuels, Herrn Dr. Thomas Mosiek, Herrn Dipl.-Ök. David Rose, Herm Jürgen Schaar, Herrn Dipl.-Kfm. Klaus Segbers und Frau Dr. Meike Tewes danken. Herrn Dipl.-Kfm. Marco Schiffner möchte ich für die Hilfe bei der grafischen Bearbeitung der Abbildungen und Tabellen danken. Hervorheben möchte ich Herrn Dr. Walter Schmitting der trotz eigener Arbeitsbelastung in konstruktiven Diskussionen und mit außerordentlichem Engagement zur Entstehung der Arbeit beigetragen hat. Für die weitreichende Hilfe insbesondere bei der Literaturbeschaffung und -verwaltung während der Entstehungszeit dieser Arbeit möchte ich meiner studentischen Hilfskraft Herrn Dipl.-Kfm. David Gaschik danken. Herrn Heinz Krüger und Frau Erika Krüger möchte ich meinen Dank für die Durchsicht des Manuskripts aussprechen.

Bei meinen Eltern und Großeltern möchte ich mich für die menschliche und finanzielle Unterstützung bei dem von mir eingeschlagenen Weg bedanken. Vor allem meiner Frau Anne möchte ich für den Rückhalt danken, den sie mir während der Bearbeitungszeit gab. 
Andreas Siemes - 978-3-631-75322-4

Downloaded from PubFactory at 01/11/2019 06:12:16AM

via free access 
ABBILDUNGSVERZEICHNIS........................................................................................ XIX

TABELLENVERZEICHNIS .......................................................................................... XXIII

SYMBOLVERZEICHNIS........................................................................................................ XXVII

ABBKÜRZUNGSVERZEICHNIS ................................................................... XXXI

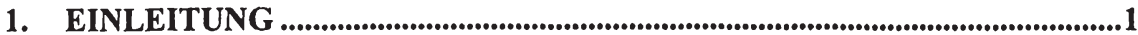

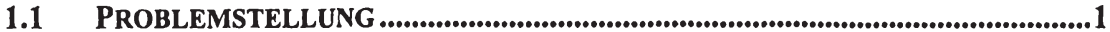

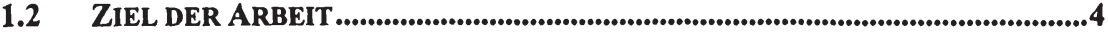

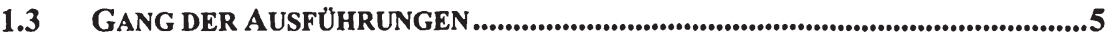

2. DIE QUANTIFIZIERUNG DES AUSFALLRISIKOS ALS

BANKBETRIEBLICHE PROBLEMSTELLUNG .................................................7

2.1 DEFINITION DES RISIKOS UND DER RISIKOEINSTELLUNGEN ..........................7

2.2 AUSFALLRISIKO ALS BANKSPEZIFISCHES RISIKO...........................................14

2.3 SYSTEMATISIERUNG DER EINZELGESCHÄFTSBEZOGENEN INSTRUMENTE ZUR RISIKOQUANTIFIZIERUNG ................................................17

2.3.1 Übersicht ...................................................................................................................17

2.3.2 Modelltheoretische Ansätze..................................................................................19

2.3.2.1 Stochastischer Ansatz .....................................................................................21

2.3.2.2 Optionspreistheoretischer Ansatz ...............................................................32

2.3.3 Heuristische Ansätze ...................................................................................................38

2.4 ANFORDERUNGEN AN VERFAHREN ZUR QUANTIFIZIERUNG DES

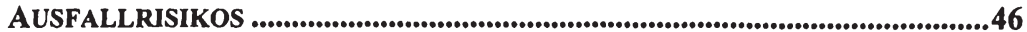

2.5 KRITISCHE WŬRDIGUNG DER EINZELNEN ANSÄTZE.......................................48 
3. DIE BILANZANALYSE ALS INSTRUMENT ZUR EINZELGESCHÄFTSBEZOGENEN RISIKOBEWERTUNG

3.1 BegrifFe, ADRESSATEN UND ZiELE DER BilanZANALYSE 59

3.2 KRITERIENKATALOG ZUR WÜRDIGUNG DER UNTERSCHIEDLICHEN METHODEN DER BILANZANALYSE..........................................................................664

3.3 TRADITIONELLE BILANZANALYSE ...................................................................65

3.3.1 Kennzahlen ...........................................................................................................................65

3.3.2 Kennzahlensysteme ..................................................................................................669

3.3.3 Scoring-Modelle....................................................................................................................72

3.3.4 Kritische Würdigung der traditionellen Analyseverfahren.....................75

3.4 MODERNE EMPIRISCH-INDUKTIVE BILANZANALYSEVERFAHREN................76

3.4.1 Die Diskriminanzanalyse als Instrument der Bilanzanalyse....................77

3.4.2 Künstliche Neuronale Netze als Instrument zur Bilanzanalyse.............81

3.5 RECHTLICHE GRUNDLAGEN DER BILANZIERUNG .............................................85

3.6 ZWISCHENFAZIT ...........................................................................................................89

4. HERLEITUNG EINES MARKTORIENTIERTEN

ANSATZES ZUR BEWERTUNG DES KREDITRISIKOS ...............................93

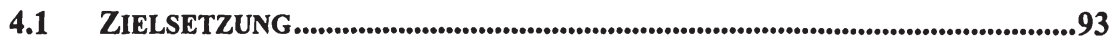

4.2 DIE EFFIZIENZHYPOTHESE ALS GRUNDLEGENDE ANNAHME FÜR DIE MARKTORIENTIERTE KREDITZINSKALKULATION..........................................96

4.3 BEWERTUNG VON RISIKOBEHAFTETEN, FESTVERZINSLICHEN ANLEIHEN.

4.3.1 Eigenschaften von Anleihen .................................................................................99

4.3.2 Risikoarten von Anleihen .......................................................................................100

4.3.2.1 Bonitätsrisiko .................................................................................................100

4.3.2.2 Zinsänderungsrisiko ....................................................................................101

4.3.2.3 Tilgungsrisiko..............................................................................................102

4.3.2.4 Liquiditătsrisiko..............................................................................................102 
4.3.2.5 Währungsrisiken............................................................................................104

4.3.3 Messung des Bonitätsrisikos.............................................................................104

4.3.3.1 Kreditderivate ............................................................................................................106

4.3.3.2 Isolation des Bonitätsrisikos über den Credit Spread.....................108

4.3.3.3 Empirische Determinanten der Bonitätsrisikoprämie ....................110

4.3.3.3.1 Makroökonomische Ebene .....................................................................111

4.3.3.3.2 Titelspezifische Ebene..............................................................................114

4.4 ABLEITUNG EINES PROGNOSEMODELLS ..............................................................117

4.5 KRITISCHE WŪRDIGUNG DES MODELLS........................................................123

5. EMPIRISCHE UNTERSUCHUNG ZUR MARKT-ORIENTIERTEN BEWERTUNG DES KREDITRISIKOS MITTELS KÜNSTLICHER NEURONALER NETZE.

5.1 KÜNSTLICHE NEURONALE NETZE ALS PROBLEMADÄQUATES INSTRUMENT

5.1.1 Instrumentenanforderung

5.1.2 Grundlegende Betrachtung von Künstlichen Neuronalen Netzen als Teilgebiet der Künstlichen Intelligenz .....................................128

5.1.3 Anwendungsgebiete............................................................................................133

5.1.4 Der strukturelle Aufbau von Künstlichen Neuronen .............................134

5.1.5 Problemspezifische Auswahl eines Netzwerktyps ...................................138

5.2 DAS MULTI-LAYER-PERCEPTRON IN KOMBINATION MIT DEM ERROR-BACKPROPAGATION-ALGORITHMUS...............................................140

5.2.1 Darstellung der Netzwerkstruktur ...................................................................140

5.2.2 Beschreibung des Lernprozesses (Error-BackpropagationAlgorithmus) 142

5.2.3 Bestimmung der Datenaufteilung 146 
5.3 Datenbasis, Datenherkunft UNd Ableitung DeS

UNTERSUCHUNGSDATENSATZES

5.3.1 Aufbau und Struktur des Datenbestandes................................................149

5.3.2 Ableitung von Bilanzkennzahlen und weiterer Inputfaktoren .............152

5.3.3 Ermittlung des Credit Spread.................................................................................156

5.3.4 Behandlung von fehlenden Daten und Extremwerten..............................158

5.4 DIE DATENANALYSE MIT DEM BACKPROPAGATION-NETZ...........................160

5.4.1 Einzustellende Parameter innerhalb der Software Neural

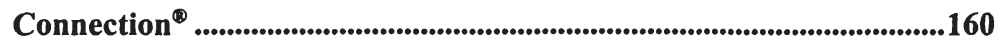

5.4.2 Ziehung der Stichproben für die Künstliche Neuronale Netzanalyse ...............................................................................................................................162

5.4.3 Das Grundmodell ...................................................................................164

5.4.3.1 Parameterkonfiguration....................................................................................164

5.4.3.2 Ergebnisdarstellung.................................................................................173

5.4.4 Sensitivitätsanalyse als Ausgangspunkt einer Faktorenreduktion ..........................................................................................................178

5.4.5 Das 13er-Modell ......................................................................................................183

5.4.5.1 Parameterkonfiguration............................................................................183

5.4.5.2 Ergebnisdarstellung..........................................................................................186

5.4.6 Einordnung des 13er-Modells in die Bilanzanalyse und in die modelltheoretischen Verfahren der Kreditzinskalkulation 191

5.5 MODELLMODIFIKATIONEN

5.5.1 Effektive Rendite vs. Credit Spread ..............................................................194

5.5.2 Untersuchungen innerhalb einzelner Risikoklassen .................................195

5.6 ERGEBNISBEWERTUNG................................................................................201

5.6.1 Definition eines Vergleichsobjektes ................................................................201

5.6.2 Vergleich der Testergebnisse ................................................................................201

5.7 ERGEBNISZUSAMMENFASSUNG ..............................................................................211 
6. BEWERTUNG VON UNTERNEHMENSKREDITEN

UND UNTERNEHMENSANLEIHEN MITTELS KÜNSTLICHER

NEURONALER NETZE

6.1 ANWENDUNGSGEBIETE DER FORSCHUNGSERGEBNISSE.............................213

6.1.1 Kreditvergabe..........................................................................................................213

6.1.2 Einsatzmöglichkeiten beim Primär- und Sekundärhandel von Anleihen ...................................................................................................................2217

6.2 ANWENDUNGSVORAUSSETZUNGEN......................................................................220

6.2.1 Vergleich der institutionellen Rahmenbedingungen.................................221

6.2.2 Transformationsmöglichkeiten der Ergebnisse auf Bilanzen nach dem HGB

7. SCHLUSSBETRACHTUNG .225

8. ANHANG

9. LITERATURVERZEICHNIS 279

10. GESETZESVERZEICHNIS 319 
Andreas Siemes - 978-3-631-75322-4

Downloaded from PubFactory at 01/11/2019 06:12:16AM

via free access 


\section{Abbildungsverzeichnis}

Abbildung 1: Entwicklung der Insolvenzzahlen in Deutschland .....................................3

Abbildung 2: Lineare Nutzenfunktion

Abbildung 3: Konkave Nutzenfunktion

Abbildung 4: Konvexe Nutzenfunktion

Abbildung 5: Nutzenfunktion mit wechselnder Risikoeinstellung..........................13

Abbildung 6: Bankspezifische Risiken.........................................................................16

Abbildung 7: Überblick über die modelltheoretischen Ansätze zur

Quantifizierung der Bonitätsrisikoprämie.

Abbildung 8: Mehrperiodiger bonitätsrisikobehafteter Zahlungsstrom

Abbildung 9: Zusammenhang zwischen Ausfallwahrscheinlichkeit und

Bonitätsrisikoprämie

Abbildung 10: Zusammenhang zwischen Rückzahlungsquote, Ausfallwahrscheinlichkeit und Bonitätsprämie.

Abbildung 11: Zusammenhang zwischen Ausfallwahrscheinlichkeit, Zinsniveau und Bonitätsprämie

Abbildung 12: Zusammenhang zwischen Restlaufzeit, Zinsstrukturkurve und Bonitätsprämie

Abbildung 13: Zusammenhang zwischen Unternehmenswert und Bonitätsprämie

Abbildung 14: Zusammenhang zwischen dem sicheren Zins und der

Bonitätsprämie

Abbildung 15: Heuristische Kalkulationsverfahren zur Ermittlung der Ausfallrisikokosten

Abbildung 16: Segmentierungskriterien für segmentspezifische Kalkulationsverfahren auf Basis normalisierter Ist-Kosten

Abbildung 17: Exemplarische Vorgehensweise bei der Ableitung von Ausfallprämien gemäß der auf externe Rating gestützten

Vorgehensweise

Abbildung 18: Beurteilungskriterien für die Verfahren der

Risikoprämienermittlung

Abbildung 19: Das ROI-Schema..................................................................................70

Abbildung 20: Das Rentabilitäts-Liquiditätssystem (RL-System) .............................71

Abbildung 21: Kennzahlen des Saarbrücker Modells 
Abbildung 22: Trennung solventer und insolvenzgefährdeter Unternehmen mittels der KNN-Analysen ............................................82

Abbildung 23: Risikoarten einer Anleihe .........................................................................106

Abbildung 24: Das Bonitätsrisiko als Bewertungskomponenten von Kreditderivaten ............................................................................................108

Abbildung 25: Einflussfaktoren des Credit Spread ......................................................111

Abbildung 26: Zeitliche Entwicklung des Credit Spread (US-Markt)....................112

Abbildung 27: Effiziente Bonitätsrisikobewertung auf Kreditmärkten ...............119

Abbildung 28: Vorgehensweise eines kreditmarktorientierten Prognosemodells als integrativer Bilanzanalyseansatz..................122

Abbildung 29: Übersicht der Methoden und Modelle der Künstlichen Intelligenz

Abbildung 30: Schematische Abbildung eines biologischen Neurons 131

Abbildung 31: Künstliches Neuron

Abbildung 32: Schematischer Kurvenverlauf der Sigmoid-Funktion....................136

Abbildung 33: Schematischer Kurvenverlauf der Tanh-Funktion..........................136

Abbildung 34: Vereinfachte Darstellung der Verarbeitung innerhalb eines Künstlichen Neurons

Abbildung 35: Grundstruktur eines Backpropagation-Netzes ...................................140

Abbildung 36: Propagation-Phase

Abbildung 37: Aufteilung der Ausgangsdaten in die unterschiedlichen

Datenbereiche

Abbildung 38: Schematischer Verlauf von Trainings- und Validierungs-

fehlern in Abhängigkeit der Lernzyklen

Abbildung 39: Branchenverteilung der untersuchten Unternehmen

Abbildung 40: Fehlerwert $S_{F}$ in Abhängigkeit von der Anzahl der Neuronen in der versteckten Schicht für das Grundmodell

Abbildung 41: Fehlerwert $s_{F}$ in Abhängigkeit von der Anzahl der Lernschritte für das Grundmodell

Abbildung 42: Schematischer Aufbau des Grundmodells

Abbildung 43: Häufigkeitsfunktion der Differenzen,

Szenario 1, Grundmodell

Abbildung 44: Häufigkeitsfunktion der Differenzen,

Szenario 2, Grundmodell

Abbildung 45: Häufigkeitsfunktion der Differenzen, Szenario 3, Grundmodell 
Abbildung 46: Differenzmessung innerhalb der Sensitivitätsanalyse am Beispiel des Inputfaktors $M_{1}$ und im Bereich des 1. Quartils......181

Abbildung 47: Fehlerwert $s_{F}$ in Abhängigkeit von der Anzahl der Inputfaktoren

Abbildung 48: Fehlerwert $s_{F}$ in Abhängigkeit von der Anzahl der Neuronen in der versteckten Schicht für das 13er-Modell.

Abbildung 49: Fehlerwert $s_{\mathrm{F}}$ in Abhängigkeit von der Anzahl an

Lernschritten für das $13 \mathrm{er}-$ Modell

Abbildung 50: Schematischer Aufbau des 13er-Modells

Abbildung 51: Häufigkeitsfunktion der Differenzen,

Szenario 1, 13er-Modell .

Abbildung 52: Häufigkeitsfunktion der Differenzen,

Szenario 2, 13er-Modell

Abbildung 53: Häufigkeitsfunktion der Differenzen,

Szenario 3, 13er-Modell

Abbildung 54: Schematischer Aufbau eines KNN zur Prognose der effektiven Rendite.

Abbildung 55: Hăufigkeitsprofil der Untersuchungsdaten hinsichtlich der Bonitätsprämie.

Abbildung 56: Lineare Regressionsanalyse von Credit Spread und der Differenz von dem prognostizierten Credit Spread und dem Credit Spread

Abbildung 57: P-P-Diagramm der standardisierten Residuen der geführten Regressionsanalyse

Abbildung 58: Klassenhäufigkeiten der Credit Spreads

Abbildung 59: Korrigierte Klassenhäufigkeiten der Credit Spreads

Abbildung 60: Standardabweichung des Credit Spread innerhalb einzelner Ratingklassen bei der Untersuchung von Longstaff/Schwartz (1995)

Abbildung 61: Standardabweichung des Credit Spread innerhalb einzelner Ratingklassen bei der Untersuchung von Heinke (1998)

Abbildung 62: Standardabweichung des Credit Spread innerhalb einzelner Ratingklassen bei der Untersuchung von Brister/Kennedy/Liu (1994)

Abbildung 63: Abweichung der Prognose des Credit Spread in Abhängigkeit des Risikopotenzials für die Lern- und Validierungsmenge 208 
Abbildung 64: Gegenuiberstellung der Prognosefähigkeit des 13er-Modells und der Prognose von Ratings......................................210

Abbildung 65: Möglichkeiten zur Übertragung der Forschungsergebnisse auf Bilanzen nach dem HGB 


\section{Tabellenverzeichnis}

Tabelle 1: Rating-Einschätzung langfristiger Verbindlichkeiten

Tabelle 2: Zusammenfassung der Würdigung verschiedener Ansätze zur Quantifizierung des Ausfallrisikos

Tabelle 3: Übersicht über externe und interne Bilanzanalytiker

Tabelle 4: Informationsbedürfnisse der unterschiedlichen Adressaten der Bilanzanalyse

Tabelle 5: Übersicht wichtiger Kennzahlen der Bilanzanalyse (Teil I) .................67

Tabelle 6: Übersicht wichtiger Kennzahlen der Bilanzanalyse (Teil II)................68

Tabelle 7: Aufbau und Kennzahlen des RSW-Modells ................................................74

Tabelle 8: Zusammenhang zwischen dem Z"'-Score-Wert und dem Kapitalmarkt-Rating

Tabelle 9: Kennzahlen des BP-14

Tabelle 10: Zuordnung der N-Werte des BP-14 zu historischen Ausfallwahrscheinlichkeiten

Tabelle 11: Gegenüberstellung der Bilanzierungsgrundsätze nach dem HGB und dem US-GAAP

Tabelle 12: Zusammenfassung der Würdigung verschiedener

Bilanzanalyseverfahren zur Kreditzinskalkulation

Tabelle 13: Deskriptive Kennzahlen der Untersuchungsmenge

hinsichtlich des Merkmales „Bilanzsumme“

Tabelle 14: Ableitung der untersuchten Kennzahlen

Tabelle 15: In der Untersuchung berücksichtigte Bilanzkennzahlen

Tabelle 16: Zusammenfassung der bilanzunabhängigen, unternehmensexternen Kennzahlen

Tabelle 17 Deskriptive Kennzahlen der Untersuchungsmenge hinsichtlichder Merkmale „Credit Spread“, "Restlaufzeit" und Nominalzins"

Tabelle 18: Einzustellende Parameter für das KNN unter der Beachtung der softwarespezifischen Möglichkeiten

Tabelle 19: Standardabweichungen des Credit Spread innerhalb verschiedener Szenarien

Tabelle 20: Fehlerwertes $S_{F}$ in Abhängigkeit der Aktivierungsfunktion für das Grundmodell 
Tabelle 21: Fehlerwert $s_{F}$ in Abhängigkeit von der Anzahl der layer, der Anzahl der Neuronen in der versteckten Schicht und der Aktivierungsfunktion für das Grundmodell.

Tabelle 22: Fehlerwert $s_{\mathrm{F}}$ in Abhängigkeit von der Aufteilung der Datenmenge für das Grundmodell

Tabelle 23: Fehlerwert $S_{\mathrm{F}}$ in Abhängigkeit von der Verteilung der Startgewichte für das Grundmodell.

Tabelle 24: Standardabweichungen und 90-Prozent-Intervall-Fehlerwert der Fehlerverteilungsfunktion des Grundmodells

Tabelle 25: Deskriptive Kennzahlen der Testmenge,

Szenario 1 im Grundmodell

Tabelle 26: Deskriptive Kennzahlen der Testmenge, Szenario 2 im Grundmodell

Tabelle 27: Deskriptive Kennzahlen der Testmenge, Szenario 3 im Grundmodell

Tabelle 28: Fehlerwert $s_{\mathrm{F}}$ in Abhängigkeit von der Aktivierungsfunktion für das 13er-Modell

Tabelle 29: Fehlerwert $s_{\mathrm{F}}$ in Abhängigkeit von der Anzahl der Schichten der Knotenpunkte und der Aktivierungsfunktion für das 13er-Modell

Tabelle 30: Fehlerwert $s_{F}$ in Abhängigkeit von der Aufteilung der

Datenmenge für das 13er-Modell

Tabelle 31: Fehlerwert $s_{\mathrm{F}}$ in Abhängigkeit der von Verteilung der Startgewichte für das 13er-Modell

Tabelle 32: Standardabweichungen und 90-Prozent-Intervall-Fehlerwert der Fehlerverteilungsfunktion des 13er-Modells

Tabelle 33: Deskriptive Kennzahlen der Testmenge, Szenario 1 im 13er-Modell

Tabelle 34: Deskriptive Kennzahlen der Testmenge, Szenario 2 im 13er-Modell

Tabelle 35: Deskriptive Kennzahlen der Testmenge, Szenario 3 im 13er-Modell

Tabelle 36: Darstellung der erklärenden Kennzahlen des 13er-Modells und ihre Einordnung in den Informationsbereich des Jahresabschlusses, hinsichtlich der bekannten Bilanzanalyseverfahren und der modelltheoretischen Ansätze zur Bepreisung des Bonitătsrisikos

Tabelle 37: Klassenaufteilung der Credit Spreads 
Tabelle 38: Korrigierte Klassenaufteilung der Credit Spreads 199

Tabelle 39: Numerische Darstellung der Untersuchungsergebnisse von Longstaff/Schwartz (1995) bezüglich der Standardabweichung des Credit Spread innerhalb einzelner Ratingklassen...........................204

Tabelle 40: Numerische Darstellung der Untersuchungsergebnisse von Heinke (1998) bezüglich der Standardabweichung des Credit Spread innerhalb einzelner Ratingklassen

Tabelle 41: Numerische Darstellung der Untersuchungsergebnisse von Brister/Kennedy/Liu (1994) bezüglich der Standardabweichung des Credit Spread innerhalb einzelner Ratingklassen.............................207

Tabelle 42: Klasseneinteilung für die Untersuchungsmenge.....................................209

Tabelle 43: Ergebnisse der Regressionsbildung für das Jahr 1995 ..........................229

Tabelle 44: Ergebnisse der Regressionsbildung für das Jahr 1996 ..........................230

Tabelle 45: Ergebnisse der Regressionsbildung für das Jahr 1997 ..........................231

Tabelle 46: Ergebnisse der Regressionsbildung für das Jahr 1998 ........................232

Tabelle 47: Ergebnisse der Regressionsbildung für das Jahr 1999 ........................233

Tabelle 48: Untersuchte Menge an Unternehmen mit dem zugehörigen Tickersymbol .............................................................................................................236

Tabelle 49: Untersuchte Menge an Unternehmensanleihen ........................................265

Tabelle 50: US-Staatanleihen zur Herleitung der Indexkurve des Sicheren Zins 
Andreas Siemes - 978-3-631-75322-4

Downloaded from PubFactory at 01/11/2019 06:12:16AM

via free access 


\section{Symbolverzeichnis}

\begin{tabular}{|c|c|}
\hline$\frac{\partial}{\partial x_{1}}$ & Erste partielle Ableitung der Funktion y nach $x_{1}$ \\
\hline$\frac{\partial^{2} y}{\partial x_{1}^{2}}$ & Zweite partielle Ableitung der Funktion y nach $x_{1}$ \\
\hline $\begin{array}{l}a_{0,1,2 \ldots} \ldots \\
C\end{array}$ & $\begin{array}{l}\text { Konstante Werte in der Diskriminanzfunktion } \\
\text { (jährliche) Zinszahlungen, Kupon }\end{array}$ \\
\hline $\mathrm{C}_{\mathrm{t}}$ & (jährliche) Zinszahlung in dem Zeitpunkt t \\
\hline$d V_{t}(t)$ & $\begin{array}{l}\text { Veränderung von V auf Grund einer infinitesimalen Veränderung } \\
\text { der Zeiteinheit }\end{array}$ \\
\hline e & Bonitätsprämie (Credit Spread) einer Anleihe bzw. eines Kredits \\
\hline$e_{i}$ & Bonitätsprämie (Credit Spread) einer Anleihe i bzw. eines Kredits i \\
\hline $\mathbf{e}_{\mathrm{i}, \mathrm{t}}$ & $\begin{array}{l}\text { Bonitätsprämie (Credit Spread) einer Anleihe i bzw. eines Kredits } \\
\text { zum Zeitpunkt t }\end{array}$ \\
\hline $\mathrm{EK}_{4}$ & Eigenkapitalmarktwert zum Zeitpunkt t \\
\hline$F(x), f(x)$ & Funktion $\mathrm{F}, \mathrm{f}$ \\
\hline $\mathrm{F}, \mathrm{f}$ & Funktion $F, f$ \\
\hline $\mathrm{FK}_{\mathrm{M}}$ & Marktwert des Kredites bzw. Fremdkapitaltitels \\
\hline $\mathrm{FK}_{\mathrm{M}, \mathrm{i}}$ & Fremdkapitalmarktwert in $\mathrm{t}$ \\
\hline $\mathrm{FK}_{\mathrm{N}}$ & Nominalbetrag eines Kredites bzw. Fremdkapitaltitels \\
\hline $\mathrm{FR}_{\mathbf{1}}$ & risikoloser $Z$ ins $r_{B}$ für die Periode $t-1$ bis $t$ \\
\hline & Zinssatz \\
\hline 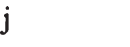 & Laufindex \\
\hline $\mathrm{K}^{\mathrm{D}}$ & $\begin{array}{l}\text { Anzahl an eingehenden Kennzahlen in die } \\
\text { Diskriminanzfunktion }\end{array}$ \\
\hline$K_{0,1,2, \ldots}^{\mathrm{D}}$ & Eingehende Kennzahlen in die Diskriminanzfunktion \\
\hline 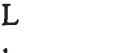 & Quasi-Verschuldungsgrad \\
\hline In & natürlicher Logarithmus \\
\hline & Anzahl der betrachteten Perioden \\
\hline 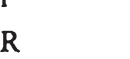 & $\begin{array}{l}\text { bonitätrisikobehafteter Zins } \\
\text { erwartete Zahlungen }\end{array}$ \\
\hline$r_{B}$ & bonitätsrisikolose Rendite \\
\hline$r_{B, L}$ & Effektive Rendite einer bonitätsrisiklosen Anleihe i zum Z \\
\hline$r_{j, j}$ & $\begin{array}{l}\text { Effektive Rendite einer risikobehafeten Anleihe i zum } \\
\text { Zeitpunkt t }\end{array}$ \\
\hline
\end{tabular}


$\mathrm{R}_{\mathrm{t}}$ tiefgestelltes $t$

$\mathrm{u}(\mathrm{x})$

$X_{1,2,3,4}$ erwartete Zahlungen in dem Zeitpunkt t

Betrachtungszeitpunkt $(t=1,2,3, \ldots)$

Nutzenfunktion

Einflussfaktoren der von ALTMAN aufgestellten Funktionsvorschrift

Speziell in Kap. 2 verwendete Symbole und Variabeln

$\begin{array}{ll}\mathrm{D} & \text { Diskriminanzwert } \\ \mathrm{N}(.) & \text { Wert der Verteilungsfunktion der Standardnormalverteilung } \\ \varnothing & \text { Arithmetisches Mittel } \\ \mathrm{p} & \text { Überlebenswahrscheinlichkeit (survival probability) } \\ \mathrm{p}_{\mathrm{t}} & \text { Überlebenswahrscheinlichkeit (survival probability) in dem } \\ \mathrm{T} & \text { Zeitpunkt t } \\ \mathrm{T}-\mathrm{t} & \text { Fälligkeitszeitpunkt } \\ \mathrm{V} & \text { Restlaufzeit einer Anleihe, eines Kredites } \\ \mathrm{V}_{\mathrm{t}} & \text { Marktwert des Unternehmens, Firmenwert, } \\ & \text { Marktwert des Unternehmens in dem Zeitpunkt t, Firmenwert in } \\ \mathrm{w} & \text { dem Zeitpunkt t, Unternehmenswert in dem Zeitpunkt t } \\ \mathrm{w}_{\mathrm{t}} & \text { Ausfallwahrscheinlichkeit (default probability) } \\ \mu & \text { Ausfallwahrscheinlichkeit (default probability) im Zeitpunkt t } \\ \sigma & \text { Momentrendite } \\ \sigma_{\mathrm{v}} & \text { Volatilität } \\ & \text { Standardabweichung der Wachstumsrate des } \\ & \text { Unternehmensvermögens V }\end{array}$

Speziell in Kap. 4 verwendete Symbole und Variabeln

$\begin{array}{ll}\mathrm{B}_{\mathrm{i}} & \begin{array}{l}\text { Menge aller bonitätserklärende Einflussfaktoren einer Anleihen } \mathrm{i}, \\ \mathrm{i}=1,2,3, \ldots\end{array} \\ \mathrm{M} & \text { Menge aller relevante Marktfaktoren einer Anleihenbewertung } \\ \text { tiefgestelltes } \mathrm{B} & \text { Bonitätsrisikoloses Wertpapier B } \\ \text { tiefgestelltes } \mathrm{i} & \text { Bonitätsrisikobehaftetes Wertpapier } \mathrm{i}, \mathrm{i}=1,2,3, \ldots \\ \mathrm{W}_{\mathrm{i} \text { bzw. B }} & \text { Menge aller wertpapierspezifischen Einflussfaktoren } \\ \mathrm{X}_{1,2,3, \ldots, \mathrm{n}} & \text { Anleihe i bzw. B }\end{array}$


Speziell in Kap. 5 und 6 verwendete Symbole und Variabeln

\begin{tabular}{|c|c|}
\hline$A(y)$ & Aktivierungsfunktion \\
\hline $\mathbf{B}_{\mathbf{i}}$ & $\begin{array}{l}\text { unternehmensspezifische Kennzahl i des Neuronalen Netzes, } \\
i=1,2,3, \ldots\end{array}$ \\
\hline $\mathrm{E}$ & Gesamter Fehlerwert eines Neuronalen Netzes \\
\hline$\hat{\mathrm{e}}_{\mathrm{i}}$ & $\begin{array}{l}\text { Vom KNN berechtete Bonitätsprämie (Credit Spread) für } \\
\text { eine Anleihe i bzw. einen Kredit i, i }=1,2,3, \ldots\end{array}$ \\
\hline I & Menge an Eingangsneuronen \\
\hline J & Restlaufzeit einer Anleihe in Jahren \\
\hline $\mathrm{K}$ & Menge an Ausgangsneuronen \\
\hline $\mathrm{M}_{\mathrm{i}}$ & makroökonomische Kennzahl i des Neuronalen Netzes, $\mathrm{i}=1,2,3, \ldots$ \\
\hline $\mathrm{n}$ & Menge an Dendriten bzw. Eingangsinformationen \\
\hline o & Ausgabewert eines Neurons bspw. auch in der Ausgabeschicht \\
\hline $\mathrm{oe}_{\mathrm{i}}$ & Ausgabewert eines Neurons $i$ in der Eingabeschicht, $i=1,2,3, \ldots$ \\
\hline $\mathrm{ov}_{\mathrm{j}}$ & Ausgabewert des Neurons $\mathrm{j}$ in der versteckten Schicht, $\mathrm{j}=1,2,3, \ldots$ \\
\hline $\mathrm{oa}_{\mathrm{k}}$ & Ausgabewert eines Neurons $\mathrm{k}$ in der Ausgabenschicht, $\mathrm{k}=1,2,3, \ldots$ \\
\hline $\mathrm{oe}_{\mathrm{pi}}$ & $\begin{array}{l}\text { Ausgabewert eines Neurons } \mathrm{i} \text { in der Eingabeschicht für den } \\
\text { Datensatz } \mathrm{p}\end{array}$ \\
\hline$o v_{p j}$ & $\begin{array}{l}\text { Ausgabewert des Neurons } j \text { in der versteckten Schicht des Datensat- } \\
\text { zes p }\end{array}$ \\
\hline $\mathrm{oa}_{\mathrm{pk}}$ & $\begin{array}{l}\text { Ausgabewert eines Neurons } \mathrm{k} \text { in der Ausgabenschicht für den } \\
\text { Datensatz p }\end{array}$ \\
\hline $\mathbf{P}$ & Menge der Datensätze in der Lernmenge bzw. Trainingsmenge \\
\hline p & Trainingsmuster bzw. Datensatz, $p=1,2,3, \ldots$ \\
\hline $\mathbf{P}_{\mathrm{T}}$ & Menge der Datensätze in der Testmenge bzw. Validierungsmenge \\
\hline $\mathrm{R}^{\mathrm{m}}$ & reelle Funktion m \\
\hline $\mathrm{R}^{\mathrm{n}}$ & reelle Funktion n \\
\hline $\mathbf{s}_{\mathrm{F}}$ & Fehlerwert eines Neuronalen Netzes \\
\hline $\mathbf{s}_{\mathbf{t}}$ & $\begin{array}{l}\text { 10-Tages-Standardabweichung der täglicher Differenzen der } \\
\text { Indexrendite }\end{array}$ \\
\hline $\mathrm{T}_{\mathbf{i}}$ & $\begin{array}{l}\text { titelspezifische Kennzahlen } \mathrm{i} \text { des Neuronalen Netzes, } \\
\mathrm{i}=1,2,3, \ldots\end{array}$ \\
\hline$T(x)$ & Identitätsfunktion \\
\hline$t_{p k}$ & Zielwert für den Datensatz $\mathrm{p}$ an dem Ausgabeneuron $\mathrm{k}$ \\
\hline $\mathrm{W}_{\mathrm{ibzw.B}}$ & $\begin{array}{l}\text { Menge aller wertpapierspezifischen Einflussfaktoren } \\
\text { Anleihe i bzw. B }\end{array}$ \\
\hline$w_{i j}$ & Gewichtungsfaktoren $w$ zwischen dem Neuron $\mathrm{i}$ und $\mathrm{j}$ \\
\hline
\end{tabular}




$\begin{array}{ll}\mathrm{w}_{\mathrm{ijp}} & \begin{array}{l}\text { Veränderung des Gewichtungsfaktoren w zwischen dem } \\ \text { Neuron } \mathrm{i} \text { und } \mathrm{j} \text { aufgrund des Datensatzes } \mathrm{p}\end{array} \\ \mathrm{x}_{\mathrm{i}} & \text { (Eingangs-)Information in der Input-Ebene } \\ \alpha & \text { Momentum } \\ \Delta & \text { Veränderung; Differenz } \\ \delta_{\mathrm{pk}} & \text { Lokaler Fehlerwert an dem Neuron } \mathrm{k} \text { für einen Datensatz } \mathrm{p} \\ \eta & \text { Lernkoeffizient, Lernrate, Lerngeschwindigkeit }\end{array}$




\section{Abkürzungsverzeichnis}

$\alpha$-Fehler

A

$\beta$-Fehler

BA

BAWe

BbankG

BP-14

BR

CAPM

CFR

CFR,

CFU

CFK

Co.

CUSIP

$\mathrm{DAX}^{\oplus}$

DCF-Methode

DCF-Methodik

DVFA/SG
BösG

Anteil der tatsächlich insolvenzgefährdeten Unternehmen

Aktivierungsfunktion

Anteil der tatsächlich gesunden Unternehmen

Bundesanzeiger

Bundesaufsichtsamt für den Wertpapierhandel

Bundesbankgesetz

Börsengesetz

Backpropagation-Algorithmus 14

Baetge-Rating

Capital Asset Pricing Model

Cash Flow Rendite

Cash Flow Rendite im Zeitpunkt t

Cash Flow zu Umsatz

Cash Flow zu Gesamtkapital

Company

Committee on Uniform Securities Identification Procedures

Deutscher Aktienindex

Discounted Cash Flow Methode

Discounted Cash Flow Methodik

Deutsche Vereinigung fur Finanzanalyse und Anlageberatung e.V. I Arbeitskreis „Externe Unternehmensrechnung“ der Schmalenbach-

Gesellschaft - Deutsche Gesellschaft für Betriebswirtschaft

\section{EBIT}

Ed.

EK

EKQ, EQ

EKQ,

EKR

$E K R_{+}$

EW

EZB

FR

GE

GuV

Ggs.

HR
Earnings before Interest and Taxes

Edition

Eigenkapital

Eigenkapitalquote

Eigenkapitalquote im Zeitpunkt $t$

Eigenkapitalrendite

Eigenkapitalrendite im Zeitpunkt t

Erwartungswert

Europäische Zentralbank

Forward Rate

Geldeinheiten

Gewinn- und Verlustrechnung

Grundsätzlich

Handelsregister 
I

IAS

ID

IdW

Inc.

ISMA

JA

KI-Methoden

KNN

KWG

L

LQ

$\mathrm{LQ}_{\mathrm{t}}$

MDA

MLP

Moody's ${ }^{\star}$

$\mathrm{N}$-Wert

o.Jg.

op

PLC

REX

RL-System

ROI

ROI-Schema

RSW-Modell

$S$

SEC

$S \& \mathrm{P}^{\otimes}$

SNNS $^{\oplus}$

$\mathrm{Sp}$.

SPSS $^{\oplus}$

st

UR

US

US-GAAP
Insolvenzgefährdete Unternehmen

International Accounting Standards

Identity

Institut der Wirtschaftsprüfer in Deutschland

Incorporation

International Securities Market Association

Jahresabschluss

Methoden der Künstlichen Intelligenz

Künstliche Neuronale Netze

Kreditwesengesetz

Quasi-Verschuldungsgrad

Liquiditätsquote

Liquiditätsquote im Zeitpunkt t

Multivariate Diskriminanzanalyse

Multi-Layer-Perceptron

Moody's Investor Service Incorporation

Neuronaler Netz-Wert

ohne Jahrgang

Optionspreismodell

Public Limited Company

Rentenindex der Deutschen Börse AG

Rentabilität-Liquiditätssystem

Return on Investment

Renturn on Investment-Schema (enstpricht dem DuPont-

Kennzahlensystem)

Rendite-Sicherheit-Wachstums-Modell

Solvente Unternehmen

Securities and Exchange Commission

Standard and Poor's Corporation

Stuttgarter Neuronaler Netz Simulator

Spalte (n)

Statistical Package for the Social Sciences

stochastisches Modell

Umsatzrentabilität

United States

US - Generally Accepted Accounting Principles 
WRBS

WRBS,

WRU

WRU,

Xetra ${ }^{\oplus}$

Z''-Score, Z-Kurve ZVEI
Wachstumsrate Bilanzsumme

Wachstumsrate Bilanzsumme im Zeitpunkt t

Wachstumsrate Umsatz

Wachstumsrate Umsatz im Zeitpunkt t

Exchange Electronic Trading

Funktionswert innerhalb von ALTMAN (1996) gefuihrten Unterschung

Zentralverband der Elektrotechnik- und Elektroindustrie e.V. 
Andreas Siemes - 978-3-631-75322-4

Downloaded from PubFactory at 01/11/2019 06:12:16AM

via free access 


\section{Einleitung}

Die Übernahme und das Management von Risiken sind die Eckpfeiler des Bankgeschäfts.'

Das US-Telekommunikationsunternehmen MCI Worldcom hält mit 12,2 Milliarden US-Dollar zur Zeit den Emissionsrekord von Unternehmensanleihen. ${ }^{2}$ Anleger waren im Mai 2001 bereit, diesen Betrag dem Unternehmen als Kredit zur Verfügung zu stellen und erhielten hierfür einen Zins in Höhe von ca. 7,5 \% p.a. Dieser Kreditzins lag deutlich über dem allgemeinen Niveau, welches (kupon-, laufzeit- und währungsabhängig) ca. 5,5\% p.a betrug. Die Differenz in Höhe von ca. 244 Millionen USDollar p.a. lässt sich auf das durch die Anleger übernommene Risiko zurückführen, welches darin bestand, dass die Gesellschaft insolvent werden und somit ihren Zahlungsverpflichtungen nicht mehr nachkommen könnte. ${ }^{3}$ Welche Faktoren determinieren derartige Differenzen? Wie können Kreditgeber das Risiko quantifizieren und damit zu ihren Entscheidungen gelangen?

\subsection{Problemstellung}

Gegenstand des Risikomanagements ist es, die bereits bestehenden und zukünftigen Risiken eines Unternehmens mit der Zielsetzung zu steuern, dass zum einen die Risikotragfähigkeit eines Unternehmens nicht überschritten und zum andern eine optimale Risiko-Rendite-Kombination erreicht wird. ${ }^{4}$ Die Strategien im Umgang mit dem Risiko gliedern sich in Risikovermeidung, Risikoverminderung, Risikoüberwälzung und Risikoakzeptanz ${ }^{5}$ wobei Unternehmen in der Regel einen Strategie-Mix verfolgen und alle vier Möglichkeiten mit unterschiedlichen Gewichten kombinieren. Aufgabe des Risikocontrollings ist es, die Informationen für die Entscheidungen des Risikomanagements bereitzustellen, wobei zunächst die Risiken identifiziert werden, um sie anschließend zu quantifizieren. Vorbereitend für die Formulierung der Risikostrategie

1 Vgl. Deutsche Bank (1996), S. 29.

2 Im Mai 2001 wurde dieser Betrag in fünf Tranchen mit jeweils unterschiedlichem Kupon und unterschiedlicher Restlaufzeit an verschiedenen Börsen platziert. Emissionsbank waren J.P. Morgan Chase \& Co. und Salomon Smith Barney. Die Anleihen wurden größtenteils in US-Dollar, aber auch in Euro und Britisch Pfund begeben. Diese und weitere Informationen zur Anleihenemissionen sind dem BLOOMBERG Service ${ }^{\circledR}$ entnommen. Vgl. www.bloomberg.de und www.bloomberg.com/de/products/prodl.html.

3 Das Unternehmen wurde von der Agentur Standard \& Poor's mit BBB+ eingeschätzt, zu der Bedeutung dieser Einschätzung bzw. des Symbols vgl. Abbildung 18.

4 Vgl. hier und im Folgenden Baetge, Jerschensky (1999), S. $171 \mathrm{ff}$.

$s$ Vgl. Hoffjan (1999), S. 23f. 
Marktorientierte Kreditrisikobewertung - Einleitung

innerhalb des Risikomanagements zeigt das Risikocontrolling die Konsequenzen und Optionen der Risikosteuerung auf. ${ }^{6}$

Kreditinstitute unterliegen in ihren Geschäften diversen Risiken, wobei die Quantifizierung des Adressenausfallrisikos einen besonders hohen Stellenwert einnimmt.' Beispielsweise allokierte die Deutsche Bank AG Ende des Jahres 1998 über $70 \%$ ihres Ökonomischen Eigenkapitals für diese Risikoart. ${ }^{8}$ Neben dem klassischen Kreditgeschäft wird das Problem der Bewertung des Kreditrisikos auch beim Handel mit Unternehmensanleihen deutlich, der in jüngster Vergangenheit deutlich zugenommen hat. ${ }^{9}$ In beiden Geschäftsfeldern obliegt dem Risikocontrolling die Aufgabe der Informationsbereitstellung.

Parallel zu dieser Entwicklung steigt die Anzahl der Unternehmensinsolvenzen an (vgl. Abbildung 1). Sowohl die Anzahl an Insolvenzfällen als auch die Höhe der Insolvenzschäden haben stark zugenommen. Die Forderungsverluste sind von 8,1 Mrd. DM im Jahre 1990 auf 37 Mrd. DM im Jahre 1995 gestiegen. Die durchschnittliche Schadenshöhe betrug 1,5 Mio. DM je Insolvenzfall. ${ }^{10}$ Die Kreditinstitute werden mit steigenden Kosten durch diese Kreditausfälle stark belastet. Nur die effiziente „Verrechnung“ dieser Kosten, d.h. die Belastung von risikoreicheren Krediten mit einem höheren Zins, sichert ein ertragsorientiertes Aktivgeschäft. Eine pauschale Erhöhung des Kreditzinses führt zwangsläufig - durch die erhöhte Preissensitivität und die abnehmende Loyalität der Kunden gegenüber der Hausbank -

6 Vgl. Baetge, Jerschensky (1999), S. 173.

7 Vgl. Deutsche Bank (1999), S. $123 \mathrm{ff}$.

8 Vgl. Deutsche Bank (1999), S. 121. Das Ökonomische Eigenkapital ist das Kapital, „das notwendig ist, um auch unerwartete Verluste mit sehr hoher Sicherheit abzufangen. Die Deutsche Bank ermittelt das Ökonomische Kapital für Ausfall-, Markt- und Geschäftsrisiken“. Deutsche Bank (1999), S. 125f. Aus Anleihen resultierende Kreditrisiken werden in diesem Fall unter dem Begriff Marktpreisrisiken subsumiert.

$9 \quad$ Erklärungsansätze für das deutlich gestiegene Volumen an gehandelten und emittierten Anleihen finden sich bei Rometsch, Kolb (1999), S. 296f. Im Einzelnen handelt es sich um einen gestiegenen Finanzierungsbedarf von Unternehmen, ausgereizte Kreditlinien der Unternehmen aufgrund von Bankenfusionen, einen gestiegenen Bedarf von Investoren an (risikoreicheren) alternativen Anlageformen aufgrund des historisch niedrigen Kapitalmarktzinsniveaus, eine wachsende private Altersvorsorge (in den USA sind die Pensionskassen der Hauptinvestorenkreis für Unternehmensanleihen) und eine erfahrungsgemäß niedrige Korrelation zwischen Aktien- und Rentenmärkten und somit wachsende Diversifikationsmöglichkeiten.

10 Vgl. Bröker (2000), S. 5., zu einer detaillierten Analyse der Insolvenzentwicklung vgl. Kirmße (1996), S. 1ff. 
zu einem Rückgang der Erträge aus dem Kreditgeschäft."

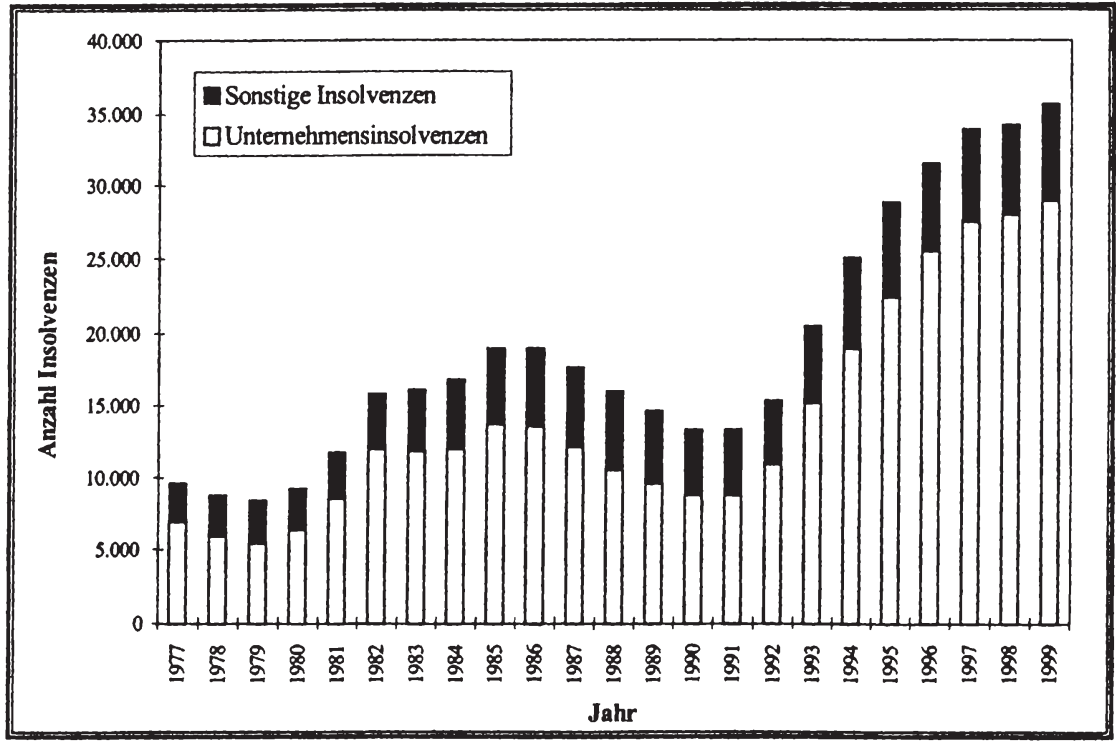

Abbildung 1: Entwicklung der Insolvenzzahlen in Deutschland ${ }^{12}$

Dieser marktwirtschaftlich bedingte Druck auf die Ertragslage der Kreditinstitute wird von einer aufsichtsrechtlichen Verpflichtung begleitet, die als „Basel II"13 bekannt ist. Hiermit werden Kreditinstitute verpflichtet, bei der Vergabe von Krediten eine individuelle Risikoprüfung durchzufuhren, die bspw. über ein Ratingsystem institutionalisiert werden kann. ${ }^{14}$ Während auf internationalen Kapitalmärkten tätige Unternehmen bereits heute ein externes Rating durch spezialisierte Unternehmen (so genannte Ratingagenturen) erwerben, stellt das Kreditrisikopapier es der Bank frei, eine Risikoprü-

"Vgl. Tegeder (1996), S. 281.

12 In Anlehnung an Bröker (2000), S. 6. Ab dem Jahr 1991 inklusive der neuen Bundesländer.

13 Das Kreditrisikopapier „A New Capital Adequacy Framework“ wird als zweite wesentliche Änderung durch das Gremium von Bankenaufsichtsbehörden mit Sekretariatssitz in Basel beschrieben. Vgl. Baseler Ausschuss für Bankenaufsicht (1999). Als „Basel I“ werden die aus der 6. Novelle des Kreditwesengesetzes und der Neufassung des Eigenkapitalgrundsatzes I abgeleiteten Bestimmungen für die Erfassung des Preis- und (teilweise) des Adressenausfallrisikos bezeichnet. Vgl. Schulte-Mattler (1999), S. 530.

Vgl. Schulte-Mattler (1999), S. 531. 
Marktorientierte Kreditrisikobewertung - Einleitung

fung durch einen internen Bewertungsprozess sicherzustellen. ${ }^{15}$ Diese Möglichkeit führt zu der Überprüfung der bankinternen Prozesse zur Kreditwürdigkeitsprüfung, da nur ein sehr kleiner Teil der Kunden deutscher Banken über ein externes Rating verfügt. ${ }^{16}$ Insbesondere bei der Kreditvergabe an mittelständische Unternehmen herrscht zur Zeit eine hohe Unsicherheit bzgl. der Konsequenzen des Kreditrisikopapiers. ${ }^{17}$ Neben Großbanken sind auch Privatbanken, Sparkassen und Genossenschaftsbanken von der Umstellung betroffen und erarbeiten zur Zeit neue Verfahren zur Bewertung des Kreditrisikos.

Diese Situationsdarstellung verdeutlicht, dass die Quantifizierung des Adressenausfallrisikos in einer modernen Risikosteuerung als Teilprozess bzw. Modul des Risikomanagements ein erfolgskritischer Faktor für Finanzinstitutionen geworden ist. Um ein ertragsorientiertes Kreditgeschäft sicherzustellen, muss eine risikoorientierte Bepreisung des einzelnen Kreditgeschäftes gewährleistet werden. ${ }^{18}$

\subsection{Ziel der Arbeit}

Einen Beitrag zur Lösung des Problems der Quantifizierung des Kreditrisikos zu leisten, ist das Oberziel der vorliegenden Arbeit. Hierbei werden drei Unterziele verfolgt:

(1) Zusammenstellung und kritische Reflexion von Verfahren zur Quantifizierung des Kreditrisikos ${ }^{19}$

Im Rahmen der Verfolgung des ersten Unterziels werden die bestehenden modelltheoretischen und heuristischen Verfahrensweisen zur Quantifizierung des Kreditrisikos untersucht. ${ }^{20}$ Diese unterschiedlichen Ansätze werden anhand konsistenter Kriterien

is Diese bankinterne Risikoprüfung setzt das Einverständnis der Bankenaufsicht voraus. Ein allgemeiner Konsens bei der Risikoprüfung hat sich bisher noch nicht herausgebildet. Vgl. Schulte-Mattler (2001), S. $62 \mathrm{f}$.

16 Vgl. Jansen (2001b), S. 101.

17 Vgl. hier und im Folgenden Völkel (2001), S. $6 \mathrm{ff}$.

18 Vgl. Flesch (1994), S. 23; Flesch (1995), S. B 9; Schüller (1995), S. $175 f$.

19 Zur Unterscheidung des Begriffspaares Adressenausfall- und Ausfall- bzw. Kreditrisiko und zu dem diesbezüglichen Verständnis der vorliegenden Arbeit vgl. Kap. 2.2.

20 Im Weiteren werden hierzu zunächst die modelltheoretischen und heuristischen Verfahrensweisen voneinander abgegrenzt. Die vorliegende Arbeit vernachlässigt den Bereich der Kreditportfoliorisiken. Offensichtlich stellt die Summe der Einzelkreditrisiken nicht das Gesamtrisiko einer Bank darstellt. Die Ansätze CreditRisk+ $+^{\star}$, Portfolio Manager ${ }^{\star}$, CreditMetrics $^{\star}$ und der CreditPortfolioManager ${ }^{\star}$ sind hier die in der Bankbetriebswirtschaftslehre aktuell diskutierten Modelle, die eine Portfoliobetrachtung explizit berücksichtigen. Da die vorliegende Arbeit ein innovatives Verfahren der einzelgeschäftsbezogenen Kreditrisikobewertung darstellt, steht eine Erweiterung der gefundenen Ergebnisse um eine Portfoliobetrachtung noch aus. 
bewertet. Dieses Unterziel stellt somit eine Zusammenfassung und Würdigung der aktuellen Diskussion zur einzelgeschäftsbezogenen Kreditzinskalkulation dar.

(2) Konzeption eines marktorientierten Kreditrisikobewertungsverfahrens

Das zweite Unterziel leitet sich aus den Ergebnissen des ersten ab. Die Kritikpunkte an den bestehenden Verfahren führen zur Konzeption eines neuen Ansatzes. Während modelltheoretische Bewertungsverfahren aus Theorien abgeleitet werden, liegen dem zu konzipierenden Verfahren Beobachtungen der Realität zugrunde. Es soll auf bereits gefundenen Zusammenhängen aufbauen und relevanten Erkenntnisse aus der empirischen Kapitalmarktforschung, den finanzmarkttheoretischen Modellen und der Bilanzanalyse zusammenführen, um eine kreditmarktorientierte Risikobewertung zu entwickeln. Durch diese Zielsetzung ergibt sich ein heuristisches Lösungsverfahren zur marktorientierten Kreditrisikobewertung.

(3) Empirische Analyse mittels des kreditmarktorientierten Bewertungsansatzes

Das dritte Unterziel ist die Ermittlung der Prognosegüte ${ }^{21}$ des entwickelten marktorientierten Bewertungsverfahrens. Eine empirische Studie, aufbauend auf den theoretischen Erkenntnissen, soll diese Güte aufzeigen. Hierzu ist es notwendig, ein geeignetes Instrument aus den konzeptionellen Ansprüchen abzuleiten. Als Ergebnis dieser Untersuchung lassen sich Aussagen über die Anwendungsmöglichkeiten des Konzeptes treffen.

\subsection{Gang der Ausführungen}

Die Abfolge der Ausfürungen in den nachfolgenden Kapiteln lehnt sich an die im Kapitel 1.2 beschriebenen Unterziele an. Zunächst wird im Kapitel 2 die Problematik der Quantifizierung des Kreditrisikos aufgegriffen. Hierzu wird der Begriff des Risikos allgemein definiert, um darauf aufbauend das Wesen und die Stellung des bankbetrieblichen Kreditrisikos zu beschreiben. Kreditzinskalkulationsverfahren ${ }^{22}$ werden erörtert, um diese kriteriengeleitet hinsichtlich ihrer Eignung zu überprüfen.

Die Bilanzanalyse wird in der Praxis regelmäßig im Rahmen der Bonitätsprüfung von Unternehmen und der damit verbundenen Risikoquantifizierung verwendet, sodass im

21 Das aus dem griechischen stammende Wort Prognose bedeutet Vorhersage. Häufig wird mit diesem Wort die Vorhersage der Zukunft in Zusammenhang gebracht. In der vorliegenden Arbeit wird der Begriff abweichend verstanden. Als Prognosegüte wird die Genauigkeit verstanden, mit der ein modelltheoretisch bestimmter Wert mit einem vorgegebenen Wert übereinstimmt.

22 Ein Kreditzinskalkulationsverfahren bestimmt den für einen Kredit vom Gläubiger zu erhebenden Kreditzins. Dieser beinhaltet neben dem ,sicheren Zins“ das bewertete Ausfallrisiko, welches der Gläubiger trägt. Zu den Begriffen ,sicherer Zins“ und Ausfallrisiko vgl. Kap. 2.2. 
Kapitel 3 dieses Instrument gesondert beschrieben wird. Hierzu wird zunächst die Bilanzanalyse allgemein vorgestellt, um anschließend auf die verschiedenen Analyseverfahren einzugehen. Zunächst werden die traditionellen Verfahren der Kennzahlenbildung und Kennzahlensysteme, dann die Scoringmodelle und statistisch basierte Verfahren der Diskriminanzanalyse sowie Künstliche Neuronale Netze beschrieben. Diese werden auf ihre Eignung als einzelgeschäftsbezogene Kreditzinskalkulationsverfahren hin untersucht.

Die zu den modelltheoretischen und heuristischen Verfahren herausgestellten Kritikpunkte und die damit in Verbindung stehenden Bilanzanalyseverfahren leiten über in das im Kapitel $4 \mathrm{zu}$ konzipierende marktorientierte Kreditbewertungsverfahren. Zunächst wird die Zielsetzung erläutert, und es werden die Grundannahmen festgelegt. Die Systematisierung bestehender kapitalmarktorientierter Untersuchungen leitet über in die detaillierte Beschreibung des Kreditrisikos von Unternehmensanleihen. Aus der Bewertung dieses Risikos am Kapitalmarkt wird ein Prognosemodell abgeleitet. Dieses wird mittels der bereits in den Kapiteln 2 und 3 angewandten Kriterien gewürdigt.

Im Kapitel 5 wird ein geeignetes Mustererkennungsverfahren zur Umsetzung des marktorientierten Bewertungsansatzes ausgewählt, indem zunächst die theoretischen Voraussetzungen beschrieben werden. Hier zeigt sich, dass sich Künstliche Neuronale Netze als Instrument eignen. Ein bestimmtes Netz, ein Multi-Layer-Perceptron, wird in den Bewertungsansatz integriert. Neben der Herleitung einer Netzstruktur werden die gewonnenen Ergebnisse der empirischen Studie aufgezeigt, um sie anschließend hinsichtlich ihrer Eignungsgüte zu bewerten. Hierzu wird die ratinggestützte Kreditzinskalkulation als ein öffentlich zugänglicher Vergleichsmaßstab verwendet.

Die Möglichkeiten der Anwendung der gefundenen Zusammenhänge und die hiermit verbundenen Forschungsfelder werden im Kapitel 6 dargestellt. Inhaltlich endet die Arbeit mit einer Schlussbetrachtung (Kap. 7). 
Marktorientierte Kreditrisikobewertung -

\section{Die Quantifizierung des Ausfallrisikos als bankbetriebliche Problemstellung}

Im folgenden Kapitel 2.1 wird zunächst der Begriff Risiko definiert und es werden die verschiedenen subjektiven Einstellungen bei der Wahrnehmung des Risikos beschrieben. Sowohl im Rahmen von Risikobewertungsmodellen als auch Erklärungsansätzen realer Sachverhalte wird häufig eine Risikoeinstellung unterstellt, sodass eine Beschreibung der verschiedenen Typen notwendig wird. Aufbauend auf der allgemeinen Risikodefinition wird in Kap. 2.2 ein spezielles Risiko, das Ausfallrisiko, definiert und seine Stellung innerhalb der bankspezifischen Risiken erklärt. Im weiteren Analyseverlauf werden in Kap. 2.3 verschiedene Instrumente zur Quantifizierung des Ausfallrisikos systematisiert und anhand einzelner Modelle Wirkungszusammenhänge zwischen dem Ausfallrisiko und seinen Einflussfaktoren erläutert. In den Kap. 2.4 und 2.5 werden die beschriebenen Instrumente hinsichtlich ihrer Eignung als Quantifizierungsinstrument gewürdigt.

\subsection{Definition des Risikos und der Risikoeinstellungen}

Der Begriff Risiko wird in der Literatur nach verschiedenen Gesichtspunkten definiert. Zunächst wird zwischen formalen und materiellen Aspekten unterschieden. ${ }^{23}$ In formaler Hinsicht wird das Begriffspaar Unsicherheit und Risiko voneinander abgegrenzt. ${ }^{24}$ Unsicherheit beschreibt die Tatsache, dass die zukünftige Entwicklung nicht gewiss, d.h. nicht absehbar ist. Es bestehen verschiedene mögliche Umweltentwicklungen. ${ }^{25}$ Falls man für den Eintritt eines Umweltzustandes eine (subjektive) Wahrscheinlichkeit angeben kann, handelt es sich um ein Risiko. Die Unsicherheit wird somit messbar ${ }^{26}$ Die Wahrscheinlichkeit kann entweder aus empirischen Häufigkeitsverteilungen oder heuristischen Regeln abgeleitet werden. ${ }^{27}$ In materieller Hinsicht wird der Begriff Risiko in eine ursachen- und eine wirkungsbezogene Betrachtung unterteilt. Als ursachenbezogen wird die Gefahr einer Entscheidung aufgrund einer Fehlinformation bezeichnet. Demgegenüber wird als wirkungsbezogen die

23 Vgl. Büschgen (1992), S. 32.

24 Vgl. hier und im Folgenden Berens, Delfmann (1995), S. 71 und S. 76.

25 Vgl. Knight (1921), S. 20; Spremann (1996), S. $91 \mathrm{f}$.

26 Vgl. Kirmße (1996), S. 9.

27 Neben dieser abgrenzenden Definition wird Risiko als Unsicherheit beschrieben, da es als ein ,Zustand des Auseinanderfalls der subjektiven Wahrscheinlichkeiten über unterschiedliche mögliche Zustände (...) [bezeichnet wird], oder insgesamt die Unterscheidung Risiko und Unsicherheit für fragwürdig [ge]halten" wird (die mit [ ] gekennzeichneten Inhalte wurden vom Verfasser hinzugefügt); Kirmße (1996), S. 9; vgl. auch Hirshleifer (1973), S. 31; Arrow (1985), S. 417; Schneider (1992), S. 35. 
Marktorientierte Kreditrisikobewertung Die Quantifizierung des Ausfallrisikos als bankbetriebliche Problemstellung

Gefahr des Eintritts eines Verlustes aufgrund der Risikowirkung verstanden. ${ }^{28}$ Die unterschiedlichen materiellen Risikoinhalte lassen sich in Anlehnung an SCHIERENBECK im prozessualen Entscheidungsvorgang einordnen. ${ }^{29}$ Hierbei besteht der Entscheidungsprozess aus Informationserhebung, Entscheidung und Kontrolle der Umsetzung durch die generierten Ergebnisse.

Eine alternative Abgrenzung von Risiko und Unsicherheit findet sich bei ADAM. ${ }^{30}$ Risiko lässt sich demnach durch die Entscheidungsrelevanz bzw. die Entscheidungselastizität von der Unsicherheit abgrenzen. Eine unsichere Datensituation wird erst dann zu einem Risiko für den Entscheider, wenn die einmal getroffene Entscheidung unveränderbar ist.

In der vorliegenden Arbeit wird der Begriff Risiko als Gefahr der negativen Abweichung der Ausprägung einer finanzwirtschaftlichen Zielgröße von einem ex-ante bestimmten Referenzwert verstanden. ${ }^{31}$ Es wird dabei kein Schwerpunkt auf eine Definition (bspw. einer Unterscheidung in einer ursachen- oder wirkungsbezogenen Betrachtung) oder einen Teilprozess im Entscheidungsvorgang ${ }^{32}$ gelegt, sondern vielmehr eine Synthese angestrebt. ${ }^{33}$

Es existieren verschiedene Formen des Umgangs mit dem Risiko. Dieser ist i.d.R. subjektiv und drückt sich in dem persönlichen Risikoempfinden aus. Dieses korrespondiert mit der Nutzenfunktion $\mathrm{u}(\mathrm{x})$, die einem risikobehafteten Ergebnis $\mathrm{x}$ einen Nutzen u (x) zuordnet. ${ }^{34}$ Hierbei werden die Wahrscheinlichkeiten für das Eintreten eines Ergebnisses als Nutzenziffern interpretiert und durch eine Transformation in eine äquivalente Nutzenfunktion überfuhrtt. ${ }^{35}$ Die Höhe des Nutzens ist eine subjekti-

28 Vgl. Büschgen (1992), S. 32.

29 Vgl. Schierenbeck (2000), S. 71ff.

30 Vgl. hier und im Folgenden Adam (1996), S. 194f.

31 Eine positive Abweichung wird als Chance bezeichnet. Diese Definition des Risikos beschränkt sich auf die negative Abweichung, sodass der Definition des so genannten „downside risk“ gefolgt wird. Vgl. Hartmann-Wendels, Pfingsten, Weber (2000), S. 540.

32 Vgl. hierzu Schierenbeck (2000), S. 71 ff. oder Adam (1996), S. 194, wobei hier kein Teilprozess betrachtet wird, sondern das Kriterium „Elastizität der einmal getroffenen Entscheidung", Adam (1996), S. 194, zur Begriffsdefinition herangezogen wird.

33 Vgl. Berger (1987), S. 253; Bitz (1993), S. 642; Mülhaupt (1980), S. 188 und Rolfes (1985), S. 9f.

34 Das Konzept des Risikonutzens geht auf die Beobachtungen des Mathematikers Daniel Bernoulli zurück (Bernoulli-Prinzip). Von Neumann und Morgenstern zogen diese Erkenntnisse zur Ableitung der Risikonutzenfunktion heran. Vgl. von Neumann, Morgenstern (1973). Vgl. Berens, Delfmann (1995), S. 84. 
Marktorientierte Kreditrisikobewertung -

ve Größe des Entscheidungsträgers. Die Risikopräferenz spielt also in dem Bewertungsvorgang eine bedeutsame Rolle. Eine Erhöhung der möglichen Ergebnisgröße (x) um einen beliebigen Multiplikator $n$ stellt nicht unbedingt eine Erhöhung des Nutzens $(\mathrm{u}(\mathrm{x}))$ um denselben Multiplikator dar. Der Nutzenzuwachs wird durch eine subjektive Bewertung des risikobehafteten Ergebnisses (Präferenz) widergespiegelt, sodass man in der Literatur häufig die Bezeichnung ,Risiko-Nutzenfunktion" findet. ${ }^{36}$

Als Sicherheitsäquivalent wird das sicher eintretende Ergebnis beschrieben, zu dem sich der Entscheidungsträger in der Wahl gegenüber „seinem“ risikobehafteten Ergebnis indifferent verhält. ${ }^{37}$ Angenommen, ein Entscheider steht vor der Wahl ein sicheres Ergebnis $\mathrm{x}_{3}$ oder ein jeweils mit 50-prozentiger Wahrscheinlichkeit eintretendes Ergebnis $x_{1}$ oder $x_{2} z u$ erhalten. Falls der subjektiv empfundene Nutzen $u\left(x_{3}\right)$ gleich dem Nutzen der risikobehafteten Alternative $\left(u\left(x_{1}\right)+u\left(x_{2}\right)\right) / 2$ ist, dann drückt $x_{3}$ die Höhe des Sicherheitsäquivalentes aus. Das Sicherheitsäquivalent entspricht dem Erwartungswert der Risikonutzenfunktion.

Innerhalb der subjektiven Bewertung werden vier verschiedene Präferenzgruppen unterschieden: ${ }^{38}$ Höhen-, Arten-, Zeit- und Sicherheitspräferenz.

Die Höhenpräferenz ist die ausschließliche Orientierung des Entscheidungsträgers an der Höhe des Ergebnisses. Als Beispiele gelten die Maximierungs- und die Minimierungsregel..$^{39}$ Falls in einer Nutzenfunktion diese Präferenz als einzige beachtet würde, resultiert daraus eine lineare Nutzenfunktion. Bei der Artenpräferenz erfährt das Ergebnis einen höheren Nutzen durch seine Klassenzugehörigkeit, d.h. der Entscheidungsträger bewertet durch seine subjektive Einstellung ein Ziel höher als ein anderes (z.B. Gewinn ist wichtiger als Umweltschutz). Die Zeitpräferenz legt eine Ordnung durch den Zeitpunkt des Eintreffens fest. Die Sicherheitspräferenz beschreibt die Vorziehungswürdigkeit, die den zu vergleichenden Ergebnissen aufgrund der Unsicherheit ihres Eintritts zukommt. ${ }^{40}$

\footnotetext{
36 Vgl. Berens, Delfmann (1995), S. 81.

37 Vgl. hier und im Folgenden Berens, Delfmann (1995), S. 82f. und S. 85.

38 Vgl. Sieben, Schildbach (1994), S. 25ff.

39 Vgl. hier und im Folgenden Bamberg, Coenenberg (2000), S. 27f.

40 Vgl. Sieben, Schildbach (1994), S. 26.
} 
Marktorientierte Kreditrisikobewertung Die Quantifizierung des Ausfallrisikos als bankbetriebliche Problemstellung

Für die weitere Untersuchung wird unterstellt, dass mittels eines Bewertungsprozesses die Arten-, Zeit und Sicherheitspräferenz in ein Ergebnis transformiert werden, ${ }^{41}$ sodass sich der Entscheidungsprozess ausschließlich an der Höhe des Ergebnisses orientiert (Höhenpräferenz).

Risikoneutralität, Risikoaversion und Risikofreude sind die drei unterschiedlichen Einstellungen eines Entscheiders, die in einer Risikonutzenfunktion zum Ausdruck kommen können. Hierzu kann die Risikonutzenfunktion in einem Koordinatensystem abgetragen werden, in dem die $\mathrm{x}$-Achse das Ergebnis aufzeigt und die $\mathrm{y}$-Achse diesem Ergebnis den zugehörigen Nutzen zuordnet. ${ }^{42}$ Der in Abbildung 2 dargestellte Funktionsverlauf entspricht dem Fall der Risikoneutralität. Einem Zuwachs des Ergebnisses $\left(\mathrm{x}_{2}-\mathrm{x}_{1}\right)$ wird aufgrund der linearen Zuordnungsvorschrift und einer unterstellten Steigung von "1" ein ebenso hoher Nutzenzuwachs $\left(u\left(x_{2}\right)-u\left(x_{1}\right)\right)$ gegenübergestellt. Folglich sind die Strecken $\overline{x_{1} x_{2}}$ und $\overline{u\left(x_{1}\right) u\left(x_{2}\right)}$ gleich groß.

Die in Abbildung 3 dargestellte konkave Funktion drückt eine risikoaverse Einstellung aus. ${ }^{43}$ Das bedeutet, der Entscheider sieht den Zuwachs eines möglichen höheren Gewinns (Gewinn im allgemeinen Sinne) im Vergleich zum höheren Risiko als weniger bedeutsam an. Es wird in diesem Fall von einem Sicherheitsdenken ausgegangen. Der Grenznutzen durch einen höheren möglichhen Gewinn fällt stetig aufgrund dieser Transformationsvorschrift. Einem Zuwachs des Ergebnisses $\left(x_{2}-x_{1}\right)$ wird aufgrund der Zuordnungsvorschrift ein geringerer Nutzenzuwachs $\left(u\left(x_{2}\right)-u\left(x_{1}\right)\right)$ gegenübergestellt. Dementsprechend ist die Strecke $\overline{x_{1} x_{2}}$ größer als

4t Bspw. wird die Zeitenpräferenz in der Investitionsrechnung durch eine Verzinsung der Zahlung ab dem Zeitpunkt der Einzahlung berücksichtigt, sodass ein Gewinn (in derselben Höhe) zu einem früheren Zeitpunkt einen höheren Wert zugeordnet bekommt als zu einem späteren. Vgl. auch zum Barwertkonzept bei der Betrachtung von Zahlungsströmen Schierenbeck (1999b), S. 178ff. Bei der Artenpräferenz könnte man als Beispiel Stammaktien und Vorzugsaktien betrachten. Diese beiden ihrer Art nach unterschiedlichen Aktientypen werden zu verschiedenen Kursen gehandelt, sodass sich der Entscheider lediglich an der Höhe des Ergebnisses orientieren muss. Bei der Sicherheitspräferenz könnte man als Beispiel den Optionspreis heranziehen. Dieser wächst bei einem Anstieg der Volatilität des unterlegten Basiswertes an (vgl. Kap. 2.3.2.2). Die erhöhte Unsicherheit wird durch einen erhöhten Optionspreis ausgeglichen, sodass der Entscheider sich wiederum ausschließlich an der Höhe orientieren muss.

42 Vgl. zu den folgenden Ausführungen Berens, Delfmann (1995), S. 85 ff. und Sieben, Schildbach (1994), S. 66f.

43 Vgl. hier und im Folgenden Berens, Delfmann (1995), S. 86. 
Marktorientierte Kreditrisikobewertung -

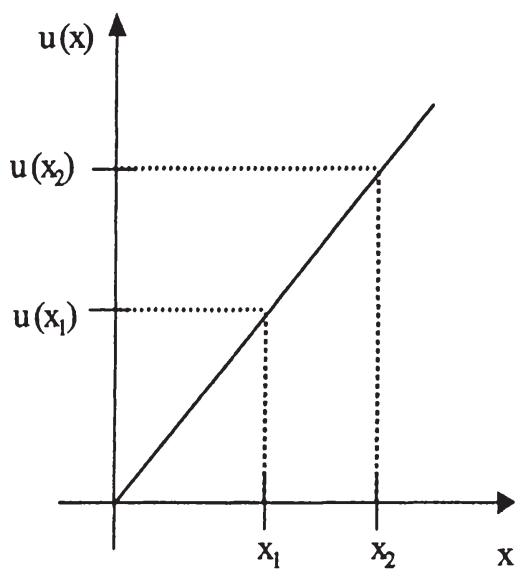

Abbildung 2: Lineare Nutzenfunktion

die Strecke $\overline{u\left(x_{1}\right) u\left(x_{2}\right)}$. Das Sicherheitsäquivalent ist bei einer streng konkaven Funktion stets kleiner als der Erwartungswert. ${ }^{45}$

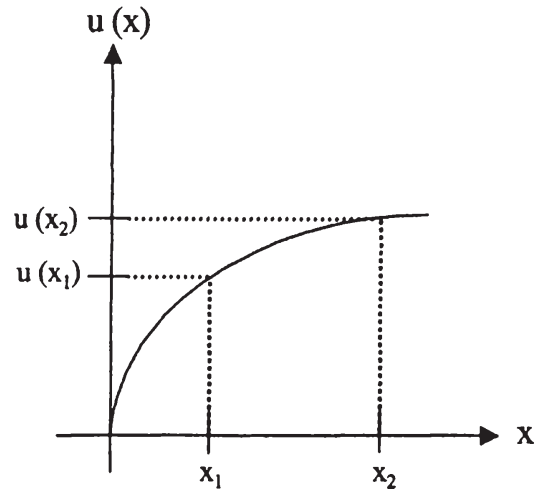

Abbildung 3: Konkave Nutzenfunktion ${ }^{46}$

44 In Anlehnung an Berens, Delfmann (1995), S. 85.

45 Vgl. Adam (1996), S. 216.

46 In Anlehnung an Berens, Delfmann (1995), S. 86. 
Die konvexe Nutzenfunktion in Abbildung 4 gibt die Einstellung eines risikofreudigen Entscheiders wieder, der umgangssprachlich auch als Spekulant oder Glücksspieler bezeichnet wird. ${ }^{47}$ Der Nutzen einer höheren Gewinnchance wird stärker gewichtet als der eigentliche Ergebniszuwachs, sodass der Grenznutzen steigt. Einem Zuwachs des Ergebnisses $\left(x_{2}-x_{1}\right)$ wird aufgrund der Zuordnungsvorschrift ein höherer Nutzenzuwachs $\left(u\left(x_{2}\right)-u\left(x_{1}\right)\right)$ gegenübergestellt. Folglich ist die Strecke $\overline{x_{1} x_{2}}$ kleiner als die Strecke $u\left(x_{1}\right) u\left(x_{2}\right)$. Das Sicherheitsäquivalent ist stets größer als der Erwartungswert. ${ }^{48}$

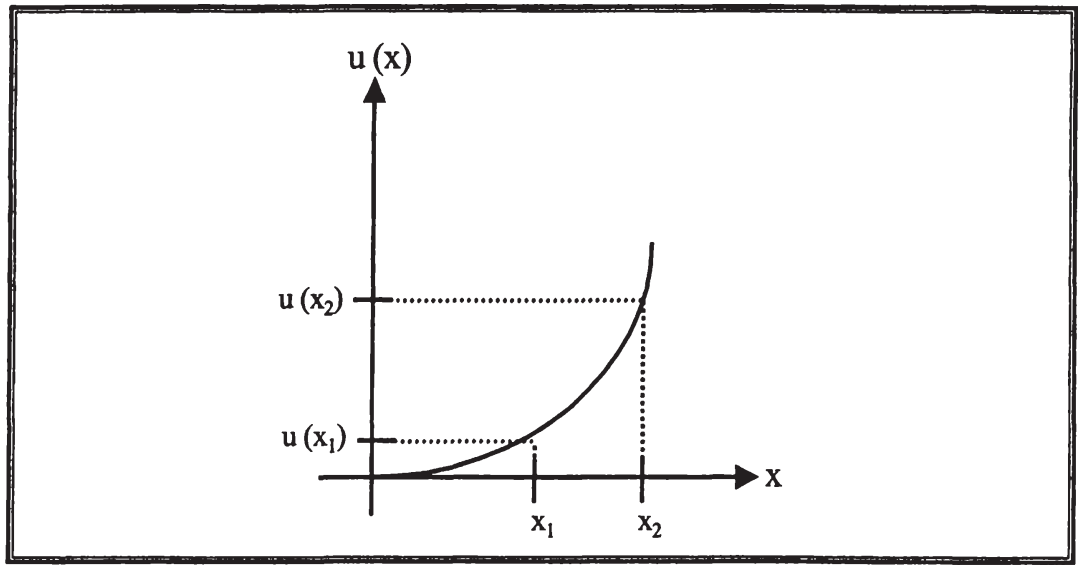

Abbildung 4: Konvexe Nutzenfunktion ${ }^{49}$

In der Realität wird man selten die alleinige Ausprägung eines Typen vorfinden. Es handelt sich bei der grafischen Darstellung der individuellen Risikoeinstellung vielmehr um eine Kombination von konkaven und konvexen Kurvenabschnitten. Die Ökonomen SAVAGE/FRIEDMAN konnten mit Hilfe einer empirischen Untersuchung

47 Vgl. hier und im Folgenden Berens, Delfmann (1995), S. 87.

48 Vgl. Adam (1996), S. 216.

49 In Anlehnung an Berens, Delfmann (1995), S. 87. 
Marktorientierte Kreditrisikobewertung -

ihre These einer sich abwechselnden Funktion nachweisen (S-förmig). ${ }^{50 / 51}$ Dieser Grundüberlegung liegt die in Abbildung 5 dargestellte Nutzenfunktion zugrunde. Sie wird auch als realitätsnahe Abbildungsvorschrift bezeichnet. ${ }^{32}$

HAHNENSTEIN/WILKENS/RÖDER weisen nach, dass die BLACK \& SCHOLES-Formel zur Optionspreisbestimmung ${ }^{53}$ mit Hilfe des Prinzips der risikoneutralen Bewertung abgeleitet werden kann.${ }^{54}$ Hieraus folgt, dass modelltheoretische Ansätze zur Kreditzinskalkulation basierend auf der Optionspreistheorie, wie sie im Weiteren vorgestellt werden, i.d.R. eine risikoneutrale Bewertung voraussetzen.

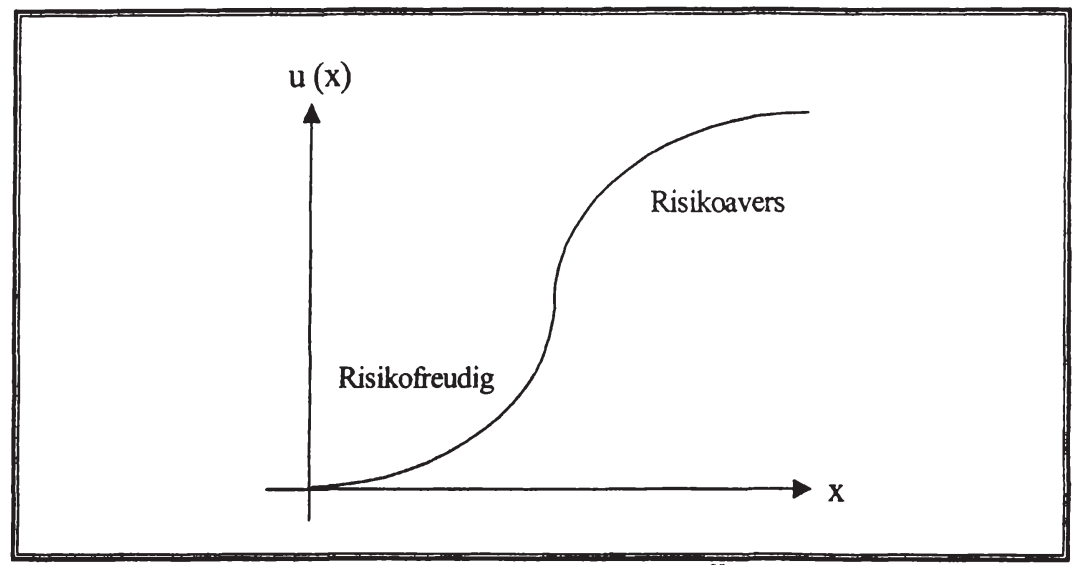

Abbildung 5: Nutzenfunktion mit wechselnder Risikoeinstellung ${ }^{35}$

so Vgl. Friedman, Savage (1948), S. 279ff., Bamberg, Coenenberg (1994), S. 76ff.

s1 Die Abhängigkeit der Risikoeinstellung von der Ergebnishöhe lässt sich an einem einfachen Beispiel deutlich machen: Es wird eine Münze geworfen. Wenn „Kopf“ erscheint, bekommt man die Summe X ausbezahlt, wenn „Zahl" erscheint bekommt man nichts. Die Summe X wird von einer dritten Person ,kostenlos“ bereitgestellt. Bei Verzicht auf das Spiel bekommt man die Hälfte der bereitgestellten Summe X ausbezahlt. Fall 1: $X=1$ EURO; Fall 2: $X=500.000$ EURO. Es kann unterstellt werden, dass man sich im ersten Fall eher für das Spiel entscheidet, sich also risikofreudig, im zweiten Fall es eher ablehnt, sich also risikoavers verhält. Vgl. zu der allgemeinen Beschreibung des oben dargestellten Beispiels Berens, Delfman (1995), S: 86.

52 Vgl. Schneeweiß (1967), S. $81 \mathrm{ff}$.

53 Vgl. zu der Bedeutung der Formel für die Bestimmung des Kreditrisikos Kap. 2.3.2.2.

54 Vgl. Hahnenstein, Wilkens, Röder (2001), S. 3ff.

35 In Anlehnung an Berens, Delfmann (1995), S. 87; von den Autoren wird eine Nutzenfunktion des Typs Savage-Friedman dargestellt. Diese setzt sich aus weiteren konvexen und konkaven Kurvenbestandteilen zusammen. 
Marktorientierte Kreditrisikobewertung Die Quantifizierung des Ausfallrisikos als bankbetriebliche Problemstellung

Für den praktischen Umgang mit dem Risiko bei Banken wiederum stehen sich verschiedene konkurrierende Hypothesen bzgl. der unterstellten Risikoeinstellung von Kreditinstituten gegenüber. ${ }^{56}$ Theoretische Ansätze deuten auf eine risikoaverse Einstellung hin. Dies widerspricht jedoch Beobachtungen der Praxis sowohl aus verhaltensorientierter als auch aus organisatorischer Sicht. ${ }^{57}$

\subsection{Ausfallrisiko als bankspezifisches Risiko}

Im Verlauf dieses Kapitels wird das Ausfallrisiko in die Risiken von Banken eingeordnet und definiert. Hierzu werden allgemeine Unternehmensrisiken genannt und ergänzend die bankbetrieblichen Risiken erläutert. Hier wird zunächst das Ausfallrisiko in Abgrenzung vom Marktpreisrisiko erklärt. Anschließend wird das für diese Arbeit unterstellte Verständnis des Ausfallrisikos als Bonitätsrisiko aufgrund von Bonitätsveränderungen beschrieben.

Allgemeine Unternehmensrisiken werden regelmäßig in die Teilbereiche Betriebs-, Personal- und Markterfolgsrisiko eingeteilt. ${ }^{58}$ Das Betriebsrisiko entsteht durch qualitative oder quantitative Abweichungen zwischen den sachlich-technischen Erfordernissen und der entsprechenden Ausstattung eines Unternehmens (technisches Risiko). Unter dem Betriebsrisiko wird ebenfalls das Organisationsrisiko subsumiert, das aus der Ablauf- oder Aufbauorganisation resultiert. ${ }^{99}$ Das Personalrisiko bezieht sich auf eine Nichtdeckung der qualitativen Ansprüche an eine Stelle und die entsprechenden Eigenschaften des Stelleninhabers. ${ }^{60}$ In quantitativer Hinsicht besteht zudem das Risiko der Über- oder Unterdimensionierung der personellen Kapazitäten. ${ }^{61}$ Das allgemeine Markterfolgsrisiko entsteht aufgrund der bestehenden Unsicherheit hinsichtlich des Absatzes der produzierten Leistungen eines Unternehmens. Analog zu dem o.g. Betriebsrisiko wird zwischen dem qualitativen und quantitativen Risiko unterschieden.

s6 Vgl. auch für die folgenden Ausführungen Hartmann-Wendels, Pfingsten, Weber (2000), S. $531 \mathrm{ff}$.

57 Vgl. zu den theoretischen Ansätzen zur Erklärung der Risikoeinstellung von Banken Santomero (1995), S. $1 \mathrm{ff}$.

$58 \mathrm{Vgl.} \mathrm{Kirmße} \mathrm{(1996),} \mathrm{S.} 10$.

59 Vgl. Schmoll (1985), S. 230f.; Brakensiek (1991) S. 18f.

60 Vgl. Kirmße (1996), S. 11.

61 Vgl. Bröker (2000), S. 10. 
Marktorientierte Kreditrisikobewertung -

Diese allgemeinen Risiken sind für Banken gleichermaßen relevant. Sie sollen im Weiteren um die bankspezifischen Risiken erweitert werden. ${ }^{62}$ Typischerweise wird furr Kreditinstitute zwischen dem Liquiditäts- und dem Erfolgsrisiko differenziert (vgl. Abbildung 6). ${ }^{63}$ Das Liquiditätsrisiko umfasst das Liquiditätsspannungs-, das Termin- und das Abrufrisiko. ${ }^{44}$ Das Liquiditätsspannungsrisiko ist die Gefahr einer fehlenden benötigten Anschlussfinanzierung. Die Möglichkeit einer Prolongation von Aktivgeschäften beschreibt das Terminrisiko. Das Abrufrisiko besteht aufgrund von außerplanmäßigen Inanspruchnahmen von Kreditzusagen bzw. unerwarteten Abzügen von Einlagen. Erfolgsrisiken unterteilen sich in das Marktpreis- und das Adressenausfallrisiko. Das „Basle Committee on Banking Supervision“ definiert das Marktpreisrisiko als die Gefahr von (negativen) Marktpreisänderungen bilanzieller oder außerbilanzieller Positionen. ${ }^{65}$ Hierbei werden regelmäßig die Risiken aufgrund von Zins-, Aktienkurs-, Währungskurs- und Warenpreisänderungen unterschieden. Als optionsspezifisches Risiko ist insbesondere das „Volatilitätsrisiko“ zu berücksichtigen, das die „Gefahr der Wertveränderung durch Schwankungen der Volatilität im Underlying der Optionsposition beschreibt" ${ }^{\text {"66. }}$.

Im Rahmen des Kreditgeschäftes einer Bank mit einem Kunden vertraut die Bank darauf, dass der Kunde die Fähigkeit und die Bereitschaft besitzt, die Schuldenverpflichtungen ordnungsgemäß (d.h. vereinbarungsgemäß) zu erfüllen. Dabei verzichtet der Kreditgeber für einen bestimmten Zeitraum (Kreditlaufzeit) auf die Nutzung eines bestimmten Kapitalbetrages (Kreditvolumen). ${ }^{67}$ Der Kreditnehmer hat fur diese Überlassung Zinsen (Kreditzins) zu bezahlen. ${ }^{68}$ Das Adressenausfallrisiko wird verstanden als die Gefahr, dass ein Schuldner seine Forderungen nicht vollständig zur vereinbarten Zeit begleicht. Der häufigste Grund ist die Zahlungsunfähigkeit, wobei auch Zahlungsunwilligkeit genannt wird ${ }^{69}$ Ein Kreditrisiko oder Ausfallrisiko beruht

Systematisierung von bankspezifischen Risiken vgl. Hagenmüller, Jacob (1988), S. 259f.; Jacob (1988), S. 6f.; Moser, Quast (1994), S. 665ff.; Schierenbeck (1999b), S. 4ff.; Schuster (1989), S. $208 f$.

Vgl. Schierenbeck (1999b), S. 7f.

Vgl. hier und im Folgenden Bröker (2000), S. $11 \mathrm{f}$.

Vgl. Basle Committee on Banking Supervision (1996), S. 1; Rolfes (1999), S. 47f.; Lister (1997), S. 7.

Bröker (2000), S. 1, innerhalb der Optionspreistheorie (vl. auch Kap. 2.3.2.2) fuhrt der Anstieg der Volatilität bspw. einer Aktie zu einem Wertzuwachs der Option.

Vgl. Grill, Perczynski (1995), S. 35.

Eine spezielle Form der Kreditvergabe ist eine festverzinsliche Anleihe. Der Kreditgeber ist der Käufer, der Kreditnehmer der Emittent der Anleihe. Die grundsätzlichen Ausführungen zu dem Kredit können auf die Anleihe übertragen werden.

Vgl. Bröker (2000), S. 12. 
Marktorientierte Kreditrisikobewertung Die Quantifizierung des Ausfallrisikos als bankbetriebliche Problemstellung

auf der Tatsache, dass die erwarteten Kreditausfälle von den Ist-Ausfällen abweichen. ${ }^{70}$

Die vorliegende Arbeit erweitert den Begriff Adressenausfallrisiko über die Insolvenz des Schuldners hinaus. Bei einer engen Auslegung des Begriffs wird das Risiko eines Wertverlustes nämlich erst in dem Moment des Ausfalls des Schuldners wirksam?" Unter dem Bonitätsrisiko ${ }^{22}$ werden die Verluste aufgrund von Bonitätsveränderungen verstanden. ${ }^{73}$ Bereits bei der Verschlechterung der Bonität des Schuldners wird das Bonitätsrisiko wirksam, da die Wahrscheinlichkeit des Ausfalls erhöht und folglich der Wert des Kredites reduziert wird. ${ }^{74}$

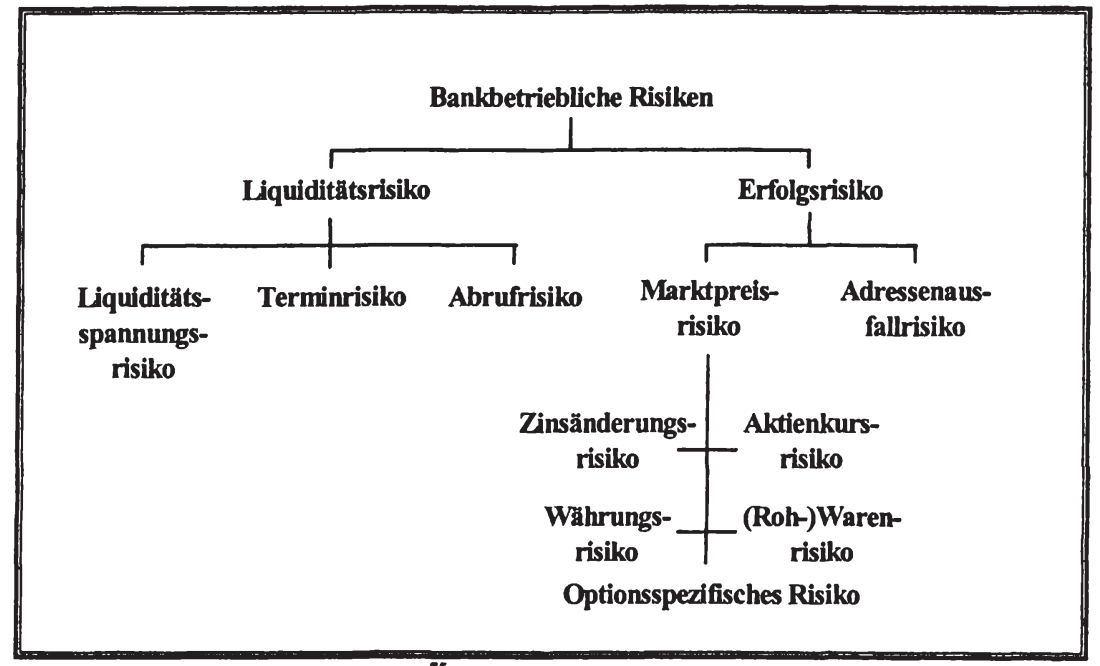

Abbildung 6: Bankspezifische Risiken ${ }^{75}$

70 Vgl. Schierenbeck (1999), S. 227.

71 Vgl. Bröker (2000), S. 12.

72 Vgl. auch zu dem Begriff Bonitätsrisiko (bei Anleihen) Kap. 4.3.2.1.

73 Vgl. Federal Reserve System Task Force on Internal Credit Risk Models (1998), S. 18ff.; Rolfes (1999), S. 332f.

74 Häufig wird in diesem Zusammenhang von einer marktorientierten Bewertung des Kredites gesprochen (vgl. Kap. 4.3).

75 In Anlehnung an Bröker (2000), S. 11, wobei die gewählte Abbildung lediglich den externen Leistungsbereich darstellt. 
Marktorientierte Kreditrisikobewertung -

Problematisch ist in diesem Zusammenhang die Operationalisierung des Adressenausfallrisikos. $^{76}$ Gemäß dem o.g. Risikoverständniss ist dies die Gefahr einer Differenz zwischen einem erwarteten und einem realen Zahlungsstrom. Analog zu dem Barwertkonzept können die Zahlungsströme in einen (Bar-)Wert übertragen werden, indem die zukünftigen Zahlungen auf einen einheitlichen Bezugszeitpunkt abdiskontiert werden. ${ }^{77}$ Die zukünftigen Zahlungen sind nicht die mit dem Kreditvertrag verbundenen, sondern um Risikokosten (Standard-Risikokosten) bereinigte Zahlungsströme. Dies liegt darin begründet, dass Kreditinstitute mit der Ausgabe von Krediten auch Verluste - resultierend aus der Nichtrückzahlung einzelner Kredite erwarten. Der Wert des Kredites verändert sich aufgrund einer bonitätsinduzierten Veränderung des Schuldners. ${ }^{78}$

Folglich tritt das Kreditrisiko nicht erst dann ein, wenn der Schuldner ganz oder teilweise nicht mehr in der Lage ist, seine Verpflichtungen zu erfüllen. Eine derartige Erweiterung des Begriffs Adressenausfallrisiko verbietet eine strikte Abgrenzung vom Marktpreisrisiko. ${ }^{79}$

\subsection{Systematisierung der einzelgeschäftsbezogenen Instrumente zur Risikoquantifizierung}

\subsection{1 Übersicht}

Während das Kapitel 2.2 das in Verbindung mit einem Kredit existierende Risiko beschrieb, wird im Weiteren aufgezeigt, mit welchen Methoden diese Risiken bewertet werden können. Die folgenden Instrumente berücksichtigen die

76 Vgl. auch Kap. 4.3.2.1.

77 Vgl. bspw. zu dem Barwertkonzept Schierenbeck (1999b), S. 178ff.

78 Die Begriffe Ausfallprämie, Bonitätsprämie, Bonitätsrisikoprämie, bepreistes Kreditrisiko bzw. Adressenausfall-, Ausfallrisiko und Kreditrisiko werden im Weiteren synonym gebraucht, wobei die Begriffe inhaltlich, gemäß dem Verständnis des Ausfallbzw. Kreditrisikos, zu verstehen sind. Zur Unterscheidung des Begriffspaares Ausfallund Bonitätsrisiko vgl. Rolfes (2001), S. 4. Als „expected loss“ wird der erwartete Verlust aufgrund der Risikokostenkalkulation bezeichnet (Standard-Risikokosten). Als unerwarteter Verlust (,unexpected loss") wird demgegenüber die Tatsache beschrieben, dass die Verluste aus dem Forderungsausfall die erwarteten Verluste übersteigen. Vgl. Bröker (2000), S. 14. Die Unterscheidung der Begriffe bezogen auf einen einzelnen Kredit findet sich bei Kirmße (1996), S. 13f.

79 Aufgrund der signifikanten Bedeutung des Firmenkundengeschäfts wird im Weiteren auf die Risikobewertung von Firmenkrediten abgestellt. Vgl. zu der Kreditrisikobewertung bei Privatkunden bspw. Hartmann-Wendels, Pfingsten, Weber (2000), S. $156 \mathrm{ff}$. oder Schierenbeck (1999), S. 253ff. 
Marktorientierte Kreditrisikobewertung Die Quantifizierung des Ausfallrisikos als bankbetriebliche Problemstellung

Eintrittswahrscheinlichkeit eines Verlustes entweder im- oder explizit. Hierzu kann man zum einen die Betrachtungsebenen und zum anderen die Modellableitungen als Unterscheidungskriterium heranziehen. Der Schwerpunkt der vorliegenden Arbeit liegt auf einer einzelgeschäftsbezogenen Betrachtungsebene, somit wird auf portfoliotheoretische Aspekte der Risikobewertung verzichtet. ${ }^{80}$ Es werden im Folgenden die modelltheoretischen Ansätze und anschließend heuristische Kalkulationsverfahren dargestellt.

Unter dem Begriff Modell versteht die betriebswirtschaftliche Theorie „Hilfsmittel

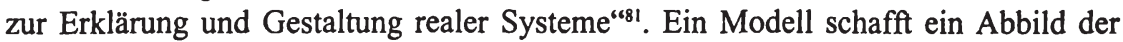
Realität. Bei diesem Vorgang wird eine gewollte Abstraktion unterstellt, d.h., die Realität wird immer nur unvollständig abgebildet ${ }^{82}$ Die Zielsetzung modelltheoretischer Ansätze zur Kreditzinskalkulation liegt in der Bewertung des Kreditrisikos.

Falls die (mathematische) Lösung des aus der Realität stammenden, im Modell vereinfacht abgebildeten Problems nicht eindeutig bestimmbar und modellimmanent nicht als optimal zu bezeichnen ist, wird das Problem als lösungsdefekt bezeichnet. ${ }^{83}$ Ein Lösungsverfahren für eine lösungsdefekte Problemstellung wird gemäß STREIM heuristisch genannt, wenn auf nicht willkürliche Art und Weise potenzielle Lösungen vom Suchprozess ausgeschlossen werden und es keine Lösungsgarantie geben kann, da ein Konvergenzbeweis nicht fuhrbar ist. ${ }^{84}$ Es sind dabei nur solche Verfahren adressiert, bei denen kein exaktes mathematisches Verfahren vorliegen kann. ${ }^{85}$ Der heuristische Lösungsprozess führt $\mathrm{zu}$ einer befriedigenden bzw. guten Lösung des Problems. ${ }^{86}$ Heuristische Kreditzinskalkulationsverfahren sind Lösungsverfahren zur Bewertung des Kreditrisikos, wobei es sich dem o.g. Verständnis folgend um eine gute, aber nicht (nachweisbar) optimale Lösung handelt.

Diese Definition bedingt, dass Heuristiken als eine Modellart verstanden werden können. Ein modelltheoretischer Ansatz zur Kreditzinskalkulation braucht eine modelldeduzierte Vorgehensweise, d.h., ausgehend von einem Bewertungsmodell wer-

80 Unter portfoliotheoretischen Aspekten werden die Diversifikations- und Risikostreuungseffekte verstanden. Vgl. Schierenbeck (1999), S. 228.

81 Adam (1996), S. 60. Vgl. zu dem Begriff Modell auch Berthel (1970), Sp. 1122ff.; Grochla (1969), S. $383 \mathrm{ff}$; Kosiol (1961), S. $318 \mathrm{ff}$.

82 Vgl. Adam (1996), S. 60.

83 Vgl. Berens (1992), S. 5. Konsequenterweise werden die im Folgenden definierten und verwendeten Begriffe Heuristik oder heuristische Planung häufig im Zusammenhang mit schlecht-strukturierten Planungsproblemen benutzt. Im Weiteren werden die Begriffe Heuristik oder heuristische Planung synonym gebraucht.

84 Vgl. Streim (1975), S. 8.

85 Vgl. Berens (1992), S. 5.

86 Vgl. Schmitting (2000), S. 37. 
Marktorientierte Kreditrisikobewertung -

Die Quantifizierung des Ausfallrisikos als bankbetriebliche Problemstellung

den die Ausfallprämien berechnet. Die heuristischen Ansätze leiten demgegenüber aus den beobachteten Gegebenheiten (wie gemessenen Ausfallwahrscheinlichkeiten) Ausfallprämien ab.

\subsubsection{Modelltheoretische Ansătze}

In Kap. 2.3.2 wird zunächst ein Überblick und eine Systematisierung der bekannten Ansätze zur modelltheoretischen Bewertung des Ausfallrisikos gegeben. Anschließend werden (Kap. 2.3.2.1 und Kap. 2.3.2.2) die Grundmodelle der stochastischen und der optionspreistheoretischen Ansătze vorgestellt. Im Rahmen der Modellbeschreibungen werden die Wirkungszusammenhänge explizit beschrieben. Zum einen werden die Einflussfaktoren erläutert und zum anderen die funktionalen Zusammenhänge zwischen diesen und dem bewerteten Ausfallrisiko aufgezeigt. Diese modelltheoretischen Zusammenhänge werden im weiteren Analyseverlauf (Kap. 4) herangezogen, um eine Prognosemodell fur die marktorientierte Bewertung von Kreditrisiken abzuleiten.

Bei der Quantifizierung des Ausfallrisikos bestehen zwei Grundprobleme: ${ }^{87}$ Zum einen wird man mit der Modellierung diskreter, nicht-linearer Ereignisse wie der Bonitätsverschlechterung konfrontiert. Zum anderen sind vergangenheitsbezogene Marktdaten zur Prognose künftiger Kreditrisikoentwicklungen nur bedingt geeignet, da Kreditrisiken besonderen schuldnerspezifischen Faktoren ausgesetzt sind. In der Literatur sind verschiedene Modelle entwickelt worden, die das Ausfallrisiko bewerten.

Die modelltheoretischen Ansätze (vgl. Abbildung 7) lassen sich in zwei Gruppen gliedern: die stochastischen ${ }^{88}$ und die optionspreistheoretischen Ansătze. ${ }^{89}$ Die stochastischen Ansätze übertragen die Wahrscheinlichkeiten des Eintritts eines bestimmten Ereignisses direkt auf die Bewertung der Ausfallrisikos (konstante Parameter), oder greifen über die Annahme stochastischer Prozesse direkt auf die Ausfallwahrscheinlichkeit zurück (variable Parameter). ${ }^{90}$ Die verschiedenen Ansätze unterscheiden sich darüber hinaus durch die Integration von verschiedenen Parametern zur Bestimmung der Ausfallprämie. Neben den Werten „Ausfallschwere" und „Steuern" wird in einigen Ansätzen auch die „Risikoeinstellung“ einbezogen. ${ }^{91}$ Die optionspreistheoretischen Ansätze modellieren das

Vgl. hier und im Folgenden Heinke (1998), S. $93 \mathrm{ff}$.

Der Begriff stochastische Ansätze lässt sich auf die ausdrückliche Verwendung von (Ausfall-) Wahrscheinlichkeiten zurückführen.

Vgl. Das, Tufano (1996), S. 162.

Vgl. bspw. Biermann, Hass (1975); Duffie (1996).

Vgl. bspw. Yawitz (1977); Yawitz, Maloney, Ederington (1985), Skinner (1995); Wu (1991). 
Marktorientierte Kreditrisikobewertung Die Quantifizierung des Ausfallrisikos als bankbetriebliche Problemstellung

Risiko anhand der Optionspreistheorie von BLACK \& SCHOLES und MERTON. ${ }^{92}$ Hierbei wird das Eigenkapital als Option auf den Unternehmenswert interpretiert, und das bonitätsrisikobehaftete Fremdkapital als Portfolio aus einer risikolosen Fremdkapitalposition und einer verkauften Verkaufsoption auf den Unternehmenswert gesehen. ${ }^{93}$ Während in den Grundmodellen ein konstantes Zinsniveau unterstellt wird, greifen neuere Ansätze auf ein stochastisch bestimmtes, variables Zinsniveau zurück. ${ }^{94}$ Ein weiterer an den Grundmodellen geäußerter Kritikpunkt ist die Definition des „Konkurses“. Es wird annahmegmäß unterstellt, dass dieser ausschließlich bei einer Fremdkapitalzahlung möglich ist, wenn der Verkaufserlös der Unternehmensaktiva hierfür nicht mehr ausreicht, sodass hierzu (nach deutschem Konkursrecht) das Unternehmen sowohl zahlungsunfähig als auch überschuldet ist. ${ }^{95}$ Durch die gesonderte Definition eines kritischen Unternehmenswertes ${ }^{96}$ wird in dem Modell von BLACK/CoX die Überschuldung als hinreichendes Kriterium für das Eintreten des Konkurses bestimmt. ${ }^{97}$

Abbildung 7 fasst die beiden Entwicklungsrichtungen der Modellansätze zur Bonitätsbewertung zusammen und ordnet ihnen die wichtigsten Vertreter $z u{ }^{98} \mathrm{Da}$ das Ziel dieser Arbeit nicht in der Validierung eines bestimmten Modellansatzes liegt, werden im Folgenden exemplarisch zwei Modelle skizziert.

92 Vgl. Black, Scholes (1973); Merton (1974). Das Modell bezieht sich ebenfalls auf stochastische Prozesse, jedoch wird keine ausdrückliche Annahme bezüglich der Ausfallwahrscheinlichkeit benötigt.

93 Vgl. Black, Scholes (1973), S. 649ff.; Merton (1974), S. 449ff.

94 Vgl. bspw. Geske (1977); Shimko, Tejima, van Deventer (1993).

95 Vgl. Longstaff, Schwartz (1995), S. 789. Für die Übertragung dieses Tatbestandes auf das deutsche Konkursrecht vgl. Gaida (1997), S. 86.

96 Falls der aktuelle Unternehmenswert den kritischen Unternehmenswert unterschreitet, tritt der Konkurs ein.

97 Vgl. Black, Cox (1976), S. $351 \mathrm{ff}$.

98 Vgl. Heinke (1998), S. 94. 
Marktorientierte Kreditrisikobewertung -

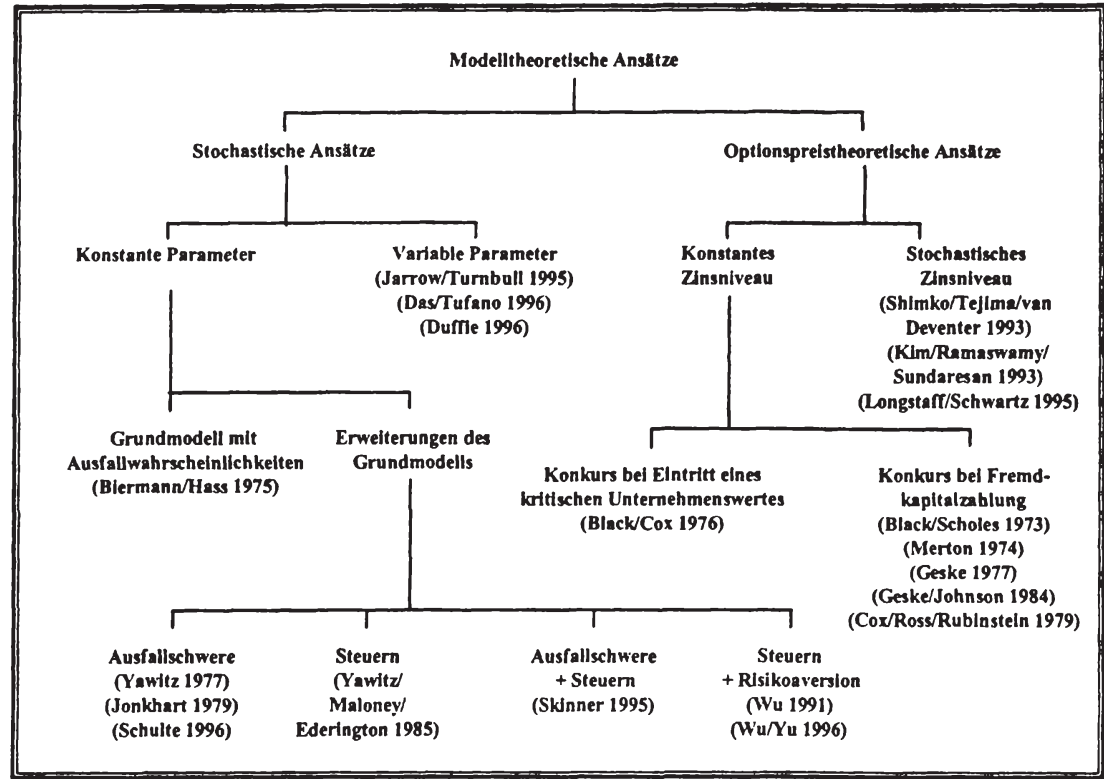

Abbildung 7:Überblick über die modelltheoretischen Ansätze zur Quantifizierung der

Bonitătsrisikoprămie 99

\subsubsection{Stochastischer Ansatz}

Eine der ersten analytischen Arbeiten, die sich mit dem stochastischen Ansatz zur Bewertung der Bonitätsrisikoprämie festverzinslicher Wertpapiere vor dem Hintergrund eines diskreten Zeitverständnisses beschäftigt, stammt von BIERMAN/HASS. ${ }^{100}$ Dieses Grundmodell untersucht den Zusammenhang zwischen der Bonitätsprämie und einer exogen gegebenen Ausfallwahrscheinlichkeit. Da die Modellerweiterung von YAWITZ ${ }^{101}$ auch die Ausfallschwere ${ }^{102}$ beachtet und somit dem Verständnis der

99 In Anlehnung an Heinke (1998), S. 94.

100 Vgl. Bierman, Hass (1975), S. 757ff., Jonkhart (1979), S. 259; Wilhelm (1983); Heinke (1998), S. 97.

101 Vgl. Yawitz (1977), S. $481 \mathrm{ff}$.

102 Die Ausfallschwere beschreibt die Möglichkeit, dass ein Kredit teilweise zurückgezahlt wird. Je höher die Ausfallschwere ist, umso geringer ist der Anteil, den der Kreditgeber vom Kreditnehmer erhält. Die Rückzahlungsquote y quantifiziert die Ausfallschwere. 
Marktorientierte Kreditrisikobewertung Die Quantifizierung des Ausfallrisikos als bankbetriebliche Problemstellung

Bonitätsprämie in dieser Arbeit folgt, wird dieser Ansatz im Weiteren beschrieben. ${ }^{103}$ Folgende Annahmen werden getroffen:

- Anleger agieren risikoneutral auf einem effizienten Kapitalmarkt. ${ }^{104}$

- Es gibt eine Überlebenswahrscheinlichkeit im Zeitpunkt $t$, mit welcher das Unternehmen alle vertraglich fixierten Zahlungen leistet; ${ }^{105}$ als Gegenwahrscheinlichkeit wird die Ausfallwahrscheinlichkeit definiert $w_{t}=1-p_{t} ;$ mit $\mathrm{p}_{\mathrm{t}}=$ Überlebenswahrscheinlichkeit (survival probability) in $t$ und $\mathrm{w}_{\mathrm{t}}=$ Ausfallwahrscheinlichkeit (default probability) in t. ${ }^{106}$

- Falls der Kredit bzw. die Anleihe (teilweise) ausfällt, erhält der Kapitalgeber bzw. Anleger unmittelbar y Prozent vom Nominalbetrag $\left(\mathrm{FK}_{\mathrm{N}}\right)$ der Forderung und y Prozent der fälligen Zinszahlung C. Vor Ausfall der Anleihe wurden alle Zahlungen pünktlich vollzogen. Die Rückzahlungsquote wird mit y beschrieben.

- T stellt den Fälligkeitszeitpunkt des Kredites bzw. der Anleihe dar, $t$ den Betrachtungszeitpunkt.

Abbildung 8 beschreibt den Zahlungsstrom. In der ersten Periode $t=1$ erhält der Investor mit einer Wahrscheinlichkeit von $\mathrm{p}_{1}$ die Zinsen und mit einer Wahrscheinlichkeit von $1-p_{1}$ die Rückzahlungsquote $y$ auf die Summe aus Zinsen und Kreditvolumen. In Periode 2 erhält er dementsprechend mit einer Wahrscheinlichkeit von $p_{1}$ mal $p_{2}$ eine Zinszahlung und mit einer Wahrscheinlichkeit von $p_{1}$ mal $1-p_{2}$ die Rückzahlungsquote multipliziert mit der Summe aus Zinsen und Kreditvolumen. ${ }^{107}$

103 Vgl. auch zu der Unterscheidung von Ausfallhöhe und Ausfallwahrscheinlichkeit Altman, Kishore (1996), S. 57.

104 Zur Definition eines effizienten Kapitalmarktes vgl. Kapitel 4.2.

105 Vgl. Bierman, Hass (1975), S. 758.

106 Die Ausfallwahrscheinlichkeit ist im Intervall $[0 ; 1]$ intervallskaliert. Festverzinsliche Wertpapiere mit $\mathrm{w}=0$ sind ausfallrisikolos. Vgl. Schulte (1996), S. 151 .

107 Die Ausfallwahrscheinlichkeit $p_{t}$ muss in den stochastischen Modellen explizit vorgegeben werden. 
Marktorientierte Kreditrisikobewertung -

Hieraus wird deutlich, dass die Wahrscheinlichkeit der vollständigen Zahlung in $t_{2}$ an die Zahlung in $\mathrm{t}_{1}$ geknüpft ist. ${ }^{108}$

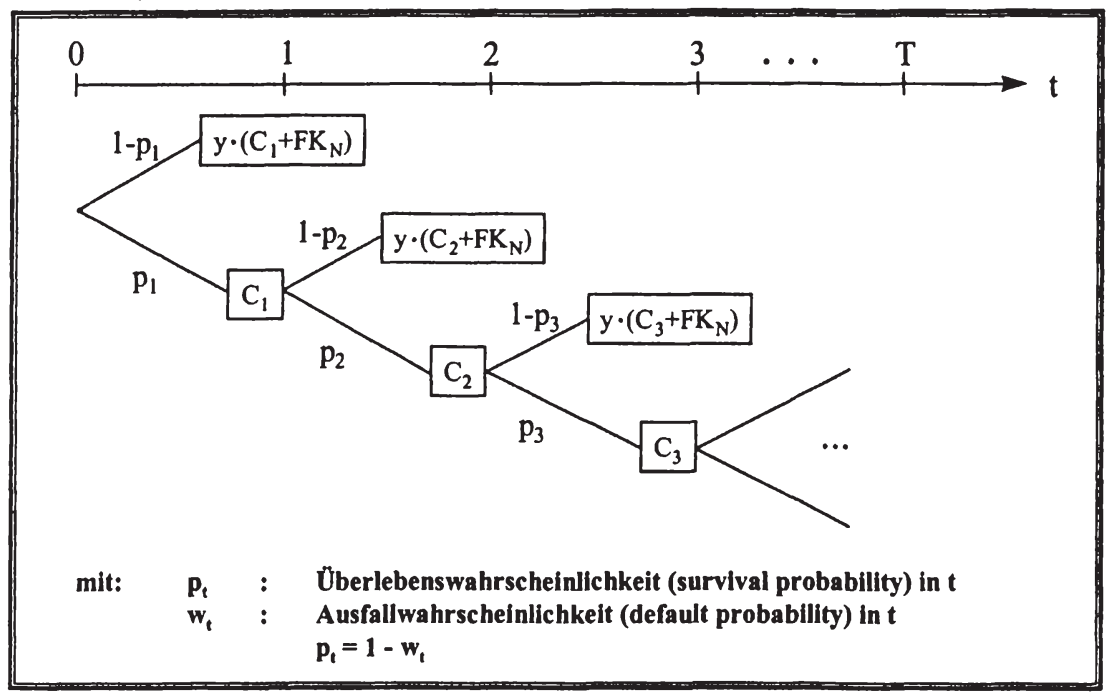

Abbildung 8: Mehrperiodiger bonitätsrisikobehafteter Zahlungsstrom ${ }^{109}$

Bei flacher Zinsstrukturkurve ${ }^{110}$, d.h. $r_{B}$ ist konstant über die Laufzeit, ergibt sich

108 Die Annahme eines vorzeitigen Laufzeitendes bei einem Ausfall notleidender Anleihen widerspricht der Wirklichkeit (vgl. Schulte (1996), S. 166). Falls eine Anleihe ausfällt, muss der Gläubiger eine Zeitlang auf die anteilige Tilgung warten. Diese Zeitverzögerung wird durch das Insolvenzverfahren begründet. Schulte kritisiert die Möglichkeit des Modells, dass der Schuldner sich unter bestimmten Parametereinstellungen im Konkursfall günstiger stellt. Nach Heinke ist dieser Fall jedoch denkbar (vgl. Heinke (1998), S. 98), und zwar, falls es sich um einen bevorrechtigten Titel handelt (vgl. Bühler (1983), S. 93 und Uhlir, Steiner (1994), S. 58). In Anlehnung an Heinke (1998), S. 99.

110 Die Zinsstrukturkurve stellt den Zusammenhang zwischen der (Rest-)Laufzeit und der Verzinsung eines Kredites bzw. einer Anleihe dar. Hierzu werden die effektiven Renditen von Nullkuponanleihen (Zerobonds) mit unterschiedlichen Laufzeiten betrachtet. Die graphische Abbildung einer Zinsstrukturkurve stellt auf der $x$-Achse aufsteigende Restlaufzeiten und auf der $y$-Achse die zugehörigen effektiven (Jahres-) Renditen der Nullkuponanleihen dar. Vgl. zu den Anleiheformen Kap. 4.3.1, zu der Definition der Zinsstrukturkurve vgl. Steiner, Bruns (1998), S. 146. 
gemäß dem Present-Value-Konzept"' (unter der Annahme, dass $p_{t}=p_{t-1}$ und $C_{t}=C_{t-1}$ ist) ein Barwert bei risikoneutraler Bewertung von:

Gleichung 1

$\mathrm{FK}_{\mathrm{M}}=\sum_{\mathrm{t}=1}^{\mathrm{T}-1} \frac{\mathrm{p}^{\mathrm{t}-1} \cdot\left(\mathrm{p} \cdot \mathrm{C}+(1-\mathrm{p}) \cdot \mathrm{y} \cdot\left(\mathrm{C}+\mathrm{FK}_{\mathrm{N}}\right)\right)}{\left(1+\mathrm{r}_{\mathrm{B}}\right)^{\mathrm{t}}}+\frac{\mathrm{p}^{\mathrm{T}-1} \cdot\left(\mathrm{p} \cdot\left(\mathrm{C}+\mathrm{FK}_{\mathrm{N}}\right)+(1-\mathrm{p}) \cdot \mathrm{y} \cdot\left(\mathrm{C}+\mathrm{FK}_{\mathrm{N}}\right)\right)}{\left(1+\mathrm{r}_{\mathrm{B}}\right)^{\mathrm{T}}}$

mit $\quad \mathrm{FK}_{\mathrm{M}}:$ Marktwert des Fremdkapitaltitels, ${ }^{112}$

$\mathrm{FK}_{\mathrm{N}}$ : Nominalwert (Rückzahlungsbetrag) des Fremdkapitaltitels,

C: Jährliche nominale Kuponzahlung der Anleihe,

$\mathrm{r}_{\mathrm{B}}$ : Bonitätsrisikoloser Zins.

Durch die vereinfachende Annahme, dass ein Agio bzw. Disagio ausgeschlossen werden kann (d.h. im Falle eines festverzinslichen Wertpapiers eine Anleihe zu pari notiert), gilt: ${ }^{113}$

Gleichung 2

$\mathrm{FK}_{\mathrm{M}}=\frac{\mathrm{C}}{\mathrm{r}}=\mathrm{FK}_{\mathrm{N}}$,

mit $\mathbf{r}$ : bonitätsrisikobehafteter Zins, Fälligkeitsrendite der Anleihe.

Die Gleichung 1 lässt sich unter Beachtung von Gleichung 2 nach $\mathrm{r}$ auflösen:

Gleichung 3

$r=\frac{1+r_{B}}{p+y-p \cdot y}-1$

und mit Gleichung 4

$e=r-r_{B}$

mit $\quad r_{\mathrm{B}}: \quad$ Rendite einer bonitätsrisikolosen Anleihe

ergibt sich

111 Vgl. Copeland, Weston (1988), S. 28ff.; Perridon, Steiner (1997), S. 212ff.; Gebhardt (1993), S. $451 \mathrm{ff}$.

112 Somit entspricht dieser Marktwert dem Barwert des Erwartungswert des Fremdkapitaltitels. Vgl. Verweise zum Present-Value-Konzept.

113 Vgl. Yawitz (1977), S. 484; Chiang, Kolb (1986), S. 195. 
Marktorientierte Kreditrisikobewertung -

Gleichung 5

$e=\frac{1+r_{B}}{p+y-p \cdot y}-1-r_{B}$

mit e: Bonitätsprämie als Differenz aus risikoloser und risikobehafteter Anleihe.

Der bonitätsrisikobehaftete Zins, welcher einer risikoadjustierten Rendite entspricht, wird mit $r$ beschrieben. Die Differenz aus $r$ und dem risikolosen Zins $r_{B}$ wird als Bonitätsprämie e definiert. ${ }^{114}$ Aus der sich ergebenden Gleichung 5 geht hervor, dass die Parameter y und $\mathrm{p}$ in einem Austauschverhältnis stehen, d.h., jede risikoadjustierte Rendite kann mittels einer Fülle von Kombinationen aus y und $p$ dargestellt werden. ${ }^{115}$ Die Kombination einer hohen Ausfallschwere mit einer niedrigen Ausfallwahrscheinlichkeit kann mit einer niedrigen Ausfallschwere und einer hohen Ausfallwahrscheinlichkeit gleichgesetzt werden. ${ }^{116}$

Aus Gleichung 5 kann man nun die verschiedenen modelltheoretischen Determinanten und deren Einflussstärken auf die Bonitätsprämie ableiten. Es werden im Weiteren die Abhängigkeiten zwischen der Bonitätsprämie und der Ausfallwahrscheinlichkeit (Fall 1), der Bonitätsprämie und der Rückzahlungsquote (Fall 2), der Bonitätsprämie und dem risikolosen Zins (Fall 3) und der Bonitätsprämie und der Restlaufzeit (Fall 4) untersucht.

Fall 1 Progressiver Funktionszusammenhang zwischen der Bonitätsrisikoprämie und der Ausfallwahrscheinlichkeiten ${ }^{117}$

Falls die Rückzahlungsquote gleich null ist $(\mathrm{y}=0)$, vereinfacht sich die Gleichung 5 dahingehend, dass e als Funktion der Ausfallwahrscheinlichkeit gesehen werden kann. Abbildung 9 zeigt unter der Annahme eines risikolosen Zinses von $r_{B}=10 \%$ den Zusammenhang zwischen Ausfallwahrscheinlichkeit und Bonitätsrisikoprämie.

Das in Abbildung 9 gezeigte progressive Steigungsverhalten drückt sich in der partiellen Ableitung erster und zweiter Ordnung nach $p$ (bzw. 1-p) aus:

114 Vgl. Yawitz (1977), S. 484; Lamy, Thompson (1988), S. 590; Chiang, Kolb (1986), S. 195.

115 Für die empirische Untersuchung im Kapitel 5 wird nicht weiter zwischen Ausfallwahrscheinlichkeit und Rückzahlungsquote unterschieden.

$116 \mathrm{Vgl}$. für die Darstellung von Kombinationen als Isoquanten Heinke (1998), S. 102f.

117 Diese Feststellung widerspricht der bekannten kapitalmarkttheoretischen Bewertungsmethodik einer linearen Risikoabgeltung. Vgl. Heinke (1998), S. 104. Für den Zusammenhang der funktionalen Beziehung und dem Rating von Agenturen, vgl. Steiner (1992), S. 514. 
Marktorientierte Kreditrisikobewertung Die Quantifizierung des Ausfallrisikos als bankbetriebliche Problemstellung

\section{Gleichung 6}

$$
\frac{\partial e}{\partial p}=-\frac{\left(1+r_{B}\right) \cdot(1-y)}{(p+y-p \cdot y)^{2}}<0
$$

Gleichung 7

$$
\frac{\partial^{2} e}{\partial p^{2}}=\frac{2 \cdot\left(1+r_{B}\right) \cdot(1-y)^{2}}{(p+y-p \cdot y)^{3}}<0 \forall(p, y) \neq(0,0) \wedge(p, y) \neq(1,1)
$$

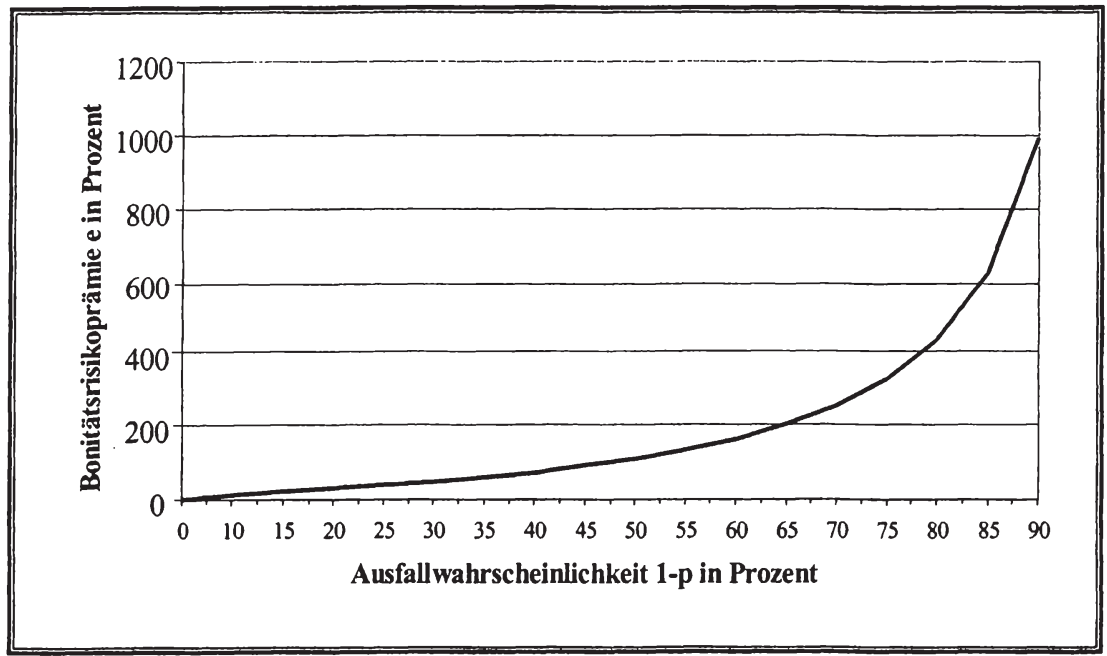

Abbildung 9: Zusammenhang zwischen Ausfallwahrscheinlichkeit und Bonitätsrisikoprämie

Fall 2 Die Steigung der Funktion „Bonitätsprämie in Abhängigkeit der Ausfallwahrscheinlichkeit" verändert sich bei variierenden Rückzahlungsquoten

Während in der Abbildung 9 eine konstante Rückzahlungsquote $(y=0)$ unterstellt wird, kann bei einer Variation $(y=] 0 ; 1[)$ dieser ein sich variierendes Steigungsverhalten der Funktion beobachtet werden. Mit steigender Rückzahlungsquote flacht die Funktion ab. ${ }^{118}$ Abbildung 10 zeigt den Funktionenraum für verschiedene Rückzahlungsquoten auf, wobei der risikolose Zins konstant bei $10 \%\left(\mathrm{r}_{\mathrm{B}}=10 \%\right)$ gehalten 
Marktorientierte Kreditrisikobewertung -

wird. ${ }^{119}$ Es wird deutlich, dass der Einfluss der Rückzahlungsquote auf die Bonitätsprämie von der unterstellten Ausfallwahrscheinlichkeit abhängt. Falls man eine niedrige (hohe) Ausfallwahrscheinlichkeit 1-p unterstellt, resultiert aus einer Veränderung der Rückzahlungsquote y eine geringe (hohe) Veränderung der Bonitätsprämie e.

Diesen formalanalytischen Zusammenhang belegen die Ableitungen erster und zweiter Ordnung, da y einen nicht-linearen Einfluss auf die Werte der zweiten Ableitung hat (Gleichung 6 und Gleichung 7).

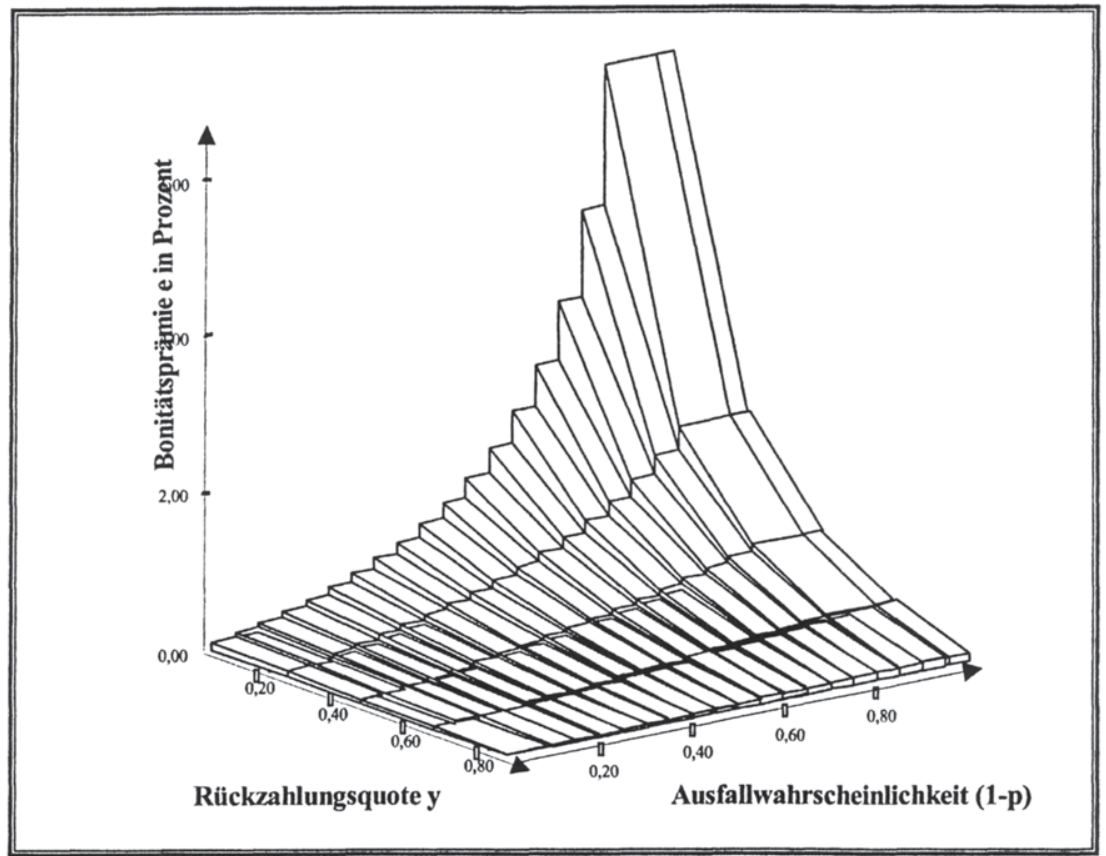

Abbildung 10: Zusammenhang zwischen Rückzahlungsquote, Ausfallwahrscheinlichkeit und Bonitätsprämie

119 Unter der Betrachtung realer Vorgänge kann man den Funktionenraum stark einschränken. So belegt eine Studie von Berblinger (1996), dass für einen Zeitraum von 21 Jahren die ermittelten Rückzahlungsquoten in Abhängigkeit von der Besicherung des Schuldtitels zwischen $16 \%$ und $53 \%$ lagen. 
Marktorientierte Kreditrisikobewertung Die Quantifizierung des Ausfallrisikos als bankbetriebliche Problemstellung

Fall 3 Linearer Zusammenhang zwischen dem risikolosen Zins und der Bonitätsrisikoprämie

Die Ableitungen der Gleichung 5 nach $r_{B}$ (risikoloser Zins) zeigt den Einfluss des Marktzinsniveaus. ${ }^{120}$

Gleichung 8

$$
\frac{\partial e}{\partial r_{B}}=\frac{1}{p+y-p \cdot y}-1>0, \text { da } p+y-p y>0 \text { für } p, y=[0 ; 1]
$$

Gleichung 9

$$
\frac{\partial^{2} \mathrm{e}}{\partial \mathrm{r}_{\mathrm{B}}^{2}}=0 \forall(\mathrm{p}, \mathrm{y}) \neq(0,0) \wedge(\mathrm{p}, \mathrm{y}) \neq(1,1) \text {. }
$$

Abbildung 11 verdeutlicht den Zusammenhang für die Variation des risikolosen Zinses und die resultierenden Bonitätsprämien. Die Rückzahlungsquote wird für die graphische Abbildung vereinfacht als $y=10 \%$ angenommen. Die Höhe der Ausfallwahrscheinlichkeit $(1-p)$ variiert in dem Bereich zwischen 10-90 Prozent. Es wird deutlich, dass ein Anstieg der Ausfallwahrscheinlichkeit zu einer größeren Steigung des Funktionsgraphen führt. Da diese jeweils partiell abgeleitet konstant ist (vgl. Gleichung 9), existiert ein linearer Zusammenhang zwischen dem risikolosen Zins und der Bonitätsprämie (bei einer unterstellten Ausfallwahrscheinlichkeit). Die Ableitung zeigt, dass ein höheres Niveau des Ausfallrisikos nicht zu unterschiedlichen Kurvenverläufen - mit Ausnahme der Steigung - führt bzw. der Wert der zweiten Ableitung (vgl. Gleichung 9) gleich Null ist.

Vgl. Schulte (1996), S. 158. 
Marktorientierte Kreditrisikobewertung -

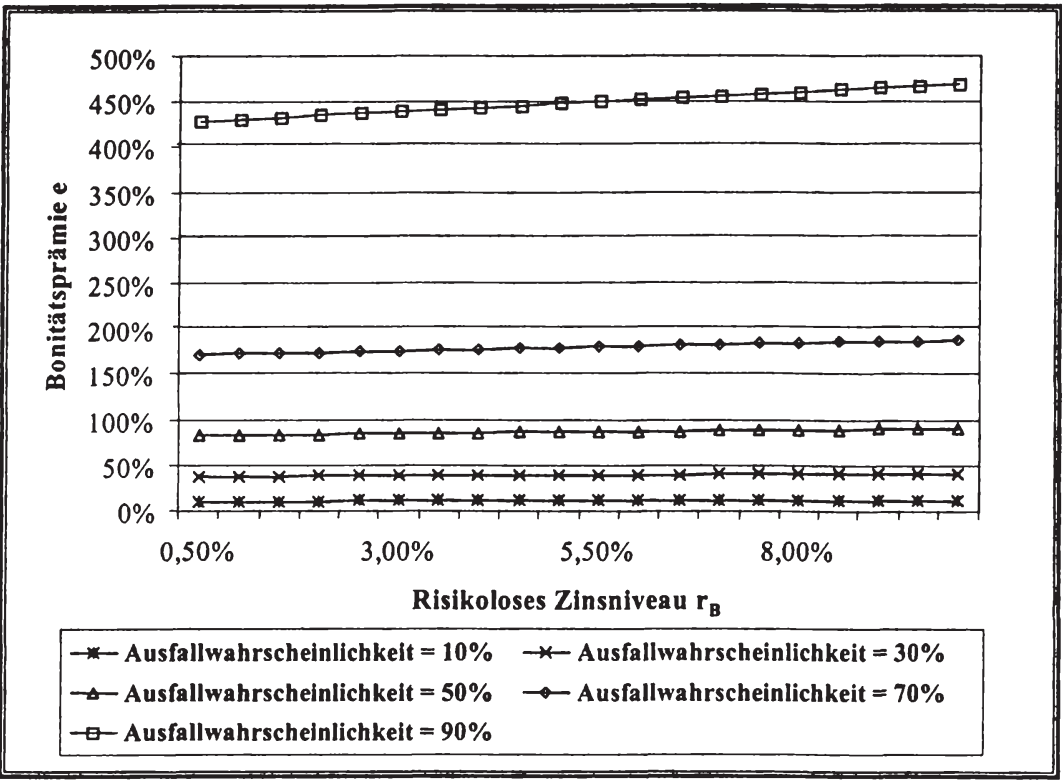

Abbildung 11: Zusammenhang zwischen Ausfallwahrscheinlichkeit, Zinsniveau und Bonitätsprämie

Fall 4 Wechselhafter funktionaler Zusammenhang zwischen der Restlaufzeit und der Bonitätsprämie

Aus Gleichung 5 geht hervor, dass kein ausdrücklicher Zusammenhang zwischen der Bonitätsprämie und der Restlaufzeit des Kredites besteht. Die Ableitung der Gleichung nach T-t (entspricht der Restlaufzeit) ist konstant null. ${ }^{121}$ Die Ausfallwahrscheinlichkeit kann jedoch als Gesamtwahrscheinlichkeit verstanden werden, d.h., die Wahrscheinlichkeit, in $\mathrm{t}$ (mit $\mathrm{t}=[0 ; \mathrm{T}])$ keine vollständige Zahlung in $\mathrm{t}_{\mathrm{T}} \mathrm{zu}$ erhalten, nimmt im Zeitablauf ab. ${ }^{122}$ Somit ist die Ausfallwahrscheinlichkeit von der Restlaufzeit abhängig; die Wahrscheinlichkeiten pro Periode $\mathrm{p}_{t}$ bleiben jedoch annahmegemäß konstant. Nach JONKHART kann der Einfluss der Restlaufzeit implizit betrachtet werden, da diese auf alle übrigen Parameter Einfluss hat. ${ }^{123}$ Die Modell-

121 Vgl. Yawitz (1977), S. 483.

122 Vgl. Schulte (1996), S. 153.

123 Vgl. hier und im Folgenden Jonkhart (1979), S. 256ff. Bei Rodriguez (1988), S. 116, kann sich der Einfluss der Restlaufzeit auf die Bonitätsprämie in Abhängigkeit von den Parametern als positiv oder negativ bzw. als monoton und nicht-monoton erweisen. 
Marktorientierte Kreditrisikobewertung Die Quantifizierung des Ausfallrisikos als bankbetriebliche Problemstellung

erweiterung erstreckt sich auf die Betrachtung der Fälligkeiten der versprochenen Zahlungen. Dazu geht die Modifikation davon aus, dass die zukünftigen Zahlungsströme reinvestiert werden. Hierzu wird der erwartete risikolose, einperiodige Zinssatz (FR) aus der aktuellen Zinsstrukturkurve abgeleitet. Somit ergibt sich die Bonitätsprämie als: ${ }^{124}$

Gleichung 10

$$
e=\frac{1-\prod_{t=1}^{T}\left(\frac{p_{t}}{1+F R_{t}}\right)-\sum_{t=1}^{T}\left(1-p_{t}\right) \cdot y_{t} \cdot \prod_{j=1}^{T}\left(\frac{p_{j-1}}{1+F R_{j}}\right)}{\sum_{t=1}^{T}\left(p_{t}+y_{t}-p_{t} \cdot y_{t}\right) \cdot \prod_{j=1}^{T}\left(\frac{p_{j-1}}{1+F R_{j}}\right)}-\frac{\prod_{t=1}^{T}\left(1+F R_{t}\right)-1}{1+\sum_{t=2 j=t}^{T} \prod_{j}^{T}\left(1+F R_{j}\right)}
$$

mit $\mathrm{FR}_{\mathrm{t}}$ : einperiodiger, risikoloser Zins für den Zeitraum $\mathrm{t}-1$ bis $\mathrm{t}$. Folglich ist der Forward Rate (FR) analog $\mathrm{zu}_{\mathrm{B}}$ aus den o.g. Gleichungen zu deuten, wobei hier die Zinsstrukturkurve eine explizite Berücksichtigung findet; der FR ist jeweils der Zins zwischen zwei unterschiedlichen Zeitpunkten,

$\mathrm{p}_{\mathrm{t}}$ : $\quad$ erwartete Überlebenswahrscheinlichkeit für das Unternehmen in $\mathrm{t}$,

$\mathrm{y}_{\mathrm{t}}$ : erwartete Rückzahlungsquote in $\mathrm{t}$,

$\mathrm{j}: \quad$ stellt einen Laufindex dar.

Falls man nun die Abhängigkeit der Bonitätsprämie von der Restlaufzeit ermitteln will, kann man die Parameter Ausfallwahrscheinlichkeit und Rückzahlungsquote konstant setzen. Dieser Zusammenhang zwischen Restlaufzeit, Zinsstrukturkurve und der Bonitätsprämie wird anhand eines numerischen Beispiels dargestellt (vgl. Abbildung 12). Hierzu werden folgende Annahmen unterstellt: feste Ausfallwahrscheinlichkeit $(1-p=0,1$; dies entspricht einer erwarteten Überlebenswahrscheinlichkeit von 90 Prozent) und feste Rückzahlungsquote $y=0,99 .{ }^{125}$

Vgl. Jonkhart (1979), S. 256.

Es gilt für die inverse Zinsstrukturkurve: $\mathrm{FR}_{1}=5 \%, \mathrm{FR}_{2}=4,5 \%, \mathrm{FR}_{3}=4,4 \%$, $\mathrm{FR}_{4}=4,3 \%, \mathrm{FR}_{5}=4,2 \% \mathrm{FR}_{6}=4,1 \%$; für die normale Zinsstrukturkurve: $\mathrm{FR}_{1}=4,1 \%$, $\mathrm{FR}_{2}=4,2 \%, \mathrm{FR}_{3}=4,3 \%, \mathrm{FR}_{4}=4,4 \%, \mathrm{FR}_{5}=4,5 \% \mathrm{FR}_{6}=5 \%$; für die flache Zinsstrukturkurve: $\mathrm{FR}_{1}=5 \%, \mathrm{FR}_{2}=5 \%, \mathrm{FR}_{3}=5 \%, \mathrm{FR}_{4}=5 \%, \mathrm{FR}_{5}=5 \% \mathrm{FR}_{6}=5 \%$. 
Marktorientierte Kreditrisikobewertung -

Es lässt sich folgendes beobachten: Je größer die Restlaufzeit ist, umso kleiner (gröBer) ist die Bonitätsrisikoprämie bei normaler (inverser) Zinserwartung. ${ }^{126 / 127} \mathrm{Bei}$ flacher Zinsstrukturkurve, d.h. $\mathrm{FR}_{\mathrm{t}}=\mathrm{FR}_{\mathrm{t}-1}$, verändert sich die Bonitätsrisikoprämie nicht durch eine Veränderung der Restlaufzeit.

Zusammenfassend kann konstatiert werden, dass im stochastischen Grundmodell nicht-lineare, multidimensionale und kontextabhängige Zusammenhänge zu beobachten sind. Die Nicht-Linearität wird beispielsweise am Zusammenhang zwischen der Ausfallwahrscheinlichkeit und der Bonitätsprämie deutlich. Das Merkmal der Multidimensionalität, d.h., die Verantwortlichkeit verschiedener Einflussfaktoren für die Wertbestimmung der Risikoprämie, verweist auf einen multivariaten Zusammenhang. Die Wirkungsweise einzelner Parameter (bspw. der Restlaufzeit) ist kontextabhängig. Dies bedeutet, dass die Wirkungsrichtung einzelner Einflussfaktoren partialanalytisch nicht eindeutig bestimmbar ist. Bspw. kann die Restlaufzeit sowohl einen positiven als auch negativen Einfluss auf die Bonitätsprämie ausüben (vgl. Abbildung 12) oder die Stärke der Einflussnahme der Rückzahlungsquote verändert sich in Abhängigkeit der Ausfallwahrscheinlichkeit (vgl. Abbildung 11). Diese Erkenntnisse werden im weiteren Analyseverlauf genutzt, um eine Prognosemodell abzuleiten (Kap. 4 und auch Kap. 5.1.1).

126 Alternativ ziehen Chiang, Kolb eine stationäre und zeitinvariante Verteilung des Firmeneinkommens heran, damit die Annahme der konstanten Überlebenswahrscheinlichkeit aufgehoben werden kann. Vgl. Chiang, Kolb (1986), S. $191 \mathrm{ff}$. Als Ergebnis erhalten sie auch bei einer flachen Zinsstrukturkurve eine abhängige Bonitätsprämie (in Abbildung 12 handelt es sich um eine konstante Funktion, wodurch die Unabhăngigkeit von Restlaufzeit und Bonitätsprämie ausgedrückt wird).

127 Vgl. zu weiteren Abhängigkeiten wie die Determinanten der Kursreagibilität oder der Einfluss des Nennwertes bspw. Heinke (1998), S. $110 \mathrm{ff}$. 


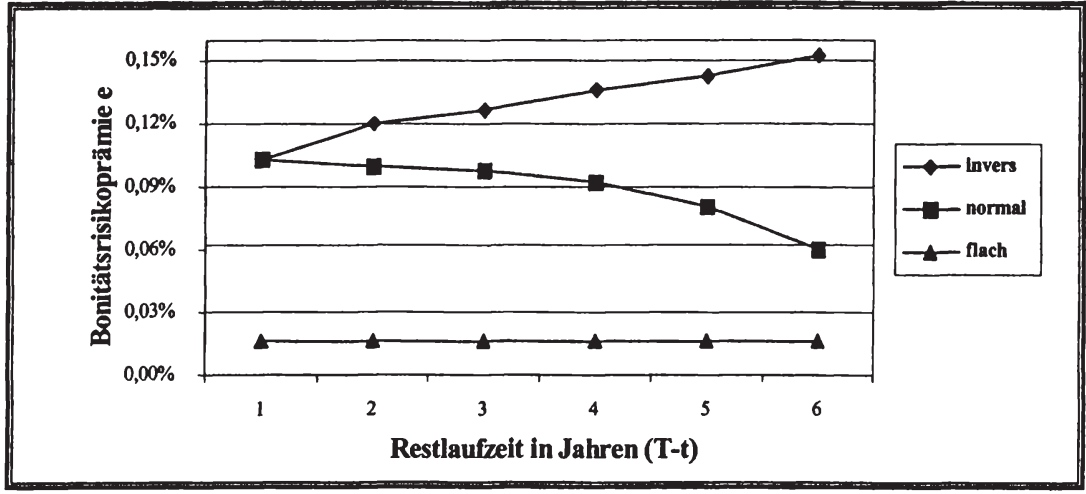

Abbildung 12: Zusammenhang zwischen Restlaufzeit, Zinsstrukturkurve und Bonitätsprămie

\subsubsection{Optionspreistheoretischer Ansatz}

Das auf dem Optionspreismodell basierende Kreditzinskalkulationsverfahren wird von KROB als der maßgebliche Ansatz zur kreditnehmerspezifischen Bewertung von Kreditrisiken bezeichnet. ${ }^{128}$ Darüber hinaus wird in der Literatur für den Bereich der marktpreisorientierten Ansätze das Capital Asset Pricing Model (CAPM) ${ }^{129}$ genannt. ${ }^{130 / 131}$

Der optionspreistheoretische Ansatz geht auf die klassische Optionspreistheorie von BLACK \& SCHOLES und MERTON zurück. ${ }^{132}$ Durch die Einbeziehung der Werte aller Vermögensgegenstände eines Unternehmens (Firmenwert) in die jeweiligen Bewertungen werden diese Modelle auch als Firmenwertmodelle bezeichnet. Die Grundüberlegung des Modells ist, dass die Eigenkapitalgeber am Tag der Kreditvergabe bzw. Fremdkapitalaufnahme das Unternehmen zum Nominalwert an die Gläubiger verkaufen, jedoch behalten sie das Recht, „ihr“ Unternehmen zu einem

Vgl. Krob (2001), S. 175. Die Idee der Deutung von Eigen- und Fremdkapitalpositionen geht auf eine ganze Reihe von Veröffentlichungen zurück. Vgl. zu einem Überblick Kirmße (2001), S. 159.

129 Das Capital Asset Pricing Model geht auf die Veröffentlichungen von Lintner (1965), Mossin (1966) und Sharpe (1964) zurïck.

130 Für einen Überblick der marktpreisorientierten Ansätze vgl. Jurgeit (1989), S. 29ff. Das CAPM wird neben dem Optionspreismodell als wichtigstes Verfahren charakterisiert. Vgl. Bröker (2000), S. 52.

131 Vgl. zu der Anwendung des CAPM als Kreditzinskalkulationsverfahren bspw. Rudolph (1994), S. 896f.; Bröker (2000), S. 53ff.; vgl. zu der Kritik Teschner (1998), S. 238. Vgl. Black, Scholes (1973) und Merton (1974). 
Marktorientierte Kreditrisikobewertung -

vereinbarten Basispreis bei Fälligkeit zurückzukaufen. ${ }^{133 / 134}$ Die Kreditaufnahme wird durch die Zahlung im Rahmen des fiktiven Unternehmenskaufes nachgestellt. Der Wert des Kredites lässt sich aus dem Wert des Rückkaufrechtes der Eigentümer ableiten. Somit unterliegt diesem Modell die Annahme, dass die Kreditgeber eine Stillhalterposition einer europäischen Kaufoption ${ }^{135}$ auf das Eigenkapital der Schuldner eingehen. ${ }^{136}$ Der Ausübungspreis der Option ist bestimmt über die Höhe des Nennwertes der Verbindlichkeit (Tilgungspreis). Der Marktwert des Unternehmens im Zeitpunkt $\mathrm{t}\left(\mathrm{V}_{\mathrm{T}}\right)$ setzt sich zusammen aus dem Marktwert des Eigenkapitals $\left(\mathrm{EK}_{\mathrm{T}}\right)$ und dem Marktwert des Fremdkapitals $\left(\mathrm{FK}_{M, T}\right)$ :

Gleichung 11

$\mathrm{V}_{\mathrm{T}}=\mathrm{EK}_{\mathrm{T}}+\mathrm{FK}_{\mathrm{M}, \mathrm{T}}$,

mit $\mathrm{V}_{\mathrm{T}}$ : Marktwert des Unternehmensvermögens in $\mathrm{t}=\mathrm{T}$;

$\mathrm{EK}_{\mathrm{T}}$ : Eigenkapitalmarktwert in $\mathrm{t}=\mathrm{T}$;

$\mathrm{FK}_{\mathrm{M}, \mathrm{T}}$ : Fremdkapitalmarktwert in $\mathrm{t}=\mathrm{T}$.

Hinsichtlich der Bestimmung des Kreditrisikos ist es wichtig, den Marktwert des Fremdkapitals zu ermitteln. Dieser ergibt sich aus der Differenz zwischen dem Marktwert des Unternehmens und des Eigenkapitals.

Gleichung 12

$\mathrm{FK}_{\mathrm{M}, \mathrm{T}}=\mathrm{V}_{\mathrm{T}}-\mathrm{EK}_{\mathrm{T}} \cdot{ }^{137}$

Die modelltheoretische Entwicklung des Firmenwertes (V), d.h. die Veränderung dV, stellt sich annahmegemäß als stochastischer Prozess in Form einer geometrischen

133 Vgl. zur Anwendung des Optionspreismodells als Kalkulationsverfahren: Black, Scholes (1973), S. 649; Merton (1974), S. 449ff.; Copeland, Weston (1988), S. $248 f$. und S. 464ff.; Perridon, Steiner (1997), S. 507ff.; Uhlir, Steiner (1994), S. $291 \mathrm{ff}$; Franke, Hax (1994), S. 495; Rudolph (1994), S. 898ff.; Jurgeit (1989) S. 34ff.; Klug (1985), S. 54ff.; Kirmße (1996), S. 76ff.; Gaida (1997), S.43ff.; Heinke (1998), S. $124 \mathrm{ff}$.

134 Alternativ kann man auch die Fremdkapitalposition als Verkauf einer Verkaufsoption in Kombination mit einer risikoreichen Kapitalanlage deuten. Vgl. bspw. Kirmße (1996), S. $80 \mathrm{ff}$.

135 Vgl. zur Unterscheidung von europäischen und amerikanischen Kaufoptionen Maltzan (2000), S. 836.

136 Folgenden Annahmen werden zugrunde gelegt: Alle Finanztitel werden kontinuierlich auf einem vollkommenen Kapitalmarkt gehandelt, es herrschen homogene Erwartungen über das Investitionsrisiko, es existiert ein risikoloser Zinssatz, es gibt über die Laufzeit keine Auszahlungen. Vgl. Bröker (2000), S. $131 \mathrm{f}$.

137 Vgl. Copeland, Weston (1988), S. 465 oder Rudolph (1994), S. 900. 
Marktorientierte Kreditrisikobewertung Die Quantifizierung des Ausfallrisikos als bankbetriebliche Problemstellung

BRowNschen Bewegung (Konzept des Wiener Prozesses W) mit einer konstanten Rendite $(\mu)$ und einer konstanten Volatilität $(\sigma)$ dar. ${ }^{138}$ Es gilt:

Gleichung 13

$d V_{t}(t)=\mu V_{t} d t+\sigma V_{t} d W(t)$.

Da das Eigenkapital als Option auf das gesamte Unternehmen interpretiert wird, kann $\mathrm{EK}_{t=0}$ mit Hilfe der Optionspreistheorie ermittelt werden:

Gleichung $14^{139}$

$\mathrm{EK}_{0}=\mathrm{V}_{0} \cdot \mathrm{N}\left(\mathrm{d}_{1}\right)-\mathrm{FK}_{\mathrm{N}} \cdot e^{-\mathrm{r}_{\mathrm{B}} \cdot \mathrm{T}} \cdot \mathrm{N}\left(\mathrm{d}_{2}\right)$,

mit $d_{1}=\frac{\ln \left(\frac{V_{0}}{F K_{N}}\right)+\left(r_{B}+0,5 \cdot \sigma_{V}{ }^{2}\right) \cdot T}{\sigma_{V} \cdot \sqrt{T}}$ und $d_{2}=d_{1}-\sigma_{V} \cdot \sqrt{T}$.

Somit ergibt sich der Marktwert des Fremdkapitals als:

Gleichung 15

$\mathrm{FK}_{\mathrm{M}, 0}=\mathrm{V}_{0}-\mathrm{V}_{0} \cdot \mathrm{N}\left(\mathrm{d}_{1}\right)+\mathrm{FK}_{\mathrm{N}} \cdot e^{-\mathrm{r}_{\mathrm{B}} \cdot \mathrm{T}} \cdot \mathrm{N}\left(\mathrm{d}_{2}\right)$

mit $\sigma_{\mathrm{v}}$ : Standardabweichung der Wachstumsrate des Unternehmensvermögens, oft vereinfachungsgemäß lediglich Volatilität des Unternehmenswertes genannt;

$\mathrm{FK}_{\mathrm{N}}:$ Nominalbetrag des Kredites;

$\mathrm{N}\left(\mathrm{d}_{\mathrm{i}}\right)$ : Verteilungsfunktion der Standardnormalverteilung, mit $\mathrm{i}=1,2$;

$e: \quad$ EULERsche Zahl, $e=\sum_{\mathrm{v}=0}^{\infty} \frac{1}{\mathrm{v} !}=2,70828182 \ldots . . .^{140}$

Während es nun im ersten Schritt möglich ist, den Marktwert des Fremdkapitals zu bestimmen, muss in einem zweiten Schritt die Verzinsung dieser risikobehafteten Position ermittelt werden. Dies geschieht, indem zunächst der Wert der Fremdkapi-

138 Vgl. Black, Scholes (1973), S. 640ff.; Mann (1995), S. 300; Grünwald (1980), S. 186f.; Hull (1997), S. 237ff.; Kjer (1981), S. $52 \mathrm{ff}$.

139 Vgl. Black, Scholes (1973), S. 637ff.; Perridon, Steiner (1997), S. 507; Rudolph (1994), S. $901 \mathrm{f}$.

140 Vgl. Reinhardt, Soeder (1987), S. 309. Die Eulersche Zahl wird in der Literatur wie auch die Bonitätsprämie mit e dargestellt. In Abgrenzung zu dieser setzt der Autor die Eulersche Zahl kursiv (e). 
Marktorientierte Kreditrisikobewertung -

talposition einer risikolosen Anlage (Nullkuponanleihe) gegenübergestellt wird. Die Differenz zwischen diesen beiden ist die Bonitätsrisikoprämie e: ${ }^{141}$

Gleichung 16

$e=r-r_{B}=\frac{1}{T} \cdot \ln \left(\frac{F K_{N}}{F K_{M, 0}}\right)-r_{B}$.

Auf Grundlage von Kapitel 2.3.2.1 leiten sich die funktionalen Zusammenhänge zwischen der Prämie für das Ausfallrisiko und den Einflussfaktoren aus dem Modell $a b .{ }^{142}$ Es werden im Weiteren die Abhängigkeiten zwischen der Bonitätsprämie und dem Unternehmenswert (Fall 1), der Bonitätsprämie und dem risikolosen Zins (Fall 2) und der Bonitätsprämie und dem Verschuldungsgrad (Fall 3) untersucht.

Fall 1 Degressiv fallender funktionaler Zusammenhang zwischen dem Unternehmenswert und dem Bonitätsrisiko

Die Ableitungen der Gleichung 16 (in Verbindung mit der Gleichung 14 und der Gleichung 15) zeigen, dass der Vermögenswert zu einem bestimmten Zeitpunkt t (V) einen negativen Einfluss auf die Bonitätsrisikoprämie besitzt. Falls V c.p. steigt, fällt e ab.

Gleichung 17

$$
\frac{\partial \mathrm{e}}{\partial \mathrm{V}_{\mathrm{t}}}<0 \text { und } \frac{\partial^{2} \mathrm{e}}{\partial \mathrm{V}_{\mathrm{t}}^{2}}>0 \text {. }
$$

Dieser Zusammenhang bedingt eine monoton fallende Funktion. Die unterstellten Annahmen in der Abbildung 13 sind ein sicherer Zins von $r_{B}=10 \%$, eine Restlaufzeit von 10 Jahren, ein nominales Kreditvolumen von 1.200 Geldeinheiten (GE) und eine Standardabweichung des Unternehmenswertes von $\sigma_{\mathrm{v}}=0,3$. Die asymptotische Annäherung von e an die Nullachse verdeutlicht, dass bei einem Unternehmen mit einem besonders hohen Vermögen c.p. so gut wie kein Kreditrisiko besteht.

141 Vgl. hier und im Folgenden Merton (1974), S. 454; Jurgeit (1989), S. 182ff.; Kirmße (1996), S. 103f.

142 Vgl. Merton (1974), S. 457ff.; vgl. zu den mathematischen Ableitungen bspw. Jurgeit (1989), S. 192ff.; Klug (1985), S. 63ff.; Gaida (1997), S. 63ff.; Kirmße (1996), S. $136 f f$. 


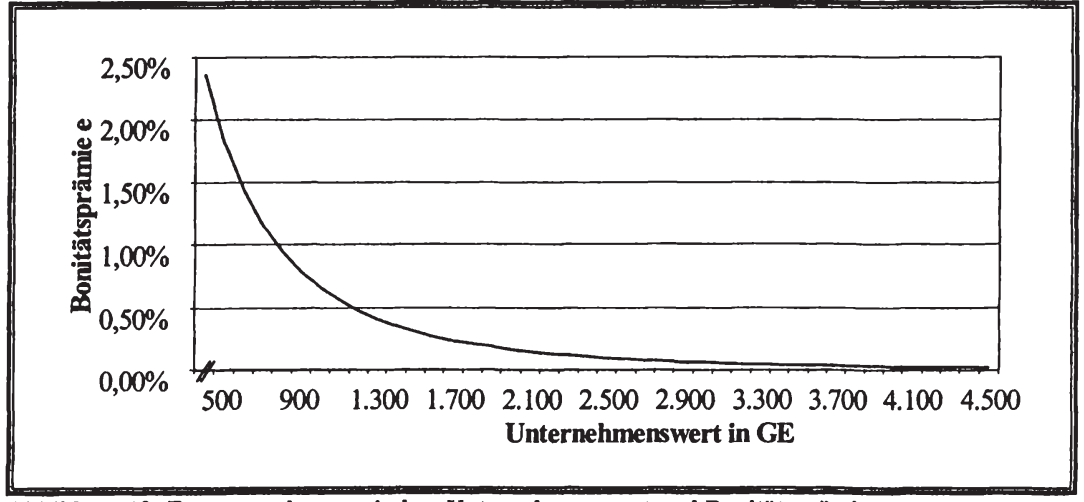

Abbildung 13: Zusammenhang zwischen Unternehmenswert und Bonitătsprämie

Fall 2 Negativer, nicht-linearer funktionaler Zusammenhang zwischen dem risikolosem Zins und der Bonitätsrisikoprämie e

Die partielle Ableitung von Gleichung 16 nach $r_{B}$ verdeutlicht den negativen Einfluss des Zinsniveaus auf den Risikoaufschlag e: ${ }^{143}$

Gleichung 18

$\frac{\partial \mathrm{e}}{\partial \mathrm{r}_{\mathrm{B}}}<0$.

Die Abbildung 14 beschreibt den Funktionsverlauf. Für die exemplarische Berechnung wurden folgende Annahmen getroffen: Der Unternehmenswert in Höhe von $1.000 \mathrm{GE}$ schwankt mit dem konstanten Wert von $\sigma_{\mathrm{v}}=0,3$. Das Kreditvolumen liegt bei $600 \mathrm{GE}$ Nominalwert. Die Restlaufzeit ist gleich 10 Jahre gesetzt.

Sowohl die erste Ableitung (Gleichung 14) als auch der exemplarisch gezeigte Funktionsverlauf (Abbildung 14) verdeutlichen den negativen Einfluss des sicheren Zinses. Falls das risikolose Zinsniveau $r_{B}$ steigt, wird eine geringere Bonitätsprämie e ausgewiesen. Die in dem Funktionsverlauf deutlich werdende negative Steigung steht den Erkenntnissen des stochastischen Ansatzes entgegen, da in dem stochastischen Modell der sichere Zins einen positiven Einfluss auf die Bonitätsprämie hat (vgl. Abbildung 11). Modellintern kann dieser Unterschied damit erklärt werden, dass der sichere Zins den Unternehmensvermögenswert Eigenkapitalwert $\mathrm{EK}_{0}$ positiv beeinflusst (vgl. Gleichung 14). Dieser gestiegene Eigenkapitalwert wiederum bedingt eine geringe Bonitätsprämie (vgl. Gleichung 16). Folglich wird durch einen höheren risikolosen Zins eine niedrigere Bonitätsprämie modelltheoretisch geschlussfolgert. 
Marktorientierte Kreditrisikobewertung -

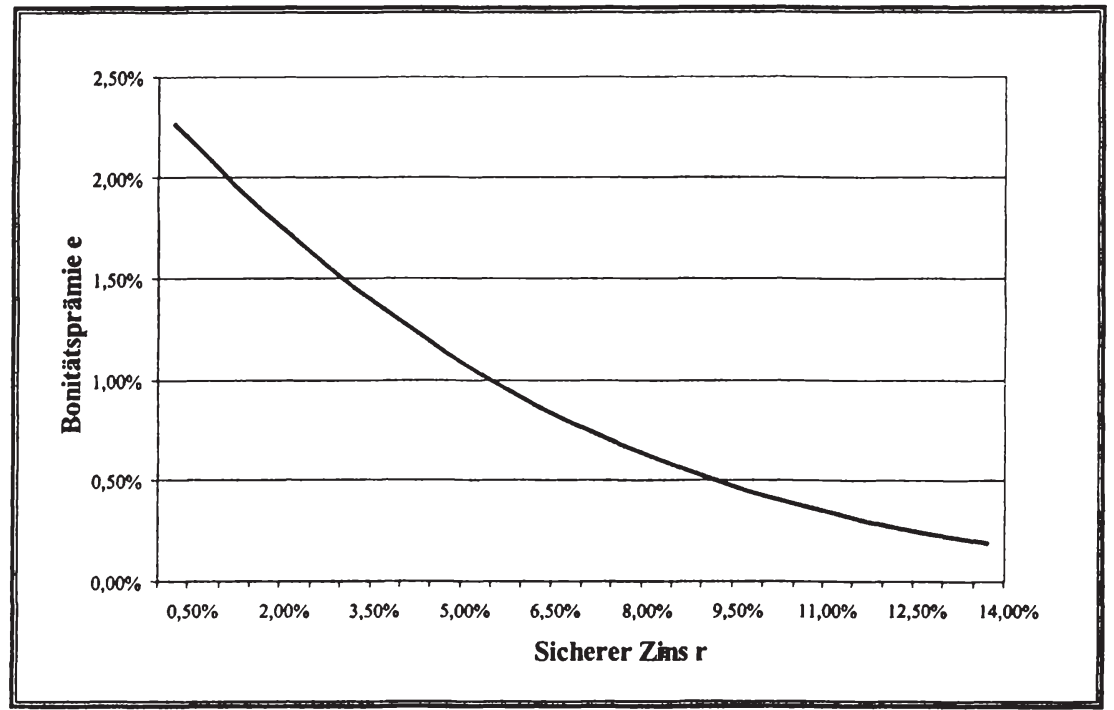

Abbildung 14: Zusammenhang zwischen dem sicheren Zins und der Bonitătsprämie

Fall 3 Die Restlaufzeit hat in Abhängigkeit von dem Verschuldungsgrad einen unterschiedlichen Einfluss auf die Risikoprämie

Die erste Ableitung von Gleichung 16 nach $T$ macht deutlich, dass für den Zusammenhang von Restlaufzeit und Bonitätsrisikoprämie keine allgemeingültige Aussage getroffen werden kann.

Gleichung 19

$\frac{\partial \mathrm{e}}{\partial \mathrm{T}}>0, \frac{\partial \mathrm{e}}{\partial \mathrm{T}}=0, \frac{\partial \mathrm{e}}{\partial \mathrm{T}}<0 .{ }^{144}$

Der Graph der Funktion kann in einem Intervall steigend, in einem anderen fallend oder in einem Intervall konstant sein. Deshalb wird von MERTON eine Fallunterscheidung vorgeschlagen. Der so genannte „Quasi-Verschuldungsgrad" L gibt den Schwellenwert der Funktionssteigungen wieder und wird definiert als: ${ }^{145}$

144 Vgl. Merton (1974), S. 457ff.

$145 \mathrm{Vgl}$. hier und im Folgenden Merton (1974), S. 455; Rudolph (1994), S. 902; Heinke (1998), S. 128f. 
Marktorientierte Kreditrisikobewertung Die Quantifizierung des Ausfallrisikos als bankbetriebliche Problemstellung

Gleichung 20

$\mathrm{L}=\frac{\mathrm{FK}_{\mathrm{N}} \cdot e^{-\mathrm{r}_{\mathrm{B}} \cdot \mathrm{T}}}{\mathrm{V}_{0}}$.

L gibt das Verhältnis zwischen dem abgezinsten Nennbetrag des Kredites $F_{N}$ und dem aktuellen Wert des Unternehmensvermögen $V_{0}$. wieder. Für den ersten Fall, mit $\mathrm{L} \geq 1$, ist die Risikoprämie stets (in Abhängigkeit von einer steigenden Restlaufzeit) fallend. Ein Wert von über $L>1$ ist einer Überschuldung gleichzusetzen, die jedoch erst bei Fälligkeit eintritt. Für den zweiten Fall - für einen Zeitpunkt ist $\mathrm{L}<1$ - ist die Funktion zunächst steigend und anschließend fallend. ${ }^{146}$

Diese partiell nicht eindeutigen Wirkungszusammenhänge zwischen Restlaufzeit und Bonitätsprämie weisen Parallelen zu den o.g. Zusammenhängen des stochastischen Modells auf. ${ }^{147}$ Analog zu den Ergebnissen des Kapitels 2.3.2.1 wird auch in den optionspreistheoretischen Modellen deutlich, dass es sich um multidimensionale, nicht-lineare Zusammenhänge handelt. Ebenfalls ist die Wirkungsweise einzelner Einflussfaktoren kontextabhängig. Dies bedeutet, dass partialanalytische Ableitungen nicht eindeutig bestimmbar sind. Bspw. bedingt der Verschuldungsgrad L, inwieweit eine höhere Restlaufzeit einen positiven oder negativen Einfluss auf die Bonitätsprämie ausübt. Wirkungsrichtungen einzelner Parameter können nur unter Kenntnis weiterer Einflussfaktoren ermittelt werden. Analog zu den Erkenntnissen des Kapitels 2.3.2.1 werden diese für die im weiteren Analyseverlauf folgende Ableitung einer Prognosemodells herangezogen.

Neben diesen modelltheoretischen Ansätzen wird im folgenden Kapitel ein Überblick über die heuristischen Ansätze zur Bestimmung des Kreditzinses bzw. der Ausfallprämie gegeben.

\subsubsection{Heuristische Ansätze}

Heuristische Ansätze im Rahmen der Kreditzinskalkulation sind dadurch gekennzeichnet, dass sich individuelle Besonderheiten und Entscheidungsparameter insbesondere der Kreditgeber auf die Höhe der Risikoprämie auswirken. ${ }^{148}$ Die Risikoprämien werden anhand dieser Spezifika abgleitet. ${ }^{149}$ Anders als die o.g. modelltheoretischen Ansätze werden die Risikoprämien nicht aus einem (abgeschlossenen) finanzierungstheoretischen Modell abgeleitet, sondern es werden mit Hilfe verschiedener

\footnotetext{
146 Vgl. Gaida (1997), S. 65.

147 Vgl. Heinke (1998), S. 128; Merton (1974), S. 457ff.; Klug (1985), S. 66ff.; Jurgeit (1989), S. 194f.

148 Vgl. Schierenbeck (1995), S. 19f.; Kirmße, (1996), S. 48.

149 Vgl. Rudolph (1994), S. 895.
} 
Marktorientierte Kreditrisikobewertung -

Die Quantifizierung des Ausfallrisikos als bankbetriebliche Problemstellung

heuristischer Lösungsverfahren, wie sie im Folgenden beschrieben werden, Ausfallrisikokosten berechnet. ${ }^{150}$ Die heuristischen Ansätze können in zwei Gruppen aufgeteilt werden: In die segmentspezifischen und die individuellen Kalkulationen (vgl. Abbildung 15)..$^{\text {s1 }}$

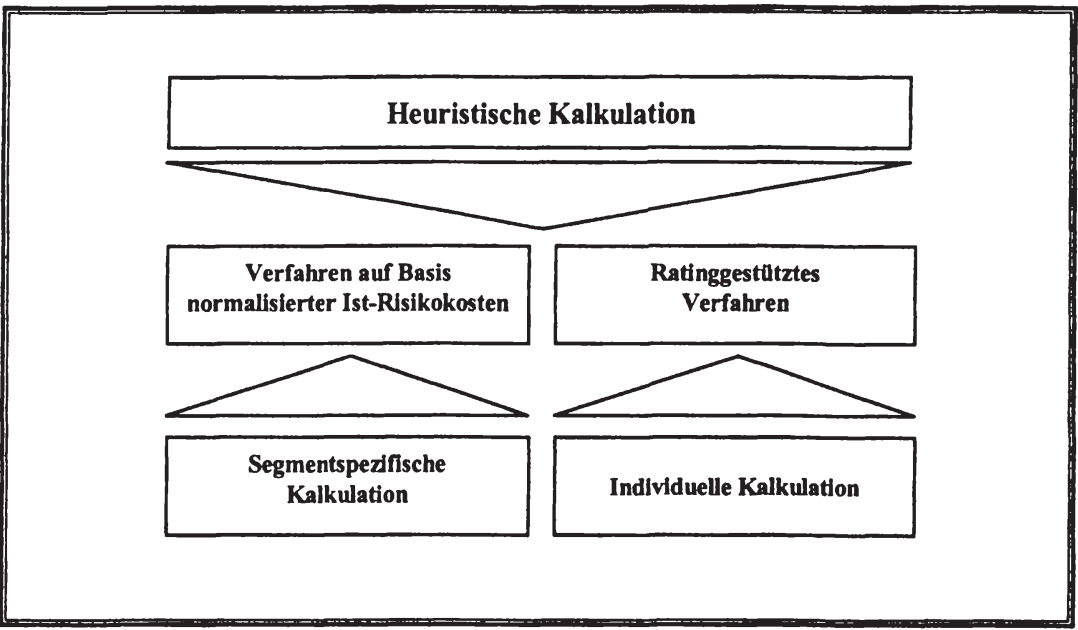

Abbildung 15: Heuristische Kalkulationsverfahren zur Ermittlung der Ausfallrisikokosten ${ }^{152}$

Die segmentspezifische Kalkulation unterteilt das gesamte Kreditportfolio einer Bank in verschiedene Segmente. Die gebildeten Segmente sollen möglichst homogen in sich und möglichst heterogen untereinander sein. Dementsprechend werden die Segmentierungskriterien abgeleitet. ${ }^{153}$ Hierzu soll ein statistischer Zusammenhang zwischen dem Segmentierungskriterium und dem Risikograd des Segmentes nachweisbar sein. Auf Basis von Ist-Risikokosten werden nach dem Versicherungsprinzip segmentspezifische Risikoprämien ermittelt. Die historischen durchschnittlichen Ausfallkosten aller Kredite in einem Segment werden im Rahmen eines Umlageverfahrens verteilt. ${ }^{154}$ Es gibt verschiedene Ansätze, das gesamte Kreditportfolio zu segmentieren. Die wichtigsten Segmentierungskriterien sind zustands-, kreditarten-, oder geschäftsfeldspezifischen Merkmalen entnommen. Abbildung 16 gibt einen Überblick.

$150 \mathrm{Zu}$ der Definition heuristischer Lösungsverfahren vgl. Kap. 2.3.1.

$151 \mathrm{Vgl}$. Kirmße (1996), S. 46ff.

152 In Anlehnung an Schierenbeck (1995), S. 19.

Is3 Vgl. Backhaus (1999), S. 159f.

154 Vgl. Rudolph (1994), S. 895; Schierenbeck, Rolfes (1988), S. 232. 


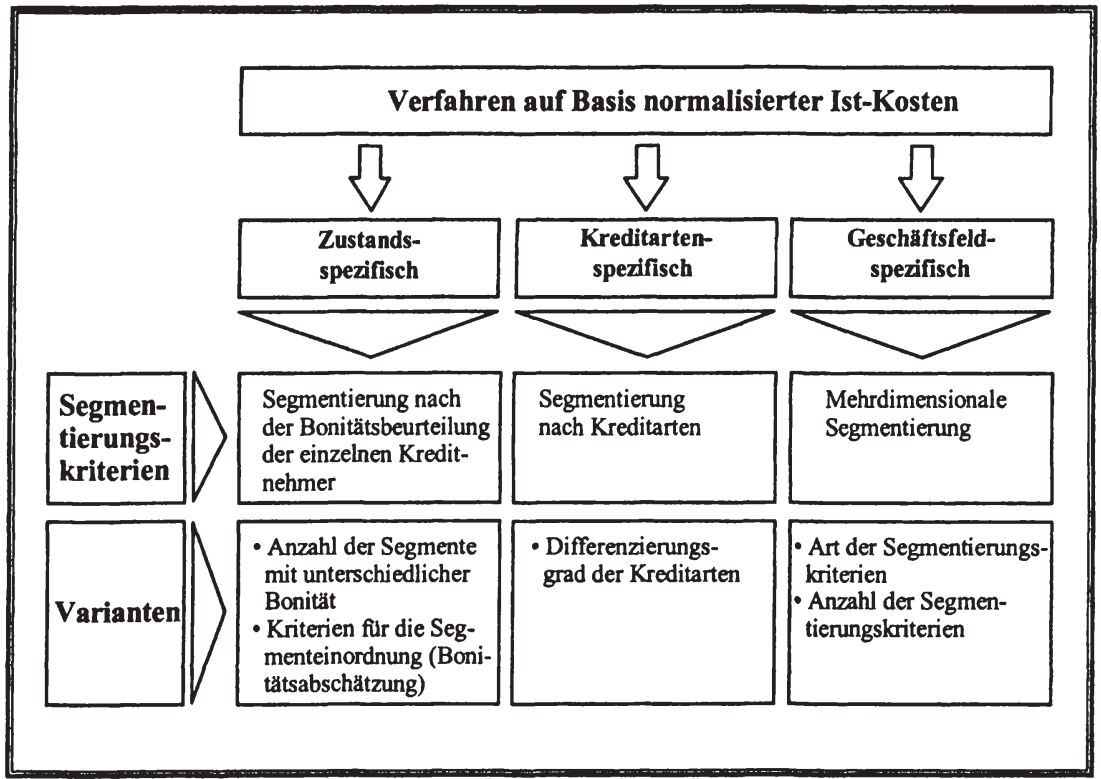

Abbildung 16: Segmentierungskriterien für segmentspezifische Kalkulationsverfahren auf Basis normalisierter Ist-Kosten ${ }^{155}$

Das zustandsspezifische Verfahren beruht auf der Bonitätseinschätzung des Kreditnehmers. Beispielsweise können die vom Institut der Wirtschaftsprüfer in Deutschland e.V. (IdW) entwickelten Risikogruppen verwendet werden und zwar: ${ }^{156}$

(1) Kredite ohne erkennbare Risiken,

(2) anmerkungsbedürftige Kredite,

(3) Not leidende Kredite und

(4) uneinbringliche Kredite.

Von verschiedenen Autoren werden Variationen bzgl. der Anzahl der gebildeten Segmente und der Segmentierungskriterien vorgeschlagen. Die Kriterien für die Bonitätseinschätzung orientieren sich dabei an den verschiedenen bekannten Verfahren der Kreditwürdigkeitsprüfung. ${ }^{157}$

155 In Anlehnung an Kirmße (1996), S. 54.

156 Vgl. Bankenfachausschuß des IdW (1978), S. 490.

157 Vgl. Süchting (1993), S. 9; Weinrich (1978), S. 184; Neuber (1988), S. 300. 
Marktorientierte Kreditrisikobewertung -

Die Quantifizierung des Ausfallrisikos als bankbetriebliche Problemstellung

Kreditarten stellen eine weitere Möglichkeit der Segmentierung dar. Beispielsweise können die Kreditarten Kontokorrent und Ratenkredit unterschieden werden, sodass sich für die unterschiedlichen Kreditarten unterschiedliche Risikoprämien ergeben, ${ }^{158}$ falls in der Vergangenheit diese verschiedenen Kreditartensegmente unterschiedliche Ist-Ausfallkosten auswiesen.

Die geschäftsfeldspezifische Verfahrensweise sieht eine mehrdimensionale Segmentierung vor. Dies bedeutet, dass verschiedene Kriterien gemeinsam einzelne Segmente beschreiben. Bspw. kann ein Segment aus den drei Kriterien Kreditnehmertypus, Produktart und Art der Besicherung gebildet werden. ${ }^{159}$ Die Kriterien wiederum können aufgrund von statistischen Untersuchungen oder auch heuristischen Ansätzen gewonnen werden. ${ }^{160}$

Zur Kalkulation der Risikoprämie innerhalb eines Segments werden regelmäßig die institutsspezifischen Ist-Risikokosten verwendet. ${ }^{161}$ Es wird das Verhältnis zwischen den angefallenen (i.d.R. normalisierten) Risikokosten und dem segmentspezifischen Kreditvolumen gebildet. ${ }^{162}$ Das Ergebnis ist somit eine segmentspezifische Ausfallrate. ${ }^{163} \mathrm{Da}$ ausschließlich nicht ausfallbedrohte Kredite die aufgetretenen Ausfälle tragen können, wird diese Ausfallquote entsprechend korrigiert. „Durch eine regelmäßige Wiederholung dieser Berechnungen und entsprechende Anpassung der Kreditkonditionen soll die Erfüllung des (...) Versicherungsprinzips zumindest in längerer Frist erreicht werden." 164

Individuelle Verfahren verzichten auf jegliche Art von Umlageverfahren ${ }^{165}$ mittels Durchschnittsbildung. Die Risikokosten bzw. die Risikoeinschätzung wird aus dem Risikogehalt des individuellen Kredites abgeleitet. Somit sind die ermittelten Risikokosten bankunabhängig und ausschließlich kreditspezifisch. ${ }^{166}$

Ein mögliches Kalkulationsverfahren unter den heuristischen Ansätzen ist das ratinggestützte Verfahren. Es wird zwischen internen und externen Ratingystemen unter-

Vgl. Kilhey (1987), S. 106f.

Vgl. Schierenbeck, Rolfes (1988), S. 235ff.; Villiez, (1989), S. 149ff.

Vgl. Schierenbeck, Rolfes, (1988), S. 234 f.

Eine Ausnahme bildet die Vorgehensweise beim marktdeduzierten Ansatz von Brakensiek. Innerhalb des Verfahrens werden segmentspezifische Ausfallraten des Marktes verwendet. Vgl. Brakensiek (1991), S. 166ff.; Kirmße (1996), S. $55 \mathrm{ff}$.

Vgl. Schierenbeck (1995), S. 19.

Vgl. Schierenbeck (1999), S. 300f.

Kirmße (1996), S. 49.

Diese Voraussetzung gilt es im Weiteren zu prüfen. Bspw. wird bei der Erhebung von einem Rating zwar zunächst eine individuelle Bewertung vorgenommen, jedoch ist die Zuordnung von Risikokosten problematisch (vgl. Kap. 2.5).

Vgl. Rudolph (1994), S. 897. 
schieden. ${ }^{167}$ Ausgangspunkt des Verfahrens ist das Rating eines Unternehmens bzw. eines bestimmten Finanztitels. Das (externe) Rating - im Fall der Beurteilung eines Schuldtitels wird von einem Credit Rating gesprochen - spiegelt die durch ein spezielles Symbol ausgedrückte Meinung wider. Diese basiert auf einer Bonitätsanalyse über einen Schuldner hinsichtlich seiner Fähigkeit und rechtlichen Bindung zur vereinbarten Rückzahlung der Zins- und Tilgungsverbindlichkeiten in einer festgelegten Art und Weise. ${ }^{168}$ Bei einem externen Rating wird diese Einschätzung von einer hierauf spezialisierten Agentur vorgenommen. ${ }^{169}$

Das Analyseobjekt kann unterschiedlichster Natur sein. ${ }^{170}$ Es wird zwischen einem Emittenten- und einem Emissionsrating unterschieden. ${ }^{171}$ Bei einer nichtnachrangigen Anleihe ohne besondere Sicherheiten ist das Emittentenrating gleich dem Emissionsrating. ${ }^{172}$

Das als Rating ausgedrückte Qualitätsurteil umfasst quantitative und qualitative Faktoren. Für den Bereich des quantitativen Rating wird methodisch meist ein computergestütztes mathematisch-statistisches Beurteilungssystem für die Jahresabschlussanalyse verwendet. ${ }^{173 / 174}$ Veröffentlichte Finanzinformationen bilden somit die Basis dieses ersten Teils des Rating. „Die Auswahl der in die Verfahren eingehenden Kriterien bleibt subjektiv“175, da sie von der Ratingagentur bestimmt werden. Es werden sowohl das Bewertungsverfahren als auch die Bewertungsgewichte agenturspezifisch festgelegt. ${ }^{176}$

Das Ziel des qualitativen Rating ist die Beurteilung von nur bedingt objektivierbaren und quantifizierbaren Faktoren wie z.B. die Beurteilung der Managementqualität, die Einschätzung zukünftig bonitätsrelevanter Erfolgspotenziale oder die Gesamtbeurtei-

167 Kirmße sieht die bankinternen Ratingysteme als Verfahren des Segmentierungsansatzes, da sie auf Basis von normalisierten Ist-Risikokosten die Risikoprämien kalkulieren. Vgl. Kirmße (1996), S. 48.

168 Vgl. Berblinger (1996), S. 31; Everling (1991), S. 29; Everling (1995), Sp. 1605.

169 Die bekanntesten Ratingagenturen sind die US-amerikanischen Unternehmen Standard \& Poor's Corporation und Moody's Investors Service Inc., jeweils mit dem Hauptsitz in New York.

170 Vgl. Steiner, Heinke (1996), S: 1694.

171 Vgl. Heinke (1998), S. $18 f$.

172 Vgl. Schulte (1996), S. 96.

$173 \mathrm{Vgl} . \mathrm{zu}$ den unterschiedlichen Verfahrensweisen der Bilanzanalyse und dem Begriffspaar Bilanz- und Jahresabschlussanalyse Kap. 3.

174 Vgl. Steiner (1992), S. 513; Everling (1991), S. 126.

175 Heinke (1998), S. 25; vgl. ebenfalls Kapitel 2.5.

176 Vgl. Kirmße (1996), S. 69. 
Marktorientierte Kreditrisikobewertung -

lung der Unternehmensstrategie. ${ }^{177}$ Während die Informationsbasis auch veröffentlichte Informationen umfasst, werden die für den qualitativen Bereich darüber hinausgehenden Informationen regelmäßig in intensiven Gesprächen mit dem Management auf verschiedenen Hierarchieebenen gewonnen. ${ }^{178}$ Von den Agenturen werden für das qualitative Rating sowohl die Auswahl als auch die Gewichtung der einzelnen qualitativen Merkmale nicht bekannt gegeben. ${ }^{179}$

Der Risikogehalt eines Kreditnehmers wird über ein Indikatorensystem ermittelt, indem einzelne Indikatoren mittels eines Punktesystems zu einem Gesamturteil (Rating) verdichtet werden. ${ }^{180}$ Hierzu wird mittels eines Top-Down-Ansatzes das Länder, Branchen-, Unternehmens- und Titelrisiko abgeschätzt. ${ }^{181}$ Zunächst werden das Länder- und das Branchenrisiko durch weitere Subkriterien ermittelt, wie z.B. Gefahr der wirtschaftlichen Instabilität, Devisen- oder Transferbeschränkungen, Marktwachstum oder konjunkturelle Ergebnisabhängigkeit. ${ }^{182}$ Das Unternehmensrisiko wird in die Bestandteile Geschäftsrisiko und finanzielles Risiko aufgeteilt. Die Bewertung des Geschäftsrisikos beinhaltet ein besonders hohes $\mathrm{Ma} ß$ an Subjektivität, da hier über eine branchenspezifische Checkliste Urteile ermittelt werden, bspw. über Qualitäten des Management oder des Marketing (qualitative Komponente des Ratingprozesses). Das finanzielle Risiko wird über die bereits o.g. Bestandteile eines quantitativen Rating beurteilt. Die Beurteilung des Titelrisikos orientiert sich an den emissionsspezifischen Besonderheiten wie der Besicherung. ${ }^{183}$ Die innerhalb dieses Indikatorensystems verwendeten Gewichte können von den Ratingagenturen verändert werden. ${ }^{184}$

Das Ergebnis des Ratingprozesses wird durch ein Symbol dargestellt. Die verschiedenen Risikoklassen werden mit unterschiedlichen Symbolen charakterisiert und werden von den Agenturen individuell definiert. Tabelle 1 zeigt die qualitative Einschätzung des Bonitätsrisikos der beiden Ratingagenturen STANDARD \& POOR'S CORPORATION (S\&P) und MOODY's INVESTOR SERVICE INC. (MOODY's) für langfristige Emissionsrating bzw. Emittentenrating auf. ${ }^{185}$ Hierbei kommt der Unterscheidung zwischen den gebildeten Klassen „Investment" und „Speculative“ eine besondere Bedeutung zu, da hierdurch ein starker Einfluß auf die Aufnahmemöglichkeiten

17 Vgl. Everling (1991), S. 125; Steiner (1992), S. 513.

178 Vgl. bspw. Berblinger (1996), S. 60.

179 Vgl. Heinke (1998), S. 26.

180 Vgl. Rudolph (1994), S. 895; Schierenbeck (1995), S. $19 f$.

181 Vgl. Berblinger (1996), S. 64ff., Steiner (1992), S. $511 \mathrm{ff}$.; Everling (1991), S. $139 \mathrm{ff}$.

182 Vgl. hier und im Folgenden Heinke (1998), S. 27ff.

183 Die Beurteilung der titelspezifischen Risiken bedingt eine emissionsspezifische Beurteilung und somit ein Emissionsrating.

184 Vgl. Schneider (1992), S. 601.

185 Vgl. Steiner (1992), S. 510. 
Marktorientierte Kreditrisikobewertung -

durch den Kapitalmarkt genommen wird. Bspw. sind einige Banken in den USA verpflichtet, keinerlei Anleihen aus der Klasse „Speculative“ in ihrem Bestand zu halten. ${ }^{186}$

\begin{tabular}{|c|c|c|c|}
\hline & $\begin{array}{c}\text { Standard \& } \\
\text { Poor's }\end{array}$ & Moody's & Bedeutung der Symbole \\
\hline \multirow{4}{*}{ 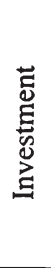 } & AAA & Aaa & Extrem starke Zinszahlungs- und Tilgungskraft des Emittenten \\
\hline & $\mathrm{AA}$ & $\mathrm{Aa}$ & Sehr starke Zinszahlungs- und Tilgungskraft \\
\hline & A & A & $\begin{array}{l}\text { Gute Zinszahlungs- und Tilgungskraft (Schuldner ist aber anfäl- } \\
\text { liger für negative Wirtschaftsentwicklung als mit AA oder Aa } \\
\text { bewertete Emittenten) }\end{array}$ \\
\hline & $\mathrm{BBB}$ & $\mathrm{Baa}$ & $\begin{array}{l}\text { Ausreichende Fähigkeit, Zins- und Tilgungszahlungen zu leisten } \\
\text { (Ungünstige Wirtschaftsentwicklung oder ein verändertes Um- } \\
\text { feld können jedoch die Fähigkeit zur Schuldenbedienung stärker } \\
\text { beeinträchtigen als in höheren Ratingklassen) }\end{array}$ \\
\hline \multirow{8}{*}{ 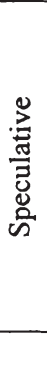 } & BB & $\mathrm{Ba}$ & $\begin{array}{l}\text { Noch ausreichende Zinszahlungs- und Tilgungskraft, aber } \\
\text { Gefährdungselemente vorhanden, die zu ungenügender Ein- } \\
\text { stufung führen können }\end{array}$ \\
\hline & B & B & $\begin{array}{l}\text { Derzeit noch ausreichende Zinszahlungs- und Tilgungskraft, aber } \\
\text { starke Gefährdungselemente vorhanden }\end{array}$ \\
\hline & CCC & & Starke Tendenz zu Zahlungsschwierigkeiten \\
\hline & $\mathrm{CC}$ & & $\begin{array}{l}\text { Symbole werden für nachrangige Verbindlichkeiten verwendet, } \\
\text { wenn Emittent z.B. mit CCC bewertet wird }\end{array}$ \\
\hline & & Caa & Zinszahlungen eingestellt oder stark gefährdet \\
\hline & & $\mathrm{Ca}$ & Zinszahlungen eingestellt \\
\hline & $\mathrm{D}$ & C & Emittent zahlungsunfähig \\
\hline & $+1-$ & $1,2,3$ & $\begin{array}{l}\text { Feinabstufungen innerhalb der Ratingkategorien, z.B. BB+ } \\
\text { (Ba 1) besser als } \mathrm{BB}-(\mathrm{Ba} 2 \text { oder } \mathrm{Ba} 3)\end{array}$ \\
\hline
\end{tabular}

Tabelle 1: Rating-Einschätzung langfristiger Verbindlichkeiten ${ }^{187}$

Während ein Rating eine qualitative Beurteilung (unter Berücksichtigung quantitativer Aspekte) der Anleihe darstellt, wird für die Kreditzinskalkulation eine Quantifizierung benötigt. Die Risikoprämie wird über den Kapitalmarkt abgeleitet, indem die Renditedifferenz zwischen einer (bonitäts-) risikolosen Anleihe und einer vom zu bewertenden Unternehmen emittierten Anleihe berechnet wird. ${ }^{188}$ Diese Vorgehensweise erfordert die Existenz eines Rating und den Handel einer emittierten Anleihe

186 Vgl. Kirmße (1996), S. 67.

187 In Anlehnung an Steiner (1992), S. 510.

$188 \mathrm{Vgl}$. Kirmße (1996), S. 96. Diese Differenz ist als Credit Spread definiert In Kap.

4.3.3.2 werden Verfahren zur Ermittlung dieser Risikoprämie vorgestellt. 
Marktorientierte Kreditrisikobewertung -

für den einzelnen Kreditnehmer. ${ }^{189}$

Falls es vom Schuldner zwar ein Rating, jedoch keine emittierte und gehandelte Anleihe gibt, kann eine vom Kapitalmarkt durchschnittlich verlangte Rendite für Schuldner einzelner Risikoklassen (Ratingklassen) bestimmt werden. Somit werden den einzelnen Ratingklassen einzelne Risikoprämien (Credit Spreads) zugeordnet. ${ }^{190}$ Abbildung 17 verdeutlicht diese Vorgehensweise exemplarisch. ${ }^{191}$ Ein Unternehmen mit einem BBB-Rating bspw. würde dementsprechend bei einem Kredit mit vier Jahren Laufzeit mit einer Ausfallprämie von 0,25\% p.a. belastet. Ein Unternehmen mit einem CC-Rating müsste demgegenüber für einen Kredit mit einer Laufzeit von neun Jahren einen jährlichen Zinsaufschlag von 1,10\% aufgrund des höheren Ausfallrisikos bezahlen.

Neben der Möglichkeit eines externen Rating besteht ebenso die Möglichkeit, ein eigenes, internes Rating für einen Schuldner vorzunehmen. Während die Zielsetzung analog zu der von Agenturen ist, d.h. die Abgabe einer in Symbolen dargestellten Meinung, kommt dabei der Bilanzanalyse eine entscheidende Rolle zu. ${ }^{192}$ Bei der einzelgeschäftsbezogenen Kreditbeurteilung wird faktisch bei allen Kreditinstituten die Bilanzanalyse verwendet, und die von ihr ermittelten Informationen zu den wirtschaftlichen Verhältnissen des Kreditnehmers bilden das zentrale Kriterium für die Kreditvergabe. ${ }^{193}$ Darüber hinaus werden weitere Kriterien wie z.B. die Zukunftsperspektiven, die Branchensituation oder die Rechtsform des Unternehmens einbezogen. ${ }^{194}$ Aufgrund der exponierten Stellung der Bilanzanalyse werden im Kapitel 3 die unterschiedlichen Möglichkeiten dieses Instrumentes aufgezeigt. ${ }^{195}$

189 Vgl. Schierenbeck (1995), S. 21. Die Möglichkeit, dass ein Unternehmen eine Anleihe emittiert hat, jedoch kein Rating aufweist, kann ausgeschlossen werden. Ein Rating wird bei der Ausgabe von Anleihen entweder aufgrund von gesetzlichen Vorschriften oder marktbezogenen Gepflogenheiten verlangt. Der Erwerb eines Rating ist somit eine faktische Verpflichtung für einen Emittenten. Vgl. Heinke (1998), S. 1.

190 Vgl. hier und im Folgenden Schierenbeck (1995), S. $21 \mathrm{ff}$.; Kirmße (1996), S. 68ff.

191 Zur detaillierten Beschreibung der Vorgehensweisen zur Ermittlung des Credit Spreads vgl. Kap. 4.3.3.2.

192 Wie bereits o.g., wird die Bilanzanalyse auch bei der Vergabe eines externen Rating regelmäßig verwendet, jedoch werden bei den marktführenden Ratingagenturen in erster Linie qualitative Ratingysteme herangezogen. Vgl. Everling (1991), S. 127. Zur Bedeutung der Bilanzanalyse bei der Vornahme von internen Rating vgl. Grabher, Klien, Stoss (1996), S. 39.

193 Vgl. Bröker (2000), S. 46.

194 Vgl. Betsch, Brümmer, Hartmann, Wittberg (1997), S. 151, vgl. auch Kap. 6.1.1.

195 Ein Überblick über Methoden und Einsatzbereiche bankinterner Ratingysteme findet sich bei Jansen (2001b), S. $100 \mathrm{ff}$. 
Marktorientierte Kreditrisikobewertung -

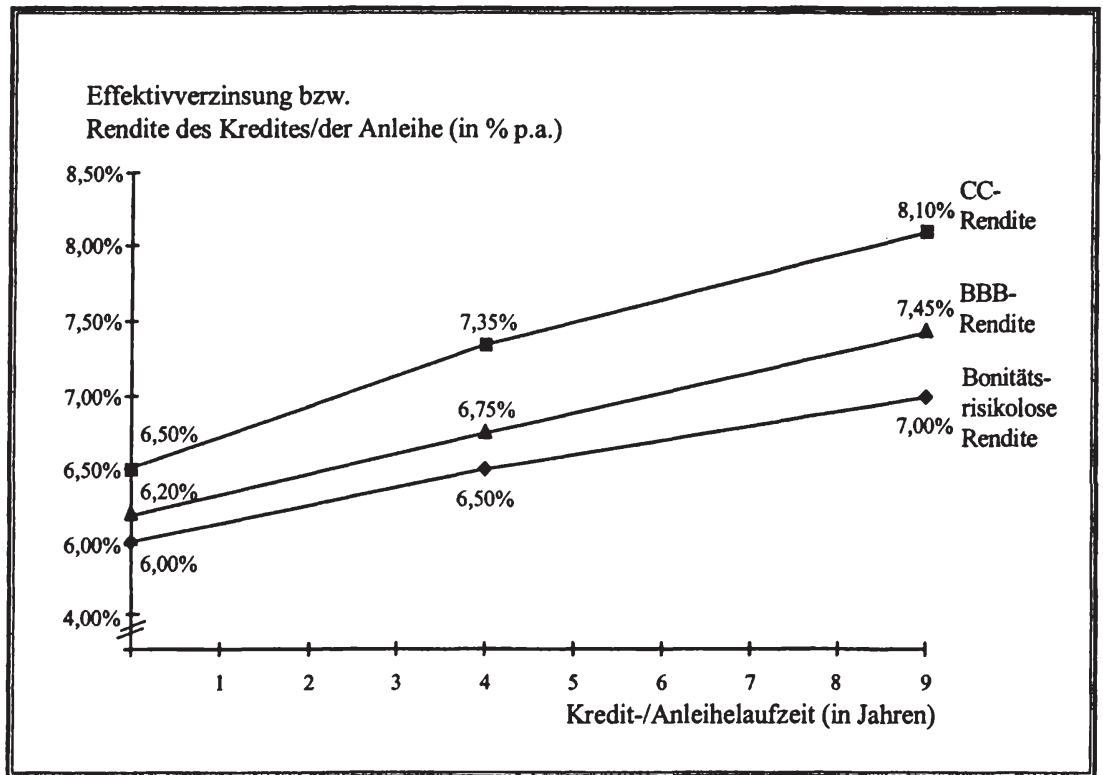

Abbildung 17: Exemplarische Vorgehensweise bei der Ableitung von Ausfallprămien gemăB der auf externe Rating gestützten Vorgehensweise

\subsection{Anforderungen an Verfahren zur Quantifizierung des Ausfallrisikos}

Die in diesem Kapitel beschriebenen Kriterien werden im Kapitel 2.5 für die kritische Würdigung der Instrumente hinsichtlich ihrer Eignung als Kreditzinskalkulationsverfahren herangezogen. Die einzelnen Vor- und Nachteile werden hierzu kriteriengeleitet verglichen. In Anlehnung an KIRMßE werden die Kriterien zur Verfahrensbewertung aufgestellt (vgl. Abbildung 18). Diese Kriterien entwickeln sich aus dem Verständnis der Kreditvergabe als partieller Investitionsentscheidung. Das Risiko ist in diesem Zusammenhang nicht aus einem Kreditprogramm, sondern bezogen auf einen Einzelkredit ableitbar. ${ }^{196}$ Somit müssen die Prinzipien der (1) Einzelbewertung und der (2) Richtigkeit zur Beurteilung herangezogen werden. Es muss ex-ante eine dem Risikogehalt des Geschäftes gerecht werdende Prämie berechnet werden.

Zunächst wird die Einhaltung des Prinzips der „Einzelbewertung “ gefordert. Dieses Prinzip besagt, dass ausschließlich die „,individuelle Risikohöhe des Einzelengagements für die Festlegung der Risikoprämie maßgeblich ist ${ }^{\text {‘1977 }}$. Andernfalls ist es nicht

\footnotetext{
196 Vgl. Schierenbeck, Rolfes (1998), S. 13; Rolfes (1992), S. $121 \mathrm{f}$.
}

197 Kirmße (1996), S. 60. 
Marktorientierte Kreditrisikobewertung -

möglich, im Rahmen einer Grenznutzenbetrachtung den durch Abschluss des Einzelgeschäftes erzielten Nutzen zu quantifizieren. Es muss der für das Unternehmen zu erwartende Mehrertrag aus dem einzelnen Geschäft ex-ante bestimmbar sein. ${ }^{198}$

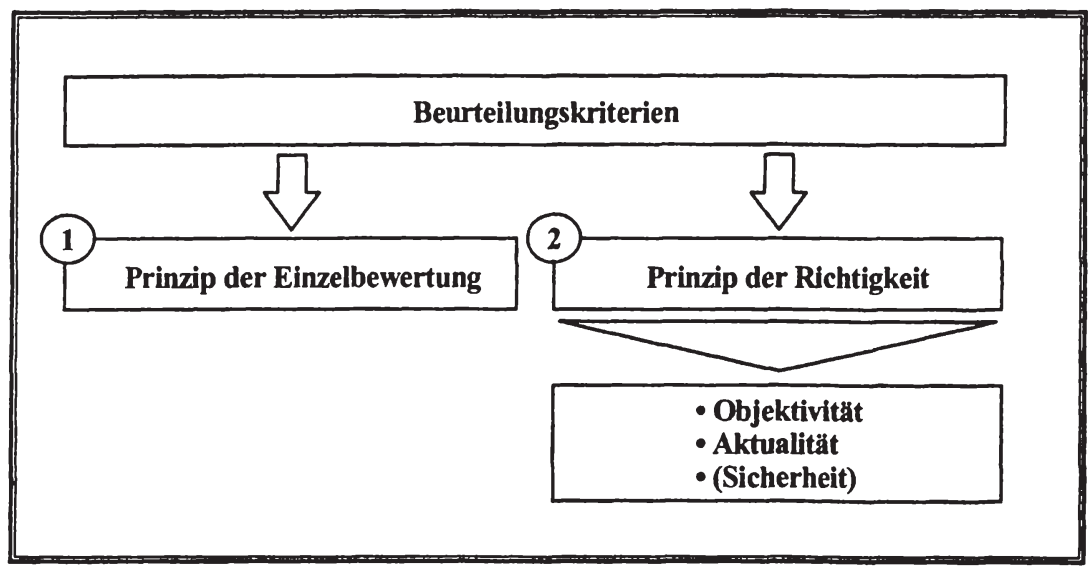

Abbildung 18: Beurteilungskriterien für die Verfahren der Risikoprămienermittlung ${ }^{199}$

Das zweite Prinzip der ,Richtigkeit" fordert einen möglichst hohen Realitätsbezug des Modells. Dies ist dann gegeben, wenn das Modell die Realität möglichst umfassend und widerspruchsfrei abbilden kann. ${ }^{200}$ Zum einen folgt aus diesem Prinzip, dass die verwendeten Maßstäbe objektiver Natur sind. Sie dürfen keine reinen institutsspezifischen Ansätze oder Rahmenbedingungen darstellen. Zum anderen wird der Anspruch der ,Aktualität “ gestellt. Hierunter wird verstanden, dass sowohl die in den Bewertungsprozess eingehenden Daten, als auch der Bewertungsprozess selbst sich an den aktuellen Gegebenheiten orientieren.

198 Vgl. Banken (1987), S. 22ff.; Rolfes (1992), S. 121f.; Schierenbeck, Rolfes (1988), S. 13.

199 In Anlehnung an Kirmße (1996), S. 61. Das Kriterium der Sicherheit bezieht sich auf das quantifizierte Risiko, das bei Erfüllung des Kriteriums über die gesamte Kreditlaufzeit in seiner Höhe Bestand haben muss. Dieses Kriterium wird vom Verfasser von der weiteren Untersuchung ausgeschlossen, da konzeptspezifische Grundlagen, wie z.B. beim optionspreistheoretischen Verfahren, keine konstante Risikoprämie ausweisen wollen. Somit ist eine gleichwertige objektive Bewertung der Kalkulationsverfahren mit dem Kriterium Sicherheit nicht möglich. Vgl. Kirmße (1996), S. 63ff.

$200 \mathrm{Vgl}$. hier und im Folgenden Rolfes (1985), S. 44; Schierenbeck, Rolfes (1988), S. 13; Kirmße (1996), S. 60. 
Marktorientierte Kreditrisikobewertung Die Quantifizierung des Ausfallrisikos als bankbetriebliche Problemstellung

\subsection{Kritische Würdigung der einzelnen Ansătze}

Basierend auf den im Kapitel 2.4 genannten Kriterien werden im Folgenden die unterschiedlichen Ansätze gewürdigt. Zunächst werden die modelltheoretischen Ansätze beurteilt (a), wobei unter (la) die Einhaltung des Prinzips der Einzelbewertung und unter (2a) die Einhaltung des Prinzips der Richtigkeit überprüft werden. Anschließend wird unter (b) die Kreditzinskalkulation mittels Segmentierung gewürdigt, wobei wiederum unter (1b) die Einhaltung des Prinzips der Einzelbewertung und unter ( $2 \mathrm{~b}$ ) die Einhaltung des Prinzips der Richtigkeit analysiert werden. Abschließend wird unter (c) die Kreditzinskalkulation mittels einzelgeschäftsbezogenen Rating beurteilt, wobei analog zu der Würdigung der o.g. Ansätze unter (1c) die Einhaltung des Prinzips der Einzelbewertung und unter (2c) die Einhaltung des Prinzips der Richtigkeit gewürdigt werden.

\section{Modelltheoretische Ansätze}

1a. Prüfung der Einhaltung des Prinzips der Einzelbewertung für die modelltheoretischen Kreditzinskalkulationsverfahren

Die modelltheoretischen Ansätze verfolgen alle einen Einzelbewertungsansatz. Basierend auf ihrem Grundgedanken sind die individuelle Situation des Kreditnehmers (Ausfallwahrscheinlichkeit, Ausfallschwere, Unternehmenswert, etc.), die kreditspezifischen Merkmale (Kreditlaufzeit, Kreditvolumen, etc.) und die Rahmenbedingungen (Zinsniveau, Zinsstruktur) entscheidend für die Risikokostenhöhe. Somit ist bei den im Kapitel 2.3.2 genannten Ansätzen das Prinzip der Einzelbewertung gewahrt.

2a. Prüfung der Einhaltung des Prinzips der Richtigkeit für die modelltheoretischen Kreditzinskalkulationsverfahren

Die (theoretische) Objektivität des stochastischen Ansatzes ist aufgrund der Ableitung aus einem Bewertungsmodell modellimmanent garantiert. Insbesondere sind die Risikoprämien bankenunabhängig und kreditspezifisch. Die praktische Objektivität, d.h. die Einhaltung des Prinzips in der Umsetzung, ist kritisch zu beurteilen.

Problematisch ist diesbezüglich die marktnahe Beobachtung von objektiven Ausfallwahrscheinlichkeiten. Somit wird das Grundproblem der Quantifizierung des Kreditrisikos auf die Ebene der Bestimmung des Ausfallrisikos transformiert. Marktorientierte Ausfallwahrscheinlichkeiten lassen sich jedoch nicht direkt bestimmen. Demnach ist die praktische Umsetzbarkeit des stochastischen Ansatzes fraglich. ${ }^{201}$ Es kann

201 Eine Möglichkeit der Ableitung von Ausfallwahrscheinlichkeiten besteht in der Erfassung historischer Ausfallraten der entsprechenden Ratingklassen. Es wird somit vorausgesetzt, dass ein Rating vorgenommen wurde, damit der stochastische Ansatz verwendet werden kann (vgl. hierzu die folgenden Ausführungen). 
Marktorientierte Kreditrisikobewertung -

Die Quantifizierung des Ausfallrisikos als bankbetriebliche Problemstellung

vermutet werden, dass dieses Objektivierungsproblem auch ein Grund für die untergeordnete Bedeutung des Ansatzes bei der Bepreisung von Krediten ist.

Die konzeptionellen Anforderungen an eine (theoretisch) objektive Ermittlung der Risikoprämie beim Kalkulationsverfahren nach der Optionspreistheorie sind erfüllt. Die Ableitung aus dem geschlossenen finanzwirtschaftlichen System ermöglicht es - unter Akzeptanz der Modellprämissen -, eine „objektive Höhe“ für die Risikoprämie zu bestimmen.

Die Aktualität ist bei dem optionspreistheoretischen Ansatz durch die vom Kapitalmarkt abgeleiteten Daten garantiert. Kritischer ist die Realitätsnähe, die sich zum einen in den modellinternen Prämissen und zum anderen in der Datenverfügbarkeit niederschlägt.

Die Modellprämissen, insbesondere die Frage nach der Vollkommenheit der Märkte und der Informationseffizienz, werden sehr häufig in der Literatur diskutiert. ${ }^{202}$ Beispielsweise wird bei HARTMANN-WENDELS/PFINGSTEN/WEBER die Existenz von Banken auf einem vollkommenen Kapitalmarkt kritisch hinterfragt. ${ }^{203}$ Darüber hinaus weisen empirische Studien unterschiedliche Ergebnisse hinsichtlich der Existenz der vollkommenen Kapitalmärkte auf. ${ }^{204}$

Für die praktische Relevanz eröffnet sich ein weiteres Problemfeld, und zwar muss im Rahmen der Informationsgewinnung überprüft werden, ob die für das Modell notwendigen Eingangsparameter ,objektiv“ ermittelbar sind. Unter objektiver Informationsgewinnung wird hier verstanden, in welchem Ausmaß subjektive Entscheidungen im Rahmen der Datenermittlung zu treffen sind und, soweit dies der Fall ist, welche Auswirkung diese auf die Zielgröße (in diesem Falle das bepreiste Risiko) haben.

Im Rahmen der Optionspreistheorie für die Bepreisung von Ausfallrisiken ist die Ermittlung eines Unternehmenswertes notwendig. Grundlegendes Problem ist die Definition dieser Größe. ${ }^{205} \mathrm{Um}$ dem Prinzip der Objektivität gerecht zu werden, muss

Vgl. Kap. 4.2.

Vgl. Hartmann-Wendels, Pfingsten, Weber (2000), S. $21 \mathrm{f}$.

An dieser Stelle wird auf eine weitere Diskussion der offenen Frage verzichtet. Vgl. zur ausfuihrlichen Darstellung Kap. 4.2

Vgl. zu den Verfahren der Unternehmensbewertung bspw. Ballwieser (1993), S. $151 \mathrm{ff}$; Dörner (1992), S. Iff.; Hafner (1993), S. 79ff.; Moxter (1983), S. 33ff.; Schierenbeck (2000), S. 395ff.; Schneider (1992), S. 520ff.; Siegel (1991), S. 231 ff. Vgl. zu dem Verfahren der Unternehmensbewertung innerhalb der Due Diligence bei Unternehmenskäufen Klein, Jonas (1999), S. 159ff.; Berens, Strauch (1999), S. 17. 
Marktorientierte Kreditrisikobewertung Die Quantifizierung des Ausfallrisikos als bankbetriebliche Problemstellung

der Vermögenswert über einen marktorientierten Ansatz erfasst werden. ${ }^{206}$ Hierzu eignen sich grundsätzlich zwei Vorgehensweisen: die Ermittlung nach dem Einzelund die nach dem Gesamtbewertungsverfahren. Die Ermittlung mittels der Substanzwert- oder Liquidationswertverfahren (als Einzelbewertungsverfahren) „sind aus unterschiedlichen Gründen für die Ermittlung des Unternehmenswertes im Rahmen des Optionsbewertungsmodells nicht geeignet ${ }^{\star 207}$. Der Liquidationswert ist nur dann sinnvoll, wenn der Ertragswert unterhalb des Substanzwertes liegt. In diesen Fällen wird jedoch kein Kredit an das Unternehmen vergeben, was wiederum die Notwendigkeit der Bepreisung eines Ausfallrisikos hinfällig macht. ${ }^{208}$ Die Substanzwertmethodik wurde bereits hinreichend in der Literatur als ökonomisch nicht sinnvolle Technik bewertet. ${ }^{209}$ Im Zusammenhang mit der Bewertung der Fähigkeit, zukünftige Zins- und Tilgungszahlungen zu leisten, ist nicht der aktuelle Wert des Nettovermögens, sondern sind vielmehr die zukünftigen Zahlungsströme relevant. ${ }^{210}$

Folglich wird der Unternehmenswert auf Basis des Gesamtbewertungsverfahrens verwendet. Hierzu werden zukünftige Zahlungen auf einen Betrachtungszeitpunkt verdichtet. Grundsätzlich werden zwei Verfahren unterschieden: Das Ertragswertverfahren und die Discounted Cash Flow Methodik (DCF-Methodik). ${ }^{211}$ Innerhalb der Anwendung zur Bestimmung des Kreditzinses mit Hilfe der Optionspreisformel wird auf die Unterscheidung der beiden Ansätze verzichtet. ${ }^{212}$ Dies liegt an der Überführbarkeit der Methoden. Darüber hinaus ist es für die Beurteilung des Objektivitätskriteriums ausreichend, sich exemplarisch auf die Probleme bei der Bestimmung mittels der DCF-Methodik zu beziehen, da diese Kritikpunkte auf die Ertragswertmethodik übertragbar sind. ${ }^{213}$

Vgl. Kirmße (1996), S. 109ff.

Kirmße (1996), S. 110.

Hierzu muss der Ertragswert zuvor mit dem Liquidationswert verglichen werden. Vgl. auch Dörner (1992), S. 120 Rn 247. Vgl. Dörner (1992), S. 124; Kümmel (1994), S. 20; Moxter (1983), S. 44f. und S. 53f.; Sieben (1963), S. $65 \mathrm{ff}$.

210 Vgl. Falter (1994), S. 259.

211 Vgl. bspw. Ballwieser (1993), S. 153; Börsig (1991), S. 84f. Die Discounted Cash Flow Methodik wird auch in der unternehmenswertorientierten Steuerung angewendet (Shareholder Value-Ansatz). Vgl. zum Shareholder Value-Ansatz bspw. Bühner (1990); Copeland, Koller, Murrin (1993); Lewis (1994); Rappaport (1995); Siegert (1995).

$212 \mathrm{Vgl}$. hier und im Folgenden Kirmße (1996), S. 111. Zur Unterscheidung der Verfahren nach der DCF- und der Ertragswertmethodik vgl. Ballwieser (1993), S. 166f.; Hafner (1993), S. 82ff.; Schmidt (1995), S. 1088ff.

213 Im Folgenden wird keine detaillierte Wiedergabe der DCF-Methodik dargestellt, sondern lediglich exemplarisch der Problemkreis bei der Umsetzung erlăutert. 
Marktorientierte Kreditrisikobewertung -

Bei der Verwendung eines einheitlichen Zinssatzes i berechnet sich der Unternehmenswert wie folgt:

Gleichung 21

$$
\mathrm{V}_{0}=\sum_{\mathrm{t}=1}^{\mathrm{n}} \frac{\mathrm{R}_{\mathrm{t}}}{(1+\mathrm{i})^{\mathrm{t}}}
$$

mit $\mathrm{V}_{0}=$ Unternehmenswert in $\mathrm{t}=0$;

$\mathrm{R}_{\mathrm{t}}=$ erwartete Zahlung in der Periode $\mathrm{t}$;

$\mathrm{n}=$ Anzahl der betrachteten Perioden.

Es besteht die Notwendigkeit, zwei Größen (die Zahlungen $\mathrm{R}$ und den Zinssatz r) objektiv im Rahmen einer Datenerhebung zu ermitteln.

Die erwarteten Zahlungen $R$ werden bspw. auf Basis des Jahresabschlusses des Unternehmens antizipiert oder geschätzt. ${ }^{214}$ An dieser Stelle soll nicht das Ermittlungsverfahren, d.h. die Definition des Cash Flows, im Vordergrund stehen, ${ }^{215}$ sondern die objektive Datenermittlung. Dementsprechend müssten die zukünftigen und somit mit Unsicherheit behafteten Zahlungsströme durch ein idealtypisches objektives Verfahren vorhergesagt werden. Eine modellhafte Vorgehensweise besteht in einer Prognose auf Basis eines Schätzverfahrens. ${ }^{216}$

$214 \mathrm{Vgl}$. zu den verschiedenen Ansätzen der Discounted Cash Flow Methode bspw. Klein, Jonas (1999), S. 166ff.

$215 \mathrm{Vgl}$. zu den verschiedenen Cash Flow Definitionen Perridon, Steiner (1997), S. 485ff.

216 Bspw. wird von dem nachhaltigen Ergebnis, das sich nach Ablauf einer konkret geschätzten Periodenbetrachtung ergibt, angenommen, „dass es entsprechend dem langjährigen Trend und der erwarteten Preissteigerung jährlich um $2 \%$ wächst" Klein, Jonas (1999), S. 170. 
Marktorientierte Kreditrisikobewertung Die Quantifizierung des Ausfallrisikos als bankbetriebliche Problemstellung

Darüber hinaus muss der Zinssatz $i$ definiert werden. Hierzu bietet sich ein risikofreier Zins oder ein risikoadjustierter Zins an. ${ }^{217}$ Während die erste Möglichkeit weniger „Spielräume“ bei der Datenermittlung lässt, ${ }^{218}$ muss einem solchen Vorgehen jedoch aufgrund einer dem Modell immanenten Kritik widersprochen werden. ${ }^{219}$ Somit wird regelmäßig ein risikoadjustierter Zins verwendet. $\mathrm{Zu}$ dessen Ermittlung eignet sich das Capital Asset Pricing Modell (CAPM), das neben der sicheren Rendite einen unternehmensindividuellen Risikoaufschlag ausweist. ${ }^{220}$ Dieser Risikoaufschlag ergibt sich aus dem Produkt des Marktpreises des Risikos und der Höhe des wertpapierspezifischen systematischen Risikos. ${ }^{221} \mathrm{Da}$ die Anwendbarkeit des CAPM wiederum auf die Grenzen der modellimmanenten Prämissen stößt, ist auch die praktische Ermittlung eines objektiven risikoadjustierten Zinses fraglich. ${ }^{222}$ „Obwohl das CAPM die Bewertung durch Einschaltung des Marktes objektivieren soll, scheitert es mit diesem Anspruch schon an den zahllosen Freiheitsgraden, die sich z.B. aus der Auswahl des in die Stichprobe eingehenden Datenmaterials ergeben. Betafaktoren sind nämlich leider keine Naturkonstanten, sondern schwanken ihrerseits im Zeitablauf.“‘223

217 Vgl. Copeland, Koller, Murrin (1993), S. 192.

218 Auch hier gibt es verschiedene Fragen, die zu beantworten sind, bspw. hinsichtlich der Bildung eines Durchschnittszinssatzes. Vgl. Gerdsmeier, Krob (1994), S. 472; Reichel (1995), S. 23.

219 Der Cash Flow ist durch die Planungsunsicherheiten mit einem gewissen Risiko verbunden. Durch die Verwendung eines risikolosen Zinssatzes wird von folgender Annahme ausgegangen: Entweder erwarten sowohl Fremd- als auch Eigenkapitalgeber keine höhere Verzinsung oder die von den Eigenkapitalgebern erwartete „Überrendite“ (Risikoaufschlag) führt bei den Fremdkapitalgebern zu einem Renditeanspruch, der unterhalb des sicheren Zinses liegen würde.

220 Vgl. hier und im Folgenden bspw. Klein, Jonas (1999), S. 166.

221 Der Marktpreis des Risikos ist die Differenz aus dem Erwartungswert der Rendite des Marktportfolios und der risikolosen Rendite; das wertpapierspezifische systematische Risiko ist das Verhältnis der Kovarianz der Rendite des untersuchten Unternehmens und des Marktportfolios und der Varianz der Rendite des Marktportfolios. Vgl. Bröker (2000), S. 53ff.

$222 \mathrm{Vgl}$. Teschner (1998), S. 243ff.

223 Hering (1999), S. 124. Vgl. auch Baetge, Krause (1994), S. 453f.; Ballwieser (1994), S. 1398 und S. 1405; Schmidt (1995), S. 1106. 
Marktorientierte Kreditrisikobewertung -

Börsennotierte Unternehmen bieten einen weiteren Ansatz zur Bestimmung des Unternehmensmarktwertes. ${ }^{224}$ Der Marktwert des Eigenkapitals ergibt sich durch die Multiplikation des Kurswertes mit der Anzahl der ausgegebenen Aktien. Hierzu muss der Wert des Fremdkapitals addiert werden. ${ }^{225}$ Diskussionswürdig ist die hohe Volatilität des Börsenwertes, sodass die Frage nach den Einflussfaktoren auf den Börsenkurs zu untersuchen ist. Die Preisbildung von Aktienkursen und die hiermit implizierte Unternehmensbewertung unterliegen ebenfalls nicht rationalen Einflussfaktoren. ${ }^{226}$

Aufgrund dieser dargestellten weitreichenden Entscheidungsspielräume, ${ }^{227}$ die einen maßgeblichen Einfluss auf den zu errechnenden Zielwert (Unternehmenswert und somit Kreditzins) durch den Anwender haben, wird vom Verfasser die Gewährleistung der Objektivität bei der Umsetzung in Frage gestellt.

Heuristische Ansätze

1b. Prüfung der Einhaltung des Prinzips der Einzelbewertung für die Kreditzinskalkulation mittels Verfahren der Segmentierung

Alle segmentspezifischen Verfahrensweisen unterlegen grundsätzlich eine einheitliche Risikoprämie. Somit weichen diese Ansätze bereits in der Konzeption von dem Prinzip der Einzelbewertung ab. ${ }^{228}$ Die Differenzierung der Segmentierungskriterien schafft eine Annäherung an das Prinzip der Einzelbewertung, doch erst bei der Be-

Da bei börsennotierten Unternchmen eine Bewertung über den Kapitalmarkt erfolgt, ist diese der individuellen Bewertung vorzuziehen. Bei Modellbewertungen wird regelmäBig versucht, die Kapitalmarktbedingungen nachzubilden. Vgl. Schneider (1992), S. 520. Diese modelltheoretischen Verzerrungen können durch den ausgewiesenen Unternehmenswert umgangen werden.

Bei börsennotierten Unternehmen wird hierzu der Buchwert der Verbindlichkeiten verwendet. Vgl. Kirmße (1996), S. 112.

Zu einer weiterführenden Diskussion der Einflussfaktoren vgl. Hoffjan, Siemes (1999) und Menkhoff, Röckmann (1994); vgl. zu empirischen Studien hinsichtlich der Einflussfaktoren bspw. Frantzmann (1989); de Long, Shleifer, Summers, Waldmann (1990, 1990b); Blume, Easley (1992); Shiller, Pound (1989); Shefrin, Statamn (1985, 1994); Shiller (1981); West (1998); Hodrick (1990), de Bondt, Thaler (1985, 1987, 1989); Shefrin (1997); Bremer, Sweeney (1991); Ripper, Kempf (1998); vgl. ebenfalls die Diskussion der Effizienzhypothese in Kap. 4.2. Zur Eignung des Optionspreismodells für die Bepreisung von Ausfallrisiken vgl. Jansen (2001b), S. $102 \mathrm{ff}$.

227 Neben dem Unternehmenswert muss die Volatilität, d.h. die Schwankungsbreite, dieser Größe bestimmt werden. Hierzu gibt es wiederum verschiedene Verfahrensweisen. Vgl. bspw. zur subjektiven Volatilitätsschätzung für börsennotierte Unternehmen Bös (1991), S. 119ff. Folglich bedingt die Bestimmung der Größe „Volatilität" ebenfalls subjektive Entscheidungsmomente.

Vgl. Kirmße (1996), S. 61. 
Marktorientierte Kreditrisikobewertung Die Quantifizierung des Ausfallrisikos als bankbetriebliche Problemstellung

trachtung eines einzelnen Kredites als ein Segment würde das Prinzip erfüllt werden. In diesem Moment würde jedoch keine Segmentierung mehr vorliegen. ${ }^{229}$

2b. Prüfung der Einhaltung des Prinzips der Richtigkeit für die Kreditzinskalkulation mittels Segmentierung

Neben dem Prinzip der Einzelbewertung gilt es, das Prinzip der Richtigkeit zu untersuchen. Hierzu wird zunächst die Frage der Objektivität gestellt. Da der Zusammenhang zwischen Segmentierungskriterium und dem Kreditrisiko messbar ist, kann dieser konzeptionell nur schwer kritisiert werden. ${ }^{230}$ Falls es sich bei dem Segmentierungsverfahren bzw. der Kalkulation der Risikokosten jedoch um eine institutsspezifische Größe handelt (Ist-Risikokosten des einzelnen Instituts), ist somit auch die Prämienfindung institutsspezifisch und unterliegt der Subjektivität, da sie von einem Dritten intersubjektiv nicht nachvollziehbar ist. ${ }^{231}$ Derselbe Kredit würde bei unterschiedlichen Instituten mit unterschiedlichen institutsspezifischen Prämien bedacht werden.

Falls jedoch marktbezogene Daten als Ausfallraten verwendet werden, kann dieser Mangel aufgehoben werden. ${ }^{232}$ Somit wird für einen Kreditnehmer bei einer einzelnen Bank die Risikoprämie in Abhängigkeit von der allgemeinen Marktsegmententwicklung kalkuliert. Es wird dann modelltheoretisch unterstellt, dass die Segmente des einzelnen Instituts identisch zu den Marktsegmenten sind. Dazu sollten annahmegemäß die institutsspezifischen Daten annähernd gleich den marktbezogenen Daten sein, sodass idealtypisch der Kreditnehmer bei jedem Kreditinstitut dieselbe Risikoprämie zu zahlen hätte.

Die Verwendung von Ist-Risikokosten erfüllt nicht das Bewertungskriterium der Aktualität. Durch die Berechnung auf Grundlage vergangenheitsorientierter Entwicklungen sind die ,aktuellen Risikoprämien nicht mehr von der Qualität des Neugeschäftes, sondern von der Qualität des Geschäftes der Vergangenheit abhängig. Die aktuelle Risikosituation spielt damit keine Rolle für die Prämienberechnung. Das Prinzip der Aktualität wird damit (...) nicht erfüllt." ${ }^{\text {(233 }}$ Leiten sich die Prämien jedoch mit Hilfe von modellhaften Prognosen hinsichtlich der unsicheren zukünftigen

$229 \mathrm{Vgl}$. hierzu auch die Ausführungen zu dem segment-of-one-approach bei Meffert, Bruhn (1995), S. 189.

230 Vgl. Brakensiek (1991), S. 158.

231 Vgl. Kirmße (1996), S. 62f.

232 Dieses Verfahren wird im marktdeduzierten Ansatz verfolgt. Vgl. hier und im Folgenden Brakensiek (1991), S. 166ff.

233 Kirmße (1996), S. 63. 
Marktorientierte Kreditrisikobewertung -

Marktsituation ab, ist in der Umsetzung das Prinzip der Aktualität erfullt, falls eine zeitnahe Datenbereitstellung gesichert werden kann. ${ }^{234}$

1c. Prüfung der Einhaltung des Prinzips der Einzelbewertung für die Kreditzinskalkulation mittels eines einzelgeschäftsbezogenen Rating

Als ein weiterer Ansatz aus dem Bereich der heuristischen Kalkulationsverfahren wurde das einzelgeschäftsbezogene Rating genannt. Wie bereits in Kapitel 2.3.3 erläutert, kann zwischen dem internen und externen Rating unterschieden werden. Gemeinsam ist beiden Verfahren, dass eine individuelle Analyse des Unternehmens zu einer individuellen qualitativen Einschätzung des Risikos führt. Somit ist das Prinzip der Einzelbewertung erfüllt. Durch die notwendige Zuordnung von Risikoprämien ist fraglich, inwieweit dieses Prinzip durchbrochen wird. ${ }^{235}$ Errechnet sich die Bonitätsprämie am Kapitalmarkt über den Vergleich mit einer emittierten Anleihe des Kreditnehmers, ist eine einzelgeschäftsbezogene Betrachtung gewährleistet. Falls jedoch keine Anleihe von diesem Unternehmen gehandelt und eine Bonitätsprämie anhand der Bewertung der Ratingklasse am Kapitalmarkt bestimmt wird, handelt es sich wiederum um eine Segmentierung. Das Segmentierungskriterium ist in diesem Fall das externe Rating. Folglich wird in diesem Fall die einzelgeschäftsbezogene Betrachtung verletzt.

2c. Prüfung der Einhaltung des Prinzips der Richtigkeit für die Kreditzinskalkulation mittels eines einzelgeschäftsbezogenen Rating

Das Prinzip der Richtigkeit bedingt zunächst die Prüfung der Einhaltung der Objektivität. Externe Ratings beziehen sich auf die Einschätzung eines Dritten. Es ist somit zu prüfen, ob diese Einschätzung objektiv ist. Zunächst legen die Ratingagenturen den Bewertungsprozess nicht offen. Dies bezieht sich sowohl auf die vollständige Darstellung der Kriterien als auch auf die unterlegten Gewichtungsfaktoren. ${ }^{236} \mathrm{Da}$ neben quantitativen auch qualitative Merkmale eingeschätzt werden, obliegt der Ratingagentur ein gewisser Ermessensspielraum. ${ }^{237}$ Dieser führt dazu, dass der gleich einzuschätzende Sachverhalt verschieden beurteilt werden kann. Es handelt sich um das so genannte "splitrating" ${ }^{238}$ Hier wird das Kreditrisiko von der Ratingagentur A anders eingeschätzt als von der Ratingagentur B, wodurch derselbe Emittent unter-

234 Schüller weist auf das Problem der Abhängigkeit von der Datenbereitstellung durch Externe hin. Vgl. Schüller (1995), S. 188.

235 Zu der Diskussion des internen Rating vgl. Kap. 3.6.

236 Vgl. Schneider (1992), S. 601.

237 Vgl. Steiner (1992) S. $514 f$.

238 Vgl. Hier und im Folgenden Cantor, Packer, Cole (1997); Ederington (1986), S. 37 und S. 41. 
Marktorientierte Kreditrisikobewertung Die Quantifizierung des Ausfallrisikos als bankbetriebliche Problemstellung

schiedliche Ratings bekommt. Somit sichern externe Ratingagenturen keine objektive Bewertung der Bonität.

Darüber hinaus ist für die Quantifizierung des Kreditrisikos durch den Kapitalmarkt zu untersuchen, inwieweit die Bewertung objektiv ${ }^{239}$ erfolgt, und die Risikoprämie über einen entsprechenden Referenzwert ${ }^{240}$ objektiv ermittelbar ist. ${ }^{241}$

Für interne Ratingysteme muss die Objektivität differenziert beurteilt werden. Der entscheidende Unterschied zu der o.g. Vorgehensweise (externes Rating) liegt darin, dass die Gewichtungsmerkmale bei der Urteilsfindung bekannt sind. Der Bonitätsanalyse mittels Bilanzanalyse kommt ein besonders hoher Stellenwert in diesem Bewertungsprozess $\mathrm{zu}^{242}$ Hierbei kann zwischen verschiedenen Instrumenten der Bilanzanalyse unterschieden werden. ${ }^{243}$ Die verwendeten Instrumente haben entscheidenden Einfluss auf die Güte des Systems. ${ }^{244}$ Eine abschließende Würdigung des internen Rating zur Kreditzinskalkulation setzt somit die Kenntnis der Bilanzanalyseinstrumente bzw. der Vorgehensweise zur Bestimmung der Gewichtungsfaktoren von einzelnen Bilanzinformationen im Rahmen der Bonitätsbeurteilung voraus. Somit ist die Frage der Objektivität der Ermittlung der Risikoeinschätzung und der Bepreisung dieser Risiken im Kapitel 3 zu diskutieren.

Bei der Beurteilung der Aktualität wird bei externen Ratings vorausgesetzt, dass eine Veränderung der Risikoeinschätzung (Herauf- oder Herabstufung bzw. up- oder downgrading des Rating durch die Agenturen) sehr zeitnah vollzogen wird. Empiri-

239 Diese Tatsache wirft wiederum die Frage der Effizienzhypothese auf.

240 Fraglich ist die Vorgehensweise zur Bestimmung der risikolosen Rendite, vgl. hierzu Kap. 4.3.3.2.

241 Beide Argumente werden im Kap. 4 vertieft und bleiben zunächst unbeantwortet.

$242 \mathrm{Vgl}$. zu verschiedenen Ansätzen der Systematisierung von Bonitätsbeurteilungen Jansen (2001), S. 38ff. In der vorliegenden Arbeit werden die weiteren Kriterien (neben der Bilanzanalyse) vernachlässigt. Es soll nicht der Eindruck entstehen, dass die Bilanzanalyse das einzige Instrument ist. Vielmehr ist sie ein Baustein, der in der vorliegenden Arbeit genauer untersucht wird. Wie im Kapitel 6.1 in Aussicht gestellt wird, sind die Ergebnisse der theoretisch abgeleiteten und empirisch belegten Möglichkeiten der Bepreisung von Kreditrisiken in einem weiteren Schritt sowohl in das bankinterne Ratingystem zu integrieren, als auch um portfoliotheoretische Erkenntnisse zu erweiterten. Um die Systematik der Analyse eindeutig zu gestalten, wird jedoch zunächst im Rahmen des bankinternen Rating die Bilanzanalyse als einziges Instrument angesetzt. Vgl. Kapitel 3.

244 Es wird im weiteren Analyseverlauf gezeigt, dass bspw. Ratingeinstufung auf Basis einer traditionellen Bilanzanalyse einem hohen $\mathrm{Ma} ß$ an Subjektivität unterliegen, bei der Verwendung von Neuronalen Netzen ein funktionaler Zusammenhang genutzt werden kann, um sich von der subjektiven Einschätzung zu lösen. Vgl. Kap. 3. 
Marktorientierte Kreditrisikobewertung -

sche Untersuchungen stellen diesen Zeitbezug jedoch in Frage. ${ }^{245}$ Somit haben Herabstufungen nachweislich keinen signifikanten Einfluss auf den am Kapitalmarkt erzielten Kreditzins. Die Veränderung der Risikoeinschätzung wurde bereits durch den Kapitalmarkt antizipiert. ${ }^{246}$ Im Rahmen der internen Ratingsysteme muss wiederum verfahrensbezogen (hinsichtlich des unterstellten Instrumentes) das Kriterium der Aktualität untersucht werden (vgl. Kap. 3.6).

Zusammenfassend kann man konstatieren, dass keines der untersuchten Kalkulationsverfahren alle Kriterien zufriedenstellend erfüllt. Tabelle 2 fasst dies zusammen und ordnet die bereits oben benutze Nummerierung $(1 \mathrm{a}, 1 \mathrm{~b}, \ldots)$ den einzelnen Fragestellungen zu.

Die modelltheoretischen Konzepte sichern zwar, wie gezeigt, eine theoretisch objektive einzelgeschäftsbezogene Quantifizierung des Kreditrisikos, jedoch weist die Ermittlung der modellrelevanten Daten subjektive Entscheidungsspielräume auf. Ein objektiver Informationsbestand ist nicht gesichert.

Segmentierungsverfahren basieren i.d.R. nicht auf einem einzelgeschäftsbezogenen Bewertungsansatz. Durch den Bezug zu den Ist-Risikokosten werden institutsspezifische Prämien berechnet, sodass die objektive Ermittlung nicht gewährleistet ist. Darüber hinaus wird, wie gezeigt, durch die Kalkulation auf Basis der Ist-Risikokosten das zukünftige Geschäft von den Ausfällen der Vergangenheit beeinflusst. Bei der Berücksichtigung von marktbezogenen Prognosedaten ist das Kreditinstitut vom externen Informationslieferanten abhängig.

Es wurde oben dargelegt, dass Ratingeinschätzungen eine einzelgeschäftsbezogene Beurteilung des Risikos vornehmen. Die einbezogenen Kriterien sind messbar und beobachtbar. Bei der Vergabe von externen Ratings ist es jedoch für den Außenstehenden nicht nachvollziehbar, welche Kriterien mit welcher Gewichtung in die Urteilsfindung einfließen. Darüber hinaus stellt ein Rating eine qualitative Einschätzung dar. Für die Bewertung des Kreditrisikos ist es notwendig, den Ratingeinschätzungen quantifizierte Bonitätsprämien zuzuordnen. Dies geschieht über die Preisbildung am

245 Vgl. bspw. Wansley, Glascock, Clauretie (1992), S. 733ff.; Hand, Holthausen, Leftwich (1992), S. 733ff.; Wansley, Clauretie (1985), S. $31 \mathrm{ff}$.

246 Vgl. Heinke (1998), S. $371 \mathrm{ff}$. 
Marktorientierte Kreditrisikobewertung Die Quantifizierung des Ausfallrisikos als bankbetriebliche Problemstellung

\begin{tabular}{|c|c|c|c|c|c|c|}
\hline \multirow{3}{*}{\multicolumn{2}{|c|}{. }} & \multicolumn{2}{|c|}{$\begin{array}{c}\text { Modelltheoretische } \\
\text { Konzepte }\end{array}$} & \multicolumn{3}{|c|}{ Heuristische Konzepte } \\
\hline & & \multirow{2}{*}{$\begin{array}{l}\text { Stochastische } \\
\text { Ansätze } \\
\text { (a) }\end{array}$} & \multirow{2}{*}{$\begin{array}{l}\text { Marktpreis- } \\
\text { orientierte } \\
\text { Ansätze } \\
\text { (a) }\end{array}$} & \multirow[t]{2}{*}{$\begin{array}{l}\text { Segmentierung } \\
\text { (b) }\end{array}$} & \multicolumn{2}{|c|}{$\begin{array}{l}\text { Individuelle Ansătze } \\
\text { (c) }\end{array}$} \\
\hline & & & & & Externes Rating & $\begin{array}{l}\text { Bilanzanalyse als } \\
\text { Instrument des } \\
\text { internen Rating }\end{array}$ \\
\hline \multirow{4}{*}{ 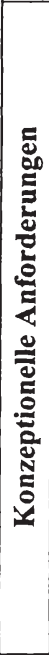 } & $\begin{array}{l}\text { Einzelbe- } \\
\text { wertung } \\
\text { (1) }\end{array}$ & $\begin{array}{l}\text { Gesichert } \\
\text { durch konzep- } \\
\text { tionellen } \\
\text { Ansatz. }\end{array}$ & $\begin{array}{l}\text { Gesichert durch } \\
\text { konzeptionellen } \\
\text { Ansatz. }\end{array}$ & $\begin{array}{l}\text { I.d.R. durch } \\
\text { konzeptionel- } \\
\text { len Ansatz } \\
\text { ausgeschlos- } \\
\text { sen. }\end{array}$ & $\begin{array}{l}\text { I.d.R. gesichert } \\
\text { durch konzeptio- } \\
\text { nellen Ansatz, } \\
\text { jedoch Kredit- } \\
\text { zinsbestimmung } \\
\text { problematisch. }\end{array}$ & $\begin{array}{l}\text { I.d.R. gesichert } \\
\text { durch konzeptio- } \\
\text { nellen Ansatz, } \\
\text { jedoch Kredit- } \\
\text { zinsbestimmung } \\
\text { problematisch. }\end{array}$ \\
\hline & Richtigkeit (2) & & & & & \\
\hline & Objektivität & $\begin{array}{l}\text { Theoretisch } \\
\text { gesichert, } \\
\text { jedoch auf- } \\
\text { grund der } \\
\text { Informations- } \\
\text { beschaffungs- } \\
\text { problematik } \\
\text { nicht haltbar. }\end{array}$ & $\begin{array}{l}\text { Prämisse der } \\
\text { vollkommenen } \\
\text { Kapitalmärkte } \\
\text { problematisch. } \\
\text { Objektiver } \\
\text { Untermehmens- } \\
\text { wert schwer } \\
\text { bestimmbar. } \\
\end{array}$ & $\begin{array}{l}\text { Muss uber } \\
\text { prognostizierte } \\
\text { Marktausfall- } \\
\text { raten innerhalb } \\
\text { der einzelnen } \\
\text { Segmente } \\
\text { gesichert } \\
\text { werden. } \\
\end{array}$ & $\begin{array}{l}\text { Gefahr der } \\
\text { Subjektivităt der } \\
\text { Rating- } \\
\text { agenturen, } \\
\text { Objektivität ist } \\
\text { nicht gesichert. }\end{array}$ & $\begin{array}{l}\text { Abhängig von } \\
\text { dem Bilanzana- } \\
\text { lyseverfahren. }\end{array}$ \\
\hline & Aktualität & $\begin{array}{l}\text { Theoretisch } \\
\text { gesichert, } \\
\text { jedoch auf- } \\
\text { grund der } \\
\text { Informations- } \\
\text { beschaffungs- } \\
\text { problematik } \\
\text { nicht unmittel- } \\
\text { bar haltbar. } \\
\end{array}$ & $\begin{array}{l}\text { Theoretisch } \\
\text { gesichert, } \\
\text { Informations- } \\
\text { beschaffung } \\
\text { aufgrund o.g. } \\
\text { Einschränkungen } \\
\text { nicht vollständig } \\
\text { gesichert. }\end{array}$ & $\begin{array}{l}\text { Abhängigkeit } \\
\text { von Dritten, da } \\
\text { keine öffent- } \\
\text { lich zugängli- } \\
\text { chen Marktda- } \\
\text { ten vorhanden } \\
\text { sind. }\end{array}$ & $\begin{array}{l}\text { Aktualităt der } \\
\text { Rating kann } \\
\text { nicht un- } \\
\text { eingeschrănkt } \\
\text { garantiert } \\
\text { werden. }\end{array}$ & $\begin{array}{l}\text { Abhängig von } \\
\text { dem Bilanzana- } \\
\text { lyseverfahren. }\end{array}$ \\
\hline
\end{tabular}

Tabelle 2: Zusammenfassung der Würdigung verschiedener Ansätze zur Quantifizierung des Ausfallrisikos

Kapitalmarkt. Empirische Untersuchungen weisen nach, dass die Aktualität des Rating kritisch ist. ${ }^{247}$ Des Weiteren besitzen nur wenige Unternehmen, vor allem in Europa, ein externes Rating. ${ }^{248}$ Offen ist die detaillierte Diskussion der Bilanzanalyse als Teil des internen Ratingystems. Es wird somit im Weiteren die Bilanzanalyse (Kap. 3) hinsichtlich der Eignung als internes Ratingystem und darüber hinaus für die Kalkulation von Ausfallrisiken diskutiert.

247 Vgl. bspw. Heinke (1998).

248 Vgl. Kirmße (1996), S. 39; Bröker (2000), S. 39. 
Marktorientierte Kreditrisikobewertung -

\section{Die Bilanzanalyse als Instrument zur einzelgeschäftsbezogenen Risikobewertung}

Wie oben gezeigt wurde, wird die Bilanzanalyse im Rahmen der Kreditzinskalkulation eingesetzt. Hierzu wird ein Rating aus den Bilanzkennzahlen abgeleitet und auf Basis dieses Urteils (dem Rating) ein Kreditzins kalkuliert (Kreditzinskalkulation mittels internem Rating). Um diese Vorgehensweise würdigen zu können, ist eine Beschreibung des angewendeten Bilanzanalyseverfahrens notwendig. In Kap. 3.1 werden die grundlegenden Aufgaben und Ziele der Bilanzanalyse in Abhängigkeit der Stellung des Bilanzanalytikers aufgezählt. Darauf aufbauend wird die für die vorliegende Arbeit relevante Stellung als „Kreditgeber" ${ }^{\text {"249 }}$ definiert. In Kap. 3.2 wird ein Kriterienkatalog aufgezeigt, mit dessen Hilfe die in Kap. 3.3 und Kap. 3.4 beschriebenen traditionellen und modernen Verfahren beurteilt werden. Diese Würdigung beschränkt sich in einem ersten Schritt auf die generelle Eignung als Bilanzanalyseverfahren. In einem zweiten Schritt in Kap. 3.6 werden die Bilanzanalyseverfahren herangezogen, welche diese Kriterien erfüllen, um ihre Anwendung als Kreditzinskalkulationsverfahren mittels internem Rating zu überprüfen. Hierzu werden wiederum die in Kap. 2.4 dargestellten Anforderungen an ein Kreditzinskalkulationsverfahren herangezogen.

\subsection{Begriffe, Adressaten und Ziele der Bilanzanalyse}

Unternehmen sind verpflichtet, mindestens jährlich Rechenschaft über ihre $\mathrm{Ge}-$ schäftstätigkeit abzulegen. ${ }^{250}$ Verschiedene nationale Gesetze oder internationale Prinzipien dokumentieren diese Aufgabe des externen Rechnungswesens. Bspw. findet diese Verpflichtung in $\S 242$ Handelsgesetzbuch (HGB) ${ }^{231}$ ihre rechtliche Grundlage, da hier der Kaufmann aufgefordert wird, „zu Beginn seines Handelsgewerbes und für den Schluss eines jeden Geschäftsjahres einen das Verhältnis seines

249 Vgl. hierzu auch die folgenden Ausführungen und die Darstellungen in Tabelle 3 und Tabelle 4.

250 Die Rechenschaftslegung ist bereits ein Jahresabschlusszweck. Vgl. hierzu bspw. Leffson (1964), S. 47f.

251 Die folgenden Ausführungen beziehen sich zunächst auf den entsprechenden Bereich des HGBs, da im weiteren Verlauf des Kapitels die grundlegende Vorgehensweise der Bilanzanalyse beschrieben wird. Die im Analyseverlauf verwendeten Kennzahlen beziehen sich auf Unternehmen, die nach den US-GAAP bilanzieren. Trotz der Diskrepanz wird bei der Herleitung der Aufgaben der Bilanzanalyse davon abstrahiert, um die Systematik der Analyse eindeutig zu gestalten. Im Kapitel 6.2.2 wird der weitere Forschungsbedarf aufgezeigt, um die gewonnenen Ergebnisse auf Bilanzen nach dem HGB zu übertragen. 
Marktorientierte Kreditrisikobewertung Die Bilanzanalyse als Instrument zur einzelgeschäftsbezogenen Risikobewertung

Vermögen und seiner Schulden darstellenden Abschluss (...) aufzustellen." ${ }^{\text {(2522253 Bei }}$ dieser Aufstellung hat der Kaufmann verschiedene Grundsätze und rechtlich festgeschriebene Normen zu beachten. Der Jahresabschluss umfasst neben der Bilanz i.e.S. ${ }^{254}$ noch eine Gewinn- und Verlustrechnung (GuV), wobei für Kapitalgesellschaften zusätzlich die Verpflichtung zur Aufstellung eines Anhangs und eines Lageberichtes besteht. ${ }^{255 / 256 / 257 / 258 / 259}$

Bilanzanalyse ist nach BALLWIESER die „Durchsicht und Auswertung von Jahresabschluß und Lagebericht zum Zweck der Informationsgewinnung "260/261. Als Bilanzanalyse wird somit die Aufbereitung bzw. Verdichtung von Informationen aus dem Jahresabschluss und dem Lagebericht mittels Kennzahlen, Kennzahlensystemen oder sonstigen Methoden und die Interpretation dieser Ergebnisse vor dem Hintergrund der wirtschaftlichen Rahmenbedingungen des Unternehmens und der allgemei-

\section{$252 \S 242(1) \mathrm{HGB}$.}

253 Nach § 44 BösG besteht für börsennotierte Unternehmen darüber hinaus die Verpflichtung, regelmäßig Zwischenberichte aufzustellen. Vgl. hierzu Bridts (1990); Coenenberg, Henes (1995), S. 969ff.; Baetge, Schlösser (1993), S. $225 \mathrm{ff.}$

254 I.e.S. bedeutet hier, dass die reine Vermögensübersicht als Bilanz bezeichnet wird. In der Praxis umfasst der Begriff Bilanz auch häufig die Gewinn- und Verlustrechnung und (bei Kapitalgesellschaften) den Lagebericht. Hier kann man von einer Bilanz i.w.S. sprechen. In dieser Arbeit bezieht sich der Begriff Bilanz sowohl auf die Bilanz i.e.S. als auch auf die GuV.

255 Große und mittlere Kapitalgesellschaften sind darüber hinaus zur Prüfung durch einen Abschlussprüfer verpflichtet ( 3316 Abs. 1 HGB). Die Beurteilung wird gemeinsam mit dem Jahresabschluss in Form eines Prüfungsberichtes und eines Bestätigungsvermerks bzw. Testats offengelegt. Vgl. Baetge (2001), S. 38ff.

$256 \mathrm{Vgl}$. Baetge (2001), S. 25ff.

$257 \mathrm{Zu}$ den verschiedenen Bilanzarten vgl. Wöhe (1996), S. 999ff. In der vorliegenden Arbeit ist mit Bilanz die gesetzlich vorgeschriebene jährlich aufzustellende Handelsbilanz bzw. deren Äquivalent nach amerikanischem Gesetz gemeint.

258 Vgl. zum Informationsgehalt des Anhangs bzw. Lageberichts Krumbholz (1994); Armeloh (1998) oder Baetge, Armeloh, Schulze (1997), S. 176ff.; Baetge, Armeloh, Schulze (1997b), S. $212 \mathrm{ff}$.

Neben der Bilanz, der GuV und dem Anlagebericht ist die Kapitalflussrechnung ein weiterer Bestandteil des Jahresabschlusses. Vgl. Käfer (1984), S. LVII und Käfer (1969), S. 9f.

261 Nicht der Begriff Bilanzanalyse ist treffend, sondern vielmehr müsste ein Begriff wie Jahresabschlussanalyse gebraucht werden (vgl. Leffson (1984), S. 3; Rehkugler, Poddig (1998), S. 9). Dies liegt daran, dass der Begriff Bilanz in der Praxis als Synonym für den Jahresabschluss gebraucht wird (vgl. Coenenberg (2000), S. 26). In dieser Arbeit werden die Begriffe Bilanzanalyse und Jahresabschlussanalyse synonym gebraucht. 
Marktorientierte Kreditrisikobewertung -

nen wirtschaftlichen Situation verstanden. ${ }^{262}$ Das Ziel der Bilanzanalyse ist die Gewinnung eines Gesamturteils über die wirtschaftliche Situation eines Unternehmens. $^{263}$

Die Vorgehensweise zur Urteilsfindung wird von KRUMNOW als „Aufgliederung und Aufbereitung von Jahresabschlüssen in deren Einzelelemente sowie [die Gewinnung von] sachlogische[n] zusammenhängende[n] Komponente[n] und Relation[en]"264 beschrieben. Ein Bilanzanalyst ist entweder ein externer oder interner Beobachter, ${ }^{265}$ wobei der externe Analytiker anhand der veröffentlichten Informationen (u.a.) im Jahresabschluss und der interne Analytiker anhand zusätzlicher Informationen aus dem internen Rechnungswesen sein Urteil bildet. ${ }^{266}$ Dabei bilden zwei Kernfragen die Basis der Gesamturteilsfindung: ${ }^{267}$

- Wie hoch ist die Ertragskraft des Unternehmens (erfolgswirtschaftliche Analyse)?

- Wie stark ist die finanzielle Stabilität des Unternehmens (finanzwirtschaftliche Analyse)?

In Abhängigkeit von den Zielen des Bilanzanalytikers bzw. seines Auftraggebers bestimmt sich die Gewichtung zwischen diesen beiden Teilfragen. Hierzu ist es von Bedeutung, den Adressaten der Bilanz bzw. Bilanzanalyse zu bestimmen. Nach der Koalitionstheorie muss dieser Kreis alle Personen einschließen, die in irgendeiner Weise mit dem Unternehmen in Kontakt stehen. ${ }^{268}$ Tabelle 3 gibt einen Überblick über potenzielle Bilanzanalytiker und damit die Jahresabschlussadressaten bzw. Informationsinteressenten.

262 Vgl. hier und im Folgenden Schierenbeck (2000), S. 582. und auch die von Baetge dargestellten sechs Schritte der Bilanzanalyse (1998), S. $16 f$.

263 Von dem Problemkreis der Konzernbilanzierung wird vereinfachend abgesehen, da hiervon die allgemeine Vorgehensweise der Bilanzanalyse nicht betroffen ist.

264 Krumnow (1985), S. 783; die mit [ ] gekennzeichneten Inhalte wurden vom Verfasser hinzugefügt.

265 Vgl. Baetge (1998), S. 6ff.

266 In der Praxis bezieht sich der Begriff Bilanzanalytiker überwiegend auf den externen Beobachter. Vgl. Küting, Weber (2000), S. $10 \mathrm{f}$.

$267 \mathrm{Vgl}$. hier und im Folgenden Baetge (1998), S. $2 \mathrm{ff}$.

268 Vgl. Coenenberg (2000), S. 1074ff. 
Marktorientierte Kreditrisikobewertung Die Bilanzanalyse als Instrument zur einzelgeschäftsbezogenen Risikobewertung

\begin{tabular}{|c|c|}
\hline Externe Bilanzanalytiker & Interne Bilanzanalytiker \\
\hline $\begin{array}{ll}\text { - } & \text { Kreditgeber, Kreditversicherer, Auskunfteien } \\
\text { - } & \text { Rlein-)Aktionäre, externe Gesellschafter } \\
\text { - } & \text { Lieferanten, Kunden } \\
\text { - } & \text { Finanzanalysten } \\
\text { - } & \text { Bundesbank } \\
\text { - } & \text { Arbeitnehmer, Gewerkschaften } \\
\text { - } & \text { Konkurrenzunternehmen } \\
\text { - } & \text { Presse }\end{array}$ & $\begin{array}{ll} & \text { Unternehmensleitung } \\
\text { - } & \text { Kontrollorgane (Aufsichtsrat, Beirat, Jahres- } \\
& \text { abschlussprüfer) } \\
\text { - } & \text { Beteiligungscontroller } \\
\text { - } & \text { Großaktionär } \\
\text { - } & \text { Großkreditgeber } \\
\text { Finanzverwaltung }\end{array}$ \\
\hline
\end{tabular}

Tabelle 3: Übersicht über externe und interne Bilanzanalytiker ${ }^{269}$

Das Informationsbedürfnis des Adressaten bestimmt die konkreten Aufgaben und Schwerpunkte der Bilanzanalyse. Tabelle 4 gibt einen Überblick über die unterschiedlichen Bedürfnisse, die eine Bilanzauswertung in Abhängigkeit vom Adressatenkreis begleiten. Die Bilanzanalyse wird somit zu einem „adressaten- und zweckspezifischen Auswertungssystem " ${ }^{\text {"270, }}$; man kann ihre Zwecksetzung lediglich deduktiv aus den Informationsbedürfnissen des Analytikers ableiten. ${ }^{271}$

Eine der bedeutsamsten Gruppen ist die der Kreditgeber. ${ }^{272} \mathrm{Zu}$ ihnen zählen neben Kreditinstituten auch Leasinggesellschaften, Kreditversicherer und Kapitalsammelstellen im Allgemeinen. Ihr Informationsbedürfnis liegt in der Ermittlung der Kreditwürdigkeit bzw. eines Bonitätsstatutes. Eine wesentliche Aufgabe der Kreditwürdigkeitsprüfung ist die Beurteilung der Zahlungsfähigkeit des Schuldners. ${ }^{273}$ Ein Schwerpunkt der Bilanzanalyse liegt somit aus der Sicht der Kreditgeber auf der Ermittlung der gegenwärtigen und zukünftigen finanziellen Fähigkeit zur Zahlung der vereinbarten Zins- und Tilgungsleistungen. ${ }^{274}$ In der Praxis der Kreditwirtschaft ist es daher selbstverständlich, dass ein Unternehmen u.a. seine Bilanz im Rahmen der Kreditwürdigkeitsprüfung einreichen muss. ${ }^{275}$ Bei der im Kap. 5 folgenden Konzepti-

In Anlehnung an Baetge (1998), S. 7.

Pellens (1989), S. 155.

Vgl. Buchner (1981), S. 195.

Vgl. hier und im Folgenden Baetge (1998), S. 8f.

Vgl. hier und im Folgenden Buchner (1981), S. 199.

Vgl. auch zu den Bestandteilen der Kreditwürdigkeitsprüfung und der Unterscheidung von Kreditfähigkeit und Kreditwürdigkeit Bröker (2000), S. 44f.

Nach § 18 KWG ist ein Kreditinstitut verpflichtet, sich bei Vergabe von einem Kredit ab 500.000 DM (bzw. 250.000 EURO) die wirtschaftlichen Verhältnisse offen legen zu lassen. Dies kann z.B. durch die Vorlage eines aktuellen Jahresabschlusses geschehen. In der Praxis wird jedoch regelmäßig auch bei kleineren Krediten eine Bilanz von den Unternehmen verlangt. In den weiteren Ausführungen wird die Position des Kreditgebers unterstellt. 
Marktorientierte Kreditrisikobewertung -

on eines marktorientierten Prognosemodells unter zu Hilfenahme der Bilanzanalyse wird die Position eines Kreditgebers unterstellt.

\begin{tabular}{|c|c|}
\hline Adressat & Informationsbedirfnis bezüglich: \\
\hline $\begin{array}{l}\text { Kreditgeber, Kreditversicherer, } \\
\text { Auskunfteien }\end{array}$ & Kreditwürdigkeit, Bonitătsstatus \\
\hline $\begin{array}{l}\text { (Klein-)Aktionäre, exteme Gesell- } \\
\text { schafter }\end{array}$ & \begin{tabular}{|c|}
$\begin{array}{c}\text { Risiko-Rendite-Struktur }{ }^{276} \text { bzw. der risikoadjustierten Verzinsung des eingesetz- } \\
\text { ten Kapitals }\end{array}$ \\
\end{tabular} \\
\hline Ratingagenturen & Bonităt im Hinblick auf eine Ratingeinstufung ${ }^{277}$ \\
\hline Lieferanten & $\begin{array}{l}\text { Bonităt im Hinblick auf die Aufnahme einer } \\
\text { Geschäftsbeziehung }\end{array}$ \\
\hline Kunden & $\begin{array}{l}\text { Bonität im Hinblick auf die Aufnahme einer } \\
\text { Geschäftsbeziehung }\end{array}$ \\
\hline Finanzanalysten & $\begin{array}{c}\text { Zukünftige Ertragskraft im Hinblick auf die Kauf- bzw. Verkaufsentscheidung } \\
\text { des entsprechenden börsennotierten } \\
\text { Wertpapiers }\end{array}$ \\
\hline Bundesbank & $\begin{array}{l}\text { Bonităt im Hinblick auf den Eingang von } \\
\text { Wechselverbindlichkeiten }\end{array}$ \\
\hline Arbeitnehmer, Gewerkschaften & Bestandskraft und Arbeitsplatzsicherheit \\
\hline Konkurrenzunternehmen & Stärken-Schwăchen-Analysen ${ }^{279}$ \\
\hline Presse & Wenige ,publikumsfahige“ Kennzahlen im Hinblick auf Pressemitteilungen ${ }^{280}$ \\
\hline Unternehmensleitung & Eigene wirtschaftliche Lage, Simulation von Bilanzwahlrechten ${ }^{281}$ \\
\hline $\begin{array}{l}\text { Kontrollorgane (Aufsichtsrat, Beirat, } \\
\text { Jahresabschlussprüfer) }\end{array}$ & $\begin{array}{l}\text { Einhaltung (gesetzlicher) Vorschriften (Rechenschaft des Vorstands), wirt- } \\
\text { schaftliche Lage des Unternehmens }{ }^{282}\end{array}$ \\
\hline Beteiligungscontroller & (Teil-)Rentabilităten ejnzelner Einheiten \\
\hline Großaktionăre / Großkreditgeber & Wirtschaftliche Entwicklung bzw. Rentabilitäten einzelner Einheiten \\
\hline Finanzverwaltung & Steuerlicher Gewinn bzw. Verlust \\
\hline
\end{tabular}

Tabelle 4: Informationsbedürfnisse der unterschiedlichen Adressaten der Bilanzanalyse ${ }^{203}$

Vgl. Perridon, Steiner (1997), S. 279f.

Vgl. Berblinger (1996), S. $63 \mathrm{f}$.

Nach $\S 19$ BbankG ist die Deutsche Bundesbank zur Bonitätsprüfung verpflichtet, falls sie mit dem entsprechenden Unternehmen ein Wechselgeschäft eingehen möchte. Die Bedeutung von Wechselgeschäften wird jedoch in Zukunft zurückgehen, da es im Rahmen der Einführung der europäischen Zentralbank zu einer Verschiebung in der Bedeutung der einzelnen geldpolitischen Instrumenten kam. Das Instrument des Rediskontkontingents wird an Bedeutung verlieren (vgl. zu den Auswirkung des Liquiditätsgrundsatzes auf die Geschäftspraktiken der Refinanzierung, Kirmße, Siemes (1999b)).

Vgl. Küting, Weber (2000), S. 11; Coenenberg (1990), S. 13; Grütter-Settele (1999).

Vgl. Helbling (1992), S. 16.

Vgl. bspw. Baetge, Ballwieser (1978), S. 511f. oder Hinz (1994).

Vgl. Krüger (1983), S. 269ff.; Potthoff (1996), S. 835ff.

Die Systematik lehnt sich an Baetge (1998) S. 8ff. an. 
Marktorientierte Kreditrisikobewertung Die Bilanzanalyse als Instrument zur einzelgeschäftsbezogenen Risikobewertung

\subsection{Kriterienkatalog zur Würdigung der unterschiedlichen Methoden der Bilanzanalyse}

Bevor die Auswertungsmethoden der Bilanzanalyse und die Vorgehensweise genauer beschrieben werden, sollen allgemeingültige Kriterien definiert werden, mit denen sich die Güte einer Bilanzanalyse oder besser die Analysestärke der Instrumente einer Bilanzanalyse messen lassen. Hierzu werden die von BAETGE formulierten Prinzipien

- das Neutralisierungsprinzip,

- das Ganzheitlichkeitsprinzip und

- das Objektivierungsprinzip verwendet. ${ }^{284}$

Das Neutralisierungsprinzip bezieht sich auf die bilanziellen Freiräume, die man im Rahmen der Bilanzierung genießt. Die Bilanzanalyse hat die Aufgabe, die Kennzahlen bzw. das Gesamturteil so zu bilden, dass diese Wahlrechte neutralisiert werden, d.h,. unabhängig von den bilanzpolitischen Gestaltungsentscheidungen muss die Bilanzanalyse zu demselben Ergebnis kommen.

Das Prinzip der Ganzheitlichkeit besagt, dass alle Bereiche des Unternehmens und folglich sowohl die Finanz- als auch Ertrags- und Vermögenslage Einfluss auf das Gesamturteil nehmen sollten ${ }^{285}$ Ferner hat der Bilanzanalytiker auch weitere quantitative Informationen, wie sie bspw. im Lagebericht erkennbar sind, in seine Urteilsfindung oder seine Urteilsbegründung im Sinne einer Ursachenanalyse einzubeziehen. Bei der Sammlung der relevanten Daten sind neben den aus der Bilanz erkennbaren Informationen nämlich auch wirtschaftliche und rechtliche Rahmenbedingungen zu beachten. ${ }^{286}$ Darüber hinaus können noch weitere Informationen wichtig sein, die nicht in quantitativer Form vorliegen.

Das Objektivierungsprinzip bezieht sich auf zwei Dimensionen: Zum einen muss das abgeleitete Urteil intersubjektiv nachvollziehbar und zum anderen müssen die benutzten Gewichtungen objektiv sein. Ein Verfahren ist genau dann als objektiv zu bezeichnen, „wenn die Auswahl, Gewichtung und Zusammenfassung der Urteilskriterien, also der Kennzahlen bei der Bilanzanalyse, unabhängig von subjektiven Empfindungen und Erfahrungen des Bilanzanalytikers - auf der Basis einer breiten empirischen Basis und mit mathematisch-statistischen Methoden ermittelt - geschieht, da bisher eine Theorie der Bilanzanalyse fehlt ${ }^{40}$. ${ }^{287}$

284 Vgl. auch für die folgenden Erläuterungen Baetge (1998), S. 54f. und S. $513 \mathrm{ff}$.

285 Vgl. auch zu den Aufgaben und Zielen einer vollständigen Datenerfassung Lachnit (1976), S. 143 und Schedelbauer (1978), S. 2425.

286

Vgl. Riebell (1996), S. 474ff.

Vgl. Baetge (1998), S. 514f. 
Marktorientierte Kreditrisikobewertung -

Die Bilanzanalyse als Instrument zur einzelgeschäftsbezogenen Risikobewertung

Diese generellen Maßstäbe eignen sich, die verschiedenen Modelle hinsichtlich der konzeptionellen Beschaffenheit und der Einhaltung der Prinzipien in der praktischen Umsetzung zu beurteilen.

\subsection{Traditionelle Bilanzanalyse}

Mit Hilfe von Kennzahlen, Kennzahlensystemen und Scoring-Modellen lassen sich Bilanzen hinsichtlich der o.g. Zielsetzung, nämlich der Beurteilung der zukünftigen Zahlungsfähigkeit eines Unternehmens, analysieren. Im Weiteren (Kap. 3.3.1, Kap. 3.3.2 und Kap. 3.3.3) werden diese drei Ansätze allgemein dargestellt und an einem oder mehreren Beispielen konkretisiert. Die Auswahl der Beispiele lehnt sich an das in Kap. 4 zu konzipierende Prognosemodell an. In Kap. 3.3.4 werden diese drei Verfahren mit Hilfe der in Kap. 3.2 beschriebenen Kriterien hinsichtlich ihrer Eignung als Bilanzanalyseverfahren gewürdigt.

\subsubsection{Kennzahlen}

Den traditionell höchsten Stellenwert innerhalb der Auswertungsmethodik haben Kennzahlen und die damit eng verbundenen Kennzahlensysteme. ${ }^{288}$ Eine Kennzahl stellt einen quantitativ erfassbaren Zusammenhang in bewusst einfacher und konzentrierter Form dar. ${ }^{289}$ Es lassen sich zum einen absolute Kennzahlen (wie Bilanzsumme) und zum anderen relative Kennzahlen, d.h. Verhältniszahlen (wie Fremdkapital / Bilanzsumme) bilden. ${ }^{290}$ BAETGE ordnet absoluten Zahlen keine große Aussagekraft zu, da sie weder Tendenzen erkennen lassen, noch Ursache-WirkungsZusammenhänge verdeutlichen können. ${ }^{291}$ Dementsprechend finden relative Kennzahlen (unter Umständen in Verbindung mit absoluten Kennzahlen) größere Beachtung. Es gibt verschiedene Kriterien, nach denen man die Fülle der Kennzahlen gliedern kann. Bspw. kann man eine Aufteilung in Gliederungs-, Beziehungs- und Indexzahlen vornehmen. ${ }^{292}$ Gliederungszahlen sind Relationen von Teilmengen zur Gesamtheit (wie die Eigenkapitalquote ${ }^{293}$ ). Beziehungszahlen drücken UrsacheWirkungs-Zusammenhänge bzw. Mittel-Zweck-Zusammenhänge aus, indem sie

288 Der Begriff Kennzahlensystem wird in Kap. 3.3.2 definiert und zum Begriff Kennzahl abgegrenzt.

289 Vgl. Makoski (1999), S. 79.

290 Vgl. Schierenbeck (2000), S. 620.

291 Vgl. Baetge (1998), S. 27f.

292 Vgl. Hauschildt (1971), S. 340.

293 Die Eigenkapitalquote sei hier vereinfachungsgemäß als Eigenkapital / Gesamtkapital verstanden. 
Marktorientierte Kreditrisikobewertung Die Bilanzanalyse als Instrument zur einzelgeschäftsbezogenen Risikobewertung

verschiedenartige Zahlen in Beziehung setzen (wie die Eigenkapitalrentabilität ${ }^{294}$ ). ${ }^{295}$ Indexzahlen schließlich zeigen die zeitliche Entwicklung einzelner Kennzahlen auf (wie das prozentuale Wachstum des Umsatzes). Neben dieser Aufteilung lassen sich Kennzahlen auch nach den Kriterien der Sensibilität hinsichtlich bilanzpolitischer Veränderungen und Reagibilität in Bezug auf Datenänderungen gliedern. ${ }^{296}$ Ein weiteres Kriterium ist die mit Hilfe der Kennzahl verfolgte Zielsetzung, nämlich die Beschreibung der Vermögens-, Finanz- oder Ertragslage.

Tabelle 5 und Tabelle 6 zeigen einen Überblick über 70 verschiedene Kennzahlen. Die Benennung in der ersten Spalte der Kennzahl $\mathrm{K}_{\mathrm{j}}$ mit $1 \leq \mathrm{j} \leq 70$ lehnt sich, wie die Auswahl, an SCHULTE an.$^{297}$ Sie bildet neben den später folgenden Kennzahlensystemen die Grundlage der Kennzahlen der empirischen Untersuchung. ${ }^{298}$ SCHULTE zog diese Kennzahlen als potenzielle Erklärungsgrößen im Rahmen einer Arbeit zur kapitalmarktorientierten Bilanzanalyse heran. ${ }^{299}$ Die Kennzahlen $\mathrm{K}_{01}-\mathrm{K}_{25}$ beziehen sich auf die finanzielle Stabilität des Unternehmens und sind einer finanzwirtschaftlichen Jahresabschlussanalyse zuzuordnen $\left(\mathrm{K}_{01}-\mathrm{K}_{05}\right.$ : Investitionsanalyse, $\mathrm{K}_{06}-\mathrm{K}_{14}$ : Finanzierungsanalyse, $K_{15}-K_{25}$ : Liquiditätsanalyse). Die Kennzahlen $K_{26}-K_{50}$ stellen eher auf die Ertragskraft und somit die erfolgswirtschaftliche Bilanzanalyse $a b$ $\left(\mathrm{K}_{26}-\mathrm{K}_{29}\right.$ : Erfolgsstrukturanalyse, $\mathrm{K}_{30}-\mathrm{K}_{50}$ : Rentabilitätsanalyse). Die Kennzahlen $\mathrm{K}_{50}-\mathrm{K}_{70}$ orientieren sich primär an der kapitalmarktorientierten Bilanzanalyse. ${ }^{300}$ Eine Möglichkeit zur Bewertung einzelner Kennzahlen hinsichtlich ihrer Eignung zur Bilanzanalyse besteht darin zu prüfen, ob es mit dieser Kennzahl gelingt, bilanzielle Spielräume zu eliminieren. ${ }^{301}$ Diese Fähigkeit wurde in Kap. 3.2 dem Neutralisierungsprinzip zugeordnet. Grundsätzlich ist die Subjektivität bei der Einschätzung der

294 Die Eigenkapitalrentabilität sei hier vereinfachungsgemäß als Jahreserfolg / Eigenkapital verstanden.

295 Vgl. hier und im Folgenden Hauschildt (1971), S. $340 \mathrm{ff}$.

296 Vgl. Rehkugler, Schindel (1994), S. 252.

297 Vgl. Schulte (1996b), S. 144f. Einzelne Positionen können als Durchschnittsgröße angegeben werden. Somit würde nicht der Bilanzstichtagswert verwendet, sondern das arithmetische Mittel aus den Werten zum Bilanzstichtag und dem Bilanzstichtag des Vorjahres. Damit kann bei der Berechnung von Kennzahlen eine zeitliche Verteilung der Stichtagsgröße auf den Betrachtungszeitraum erreicht werden. Aus Gründen der vereinfachten Darstellung wird im Weiteren darauf verzichtet. Bei der im Kap. 5 folgenden empirischen Untersuchung soll durch einen möglichst umfassenden Kennzahlenkatalog nicht bereits in der Kennzahlenauswahl eine Ergebnisbeeinträchtigung erzielt werden.

Vgl. Schulte (1996b). Zur Definition des Begriffs „kapitalmarktorientierte Bilanzanalyse" vgl. Kap. 4 und Coenenberg (2000), S. $1121 \mathrm{f}$.

Vgl. Schulte (1996b), S. 144f.

301 Vgl. hier und im Folgenden Baetge (1998), S. $155 f$. 
Marktorientierte Kreditrisikobewertung -

Die Bilanzanalyse als Instrument zur einzelgeschäftsbezogenen Risikobewertung

Unternehmenslage aufgrund von Kennzahlen problematisch. Obwohl die Urteilsfindung intersubjektiv nachvollzogen werden kann, d.h., das anhand von Kennzahlen abgeleitete Urteil kann auch von einem Dritten gebildet werden, ist das Verfahren der Urteilsbildung selbst subjektiv. Die Urteilsbildung ist der Auswahl und Gewichtung einzelner Kennzahlen für die Urteilsfindung gleichzusetzen. Dem Bilanzanalytiker obliegt eben diese Urteilsbildung, die aufgrund der subjektiven Einschätzung vorgenommen wird.

\begin{tabular}{|c|c|c|}
\hline $\mathrm{Nr}$. & Kennzah! & Kennzahlendefinition \\
\hline $\mathrm{K}_{01}$ & Umschlagshauufigkeit der Vorrăte & Umsatzerlóse / Vorrate \\
\hline $\mathrm{K}_{02}$ & Umschlagshāufigkeit der Fertigerzeugnisse u. Waren & Umsatzerlöse / (Fertigerzeugnisse + Waren) \\
\hline $\mathrm{K}_{03}$ & Umschlagshăufigkeit des Gesamtkapitals & Umsatzerlose / Gesamtkapital \\
\hline $\mathrm{K}_{04}$ & Investitionsdeckung & $\begin{array}{l}\text { Abschreibungen Sachanlagevermögen / Investitionen Sachan- } \\
\text { lageverm } 8 g e n\end{array}$ \\
\hline $\mathrm{K}_{\mathrm{os}}$ & Abschreibungsquote & $\begin{array}{l}\text { Abschreibungen Sachanlagevermögen / Sachanlagevermogen } \\
\text { (zu historischen Anschaffungs- / Herstellungskosten) }\end{array}$ \\
\hline $\mathrm{K}_{06}$ & Eigenkapitalquote I & Eigenkapital / Gesamtkapital \\
\hline $\mathrm{K}_{07}$ & Eigenkapitalquote II & $\begin{array}{l}\text { (Eigenkapital - Immaterielle Vermoggensgegen- } \\
\text { stănde) / (Gesamtkapital - Immaterielle Vermogensgegenstănde } \\
\text { - Liquide Mittel - Grundstucke und Gebaude) }\end{array}$ \\
\hline $\mathrm{K}_{08}$ & Statischer Verschuldungsgrad II & Kurzfristiges Fremdkapital / Eigenkapital \\
\hline $\mathrm{K}_{09}$ & Statischer Verschuldungsgrad V & $\begin{array}{l}\text { (Fremdkapital - Pensionsrackstellungen) / (Eigenkapital + } \\
\text { Pensionsrülckstellungen) }\end{array}$ \\
\hline $\mathrm{K}_{10}$ & Anspannungsgrad II & Kurzfristiges Fremdkapital / Gesamtkapital \\
\hline $\mathrm{K}_{11}$ & Fremdkapitalstruktur I & $\begin{array}{l}\text { (Verbindlichkeiten aus Lieferungen und Leistungen + Wech- } \\
\text { selverbindlichkeiten) / Fremdkapital }\end{array}$ \\
\hline $\mathrm{K}_{12}$ & Fremdkapitalstruktur II & $\begin{array}{l}\text { (Verbindlichkeiten aus Lieferungen und Leistungen + Wech- } \\
\text { selverbindlichkeiten + Bankverbindlichkeiten) / Fremdkapital }\end{array}$ \\
\hline $\mathrm{K}_{13}$ & Selbstfinanzierungsgrad II & Gewinnrlcklagen / Gesamtkapital \\
\hline $\mathrm{K}_{14}$ & Bilanzkurs & Eigenkapital / Gezeichnetes Kapital \\
\hline $\mathrm{K}_{1 \mathrm{~S}}$ & Anlagendeckungsgrad I & Eigenkapital/ Anlagevermógen \\
\hline $\mathrm{K}_{16}$ & Anlagendeckungsgrad II & (Eigenkapital + Langfristiges Fremdkapital) / Anlagevermogen \\
\hline$K_{17}$ & Anlagendeckungsgrad IV & $\begin{array}{l}\text { Eigenkapital / ( Sachanlagevermógen - Grundstocke und } \\
\text { Gebăude) }\end{array}$ \\
\hline $\mathrm{K}_{18}$ & Liquiditat 2. Grades (quick ratio) & Monetâres Umlaufvermogen / Kurzfristiges Fremdkapital \\
\hline $\mathrm{K}_{19}$ & Dynamische Investitionsdeckung & $\begin{array}{l}\text { (Netto Cash Flow - Ausschuttungen) / Investitionen Sachanla- } \\
\text { gevermogen }\end{array}$ \\
\hline $\mathrm{K}_{20}$ & Dynamische Fremdkapitaldeckung & Netto Cash Flow / Fremdkapital \\
\hline $\mathrm{K}_{21}$ & \begin{tabular}{|l|} 
Kapitalrückflussquote I \\
\end{tabular} & Betriebsbedingter Brutto Cash Flow / Kurzfristiges Fremdkap. \\
\hline $\mathrm{K}_{22}$ & Kapitalrulckflussquote II & Netto Cash Flow / (Fremdkapital - Erhaltene Anzahlungen) \\
\hline $\mathrm{K}_{23}$ & Fremdfinanzierung des Umsatzes I & Effektivverschuldung / Umsatzerlose \\
\hline $\mathrm{K}_{24}$ & Fremdfinanzierung des Umsatzes II & (Effektivverschuldung - Erhaltene Anzahlugen) / Umsatzerlose \\
\hline $\mathrm{K}_{22}$ & Fremdfinanzierung des Umsatzes III & Kurzfristiges Fremdkapital / Umsatzerlose \\
\hline $\mathrm{K}_{26}$ & Ergebnisstruktur I & Betriebsergebnis / Jahresergebnis vor Gewinnsteuem \\
\hline $\mathrm{K}_{27}$ & Ergebnisstruktur II & Finanzergebnis / Jahresergebnis vor Gewinnsteuem \\
\hline $\mathrm{K}_{28}$ & Ergebnisstruktur III & $\begin{array}{l}\text { (Betriebsergebnis + Finanzergebnis) / Jahresergebnis vor } \\
\text { Gewinnsteuern }\end{array}$ \\
\hline
\end{tabular}

Tabelle 5: Uีbersicht wichtiger Kennzahlen der Bilanzanalyse (Teil I) 


\begin{tabular}{|c|c|c|}
\hline Nr. & Kennzahl & Kennzahlendefinition \\
\hline $\mathrm{K}_{29}$ & Ergebnisstruktur IV & Außerordentliches Ergebnis / Jahresergebnis v. Gewinnsteuem \\
\hline $\mathrm{K}_{30}$ & Eigenkapitalrentabilităt vor Gewinnsteuem & (Jahresergebnis + Gewinnsteuern) / Eigenkapital \\
\hline $\mathrm{K}_{31}$ & Eigenkapitalrentabilităt nach Gewinnsteuem & Jahresergebnis / Eigenkapital \\
\hline $\mathrm{K}_{32}$ & Cash Flow-Eigenkapitalrentabilităt I v. Gewinnsteuern & Brutto Cash Flow / Eigenkapital \\
\hline $\mathrm{K}_{33}$ & Cash Flow-Eigenkapitalrentabilităt I nach Gewinnsteuern & Netto Cash Flow / Eigenkapital \\
\hline $\mathrm{K}_{34}$ & Cash Flow-Eigenkapitalrentabilităt II vor Gewinnsteuern & Betriebsbedingter Brutto Cash Flow / Eigenkapital \\
\hline $\mathrm{K}_{35}$ & $\begin{array}{l}\text { Cash Flow-Eigenkapitalrentabilitat II nach Gewinnsteu- } \\
\text { em }\end{array}$ & Betriebsbedingter Netto Cash Flow / Eigenkapital \\
\hline $\mathrm{K}_{36}$ & Fremdkapitalrentabilităt & Zinsaufwand / Fremdkapital \\
\hline $\mathrm{K}_{37}$ & Gesamtkapitalrentabilităt vor Gewinnsteuem & $\begin{array}{l}\text { (Jahresergebnis + Gewinnsteuern + Zinsauf- } \\
\text { wand)/Gesamtkapital }\end{array}$ \\
\hline $\mathrm{K}_{38}$ & Gesamtkapitalrentabilităt nach Gewinnsteuem & (Jahresergebnis + Zinsaufwand) / Gesamtkapital \\
\hline $\mathrm{K}_{39}$ & $\begin{array}{l}\text { Cash Flow-Gesamtkapitalrentabilităt I vor Gewinnsteu- } \\
\text { em }\end{array}$ & Brutto Cash Flow / Gesamtkapital \\
\hline $\mathrm{K}_{40}$ & $\begin{array}{l}\text { Cash Flow-Gesamtkapitalrentabilităt I nach Gewinnsteu- } \\
\text { em }\end{array}$ & Netto Cash Flow / Gesamtkapital \\
\hline$K_{41}$ & $\begin{array}{l}\text { Cash Flow-Gesamtkapitairentabilităt II vor Gewinnsteu- } \\
\text { em }\end{array}$ & Betriebsbedingter Brutto OCash Flow / Gesam(kapital \\
\hline $\mathrm{K}_{42}$ & $\begin{array}{l}\text { Cash Flow-Gesamtkapitalrentabilităt II nach Gewinn- } \\
\text { steuem }\end{array}$ & Betriebsbedingter Netto Cash Flow / Gesamtkapital \\
\hline$\overline{K_{43}}$ & $\begin{array}{l}\text { Gesamtkapitalrentabilităt nach Zinsen, vor Gewinnsteu- } \\
\text { em }\end{array}$ & (Jahresergebnis + Gewinnsteuem)/ Gesamtkapital \\
\hline $\mathrm{K}_{44}$ & $\begin{array}{l}\text { Gesamtkapitalrentabilităt nach Zinsen, nach Gewinn- } \\
\text { steuem }\end{array}$ & Jahresergebnis / Gesamtkapital \\
\hline $\mathrm{K}_{45}$ & Umsatzrentabilităt vor Gewinnsteuem & Betriebsergebnis / Umsatzerlose \\
\hline $\mathrm{K}_{46}$ & Umsatzrentabilităt nach Gewinnsteuern & (Betriebsergebnis - Gewinnsteuem)/ Umsatzerlöse \\
\hline $\mathrm{K}_{47}$ & Cash Flow-Umsatzrentabilitat I vor Gewinnsteuem & Brutto Cash Flow / Umsatzerlöse \\
\hline $\mathrm{K}_{48}$ & Cash Flow-Umsatzrentabilität I nach Gewinnsteuem & Netto Cash Flow / Umsatzerlöse \\
\hline $\mathrm{K}_{49}$ & Cash Flow-Umsatzrentabilităt II vor Gewinnsteuem & Betriebsbedingter Brutto Cash Flow / Umsatzerlöse \\
\hline $\mathrm{K}_{50}$ & Cash Flow-Umsatzrentabilităt II nach Gewinnsteuern & $\begin{array}{l}\text { (Betriebsbedingter Brutto Cash Flow - Gewinnsteuem) } \\
\text { /Umsatzerlose } \\
\end{array}$ \\
\hline $\mathrm{K}_{\mathrm{gi}}$ & Jahresergebnis je Aktie & Jahresergebnis / Aktienanzahl \\
\hline $\mathrm{K}_{52}$ & Veränderungsrate Jahresergebnis je Aktie & Verănderungsrate Jahresergebnis / Aktienanzahl \\
\hline $\mathrm{K}_{93}$ & Betriebsergebnis je Aktie & Betriebsergebnis / Aktienanzahl \\
\hline $\mathrm{K}_{54}$ & Verănderungsrate Betriebsergebnis je Aktie & Verănderungsrate Betriebsergebnis / Aktienanzahl \\
\hline $\mathrm{K}_{\mathrm{ss}}$ & Finanzergebnis je Aktie & Finanzergebnis / Aktienanzahl \\
\hline $\mathrm{K}_{56}$ & Verănderungsrate Finanzergebnis je Aktie & Verănderungsrate Finanzergebnis / Aktienanzahl \\
\hline $\mathrm{K}_{57}$ & Betriebs- und Finanzergebnis je Aktie & (Betriebsergebnis + Finanzergebnis)/ Aktienanzahl \\
\hline$K_{s 8}$ & Verănderungsrate Betriebs- und Finanzergebnis je Aktie & $\begin{array}{l}\text { Verănderungsrate (Betriebsergebnis + Finanzergeb- } \\
\text { nis)/ Aktienanzahl }\end{array}$ \\
\hline $\mathrm{K}_{\mathrm{g9}}$ & Gewinn nach DVFA / SG je Aktie & Bereinigtes Betriebsergebnis / Aktienanzahl \\
\hline$K_{60}$ & Verănderungsrate Gewinn nach DVFA / SG je Aktie & Veranderungsrate Bereinigtes Betriebsergebnis / Aktienanzahl \\
\hline $\mathrm{K}_{61}$ & Cash Flow nach DVFA / SG je Aktie & Cash Flow nach DVFA / SG Aktienanzahl \\
\hline $\mathrm{K}_{62}$ & Verănderungsrate Cash Flow nach DVFA / SG je Aktie & Verănderungsrate Cash Flow nach DVFA / SG Aktienanzahl \\
\hline $\mathrm{K}_{63}$ & Kurs-Gewinn-Verhăltnis & Kurs / Gewinn nach DVFA / SG je Aktie \\
\hline $\mathrm{K}_{64}$ & Verănderungsrate Kurs-Gewinn-Verhaltnis & Verănderungsrate Kurs / Gewinn nach DVFA / SG je Aktie \\
\hline $\mathrm{K}_{65}$ & Kurs Cash Flow-Verhăltnis & Kurs / Cash Flow nach DFVA / SG je Aktie \\
\hline $\mathrm{K}_{66}$ & Veranderungsrate Kurs-Cash Flow-Verhalitnis & Verănderungsrate Kurs / Cash Flow nach DFVA / SG je Aktie \\
\hline$K_{67}$ & Ausschüttungsquote II & Bilanzgewinn (bzw. Dividendensumme) / Eigenkapital \\
\hline $\mathrm{K}_{68}$ & Verănderungsrate Ausschüttungsquote II & $\begin{array}{l}\text { Verănderungsrate Bilanzgewinn (bzw. Dividendensum- } \\
\text { me)/Eigenkapital }\end{array}$ \\
\hline $\mathrm{K}_{69}$ & Dividendenrendite & (Nettodividenden + Steuergutschrift)/ Kurs \\
\hline $\mathrm{K}_{70}$ & Verd̆nderungsrate Dividendenrendite & Verănderungsrate (Nettodividenden + Stevergutschrift) / Kurs \\
\hline
\end{tabular}

Tabelle 6: Übersicht wichtiger Kennzahlen der Bilanzanalyse (Teil II) 
Marktorientierte Kreditrisikobewertung -

\subsubsection{Kennzahlensysteme}

Aufgrund der geringen Aussagekraft und widersprüchlichen Interpretationsmöglichkeiten einzelner Kennzahlen sind Systeme von Kennzahlen zu bilden, ${ }^{302}$ die komplexe Sachverhalte aus unterschiedlichen Blickwinkeln beleuchten ${ }^{303}$ Falls man nicht einzelne Kennzahlen betrachten möchte, sondern diese verdichtet in ein System überführt, spricht man i.d.R. von so genannten Kennzahlensystemen. ${ }^{304}$ Häufig bilden diese Systeme eine Spitzenkennzahl, welche anhand analytischer Überlegungen in Unterkennzahlen zerlegt bzw. sich aus der Aggregation und Verknüpfung der verschiedenen Unterkennzahlen ergibt. Hierdurch entstehen Kennzahlenpyramiden. Der Vorteil liegt in der Darstellung der Ursache-Wirkungs-Zusammenhänge, welche nun nicht mehr mono- sondern multikausale Verknüpfungen abbilden können. ${ }^{305}$ Falls ein Kennzahlensystem dieses Aufbrechen in Unterkennzahlen leistet, spricht man von einem analytisch-deduktiven System.

Den drei o.g. Prinzipien (Neutralisierungs-, Ganzheitlichkeits- und Objektivierungsprinzip) wird bei der Überleitung einzelner Kennzahlen in ein System eine größere Beachtung als bei der einzelnen Kennzahlenanalyse geschenkt. ${ }^{306}$ Dies geschieht dadurch, dass erstens bilanzpolitische Manipulationsmöglichkeiten besser neutralisiert werden können, zweitens alle Bereiche (Vermögens-, Finanz- und Ertragssituation) beachtet und drittens die Verdichtung durch eine festgelgte Vorgehensweise nachvollziehbarer gemacht werden kann. Genau wie im Bereich der Kennzahlen finden sich auch im Bereich der Kennzahlensysteme verschiedene Ansätze: hierzu

Vgl. Küting, Weber (2000), S. 27.

Vgl. Reichmann (1988), S. 382.

Vgl. hier und im Folgenden Baetge (998), S. 36ff.

Vgl. Hauschildt (1971), S. 342f.

Vgl. Baetge (1998), S. 36f. 
Marktorientierte Kreditrisikobewertung Die Bilanzanalyse als Instrument zur einzelgeschäftsbezogenen Risikobewertung

zählen bspw. das ZVEI-System, das DuPont-Kennzahlensystem (ROI) und das Rentabilitäts-Liquiditätssystem (RL-System). ${ }^{307 / 308}$

In Abbildung 19 und Abbildung 20 sind die oben genannten Systeme ROI und RL aufgezeigt.

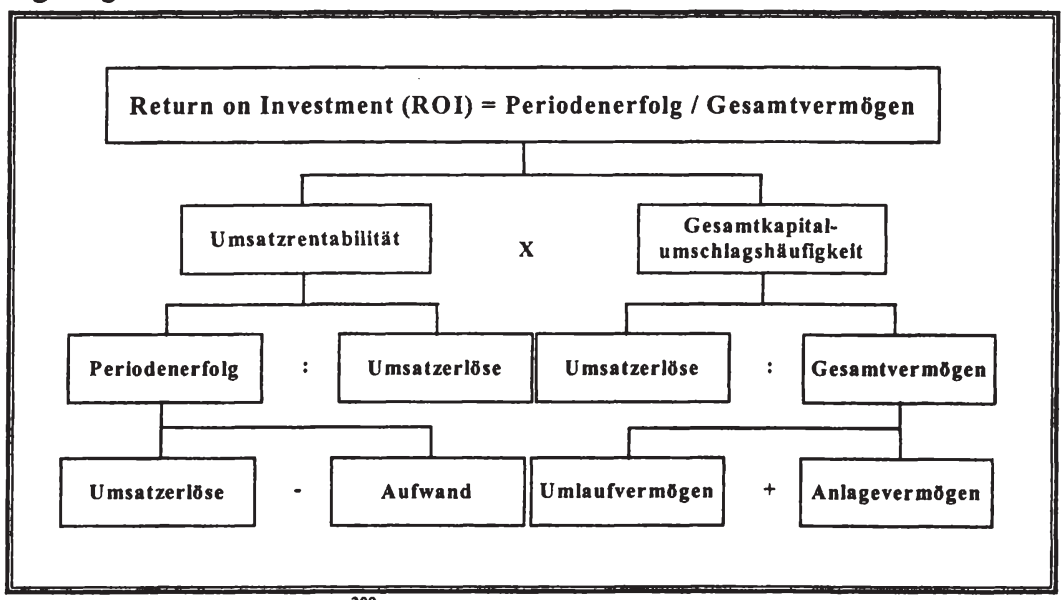

Abbildung 19: Das ROI-Schema ${ }^{309}$

Im ROI-System wird der Quotient aus dem Periodenerfolg und dem Gesamtvermögen als Spitzenkennzahl verwendet. Er stellt eine statische Rentabilitätskennzahl dar. ${ }^{310}$ Diese wird durch eine einfache Erweiterungen von Zähler und Nenner mit dem Wert „Umsatz" modifiziert und anschließend werden die Teilgrößen Periodenerfolg

307 Vgl. zum ZVEI: Zentralverband der Elektrotechnik-Elektroindustrie (1989) sowie Perridon, Steiner (1997), S. 566f.; zum DuPont-Kennzahlensystem: E.I. DuPont Nemours and Company (1959) sowie Staehle (1975), S. 69ff.; zum RL-System: Reichmann, Lachnit (1976), S. 53ff. Im Weiteren werden das ROI- und das RL-System skizziert, da diese beiden Ansätze die Grundlage der in Kap. 5 geführten Untersuchung bilden.

308 Schwächen der traditionellen Kennzahlensysteme, wie bspw. eine Fokussierung auf monetäre Kennzahlen, werden in Ansätzen wie der Balanced Scorecard aufgehoben (vgl. zu einer Gegenüberstellung von traditionellen Kennzahlensystemen und Performance-Measurement-Systemen Lynch, Cross (1995), S. 38; vgl. zum Konzept der Balanced Scorecard Kaplan, Norton, (1992), S. 71ff.). Da der Fokus der vorliegenden Arbeit auf Kennzahlensystemen zur Abschätzung des Bonitätsrisikos mittels der Bilanzanalyse liegt, wird auf die detaillierte Beschreibung nicht-monetärer Kennzahlensysteme verzichtet.

310 Vgl. hier und im Folgenden Baetge (1989), S. 55. 
Marktorientierte Kreditrisikobewertung -

Die Bilanzanalyse als Instrument zur einzelgeschäftsbezogenen Risikobewertung

und Gesamtvermögen in die Bestandteile Umsatzerlöse und Aufwand bzw. Umlaufvermögen und Anlagevermögen aufgeteilt.

Das RL-System teilt die Analyse in zwei Gruppen. Das ordentliche Ergebnis und seine Bestandteile beschreiben die Erfolgslage. Die liquiden Mittel und die untergeordneten Kennzahlen geben Aufschluss über die Finanzlage. Gemeinsam erklären sie somit die Rentabilitäts- und die Liquiditätssituation eines Unternehmens (RLSystem). ${ }^{31 \prime}$

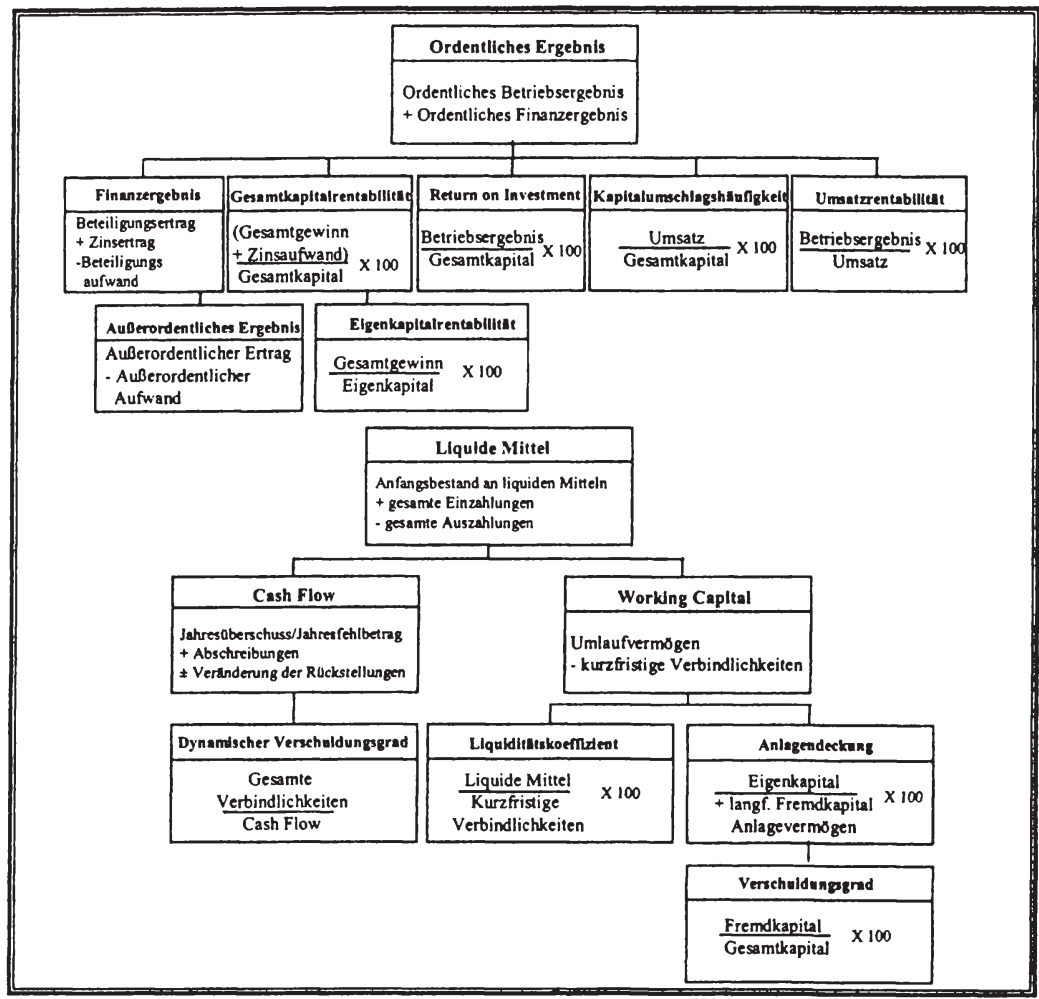

Abbildung 20: Das Rentabilitäts-Liquiditătssystem (RL-System) ${ }^{312}$

In Anlehnung an die Aufzählung der einzelnen Kennzahlen in Tabelle 6 bilden auch diese beiden ausgewählten Systeme die Grundlage der Auswahl der im empirischen

311 Vgl. Reichmann (1997), S. 32ff.

312 In Anlehnung an Reichmann (1997), S. 59. 
Marktorientierte Kreditrisikobewertung Die Bilanzanalyse als Instrument zur einzelgeschäftsbezogenen Risikobewertung

Teil untersuchten Größen. Auch an dieser Stelle soll, wie bei den Kennzahlen, nicht auf die Würdigung einzelner Methoden und deren Anwendungstechniken eingegangen werden. ${ }^{313}$

\subsubsection{Scoring-Modelle}

Bei Scoring-Modellen, auch Nutzwertanalyse bzw. Punktbewertungsmodelle ${ }^{314}$ genannt, werden verschiedene Handlungsalternativen nebeneinander gestellt und mittels eines standardisierten Verfahrens bewertet, sodass ihnen Punktwerte zugeordnet werden. ${ }^{315}$ Anschließend kann man die bewerteten Alternativen in eine Rangfolge bringen. ${ }^{316}$

Das Ziel eines Scoring-Modells im Rahmen der Bilanzanalyse ist die vergleichende Beurteilung verschiedener Bilanzen mittels zugeordneter Punktwerte. ${ }^{317}$ Ein ScoringModell setzt voraus, dass im Vorfeld eine Gewichtung einzelner Kennzahlen vorgenommen wurde. Diese festgelegte Gewichtung ist verbindlich. Im Rahmen der Aufstellung eines Scoring-Modells wird ein Experte bzw. ein Expertenteam bestimmt, das insbesondere über die Auswahl der Merkmale (in dem Fall der Bilanzanalyse sind dies die Kennzahlen) und deren Gewichtung entscheidet. Neben quantitativen können auch qualitative Größen (wie z.B. die Qualität des Managements) in den Modellen berücksichtigt werden. Die beiden im deutschsprachigen Raum bekanntesten Modelle sind das Saarbrücker Modell ${ }^{18}$ und das Rendite-Sicherheit-WachstumsModell (RSW-Modell) $)^{319}$.

Die vier Kennzahlen des Saarbrücker Modell ${ }^{320}$ werden explizit innerhalb des Modells definiert (vgl. Abbildung 21). Falls man nun zwei Bilanzen miteinander vergleichen möchte, muss man in einem ersten Schritt die vier Kennzahlen EQ, ROI, CFU und CFK berechnen. Anschließend kann man diesen unternehmensindividuellen Werte Punkte zuordnen. Diese Punktwerte werden durch eine entsprechende Inter-

313 Vgl. zur Technik der ROI-Systematik Baetge (1998), S. 522ff.; Wöhe (1996), S. 751f.; vgl. zur detaillierten Beschreibung des RL-Systems Reichmann (1997), S. 59ff.; Meyer (1994), S. $117 \mathrm{ff}$.

314 Vgl. Adam (1996), S. 413.

315 Vgl. Weber, Krahnen, Weber (1995), S. 1621.

316 Vgl. Adam (1996), S. 412.

317 Vgl. hier und im Folgenden Baetge (1998), S. 539.

318 Vgl. Küting (1993).

319 Vgl. Schmidt, Wilhelm (1987).

320 Im Folgenden wird nur der erste Teil des Saarbrückers Modells erläutert Der qualitative, zweite Bereich umfasst eine Analyse der Bilanzpolitik. Vgl. Küting (1993), S. 7; Küting (1996), S. 62. 
Marktorientierte Kreditrisikobewertung -

Die Bilanzanalyse als Instrument zur einzelgeschäftsbezogenen Risikobewertung

vallfunktion gebildet. ${ }^{321}$ Bspw. führt ein $\mathrm{EQ}$ von $10 \mathrm{zu}$ einem Wert von 0,5. Die

\begin{tabular}{|c|c|}
\hline $\begin{array}{l}\text { Bezeichnung } \\
\text { Eigenkapitalquote (EQ) } \\
\text { Return on Investment (ROI) } \\
\text { Cash Flow zu Umsatz (CFU) } \\
\text { Cash Flow zu Gesamtkapital (CFK) }\end{array}$ & $\begin{array}{l}\text { Definition } \\
\text { Eigenkapital / Gesamtkapital } \\
\text { Korrigierter Jahresüberschuss / Gesamtkapital } \\
\text { Cash Flow / Nettoumsatzerlöse } \\
\text { Cash Flow zu Gesamtkapital; }\end{array}$ \\
\hline $\begin{array}{l}\text { Mit: } \\
\text { Eigenkapital laut Bilanz } \\
\text { - Ausstehende Einlagen auf das gezeichnet } \\
+50 \% \text { des Sonderpostens mit Rücklagenan } \\
\text { - Dividendenausschüttung des Mutterunter } \\
\text { - Restbuchwert aktivierter Geschäfts- und } \\
\text { - } 50 \% \text { der nicht gedeckten Pensionsverpfli } \\
\text { = Eigenkapital; }\end{array}$ & $\begin{array}{l}\text { Kapital } \\
\text { teil } \\
\text { gehmens } \\
\text { irmenwerte } \\
\text { htungen }\end{array}$ \\
\hline $\begin{array}{l}\text { Bilanzsumme laut Bilanz } \\
\text { - Ausstehende Einlagen auf das gezeichnet } \\
+ \text { Offen von Vorräten abgesetzte erhaltene } \\
\text { - Restbuchwert aktivierter Geschäfts- und } \\
\text { - } 50 \% \text { der nicht gedeckten Pensionsverpfli } \\
=\text { Gesamtkapital; }\end{array}$ & $\begin{array}{l}\text { Kapital } \\
\text { Anzahlungen } \\
\text { irmenwerte } \\
\text { htungen }\end{array}$ \\
\hline $\begin{array}{l}\text { Jahresüberschuss vor Steuern vom Einkomn } \\
+ \text { Außerordentliche Aufwendungen } \\
\text { - Außerordentliche Erträge } \\
+ \text { Fremdkapitalzinsen } \\
+ \text { Abschreibungen des Geschäftsjahres auf } \\
\text { = korrigierter Jahresüberschuss; }\end{array}$ & $\begin{array}{l}\text { hen und Ertrag } \\
\text { Geschäfts- oder Firmenwerte }\end{array}$ \\
\hline $\begin{array}{l}\text { Jahresüberschuss vor Steuern vom Einkomn } \\
+ \text { Außerordentliche Aufwendungen } \\
\text { - Außerordentliche Erträge } \\
+ \text { Abschreibungen des Geschäftsjahres } \\
\text { - Zuschreibungen } \\
\pm \text { Veränderungen des Sonderpostens mit Ri } \\
\pm \text { Veränderungen der Rückstellungen für Pe }\end{array}$ & $\begin{array}{l}\text { en und Ertrag } \\
\text { klagenanteil } \\
\text { sionen und ähnliche Verpflichtungen }\end{array}$ \\
\hline$=$ Cash Flow & \\
\hline
\end{tabular}

Abbildung 21: Kennzahlen des Saarbrücker Modells ${ }^{322}$

Wahl der Punktwerte, d.h. die Konstruktion der Intervallfunktion, begründen KÜTING/WEBER mit langjähriger Erfahrung. ${ }^{323}$ Die unternehmensindividuellen Summen

321 Diese Zuordnungsvorschriften für die Transformation der unternehmensindividuellen Werte (EQ, ROI, CFU und CFK) in Punkte bewirken die Einordnung des Systems in den Bereich der Scoring-Modelle.

322 In Anlehnung an Küting, Weber (2000), S. 416f.

323 Vgl. Küting, Weber (2000), S. 418; Küting (1996), S. 62. 
Marktorientierte Kreditrisikobewertung -

Die Bilanzanalyse als Instrument zur einzelgeschäftsbezogenen Risikobewertung

führen jeweils zu einer Punktzahl zwischen 0 und 2,5. Anschließend werden die vier Punktzahlen addiert. Die Gesamtpunktzahl liegt somit zwischen 0 und 10 Punkten und wird in ein Gesamturteil transformiert (von außergewöhnlich geringe bis außergewöhnlich hohe Ertragsstärke). ${ }^{324}$ Das Unternehmen mit der höheren Punktzahl erlangt eine bessere Burteilung.

Im Rahmen des von SCHMIDT vertretenen RSW-Modells (Tabelle 7) wird eine Spitzenkennzahl (Fundamentalscore) auf Basis von Kennzahlen aus den Bereichen Rendite, Sicherheit und Wachstum generiert. ${ }^{325}$

\begin{tabular}{|c|c|c|}
\hline Bereich & Bezeichnung & Definition \\
\hline \multirow{2}{*}{ Rendite } & $\begin{array}{l}\text { Eigenkapitalrendite } \\
\text { (EKR) }\end{array}$ & $\begin{array}{c}\left(3 \times \mathrm{EKR}_{1}+2 \times \mathrm{EKR}_{\mathrm{t}-1}+1 \times \mathrm{EKR}_{\mathrm{t}-2}\right) / 6, \mathrm{mit}^{2} \mathrm{EKR}_{\mathrm{t}}=\text { Jahres } \\
\text { uberschus }\end{array}$ \\
\hline & Cash Flow Rendite (CFR) & $\begin{array}{c}\left(3 \times \mathrm{CFR}_{1}+2 \times \mathrm{CFR}_{1-1}+1 \times \mathrm{CFR}_{1-2}\right) / 6, \mathrm{mit}_{\mathrm{CFR}}=\mathrm{Cash} \\
\text { Flow }_{1} / \mathrm{Umsatz}_{1}\end{array}$ \\
\hline \multirow{2}{*}{$\begin{array}{c}\text { Sicher- } \\
\text { heit }\end{array}$} & Eigenkapitalquote (EKQ) & Eigenkapital / Gesamtkapital. \\
\hline & Liquiditätsquote ( (LQ) & $\begin{array}{c}\text { (Liquide Mittel }{ }_{t}+\begin{array}{l}\text { Wertpapiere des Umlaufvermogens } \\
\text { Gesamtkapital }\end{array} \text { ) }\end{array}$ \\
\hline \multirow{2}{*}{$\begin{array}{l}\text { Wachs- } \\
\text { tum }\end{array}$} & $\begin{array}{l}\text { Wachstumsrate Bilanz- } \\
\text { summe (WRBS) }\end{array}$ & / Bilanzsumme $\left._{t-2}\right)^{-2}-1$ \\
\hline & $\begin{array}{l}\text { Wachstumsrate Umsatz } \\
\text { (WRU) }\end{array}$ & $\left(\text { Umsatz }_{1} / \text { Bilanzsumme }_{1-2}\right)^{-2}-1$ \\
\hline
\end{tabular}

Tabelle 7: Aufbau und Kennzahlen des RSW-Modells ${ }^{326}$

Die Ausprägungen der sechs Kennzahlen (zwei je Bereich, d.h., für die Rendite sind dies EKR und CFR, für die Sicherheit, EKQ und LQ und für den Wachstum WRBS und WRU) werden zunächst standardisiert (Z-Transformation). ${ }^{327 / 328}$ Dieser Vorgang bedingt, dass eine (statistische) Grundgesamtheit an untersuchten Bilanzen bzw. erhobenen Kennzahlen vorliegt. Die standardisierten Kennzahlen werden anschlieBend gewichtet. Der Renditebereich wird hierbei viermal so hoch gewichtet wie die

Vgl. Küting, Weber (2000), S. 418.

In Abhängigkeit von der Branche werden die einzelnen Kennzahlen (jeweils die zweite eines jeden Bereiches) leicht modifiziert zu der im Weiteren dargestellten Vorgehensweise berechnet. Im Weiteren wird Bezug genommen auf die Branche Industrie/Handel/Verkehr. Auf die explizite Betrachtung der Modifikation innerhalb der Kennzahlen wurde verzichtet. In Anlehnung an Schmidt (1991), S. 32ff., zur detaillierten Beschreibung der Kennzahlendefinition vgl. auch Baden (1992), S. 112.

327 Die Standardisierung erfolgt, da die Dimensionen der einzelnen Kennzahlen unterschiedlich sind, bspw. nehmen die Kennzahlen aus dem Bereich Sicherheit Werte aus dem Bereich 0 bis 1 an, die Kennzahlen aus dem Bereich Rendite hingegen sind nicht auf ein Intervall beschränkt. Durch die Standardisierung werden die verschiedenen Dimension vergleichbar. Vgl. auch Baetge (1998), S. 554f.

Vgl. hier und im Folgenden Balzer (1997), S. 162. 
Marktorientierte Kreditrisikobewertung -

Bereiche Sicherheit und Wachstum. ${ }^{329}$ Darüber hinaus wird jeweils die erste Kennzahl (EKR, EKQ, WRBS) eines Bereiches doppelt so hoch gewichtet wie die zweite (CFR, LQ, WRU). Insgesamt ergibt sich somit für die Kennzahl EKR ein Gewichtungsfaktor von 0,444, für die Kennzahl CFR von 0,222, für die Kennzahlen EKQ und WRBS von jeweils 0,111 und die Kennzahlen LQ und WRU von jeweils 0,056. Die standardisierten und gewichteten sechs Kennzahlen werden anschließend addiert und die Summe ergibt den Fundamentalscore.

Der Fundamentalscore als unternehmensindividuelle Summe der einzelnen Kennzahlen kann durch eine Rangliste zwei (oder mehrere) Unternehmen miteinander vergleichen. ${ }^{330}$ Falls die Untersuchungsmasse groß genug ist, kann mittels einer prozentualen Aufteilung ein Rating vergeben werden. ${ }^{331}$ Bspw. können die besten fünf Prozent der Unternehmen (d.h. die mit dem höchsten Fundamentalscore) einen Ratingwert von A erhalten. Somit ist die Beurteilung eines Unternehmens anhand des Fundamentalscores immer relativ, da sie sich an der Gesamtheit der untersuchten Unternehmen orientiert. Die isolierte Information des Fundamentalscores liefert keine Einschätzung der Güte des Unternehmens.

Entscheidend bei beiden Modellen ist, dass die Bilanzanalyse intersubjektiv nachvollziehbar wird. Der Grundsatz der Objektivierung wird jedoch nicht erfüllt, da der Entwickler des Modells subjektiv über die Auswahl und die Gewichtung der Merkmale entscheiden muss. ${ }^{332}$

\subsubsection{Kritische Würdigung der traditionellen Analyseverfahren}

Als traditionelle Verfahren werden in dieser Arbeit die Kennzahlenanalyse bzw. Kennzahlensysteme und die Scoring-Modelle bezeichnet, da ihre historischen Wurzeln zeitlich vor den im weiteren Analyseverlauf noch zu definierenden modernen Verfahren liegen. Im Rahmen der Würdigung dieser Methoden fällt auf, dass insbesondere Kennzahlen und Kennzahlensysteme gegen das Prinzip der Objektivierung verstoßen. Es ist zwar möglich, das Urteil intersubjektiv nachzuvollziehen; warum jedoch gerade diese Kennzahlen für die Analyse gewählt wurden bzw. wie die Wahl der Gewichtungsfaktoren getroffen wurde, kann nicht objektiv hinreichend begründet werden. Es handelt sich somit um subjektive Systeme. ${ }^{333}$

329 Zur (subjektiven) Begründung dieser Gewichtungsfaktoren vgl. Schmidt (1990), S. $71 \mathrm{ff}$.

330 Vgl. hier und im Folgenden Balzer (1997), S. 196ff.

331 Vgl. Baden, Wilhelm (1995), S. 160.

332 Vgl. Baetge (1998), S. 549f. und 558f.

333 Vgl. Baetge (1998), S. $518 \mathrm{ff}$. 
Marktorientierte Kreditrisikobewertung -

Im Rahmen der Scoring-Modelle findet zwar ein standardisiertes Vorgehen innerhalb der Bilanzanalyse statt, das Aufstellen dieser Modelle beruht jedoch auf Erfahrungen der „Experten“. Eine sachlich objektive Auswahl der Merkmale und der Gewichte und somit auch eine sachlich objektive Zusammenfassung zu einem Gesamturteil kann nicht gewährleistet werden, sodass diese Vorgehensweise als quasi-objektiv bezeichnet werden kann. ${ }^{334}$ Ein weiteres Problem ist der fehlende empirische Nachweis bezüglich der Relevanz der aufgestellten Kennzahlen. Hiermit ist zum einen das Problem der Vollständigkeit angesprochen, da subjektiv beurteilt wird, welche Kennzahlen die gesamte Unternehmenssituation darstellen und somit das Prinzip der Ganzheitlichkeit nicht uneingeschränkt erfüllt werden kann, ${ }^{335}$ und zum anderen fehlt eine Validierung der "Treffergenauigkeit“. Es gibt keinen statistischen Nachweis bezüglich der Qualität des Modells.

Inwieweit das Prinzip der Neutralisierung eingehalten wird, hängt sehr stark von der Vorgehensweise des Kennzahlensystems bzw. des Scoring-Modells ab. Es kann nicht behauptet werden, dass dieser Grundsatz immer vollständig erfüllt ist. ${ }^{336}$ Des Weiteren gibt es keine ausreichende statistische Begründung für die Stabilität des Modells bezüglich einer Änderungen der Rahmenbedingungen.

Trotz dieser konzeptionellen Schwächen werden bei der Vergabe von Krediten häufig institutsspezifische traditionelle Bilanzanalyseverfahren eingesetzt. Dies lässt sich darauf zurückführen, dass die praktische Anwendbarkeit und die schnelle Ergebnisverarbeitung für die Institute zwei hinreichende Kriterien für die Umsetzung sind. ${ }^{337}$

\subsection{Moderne empirisch-induktive Bilanzanalyseverfahren}

Insbesondere die Kritik der Verletzung der Objektivität hat dazu geführt, dass neue Methoden und Konzepte zur Beurteilung des Jahresabschlusses entwickelt wurden, welche im Weiteren als moderne Analyseverfahren bezeichnet werden. ${ }^{338}$ Im Weiteren werden die Instrumente der Diskriminanzanalyse und der Künstlichen Neuronalen Netze als Bilanzanalyseverfahren dargestellt (Kap. 3.4.1 und Kap. 3.4.2). Anlog zu der Vorgehensweise in den Kapiteln 3.3.1 bis 3.3.3 wird wiederum zunächst die Vorgehensweise im Allgemeinen beschrieben und anschließend an einem Beispiel

334 Vgl. Baetge (1998), S. 539, S. 549 und S. 549.

335 Vgl. Baetge (1998), S. 558f. und 549f.

336 Vgl. bspw. die von Baetge geäußerte Kritik am Saarbrücker Modell, Baetge (1998), S. 559.

337 Eine Studie von Grabher, Klien, Stoss fuir das Jahr 1993 konnte nachweisen, dass bei 513 untersuchten Banken die Bilanzanalyse immer von den Instituten bei der Kreditvergabe im Rahmen der Bonitätsanalyse verwendet wurde (vgl. Grabher, Klien, Stoss (1996), S. 39).

338 Vgl. Rehkugler, Poddig (1998), S. 233. 
Marktorientierte Kreditrisikobewertung -

detailliert erläutert. Die Auswahl der Beispiele erfolgt wiederum in Anlehnung an das in Kap. 4 zu konzipierende Prognosemodell.

Der neue Gedanke an modernen empirisch-induktiven Verfahren war, statistische Verfahren zu nutzen, um Zusammenhänge zwischen einzelnen Merkmalen und der Unternehmenssituation zu belegen. ${ }^{339}$ Angewendet wird ein Klassifikationsmodell, das Objekte anhand von Merkmalsausprägungen in bestimmte Klassen einordnet. Objekt ist immer der Jahresabschluss, ${ }^{340}$ und die Merkmale sind die einzelnen Bilanzkennzahlen bzw. deren Ausprägungen. Als Klassen sind verschiedene Ansätze möglich, bspw. kann man bzgl. der Objekte zwischen „,solvent“ und ,insolvenzgefährdet“" unterscheiden. Es sind jedoch auch feinere Abstufungen denkbar und bei einer ausreichenden Datengrundlage anwendbar. ${ }^{341}$

Das Klassifikationsmodell errechnet den bestmöglichen in der Vergangenheit deutlich gewordenen Zusammenhang zwischen den Klassen und den Merkmalausprägungen. ${ }^{342}$ Dabei wird unterstellt, dass erstens dieser Zusammenhang zeitlich stabil bleibt und zweitens die Beispieldaten diesen Zusammenhang sehr gut (,exakt") wiedergeben können. ${ }^{343}$ Ein Problem bei der Anwendung dieser Klassifikationsmodelle für die Bilanzanalyse liegt darin, dass eine Vorauswahl an Merkmalen getroffen werden muss. ${ }^{344}$ Dies sind häufig die bekanntesten Kennzahlen. Die Qualität eines Klassifikationsmodells wird somit durch eine breitere Vorauswahl an Kennzahlen gestärkt. Es werden zwei Verfahrensweisen besonderes häufig in der Literatur diskutiert und von Kreditinstituten angewendet: ${ }^{345}$ Die Diskriminanzanalyse und Künstliche Neuronale Netze (KNN).

\subsubsection{Die Diskriminanzanalyse als Instrument der Bilanzanalyse}

Das Verfahren der Diskriminanzanalyse untersucht den statistischen Zusammenhang zwischen der Klassenzugehörigkeit eines Objektes und einer Anzahl von Variablen. Im Rahmen der Bilanzanalyse wird regelmäßig die gesamte Menge an zu untersuchenden Unternehmen in die Klassen „solvent" und ,insolvenzgefährdet“ eingeteilt. ${ }^{346}$ Die in die Untersuchung eingehenden Variablen sind die zugehörigen Bilanzkennzahlen der zu klassifizierenden Unternehmen. Die grundsätzliche Vorgehenswei-

\footnotetext{
339 Vgl. hier und im Folgenden Rehkugler, Poddig (1998), S. 233 und S. $245 \mathrm{ff}$.

340 Falls neben den Bilanzdaten noch weitere Informationen einbezogen werden, ist es treffender, das Unternehmen als Objekt zu bezeichnen.

${ }^{341}$ Vgl. Uthoff (1997), S. 192f.

342 Vgl. hier und im Folgenden Rehkugler, Poddig (1998), S. $246 \mathrm{ff}$.

343 Vgl. Bröker (2000), S. 57.

344 Vgl. hier und im Folgenden Baetge (1998), S. 562 und S. 580.

345 Vgl. Bröker (2000), S. 56.

346 Vgl. Glormann (2000), S. $127 \mathrm{f}$.
} 
Marktorientierte Kreditrisikobewertung Die Bilanzanalyse als Instrument zur einzelgeschäftsbezogenen Risikobewertung

se besteht in einem ersten Schritt darin, ein Insolvenzkriterium zu operationalisieren und eine möglichst repräsentative Menge an Jahresabschlüssen zu erheben, für die eine Klassifizierung hinsichtlich dieses Kriteriums bereits bekannt ist. ${ }^{347}$ Als Insolvenzkriterium eignen sich Tatbestände wie bspw. Konkurs, Vergleich oder die Abgabe einer eidesstattlichen Versicherung. ${ }^{348}$ Untersuchungen haben gezeigt, dass lediglich für den Zeitraum von fünf Jahren vor der Insolvenz es sinnvoll erscheint, zwischen „solvent" und ,insolvenzgefährdet" zu unterscheiden. ${ }^{349}$ In einem zweiten Schritt wird die Untersuchungsgruppe in eine Analysestichprobe (zur Ermittlung der Diskriminanzfunktion) und eine Kontrollgruppe (zur Verifizierung der Diskriminanzfunktion) aufgespalten. ${ }^{350}$

Die Analysestichprobe besteht, wie auch die Kontrollgruppe, aus verschiedenen Jahresabschlüssen, welche jeweils dieselben Kennzahlen $\left(\mathrm{K}^{\mathrm{D}}\right)$ ausweisen. Die Diskriminanzfunktion verknüpft nun diese einzelnen Kennzahlen linear miteinander, nachdem sie jeweils gewichtet wurden, und addiert eine feste Konstante $a_{0}$ hinzu, um den Diskriminanzwert D zu berechnen. Somit ergibt sich folgende

Gleichung 22: : $^{351}$

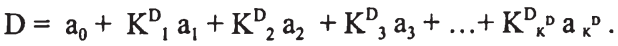

Für jedes einzelne Unternehmen wird nun der Diskriminanzwert berechnet und falls dieser über bzw. unter einem kritischen Wert liegt, wird das Unternehmen in die o.g. Klasse der ,solventen“ bzw. ,insolvenzgefährdeten“ Unternehmen klassifiziert. Die einzelnen Gewichte werden solange variiert, bis keine bessere Klassifizierung erreicht werden kann. ${ }^{352}$ Grundsätzlich können zwei Fehlklassifizierungen resultieren: Entweder wird ein „insolvenzgefährdetes“ Unternehmen der Klasse der „solventen“ $(\alpha$-Fehler) Unternehmen oder ein „solventes“ Unternehmen wird der Klasse der „,insolvenzgefährdeten" Unternehmen zugeordnet $\left(\beta\right.$-Fehler). ${ }^{353}$ Durch das Verschieben des Wertes D kann bewusst ein höherer $\alpha$-Fehler ( $\beta$-Fehler) zu Gunsten eines niedrigeren $\beta$-Fehler ( $\alpha$-Fehler) erreicht werden. Da der Kreditausfall sich für ein Kreditinstitut in höheren Kosten niederschlägt als die Ablehnung der Kreditvergabe an ein solventes Unternehmen, wird regelmäßig ein höherer $\beta$-Fehler zugunsten eines nied-

\footnotetext{
347 Vgl. Bröker (2000), S. 57.

348 Vgl. Uthoff (1997), S. 192f.

349 Vgl. Rehkugler, Poddig (1998), S. 255.

350 Vgl. hier und im Folgenden Bröker (2000), S. $57 \mathrm{ff}$.

351 Vgl. Bröker (2000), S. 59.

352 Vgl. bspw. Hüls (1995), S. $169 f f$.

353 Vgl. hier und im Folgenden Bröker (2000), S. 60.
} 
Marktorientierte Kreditrisikobewertung -

rigeren $\alpha$-Fehlers in Kauf genommen. ${ }^{354}$ Der kostenoptimale Diskriminanzwert wird in Richtung der solventen Kreditnehmer verschoben. In einer Modellerweiterung kann der Diskriminanzwert darüber hinaus genutzt werden, um eine Einstufung in verschiedene Risikoklassen vorzunehmen. ${ }^{355}$

Die Kontrollgruppe dient der Berechnung des Fehlerwertes mittels der zuvor gefundenen Funktionsgleichung und dem festgelegten Diskriminanzwert. Diese Gruppe eignet sich deshalb, weil an einer für die Diskriminanzfunktion ,unbekannten“ Gruppe, von der man jedoch die ,wahre“ Klassenzugehörigkeit kennt, die Funktion getestet werden kann.

Das dargestellte Verfahren der Diskriminanzanalyse unterliegt strengen Annahmen. Die wichtigsten theoretischen Voraussetzungen sind: Normalverteilung der Kennzahlenwerte, Unabhängigkeit der Kennzahlenwerte, Gleichheit der Varianz-KovarianzMatrizen. ${ }^{356}$

Während bereits seit den 30er-Jahren in der Wissenschaft Untersuchungen aus diesem Analyseumfeld publiziert wurden, hat insbesondere ALTMAN 1968 mit der ersten Untersuchung im Bereich der multivariaten Verfahrensweise für eine neue Dynamik gesorgt. ${ }^{357}$ Einen Überblick bezüglich der Untersuchungen und der Ergebnisse findet sich bei REHKUGLER/PODDIG. ${ }^{358}$

Eine in den USA verbreitete Diskriminanzfunktion ist das Z' '-Score-Modell. ${ }^{359}$. Die Untersuchung von ALTMAN aus dem Jahre 1994 ist zu folgenden Ergebnissen gekommen, wobei ein Unternehmen mit einem Z''Score-Wert von unter 1,81 in die Klasse der „insolvenzgefährdeten" Unternehmen klassifiziert wird: ${ }^{360}$

Vgl. bspw. Baetge (1998), S. 565; Feidicker (1992), S. 212. Die hier zitierten Untersuchungen konnten nachweisen, dass ein $\alpha$-Fehler einer Kostenbelastung entspricht, die fünfmal so groß ist wie die Kostenbelastung bedingt durch einen $\beta$-Fehlers. Vgl. Hüls (1995), S. 282ff. und die folgenden Ausführungen.

356 Vgl. Rehkugler, Poddig (1998), S. 334

357 Vgl. Altman (1968).

358 Vgl. Rehkugler, Poddig (1998), S. 322ff.

359 Vgl. Altmann (1996); Altman, Saunders (1997).

360 Vgl. hier und im Folgenden Altman (1996), S. 16ff.; Altman, Saunders (1997), S. 1736f. Die hier dargestellte Untersuchung bezieht sich auf nach dem US - Generally Accepted Accounting Principles aufgestellten Jahresabschlüsse. Die grundsätzliche Vorgehensweise lässt sich jedoch auch auf nach dem HGB aufgestellte Jahresabschlüsse übertragen. Vgl. zu der Problematik der Rechenlegungssysteme Kap. 6.2.2. 
Marktorientierte Kreditrisikobewertung Die Bilanzanalyse als Instrument zur einzelgeschäftsbezogenen Risikobewertung

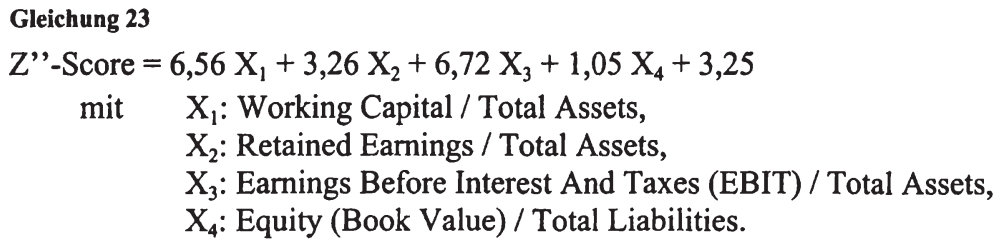

Der Wert „Z”'-Score“ stellt hierbei eine Größe dar, die die Zuordnung des Unternehmens zu einer Ratingklasse leisten kann, indem dieser in ein Ratingäquivalent transformiert wird. Hierzu wurde von ALTMAN auf Datenbasis von 750 kommerziellen Anleihen der Z"'-Score-Wert für jedes Unternehmen ermittelt und dieser dem Rating gegenübergestellt. Die Tabelle 8 zeigt die von ALTMAN untersuchten Anleihen hinsichtlich des Rating und des Z"'-Score-Wertes auf. Bspw. besitzen acht Anleihen ein Rating der Klasse „AAA“. ${ }^{361}$ Den zugehörigen Unternehmen bzw. den Bilanzen dieser werden gemäß der in Gleichung 23 dargestellten Funktion Z' Score-Werte zugeordnet, die im Durchschnitt (Ø Z"-Score) einen Wert von 8,15 erreichen. Somit kann einem Unternehmen mit Hilfe dieser Diskriminanzfunktion genau dann ein Rating von „AAA“ zugeordnet werden, falls es einen Z"'-Score-Wert von 8,15 aufweist. Für eine lückenlose Zuordnung ist es notwendig, Klassengrenzen für die unterschiedlichen Z"'-Score-Werte einzuführen. Wie zu erkennen ist, hängt der Z"'-Score-Wert von den vier Kennzahlen des Jahresabschlusses ab. Die gefundenen Kennzahlen $X_{1}$ bis $\mathrm{X}_{4}$ werden im empirischen Teil der vorliegenden Arbeit wieder aufgegriffen.

\begin{tabular}{|c|c|c|c|c|c|c|c|c|c|c|}
\hline $\begin{array}{c}\text { Rating Investment } \\
\text { Grade }\end{array}$ & AAA & $\mathrm{AA}+$ & AA & AA- & A+ & A & A- & $\mathrm{BBB}+$ & BBB & BBB- \\
\hline Ø Z"-Score & 8,15 & 7,60 & 7,30 & 7,00 & 6,85 & 6,65 & 6,40 & 6,25 & 5,85 & 5,65 \\
\hline Stichprobengroßße & 8 & - & 18 & 15 & 24 & 42 & 38 & 38 & 59 & 52 \\
\hline $\begin{array}{c}\text { Rating Speculative } \\
\text { Grade }\end{array}$ & $\mathrm{BB}+$ & BB & BB- & $\mathrm{B}+$ & B & B- & $\mathrm{CCC}+$ & $\mathrm{CCC}$ & CCC- & D \\
\hline Ø Z"-Score & 5,25 & 4,95 & 4,75 & 4,50 & 4,15 & 3,75 & 3,20 & 2,50 & 1,75 & 0,00 \\
\hline Stichprobengröße & 34 & 25 & 65 & 78 & 115 & 95 & 23 & 10 & 6 & 14 \\
\hline
\end{tabular}

Tabelle 8: Zusammenhang zwischen dem Z'-Score-Wert und dem Kapitalmarkt-Rating ${ }^{362}$

Vgl. zu der Bedeutung der Ratingklassen Kap. 2.3.3.

In Anlehnung an Altman (1996), S. 18. 
Marktorientierte Kreditrisikobewertung -

Die Bilanzanalyse als Instrument zur einzelgeschäftsbezogenen Risikobewertung

\subsubsection{Künstliche Neuronale Netze als Instrument zur Bilanzanalyse}

Der Begriff Künstliche Neuronale Netze (KNN) stammt aus dem Forschungsbereich der Künstlichen Intelligenz. ${ }^{363}$ Ein wesentlicher Vorteil dieses Verfahrens ist die Befreiung von Prämissen, wie sie die MDA hat. ${ }^{364}$ Die Modelle sind der Biologie entlehnt. Sie sind mathematische Abbildungen der natürlichen neuronalen Netzwerke, die für die Verarbeitung von Reizen und deren Steuerung in biologischen Organismen verantwortlich sind. ${ }^{365}$ Insbesondere seit Anfang der 90er-Jahre wurde diese Technik zur Klassifikation von Jahresabschlüssen eingesetzt. ${ }^{366} \mathrm{KNN}$ werden wie auch die Diskriminanzanalyse im Bereich der Bilanzanalyse zur Entscheidung, ob ein Unternehmen ,solvent“ oder ,insolvenzgefährdet“ ist, eingesetzt.

Durch eine möglichst große Zahl an Beispielfällen lernt das System eigenständig, welche Zusammenhänge zwischen den Inputfaktoren (Kennzahlen) und dem Objekt (solventes bzw. gefährdetes Unternehmen) bestehen. Diese Beispieldaten werden in einem Trainingsprozess genutzt, um einen bestmöglichen funktionalen Zusammenhang abzuleiten. Anschließend werden neue unbekannte Datensätze mit den bekannten und gefundenen Verknüpfungen ausgewertet. ${ }^{367}$

Ein wesentlicher Vorteil bei dieser Vorgehensweise liegt in der Abbildung von nichtlinearen Zusammenhängen. ${ }^{368}$ Das Ergebnis des Lernprozesses ist eine (nicht-lineare) Trennfunktion, welche anhand von Eingangsparametern (bspw. Umsatzrentabilität und Eigenkapitalquote) den Datensatz in die Klassen solvent (,1") oder insolvenzgefährdet (,,0) einteilt (Abbildung 22). Hierzu ordnet das KNN jedem Jahresabschluss mittels des gefundenen funktionalen Zusammenhangs einen Wert zu. Falls dieser Trennwert über- bzw. unterhalb des kritischen Wertes (abgeleitet aus der Trennfunktion) liegt, wird das Unternehmen als ,solvent“ bzw. ,insolvenzgefährdet“ klassifiziert.

363 Das Thema wird im Kapitel 5 eingehender erörtert. Dort wird die Funktionsweise detailliert dargestellt.

364 Vgl. bspw. Baetge (1995), $191 \mathrm{ff}$.

365 Vgl. Rehkugler, Poddig (1998), S. 334.

366 Vgl. Rehkugler, Poddig (1998), S. 335.

367 Vgl. Baetge (1998), S. 575.

$368 \mathrm{Zu}$ den Vorteilen Neuronaler Netze gegenüber der Diskriminanzanalyse vgl. Lachenbruch, Sneeringer, Revo (1973), S. 53. 
Marktorientierte Kreditrisikobewertung -

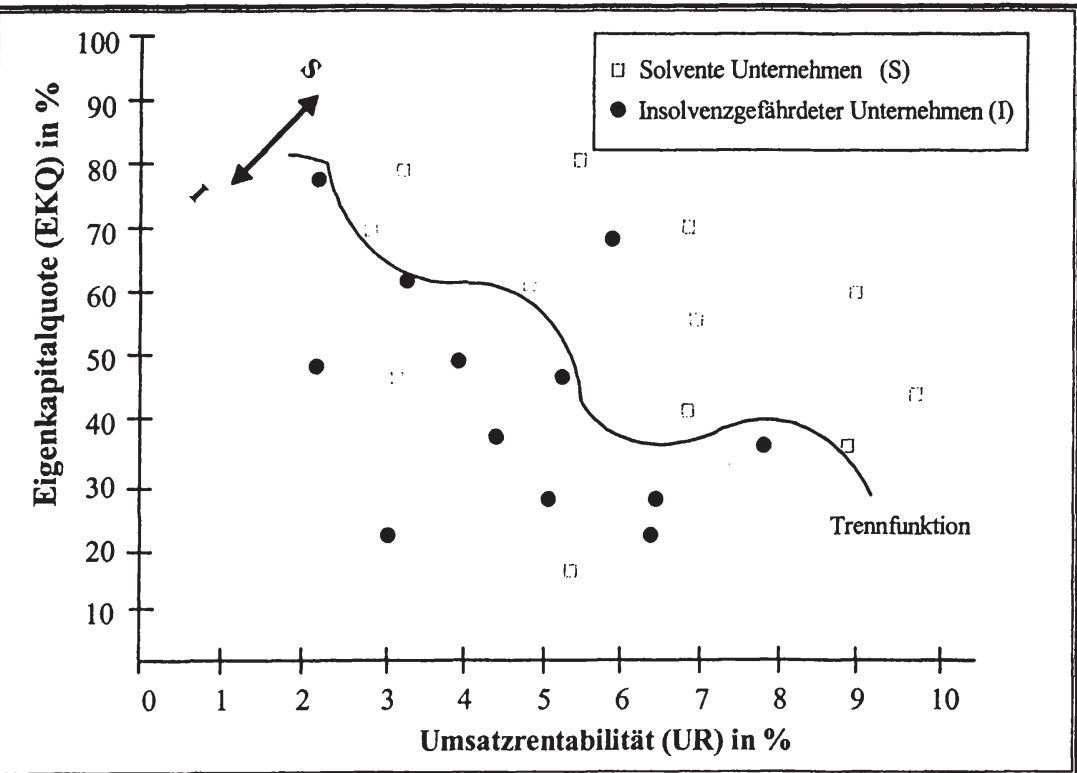

Abbildung 22: Trennung solventer und insolvenzgefährdeter Unternehmen mittels der KNN-Analysen ${ }^{369}$

In der Wissenschaft sind bereits verschiedene Untersuchungen zur Bilanzanalyse mittels KNN geführt worden. Einen Überblick geben REHKUGLER/PODDIG. ${ }^{370}$ Die Untersuchung von BAETGE u.a. ist bezüglich ihrer Datengrundlage eine der umfangreichsten Erhebungen (10.512 ,gesunde“ und 912 „kranke“ Unternehmen) ${ }^{371 / 372}$. Aus ihr ging der so genannte Backpropagation-Algorithmus (BP) mit 14 Kennzahlen (BP14) hervor (Tabelle 9 zeigt die 14 Kennzahlen). ${ }^{373}$ Es wird deutlich, dass die 14 Kennzahlen sowohl den Bereich der Vermögens-, Finanz- als auch Ertragslage beschreiben.

369 In Anlehnung an Baetge (1998b), S. 11; Krause (1993), S. 127; Rolfes (1999), S. 349.

370 Vgl. Rehkugler, Poddig (1998), S. 385ff.

371 Vgl. Rehkugler, Poddig (1998), S. 396; zur Untersuchung vgl. bspw. Baetge, Hüls, Uthoff (1995); Baetge (1998); Uthoff (1997).

372 Als gesundes Unternehmen wird ein im folgenden Zeitraum solventes, als krankes ein im folgenden Zeitraum insolventes Unternehmen definiert.

373 Der Begriff BP Backpropagation wurde durch den Begriff Baetge-Bilanz-Rating (BBR) ersetzt. 
Marktorientierte Kreditrisikobewertung -

Die Bilanzanalyse als Instrument zur einzelgeschäftsbezogenen Risikobewertung

Das BP-14 erzielt eine Alpha-Beta-Fehler-Relation von 8,75 \% zu 33,55 \%. ${ }^{374}$ Analog zu der Interpretation in Kap. 3.4.1 bedeutet dies, das 8,75\% (33,55\%) der insolvenzgefährdeten (solventen) Unternehmen als solvent (insolvenzgefährdet) klassifiziert werden. Neben der reinen Klassifikation in solvent/insolvenzgefährdet werden den einzelnen Unternehmen auch Ratings zugeordnet. Hierzu wird der vom KNN als Trennwert zur Klassifikation verwendete $\mathrm{N}$-Wert in 10 Klassen eingeteilt. Der

\begin{tabular}{|c|c|c|c|c|}
\hline & & $\begin{array}{l}\text { Informations- } \\
\text { bereich }\end{array}$ & Bezeichnung & Definition \\
\hline & & Kapitalbindungs & $\begin{array}{l}\text { Kapitalbindungs- } \\
\text { dauer } 1\end{array}$ & $\begin{array}{l}\text { ((Akzepte + Verbindlichkeiten aus Lieferungen und Leistun- } \\
\text { gen }) \times 360) / \text { Gesamtleistung }\end{array}$ \\
\hline & & dauer & $\begin{array}{c}\text { Kapitalbindungs- } \\
\text { dauer2 }\end{array}$ & $\begin{array}{l}\text { ((Akzepte + Verbindlichkeiten aus Lieferungen und Leistun- } \\
\text { gen) } \times 360) / \text { Umsatz }\end{array}$ \\
\hline & 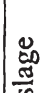 & Kapitalbindung & Kapitalbindung & $\begin{array}{c}\text { (Kurzfristige Bankverbindlichkeiten + Kurzfristige Verbind- } \\
\text { lichkeiten aus Lieferungen und Leistungen + Akzepte + } \\
\text { Kurzfristige Sonstige Verbindlichkeiten) / Umsatz }\end{array}$ \\
\hline & בֶ̃ & & Fremdkapitalquote & $\begin{array}{c}\text { (Kurzfristiges Fremdkapital - Erhaltene Anzahlungen) / } \\
\text { Bilanzsumme }\end{array}$ \\
\hline & $\stackrel{:}{E}$ & Verschuldung & Fremdkapitalstruktur & $\begin{array}{c}\begin{array}{c}\text { (Verbindlichkeiten aus Lieferungen und Leistungen + Akzepte } \\
+ \text { Bankverbindlichkeiten) / (Fremdkapital - Erhaltene Anzah- } \\
\text { lungen) }\end{array} \\
\end{array}$ \\
\hline$\frac{2}{2}$ & & Kapitalstruktur & Eigenkapitalquote 1 & $\begin{array}{c}\text { (Wirtschaftliches Eigenkapital - Immaterielle Vermögens- } \\
\text { gegenstănde) / ( Bilanzsumme - Immaterielle Ver- } \\
\text { mögensgegenstände - Flüssige Mittel - Grundstucke u. Bauten) }\end{array}$ \\
\hline בี & & & Eigenkapitalquote2 & $\begin{array}{l}\text { (Wirtschaftliches Eigenkapital + Rückstellungen) / (Bilanz- } \\
\text { summe - Flüssige Mittel - Grundstück und Bauten) }\end{array}$ \\
\hline$\sum_{\underline{c}}^{E}$ & 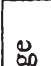 & Finanzkraf & Finanzkraftl & $\begin{array}{c}\text { Ertragswirtschaftlicher Cash Flow / (Fremdkapital - Erhaltene } \\
\text { Anzahlungen) }\end{array}$ \\
\hline & $\frac{0}{\mathrm{a}}$ & Finanzkrait & Finanzkraft2 & $\begin{array}{c}\text { Ertragswirtschaftlicher Cash Flow / (Kurzfristiges Fremdkapital } \\
\text { + Mittelfristiges Fremdkapital - Erhaltene Anzahlungen) }\end{array}$ \\
\hline & 惡 & Deckungsstruktur & Anlagendeckung & $\begin{array}{l}\text { Wirtschaftiches Eigenkapital / (Sachanlagevermögen - } \\
\text { Grundstucke und Bauten) }\end{array}$ \\
\hline & & & Umsatzrentabilităt & Ordentliches Ergebnis / Umsatz \\
\hline & $\stackrel{\leftrightarrow}{0}$ & & Cash Flow1 - ROI & Ertragswirtschaftlicher Cash Flow / Bilanzsumme \\
\hline & $\frac{\pi}{\sqrt[n]{\infty}}$ & Rentabilităt & Cash Flow2 - ROI & $\begin{array}{c}\text { (Ertragswirtschaftlicher Cash Flow + Zufuhnung zu den } \\
\text { Pensionsrackstellungen)/Bilanzsumme }\end{array}$ \\
\hline & 壳 & Aufwandsstruktur & $\begin{array}{l}\text { Personalaufwands- } \\
\text { quote }\end{array}$ & Personalaufwand / Gesamtleistung \\
\hline
\end{tabular}

Tabelle 9: Kennzahlen des BP-14

374 Vgl. hier und im Folgenden Baetge, Hüls, Uthoff (1995), S: 24ff.; Baetge (1998), S. $582 \mathrm{ff}$., Baetge (1995), S. $191 \mathrm{ff}$.

375 In Anlehnung an Baetge (1998), S. 584. Zur detaillierten Beschreibung der Kennzahlen auf Einzelebene vgl. Baetge (1998), S. 584ff. 
Marktorientierte Kreditrisikobewertung Die Bilanzanalyse als Instrument zur einzelgeschäftsbezogenen Risikobewertung

$\mathrm{N}$-Wert ist der durch das $\mathrm{KNN}$ berechnete Wert für ein Unternehmen, der zur Trennung in solvent/insolvenzgefährdet herangezogen wird. Diese Vorgehensweise unterstellt, dass Unternehmen mit sehr stark ausgeprägten Solvenz- oder Insolvenzstrukturen durch den Klassifikator einen N-Wert nahe am oberen bzw. unteren Ende der NWert-Skala ${ }^{376}$ zugewiesen bekommen. ${ }^{377}$ Ein Unternehmen mit einem $\mathrm{N}$-Wert von +6 gilt folglich als solventer als ein Unternehmen mit einem zwar über dem kritischen Wert (abgeleitet aus der Trennfunktion) aber unter dem Wert von +6 liegenden $\mathrm{N}$-Wert. Anschließend können gleichverteilte Klassen über das mögliche Ausprägungsintervall, in diesem Fall von -10 bis +10 , gebildet werden. Den einzelnen Klassen können abschließend historische Ausfallwahrscheinlichkeiten zugeordnet werden.

Die Zuordnung von historischen Ausfallwahrscheinlichkeiten zu den Klassen geschieht, indem eine hinreichend große Anzahl (,einige Tausend repräsentative Jahresabschlüsse ${ }^{(378)}$ ) von Unternehmen vom BP-14 klassifiziert und anschließend diesen Klassen die ablesbaren, eingetretenen Insolvenzen der Vergangenheit zugeteilt wurden. ${ }^{379}$ Tabelle 10 zeigt die Zuordnung von N-Werten vom BP-14 zu historischen Ausfallraten in den einzelnen Ratingstufen. Somit kann der BP-14 auf Basis eines Jahresabschlusses zunächst die Ratingklasse der Unternehmens bestimmen (bspw. BB) und darauf aufbauend idealtypisch diesem Unternehmen eine Ausfallwahrscheinlichkeit zuordnen (für das Beispiel 0,12 \%). Auf Basis dieser Zuordnung könnte in einem weiteren Schritt ein Kreditzins abgeleitet werden (vgl. Kap. 3.6).

376 Als N-Wert-Skala wird ein reellwertiges Intervall von -10 bis +10 beschrieben. Vgl. Glormann (2001), S. 288.

377 Vgl. Glormann (2001), S. 288.

378 Baetge (1998), S. 597.

$379 \mathrm{Vgl}$. Baetge (1998), S. $596 \mathrm{f}$. 
Marktorientierte Kreditrisikobewertung -

Die Bilanzanalyse als Instrument zur einzelgeschäftsbezogenen Risikobewertung

\begin{tabular}{|c|c|c|c|c|}
\hline & $\begin{array}{l}\text { N-Wert- } \\
\text { Bereich }\end{array}$ & $\begin{array}{l}\text { BP-14 } \\
\text { Rating- } \\
\text { stufe }\end{array}$ & $\begin{array}{l}\text { Rating auf Basis der KNN-Bilanzanalyse BP-14 } 1995 \\
\qquad(1994)^{380}\end{array}$ & $\begin{array}{l}\text { Historische } \\
\text { Ausfallrate }\end{array}$ \\
\hline \multirow{6}{*}{ 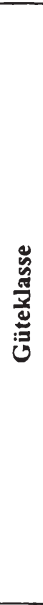 } & 8 bis 10 & AA & $\begin{array}{l}\text { 2.B. Simona, (Hach), Kampa-Haus, Weru, Bien-Haus, Westag \& Getalit, } \\
\text { Leifheit }\end{array}$ & $0,02 \%$ \\
\hline & 6 bis 8 & A & $\begin{array}{l}\text { 2.B. Schering, Bayer, Linde, Gelsenwasser, Energie-Vers. Schwaben, } \\
\text { (Bewag), J. Vogele, Linde, Kögel Fahrz., Robert Bosch GmbH, Altana, } \\
\text { Boehr. Ingclh., Beiersdorf, Fielmann, A. WUrth, Douglas Hold., (Hor- } \\
\text { ten), F. Grohe, Dyckerhoff, (P. Hartmann), Heraeus Hold., (Schaerf) } \\
\end{array}$ & $0,12 \%$ \\
\hline & 4 bis 6 & BB & $\begin{array}{l}\text { z.B. Siemens, Thyssen, Preussag, Mannesmann, Henkel, Lufthansa, } \\
\text { Badenwerk, Allweiler, (Drăgerwerk), (Benteler), Porsche, Leonische } \\
\text { Drahtwerke, Fresenius, Süd-Chemic, Th. Goldschmidt, Merck, Phoenix, } \\
\text { Binding, Holsten, Tschibo, Heidelberger Zement, Freudenberg, Vossloh, } \\
\text { A. Springer, Salamander, Hapag-Lloyd, (Bertelsmann), Norddeutsche } \\
\text { Raffinerie }\end{array}$ & $0,12 \%$ \\
\hline & 2 bis 4 & B & $\begin{array}{l}\text { z.B. Höchst, Degussa, Karstadt, Continental, Bayrische Motorenwerke, } \\
\text { Volkswagen, Kaufhof, Ruhrkohlc, (Krones), Jungheinrich, Hoesch- } \\
\text { Kupp, Max-Holding, J.M. Voith, Berentzen, Sudzucker, Spar, (Mode } \\
\text { Hold.), Escada, Dyckerhoff \& Widmann, Bilfinger \& Berger, F. Haniel, } \\
\text { Villeroy \& Boch, Stöhr \& Co. }\end{array}$ & $0,35 \%$ \\
\hline & 0 bis 2 & $\mathrm{CC}$ & $\begin{array}{l}\text { z.B. Daimler-Benz, Metallgesellschaft, Linotype-Hell, Varta, (Compu- } \\
\text { Net), Berliner Elektro.Hold., Fuchs Petrolub Oel + Chemie, Wella, Brau } \\
\text { und Brunnen, Kaufring, Wūnsche, (Markant-Suldwest), Walter Bau, } \\
\text { Rheinhold \& Mahla, Heilit \& Woemer Bau, Strabag, Verseidag, } \\
\text { Rosenthal, Großkraftwerk Mannheim }\end{array}$ & $0,66 \%$ \\
\hline & -2 bis 0 & $\mathrm{C}$ & $\begin{array}{l}\text { z.B. Knort-Bremse, Langheim-Pfannhauser, Klöckner-Werke, Sixt, } \\
\text { VBH, (Glunz), Steffen }\end{array}$ & $0,86 \%$ \\
\hline \multirow{4}{*}{ 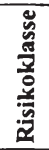 } & -4 bis -2 & I & $\begin{array}{l}\text { z.B. Wanderer-Werke, Computer 2000, A. Moksel, Philipp Holzmann, } \\
\text { Herlitz, (Neue Baumwoll-Spinnerei u. Webcrei) }\end{array}$ & $2,09 \%$ \\
\hline & -6 bis -4 & II & z.B. Greiffenberger, (Pittler Maschincnfabrik) & $3,09 \%$ \\
\hline & -8 bis -6 & III & 2.B. Traub, (Gildemeister) & $7,44 \%$ \\
\hline & -10 bis -8 & IV & & $15,23 \%$ \\
\hline
\end{tabular}

Tabelle 10: Zuordnung der N-Werte des BP-14 zu historischen Ausfallwahrscheinlichkeiten ${ }^{381}$

\subsection{Rechtliche Grundlagen der Bilanzierung}

Die im Kapitel 3.1 und in den folgenden Kapiteln verwendeten HGB-Normen als rechtliche Grundlage stehen neben weiteren rechtlichen Vorschriften im übrigen europäischen und aussereuropäischen Rechtsraum. Insbesondere die US - Generally Accepted Accounting Principles (US-GAAP) erlangen eine immer größer werdende Bedeutung. ${ }^{382}$ Somit gilt es zu untersuchen, inwieweit rechtliche Rahmenbedingungen einen Einfluss auf die dargestellten Bilanzanalyseverfahren besitzen. Zunächst wird

380 Unternehmen aus dem Jahre 1994 werden durch eine Klammersetzung gekennzeichnet.

381 In Anlehnung an Baetge, Sieringhaus (1996), S. 247; Baetge (1998), S. 597; ähnlich auch bei Bröker (2000), S. 64.

382 Die International Accounting Standards (IAS) erlangen ebenso wie das US-GAAP eine steigende Bedeutung. Da sie jedoch in der weiteren Analyse nur eine untergeordnete Stellung einnehmen, werden sie nicht detailliert beschrieben. Zur Beschreibung der IAS vgl. bspw. Pellens (1998), S. 367ff. und die dort angegebenen Quellen. 
Marktorientierte Kreditrisikobewertung -

hierzu der grundsätzliche Aufbau des Jahresabschlusses nach dem US-GAAP skizziert, um anschließend die konzeptionellen Unterschiede aufzuzeigen.

Im Folgenden wird der Aufbau des Annual Reports aufgezeigt. ${ }^{383}$ Demnach gliedert sich der Jahresabschluss nach dem US-GAAP in folgenden Teile:

(1) Balance Sheet / Statement of Financial Position (Bilanz),

(2) Statement of Retained Earnings (Entwicklung der Gewinnrücklagen),

(3) Statement of Income / Statement of Operations / Statement of Earnings (Gewinn- und Verlustrechnung),

(4) Statement of Cash Flows (Finanzrechnung / Kapitalflussrechnung),

(5) Notes to Financial Statements (Anhang),

(6) Report of Independent Auditors (Prüfungsbericht / Bestätigungsvermerk),

(7) Management's Letter / Report of Management (Lagebericht).

Die in dem empirischen Teil dieser Arbeit vorgenommene Untersuchung bezieht sich auf Bilanzkennzahlen aus den Bereichen (1), (3) und (4) und somit auf Kennzahlen aus der Bilanz, inklusive Gewinn- und Verlustrechnung, und der Kapitalflussrechnung. ${ }^{384}$ Konzeptionelle Unterschiede zu HGB-Vorschriften werden hinsichtlich der folgenden Kriterien festgestellt: Rechnungslegungsziele, Bedeutung der Generalnormen, dominierende Rechenlegungsgrundsätze, Auslegung des Realisationsprinzips, Möglichkeiten der Bildung stiller Reserven, Stetigkeitsgrundsatz und Maßgeblichkeit zwischen Handels- und Steuerbilanz. ${ }^{385 / 386}$

Die nach dem HGB aufzustellende Bilanz hat das Ziel, die Vermögens-, Finanz- und Ertragslage eines Unternehmens unter der Beachtung der Grundsätze Ordnungsgemäßer Buchführung darzustellen. ${ }^{387}$

Eine ,fair presentation" (tatsachengetreue Darstellung) der "decision usefulness information" (entscheidungsrelevanten Informationen) für die Investoren und Gläubiger ist die Zielsetzung der Bilanzierung nach dem US-GAAP. ${ }^{388}$

Die folgende Gliederung und die Ausführungen wurden entnommen aus Gräfer (1992), S. $17 \mathrm{f}$.

Die Kapitalflussrechnung untersucht die Zahlungsströme des vergangenen Jahres und wird auch als dynamische Liquiditätsanalyse bezeichnet. Vgl. Bartram (1991), S. 614.

Vgl. zur detaillierten Beschreibung der Auswirkung der unterschiedlichen Systeme auf die Interpretationsmöglichkeiten für den Bilanzanalytiker Born (2001).

Vgl. zu den weiteren Ausführungen Goebel (1995), S. 2489ff.

387 Vgl. $\S \S 243$ Abs. 1 und $\S 264$ Abs. 2 Satz 1 HGB.

388 Vgl. Hier und im Folgenden Gräfer (1992), S. 3. 
Marktorientierte Kreditrisikobewertung -

In beiden Vorschriften wird ein übergeordneter Grundsatz formuliert, dessen Maßgeblichkeit für die untergeordneten Spezialnormen jedoch unterschiedlich ist. ${ }^{389}$ Während im HGB der "true and fair view"-Grundsatz durch die Spezialnormen ausgehebelt werden kann, ist in der Rechnungslegung nach US-GAAP dieser Grundsatz ein „overriding principle“ (übergeordneter Grundsatz). ${ }^{390}$ In diesem Zusammenhang erfolgt bei der Gewinnermittlung nach dem US-GAAP eine periodengerechte Ermittlung (,accrual and matching principle“) ${ }^{391}$ Demgegenüber ermitteln Kaufleute den Gewinn nach HGB nach dem Vorsichts- und Realisationsprinzip. ${ }^{392}$ Hierdurch wird der Schutz des Gläubigers in den Vordergrund gestellt.

Aus diesem Vorsichtsprinzip abgeleitet, wird bei der Auslegung des Realisationsprinzips bestimmt, dass i.d.R. nichtrealisierte Verluste (Gewinne) in der Gewinnberechnung berücksichtigt (nicht berücksichtigt) (Imparitätsprinzip) werden. ${ }^{393}$ Für den Bereich der US-GAAP findet eine Orientierung an der periodengerechten Gewinnermittlung statt, sodass u.U. nicht realisierte Gewinne und Verluste ergebniswirksam (periodengerecht) ausgewiesen werden müssen. ${ }^{394}$

Analog zu der Auslegung des Realisationsprinzips wird bei der Möglichkeit zur Bildung stiller Reserven verfahren. ${ }^{395}$ Im HGB findet sich ein relativ großer Gestaltungsraum bei der Auslegung von Wertansätzen und der Einräumung von Wahlrechten. Diese großen Spielräume können wiederum mit dem Gläubigerschutz motiviert werden. Durch die Bildung von Stillen Reserven wird der Gewinnabfluss verhindert. Aufgrund des Prinzips der fair presentation würde die Bildung von Stillen Reserven dagegen das Bild verfälschen. Somit schränkt das US-GAAP die Bildung von Stillen Reserven stark ein.

Unter dem Kriterium der Stetigkeit wird die Beibehaltung von getroffenen Bilanzierungsentscheidungen verstanden. ${ }^{396} \mathrm{Um}$ eine (zeitliche) Vergleichbarkeit von Abschlüssen garantieren zu können, müssen diese Ansätze beibehalten werden. Der Grundsatz der Stetigkeit wird im US-GAAP-System sehr streng verfolgt. Im Rahmen des HGB gibt es zahlreiche Möglichkeiten, vom Grundsatz der Stetigkeit abzuweichen. ${ }^{397}$ Formell wird hier dieser Grundsatz auch verankert, jedoch wird er durch die Einräumung von Ausnahmen im Vergleich zum US-GAAP nicht so stringent ver-

Vgl. Gräfer (1995), S. 104f.

Vgl. Goebel (1995), S. 250ff.

Vgl. Glormann (2001), S. $41 \mathrm{f}$.

Vgl. § 252 Abs. 1 Nr. 4 HGB.

Vgl. § 252 Abs. 1 Nr. 4 HGB.

Vgl. bspw. Pellens (1989), S. 174.

Vgl. hier und im Folgenden Goebel (1995), S. 2489ff.

Vgl. hier und im Folgenden Gräfer (1992), S. 104f.

Vgl. hier und im Folgenden Müller (1988), S. 18. 
Marktorientierte Kreditrisikobewertung Die Bilanzanalyse als Instrument zur einzelgeschäftsbezogenen Risikobewertung

folgt. Die so genannte einfache und umgekehrte Maßgeblichkeit zwischen der Handels- und der Steuerbilanz ist z.B. im US-GAAP nicht implementiert. ${ }^{398}$ Im Rahmen von Konzernabschlüssen besteht auch nach deutschen Recht die Möglichkeit, sich von dieser Maßgeblichkeit zu lösen (Entkopplung).

Die unterschiedlichen Bilanzierungsvorschriften (zusammengefasst in Tabelle 11) machen deutlich, dass sich das durch den Abschluss vermittelte Unternehmensbild aufgrund der Vorschriften unterscheiden kann. Somit ist offensichtlich, dass die aus der Bilanzanalyse gewonnenen Erkenntnisse sehr detailliert vor dem entsprechenden rechtlichen Hintergrund gedeutet werden müssen. Die nach derselben mathematischen Vorschrift erhobene Kennzahl kann durch die unterschiedlichen Bilanzierungsvorschriften verschiedene Interpretationsmöglichkeiten ermöglichen bzw. nötig machen.

Das o.g. Neutralisierungsprinzip besagt, dass eine Bilanzanalyse nicht von den gewählten Spielräumen durch den Bilanzersteller abhängig sein darf. Im Rahmen eines Vergleiches der unterschiedlichen Rechnungslegungssysteme bedeutet die Einhaltung des Prinzips, dass das Ergebnis der Bilanzanalyse nicht vom Rechnungslegungssystem abhängen darf. ${ }^{399}$ Es bestehen somit keine zwingenden Gründe, die eine Verwendung der o.g. dargestellten Bilanzanalyseverfahren auf ein Rechnungslegungssystem beschränken.

\begin{tabular}{|c|c|l||}
\hline $\begin{array}{c}\text { Bedeutung der } \\
\text { Generalnorm }\end{array}$ & $\begin{array}{l}\text { HGB } \\
\text { keine übergeordnete Funktion, } \\
\text { Spezialnormen durchbrechen die } \\
\text { Generalnorm }\end{array}$ & $\begin{array}{l}\text { US-GAAP } \\
\text { Grundsatz (overriding principle) }\end{array}$ \\
\hline $\begin{array}{c}\text { Dominierende Rechnungsle- } \\
\text { gungsgrundsätze }\end{array}$ & Vorsichtsprinzip & $\begin{array}{l}\text { Grundsatz der perioden-gerechten } \\
\text { Gewinnermittlung (accrual principle) }\end{array}$ \\
\hline $\begin{array}{c}\text { Auslegung des } \\
\text { Realisationsprinzips }\end{array}$ & $\begin{array}{l}\text { Realisationszeitpunkt orientiert } \\
\text { sich an rechtlichen Kriterien; } \\
\text { Ableitung aus dem Vorsichts- } \\
\text { prinzip }\end{array}$ & $\begin{array}{l}\text { (Weniger restriktive)Ableitung aus } \\
\text { Grundsatz der periodengerechten } \\
\text { Gewinnermittlung }\end{array}$ \\
\hline $\begin{array}{c}\text { Möglichkeit der Bildung } \\
\text { stiller Reserven }\end{array}$ & $\begin{array}{l}\text { In relativ großen Umfang durch } \\
\text { Wahlrechte und Ermessensspiel- } \\
\text { räume gegeben }\end{array}$ & $\begin{array}{l}\text { In eingeschränktem Umfang gegeben, da } \\
\text { stille Reserven dem Grundsatz der fair } \\
\text { presentation widersprechen }\end{array}$ \\
\hline Stetigkeit & $\begin{array}{l}\text { Formelles Stetigkeitsgebot mit } \\
\text { zahlreichen Durchbrechungen }\end{array}$ & $\begin{array}{l}\text { Strenger Grundsatz der Stetigkeit } \\
\text { aufgrund des Grundsatzes der Ver- } \\
\text { gleichbarkeit (comparability) }\end{array}$ \\
\hline
\end{tabular}

Tabelle 11: Gegenubberstellung der Bilanzierungsgrundsătze nach dem HGB und dem US-GAAP ${ }^{400}$

$398 \mathrm{Zu}$ der Maßgeblichkeit nach dem HGB vgl. bspw. Coenenberg (2000), S. 39ff.

In Anlehnung an Goebel (1995), S. 2490ff. 
Marktorientierte Kreditrisikobewertung -

\subsection{Zwischenfazit}

Als Zwischenfazit lässt sich konstatieren, dass die neueren Ansätze zur Bilanzanalyse versuchen, die Schwächen der traditionellen Analyse (teilweise) zu überwinden. ${ }^{401}$ Dies gelingt insbesondere in dem Bereich der Objektivierung. Es werden statistische Zusammenhänge zwischen Kennzahlen und Unternehmenskrisen gefunden. Somit handelt es sich um Verfahren, die „die Ursachen der Insolvenzgefährdung nicht (...) [erkennen], sondern nur Symptome "“402 messen können. Nach BURGER ist es nicht gelungen, eine theoretische Fundierung der in den verschiedenen empirischen Ergebnissen gewonnenen Zusammenhänge zu leisten. ${ }^{403}$ Das Prinzip der Objektivierung wird somit auf einer statistischen Ebene erfüllt. Inwieweit die Modellstrukturen für den Anwender, d.h. den Bilanzanalytiker, nachvollziehbar sind, bleibt offen. Die Methodik der KNN wird häufig als „Black Box“ bezeichnet. ${ }^{404}$ Außerdem liefern die unterschiedlichen Untersuchungen verschiedene Ergebnisse, wodurch sich für den Bilanzanalytiker die Notwendigkeit zur Auswahl eines Systems stellt. Hierfür könnte der Umfang der Datengrundlage ein gutes Argument sein, weil sich dadurch die Güte des Modells erhöht. ${ }^{405}$ Das BP-14 weist in diesem Punkt die umfangreichste Datenbasis auf und könnte sich deshalb als Standard- bzw. Referenzmodell anbieten. Trotzdem liegt auch hier ein $\beta$-Fehler von 33,55\% vor, was bedeutet, dass $33,55 \%$ der solventen Unternehmen als insolvenzgefährdet eingestuft werden.

Der Forderung nach dem Prinzip der Ganzheitlichkeit und der Neutralisierung scheinen die empirisch-induktiven Ansätze zu genügen. Das Prinzip der Ganzheitlichkeit ist dann erfültt, wenn die Berechnung der Kennzahlen alle Unternehmensbereiche einbezieht. Vor diesem Hintergrund muss der Kennzahlenkatalog, der bei der Modellkonzeption hinterlegt wird, ausreichend groß sein. Beispielsweise leistet das BP14 diese Ganzheitlichkeit, da alle Bereiche des Unternehmens durch eine geschickte Kennzahlenauswahl eingeschlossen werden. ${ }^{406}$ Darüber hinaus sorgen diese Kennzahlen dafür, dass bilanzpolitische Maßnahmen keinen Einfluss auf das Gesamturteil haben, da sich Bilanzmanipulationen nicht isoliert in einer Kennzahl äußern können,

401 Da das KNN als Weiterentwicklung der MDA aufgrund der Reduktion statistischer Prämissen gesehen wird, bildet es die Grundlage der Diskussion in Kap. 3.6. Die im Weiteren folgende Diskussion insbesondere hinsichtlich der Eignung als Kreditzinskalkulationsverfahren kann analog geführt werden. Baetge, Beuter, Feidicker (1992), S. 263; (die mit [ ] gekennzeichneten Inhalte wurden vom Verfasser hinzugefügt).

Vgl. Burger (1995), S. 335.

Vgl. bspw. Bröker (2000), S. 65.

Vgl. hier und im Folgenden Baetge (1998), S. 583.

Vgl. hier und im Folgenden Baetge (1998), S. $585 f f$. 
Marktorientierte Kreditrisikobewertung -

Die Bilanzanalyse als Instrument zur einzelgeschäftsbezogenen Risikobewertung

sondern durch die gleichzeitige Variation verschiedener Kennzahlen neutralisiert werden. Das BP-14 genügt somit dem Anspruch der Neutralisierung.

Hinsichtlich der natürlichen Grenzen der Bilanzanalyse lässt sich konstatieren, dass der Vergangenheitsbezug der Bilanzkennzahlen nicht aufgehoben werden kann. Ebenso ist die Reduktion der komplexen Umwelt auf wenige Kennzahlen problematisch, jedoch werden beide Prozesse durch statistisch-empirisch nachgewiesene $\mathrm{Zu}$ sammenhänge gestützt. Fraglich ist, inwieweit die gefundenen Zusammenhänge sich fortschreiben lassen. Hierzu könnte man das Modell regelmäßig überprüfen und mit aktuellen Daten trainieren, sodass sich bspw. das Neuronale Netz auf Veränderungen einstellen kann. Offen bleibt jedoch der Umgang mit Diskontinuitäten, dass bedeutet, falls historische Strukturen, in diesem Fall der Zusammenhang zwischen verschiedenen Bilanzkennzahlen und dem Bonitätsurteil, aufgrund realwirtschaftlicher Veränderungen instabil sind.

$\mathrm{Zu}$ untersuchen ist somit, falls ein System die oben genannten Kriterien erfüllt, inwieweit die Bilanzanalyse als internes Ratingystem den Anforderungen an ein Kalkulationsverfahren zur Bepreisung von Kreditrisiken genügt. Analog zu der Übertragung eines externen Rating muss auch in dem Fall des internen Rating eine Übertragung der qualitativen Einschätzung in eine quantitative Größe (Kreditzins oder Ausfallwahrscheinlichkeit) erfolgen. Bspw. könnten bei dieser Vorgehensweise die Ausfallwahrscheinlichkeiten der zugeordneten Ratingklassen verwendet werden (vgl. Tabelle 10). Um von der Ausfallwahrscheinlichkeit auf das bepreiste Risiko schlieBen zu können, müsste ein weiterer Arbeitsschritt folgen. Beispielsweise könnte das unter Kapitel 2.3.2.1 dargestellte stochastische Modell genutzt werden, um einen Kreditzins zu ermitteln, da dieses Modell explizit auf Ausfallwahrscheinlichkeiten zurückgreift und mit Hilfe dieser eine individuelle Bonitätsprämie berechnen kann. ${ }^{407}$

Das Prinzip der Einzelbewertung wird bei dieser Vorgehensweise verletzt, da wiederum auf die Ausfallwahrscheinlichkeiten einer Klasse und somit einzelner Segmente zurückgegriffen wird. Es wird zwar eine unternehmensindividuelle Bonitätsanalyse mit Hilfe der KNN-Analyse sichergestellt, unternehmensindividuelle Ausfallwahrscheinlichkeiten liegen jedoch nicht vor. Vielmehr werden den Unternehmen in einem Segment gleiche Ausfallwahrscheinlichkeiten zugeordnet. Das Segmentierungskriterium ist der aus der Bilanzanalyse abgeleitete Wert (in dem o.g. Verfahren der $\mathrm{N}$-Wert).

407 Alternativ können der Z"'-Score und die zugehörige Ratingklasse verwendet werden. Im Ergebnis wird die Bonitätsprämie (Credit Spread) der korrespondierenden Ratingklasse benutzt, um den Kreditzins für den Schuldner zu berechnen. Die Würdigung dieser Vorgehensweise kann analog zu der Betrachtung der Eignung der Bilanzanalyse mittels KNN für die Kreditzinskalkulation geführt werden. 
Marktorientierte Kreditrisikobewertung -

Im folgenden Schritt wird das Prinzip der Richtigkeit geprüft. Hierzu ist zunächst die Frage nach der Objektivität zu stellen. Damit können die Bilanzanalyseverfahren, die das oben genannte Objektivierungsprinzip nicht erfüllen, auch als Kalkulationsverfahren nur bedingt geeignet sein. ${ }^{408} \mathrm{Als}$ (bedingt) objektive Instrumente wurden die empirisch-induktiven Bilanzanalyseverfahren genannt. Bei diesen war die Ausgangsfragestellung eine Klassifikation von gesunden und nichtgesunden Unternehmen. Wie o.g. werden nun den einzelnen Segmenten (Segmentierungskriterium ist in dem o.g. Verfahren der N-Wert) ein Rating und eine historische Ausfallrate zugeordnet. Folglich kann die Bilanzanalyse für das einzelne Unternehmen eine Ausfallwahrscheinlichkeit ausweisen. Während der ausgewiesene $\alpha$ - $\beta$-Fehler das Gütema $\beta$ für eine falsche Klassifikation darstellt, müssen parallel die Unsicherheiten in der ermittelten Ausfallwahrscheinlichkeit ausgewiesen werden. ${ }^{409}$

Das Kriterium der Aktualität hinsichtlich der Datengrundlage kann nur bedingt erfullt werden. Während die Zusammenhänge, welche durch die statistischen Untersuchungen analysiert wurden, regelmäßig durch die Erweiterung der Datenbasis aktualisiert werden können, ${ }^{410}$ erweist sich die Bepreisung des Kreditrisikos analog zu dem Segmentierungsverfahren als problematisch. Die ermittelten historischen Ausfallraten bedingen, dass die aktuelle Risikosituation keinen direkten Einfluss auf die Ausfallwahrscheinlichkeit besitzt. ${ }^{411}$ Falls es gelingen würde, mit Hilfe von Prognosen die Ausfallwahrscheinlichkeiten zu bestimmen, wäre dieses Problem aufgehoben. ${ }^{412}$

Neben dem spezifischen Problem der Ausfallquoten wird auch die ausschließliche Erhebung von Bilanzkennzahlen zur Bepreisung des Ausfallrisikos unter dem Aspekt der Aktualität als problematisch angesehen. „Bilanz und GuV sind doppelt veraltet: Zum einen bezieht sich das Zahlenwerk des Jahresabschlusses auf vergangene Ab-

408 Bei der weiteren Diskussion wird auf die Bilanzanalyseinstrumente verzichtet, die das Objektivitätskriterium nicht erfüllen. Die folgende Analyse beschränkt sich auf die Bilanzanalyse mittels KNN. Die Argumentation lässt sich jedoch auf die Bilanzanalyse mittels MDA übertragen.

409 Dieser Wert beschreibt die Unsicherheit bei der Zuordnung einer Ausfallwahrscheinlichkeit zu einer Klasse. Falls man einer Klasse eine (durchschnittliche) Ausfallwahrscheinlichkeit zuordnet, besteht die Ungenauigkeit der Streuung der ermittelten Wahrscheinlichkeiten in dieser Klasse.

${ }^{410} \mathrm{Zu}$ untersuchen ist die zeitliche Diskrepanz (time lag) zwischen der Strukturänderung und der möglichen Aufnahme in den ermittelten Strukturen.

411 Vgl. zu dieser Problematik die im Kap. 2.5 dargestellte Diskussion der Segmentierungsverfahren, die ebenfalls die Ist-Risikokosten der Vergangenheit heranzeihen, um das heutige Kreditgeschäft zu kalkulieren.

${ }^{412}$ Zur Problematik der Zuordnung vgl. Jansen (2001), S. 55ff. und Baetge, Jerschensky (1996), S. 1584. 
Marktorientierte Kreditrisikobewertung Die Bilanzanalyse als Instrument zur einzelgeschäftsbezogenen Risikobewertung

rechnungsperioden, zum anderen liegt der Jahresabschluss i.d.R. erst eine geraume Zeit nach dem Abschlussstichtag vor. ${ }^{6413}$

Zusammenfassend kann man eine bedingte Eignung der Bilanzanalyse als internes Ratingsystem zur Kalkulation von Kreditrisiken konstatieren (vgl. Tabelle 12). Es wird deutlich, dass die empirisch-induktiven Verfahren den traditionellen Verfahren der Kennzahlen, Kennzahlensysteme und Scoring-Modelle insbesondere auf Grund einer objektiven Urteilsfindung überlegen sind. Eine hinreichende Übertragung dieser empirisch-induktiven Verfahren auf die Kreditzinskalkulation ist jedoch noch nicht gelungen. Weder das Prinzip der Einzelbewertung noch das Prinzip der Richtigkeit werden vollständig erfüllt. Da die moderne Bilanzanalyse die an die Bilanzanalyse gestellten Prinzipien (Neutralisierung, Ganzheitlichkeit, Objektivierung) erfüllt, besteht der Anlass, weitere Methoden zu entwickeln, welche insbesondere die im Rahmen der Anwendung als Kalkulationsverfahren entstehenden Probleme (bspw. die Aktualität) beachten.

\begin{tabular}{|c|c|c|c|c|c|c|}
\hline \multirow{3}{*}{$\begin{array}{c}\text { Bilanzanalyse- } \\
\text { Instrumente }\end{array}$} & \multicolumn{3}{|c|}{ Prinzipien der Bilanzanalyse } & \multicolumn{3}{|c|}{ Eignung als Kreditzinskalkulationsverfahren } \\
\hline & \multirow{2}{*}{$\begin{array}{l}\text { Neutrali- } \\
\text { sierung }\end{array}$} & \multirow{2}{*}{$\begin{array}{l}\text { Ganzheit- } \\
\text { lichkeit }\end{array}$} & \multirow{2}{*}{$\begin{array}{l}\text { Objektivie- } \\
\text { rung }\end{array}$} & \multirow{2}{*}{$\begin{array}{c}\text { Einzel- } \\
\text { bewertung }\end{array}$} & \multicolumn{2}{|c|}{ Richtigkeit } \\
\hline & & & & & Objektivität & Aktualität \\
\hline Kennzahlen & $\begin{array}{l}\text { Eher nicht } \\
\text { gegeben, } \\
\text { Einzelfall- } \\
\text { prüung }\end{array}$ & $\begin{array}{l}\text { Eher nicht } \\
\text { gegeben, } \\
\text { Einzelfall- } \\
\text { prüfung }\end{array}$ & $\begin{array}{c}\text { Unsystemati- } \\
\text { sche, subjektive } \\
\text { Gesamturteils- } \\
\text { bildung }\end{array}$ & $\begin{array}{l}\text { Problem der } \\
\text { Segmentie- } \\
\text { rung }\end{array}$ & $\begin{array}{c}\text { Keine (objektive) } \\
\text { Bestimmung von } \\
\text { Ausfallpramien }\end{array}$ & $\begin{array}{l}\text { Grundsătzliches } \\
\text { Problem der } \\
\text { Bilanzanalyse } \\
\text { (doppelte } \\
\text { Vergangenheit) }\end{array}$ \\
\hline $\begin{array}{l}\text { Kennzahlen- } \\
\text { systeme }\end{array}$ & $\begin{array}{l}\text { Eher } \\
\text { gegeben, } \\
\text { Einzel- } \\
\text { fallprufung }\end{array}$ & $\begin{array}{c}\text { Eher gegeben, } \\
\text { Einzelfall- } \\
\text { prüfung }\end{array}$ & $\begin{array}{l}\text { Systematische, } \\
\text { subjektive } \\
\text { Gesamturteils- } \\
\text { bildung }\end{array}$ & $\begin{array}{l}\text { Problem der } \\
\text { Segmentie- } \\
\text { rung }\end{array}$ & $\begin{array}{c}\text { Keine (objektive) } \\
\text { Bestimmung von } \\
\text { Ausfallprämien }\end{array}$ & $\begin{array}{l}\text { Grundsatzliches } \\
\text { Problem der } \\
\text { Bilanzanalyse } \\
\text { (doppelte } \\
\text { Vergangenheit }\end{array}$ \\
\hline $\begin{array}{l}\text { Scoring- } \\
\text { Modelle }\end{array}$ & $\begin{array}{c}\text { Eher } \\
\text { gegeben, } \\
\text { Einzelfallpr } \\
\text { ufung }\end{array}$ & $\begin{array}{c}\text { Eher gegeben, } \\
\text { Einzelfall- } \\
\text { prufung }\end{array}$ & $\begin{array}{l}\text { Quasi-objektive } \\
\text { Gesamturteils- } \\
\text { bildung }\end{array}$ & $\begin{array}{l}\text { Problem der } \\
\text { Segmentie- } \\
\text { rung }\end{array}$ & $\begin{array}{l}\text { Aufgrund der } \\
\text { quasi-objektiven } \\
\text { Urteilsbildung } \\
\text { nicht gesichert }\end{array}$ & $\begin{array}{l}\text { Grundsätzliches } \\
\text { Problem der } \\
\text { Bilanzanalyse } \\
\text { (doppelte } \\
\text { Vergangenheit) }\end{array}$ \\
\hline $\begin{array}{l}\text { Empirisch- } \\
\text { induktive } \\
\text { Verfahren }\end{array}$ & $\begin{array}{l}\text { Eher } \\
\text { gegeben, } \\
\text { Einzelfallpr } \\
\text { ufung }\end{array}$ & $\begin{array}{l}\text { Eher gegeben, } \\
\text { Einzelfall- } \\
\text { prüfung }\end{array}$ & $\begin{array}{l}\text { Objektive } \\
\text { Gesamturteils- } \\
\text { bildung }\end{array}$ & $\begin{array}{l}\text { Problem der } \\
\text { Segmentie- } \\
\text { rung }\end{array}$ & $\begin{array}{c}\text { Anhand von } \\
\text { marktbezogenen } \\
\text { Ausfall- } \\
\text { wahrscheinlich- } \\
\text { keiten möglich }\end{array}$ & $\begin{array}{c}\text { Historische } \\
\text { Ausfallraten } \\
\text { weisen Vergan- } \\
\text { genheitsbezug } \\
\text { auf }\end{array}$ \\
\hline
\end{tabular}

Tabelle 12: $\quad$ Zusammenfassung der Würdigung verschiedener Bilanzanalyseverfahren zur Kreditzinskalkulation

413 Jansen (2001), S. 52f., vgl. auch Baetge (1998b) S. 27. 
Marktorientierte Kreditrisikobewertung -

\section{Herleitung eines marktorientierten Ansatzes zur Bewertung des Kreditrisikos}

\subsection{Zielsetzung}

Wie in Kap. 2.5 gezeigt wurde, erfüllen sowohl modelltheoretische als auch heuristische Kreditzinskalkulationsverfahren die geforderten Kriterien an ein solches nicht vollständig. Auch ist bislang ein idealtypisches Instrument zur Bepreisung von Ausfallrisiken unter Heranziehung der Bilanzanalyse nicht identifiziert worden (vgl. Kap. 3.6). Vor diesem Hintergrund stellt sich die Anforderung, ein Prognosemodell ${ }^{414} \mathrm{zu}$ entwickeln, dessen Zielgröße der objektive Kreditzins bzw. das objektiv bepreiste Bonitätsrisiko ist. Aus dem in Kap. 2.4 aufgestellten Objektivitätskriterium folgt, dass ein Kreditzins genau dann objektiv ist, wenn ,,institutsinterne Maßstäbe oder Rahmenbedingungen die Höhe der Risikoprämie nicht beeinflussen (...) und [für] (...) die Bewertung des Risikos (...) ein objektivierter Maßstab [herangezogen wird]. Idealerweise handelt es sich dabei um einen marktlichen Maßstab“415. Folglich muss dieses Prognosemodell funktionale Zusammenhänge integrieren, die aus objektiven Kriterien abgeleitet werden. In konzentrierter Form kann man somit die Aufgabe der Entwicklung eines diesbezüglich neuen Konzeptes formulieren als:

Entwicklung eines marktorientierten Kreditzinskalkulationsverfahrens auf Basis der Enwartungsbildung eines rational urteilenden ${ }^{46}$, externen Bilanzanalytikers.

Wie bereits in Kap. 3.1 dargestellt wurde, werten externe Bilanzanalytiker die Bilanzinformationen zur Urteilsfindung hinsichtlich der Kreditwürdigkeit und der Bonität eines Unternehmens aus. Diese Urteilsfindung wird für das Prognosemodell konkretisiert, indem eine quantitative Bewertung der Bonität in Form eines Kreditzinses abgeleitet wird. Da dieses Urteil mit Hilfe von rationalen Erwartungen gebildet wird, darf es keine systematischen Fehler beinhalten und muss auf Basis eines Modells erfol-

$414 \mathrm{Zu}$ der Verwendung des Begriffs Prognosemodell vgl. die Ausführungen im Kapitel 1.2. In Anlehnung an die griechische Herkunft des Wortes wird als Prognose eine Vorhersage verstanden. Als Zielgröße wird in diesem Fall die Bestimmung eines objektiven Kreditzinses definiert, wobei dieser im Weiteren abgleitet wird. Das Wort Modell bezieht sich hierbei auf die vereinfachte Abbildung des Kapitalmarktes, sodass als Prognosemodell die modelltheoretische Vorhersage des realwirtschaftlichen Ergebnisses „objektiver Kreditzins“ verstanden wird. Zur alternativen Definition von Finanzprognose als systematische Ursachen-Wirkungs-Analyse vgl. Storm, Baun (1992), S. 534.

415 Kirmße (1996), S. 61; (die mit [ ] gekennzeichneten Inhalte wurden vom Verfasser hinzugefügt). Ähnlich auch bei Bröker (2000), S. 188.

416 Vgl. zu dem Begriff der ,rationalen Erwartungsbildung“ Muth (1961), S. 316; Lucas (1972, 1975, 1981), Schlieper (1995), S. 32f.; Cassel (1992), S. 297. 
Marktorientierte Kreditrisikobewertung Herleitung eines marktorientierten Ansatzes zur Bewertung des Kreditrisikos

gen. ${ }^{417}$ Eine marktorientierte Bewertung, d.h. eine Anlehnung an marktliche Maßstäbe, wird als Teilaufgabe festgelegt, da sie, wie bereits oben erläutert, eine objektive Bewertung ermöglicht.

Die marktorientierte Einschätzung des Kreditrisikos bedingt den Bezug auf einen Markt, der dieses Risiko bewertet. Als Kapitalmarkt im weiteren Sinne wird der „organisierte Kapitalmarkt" verstanden, auf dem Aktien und Anleihen entweder als Neuemissionen oder aus Beständen nachgefragt und angeboten werden. ${ }^{418}$ Es stehen sich hier eine (theoretisch) unendlich große Zahl von Käufern und Verkäufern gegenüber. Das Ergebnis des Handels ist eine Bepreisung bzw. Bewertung eines Finanztitels, welche dem o.g. Objektivitätskriterium folgend als objektiv bezeichnet werden kann. Es ist zu untersuchen, welche Informationen im Rahmen der marktorientierten Risikobewertung benötigt und wie diese verwertet werden.

Der Informationsgehalt der Bilanz für den Kapitalmarkt wurde in der Wissenschaft bereits im Rahmen der empirischen, kapitalmarktorientierten Bilanzforschung untersucht. ${ }^{419 / 420}$ Diese beschäftigt sich u.a. mit der Fragestellung, welchen Informationsgehalt der Jahresabschluss besitzt. ${ }^{421}$ Im Rahmen dieses Forschungszweigs werden aus dem Blickwinkel eines Anteilseigners die informatorischen Eigenschaften veröffentlichter Jahresabschlüsse untersucht. Die empirische Forschung konnte im Ergebnis einen Zusammenhang zwischen den Bilanzen als öffentlich zugängliche Information und Kursbewegungen von Aktien als resultierende Kapitalmarktreaktion identifizieren. Dies legt den Schluss nahe, dass die Bilanzanalyse ein Instrument zur Entscheidungsfindung auf Kapitalmärkten ist.

Die Notwendigkeit einer Kreditzinskalkulation im Rahmen einer Kreditvergabe folgt aus der Schuldner-Gläubiger-Beziehung, wie sie zwischen einem Unternehmen und einem Kreditinstitut besteht. ${ }^{422}$ Eine eben solche Beziehung findet sich bei den auf dem

417 Als systematischer Fehler wird ein das System betreffender und somit planbarer Fehler verstanden. Demgegenüber sind unsystematische Fehler zufallsbedingt. Vgl. zur Entstehung von systematischen bzw. unsystematischen Prognosefehlern innerhalb der rationalen Erwartungsbildung Schlieper (1995), S. 34.

418 Vgl. Borchert (1997), S. 254. Im Kap. 4.4 wird das Begriffspaar Kreditmarkt und Kapitalmarkt voneinander abgegrenzt.

$419 \mathrm{Zu}$ der Definition des Begriffs ,empirische Bilanzforschung“ und der Einordnung des kapitalmarktorientierten Ansatzes vgl. auch Coenenberg (2000), S. $1121 \mathrm{f}$.

420 Vgl. Coenenberg (2000), S. 1124ff. Weitere Übersichten finden sich bei Coenenberg (1974); Lev, Ohlsen (1982); Forster (1986); Möller (1983, 1985, 1986); Haller (1989); Beaver (1981); Schmidt (1982); Ballwieser (1982, 1985).

421 Vgl. hier und im Folgenden Coenenberg (2000), S. 1119 und auch S. $1121 \mathrm{ff}$.

422 Vgl. Kap. 2.2. 
Marktorientierte Kreditrisikobewertung -

Kreditmark $t^{423}$, als Teilmarkt des Kapitalmarktes, gehandelten Anleihen wieder. Das Ergebnis der Bewertung des Ausfallrisikos durch den Kapitalmarkt spiegelt sich in dem gehandelten Kurs, dem Preis der Anleihe, wider. ${ }^{424}$ Dieser Kurs kann in eine Rendite (die so genannte Anleihenrendite) überführt werden ${ }^{425}$, die als Kreditzins gedeutet werden kann. ${ }^{426}$ Aus dem Blickwinkel des Gläubigers ist es besonders interessant, die Einflussfaktoren des Bewertungsprozesses zu kennen. Aufgrund der o.g. Ergebnisse der kapitalmarktorientierten Bilanzanalyse lässt sich vermuten, dass auch bei Anleihen Bilanzen eine wesentliche Entscheidungsgrundlage zur Bewertung des enthaltenen Bonitätsrisikos bilden. Folglich gilt es, einen Zusammenhang zwischen dem Preis einer Anleihe und einzelnen Bilanzkennzahlen bzw. Kennzahlensystemen als potenzielle Einflussfaktoren zu untersuchen, um die für die Kreditzinskalkulation relevanten Einflussfaktoren und deren Stellung zum Kreditzins ableiten zu können. Die Fragestellungen der Untersuchung lauten:

(1) Inwieweit benutzen Kreditgeber auf organisierten Kreditmärkten Bilanzen und die Bilanzanalyse zur Dispositionsentscheidung?

(2) Welche Kennzahlen bzw. Kennzahlensysteme werden innerhalb des Entscheidungsprozesses herangezogen?

(3) Lässt sich der im Anleihenkurs enthaltene Kreditzins in Abhängigkeit von den Bilanzkennzahlen und wenn ja mit welcher Genauigkeit quantifizieren?

Die dargestellte Aufgabe der Entwicklung eines marktorientierten Kreditzinskalkulationsverfahrens wird in den folgenden Kapiteln durch die Ableitung eines Prognosemodells aufgegriffen. Hierzu wird zunächst im Kap. 4.2 die Effizienzhypothese als grundlegende Annahme beschrieben. Anschließend wird in Kap. 4.3 das Untersuchungsobjekt „Anleihe“ insbesondere hinsichtlich der enthaltenen Risikoarten charakterisiert. In diesem Zusammenhang werden ebenfalls Verfahren zur Quantifizierung des in der Anleihenrendite enthaltenen Ausfallrisikos beschrieben. Auf Basis dieser Grundlagen wird in Kap. 4.4 das Prognosemodell abgeleitet und abschließend in Kap. 4.5 mit den dargestellten Prinzipien der Bilanzanalyse und den Kriterien zur Eignung als Kreditzinskalkulationsverfahrens gewürdigt.

\footnotetext{
$423 \mathrm{Vgl}$. zur Definition Kap. 4.4.

424 Zur Bewertung von Anleihen vgl. 4.3.

425 Vgl. zur Renditeberechnung bspw. Frühwirth (1997), S. 60ff. und Kap. 4.3.3.

426 Zur genauerer Interpretation der Rendite vgl. Kap. 4.3.2 und Kap. 4.3.3.
} 
Marktorientierte Kreditrisikobewertung Herleitung eines marktorientierten Ansatzes zur Bewertung des Kreditrisikos

\subsection{Die Effizienzhypothese als grundlegende Annahme für die marktorientierte Kreditzinskalkulation}

Die in Kap. 4.1 gefolgerte Beziehung, dass ein objektiver Kreditzins sich aus einem marktlichen Maßstab ableitet, unterstellt die Richtigkeit dieses Maßstabes. Dies bedeutet, dass die für die Kreditzinsbestimmung notwendigen Informationen durch den Markt bzw. durch die Marktteilnehmer ,richtig“ ausgewertet werden. Diese Annahme spiegelt sich in der Effizienz von Kapitalmärkten wider. ${ }^{427}$ Die Effizienztheorie geht der Frage nach, welche Informationen im am Markt realisierten Preis enthalten sind. Folgende Bedingungen sind nach FAMA für einen effizienten Markt konstitutiv: ${ }^{428}$

- Sämtliche Informationen sind für jeden Marktteilnehmer ohne Beanspruchung von

Ressourcen zugänglich,

- es kommt zu keinerlei Transaktionskosten,

- alle Marktteilnehmer sind Preisnehmer und

- alle Marktteilnehmer handeln rational.

Auf einem effizienten Markt muss zu jedem Zeitpunkt die verarbeitete Menge an Informationen gleich der Menge aller verfügbaren Informationen sein. ${ }^{429}$ Gleichzeitig muss die subjektive Erwartung eines Marktteilnehmers, basierend auf seinen Informationen, gleich der „wahren“ Erwartung, aufbauend auf allen Informationen, sein. Eine Abweichung zwischen der erwarteten und der tatsächlichen Marktentwicklung beruht auf einem unerwarteten Ereignis (Schock).

Die Effizienz von Märkten kann jeweils in Abhängigkeit von der Menge der verarbeiteten Informationen in die drei Klassen eingeteilt werden:

- schwache (,weak-form efficiency“),

- mittelstrenge (,, semistrong-form efficiency“) und

- strenge Effizienzform (,strong-form efficiency“).

Die schwache Form besagt, dass lediglich Informationen über die vergangene Kursentwicklung im aktuellen Kurs enthalten sind. ${ }^{430}$

Neben der Gültigkeit der schwachen Effizienz gilt für die mittelstrenge Form zusätzlich, dass alle preisrelevanten öffentlichen Informationen unverzüglich im Preis be-

427 Vgl. Coenenberg (2000), S. 1124.

428 Vgl. Fama, Miller (1972), S. 335 sowie Fama (1970), S. 387.

429 Vgl. hier und im Folgenden Granziol (1981), S. 7.

430 Vgl. Sawazki (1996), S. 99. 
Marktorientierte Kreditrisikobewertung -

rücksichtigt werden. ${ }^{431}$ Die amerikanische Literatur bezeichnet z.B. eine Information, die im Wall Street Journal veröffentlicht ist, als ,publicly available information““432 Nach der hier vertretenen Meinung sollte man über eine Publikation in den Printmedien hinausgehen und die Information mit der Bekanntgabe durch einen Informationsdienst, wie z.B. dem Informationsdienst der REUTERS $\mathrm{GMBH}^{433}$, als öffentlich bezeichnen. ${ }^{434}$

Die strenge Form verlangt, dass alle preisrelevanten Informationen, d.h. auch nicht öffentlich zugängliche Informationen wie Insiderinformationen, im Kurs enthalten sind ${ }^{435}$ Im Rahmen dieser Stufe können selbst besser informierte Marktteilnehmer ihre Position nicht gewinnbringend ausnutzen, da der Preis bereits ihren Informationsvorsprung beinhaltet.

Über die Effizienztheorie ist in der Literatur häufig diskutiert worden. ${ }^{436}$ Die bekanntesten Kritikpunkte sind das Informationsparadoxon, die Nichtbeachtung der Informationskosten und die Vernachlässigung der Verarbeitungszeit.

Bei Anwendung der Definition der "strong-form efficiency“ kommt es zu dem so genannten Informationsparadoxon. Unter der Annahme, der Marktpreis drücke alle Informationen aus, stellt sich die Frage nach der Motivation der Marktteilnehmer, sich neue Informationen zu beschaffen, da sie dadurch keinen zusätzlichen Gewinn erzielen können. Ein weiterer Kritikpunkt ist die Vernachlässigung der Informationskosten. ${ }^{47}$ In der Definition effizienter Märkte nach FAMA wird die Prämisse gesetzt, es entstehen keine Informationskosten, eine nach Meinung des Verfassers sehr restriktive und realitätsfremde Einschränkung. Das Informationsparadoxon gewinnt unter der Beachtung von Informationsbeschaffungskosten noch an Bedeutung. Der Marktpreis stellt nämlich durch seine Veröffentlichung ein kostengünstiges Informationsgut dar. ${ }^{438}$ Falls dieser bereits alle relevanten Informationen enthält, sind Marktteilnehmer nicht motiviert, neue Informationen zu sammeln und zu verarbeiten. Es ist von daher unerlässlich, dass es mindestens zu einer zwischenzeitlichen Ineffizienz des Marktes kommt,

431 Vgl. Heri (1982), S. 129.

432 Vgl. Uhlir (1979), S. 42.

433 Die Reuters Holdings PLC ist ein international ausgerichteter Informationsdienst, der sich auf die Übermittlung von Finanzmarktdaten spezialisiert hat, vgl. http://www.reuters.com.

434 Die Frage nach dem Zeitpunkt der Bekanntgabe ist sehr schwer zu beantworten. Vgl. Möller (1985), S. 513. Die Publikation stellt den spätesten Zeitpunkt dar.

${ }_{435}$ Vgl. hier und im Folgenden Coenenberg (2000), S. $1124 \mathrm{ff}$.

436 Vgl. hier und im Folgenden Heinke (1998), S. 139.

${ }^{437}$ Der Begriff Informationskosten wird teilweise implizit mit dem Begriff Transaktionskosten gleichgesetzt. Vgl. bspw. die originäre Definition bei Fama (1970), S. 387ff.

438

Vgl. hier und im Folgenden Sawazki (1996), S. 100. 
Marktorientierte Kreditrisikobewertung Herleitung eines marktorientierten Ansatzes zur Bewertung des Kreditrisikos

indem zunächst alle relevanten Informationen gesammelt und aufbereitet werden, bevor sie sich in dem Preis niederschlagen können. Somit wird sich langfristig der effiziente Preis wieder einstellen. In der Theorie der effizienten Märkte jedoch besteht die Prämisse, dass Informationen ohne Verarbeitungszeit in neue Preise transformiert werden. Diese theoretische Voraussetzung kann so nicht in der Realität bestătigt werden. ${ }^{439}$

Diese Kritikpunkte sollen nicht den Eindruck erwecken, man könne eine Effizienz an den Börsen grundsätzlich verneinen. Es sind zur Effizienzfrage an den Kredit- und Kapitalmärkten weltweit zahlreiche Tests und Studien betrieben worden, wobei ein Schwerpunkt auf den amerikanischen Kapitalmärkten liegt. ${ }^{440}$ Im Ergebnis lässt sich Folgendes festhalten: ${ }^{441}$ Die Gültigkeit der starken Form wird i.a. abgelehnt, die schwache Form in den meisten Fällen akzeptiert, es herrscht Uneinigkeit bezüglich der mittelstrengen Form. Die Ablehnung der starken Form kann bereits aufgrund des oben erläuterten Informationsparadoxons begründet werden. ${ }^{442}$ Der Nachweis der schwachen Form der Effizienz wird anhand von Kursreihenanalysen geführt. Gemäß der o.g. Definition dürfen Informationen aus der historischen Kursentwicklung nicht auf den weiteren Verlauf schließen lassen (keine Trendbildungen). Untersuchungen in den 60 er- und 70er-Jahren belegen, dass es keine Trends gibt. ${ }^{443}$ Insgesamt spricht für die schwache Informationseffizienz, dass auf Basis von Trenduntersuchungen keine dauerhaft erfolgreichen Strategien entwickelt werden konnten. ${ }^{444}$ Die Uneinigkeit hinsichtlich der Gültigkeit der mittelstrengen Effizienzform lässt sich auf die Schwierigkeiten bei der Überprüfung dieser Hypothese zurückführen. ${ }^{45}$ Die Beobachtung, dass professionelle Anleger (wie Investmentfonds) im Durchschnitt keine Überperformance erzielen konnten, belegt die Gültigkeit der mittelstrengen Effizienzform. ${ }^{46}$

Die Gültigkeit der mittelstrengen Effizienzform wird als Grundannahme für die folgende Untersuchung unterstellt. Dies bedeutet, dass alle Marktteilnehmer die Bilanz zur Entscheidungsfindung nutzen, falls sie den Informationsgehalt als sinnvoll erachten. Der am Markt realisierte Preis für eine Anleihe reflektiert alle verfügbaren und

439

Vgl. Schäfer (1994), S. 18f.

Vgl. Steiner, Bruns (1998), S. 44; vgl. zu Untersuchungen der Informationseffizienz des deutschen Aktienmarktes Möller (1985), S. 500ff. Vgl. Sawazki (1996), S. 119f.

„Die größte Einigkeit besteht dabei in der Feststellung, dass strenge Informationseffizienz auf Wertpapiermärkten nicht vorliegt", Steiner, Bruns (1998), S. 44.

Vgl. Fama (1970), S. 383ff.; Steiner, Bruns (1998), S. 45ff. Eine gegensätzliche Aussage findet sich bei Loistl (1990), S. 67ff.

Vgl. Steiner, Bruns (1998), S. 48.

Vgl. Steiner, Bruns (1998), S. 46.

Vgl. Malkiel (1990), S. 169ff.; Sharpe (1992), S. 18; Lerbinger (1984), S. $60 \mathrm{ff}$. 
Marktorientierte Kreditrisikobewertung -

relevanten Informationen über das Unternehmen. Die resultierende Preisfindung genügt dem Anspruch eines objektiven Maßstabes, da der Preis von der „wahren“ bzw. „richtigen“ Erwartung abgeleitet wird. ${ }^{447}$

\subsection{Bewertung von risikobehafteten, festverzinslichen Anleihen}

Anleihen sind Schuldverschreibungen, mit denen sich der Emittent i.d.R. langfristiges Kapital beschafft ${ }^{48}$ Die Emission dieser Papiere erfolgt über den anonymen, annahmegemäß effizienten Kapitalmarkt. ${ }^{49}$ Ein wesentlicher Unterschied zwischen zwei Anleihen unterschiedlicher Schuldner ist das mit der Anleihe verbundene Risiko der Nichtrückzahlung des aufgenommenen Kapitals aufgrund bspw. der Insolvenz des Emittenten. ${ }^{450}$ Dieses Risiko entspricht dem unter Kap. 2.2 dargestellten Ausfallrisikos eines Kredites. Das mit einer Anleihe verbundene Ausfallrisiko wird durch die Bonität des Schuldners bedingt und kommt im Marktpreis der Anleihe zum Ausdruck. Um dieses Risiko messen zu können, ist es wichtig, zunächst die Charakteristika der Anleihe und die mit dieser verbundenen Risikoarten zu beschreiben (Kap. 4.3.1 und Kap. 4.3.2). Aufbauend hierauf werden verschiedene Ansätze zur Messung des Ausfallrisikos (Kap. 4.3.3) vorgestellt.

\subsubsection{Eigenschaften von Anleihen}

Das im Folgenden untersuchte Objekt ist die festverzinsliche Anleihe. ${ }^{41}$ Die vorliegende Arbeit unterscheidet dabei zunächst nach dem Kriterium feste vs. variable Zinszahlung. ${ }^{42}$ Der überwiegende Teil der international emittierten Anleihen findet sich in der erstgenannten Kategorie wieder. Die Zinszahlung erfolgt nach vereinbarten Perioden (bspw. in den USA in einem halbjährlichen Rhythmus). Der Nominalzins wird dabei auf das Nominalvolumen der Anleihe gezahlt. Zerobonds oder Nullkuponanleihen besitzen einen Nominalzins von Null. Dementsprechend erfährt der Käufer seine Rendite lediglich über Kurssteigerungen zwischen dem Kauf- und dem Verkaufskurs. Falls der Schuldner (Emittent) der Anleihe im Land des Käufers seinen Sitz hat, handelt es sich um eine so genannte Inlandsanleihe (vs. Auslandsanleihe). Unter Umständen

447 Neben dieser „Grundannahme“ werden für das Prognosemodell weitere Prämissen (statistische Annahmen) getroffen, die jedoch in Abhängigkeit von der gewählten Untersuchungsmethode voneinander abweichen. Vgl. Kap. 5.1.1 und Kap. 5.2.3.

448 Vgl. hier und im Folgenden bspw. Grill, Perczynski (1995), S. 366ff. und S. $373 \mathrm{ff}$.

449 Vgl. auch Kap. 6.1.2.

450 Vgl. Kap. 4.3.2.1.

451 Vgl. hier und im Folgenden Steiner, Bruns (1998), S. 133. Die hier aufgezeigte Einordnung stellt keine abschließende Auflistung aller Merkmale dar, sondern benennt zielführend die in der weiteren Untersuchung relevanten Merkmale.

452 Variabel verzinsliche Wertpapiere (Floating Rate Notes) werden aus der Untersuchung ausgeklammert. 
werden die Zahlungsströme bei einer Anleihe (Zins- oder Tilgungszahlungen) in unterschiedlichen Währungen vereinbart, in diesen Fällen spricht man von Doppelwährungsanleihen. Darüber hinaus kann es sich um einen privaten (Industrieanleihen) oder öffentlichen Schuldner (Staatsanleihen) handeln. In der Regel wird eine Anleihe vom Emittenten zu einem fest vereinbarten Termin eingelöst. Falls dieser Termin vom Emittenten aufgehoben und die Anleihe frühzeitiger zurückgezahlt werden kann (Tilgungsrechte), spricht man von einer kündbaren Anleihe.

Im Rahmen der weiteren Untersuchung werden lediglich Anleihen, die an organisierten Finanzmärkten gehandelt werden, analysiert. Diese Papiere werden somit auf einem Markt bewertet. Als Ansatz kann hierzu ein Barwertmodell verwendet werden. ${ }^{453}$ Ziel dieses Modells ist es, zukünftige Zahlungsströme auf den heutigen Wert zu ,transformieren". Die generelle Schwierigkeit des Modells liegt in der Wahl des Diskontierungszinses zur Bewertung zukünftiger, risikobehafteter Zahlungsströme. Aus diesem Grund werden im Folgenden die unterschiedlichen Risikoarten von festverzinslichen Wertpapieren erläutert, die diesen Bewertungsvorgang beeinflussen. Hierbei wird eine festverzinsliche, mit Tilgungsrechten ausgestattete ausländische Industrieanleihe unterstellt.

\subsubsection{Risikoarten von Anleihen}

Als Risiko (Chance) wird - analog zu der allgemeinen Definition im Kapitel 2.1 - die Möglichkeit verstanden, dass ein zukünftiger Umweltzustand von einem erwarteten Zustand negativ (positiv) abweicht und die getroffene Entscheidung nicht (ohne Kostenbelastung) verändert werden kann. ${ }^{454}$ Die Gründe für die potenzielle Abweichung definieren die verschiedenen Risikoarten. Die im Folgenden aufgezählten Risiken (Bonitäts-, Zinsänderungs-, Tilgungs-, Währungs-, und Liquiditätsrisiko) werden als die wichtigsten Risiken im Bereich der festverzinslichen Wertpapiere genannt. ${ }^{455}$ Alle Risiken beziehen sich auf die Sichtweise des Gläubigers, der die Bewertung des Risikos in die Bewertung der Anleihe einfließen lässt.

\subsubsection{Bonitätsrisiko}

In Anlehnung an Kap. 2.2 wird im Weiteren der Begriff Bonitätsrisiko ursachenbezogen als Bestandteil eines Wertpapierrisikos beschrieben. Der Kreditgeber (Anleihekäufer) ist gewillt, dem Kreditnehmer (Emittent der Anleihe) finanzielle Mittel zu überlassen (klassische Gläubiger-Schuldner-Beziehung). Sie vereinbaren vertraglich Zinsund Rückzahlung. Als Bonität wird die Fähigkeit und Eigenschaft der termingerechten

433 Vgl. bspw. Bühler, Uhrig-Homburg (2000), S. 299.

454 Vgl. Adam (1996), S. 194f.; Perridon, Steiner (1997), S. 98.

455 Vgl. Zapotocky (2000), S. 1084ff.; Holzer (1990), S. 11. 
Marktorientierte Kreditrisikobewertung -

Zins- und Rückzahlung verstanden. ${ }^{456}$ Die Gründe für die Unfähigkeit zur vereinbarten Zins- und Rückzahlung liegen: ${ }^{47}$

- im technologischen Risiko des Schuldners,

- im Informationsrisiko des Gläubigers und

- im Moral-Hazard-Risiko des Kapitalgebers.

Die Einzahlungsüberschüsse, die aus den kreditfinanzierten Investitionen generiert werden, unterliegen konjunkturell oder strukturell begründeten Schwankungen. Die hieraus resultierende Gefahr der Nicht-Rückzahlung ist das technologische Risiko, das vom Gläubiger zu tragen ist. I.d.R. ist dieser im Vergleich zum Schuldner in der ungünstigeren Position zur Einschätzung dieses technologischen Risikos. Falls der Schaden des Gläubigers aus einer schlechten Informationsversorgung heraus resultiert, handelt es sich um das Informationsrisiko. Falls aufgrund der Gläubiger-SchuldnerBeziehung Anreize für den Schuldner entstehen, den Schaden aus einer negativen Abweichung zwischen den geplanten und den tatsächlichen Umweltzuständen in Richtung Gläubiger zu verschieben, handelt es sich um das Moral-Hazard-Risiko.

Beschreibend für die Bonität ist ihr qualitativer, unstrukturierbarer und nur begrenzt objektivierbarer Charakter. Hierdurch wird die Bedeutung eines zweckmäßigen Messkonzeptes zur Beschreibung des hieraus erwachsenden Risikos deutlich. ${ }^{458}$

Die vorliegende Arbeit analysiert das Bonitätsrisiko von festverzinslichen Wertpapieren. Hier besteht das Risiko darin, dass der Emittent die Zinsen und Tilgungen nicht zu dem vereinbarten Zeitpunkt und / oder in der vereinbarten Höhe leistet. Als Bonitätsänderungsrisiko wird die negative Kursentwicklung eines Wertpapiers aufgrund der Änderung der Bonitätseinschätzung des Emittenten verstanden. ${ }^{459}$ Falls die Bonität des Emittenten herabgestuft wird, steigt die Wahrscheinlichkeit, dass es aufgrund der gesunkenen Zahlungsfähigkeit zu Störungen bei den erwarteten Zahlungsströmen (Zinsund Tilgungszahlungen) kommt. ${ }^{460}$ Folglich sinkt der Marktwert der Anleihe.

\subsubsection{Zinsänderungsrisiko}

Allgemein wird das Zinsänderungsrisiko als Gefahr verstanden, die darin besteht, dass sich aufgrund einer Veränderung des Marktzinses eine negative Abweichung einer realisierten von einer erwarteten Ergebnisgröße (Wert der Anleihe) ergibt. Das Zins-

Haufig wird die Unfahigkeit der Rückzahlung mit der Insolvenz in Verbindung gebracht. Vgl. zum Begriff „Insolvenz“ Steiner (1980), S. 4f.; vgl. zu einer Integration der Insolvenz in ein Messkonzept des Bonitätsrisikos Heinke (1998), S. 9f.

457 Vgl. hier und im Folgenden Schmidt (1981), S. 205f. und auch Heinke (1998), S. 7f.

458 Vgl. Schulte (1996), S. 89f.

459 Vgl. Kap. 2.2.

460 Vgl. hier und im Folgenden Schulte (1996), S. $131 \mathrm{ff}$. 
Marktorientierte Kreditrisikobewertung Herleitung eines marktorientierten Ansatzes zur Bewertung des Kreditrisikos

änderungsrisiko für Anleihen resultiert aus nicht antizipierten Schwankungen des allgemeinen Zinsniveaus ${ }^{461}$ Falls das allgemeine Zinsniveau steigt (fällt), fällt (steigt) der Kurs der Anleihe. Eine enge Begriffsdefinition stellt auf das Endwertänderungsrisiko ab und beschreibt die Gefahr, „daß ein bei bestehendem Zinsniveau zu erwartender Endwert einer Anleihe im Tilgungszeitpunkt nicht erzielt wird“4662. Der Grund dafür liegt in der möglichen Wiederanlage der Zinserträge. Somit steigt der Endwert (als Summe aus der originären Kapitalanlage, den Zinsen und den Zinseszinsen), falls sich das Zinsniveau nach oben verschiebt. Gleichzeitig verringert sich der aktuelle Marktwert der Anleihe, sodass man von dem Zinsänderungsrisiko im weiteren Sinne oder auch Vermögensänderungsrisiko spricht. Diese gegenläufigen Effekte aufgrund einer Zinsniveauänderung finden in der Kennzahl „Duration“ nach MACAULAY ${ }^{463}$ ihren Niederschlag, indem der Zeitpunkt berechnet wird, zu welchem sich die beiden Effekte gegenseitig neutralisieren (durchschnittliche Kapitalbindungsdauer). ${ }^{464}$

\subsubsection{Tilgungsrisiko}

Wie im Kapitel 4.3.1 erklärt, besitzen verschiedene Anleihen Kündigungs- oder Tilgungsrechte. Diese Option als vertraglich gesichertes Recht ermächtigt den Emittenten vorzeitig (vor dem vereinbarten Fälligkeitstermin), die komplette Anleihe oder einen Teil zu tilgen. ${ }^{465}$ Die Nachteile für den Gläubiger / Anleger liegen in den nicht sicheren Zahlungsströmen, bedingt durch die Möglichkeit der Ausübung dieses Rechtes durch den Schuldner. Im Allgemeinen wird der Emittent die Anleihe genau dann kündigen, wenn der relevante Opportunitätszins zur Refinanzierung gesunken ist. In diesem Augenblick ist der Marktwert größer als der Nominalwert. Folglich erleidet der Investor einen Verlust, und er muss nun sein Kapital zu ungünstigeren Bedingungen anlegen.

\subsubsection{Liquiditätsrisiko}

Das Liquiditätsrisiko besteht darin, dass eine Anleihe aufgrund eines zu geringen Angebotes bzw. einer zu geringen Nachfrage nicht jederzeit am Markt gehandelt wer-

461 Vgl. hier und im Folgenden Fabozzi (1996), S. 5f. Neben dem reinen Zinsniveau als Durchschnittswert von Anleihen mit verschiedenen Restlaufzeiten, besitzt die Steigung der Zinsstrukturkurve ebenfalls einen analogen Einfluss auf den Anleihenwert.

462 Steiner, Bruns (1998), S. 154.

463 Vgl. Macaulay (1938), S. 44ff.; Hicks (1939), S. $12 \mathrm{ff}$.

464 Nach der Theorie von Fisher setzt sich das heutige nominale Zinsniveau aus einem „realen Zins" und der antizipierten Inflationsrate zusammen. Somit besteht ein Inflationsrisiko, da sich die Inflationsrate von der erwarteten Inflationsrate unterscheiden kann (Fisher (1911, 1937)). Demzufolge kann sich das Inflationsrisiko auf das Zinsänderungsrisiko auswirken, da sich das nominale Zinsniveau in Abhängigkeit der Inflationsrate verändert.

Vgl. hier und im Folgenden Fabozzi (1996), S. 324f. und auch S. 6. 
den kann. ${ }^{466}$ Die Folge ist, dass dieser Titel „nicht oder zu keinem vernünftiger Preis ${ }^{\text {‘467 }}$ gehandelt wird. Als vernünftiger Preis kann der faire Preis eines effizienter Kapitalmarktes definiert werden. Als fairer Kurs wird der Wert bezeichnet, bei der keine Arbitragegeschäfte möglich sind. Bei diesem Geschäft würde ein Gewinn durct den Kauf und Verkauf von Wertpapieren zu unterschiedlichen Kursen erzielt. Ent. scheidend dafür ist, dass es ohne Risiko und Liquiditätseinsatz erfolgt. ${ }^{468}$ Eir Arbitragehandel ist somit die risikolose Ausnutzung von Differenzen in der Preisnotizen für gleiche Werte. ${ }^{469}$ Der faire Kurs resultiert aus einem unterstellter Bewertungsmodell (bspw. dem Barwertmodell) und bevorzugt weder Käufer noch Verkäufer. ${ }^{470}$ In der Realität existieren jedoch unterschiedliche Kurse für Kauf- odes Verkaufsgeschäfte. ${ }^{471}$ Ein Makler beispielsweise kauft ein Papier zu einem niedrigerer Kurs als er es verkauft. Die Differenz aus Kauf- und Verkaufskurs drückt die Gewinnspanne des Händlers aus und stellt bei einem zeitgleichen Kauf- und Verkauf einen Arbitragegewinn dar. Je größer die Spanne zwischen Kauf- und Verkaufskurs ist. umso größer ist das Risiko, das Wertpapier nicht zu einem „vernünftigen Preis“ kaufer bzw. verkaufen zu können. Im Extremfall findet man keinen Käufer bzw. Verkäufer. der bereit ist, dieses Papier zu kaufen bzw. verkaufen.

Darüber hinaus erstreckt sich das Liquiditätsrisiko auf eine Zeitdimension. Während zum einen der Preis vom fairen Kurs abweicht, wird zum anderen auch die Ausübungsdauer unter dem Liquiditätsrisiko / Marktgängigkeitsrisiko subsumiert. ${ }^{472}$ Unter der Ausübungsdauer wird die Zeitspanne zwischen der Kauf- bzw. Verkaufsentscheidung und der Ausübung des Geschäftes, d.h. der Kursfixierung zwischen den beiden Parteien, verstanden. Der Kurs kann sich während dieser Zeitspanne verändern und der Käufer bzw. Verkäufer des Wertpapiers erleidet einen Verlust. ${ }^{473}$ Je größer das gehandelte Volumen eines Wertpapiers ist, umso geringer ist die Ausübungsdauer. Das Marktliquiditätsrisiko entsteht aufgrund einer unzulänglichen Tiefe des Marktes ${ }^{474}$ und kann durch den einzelnen Marktteilnehmer nicht beeinflusst werden. Ein etablier-

Vgl. hier und im Folgenden Fabozzi (1996), S. 7.

Zapotocky (2000), S. 1088.

Vgl. Friedman (1953), S. 175.

Vgl. Zapotocky (2000), S. 1082.

Vgl. auch zu der Bestimmung des fairen Preises Bühler, Uhrig-Homburg (2000), S. 304f.

Bspw. wird im Devisenhandel zwischen dem Geld- und dem Briefkurs unterschieden.

Vgl. Grill, Perczynski (1995), S. 571. Ebenfalls stellen Market Maker für die von ihnen betreuten Wertpapiere fortlaufend Kauf- und Verkaufkurse, die leicht voneinander abweichen. Vgl. Zapotocky (2000), S. 1081.

Vgl. Fisher (1959), S. $217 \mathrm{ff}$.

Unterstellt wird hierbei wiederum die Inflexibilität der Entscheidung. Vgl. Perrdion, Steiner (1997), S. 98.

Als Markttiefe wird das gehandelte Volumen eines Wertpapiers verstanden. 
Marktorientierte Kreditrisikobewertung Herleitung eines marktorientierten Ansatzes zur Bewertung des Kreditrisikos

ter und liquider Sekundärmarkt $t^{45}$ lässt das Marktliquiditätsrisiko sinken, da die Ausübungsdauer geringer ist und die Differenzen zwischen dem gehandelten und dem fairen Kurs abnehmen.

\subsubsection{Währungsrisiken}

Ein Währungsrisiko benötigt das Zusammenspiel zweier Währungen, weil ansonsten Wechselkursschwankungen für die Bewertung der Anleihe irrelevant wären. Voraussetzung ist, dass die Zahlungsströme in einer für den Betrachtungsstandpunkt ,fremden Währung" vereinbart sind. HÖLSCHER bezeichnet als Währungsrisiko die Gefahr, dass das erzielte Ergebnis das erwartete unterschreitet, wobei das zugrunde liegende Geschäft den Übergang von einer in eine andere Währung bedingt. ${ }^{476}$ Die Ursache des Risikos liegt in der Ungewissheit, in welchem Ausmaß und in welche Richtung sich das Austauschverhältnis (Wechselkurs) zwischen der inländischen und der ausländischen Währung entwickelt. Die Zinszahlungen und / oder die Tilgungen sind (bedingt durch den unterstellten Betrachtungsstandpunkt) an die Entwicklung eines Wechselkursverhältnisses gekoppelt. Ein Verlust entsteht in dem Augenblick, wenn die heimische Währung aufgewertet (abgewertet) wird und man einen Zahlungsstrom in der Fremdwährung erwartet (zu leisten hat). ${ }^{477}$ Bei Doppelwährungsanleihen tritt folglich regelmäßig ein Währungsrisiko ein.

\subsubsection{Messung des Bonitätsrisikos}

In den vorangegangenen Kapiteln wurden ursachebezogen die verschiedenen Risikoarten einer Anleihe beschrieben. Die Bewertung der Risiken schlägt sich in dem am Markt erzielten Kurs einer Anleihe wieder. Falls bspw. das Bonitätsrisiko steigt, wird der aktuelle Kurs der Anleihe sinken. ${ }^{478}$ Dieser Kurs der Anleihe (ausgedrückt in Geldeinheiten) lässt sich in eine Rendite (ausgedrückt in Prozent) überführen. Hierzu ist zunächst eine Grundannahme bezüglich der Haltedauer, d.h. dem Verbleib der Anleihe im Besitz des Käufers, zu treffen. Falls davon ausgegangen wird, dass die Anleihe bis zur Fälligkeit gehalten wird, kann die entsprechende Fälligkeitsrendite (yield to maturity) berechnet werden.$^{49}$ Falls unterstellt wird, dass die Anleihe nur für eine bestimmte Zeit (kürzer als die Restlaufzeit) bei dem Anleger verbleibt, kann die Halterendite

475 „Am Sekundärmarkt werden die bereits emittierten Wertpapiere zwischen den Marktteilnehmern gehandelt", Steiner, Bruns (1998), S. 2.

476 Vgl. Hölscher (1987).

477 Vgl. Steiner, Bruns (1998), S. 134.

478 Vgl. hierzu die in Kap. 2.3 dargestellten modelltheoretischen Verfahren der Bonitätsprämienermittlung.

479 Vgl. Bühler, Uhrig-Homburg (2000), S. 298f. 
Marktorientierte Kreditrisikobewertung -

(holding period return) berechnet werden. ${ }^{480}$ Die erstgenannte Haltedauerhypothese ist die gebräuchlichere und wird auch in den folgenden Ausführungen zugrunde gelegt. ${ }^{481}$ Grundlegend für die Berechnung einer Rendite ist die Annahme sicherer Zahlungsströme, d.h. die unsicheren zukünftigen Zahlungen werden über geeignete Annahmen (z.B. eine unterstellte Wahrscheinlichkeit), wie sichere Zahlungen betrachtet. ${ }^{482}$ Die Rendite einer Anleihe ist genau der Zinssatz, zu dem der Barwert der zukünftigen Zahlungen gleich dem heutigen Kurs ist. Die Rendite bedingt die Kenntnis des heutigen Marktwertes und fungiert als Diskontierungssatz im Rahmen der Barwertbetrachtung der zukünftigen Zahlungen. Dieser Vorgehensweise entspricht der Berechnung des internen Zinsfusses bzw. der Effektivverzinsung. ${ }^{483}$

Die Gesamtrendite einer Anleihe kann als Summe aus dem risikofreien Tageszins und den einzelnen bewerteten Risikoarten verstanden werden (vgl. Abbildung 23). Die reine Summierung unterstellt eine (realitätsfremde) Unabhängigkeit der einzelnen Risiken. ${ }^{484}$

Gemäß der im Kapitel 4.1 beschriebenen Zielsetzung muss für die vorliegende Untersuchung das Bonitätsrisiko bewertet und innerhalb eines Kreditzinses quantifiziert werden. Hierzu eignen sich zwei Vorgehensweisen. Zum einen besteht die Möglichkeit, das Bonitätsrisiko isoliert zu bewerten. Dies ist durch so genannte Kreditderivate möglich (Kapitel 4.3.3.1). Dabei wird das in der Anleihenrendite enthaltene Risiko separat in einem zweiten Kapitalmarktgeschäft betrachtet. Zum anderen kann das bewertete Bonitätsrisiko aus der Anleihenrendite isoliert werden. Dies bedeutet, man gliedert den beobachteten Preis eines Wertpapiers in Einzelprämien für die unterschiedlichen Risikoarten (Kapitel 4.3.3.2).

Vgl. Bühler, Uhrig-Homburg (2000), S. 298; Heinke (1998), S. 380f.

482 Vgl. hier und im Folgenden Bühler, Uhrig-Homburg (2000), S. $298 \mathrm{ff}$.

483 Vgl. Perridon, Steiner, (1997), S. 185.

484 Bspw. werden im weiteren Verlauf der Analyse empirisch belegte Abhängigkeiten von dem Bonitäts- und dem Zinsänderungsrisiko erläutert. 
Marktorientierte Kreditrisikobewertung Herleitung eines marktorientierten Ansatzes zur Bewertung des Kreditrisikos

\section{Gesamtrendite}

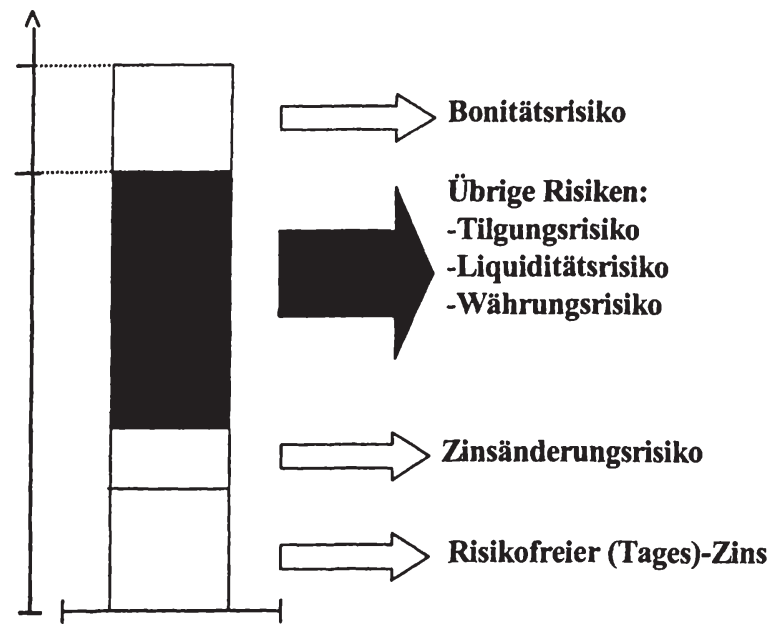

Abbildung 23: Risikoarten einer Anleihe ${ }^{485}$

\subsubsection{Kreditderivate}

Derivate $^{486}$ sind Rechte, deren Werte (Börsen- oder Marktpreise) sich unmittelbar oder mittelbar aus einem zugrunde liegenden Basiswert ableiten lassen. ${ }^{487}$ Als Basiswerte kommen Aktien, festverzinsliche Wertpapiere, (zukünftige) Zinssätze, Devisenkurse oder auch ein zweites Derivat oder dessen Preis in Frage. ${ }^{488}$ Ein Derivat ist ein Termingeschäft, deren Erfüllung erst nach Vertragsabschluss anfällt. ${ }^{489}$ Sie haben hierbei die Eigenschaft, dass sie die Risikokomponenten des Basiswertes aufspalten, bewerten, neu bündeln und handelbar machen können. ${ }^{490}$

In Anlehnung an Heinke (1998), S. 78.

Das lateinische Wort „derivare“ bedeutet „ableiten“.

Vgl. Eller (1996), S. 8; Grill, Perczynski (1995), S. 400. Diese Definition lehnt sich an die Begriffsbestimmung im Wertpapierhandelsgesetz an.

Vgl. Hartmann-Wendels, Pfingsten, Weber (2000), S. 296.

Vgl. Maltzan (2000), S. 833f.

Vgl. hier und im Folgenden Hattori (1996), S. 14ff. 
Als Kreditderivat versteht man eine individuelle Vereinbarung zwischen zwei Parteien, deren Erfüllung an eine Messgröße geknüpft wird, welche die Bonität des Basiswertes quantifiziert. Ein Basiswert kann bspw. ein Bankkredit sein. Falls die MessgröBe einen vereinbarten Wert erreicht, findet eine Prämienzahlung zwischen den beiden Parteien statt. Als auslösendes Ereignis für diese Prämienzahlung ist die Verschlechterung der Kreditqualität des Basiswertes definiert. Ein Referenzwert wird dann eingesetzt, wenn ein Basiswert nicht vorhanden ist bzw. das Risiko für den Basiswert nicht objektiv bestimmbar ist. Voraussetzung ist, dass der Basiswert und der Referenzwert hinsichtlich des Kreditrisikos signifikant stark korrelieren. Ein Referenzwert kann beispielsweise eine Unternehmensanleihe sein. Als Messgröße muss ein Kreditereignis bestimmt werden, welches als objektiver Maßstab der Bonitätsverschlechterung verwendet werden kann. Voraussetzung für die Objektivität ist die öffentliche Nachprüfbarkeit.

Hierzu eignen sich zum ersten als direkt beobachtbare Ereignisse bspw.:.491

- Preisverfall des Referenztitels oder

- schlechteres Rating des Referenzinstrumentes.

Als indirekte Kreditereignisse gelten zum zweiten: ${ }^{492}$

- Schlechteres Rating des Schuldners,

- Erfolglose Zwangsvollstreckung,

- Zahlungsunfähigkeit durch Zahlungseinstellung,

- Konkurseröffnung,

- Ablehnung der Konkurseröffnung mangels Masse und

- Abschluss eines gerichtlichen oder außergerichtlichen Vergleichsverfahrens.

Durch die losgelöste Betrachtung des Bonitätsrisikos wird dieses Risiko (vgl. Abbildung 24) isoliert von den anderen Risiken am Markt bewertet, sodass sich Kreditderviate von ihrer Konzeption her bestens eignen, um das Bonitätsrisiko mit einem marktlichen Maßstab zu bewerten. Die aktuelle Situation erlaubt es jedoch nicht, eine Untersuchung mit einer statistisch ausreichenden Stichprobengröße durchzuführen. ${ }^{493}$ Obwohl der Markt für Kreditderviate seit der Entstehung Anfang der 90er-Jahre enorm gewachsen ist, wird es noch einige Jahre dauern, bis eine breite Grundgesamtheit vorhanden ist. ${ }^{44}$ Problematisch erscheint insbesondere die mangelnde Streuweite der

\footnotetext{
491 Vgl. Smithson (1995), S. 38.

492 Vgl. Kirmße (1996), S. 258.

493 Vgl. Sommerfeld (2001), S. 88f.

494 Vgl. Green, Locke, Paul-Choudhury (1998), S. $6 \mathrm{ff}$.
} 
Marktorientierte Kreditrisikobewertung Herleitung eines marktorientierten Ansatzes zur Bewertung des Kreditrisikos

Kreditderivate über die verschiedenen Unternehmen und die fehlende Standardisierung der Derivate, wodurch keine gesicherte Auswertung ermöglicht werden. ${ }^{495}$

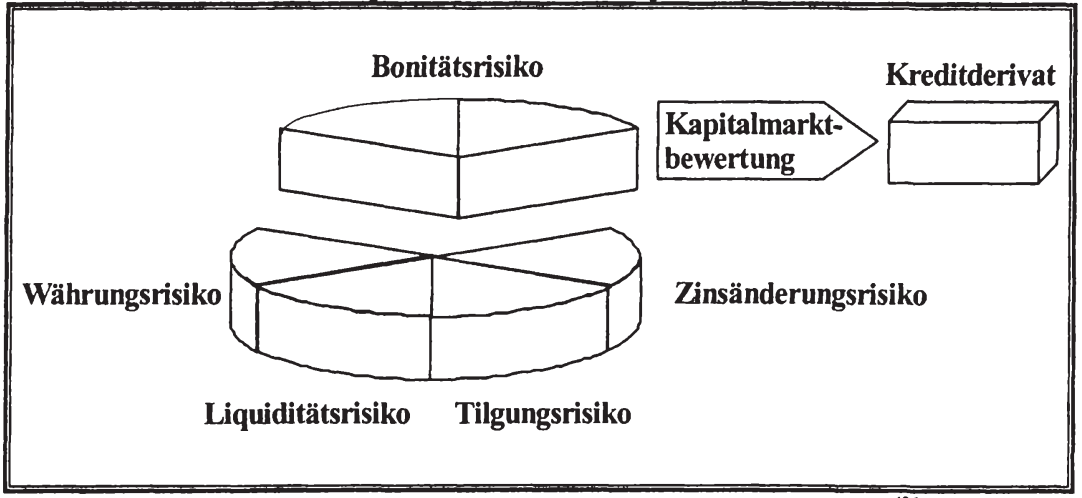

Abbildung 24: Das Bonitătsrisiko als Bewertungskomponenten von Kreditderivaten ${ }^{496}$

\subsubsection{Isolation des Bonitätsrisikos über den Credit Spread}

Während eine statistische Untersuchung der Bewertung des Bonitätsrisikos durch Kreditderivate an der fehlenden Grundgesamtheit scheitert, ist die Möglichkeit einer empirischen Untersuchung des Anleihenmarktes aufgrund der Größe möglich. ${ }^{497}$ Wie bereits in Kap. 4.3.2 dargestellt, lässt sich ein Bestandteil der Gesamtrendite einer Unternehmensanleihe auf das bewertete Bonitätsrisiko zurückführen. Diese Prämie wird als Credit Spread bezeichnet und definiert sich als Renditeunterschied zweier festverzinslicher Wertpapiere mit gleichen Ausstattungsmerkmalen, die jedoch von unterschiedlichen Emittenten begeben wurden. ${ }^{498}$ Entscheidend ist, dass einer dieser Emittenten kein Ausfallrisiko besitzt. Dies bedeutet, der Renditeunterschied lässt sich ausschließlich auf Unterschiede in der Bonität zurückführen.

Im Rahmen der Ermittlung des Credit Spread für eine einzelne Anleihe i teilt man nun die für die Anleihenbewertung am Markt relevanten Einflussfaktoren in drei Kategorien ein. ${ }^{499}$ Mit $\mathrm{M}$ werden die bewertungsrelevanten Marktfaktoren beschrieben, die

Vgl. Arditti, Curran (1998), S. $30 \mathrm{ff}$.

496 Die relative Verteilung der einzelnen Risiken ist abhängig von dem einzelnen Wertpapier. Die in der Abbildung gewählte Verteilung entspricht nicht dem Ergebnis von empirischeren Untersuchungen.

497 Vgl. Bohl, Alexander (2000), S. 468.

498 Vgl. hier und im Folgenden Hartmann-Wendels, Pfingsten, Weber (2000), S. 428; Rolfes (1999), S. 337.

499 Vgl. hier und im Folgenden Heinke (1998), S. $77 \mathrm{ff}$. 
Marktorientierte Kreditrisikobewertung -

auf alle Anleihen gleichzeitig einen Einfluss haben (bspw. Inflationsrate oder Marktzinsniveau). $\mathrm{W}_{\mathrm{i}}$ ist die Menge aller wertpapierspezifischen Einflussfaktoren, wie z.B. die Restlaufzeit oder der Kupon. Diese Ausstattungsmerkmale einer Anleihe i bedingen die in den Kap. 4.3.2.2 bis Kap. 4.3.2.5 dargestellten Risiken. $B_{i}$ wiederum ist die Menge aller das Bonitätsrisiko der Anleihe $i$ bewertenden Faktoren $X_{1}$ bis $X_{n}$. Die Rendite $r$ eines Wertpapiers i kann durch

Gleichung $24^{500}$

$r_{i}=r_{i}\left(M, W_{i}, B_{i}\right)$,

mit $B_{i}=\left(X_{1}, X_{2}, X_{3}, \ldots, X_{n}\right)$,

bestimmt werden.

Der Credit Spread e einer Anleihe i stellt sich dar als Renditedifferenz von grundsätzlich identischen Anleihen $\mathrm{i}$ und $\mathrm{B}$ (somit gilt $\mathrm{W}_{\mathrm{i}}=\mathrm{W}_{\mathrm{B}}$ ), wobei die Anleihe $\mathrm{B}$ (der sogenannte Benchmark) kein Bonitätsrisiko beinhaltet und folglich die Menge $B_{B}$ leer ist:

Gleichung 25

$e_{i}=r_{i}\left(M, W_{i}, B_{i}\right)-r_{B}\left(M, W_{B}, 0\right)$, mit $W_{i}=W_{B}$.

Bei der empirischen Ermittlung von $e_{i}$ stellt die Bestimmung der Rendite $r_{B}$ ein besonderes Problem dar. Zunächst erweist es sich als äußerst schwer, eine Anleihe bzw. einen Anleihentyp ohne Ausfallrisiko zu finden. Vereinfachungsgemäß geht man jedoch davon aus, dass Staatstitel mit der US-Regierung als Emittenten oder von der deutschen Regierung begebene Titel kein Ausfallrisiko implizieren. ${ }^{501}$ Somit muss man nun entweder eine äquivalente Staatsanleihe mit den gleichen Ausstattungsmerkmalen $\left(\mathrm{W}_{\mathrm{i}}=\mathrm{W}_{\mathrm{B}}\right)$ finden, oder man verwendet einen Index aus verschiedenen risikolosen Anleihen.

Die Möglichkeit der Suche einer adäquaten Bundesanleihe ${ }^{502}$ als Benchmark wird durch die Vielzahl der Einflussfaktoren aus der Menge $W_{i}$ erschwert. Während man einen Teil der Einflussfaktoren durch die Auswahl einer entsprechend ausgestatteten Anleihe i ausschließen kann (bspw. keine Tilgungs- und Kündigungsrechte), muss das Zinsänderungsrisiko, welches durch die Restlaufzeit und den Kupon bestimmt wird, explizit durch die Rendite der Bundesanleihe bewertet werden. Dies bedeutet, man muss eine Bundesanleihe mit gleicher Restlaufzeit und gleichem Kupon wie die Anleihe $\mathrm{i}$ finden. In der Regel ist es nicht immer möglich, eine passende Anleihe (Lauf-

Vgl. hier und im Folgenden Lamy, Thompson (1988), S. 587.

Vgl. bspw. Perry, Liu, Evans (1988); Liu, Moore (1987); Barret, Heuson, Kolb (1986), Heinke (1998).

502

Die Begriffe Bundes- und Staatsanleihen werden synonym benutzt. 
Marktorientierte Kreditrisikobewertung Herleitung eines marktorientierten Ansatzes zur Bewertung des Kreditrisikos

zeit- und Kupongleichheit) zu finden, da die Menge an Staatstiteln begrenzt ist. ${ }^{503}$ Somit muss eine Ungenauigkeit bei einem der beiden Faktoren (Laufzeit vs. Kupon) in Kauf genommen werden. ${ }^{504} \mathrm{Im}$ Rahmen von Neuemissionen bspw. ist es üblich, lediglich eine laufzeitäquivalente Bundesanleihe zur Bestimmung des Credit Spread heranzuziehen. ${ }^{505}$

Bei der Bildung eines Indexes wird aus einer Menge an bonitätsrisikolosen Wertpapieren, welche jedoch alle unterschiedliche Ausstattungsmerkmale $\left(W_{B} \neq W_{B+1}\right)$ besitzen, eine modellhafte Anleihe berechnet (Benchmark), die die entsprechenden Ausstattungsmerkmale $\mathrm{W}_{\mathrm{i}}$ (der zu untersuchenden Anleihe i) aufweisen kann. Die Indexmenge beinhaltet die Renditen der bonitätsrisikolosen Wertpapiere als zu erklärende Größe $\left(\mathrm{r}_{\mathrm{B}}\right)$ und die Einflussfaktoren Restlaufzeit und Kupon als erklärende Größen. Hierzu wird die Methodik der Schätzung einer Regressionsgleichung angewendet, wobei es kein einheitliches Regressionsmodell gibt. ${ }^{506}$ Beispielsweise wird der $R E X^{\circledR}$ Rentenindex über eine nicht-lineare Regressionsgleichung ${ }^{507}$ mit den Einflussfaktoren Kupon und Restlaufzeit abgebildet, indem die Renditen aller Anleihen, Obligationen und Schatzanweisungen der Bundesrepublik Deutschland, des Fonds Deutsche Einheit und der Treuhandanstalt mit einer Restlaufzeit von 0,5 bis 10,5 Jahren herangezogen werden. ${ }^{508 / 509}$

\subsubsection{Empirische Determinanten der Bonitätsrisikoprämie}

Während Kapitel 4.3.3.2 Möglichkeiten der Isolation des Credit Spread aufgezeigt hat, werden im Weiteren die empirischen Einflussfaktoren dieser vom Markt bewerteten Bonitätsrisikoprämie erläutert. ${ }^{510}$ Häufig werden im Rahmen von Untersuchungen zum Credit Spread makroökonomische und titelspezifische Einflussfaktoren analysiert. ${ }^{511}$ In

Vgl. Heinke (1998), S. 17.

Bspw. akzeptieren Liu, Moore (1987) eine Abweichung zwischen den beiden Anleihen in der Restlaufzeit in Höhe von 6 Monaten. Vgl. Liu, Moore (1987), S. 544f.

Vgl. Lamy, Thompson (1988), S. 587; Jacob, Klein (1996), S. 143f.; Heinke (1998), S. 317

Vgl. Perry, Liu, Evans (1988), S. 233f.

Vgl. Kap. 5.3.3.

Vgl. zur Beschreibung des REX ${ }^{\oplus}$ Deutsche Börse AG (1997).

Als Alternative kann ein Index auf Basis der Kennzahl „Duration“ gebildet werden. Dadurch gelingt es ebenfalls, einen Benchmark zu berechnen, die sowohl den Kupon als auch die Restlaufzeit beachtet.Vgl. Fung, Rudd (1986), S. 633f.; Barrett, Heuson, Kolb (1986), S. 256ff.; Barrett, Heuson, Kolb (1986b), S. 306.

Vgl. Heinke (1998), S. 83ff.

Vgl. zur Systematik Heinke (1998), S. 83. Vgl. zu einer weiteren Auflistung empirischer Größen Kolb (1992), S. 290; Ma, Rao, Peterson (1990), S. 61. 
Marktorientierte Kreditrisikobewertung -

der vorliegenden Arbeit wird dieser Zweiteilung gefolgt. Abbildung 25 fasst die in den folgenden Kapiteln beschriebenen Einflussfaktoren des Credit Spread zusammen:

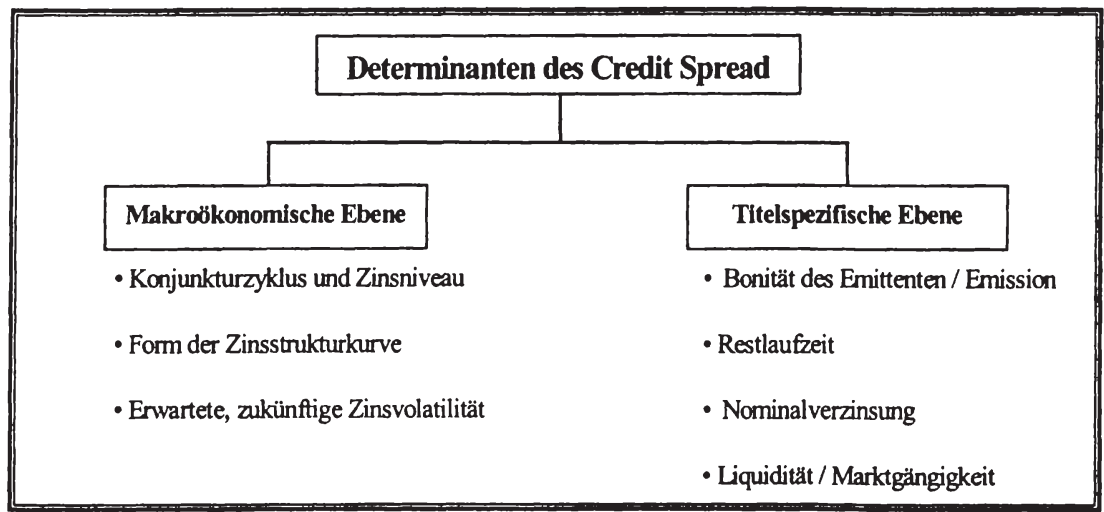

Abbildung 25: Einflussfaktoren des Credit Spread

\subsection{Makroökonomische Ebene}

In einer Vielzahl von Untersuchungen ${ }^{512}$ wurde der Konjunkturzyklus (Business Cycle) oder das allgemeine Zinsniveau als wesentlicher und bestimmender Einflussfaktor für den Credit Spread herausgestellt. Die Risikoprämie nimmt demnach in der wirtschaftlichen Rezessionsphase zu, während sie in Aufschwungphasen abnimmt. ${ }^{513}$ Abbildung 26 verdeutlicht die Entwicklung des Credit Spread im Zeitablauf. Es lässt sich erkennen, dass die Höhe des Credit Spread einer zyklischen Bewegung folgt, wie sie auch in der Konjunkturtheorie beschrieben wird.

Eine erste Begründung für diesen Zusammenhang findet sich in der veränderten fundamentalen Situation. Da sich in rezessiven Konjunkturphasen die Ertrags- und Liquiditätslage von Unternehmen grundsätzlich verschlechtern, erhöht sich die Wahrschein-

S12 Vgl. bspw. Jaffee (1975), S. 309ff.; Boardman, McEnally (1981), S. 207ff.; Kidwell, Koch (1982), S. 73; Kolb (1992), S. 290; Fabozzi (1996), S. 182; Ederington, Yawitz, Roberts (1987), S. 23ff.; van Horne (1979), S. 301; Dialynas (1988), S. 58; Dialynas, Edington (1992), S. 68.

513 Vgl. hier und im Folgenden bspw. Dialynas, Edington (1992), S. 68. Vgl. hier und im Folgenden zu den volkswirtschaftlichen Begriffen Woll (2000) S. 558. 
Marktorientierte Kreditrisikobewertung Herleitung eines marktorientierten Ansatzes zur Bewertung des Kreditrisikos

lichkeit, dass Tilgungs- bzw. Zinszahlungen ausfallen. ${ }^{514}$ Dementsprechend steigt der Credit Spread (et vice versa). ${ }^{515}$ Somit wird eine fundamentale Begründung für die beobachtete Entwicklung geliefert. Diese Zusammenhänge sollten sich langfristig auch in der Bilanz niederschlagen, und es kann somit argumentiert werden, dass sich die in Bilanzkennzahlen gemessene Bonität des Unternehmens aus fundamentalen Gründen ändert und sich folglich der Credit Spread im Sinne einer Marktbewertung der Bonität anpasst. ${ }^{516}$

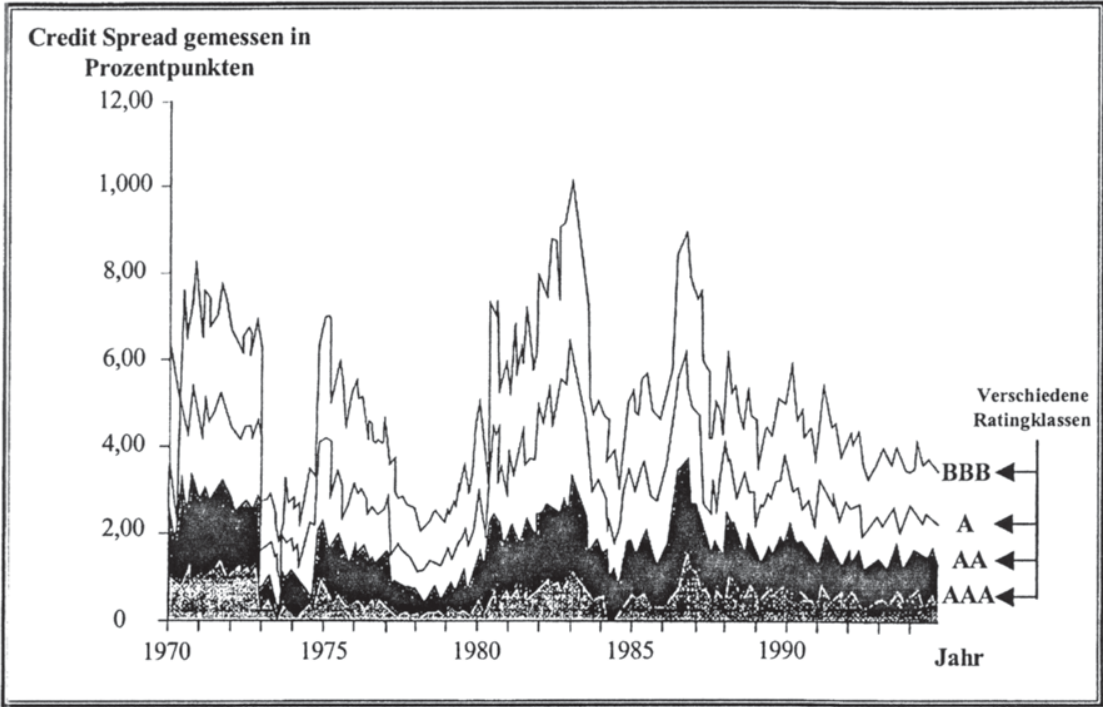

Abbildung 26: Zeitliche Entwicklung des Credit Spread (US-Markt) ${ }^{517}$

Eine zweite Begründung für den schwankenden Credit Spread lässt sich in der Risikoeinstellung von Investoren bzw. Gläubigern finden. ${ }^{518} \mathrm{Da}$ in rezessiven Phasen das allgemeine Zinsniveau höher liegt, sind Investoren mit einem „sichereren hohen Zins“

514 Dieser Effekt wird dadurch verstärkt, dass Kreditbelastungen in rezessiven Phasen durch ein erhöhtes Zinsniveau die Erträge der Unternehmen stärker belasten, als dies in expansiven Phasen der Fall ist.

S15 Vgl. Jaffee (1975), S. 312; Fuller, Farrel (1987), S. 416; Kolb (1992), S. 290; Dialynas (1988), S. 58; Dialynas, Edington (1992), S. 68; Behrenwaldt (1996), S. 301.

516 Vgl. bspw. Weinstein (1981), S. 258; für den Verschuldungsgrad als Bonitätsindikator vgl. Perridon, Steiner (1997), S. 478ff.

s17 In Anlehnung an Berblinger (1986), S. 49.

518 Vgl. hier und im Folgenden Barrett, Heuson, Kolb (1986a), S. 256; Kolb (1992), S. 290;

Heinke (1998), S. 87. 
Marktorientierte Kreditrisikobewertung -

zufrieden. Sie meiden risikoreichere Papiere, wodurch die Nachfrage nach diesen Anleihen abnimmt. Folglich nimmt auch der Kurs dieser Anleihen ab. Dies bedingt eine tendenzielle Erhöhung des Credit Spread (Anleihenrendite steigt aufgrund des niedrigeren Kurses). In expansiven Konjunkturphasen sinkt das allgemeine Zinsniveau, und Investoren bzw. Gläubiger versuchen, durch den Kauf von risikoreicheren Papieren eine akzeptable Rendite (unter Inkaufnahme eines größeren Risikos) zu erzielen. Somit fält der Credit Spread tendenziell ab. Unterstellt wird dabei eine Risikonutzenfunktion mit konkaven und konvexen Kurvenabschnitten. ${ }^{519}$ Insgesamt lässt sich ein positiver Zusammenhang zwischen Credit Spread und Zinsniveau konstatieren.

Die Erwartungen bezüglich der zukünftigen volkswirtschaftlichen Entwicklung werden u.a. in der Form der Zinsstrukturkurve sichtbar. ${ }^{520}$ Nach der Erwartungshypothese bilden die langfristigen Zinssätze die Investorenerwartung bezüglich der zukünftigen kurzfristigen Zinssätze ab. ${ }^{521}$ Eine normale (inverse) Kurve liegt vor, falls die Zinssätze mit der Laufzeit steigen (fallen). Somit erwarten die Investoren bei einer normalen (inversen) Kurve eine expansive (rezessive) Konjunkturphase. Wie oben dargelegt, wird sich folglich der Credit Spread bei einer normalen (inversen) Zinsstrukturkurve verringern (erhöhen). ${ }^{522}$ Im Falle eines stärkeren (schwächeren) Anstiegs der Zinsstrukturkurve wird ein niedrigerer (höherer) Credit Spread erwartet. ${ }^{323}$

519 Eine Nutzenfunktion mit wechselndem Steigungsverhalten wurde in Kap. 2.1 beschrieben. Vgl. auch Berens, Delfmann (1995), S. 67.

520 „Werden die zu beobachtenden Marktzinsen für einen bestimmten Laufzeitbereich in ein Diagramm eingetragen, so ergibt sich daraus die Zins- bzw. Kupon- oder Renditestruktur", Steiner, Bruns (1998), S. 146.

521 Vgl. hier und im Folgenden bspw. van Horne (1990), S. 104ff.; Kolb (1992), S. 279ff.

522 Vgl. Dialynas, Edington (1992), S. 72,

s23 Vgl. Duffee (1996b), S. 2. 
Marktorientierte Kreditrisikobewertung Herleitung eines marktorientierten Ansatzes zur Bewertung des Kreditrisikos

Die erwartete zukünftige Zinsvolatilität kann als Kennzahl für die von den Gläubigern empfundene Unsicherheit verstanden werden, die sich auf die zukünftige ökonomische Entwicklung bezieht. ${ }^{524}$ Eine höhere Volatilität bedeutet in diesem Zusammenhang eine größere Unsicherheit bezüglich zukünftiger Zahlungsströme und damit eine verbundene erhöhte Unsicherheit bezüglich der zukünftigen Zahlungsfähigkeit des Unternehmens. Die größere Unsicherheit führt zu einer Erhöhung des bewerteten Risikos. Somit ergibt sich ein positiver Zusammenhang zwischen der Zinsvolatilität und dem Credit Spread. ${ }^{525 / 526}$

\subsection{Titelspezifische Ebene}

Die Bonität eines Unternehmens ist offensichtlich für das Bonitätsrisiko eine wichtige Einflussgröße. In wissenschaftlichen Untersuchungen wird dieses Risiko regelmäßig entweder in Form von Ratings ${ }^{527}$ oder über fundamentalen Faktoren ${ }^{528}$ (wie auch in der vorliegenden Arbeit) operationalisiert. Grundsätzlich besteht ein negativer Zusammenhang zwischen Bonität und Bonitätsprämie, d.h. falls die Bonität eines Unternehmens sinkt, steigt der Credit Spread. Dieser recht offensichtliche Zusammenhang ergibt sich aus der definitorischen Beschreibung der Bonitätsprämie (bzw. des Credit Spread). Schwieriger erweist es sich, die die Bonität beschreibenden Faktoren zu identifizieren, wobei in den folgenden Kapiteln diese Faktoren detailliert analysiert werden.

Während auf der makroökonomischen Ebene die Konjunkturzyklen (Business Cycle) häufig als wichtigster Einfluss genannt werden, heben viele Untersuchungen die Restlaufzeit (neben der Bonität) als wesentlichen titelspezifischen Einflussfaktor hervor. ${ }^{529}$ Vor diesem Hintergrund spricht MERTON von der ,risk structure of interest rate ${ }^{4530}$. Hierbei wird die Struktur der Credit Spread innerhalb einer Bonitätsklasse untersucht. Während ein grundsätzlicher Zusammenhang zwischen Restlaufzeit und Credit Spread in vielen Untersuchungen bestätigt werden konnte, ist die Richtung und dementspre-

524 Vgl. bspw. Litterman, Iben (1991), S. 52 oder Dialynas (1988), S. 58.

525 Vgl. bspw. Lamy, Thompson (1988), S. 593 oder Barrett, Heuson, Kolb (1986), S. 110.

526 Vgl. für einen fundamentalen Erklärungsansatz bezüglich des Zusammenhangs zwischen der Zinsvolatilität, der Ertragskraft bzw. der erwarteten Deckungsquote des Unternehmens und dem Credit Spread Arnoldi (1993), S. 85 und auch Heinke (1998), S. 88. Vgl. bspw. die Untersuchung von Heinke (1998).

528 Vgl. bspw. die Untersuchung von Fisher (1959).

529 Vgl. bspw. Kolb (1992), S. 290; van Horne (1979), S. 301; Litterman, Iben (1991), S. 52.

330 Merton (1974), S. 449; vgl. auch Fabozzi (1996), S. 150. 
Marktorientierte Kreditrisikobewertung -

chend die Argumentation bezüglich des Zusammenhangs verschieden. ${ }^{531}$ HEINKE unterscheidet hierbei zwischen einem fundamentalen (negativer Zusammenhang) und einem anlegerorientierten (positiver Zusammenhang) Erklärungsansatz. ${ }^{532}$

Der fundamentale Erklärungsansatz bezieht die Restlaufzeit auf die Rückzahlungsfähigkeit des Unternehmens. ${ }^{533}$ Die Argumentation unterstellt, dass bei Erreichen des Fälligkeitszeitpunkts der Schuldner unter Umständen einen Liquiditätsengpass hat. Dies liegt darin begründet, dass aufgrund der notwendigen Anschlussfinanzierung bei ungünstigen Marktbedingungen die benötigten Kapitalvolumina zur Tilgung nicht zur Verfügung stehen (insbesondere bei schwachen Bonitätsklassen). Das Bonitätsrisiko steigt bei der am Ende der Laufzeit zu erbringenden Kapitalrückzahlung an. Somit entsteht ein negativer Zusammenhang zwischen Restlaufzeit und Credit Spread. Dieser wird als „Crisis at Maturity“ (oder Fälligkeitskrise) bezeichnet.

Der anlegerorientierte Ansatz verfolgt eine Argumentationslinie in Anlehnung an die aus der Volkswirtschaft bekannte Liquiditätspräferenztheorie. ${ }^{34}$ Der Anleger verlangt für eine längerfristig ausgeliehene Liquidität eine höhere Prämie, da die Wahrscheinlichkeit von Zahlungsstörungen größer wird. Er präferiert somit die heutige Liquidität. Aufgrund dieser Präferenz werden längerfristig laufende Anleihen mit einem höheren Credit Spread bewertet, und es ergibt sich ein positiver Zusammenhang zwischen Credit Spread und der Restlaufzeit. ${ }^{535}$

Während diese beiden Argumentationsstränge zunächst widersprüchlich erscheinen, kann der Gegensatz über einen klassifizierendern Faktor, die „Bonität“, aufgelöst werden. Die Untersuchung von HICKMAN konnte belegen, dass lediglich bei bonitätsschwächeren Unternehmen die Fälligkeitskrise zum Tragen kommt, die Liquiditätspräferenz wirkt sich auf bonitätsstarke Unternehmen aus. ${ }^{536}$ Dementsprechend nimmt bei bonitätsschwächeren Unternehmen der Credit Spread mit längerer Restlaufzeit der Anleihe ab, bei bonitätsstärkeren Unternehmen jedoch zu. Aus der konjunkturellen Abhängigkeit des Credit Spread folgt, ${ }^{537}$ dass die Bonitätshöhe (als Klassifikationsmerkmal) in wirtschaftlich rezessiven Phasen niedriger ist. Das bedeutet, dass die

531 Vgl. bspw. Johnson (1967), S. 318; Kolb (1992), S. 290; van Horne (1979), S. 301; Litterman, Iben (1991), S. 52; Schulte (1996), S. 109ff.

532 Vgl. hier und im Folgenden Heinke (1998), S. 89ff.

533 Vgl. hier und im Folgenden bspw. van Horne (1990), S. 193f.; van Horne (1979), S. 307.

534 Vgl. hier und im Folgenden Felderer, Homburg (1994), S. 120.

s3s Vgl. bspw. Fabozzi (1996), S. 150; Kolb (1992), S. 290; Fons (1994), S. 28.

536 Vgl. Hickman (1958), S. 109ff.

337 Vgl. Kap. 4.3.3.3.1. 
Marktorientierte Kreditrisikobewertung Herleitung eines marktorientierten Ansatzes zur Bewertung des Kreditrisikos

Fälligkeitskrise in rezessiven Phasen bereits bei Unternehmen mit mittleren Bonitäten greift. $^{338}$

Eine gleichartige Argumentation kann zur Begründung des Zusammenhangs zwischen Credit Spread und Nominalverzinsung verwendet werden, da die Höhe der Nominalverzinsung als Zahlungszeitpunkt interpretiert werden kann. Eine hohe Nominalverzinsung bedingt eine hohe Zinszahlung während der Laufzeit der Anleihe. Demgegenüber werden bei einer Nullkuponanleihe die Zinsen erst bei Fälligkeit ausgezahlt. Für eine Anleihe mit einem hohen Kupon besteht kein Bonitätsrisiko für bereits ausgezahlte Zinsen. Bei einer Nullkuponanleihe wiederum besteht ein Bonitätsrisiko sowohl für die Tilgungs- als auch alle Zinszahlungen über die gesamte Laufzeit. Diese Interpretation des Nominalzinses bedingt einen analogen Zusammenhang zwischen Nominalzins und Bonitätsprämie zu dem o.g. Zusammenhang zwischen Restlaufzeit und Bonitätsprämie. Aufgrund der Liquiditätspräferenz der Anleger wird ein negativer Zusammenhang zwischen Credit Spread und Nominalverzinsung begründet. ${ }^{539}$ Ein positiver Zusammenhang zwischen Credit Spread und Nominalverzinsung hingegen wird aus fundamentaler Sicht (gemäß der Fälligkeitskrise) durch das erhöhte Risiko bei fixen Zinszahlungen am Laufzeitende gefolgert. ${ }^{540}$

Als ein weiterer Einflussfaktor des Credit Spread gilt die Liquidität einer Anleihe, die das gehandelte Volumen umschreibt. ${ }^{541} \mathrm{Je}$ höher die Liquidität (oder Marktgängigkeit) ist, umso schneller kann das Papier zu einem fairen Preis verkauft werden. Man spricht in diesem Zusammenhang auch von der Markttiefe. Bei mangelnder Nachfrage kann der Handel unter Umständen nicht unverzüglich zum fairen Preis zustande kommen. Die Liquiditätseigenschaft gewinnt bei bonitätsschwächeren Unternehmen an Bedeutung, da mit höherem Bonitätsrisiko die Gefahr einer Zahlungsstörung und damit eines

Vgl. Johnson (1967), S. 323.

Die Kennzahl Duration kann den Zusammenhang ebenso beschreiben. „Während die Höhe der Restlaufzeit die Duration erhöht, wird sie durch höhere Kupons gesenkt", Heinke (1998), S. 91.

Vgl. Bierman, Hass (1975), S. 761 und van Horne (1990), S. 185.

Vgl. hier und Folgenden Fisher (1959), S. $217 \mathrm{ff}$. 
Marktorientierte Kreditrisikobewertung -

plötzlichen Werteverlustes größer ist. ${ }^{542} \mathrm{Um}$ diesen Verlust rechtzeitig begrenzen zu können, ist eine ausreichende Marktgängigkeit der Anleihe erforderlich. Insgesamt ist somit ein negativer Zusammenhang zwischen der Liquidität und dem Credit Spread zu erwarten. ${ }^{543}$

\subsection{Ableitung eines Prognosemodells}

Das in Kap. 4.1 genannte Ziel der Konzeption eines marktorientierten Kreditzinskalkulationsverfahrens wird im weiteren Verlauf des Kapitels durch die Ableitung eines Prognosemodells angestrebt. Zielgröße dieses Modells ist, wie oben beschrieben, die Schätzung eines objektiven Kreditzinses. Objektiv ist ein Kreditzins, falls das Kreditrisiko mit einem marktlichen Maßstab bewertet und anschließend in einem Zinssatz quantifiziert wird. ${ }^{\text {st }}$ Dieses Verständnis unterstellt die „Richtigkeit" der Marktbewertung, die in der Effizienztheorie zum Ausdruck kommt. Ein Markt ist genau dann effizient, wenn aufbauend auf allen relevanten Informationen die „wahren“ Erwartungen der Markteilnehmer im gehandelten Preis widergespiegelt werden. ${ }^{\text {Hs }}$ Übertragen auf einen effizienten Kreditzins bedeutet dies, die Markterwartung über die zukünftige Zahlungsfähigkeit (und Zahlungswilligkeit) eines Unternehmens zu bilden und das Risiko der Nichtrückzahlung des Kredites zu bewerten. Folglich wird die Bonität eines Unternehmens durch den einen marktorientierten Kreditzins objektiv bewertet.

Der in den volkswirtschaftlichen, vermögenstheoretischen Theorien beschriebene Kreditmarkt umfasst ,neben den in staatlichen Wertpapieren verbrieften Krediten (...) auch die Buchkredite der Kreditinstitute an die privaten Nichtbanken"s46. Aufbauend auf diesem Verständnis wird hier der Kreditmarkt im weiteren Sinne definiert als Ort des Zusammenkommens von Kreditnachfrage und -angebot. Regelmäßig überlässt im Rahmen eines Kredites der Kreditgeber im Vertrauen auf die Zahlungsfähigkeit des Kreditnehmers diesem das Geld. ${ }^{547}$ Diese Fähigkeit wird durch die Bonität beschrieben. Bei der Bestimmung des Kreditzinses (im Sinne eines Gleichgewichtspreises für das Kreditangebot und die -nachfrage) wird die Bonität und das hiermit verbundene

542 Vgl. hier und im Folgenden Heinke (1998), S. 92; van Home (1990), S. 185.

\$43 Eine Trennung des Credit Spread von der Gesamtrendite einer Anleihe bedingt, dass die zu untersuchende Anleihe im Vergleich zur Benchmark gleich liquide ist (Kap. 4.3.3.2). Staatstitel sind jedoch regelmäßig liquider als Unternehmensanleihen, vgl. Heinke (1998), S. 92. Folglich ist eine Trennung von Bonitätsrisiko und Liquiditätsrisiko in empirischen Untersuchungen kaum möglich, vgl. Duffee (1996), S. 16.

544 Vgl. Kirmße (1996), S. 61; Bröker (2000), S. 188 und Kap. 4.1.

345 Vgl. hier und im Folgenden Granziol (1981), S. 7 und Kap. 4.2.

546 Kath (1992), S. 199f.

547 Vgl. hier und im Folgenden Kap. 2.2 und auch Grill, Preczynski (1995), S. 233. 
Marktorientierte Kreditrisikobewertung Herleitung eines marktorientierten Ansatzes zur Bewertung des Kreditrisikos

Bonitätsrisiko bewertet. Der Kreditmarkt im weiteren Sinne steht somit vor der Aufgabe der Bewertung des mit dem Kredit verbundenen Bonitätsrisikos. Die auf dem Kreditmarkt gehandelten Kredite können durch eine Fülle von Kriterien systematisiert werden. ${ }^{548}$ Für die vorliegende Arbeit wird zwischen handelbaren und nichthandelbaren Titeln unterschieden. Ein Finanztitel ist genau dann handelbar, wenn man die Forderung unmittelbar an einen Dritten verkaufen kann. Diese Eigenschaft wird auch als Vertretbarkeit oder Fungibilität bezeichnet. ${ }^{549}$ Die in Kap. 4.3.1 beschriebenen Unternehmensanleihen fallen in diese Kategorie, da sie regelmäßig auf einem Markt gehandelt werden..$^{550}$

Auf dem organisierten Finanzmarkt werden Wertpapiere (Effekten) gehandelt. ${ }^{511}$ Diese Papiere verdeutlichen i.d.R. die Forderung der Eigentümer gegenüber den Emittenten der Wertpapiere und können in die Kategorien Eigen- und Fremdkapital unterteilt werden, indem die rechtliche Stellung des Eigentümers der einzelnen Forderung herangezogen wird. Falls es sich um ein eigenkapitalbezogenes Wertpapier (bspw. eine Aktie) handelt, ist der Eigentümer des Wertpapiers auch gleichzeitig (Mit)Eigentümer des Unternehmens. ${ }^{552}$ Dieser Teilmarkt wird in der vorliegenden Arbeit als Kapitalmarkt im engeren Sinne bezeichnet. Falls es sich um ein fremdkapitalbezogenes Wertpapier handelt, besitzt der Eigentümer des Wertpapiers einen Gläubigeranspruch gegenüber dem Unternehmen. Auf dem Kreditmarkt im engeren Sinne nach der hier verwendeten Definition werden Wertpapiere gehandelt, die sich auf Fremdkapitalforderungen beziehen. Ein Beispiel sind die in Kap. 4.3.1 beschriebenen Unternehmensanleihen. Sowohl auf dem Kredit- als auch dem Kapitalmarkt werden Risiken bewertet. Soweit man einen effizienten Markt ${ }^{553}$ unterstellt, wird somit auch das Risiko effizient bewertet.

Die Zielgröße des zu entwickelnden Prognosemodells ist der objektive Kreditzins, der das effizient bewertete Bonitätsrisiko eines Schuldners ausdrückt. Voraussetzung ist somit, dass es sich um die effiziente Marktbewertung eines bonitätsrisikobehafteten Titels handelt. Diese Forderung wird auf dem Kreditmarkt i.e.S. erfült (Abbildung 27). ${ }^{54}$ Die Schnittmenge von effizienter Risikobewertung und bonitätsrisikobehafteten

Bspw. können Kredite auch nach der Art der Kreditnehmer oder nach der Fristigkeit der Kredite gegliedert werden (vgl. Hartmann-Wendels, Pfingsten, Weber (2000), S. 193ff.) Vgl. Grill, Preczynski (1995), S. 359.

Vgl. Perridon, Steiner (1997), S. $157 \mathrm{ff}$.

Nach dem o.g. Verständnis wird hiermit der Kapitalmarkt im weiteren Sinne bezeichnet, vgl. auch Perridon, Steiner (1997), S. 157.

Vgl. hier und im Folgenden Grill, Preczynski (1995), S. 387.

Zur Definition eines effizienten Marktes vgl. Kap. 4.2.

Auf die Einbeziehung der Schnittmengen wie z.B. Wandelanleihen wird an dieser Stelle vereinfachend verzichtet. 
Marktorientierte Kreditrisikobewertung -

Herleitung eines marktorientierten Ansatzes zur Bewertung des Kreditrisikos

Titeln kann hinsichtlich einer marktorientierten Kreditzinskalkulation analysiert werden.

Die Grundidee des Prognosemodells ist es, aus empirischen Beobachtungen der effizienten Kreditrisikobewertung durch den Kreditmarkt i.e.S. mit Hilfe eines noch zu bestimmenden Instrumentes funktionale Zusammenhänge zwischen der effizient bewerteten Bonitätsrisikoprämie und den erklärenden Einflussfaktoren abzuleiten.

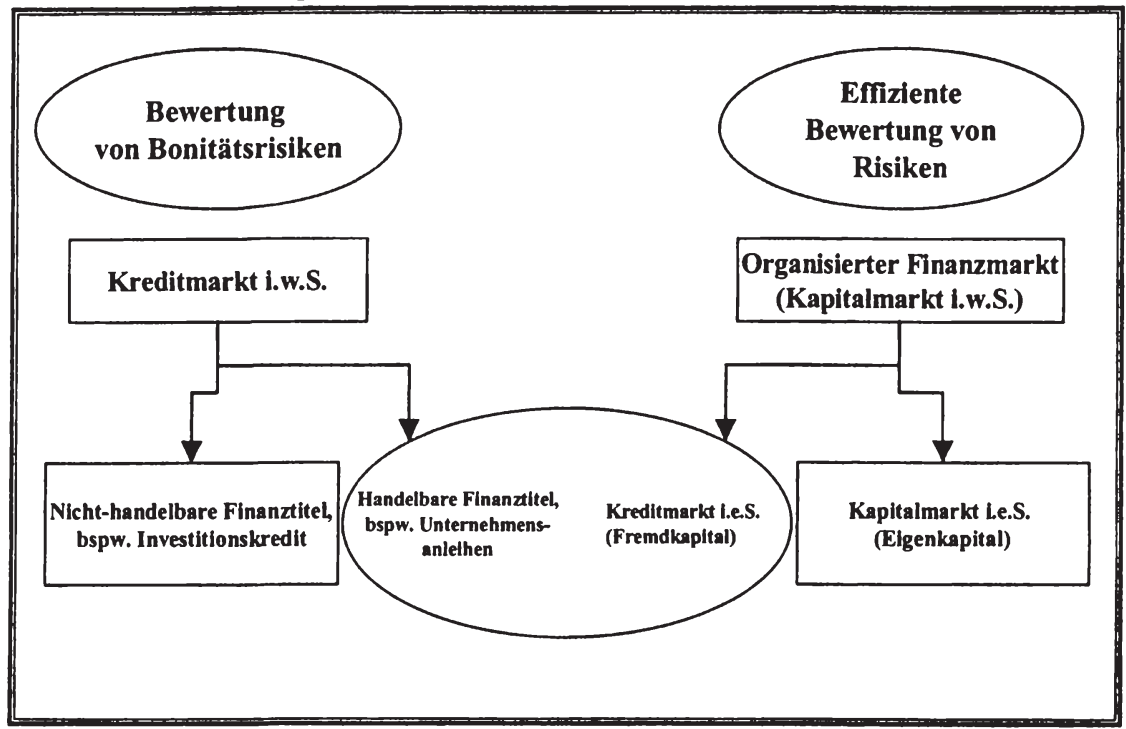

Abbildung 27: Effiziente Bonitătsrisikobewertung auf Kreditmărkten

Während die Zielgröße des Prognosemodells das innerhalb des objektiven Kreditzins enthaltene marktbewertete Bonitätsrisiko ist, müssen im weiteren Analyseverlauf die potenziellen Einflussfaktoren dieses bewerteten Risikos herausgestellt werden. Hier sind zunächst die in Kap. 2.3.2 dargestellten modelltheoretisch abgeleiteten Einflussfaktoren zu nennen. Im Einzelnen handelt es sich um:

- die Restlaufzeit des Kredites,

- das allgemeine Zinsniveau (sicherer Zins)

- die Form der Zinsstrukturkurve und

- das (nominale) Kreditvolumen.

Bewusst verzichtet wird auf die Modellparameter „Vermögenswert“, „Standardabweichung der Wachstumsrate des Vermögenswertes“, „Rückzahlungsquote“ und „Ausfallwahrscheinlichkeit“. Die Bestimmung des Vermögenswertes bzw. der hiermit 
Marktorientierte Kreditrisikobewertung Herleitung eines marktorientierten Ansatzes zur Bewertung des Kreditrisikos

verbundenen Standardabweichung der entsprechenden Wachstumsrate stößt bei der Umsetzung auf große Schwierigkeiten. ${ }^{5 s 5}$ Dem Ansatz, den Unternehmenswert von börsennotierten Gesellschaften gleich dem kapitalisierten Marktwert zu setzen, kann man nicht folgen, da die Schwankungen des Unternehmenswertes (kurzfristig) nicht mit fundamentalen Faktoren erklärbar sind. Es handelt sich vielmehr um weitergehende (psychologische) Bestimmungsgründe, die den Aktienkurs beeinflussen. ${ }^{566}$ Die Ausfallwahrscheinlichkeit und Rückzahlungsquote werden aus ähnlichen Gründen nicht mit aufgenommen. ${ }^{557}$ Falls sich eine Wahrscheinlichkeit für den Ausfall eines Kredites finden lassen würde, wäre die Bestimmung des Kreditrisikos bzw. die monetäre Quantifizierung eine rein mathematische Aufgabe. Da dies in der Realität nicht gegeben ist, muss mittels weiterer Inputfaktoren die Ausfallwahrscheinlichkeit implizit abgebildet werden.

Neben diesen modelltheoretischen Einflussfaktoren der Bonitätsrisikoprämie haben empirische Untersuchungen weitere Einflussfaktoren des Credit Spread bereits bestimmen können. ${ }^{558}$ Diese lassen sich in makroökonomische und titelspezifische Faktoren einteilen. Im Einzelnen handelt es sich hierbei um:

- den Konjunkturzyklus und das Zinsniveau,

- die Form der Zinsstrukturkurve,

- die erwartete zukünftige Zinsvolatilität,

- die Restlaufzeit,

- die Nominalverzinsung und

- die Liquidität bzw. Marktgängigkeit einer Anleihe.

Sowohl die modelltheoretischen als auch die empirischen Einflussfaktoren werden als den Credit Spread potenziell erklärende Faktoren in das Modell integriert.

Die Bonität des Unternehmens, die sich auch in dem Unternehmenswert und der Ausfallwahrscheinlichkeit reflektiert, als weiterer Einflussfaktor des Credit Spread lässt sich schwerer operationalisieren. Die in Kap. 3 beschriebenen Bilanzanalyseverfahren sind dazu in der Lage, Unternehmen hinsichtlich verschiedener Fragestellungen zu beurteilen. Insbesondere sind die dargestellten Verfahren in der Lage, Aussagen über die Bonität zu treffen. Innerhalb des Prognosemodells werden die vorgestellten Ver-

Vgl. hier und im Folgenden Kap. 2.5.

Vgl. Hoffjan, Siemes (1999).

Vgl. zur Substituierbarkeit der beiden Einflussfaktoren Ausfallwahrscheinlichkeit und Rückzahlungsquote Heinke (1998), S. 102.

Vgl. Kap. 4.3.3.3. 
Marktorientierte Kreditrisikobewertung -

fahren dazu genutzt, die Bonität zu bewerten. Dies geschieht, indem zunächst alle potenziell erklärende Faktoren abgeleitet aus den verschiedenen Bilanzanalyseverfahren aufgenommen werden. Das später zu integrierende Analyseinstrument muss eigenständig und objektiv die am besten zur Bonitätsbeschreibung geeigneten fundamentalen Kennzahlen auswählen. Dieser Auswahlprozess muss auf objektive Maßstäbe zurückgreifen. Wie bereits oben beschrieben wurde, ermöglicht eine Anlehnung an einen marktlichen Maßstab eine objektive Auswahl. Somit werden die im Rahmen einer Marktbewertung des Bonitätsrisikos relevanten Kennzahlen gesucht. Grundsätzlich kann dies durch eine an marktlichen Grundsätzen angelehnte Gewichtung der einzelnen Kennzahlen geschehen. Es besteht für den weiteren Analyseverlauf die Notwendigkeit der Integration eines Instruments, das diesem Anspruch genügt. ${ }^{559}$

Darüber hinaus muss dieses Instrument auch nicht-lineare, multidimensionale und kontextabhängige Zusammenhänge zwischen der Zielgröße und den Einflussfaktoren beachten. ${ }^{560}$ Die Nichtlinearität bezieht sich auf den unterstellten Funktionszusammenhang zwischen dem Credit Spread und den Einflussfaktoren, der - wie unter Kapitel 2.3.2 und 4.3.3.3 erläutert - sowohl innerhalb modelltheoretischer Überlegungen als auch empirischer Beobachtungen nicht-linear verläuft. Die Multidimensionalität bedeutet, dass mehrere Einflussfaktoren gleichzeitig bedacht werden müssen. Die Kontextabhängigkeit bezieht sich auf die Wirkungsweise einzelner Einflussfaktoren, d.h. die Wirkungsrichtung und die Wirkungsstärke eines Einflussfaktors wird durch die Konstellation mit den anderen Faktoren bestimmt. Bspw. wurde in Kap. 2.3.2.1 gezeigt, dass die Restlaufzeit sowohl einen positiven, keinen oder einen negativen Einfluss auf die Bonitätsprämie besitzt. Entscheidend für die Wirkungsrichtung ist die Ausprägung der Zinsstrukturkurve, sodass eine Kontextabhängigkeit des Einflussfaktors „Restlaufzeit“ konstatiert werden kann.

Abbildung 28 zeigt die Vorgehensweise bei der Aufstellung eines kreditmarktorientierten Prognosemodells. Während der objektive Kreditzins, die potenziell bonitätsbeschreibenden Bilanzkennzahlen und die verschiedenen Einflussfaktoren bereits konkretisiert sind, bedarf es im weiteren Analyseverlauf der Auswahl eines geeigneten Analyseinstrumentes. ${ }^{561}$ Die Einordnung der Vorgehensweise des Prognosemodells in die Kreditzinskalkulationsverfahren macht deutlich, dass es sich um ein heuristisches Lösungsverfahren handelt, da auf nicht willkürliche Art und Weise (Validierung mit geführten Untersuchungen) potenzielle Lösungen vom Suchprozess ausgeschlossen werden (Einschränkungen innerhalb der erklärenden Parameter) und es keine Garantie geben kann, da ein Konvergenzbeweis nicht führbar ist (funktionaler Zusammenhang

559 Vgl. zum Auswahlprozess eines geeigneten Instrumentes Kap. 5.1.

$560 \mathrm{Vgl}$. hier und im Folgenden Kap. 2.3.2.

561 Vgl. Kap. 5.1. 
ist nicht beweisbar, sondern stellt eine gute Lösung dar). ${ }^{52}$ Dies bedeutet für die unter Kap. 2.3.1 vorgenommene Systematisierung, dass es sich um ein heuristisches Kreditzinskalkulationsverfahren handelt.

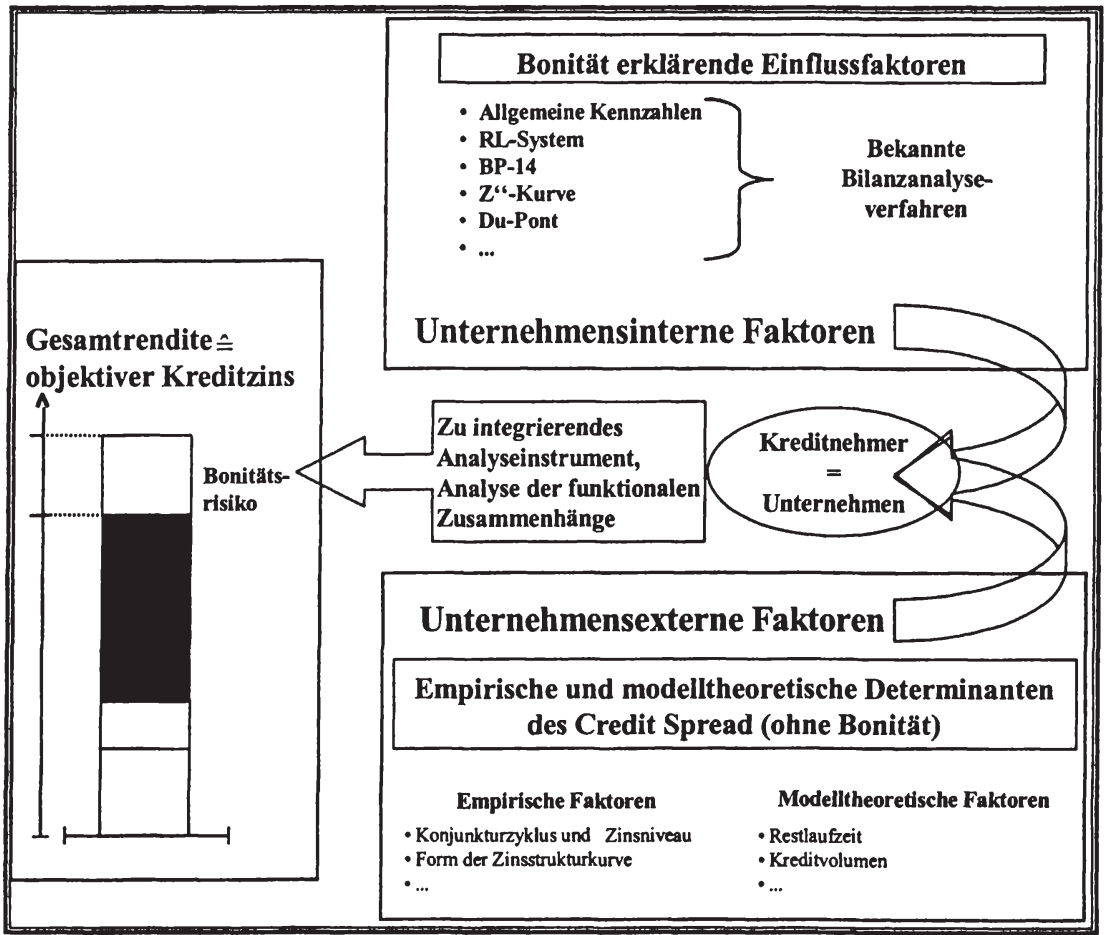

Abbildung 28: Vorgehensweise eines kreditmarktorientierten Prognosemodells als integrativer Bilanzanalyseansatz 
Marktorientierte Kreditrisikobewertung -

\subsection{Kritische Würdigung des Modells}

Nachdem im vorangegangenen Kapitel die Grundidee zur Entwicklung eines empirisch-induktiven Kreditzinskalkulationsverfahren dargelegt wurde, wird dieses im Weiteren mit den in Kap. 2.4 und Kap. 3.2 dargestellten Kriterien hinsichtlich der Eignung als Kreditzinskalkulation gewürdigt. ${ }^{563}$ Dieser theoretischen Beurteilung muss eine Überprüfung der Modellgüte des integrierten Analyseinstrumentes folgen. Das Analyseinstrument dient zur Abbildung beobachtbarer Zusammenhänge. Dementsprechend ist eine hohe Modellgüte erreicht, wenn diese Zusammenhänge besonders gut abgebildet werden. Folglich kann die Modellgüte über den Fehlerwert zwischen dem beobachteten Zielwert und dem vom Prognosemodell bestimmten Wert abgeleitet werden. ${ }^{564}$

Im Rahmen der Bilanzanalyse (Kapitel 3.2) wurden die folgenden Beurteilungskriterien für die Bilanzanalyse vorgestellt:

- Das Neutralisierungsprinzip,

- das Ganzheitlichkeitsprinzip und

- das Objektivierungsprinzip.

Das Neutralisierungsprinzip fordert, dass bilanzpolitische Ermessensspielräume nicht zu einem veränderten Bonitätsbild führen. Grundsätzlich ist das Prinzip erfüllt, da auf Basis einer effizienten Beurteilung entweder „neutralisierte“ Kennzahlen herangezogen bzw. die Ermessensspielräume erkannt und neutralisiert werden. Im Einzelfall kann eine weitere Prüfung der abgeleiteten Kennzahlen hinsichtlich der Erfüllung des Prinzips erfolgen. Hierzu könnten experimentell verschiedene Bilanzen „manipuliert“ werden und die Sensitivität in den durch das Modell abgeleiteten Risikoprämien gemessen werden. ${ }^{.65}$

Das Ganzheitlichkeitsprinzip besagt, dass ein Bilanzanalytiker sich ein umfassendes Bild des Unternehmens verschaffen soll. Dies kann in dem empirisch-induktiven Verfahren sichergestellt werden. Zunächst werden alle Kennzahlen aus den Bereichen der Ertrags-, Finanz- und Vermögenslage zur Urteilsbildung verwendet. Während in der Bilanzanalyse lediglich Bilanzzahlen verwendet werden, weist die Integration der Erwartungsbildungsmechanismen des Kreditmarktes für die Quantifizierung des Kre-

563 Es werden die vertragsspezifischen Besonderheiten einer Anleihe im Vergleich zu einem Bankkredit vernachlässigt. Die Beurteilung bezieht sich somit auf ein allgemeines Kalkulationsverfahren. In Kap. 6.1.1 wird die Anwendungsmöglichkeit des Prognosemodells als Bewertungsmodell für Bankkredite analysiert.

s6s Vgl. Baetge (1998), S. 602ff. und Baetge (1997), S. 42ff. 
Marktorientierte Kreditrisikobewertung Herleitung eines marktorientierten Ansatzes zur Bewertung des Kreditrisikos

ditrisikos den Vorteil auf, dass weitergehende Informationen (wie das Kreditvolumen oder der Nominalzins) mit in die Beurteilung einbezogen werden. Der vom effizienten Markt bestimmte Preis reflektiert alle verfügbaren Informationen. Somit weist dieser Ansatz einen höheren Anspruch an die Ganzheitlichkeit (hinsichtlich der Quantifizierung von Kreditrisiken) auf als die bekannten Bilanzanalyseverfahren.

Neben dem Neutralisierungs- und dem Ganzheitlichkeitsprinzip ist das Objektivierungsprinzip im Rahmen der konzeptionellen Ansprüche an die Bilanzanalyse zu überprüfen. Hierbei ist zunächst der Weg der Bilanzanalyse zu analysieren. Dies bedeutet, dass das Gesamturteil durch konstante Kriterien und gleiche Gewichtungen zustande kommt. Dies kann durch die Fixierung innerhalb eines funktionalen Zusammenhanges als gesichert angesehen werden. Die Festlegung von Parametern in einem funktionalen Zusammenhangs gewährleistet konstante Kriterien und eine gleiche Gewichtung. Anschließend muss das Verfahren der Bilanzanalyse hinsichtlich der Einhaltung der Objektivität überprüft werden. Dies kann durch den Bewertungsmechanismus eines effizienten Kreditmarktes sichergestellt werden. Folglich ist das Objektivitätskriterium durch die Konzeption einer marktorientierten Kalkulation erfüllt.

Während sich die Überprüfung dieser drei Kriterien an den Prinzipien der Bilanzanalyse orientiert hat, wird im Weiteren die Eignung als Kreditzinskalkulationsverfahren gewürdigt. Hierzu wird die Einhaltung der Prinzipien der Einzelbewertung und der Richtigkeit überprüft. ${ }^{566}$

Dem Prinzip der Einzelbewertung wird entsprochen, da der Ausgangspunkt für die Kalkulation der Ausfallprämie das individuelle Unternehmen ist. Es findet eine Kalkulation auf Basis eines einzelnen Kredites statt. Das Kriterium der Objektivität ist ebenfalls erfüllt, da jedes Kreditinstitut losgelöst zu demselben Kalkulationsergebnis gelangt, falls dieselbe Datenbasis die Grundlage der Untersuchung bildet. ${ }^{567} \mathrm{Da}$ marktrelevante, veröffentlichte und für jeden zugängliche Daten die Ausgangsbasis bilden, kann unterstellt werden, dass eine identische Datenbasis vorliegt. Somit kann jeder eigenständig die funktionalen Zusammenhänge nachbilden. Wichtig ist hierfür die Kenntnis des noch zu integrierenden Analyseinstrumentes. Durch den Bezug auf den Kredit- und Kapitalmarkt handelt es sich um einen marktlichen Bewertungsmaßstab, der als objektiv bezeichnet werden kann.

Die weitere Prüfung bezieht sich auf das Kriterium der Aktualität. Sicherlich sind die markt- und titelspezifischen Daten tagesaktuell erfassbar. Die Daten aus dem Jahresabschluss weisen jedoch die Problematik auf, dass sie, wie im Kap. 3.6 gezeigt, jährlich, i.d.R. zeitversetzt zur Verfügung stehen und einen vergangenheitsorientierten

Vgl. Kap. 2.4.

567 Unterstellt wird hier die Nutzung desselben Instruments zur Ableitung des funktionalen Zusammenhangs. 
Marktorientierte Kreditrisikobewertung -

Aussagegehalt besitzen. Die Problematik ,veralteter“ Informationen könnte sich in der Modellgüte niederschlagen. Somit kann nicht ausgeschlossen werden, dass die Bilanzkennzahlen keinen signifikanten Einfluss auf die Anleihenrendite haben, jedoch würde eine Irrelevanz dieser Kennzahlen in dem empirisch-induktiven Verfahren durch eine schlechte Modellgüte sichtbar. Die Vergangenheitsorientierung des Datenmaterials wird am Kreditmarkt in Erwartungen transformiert, sodass zukunftsweisende Aussagen abgeleitet werden. Darüber hinaus stehen neben dem Jahresabschluss auch unterjährige Berichte zur Verfügung, die grundsätzlich zur Herleitung der fundamentalen Kennzahlen innerhalb der Bilanzanalyse geeignet sind, wodurch die Problematik nicht aufgehoben, jedoch entschärft wird.

Zusammenfassend lässt sich konstatieren, dass sich die kreditmarktorientierte Bilanzanalyse in ihrer Konzeption als Kalkulationsverfahren eignet. Sie entspricht einem heuristischen Lösungsverfahren. Die Umsetzung durch die Integration eines Analyseinstruments und die Ableitung eines funktionalen Zusammenhangs muss auf ihre praktische Relevanz hin überprüft werden. Die bereits oben beschriebene Modellgüte eignet sich hierzu, um das heuristische Lösungsverfahren zu bewerten. An ihr kann überprüft werden, inwieweit es sich um eine gute Lösung im Sinne eines heuristischen Kreditzinskalkulationsverfahrens handelt. ${ }^{568}$

${ }^{568}$ Ebenfalls muss dieser Zusammenhang hinsichtlich der Erfüllungen des Ganzheitlichkeits- und des Neutralisierungsprinzips geprüft werden. 
Andreas Siemes - 978-3-631-75322-4

Downloaded from PubFactory at 01/11/2019 06:12:16AM

via free access 
Marktorientierte Kreditrisikobewertung - Empirische Untersuchung zur marktorientierten Bewertung des Kreditrisikos mittels Künstlicher Neuronaler Netze 127

\section{Empirische Untersuchung zur marktorientierten Bewertung des Kreditrisikos mittels Künstlicher Neuronaler Netze}

Das in Kap. 4.4 vorgestellte Konzept eines Prognosemodells benötigt zur Untersuchung der Zusammenhänge zwischen der marktorientierten Kreditbewertung (Credit Spread) und den potenziellen unternehmensinternen und unternehmensexternen Faktoren ein Analyseinstrument. Im folgenden Kap. 5.1.1 werden zunächst die Anforderung an ein solches Instrument aufgezeigt, um darauf aufbauend in den Kap. 5.1.2 bis Kap. 5.2.3 ein Analyseinstrument vorzustellen. Es wird dargelegt, dass sich Künstliche Neuronale Netze grundsätzlich und ein spezieller Netzwerktyp im Besonderen (das Multi-Layer-Perceptron in Kombination mit dem Error-BackpropagationAlgorithmus) hierzu eignen. Nachdem das Analyseinstrument erklärt wurde, wird im Kap. 5.3 der zu untersuchende Datenbestand beschrieben. Dieser wird im anschließend Kap. 5.4 mittels des vorgestellten Künstlichen Neuronalen Netzes analysiert. Im Rahmen der Analyse wird zunächst ein „Grundmodell“ (Kap. 5.4.3) und anschließend als Modellerweiterung ein „13er-Modell“ (Kap. 5.4.5) abgeleitet. Abschließend werden in Kap. 5.6 die gefundenen Ergebnisse bewertet.

\subsection{Künstliche Neuronale Netze als problemadäquates Instrument}

\subsubsection{Instrumentenanforderung}

Das im vorangestellten Kapitel vorgestellte Konzept eines Prognosemodells, ist - wie jedes Modell - eine vereinfachte Abbildung der Realität. Der unterstellte Zusammenhang zwischen der Zielgröße (dem Credit Spread) und den erklärenden Werten (unternehmensinterne, bonitätsbeschreibende Faktoren und unternehmensexterne Marktfaktoren) zeichnet sich durch drei Merkmale aus: ${ }^{569}$

Erstens liegen multidimensionale, kontextabhängige Wirkungsverknüpfungen vor. Beispielsweise kann aus einer modelltheoretischen Betrachtung heraus die Restlaufzeit sowohl einen positiven als auch einen negativen Einfluss auf die Kreditprämie haben. Partielle Ableitungen bieten keine eindeutigen Lösungen.

569 Vgl. zur Herleitung der unterstellen Zusammenhänge Kap. 2.3.2, Kap. 4.3.3.3 und Kap. 4.4. 
Marktorientierte Kreditrisikobewertung - Empirische Untersuchung 128 zur marktorientierten Bewertung des Kreditrisikos mittels Künstlicher Neuronaler Netze

Zweitens liegen nicht-lineare Zusammenhänge vor. Exemplarisch wurden im Kapitel 2.3.2 modelltheoretische Kurvenverläufe aufgezeigt, die einen nicht-linearen Zusammenhang zwischen dem Credit Spread und den Faktoren wie bspw. der Restlaufzeit oder dem allgemeinen Zinsniveau beschrieben. ${ }^{570}$

Drittens wird das Konzept eines Prognosemodells entworfen, da (in Abgrenzung zu den meisten wissenschaftlichen Untersuchungen im Bereich der Kreditwürdigkeitsprüfung $)^{571}$ nicht nach einer Klassifikation in bspw. „solvent und insolvenzgefährdet" gesucht wird, sondern die Lösung eine quantifizierte Kreditprämie darstellt.

Es eignen sich verschiedene statistische Instrumente zur Prognose des Credit Spread. Ein mögliches Verfahren ist der Einsatz von Künstlichen Neuronalen Netzen. Der Vorteil dieses Einsatzes liegt insbesondere in der Möglichkeit, nicht-lineare, komplexe Probleme abzubilden und zu untersuchen..$^{572}$ Im Folgenden wird zunächst gezeigt, dass sich Künstliche Neuronale Netze für die vorliegende Problemstellung eignen, indem die Grundlagen und Anwendungsgebiete aufgezeigt werden, um anschließend einen speziellen Netzwerktyp für die weiteren Untersuchungen auszuwählen.

\subsubsection{Grundlegende Betrachtung von Künstlichen Neuronalen Netzen als Teilgebiet der Künstlichen Intelligenz}

Künstliche Neuronale ${ }^{573}$ Netze sind im weitesten Sinne dem Forschungsgebiet der Künstlichen Intelligenz zuzuordnen (KI-Methoden). Künstliche Intelligenz wird nicht als Wissensgebiet verstanden, sondern vielmehr als „Konglomerat aus mehr oder weniger zufällig zusammengekommenen Forschungsbereichen, Programmiersprachen und zielorientierten Lösungsansätzen" ${ }^{\text {‘574 }}$ mit der Gemeinsamkeit, eine Problemstellung computergestützt zu lösen. Gleichzeitig ist die Struktur der Problemlösung an biologische Vorbilder angelehnt. ${ }^{575}$ Der Forschungszweig Künstliche Intelligenz wurde durch die Verfügbarkeit entsprechender Computersysteme (computergestützte Problemlö-

570 Eine nicht-lineare und komplexe Problemstruktur ist regelmäßig in der Ökonomie zu finden, bspw. die Ertragsfunktion, das Lebenszykluskonzept, das Konzept der Erfahrungskurven, Ist-Kosten bei intensitätsmäßiger Anpassung, ISO-Gewinnkurven, Kurven bei degressiver und digitaler Abschreibung, Risikopräferenzkurven oder Analysen der zulässigen effizienten Wertpapierdepots. Vgl. Enders (2001), S. 372.

571 Vgl. Kap. 3.6.

572 In ihrer empirischen Studie belegen Fadlalla, Lin die Vorteile von Neuronalen Netzen gegenüber anderen statistischen Instrumenten für finanzwissenschaftliche Fragestellungen. Vgl. Fadlalla, Lin (2001), S. $112 \mathrm{ff}$.

Der Begriff Neuron stammt aus dem griechischen und bedeutet Sehne oder Nerv.

Hürlimann (1987), S. 396.

Vgl. Blien, Lindner (1993), S. 497. 
Marktorientierte Kreditrisikobewertung - Empirische Untersuchung zur marktorientierten Bewertung des Kreditrisikos mittels Künstlicher Neuronaler Netze

sung) ermöglicht. ${ }^{576}$ Somit ist die Entwicklungsgeschichte eng mit der Computertechnologie verbunden. Während konzeptionelle Grundlagen bereits in den 60er-Jahren entwickelt wurden ${ }^{577}$ genießen diese Instrumente in der jüngsten Vergangenheit aufgrund von leistungsstärkeren elektronischen Datenverarbeitungssystemen eine gewachsene Aufmerksamkeit. Daher ist auch zukünftig eine extrem rasche Weiterentwicklung der KI-Methoden zu erwarten, wobei die Leistungsfähigkeit von Computern sich nicht ins Unendliche, gemessen an den Dimensionen der biologischen Vorbilder, steigern lässt. ${ }^{578}$ Diese rasanten Entwicklungsmöglichkeiten erschweren eine vollständige Übersicht der KI-Methoden. Exemplarisch sein hier die Verfahren Fuzzy Logic, Genetische Programmierung bzw. Genetische Algorithmen und Künstliche Neuronale Netze genannt (Abbildung 29).

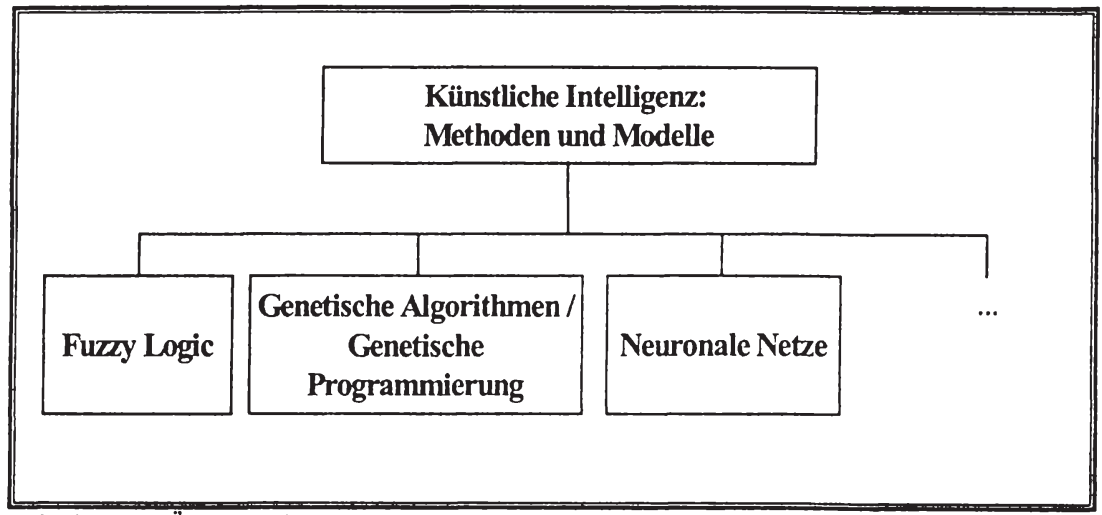

Abbildung 29: Übersicht der Methoden und Modelle der Künstlichen Intelligenz

Die Fuzzy Logic kann als die Verallgemeinerung der klassischen Mengentheorie dargestellt werden. Sie ahmt das menschliche Schließen und Kommunizieren nach. ${ }^{579}$ Die

576 Vgl. Ritter, Martinetz, Schulten (1991), S. 17ff. oder Köhle (1990), S. $35 \mathrm{ff}$.

577 Roseblatt stellte bereits 1958 das erste abgeschlossene Modell vor. Vgl. Carpenter (1989), S. 245f. Vgl. auch Füser (1995), S. 15.

578 Vgl. Füser (1995), S. 4f.

$579 \mathrm{Vgl}$. Zimmermann (1999), S. 24; alternativ kann man sie auch als Verallgemeinerung der zweiwertigen Logik deuten. 
Marktorientierte Kreditrisikobewertung - Empirische Untersuchung 130 zur marktorientierten Bewertung des Kreditrisikos mittels Künstlicher Neuronaler Netze

genetischen Algorithmen (oder die genetische Programmierung) bilden den biologischen Evolutionsprozess nach und wenden dessen Problemlösungstechnik zur Bewältigung technischer und ökonomischer Probleme an..$^{580 / 581}$

Neuronale Netze ,erheben den Anspruch, die Denkprozesse des Menschen auf dem Computer abzubilden" ${ }^{\text {“582 }}$. Grundlage bildet somit das menschliche Gehirn bzw. die menschlichen Neuronen als dessen Bestandteile. Der Mensch besitzt über 100 Milliarden dieser Neuronen, die untereinander hochgradig vernetzt sind. ${ }^{583}$ Fraglich ist es jedoch, inwieweit Neuronale Netze in der Lage sind, diese hochkomplizierten Vernetzungen abzubilden..$^{54}$ Dementsprechend kann man die Begriffswahl „Neuronale Netze" mit der historischen Entwicklung begründen und aus heutiger Sicht ist es sinnvoller, von „mathematischen Modellen über Verbindungen von Berechnungseinheiten“"s85 zu sprechen. Schematisch lässt sich ein natürliches Neuron wie in Abbildung 30 darstellen.

Ein Neuron besitzt einen Zellkörper (Soma), der den Zellkern und die anderen Zellorgane enthält. ${ }^{586}$ Der Zellkern besitzt zwei Typen von Fortsätzen. Die Dendriten leiten Informationen in Richtung des Zellkörpers und die Axone in Richtung ihres Ausgangs. Die Neuronen geben hierbei Informationen in Form von elektrischen Impulsen weiter. In der Regel enthält ein Neuron genau ein einziges Axon jedoch eine Vielzahl von stark verzweigten Dendriten (Dendritenbaum). Die synaptischen Eingänge an den Dendriten funktionieren wie Analogschalter. Sie reagieren entweder erregend oder hemmend auf Eingangsinformationen. ${ }^{587}$ Ihnen wird eine besondere Bedeutung beim Lernen zugesprochen. Ein Lernerfolg findet durch die Gewichtungsmöglichkeit von Informationen statt. Durch die parallele Verarbeitung von Informationen besitzt das

Vgl. Zimmermann (1999), S. 28.

Vgl. für eine Gegenüberstellung der Anwendungen der Genetischen Programmierung und der Künstlichen Neuronalen Netze zur Bewertung von Optionen Berens, Thonemann, Siemes, Kleinau (2001).

Füser (1995), S. 11.

Vgl. Wittkemper (1994), S. 11.

Auf Grund von Modifikationen und Weiterentwicklungen der Neuronalen Netze weisen einige Verfahren keine großen Parallelen zu dem biologischen Lernverhalten der natürlichen Neuronen auf (vgl. Schmitting (2000), S. 74). Somit wird von Potvin der Ausdruck „neurally's inspired problem-solving approaches“ (Potvin (1993), S. 328) gewählt.

Enders (2001), S. 372.

Vgl. hier und im Folgenden Campell (1997), S. 1082f.

Vgl. hier und im Folgenden Stanley, Bak (1991), S. 20ff.; Brause (1991), S. $31 \mathrm{ff}$;

Dorfner (1991), S. 16f., Füser (1995), S. 13f.; Uthoff (1997), S. $170 f$. 


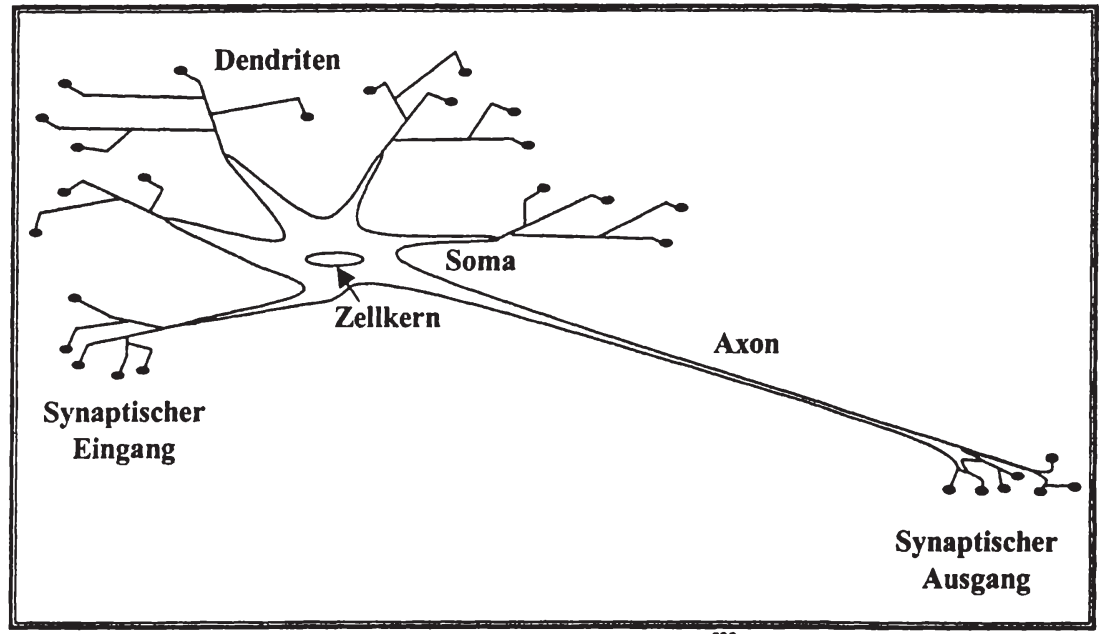

Abblidung 30: Schematische Abbildung eines biologischen Neurons ${ }^{588}$

Gehim eine sehr hohe Verarbeitungsgeschwindigkeit und Lernfähigkeit. Die Idee, das biologische Vorbild zu adaptieren, geht auf MCCULLOCH und PITTS zurück. ${ }^{589} \mathrm{Der}$ entscheidende Schritt gelang 1943, als das mathematische Modell zum Aufbau eines Neurons entwickelt wurde. ${ }^{500}$ Grundsätzlich kann jeder arithmetische oder logistische Zusammenhang durch die „McCulloch-Pitts-Neuronen“ abgebildet werden. ${ }^{591}$ Mit der Weiterentwicklung von ROSEBLATT 1958 wurde das erste abgeschlossene Modell vorgestellt (Perzeptron), welches die Realisierung eines adaptiven klassifizierenden Systems darstellt. ${ }^{392}$ MINSKY/PAPERT konnten jedoch Ende der 60er-Jahre die (aus ihrer Sicht) fundamentale Beschränkung des Modells durch das Unvermögen zur Lösung bekannter Fragestellungen nachweisen, sodass die KNN ins wissenschaftliche Abseits gerieten. ${ }^{593}$ Das bis heute andauernde Interesse wurde 1985 durch die Veröffentlichung von RUMELHART/HINTON/WILLIAMS eines Error-Backpropagation-

In Anlehnung an Campell (1997), S. 1083.

Vgl. McCulloch, Pitts (1943), S. 115ff. Ansätze zur Untersuchung des menschlichen Lernverhaltens bzw. von Gehirnfunktionen gehen bereits auf erste Schriften 3000 vor Christus zurück. Darüber hinaus wurde bspw. in der Arbeit von Alan Turing 1936 bereits das Gehim als Vorbild furr einen Computer beschrieben. Vgl. Füser (1995), S. 15.

Vgl. Pytlik (1995), S. $151 \mathrm{ff}$.

Vgl. Uthoff (1997), S. 170.

Vgl. Carpenter (1989), S. 245f.

Vgl. Rehkugler, Poddig (1992), S. 50. 
Marktorientierte Kreditrisikobewertung - Empirische Untersuchung 132 zur marktorientierten Bewertung des Kreditrisikos mittels Künstlicher Neuronaler Netze

Algorithmus wieder geweckt. ${ }^{594}$ Bei Verwendung desselben konnten nun auch komplexere Netzwerke ein gewünschtes Lernverhalten zeigen. ${ }^{.95}$ Das Lernverhalten von KNN liegt darin begründet, dass das Netz ,selbstständig“ Strukturen erkennen kann, wobei man im Vorfeld „kein exakt beschriebenes Wissen zur Problemlösung “5966 benötigt. Dieses Wissen kann als Strukturwissen bezeichnet werden. Neuronale Netze werden trainiert und bilden selbständig Annahmen über strukturelle Zusammenhänge und optimieren diese. Dieser Prozess geschieht anhand von Beispielfällen. Ein Datensatz (Bspw. eine Person) mit einzelnen Informationen (bspw. Angaben über das Alter, Einkommen und dem Geschlecht) entspricht einem Beispielfall, und die Summe aller Beispielfälle entspricht der zu untersuchenden Datenmenge (bspw. Personengruppe). Das gelernte Wissen wird gespeichert und steht anschließend für neue, „unbekannte“ Fälle zur Verfügung.

Der Begriff „Neuronales Netz“ oder „Künstliches Neuronales Netz“ ist hierbei nur als Sammelbegriff zu verstehen. Hierunter werden verschiedene Netzwerktypen verstanden. Jedes Neuronale Netz kann als Abbildungsmaschine der Realität verstanden werden, indem eine reelle Funktion $f: \mathrm{R}^{\mathrm{n}} \rightarrow \mathrm{R}^{\mathrm{m}}$ modelliert wird. ${ }^{597}$ Es wird ein $\mathrm{n}$-dimensionaler Eingabevektor in einen m-dimensionalen Ausgabevektor transformiert. Der funktionale Zusammenhang ist die abgebildete Realität. Unter einem Künstlichen Neuronalen Netz wird

„a system composed of many simple processing elements operating parallel whose function is determined by network structure, connection strengths, and the processing performed at computing elements or nodes "s998

verstanden. Künstliche Neuronale Netze können verschiedene Problemstellungen anhand eines eigenständigen Lernverhaltens lösen. Im folgenden Kapitel werden diese Problemstellungen, welche die Anwendungsgebiete der $\mathrm{KNN}$ darstellen, verdeutlicht.

Vgl. Rumelhardt, Hinton, Williams (1986), S. 318ff.; vgl. auch Kapitel 5.2.2.

Zur historischen Entwicklung vgl. Zell (1994), S. 28ff.

Schumann (1991), S. 30; vgl. auch im Folgenden Schumann (1991), S. $30 f$.

Vgl. hier und im Folgenden Füser (1995), S. 22f.

DRAPA Neural Network Study, zitiert bei Strecker (1997), S. 1. 
Marktorientierte Kreditrisikobewertung - Empirische Untersuchung zur marktorientierten Bewertung des Kreditrisikos mittels Künstlicher Neuronaler Netze

\subsubsection{Anwendungsgebiete}

Hauptanwendungsgebiet von Künstlichen Neuronalen Netzen ist die Mustererkennung. ${ }^{599}$ Hierbei wird untersucht, inwieweit sich für eine gegebene Menge an Daten spezifische Muster erkennen lassen. Als Muster wird ein sich wiederholender (erkennbarer) Zusammenhang zwischen einzelnen Daten bezeichnet. Darüber hinaus können Neuronale Netze Muster vervollständigen oder zuordnen. ${ }^{600}$ Die Klassifikation ist ein typischer Mustererkennungsvorgang. ${ }^{601}$ Hierbei werden einzelne Merkmale von Daten dazu genutzt, um diese Datensätze bestimmten Klassen zuzuordnen. Der Klassifikator approximiert die funktionalen Zusammenhänge, die die Entscheidungsgrenzen in einem Musterraum definieren. Reale Problemstellungen zeichnen sich durch einen komplexen Musterraum aus, der sich nicht durch lineare Entscheidungsgrenzen einschränken lässt. Wie im weiteren Analyseverlauf gezeigt wird, zeichnen sich Neuronale Netze insbesondere dadurch aus, nicht-lineare Entscheidungsgrenzen anhand von Beispieldaten zu erkennen. Neben der Klassifikation ist auch die Prognose ein Vorgang der Mustererkennung. Falls bspw. ein Aktienkurs prognostiziert werden soll, geschieht dies auf Basis gefundener vergangenheitsorientierter Muster zwischen dem Aktienkurs und den erklärenden Faktoren wie bspw. dem Umsatz des Unternehmens oder der allgemeinen Marktentwicklung. Insgesamt lassen sich die folgende Anwendungsbereiche finden: ${ }^{602}$

- Prüfung und Beurteilung: Kreditwürdigkeitsprüfung, Insolvenzprüfung, Bilderkennung;

- Klassenbildung: $\quad$ Marktsegmentierung, Data Mining;

- Prognose: Kursprognose, Absatzprognose, Kostenprognose;

- Optimierung: Transportoptimierung (Traveling-Salesman-Problem) ${ }^{603}$, Reihenfolgeplanung.

Der Lösungsprozess für diese Problemstellungen vollzieht sich innerhalb der Künstlichen Neuronen, welche im Weiteren erläutert werden.

599 Vgl. Corsten, May (1996), S. 3.

600 Vgl. Füser (1995), S. 10.

601 Vgl. hier und im Folgenden Corsten, May (1996), S. 3f.

602 Einen Überblick hinsichtlich der überzeugenden Leistungsfähigkeit Neuronaler Netze für verschiedene finanzwissenschaftliche Fragestellungen aus den Bereichen Prüfung und Beurteilung, Klassenbildung und Prognose findet sich bei Fadlalla, Lin (2001).

$603 \mathrm{Vgl}$. zu den bislang nur wenig überzeugenden Leistungen von Neuronalen Netzen für diesen Anwendungsbereich Schmitting (2000), S. 73. 
Marktorientierte Kreditrisikobewertung - Empirische Untersuchung 134 zur marktorientierten Bewertung des Kreditrisikos mittels Künstlicher Neuronaler Netze

\subsubsection{Der strukturelle Aufbau von Künstlichen Neuronen}

Wie bereits oben ausgeführt, werden Künstliche Neuronen in Anlehnung an biologische Neuronen strukturiert. Sie besitzen n Eingänge (Dendriten), die mittels n Gewichten (Synapsen) die Eingangsinformationen zur Informationsverarbeitung weiterleiten (Soma). Die Verarbeitung innerhalb eines Neurons vollzieht sich in drei Schritten (vgl. Abbildung 31): 604

1) Jede Eingangsinformation $\left(x_{i}\right)$ wird mit einem speziellen Gewichtungsfaktor $\left(w_{i}\right)$ multipliziert. Hierbei stellt $i=[1 ; n]$ ein Dendrit dar. Die Gewichte sind unterschiedlich hoch, sodass die Eingangsinformationen einen unterschiedlichen Einfluss auf die später zu berechnende Ausgangsinformation (o) besitzen. ${ }^{605}$ Die Gewichtungsfaktoren stellen die Synapsengewichte dar. Anschließend wird die Summe über alle Produkte gebildet (Skalarprodukt aus dem Vektor der Eingangsinformationen und dem Gewichtungsvektor als:

$$
\left.y=F\left(\sum_{i=1}^{n} w_{i} x_{i}\right)\right) .
$$

2) Im folgenden Schritt erfolgt die Berechnung mittels der Aktivierungsfunktion A. Die im ersten Schritt gebildete Summe y wird hierzu mit Hilfe der Aktivierungsfunktion bewertet: A(y). Die Intensität des Ausgangssignals wird somit durch diese Funktion bestimmt.

3) Abschließend wird mit Hilfe der Transferfunktion $\mathrm{T}$ das Ausgangssignal $A(y)$ weitergeleitet. $T(A(y))$ ergibt den Ausgabewert o. Diese Transferfunktion ist in der Regel die Identitätsfunktion. ${ }^{606}$

Insgesamt gilt somit (falls die Transferfunktion die Identitätsfunktion ist):

Gleichung 26

$$
T\left(A\left(F\left(\sum_{i=1}^{n} w_{i} x_{i}\right)\right)\right)=A\left(F\left(\sum_{i=1}^{n} w_{i} x_{i}\right)\right)=A(y)=0 .
$$

604 Vgl. Füser (1995), S. 24f.

605 Vereinfachungsgemäß wird zunächst eine Unterschicht in der Hidden-Ebene und ein Ausgabewert o unterstellt (vgl. Kap. 5.2.1).

606 Die Identitätsfunktion bildet einen Wert $x$ auf einen Wert $y$ identisch $a b, y=T(x)=x$. 
Marktorientierte Kreditrisikobewertung - Empirische Untersuchung zur marktorientierten Bewertung des Kreditrisikos mittels Künstlicher Neuronaler Netze 135

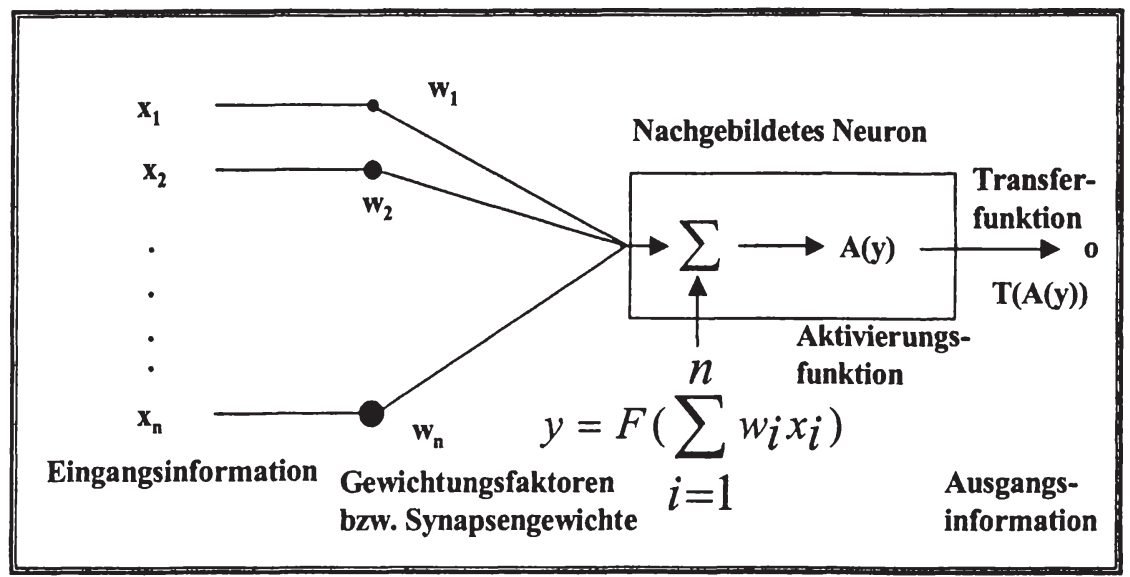

Abbildung 31: Künstliches Neuron ${ }^{607}$

Wie o.g. werden die Eingangsinformationen durch die Gewichte unterschiedlich stark bewertet. Innerhalb des im Weiteren dargestellten Lernprozesses, findet eine Veränderung der Gewichte statt. Grundlage des Lemprozesses sind die realen Strukturen, die in den zu untersuchenden Daten enthalten sind. Eine optimale Gewichtung sorgt dafur, dass die realen Strukturen bestmöglich durch die Gewichte innerhalb der Neuronen dargestellt werden. ${ }^{608}$ Wie im weiteren Untersuchungsverlauf gezeigt wird, werden die Gewichte auf Basis der beobachtbaren Zusammenhänge gebildet, sodass erkennbare Muster in Gewichten abgespeichert und in funktionalen Zusammenhängen sichtbar werden. Ein Künstliches Neuron ist in der Lage, multidimensionale, hochkomplexe Zusammenhänge durch die Bestimmung entsprechender Gewichte abzubilden ${ }^{609}$ Die Aktivierungsfunktion dient hierbei der Abbildung nicht-linearer Zusammenhänge, da diese Funktion sich auf beliebige mathematische Zuordnungsvorschriften beziehen kann. Beispiele für Aktivierungsfunktionen, die auch nicht-lineare Zusammenhänge abbilden können, sind die Sigmoid-Funktion (vgl. Abbildung 32) und die Tanh-Funktion (vgl. Abbildung 33). Diese Aktivierungsfunktionstypen werden auch im weiteren Verlauf der Untersuchung herangezogen.

\footnotetext{
607 In Anlehnung an Füser (1995), S. 24.

608 Zur Findung der Gewichte mittels eines Lemvorgangs vgl. Kap. 5.2.2.

Vgl. bspw. Glormann (2001), S. 195f. oder Füser (1995), S. $16 f$.
} 
Marktorientierte Kreditrisikobewertung - Empirische Untersuchung 136 zur marktorientierten Bewertung des Kreditrisikos mittels Künstlicher Neuronaler Netze

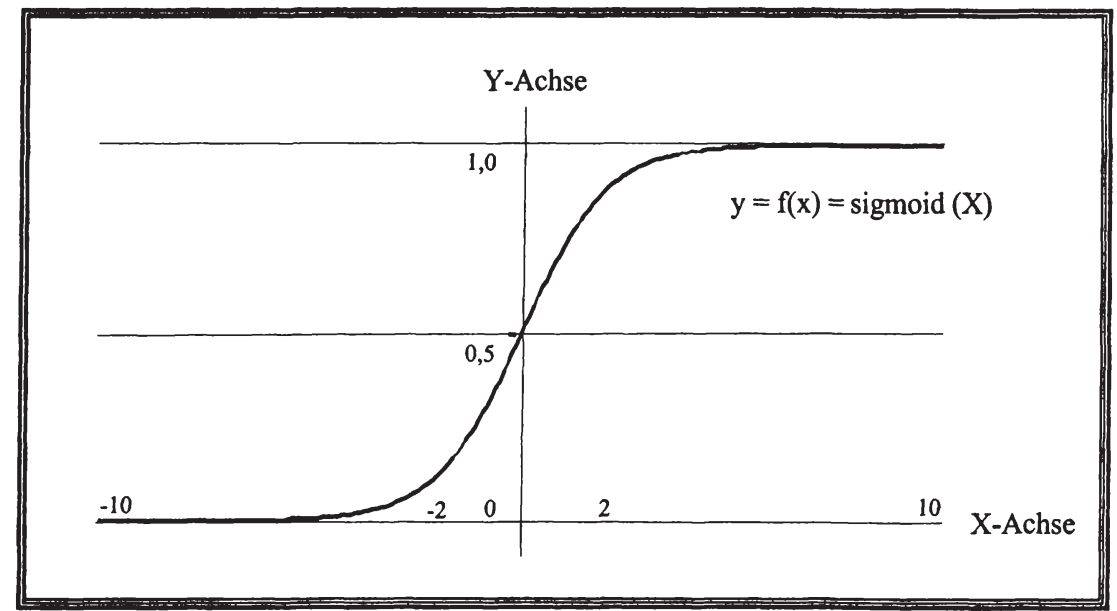

Abbildung 32: Schematischer Kurvenverlauf der Sigmoid-Funktion

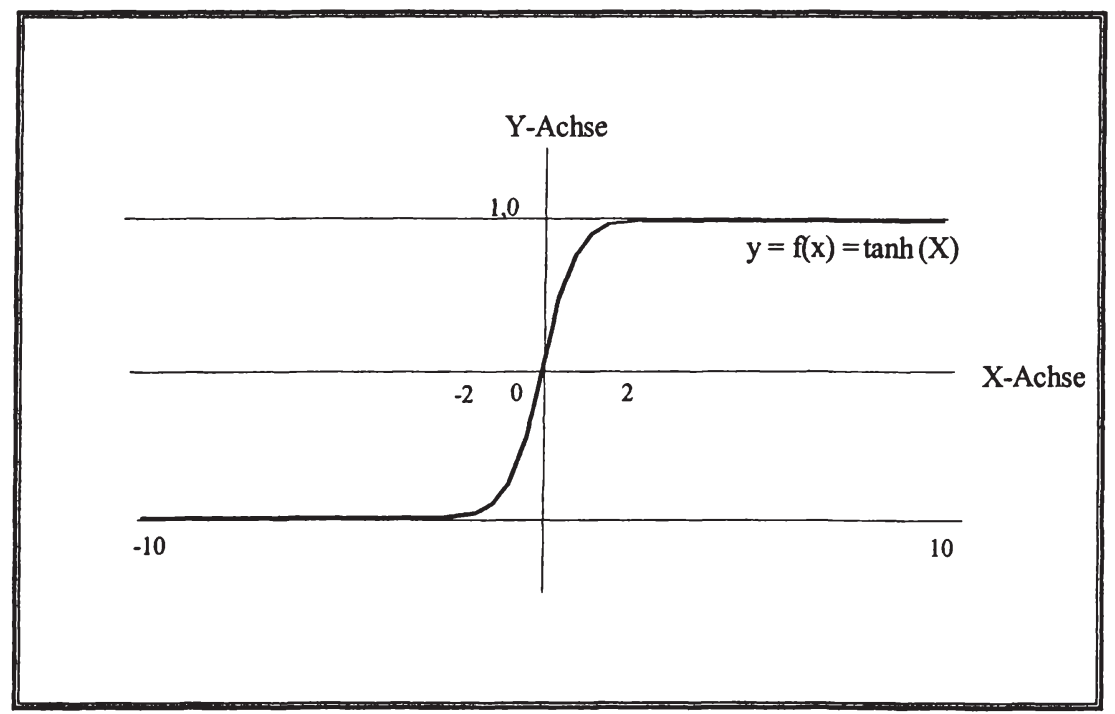

Abbildung 33: Schematischer Kurvenverlauf der Tanh-Funktion

Abbildung 34 veranschaulicht die oben allgemein beschriebenen Prozesse innerhalb der Neuronen anhand fiktiver Gewichte. Die unterschiedlich starken Eingabeinformationen werden zunächst mit den Gewichten multipliziert, sodass je Eingabewert ein gewichteter Eingabewert berechnet wird. Diese werden anschließend summiert. Das Ergebnis stellt die gewichtete Gesamteingabe dar. Diese wird mittels einer Aktivie- 
Marktorientierte Kreditrisikobewertung - Empirische Untersuchung

rungsfunktion bewertet, sodass als Endergebnis ein Ausgabewert von dem Künstlichen Neuron berechnet wird. Im Weiteren wird - falls nicht ausdrücklich eine bestimmte Funktion genannt wird - die Sigmoid-Funktion als Aktivierungsfunktion unterstellt.

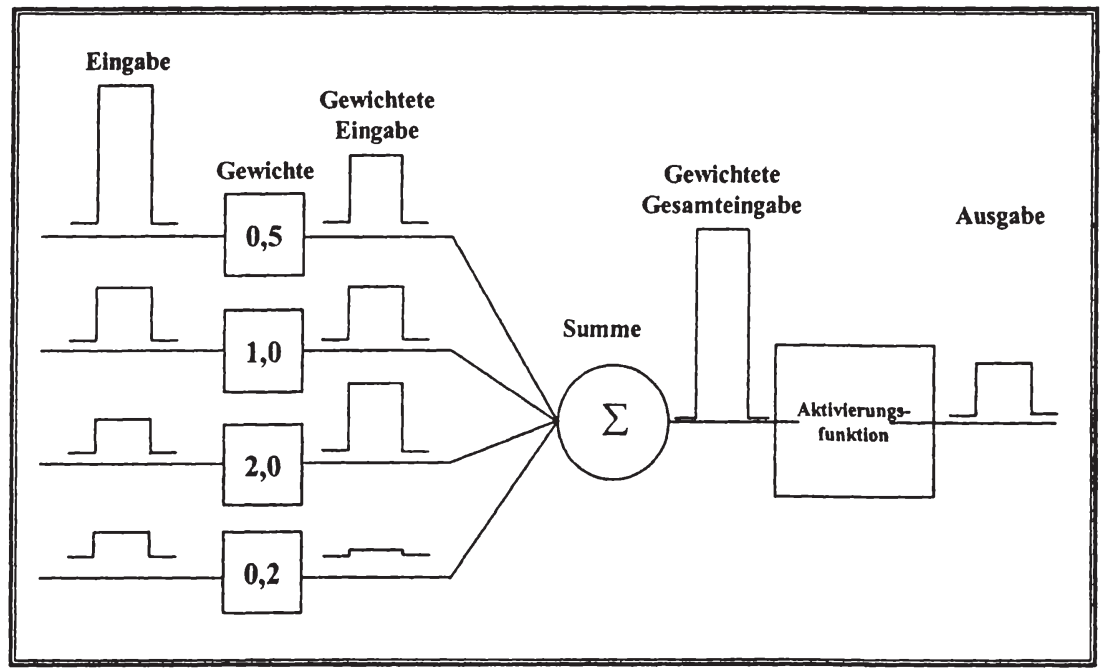

Abbildung 34: Vereinfachte Darstellung der Verarbeitung innerhalb eines Künstlichen Neurons ${ }^{610}$ 
Marktorientierte Kreditrisikobewertung - Empirische Untersuchung 138 zur marktorientierten Bewertung des Kreditrisikos mittels Künstlicher Neuronaler Netze

\subsubsection{Problemspezifische Auswahl eines Netzwerktyps}

Die Kapitel 5.1.2 bis 5.1.4 machen deutlich, dass Künstliche Neuronale Netze grundsätzlich in der Lage sind, multidimensionale und kontextabhängige und somit komplexe Wirkungszusammenhänge abzubilden. Sie sind durch die Verwendung nichtlinearer Aktivierungsfunktionen in der Lage, nicht-lineare Wirkungszusammenhänge zu integrieren. Mittels der Vorgehensweise einer Mustererkennung können neben Klassifizierungsproblemen auch Prognoseprobleme wie die Bestimmung eines konkreten Aktienkurses ${ }^{611}$ oder einer Rendite von Staatsanleihen ${ }^{612}$ gelöst werden. Darüber hinaus wurde im Rahmen der Bond-Rating-Klassifikation, d.h. der Zuordnung von Unternehmensanleihen zu bestimmten Ratingklassen, ebenfalls auf das Instrument der KNN zurückgegriffen. ${ }^{613}$ Die bereits in Kap. 4.4 vorgestellten Anforderungen an ein zu integrierendes Analyseinstrument in das vorgestellte Prognosemodell liegen in diesen empirischen Untersuchungen (Prognose von Aktienkursen bzw. Staatsanleihenrendite und der Bond-Rating-Klassifikation) ebenfalls vor. Folglich belegen die bereits geführten Untersuchungen die grundsätzliche Eignung der KNN zur Verwendung als Analyseinstrument in das in Kap. 4.4 vorgestellte Konzept eines Prognosemodells. ${ }^{614} \mathrm{Im}$ Weiteren wird ein spezieller Netzwerktyp ausgewählt, der in das vorgestellte Konzept integriert wird.

Die oben dargestellten Zusammenhänge beschränken sich auf ein einzelnes Neuron, in einem Neuronalen Netz sind jedoch regelmäßig verschiedene Neuronen vorhanden und miteinander vernetzt. ${ }^{615}$ Sowohl die Möglichkeiten der Vernetzung als auch die Arten der Informationsweitergabe erlauben es, verschiedene Netzwerktypen zu entwickeln. ${ }^{616}$ Dementsprechend ist zur Verwendung eines KNN eine Auswahl zwischen diesen verschiedenen Netzwerktypen notwendig.

611 Vgl. zur Verwendung von KNN im Rahmen der Aktienkursprognose Uhlig (1995); Baun (1994); Rehkugler, Poddig (1990, 1992); Wittkemper (1994); White (1988); Schöneburg, Gantert, Reiner (1989); Schöneburg, Hansen, Gawelczyk (1990); Kimoto, Asakawa, Yoda, Takeoka (1990); Deutsche Bank Research zitiert bei Chaouli, Froitzheim (1992); SGS-Bank zitiert bei Graf $(1991,1993)$ und bei Werner (1993); Schumann, Lohrbach (1993); Malliaris (1994); Ruggiero (1994); Rhee (1994); Chenoweth, Obradovic (1994); Hiemstra (1994); Bentz, Refenes (1994); Baestaens, van den Bergh (1995).

612 Vgl. zur Verwendung von KNN im Rahmen der Prognose von Staatsanleihen Uhlig (1995); Poddig (1994); Landesbank Hessen-Thüringen zitiert bei Matthes (1994); Wild (1991), Allianz-Lebensversicherung zitiert bei Riess (1992).

613 Vgl. Dutta, Shekar (1988); Dutta, Shekar, Wong (1994); Utans, Moody (1991).

614 Die Autoren Fadlalla, Lin zeigen die Vorteilhaftigkeit von Neuronalen Netzen im Vergleich zu anderen Instrumenten. Vgl. Fadlalla, Lin (2001), S. 118.

615 Vgl. hier und im Folgenden bspw. Füser (1995), S. 31.

616 Zur Systematisierung verschiedener Netzwerktypen vgl. Füser (1995), S. $31 \mathrm{ff}$. 
Marktorientierte Kreditrisikobewertung - Empirische Untersuchung zur marktorientierten Bewertung des Kreditrisikos mittels Künstlicher Neuronaler Netze 139

Untersuchungen mit Künstlichen Neuronalen Netzen bedingen im Analyseverlauf verschiedene Entscheidungen hinsichtlich der verwendeten Parametereinstellungen innerhalb der Netzwerkarchitektur. ${ }^{617}$ Diese Entscheidungen können nicht immer objektiv getroffen werden, sondern mitunter werden Faustregeln oder vergleichbare Analyseverläufe herangezogen. Für den erfolgreichen Einsatz von KNN ist es wichtig, auf erprobte Verfahren aufzubauen, um somit Erfahrungswerte bei den Parametereinstellungen zu nutzen. Ein sehr weit verbreitetes Verfahren ist ein Multi-LayerPerceptron $^{618}(M L P)$ mit einem Error-Backpropagation-Lernverhalten ${ }^{619}$. Dieses Instrument kann wie die meisten Netzwerktypen dazu genutzt werden, Prognoseprobleme zu lösen. ${ }^{620}$ Die Problemstellung aus Kapitel 4.1 entspricht einem Prognoseproblem (Prognose des Credit Spread). Zusammenfassend kann konstatiert werden, dass ein MLP in der Lage ist, dass in Kap. 4.1 dargestellte Problem grundsätzlich zu lösen. Darüber hinaus erfüllt es (wie die meisten Netzwerktypen aus dem Bereich der KNN) die gestellten Anforderungen und weist den größten Erfahrungswert bei vergleichbaren Studien auf. ${ }^{61}$ Vor diesem Hintergrund wird im weiteren Analyseverlauf dieses Instrument in das Prognosemodell integriert (Kap. 5.4). Vor der Anwendung des Instrumentes wird dieser spezielle Netzwerktyp im Weiteren zunächst detailliert beschrieben (Kap. 5.2), um ihn anschließend zur Analyse der in Kap. 5.3 darzustellenden empirischen Untersuchungsmenge heranzuziehen.

617 Vgl. hier und im Folgenden bspw. Uhlig (1995), S. 48 und auch Kap. 5.4.1.

618 Nach Rehkugler, Poddig ist dies das am hăufigsten eingesetzte Instrument der Neuronalen Netze. Vgl. Rehkugler, Poddig (1998), S. 381.

619 Das Error-Backpropagation-Verfahren stellt nach Füser die am weitesten verbreitete Lernmethode dar. Vgl. Füser (1995), S. 57.

620 Zur Beschreibung des MLP vgl. die Ausführungen in den folgenden Kapiteln.

621 Vgl. Rehkugler, Poddig (1998), S. 381; Füser (1995), S. 57; Fadlalla, Lin (2001), S. $112 \mathrm{ff}$. 
Marktorientierte Kreditrisikobewertung - Empirische Untersuchung 140 zur marktorientierten Bewertung des Kreditrisikos mittels Künstlicher Neuronaler Netze

\subsection{Das Multi-Layer-Perceptron in Kombination mit dem Error-Backpropagation-Algorithmus}

\subsubsection{Darstellung der Netzwerkstruktur}

Wie bereits o.g., ist das „Multi-Layer-Perceptron“ ein verbreitetes Instrument zur KNN-Analyse. Grundsätzlich werden in diesem Netzwerktyp Muster mittels eines überwachten Lernverhaltens ${ }^{622}$ erkannt. Dies gelingt, indem das MLP eingeständig ein Muster bestimmt und dieses mit einem vorgegebenen Muster vergleicht (Soll-IstPaare). Diese Vorgehensweise wird als überwachtes Lernen bezeichnet. Ein MLP besteht (vgl. Abbildung 35) aus drei Schichten (auch Ebenen genannt), der Eingabeschicht (Input-Ebene), der versteckten Schicht (Hidden-Ebene) und der Ausgabeschicht (Output-Ebene). ${ }^{623}$ Die einzelnen Neuronen innerhalb dieser Schichten wurden bereits in Kapitel 5.1.4 dargestellt. Wie in Abbildung 35 deutlich wird, kann die HiddenEbene weitere Unterschichten (in diesem Fall drei) besitzen. ${ }^{624}$ Diese sogenannten layers bestehen aus einzelnen Neuronen (in diesem Fall je drei), auch als Knotenpunkte bezeichnet, und leiten die Informationen jeweils an die nachgelagerten Neuronen weiter.

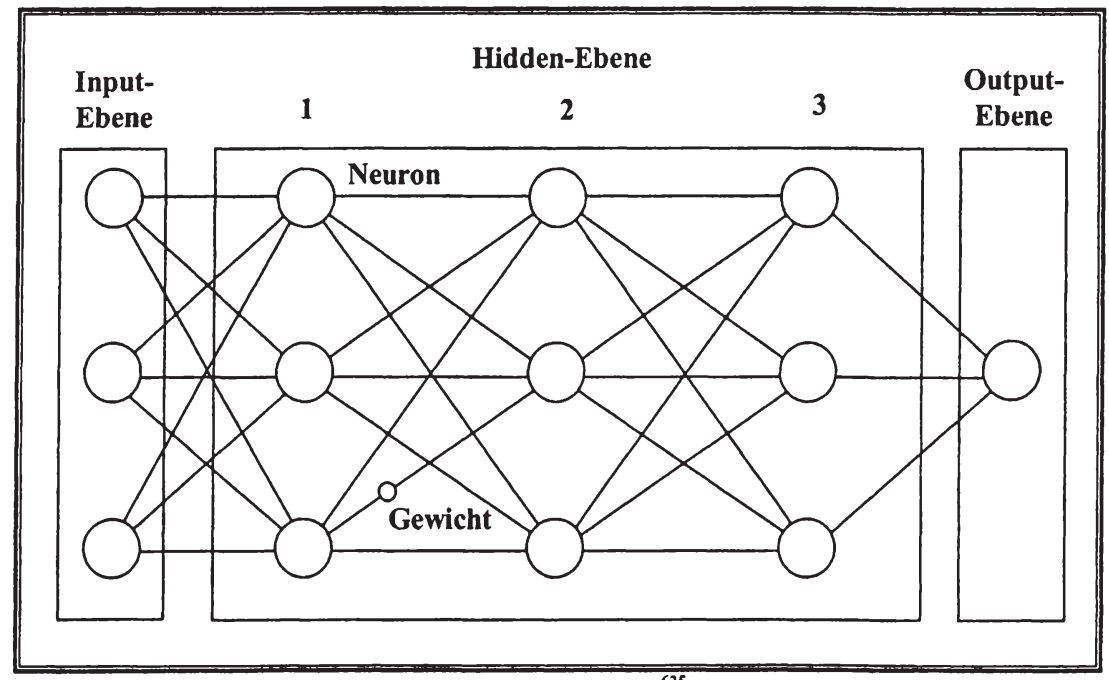

Abbildung 35: Grundstruktur eines Backpropagation-Netzes ${ }^{625}$

Vgl. bspw. Hecht-Nilsen (1990), S. $48 \mathrm{f}$.

Vgl. Füser (1995), S. 31.

Vgl. hier und im Folgenden Füser (1995), S. 31 f.

In Anlehnung an Füser (1995), S. 31. 
Marktorientierte Kreditrisikobewertung - Empirische Untersuchung

Da Neuronen einer Schicht ausschließlich mit Neuronen einer nachgelagerten Schicht positiv verbunden sind, handelt es sich um ein vomuärtsgerichtetes Netz hinsichtlich der Informationsverarbeitungsrichtung. ${ }^{626}$ Die Eingangsneuronen (im Weiteren mit oe ${ }_{\mathrm{i}}$ gekennzeichnet) nehmen die verschiedenen Eingangsinformationen aus den Inputfaktoren auf. Häufig werden diese mit Hilfe einer Z-Transformation ${ }^{627}$ normiert, damit Niveauunterschiede ausgeglichen werden. ${ }^{628}$ Die Zahl der Eingangsneuronen hängt von der Anzahl der Informationen ab, die das Neuronale Netz verarbeiten soll. Dies bedeutet, dass für einen Datensatz mit drei Informationen (bspw. Geschlecht, Alter und Einkommen) drei Eingangsneuronen benötigt werden. Die nachgelagerte Schicht wird von der Eingabe- und Ausgabeschicht eingeschlossen. Da diese Verarbeitungsebene keine direkten Informationen von der Umwelt erhält, wird sie als versteckte Schicht bezeichnet. ${ }^{629}$ Die Verarbeitung in den einzelnen Neuronen (im Weiteren mit ov gekennzeichnet) wird gemäß der im Kapitel 5.1.4 dargestellten Arbeitsweise Künstlicher Neuronen durchgeführt. Da die verarbeitende Schicht (Hidden-Ebene) sich wiederum in Unterschichten (layers) gliedert, wird der Ausgabewert eines Künstlichen Neurons in der versteckten Schicht u.U. an ein weiteres Künstliches Neuron weitergereicht. Der Ausgabewert eines Neurons wird von dem empfangenden Neuron gewichtet und als Summand in die Aktivierungsfunktion überführt. Der Ausgabewert (Summe aller gewichteten Eingabewerte transformiert mittels der Aktivierungsfunktion) wird anschließend wiederum an das nachgelagerte Neuron übermittelt. Die Anzahl der Künstlichen Neuronen und die Anzahl der versteckten Schichten sind nicht durch das zu analysierende Problem vordefiniert, sondern müssen im Rahmen der Untersuchungen festgelegt werden. Ebenso ist die Aktivierungsfunktion frei wählbar. ${ }^{630}$

Jedes Neuron in der Hidden-Ebene besitzt ein so genanntes Bias-Neuron, welches als Konstante in die jeweilige Aktivierungsfunktion einfließt. ${ }^{631}$ Dieses Bias-Neuron ist nicht mit einem Neuron der vorgelagerten Schicht verbunden und somit in der Lage unabhängig von den Eingangsinformationen einen konstanten Wert, d.h. einen bei

626 Vgl. Dorfner (1991), S. 36ff.

${ }^{627}$ Der Z-Wert stellt die lineare Transformation einer normalverteilten Zufallsvariablen X dar und berechnet sich nach der Gleichung als: Z-Wert $=(X-$ Erwartungswert) / Standardabweichung (vgl. Schierenbeck (1999), S. 34).

628 Die Normalisierung kann auch mit Hilfe der Minmax-Tabelle vorgenommen werden. Vgl. bspw. Uthoff (1997), S. 174f.

629 Vgl. hier und im Folgenden Uthoff (1997), S. 175ff.

630 Vgl. zur Vorgehensweise bei der Bestimmung dieser Parametereinstellungen Kap. 5.4.1, Kap. 5.4.3 und auch Kap. 5.4.5.

631 Vgl. Uthoff (1997), S. 176. 
Marktorientierte Kreditrisikobewertung - Empirische Untersuchung 142 zur marktorientierten Bewertung des Kreditrisikos mittels Künstlicher Neuronaler Netze

jedem Datensatz identischen Wert, in die Aktivierungsfunktion einfließen zu lassen. Im Rahmen der Lernphase (vgl. Kap. 5.2.2) wird der eingehende Wert ermittelt und vom Netz eigenständig optimiert. Häufig wird dieses Bias-Neuron, wie auch in Abbildung 36 und den folgenden Abbildungen, nicht explizit im Netzwerkdesign aufgefuihrt.

Die Ausgabeneuronen (im Weiteren mit oa ${ }_{k}$ gekennzeichnet) in der Ausgabeschicht empfangen die einzelnen Ausgabewerte der Neuronen in dem letzten vorgelagerten layer und transformieren diese in den Zielwert. Die Anzahl der Ausgabeneuronen richtet sich nach der Problemstellung. Ist bspw. ein Zielwert (Kreditrisikoprämie) gesucht, so handelt es sich um ein Ausgangsneuron. Während im Kap. 5.2.1 die Netzwerkstruktur dargestellt wurde, wird im weiteren Verlauf der Analyse der innerhalb dieser Struktur ablaufende Lernprozess beschrieben.

\subsubsection{Beschreibung des Lernprozesses (Error-Backpropagation-Algorithmus)}

Zweck des MLP ist es, die Muster zwischen beobachtbaren Datensätzen zu erkennen bzw. zu erlernen. Ein Datensatz stellt hierbei eine Sammlung von Informationen zu einem Objekt (bspw. einer Aktie) dar. Zwecks Mustererkennungen wird der ErrorBackpropagation-Algorithmus, ein Zwei-Phasen-Algorithmus, herangezogen ${ }^{632}$ In der ersten Phase bestimmt das Netz den Wert für einen Datensatz p. Basis dieser Wertbestimmung sind Gewichtungsfaktoren, wie sie in der ersten Phase (in der Regel zufallsbedingt) vorliegen. Diese ersten Gewichtungsfaktoren werden Initialgewichtungsfaktoren genannt. Der Wert des Ausgabeneurons $k$ für den Datensatz $\mathrm{p}\left(\mathrm{oa}_{\mathrm{pk}}\right)$ wird bestimmt, indem die hinter dem Datensatz $\mathrm{p}$ liegenden Informationen mittels Eingangsneuronen (bspw. $\mathrm{i}-1$ bis $\mathrm{i}+\mathrm{n}$ ) propagiert werden (Propagation-Phase, dabei stellt $\mathrm{P}$, mit $\mathrm{p}=1,2,3, \ldots, \mathrm{P}$, die Anzahl aller Datensätze dar). Hierzu werden schichtweise in jedem Neuron mittels der in Kap. 5.1.4 vorgestellten Berechnungsvorschrift die Ausgabewerte der einzelnen Neuronen berechnet. Die in den einzelnen Neuronen herangezogenen Gewichte ${ }^{633}$ werden mit $w_{i j}$ (Gewicht zwischen Eingabe- und versteckter Schicht) und $\mathrm{w}_{\mathrm{jk}}$ (Gewicht $\mathrm{zwischen}$ versteckter und Ausgabeschicht) bezeichnet. Abbildung 36 stellt die Propagation-Phase dar.

Vgl. hier und im Folgenden Füser (1994), S. 252ff. und auch Füser (1995), S. 58ff. Vereinfachungsgemäß wird eine versteckte Schicht mit genau einer Unterschicht (layer) unterstellt. 


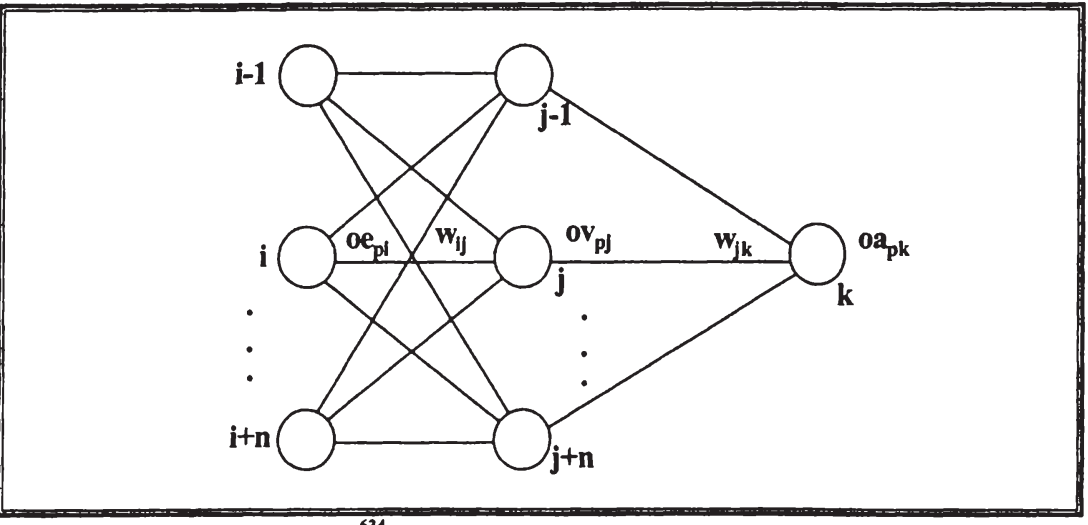

Abbildung 36: Propagation-Phase ${ }^{634}$

In der zweiten Phase wird mittels der messbaren Differenz aus dem Ausgabewert des Ausgabeneurons und dem beobachteten Istwert des entsprechenden Datensatzes eine Optimierung der verwendeten Gewichte erzielt. ${ }^{635}$ Hierzu wird der für den Datensatz $p$ durch das KNN bestimmte Wert oe $e_{\mathrm{pk}}$ mit dem beobachteten Wert (Zielwert) $t_{\mathrm{pk}}$ verglichen. ${ }^{636}$ Dieser Fehlerwert berechnet sich: ${ }^{637}$

a) für einen bestimmten Datensatz $p$ als $E_{p}=\frac{1}{2} \sum_{k}\left(t_{p k}-o e_{p k}\right)^{2}$. Der Proportionalitätsfaktor $1 / 2$ wurde wegen der vereinfachten Herleitung der Lernregel eingeführt, $\mathrm{k}$ ist für das vorliegende Beispiel gleich „1“

b) für alle Datensätze als: $E=\sum^{P} E_{p} \cdot{ }^{639}$

$$
\mathrm{p}=1
$$

In Anlehnung an Füser (1995), S. 59. angepasst werden, spricht man vom Error-Back-Algorithmus. Vgl. Rumelhart, Hinton, Williams (1986), S. 322ff.; Ritter, Martinetz, Schulten (1991), S. 53ff.; Zell (1994), S. 105ff.; Rojas (1992), S. 149ff.

Mit $t_{p}$ wird der aus Beobachtungen dokumentierte Zielwert hinsichtlich eines speziellen Trainingsmusters $p$ bezeichnet.

637 Vgl. hier und im Folgenden Füser (1995), S. 60f.

638 Vgl. Füser (1995), S. 60.

639 Vgl. Uthoff (1997, S. 177. 
Marktorientierte Kreditrisikobewertung - Empirische Untersuchung 144 zur marktorientierten Bewertung des Kreditrisikos mittels Künstlicher Neuronaler Netze

Lernen bedeutet, die Differenz über alle Datensätze zu vermindern, dass heißt durch die Anpassung der Gewichtungsfaktoren diesen über alle Datensätze resultierenden Fehlerwert zu minimieren. Aus der Differenz $\left(\left|t_{\mathrm{pk}}-\mathrm{oa}_{\mathrm{pk}}\right|\right)$ eines Trainingsmusters $\mathrm{p}$ an dem Output-Neuron k leitet sich systematisch der Korrekturvorgang für die Gewichtungsfaktoren ab. Ziel der einzelnen Modifikationen der Gewichte ist die Minimierung des Gesamtfehlers des Netzes. Schlussfolgernd kann man somit das Lernen des Netzes als Versuch der systematischen Fehlerminimierung bezeichnen. ${ }^{640}$

Es ist nun zu untersuchen, wie diese Fehlerminimierung bzw. Modifikation der Gewichte verläuft. Zunächst wird der Anpassungsprozess der Gewichte zwischen dem Ausgabeneuron und den Neuronen in der versteckten Schicht untersucht. Hierzu wird ein neuer Parameter $\eta$ eingeführt, der für die Gewichtsanpassung aufgrund des berechneten Fehlers verantwortlich ist. ${ }^{641}$ Je größer dieser Wert ist, umso schneller vollzieht sich die Anpassung. Deshalb wird dieser Parameter als Lernkoeffizient (oder Lernrate bzw. Lerngeschwindigkeit) bezeichnet. Als Korrekturwert für den Gewichtungsfaktor $\mathrm{w}_{\mathrm{jk}}$, der zwischen einem Neuron in der versteckten Schicht und dem Ausgabeneuron $\mathrm{k}$ liegt, gilt:

\section{Gleichung 27}

$\Delta \mathrm{w}_{\mathrm{jkp}}=\delta_{\mathrm{pk}} \cdot \eta \cdot \mathrm{o}_{\mathrm{pj}}, \mathrm{mit}_{\mathrm{pk}}=0 \mathrm{a}_{\mathrm{pk}} \cdot\left(1-\mathrm{oa}_{\mathrm{pk}}\right) \cdot\left(\mathrm{t}_{\mathrm{pk}}-\mathrm{oa}_{\mathrm{pk}}\right)^{642}$.

Der lokale Fehlerwert $\delta_{\mathrm{k}}$ eines Neurons $\mathrm{k}$ berechnet sich als Produkt aus der Differenz zwischen Ziel- und Ist-Wert $\left(\mathrm{t}_{\mathrm{pk}}-\mathrm{oa}_{\mathrm{pk}}\right)$ und der Ableitung der Aktivierungsfunktion ${ }^{643}$ $\left(\mathrm{oa}_{\mathrm{pk}} \cdot\left(1-\mathrm{oa}_{\mathrm{pk}}\right)\right)^{644}$ Typische Werte für den Parameter $\eta$ liegen zwischen 0,70 und $0,90 .^{645}$ Es muss somit festgehalten werden, dass der Wert der Lerngeschwindigkeit nicht von der Problemstellung abhängig ist, sondern vielmehr vom Netzwerkarchitekten festgelegt wird. Der beschriebene Anpassungsprozess der Gewichte bezieht sich auf Ausgabeneuronen, da hier der Fehler zwischen gewünschtem und errechnetem Output ableitbar ist. Bei Neuronen in der versteckten Schicht ist dies jedoch nicht direkt möglich. Hierzu muss man den Fehlerwert zurückrechnen, indem man: ${ }^{646}$

${ }_{640}$ Vgl. Rehkugler, Poddig (1992), S. 51.

641 Vgl. hier und im Folgenden Füser (1995), S. 61ff.

642 Vgl. Ritter, Martinetz, Schulten (1991), S. 54ff.; Füser (1995), S. 61.

${ }_{643}$ Vgl. Füser (1995), S. 28.

644 Wie oben bereits genannt, wird als Aktivierungsfunktion die Sigmoid-Funktion gewählt.

645 Vgl. Füser (1995), S. 61.

646 Vgl. hier und im Folgenden Füser (1995), S. 62 und auch Uthoff (1997), S. 178. Refenes erläutert, dass, falls alle Gewichte dieselben Ausgangsgewichtungsfaktoren - dies sind die in der Propagationphase unterstellten Gewichte - besitzen, es nicht zu einem Lerneffekt kommen wird. Vgl. Refenes (1995), S. 29. Deshalb ist es wichtig, dass die Startgewichte in der Propagationsphase zufallsverteilt oder mindestens verschieden sind. 
Marktorientierte Kreditrisikobewertung - Empirische Untersuchung zur marktorientierten Bewertung des Kreditrisikos mittels Künstlicher Neuronaler Netze 145

Gleichung 28

$\Delta w_{i j p}=\delta_{j p} \cdot \eta \cdot o_{p i}$, mit $\delta_{j p}=o_{p j} \cdot\left(1-o_{p j}\right) \cdot \sum_{k}\left(\delta_{p k} \cdot w_{j k}\right)$ bestimmt.

Der Fehlerwert des Neurons $\mathrm{k}$ in der Ausgabeschicht $\delta_{\mathrm{k}}$ wird herangezogen, um den Fehlerwert des Neurons $\mathrm{j}$ in der versteckten Schicht $\delta_{\mathrm{j}} \mathrm{zu}$ bestimmten. Dieses Verfahren stellt die Fehlerrückführungstechnik (Error-Backpropagation-Methodik) dar. Ein Lernschritt oder Lernzyklus ist dann abgeschlossen, wenn der Fehlerwert ,vollständig“, d.h. über alle Neuronen, zurückgerechnet und eine anschließende Gewichtsanpassung vollzogen wurde. Dementsprechend müssen für einen Lernschritt alle Datensätze $\mathrm{p}$ aus der Menge $[1 ; \mathrm{P}]$ das Netz durchlaufen haben.

Die Schritte des Error-Backpropagation-Algorithmuses werden so lange wiederholt, bis der Fehler des Netzes ausreichend klein ist. Es handelt sich hierbei um ,die Lösung eines nicht-linearen Optimierungsproblems. Der Optimierungsalgorithmus arbeitet nach dem Gradientenabstiegsverfahren. "647 Das Netz bewegt sich in die Richtung, in der die höchste Fehlerminimierung erreicht wird. Nach RoJAS wird die „Kombination derjenigen Gewichte eines Netzes, die den Berechnungsfehler minimiert, (...) als Lösung des Lernproblems betrachtet" ${ }^{\text {"648 }}$. Das optimale Ergebnis eines KNN kann dementsprechend als die Kombination von Gewichten gesehen werden, die den geringsten Gesamtfehler ausweist. Diese Gewichte werden im Weiteren als optimale Gewichte bezeichnet und ergeben in ihrer Kombination die optimale Netzstruktur. ${ }^{649}$ Das durch den Error-Backpropagation-Algorithmus unter zu Hilfenahme der Datensätze ,gelernte Wissen" spiegelt sich somit in den Gewichtungsfaktoren wider.

Inwieweit eine Struktur als optimale Struktur bezeichnet werden kann, ist fraglich. Nach Meinung des Verfassers können die Gewichte lediglich suboptimale Strukturen erzeugen, da durch eine sehr große Anzahl von Parametereinstellungen und Netzmodifikationen (vgl. Kap. 5.4) es nicht gewährleistet werden kann, dass alle möglichen Netzstrukturen getestet werden können. Eine suboptimale Struktur weist somit eine sehr gute Lösung auf, deren Fehlerwert in den Analysen nicht unterboten werden konnte. Wenn im Weiteren der Begriff „optimal“ verwendet wird, ist dieser immer als beschränkt optimal, d.h. suboptimal, zu verstehen. Es ist keine bessere Lösung bekannt, jedoch kann die Existenz dieser nicht ausgeschlossen werden.

647 Füser (1995), S. 65, vgl. auch Ritter, Martinetz, Schulten (1991), S. 43.

648 Rojas (1992), S. 149.

649 Als Netzstruktur wird im Weiteren die gesamte Netzkonfiguration bezeichnet. 
Marktorientierte Kreditrisikobewertung - Empirische Untersuchung 146 zur marktorientierten Bewertung des Kreditrisikos mittels Künstlicher Neuronaler Netze

Während diese Darstellung die grundsätzliche Vorgehensweise der Fehlerkorrektur bzw. der Gewichtsanpassung erklärt, wird zwecks Performanceverbesserung ${ }^{650}$ ein weiterer Parameter in den Anpassungsprozess eingefügt. ${ }^{651}$ Das so genannte Momentum $\alpha$ berücksichtigt den historischen Lernanpassungsprozess. ${ }^{652}$ Dies bedeutet im Einzelnen, dass die Gewichtungsfaktorenanpassung (d.h. die Veränderung des einzelnen Gewichtes w) im vorausgegangenen Lernschritt $(L-1)$ für den aktuellen Lernschritt (L) Verwendung findet. Während bisher die Gewichtsanpassung zwischen zwei Neuronen sich ausschließlich an dem Fehlerwert des jeweiligen Neurons $\delta$ orientiert hat, wird durch die Erweiterung um den Summanden bestehend aus dem Produkt von Momentum und Gewichtsveränderung des vorangegangenen Lernschrittes eine Orientierung an den letzten Error-Backpropagation-Vorgang geschafft. Somit ergibt sich die rekursive Anpassung der Gewichtungsfaktoren am Beispiel eines Datensatzes $p$ als:

Gleichung 29

$\Delta \mathrm{w}_{\mathrm{ijp}}(\mathrm{L})=\delta_{j p} \cdot \eta \cdot \mathrm{o}_{\mathrm{pj}}+\alpha \cdot \Delta \mathrm{w}_{\mathrm{ijp}}(\mathrm{L}-1)^{653}$.

\subsubsection{Bestimmung der Datenaufteilung}

Während in den vorstehenden Ausführungen die Datensätze als gegeben betrachtet wurden, wird im Folgenden auf die Behandlung der Datengrundlage eingegangen. In den Beschreibungen zum Lernprozess wurden alle Datensätze $[1 ; \mathrm{P}]$ herangezogen, um das Lernen in den Neuronen zu ermöglichen. Im Trainings- oder Lernprozess wird mit den oben dargestellten Verfahren eine Funktion abgeleitet, die zwischen einer Menge an Inputfaktoren und einem Ausgangswert einen Bezug herstellt. Diese wird mittels Fehleroptimierung solange modifiziert, bis die Abweichungen zwischen dem errechne-

650 Unter Performance wird in der vorliegenden Arbeit die Lernzeit des KNN verstanden. Als Lernzeit wird die Zeitdauer bezeichnet, die das KNN benötigt, um die Gewichtsanpassungen vorzunehmen. Insbesondere von den Parametereinstellungen und den informationstechnologisch bedingten Voraussetzungen sind Lernzeiten von vielen Stunden bis hin zu Tagen zu beobachten. Deshalb weist sich ein besonders gutes Netz sowohl durch einen minimalen Fehlerwert als auch eine kurzen Lernzeit aus. Falls man einen der beiden Zielwerte (niedrige Bearbeitungszeiten oder Fehlerminimierung) stärker gewichten muss, so sollte man nach Meinung des Verfassers der Fehlerminimierung eine größere Gewichtung zukommen lassen, da insbesondere durch die technologische Weiterentwicklung kürzere Lernzeiten zu erwarten sind.

651 Diese Modifikation wird erläutert, da sie in der hier verwendeten empirischen Anwendung benutzt wurde. In der Literatur werden darüber hinaus weitere Vorschläge zur Performanceverbesserung angeführt. Vgl. bspw. Rojas (1992), S. 168ff.

652 Vgl. hier und im Folgenden bspw. Köhle (1990), S. 97, Kohonen (1991), S. 617ff.; Füser (1995), S. 71.

653 Vgl. Füser (1995), S. 71 und die dort dargestellten Anpassungsprozesse für Neuronen in der versteckten Schicht. 
Marktorientierte Kreditrisikobewertung - Empirische Untersuchung zur marktorientierten Bewertung des Kreditrisikos mittels Künstlicher Neuronaler Netze 147

ten und dem tatsächlichen Wert minimal sind. Fraglich ist nun, ob diese Zusammenhänge auch auf einen Datensatz übertragbar sind, der nicht in den Lernprozess einbezogen wurde. Hierzu wird die Inputdatenmenge in zwei Mengen aufgeteilt:654 Die Lernmenge, mit den Trainingsdaten, (Feststellung der optimalen Gewichte) und die Testmenge, mit den Generalisierungsdaten, (Überprüfung der optimalen Gewichte). Somit lässt sich die gesamte Datenmenge in Lern- und Testdaten aufteilen. Die oben beschriebenen Algorithmen bewirken eine Optimierung der Gewichte hinsichtlich der Fehlerminimierung für die Lerndaten. Gewünscht ist jedoch nicht ein möglichst geringer Fehler für die Lerndaten, sondern vielmehr eine „fehlerfreie“ Abbildung der Testdaten, weil hierdurch eine generalisierte Aussage (Modellbildung) erst möglich wird. Falls man ein Netz trainiert, wird sich der Gesamtfehler stetig verringern. Falls man eine reine Optimierung des Fehlers der Trainingsdaten als Zielsetzung verfolgt, würde man unendlich viele Lernzyklen durchführen. Hier wird die Gefahr des „overlearning“ deutlich. Naturgemäß reduziert das Netz im Lernprozess den Gesamtfehler mit Bezug auf die Lernmenge kontinuierlich. Die Gewichtsanpassungen werden ausschließlich in Bezug auf die Trainingsdaten optimiert. Sobald diese Gewichtsanpassungen zwar den Fehlerwert der Trainingsdaten reduzieren können, jedoch nicht den der unbekannten Datensätze (Generalisierungsfrage), spricht man vom „Auswendiglernen der Trainingsdaten"6s5s. Um diesen nicht erwünschten Effekt zu vermeiden, wird ein dritter Datenbereich eingefügt: die Validierungsdaten. Aufgabe dieser ist die Festlegung des ,early-stopping-Zeitpunktes", nämlich des Zustandes, ab dem sich der Fehlerwert der Validierungsdaten nicht weiter reduziert lässt. Die Validierungsdaten sind kein Bestandteil des Lernprozesses, d.h. sie wirken nicht auf die Gewichtsveränderungen ein, sondern sie dienen dazu, den Fehlerwert an für das KNN unbekannten Datensätzen zu messen. Die Testmenge dient ausschließlich zur Fehlermessung. Dies bedeutet, dass die mit Hilfe der Lernmenge gefundene bestmögliche Netzstruktur an dieser Menge gemessen und die Güte des KNN gemessen durch den Fehlerwert anhand dieser Datensätze bestimmt wird. Abbildung 37 zeigt die drei Datenbereiche auf und ordnet die Begriffe Trainings-, Validierungs- und Generalisierungsdaten den Begriffspaaren Lern- und Testmenge zu.

$654 \mathrm{Vgl} \mathrm{hier} \mathrm{und} \mathrm{im} \mathrm{Folgenden} \mathrm{Füser} \mathrm{(1995),} \mathrm{S.} \mathrm{75ff.}$

6ss Füser (1995), S. 76. 


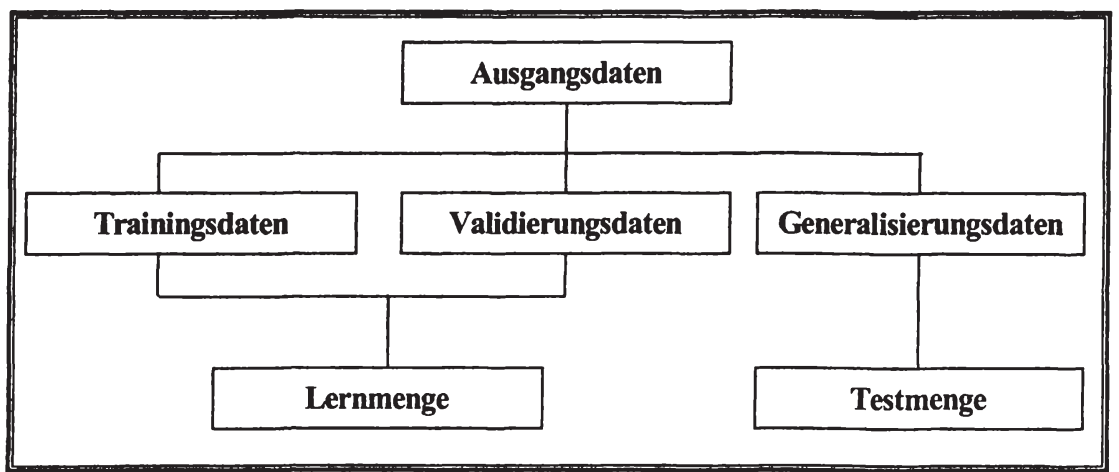

Abbildung 37: Aufteilung der Ausgangsdaten in die unterschiedlichen Datenbereiche

Die optimale Netzstruktur wird an dem „early-stopping-Zeitpunkt“ festgemacht, indem der Fehlerwert fortlaufend für die Trainings- und Validierungsdaten berechnet wird. Das optimale Netz (die optimalen Gewichte) liegt im Minimum der Fehlerfunktion der Valdierungsdaten vor. ${ }^{656}$ Abbildung 38 verdeutlicht das Kurvenverhalten.

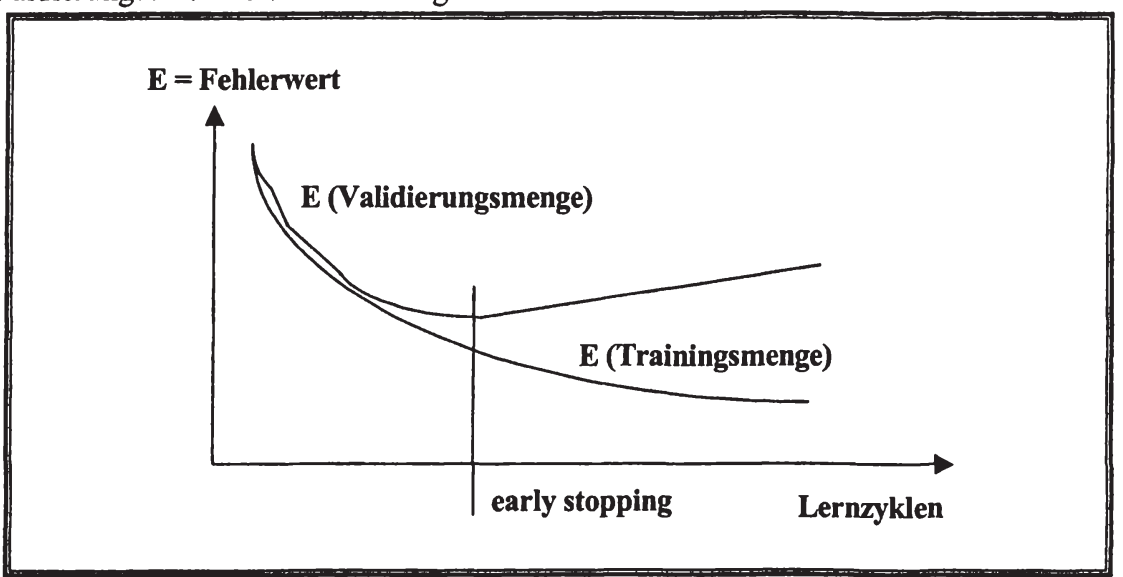

Abbildung 38: Schematischer Verlauf von Trainings- und Validierungsfehlern in Abhängigkeit der LernzykJen $^{657}$

656 Vgl. Schiffmann (1999), S. 48f.

657 In Anlehnung an Schiffmann (1999), S. 48. 
Marktorientierte Kreditrisikobewertung - Empirische Untersuchung zur marktorientierten Bewertung des Kreditrisikos mittels Künstlicher Neuronaler Netze

\subsection{Datenbasis, Datenherkunft und Ableitung des Untersuchungsdatensatzes}

Nachdem in Kap. 5.1 dargestellt wurde, dass KNN im Allgemeinen sich als Analyseinstrument für das im Kap. 4.4 dargestellte Konzept eines Prognosemodells eignen, wurde im Kap. 5.2 die wohl bekannteste Netzstruktur, das Multi-Layer-Perceptron, in Kombination mit dem Error-Backpropagation-Algorithmus vorgestellt. Um dieses Instrument einsetzen zu können, ist das Vorliegen einer Datenmenge zwingend erforderlich, da das KNN die in den Daten enthaltenen Muster erlernen soll. Wie im Kap. 4.4 dargestellt wurde, soll das $\mathrm{KNN}$ als Analyseinstrument herangezogen werden, um die Zusammenhänge zwischen unternehmensinternen und - externen Faktoren und eines bewerteten Kreditrisikos (Credit Spread) zu analysieren. Im Weiteren werden nun die von der REUTERS GMBH bereitgestellten Datensätze und die für die Untersuchung aufbereitete Datenmenge erläutert. ${ }^{658 / 659}$ Zunächst wird der Datenbestand dargestellt und mittels deskriptiver Statistikkennzahlen beschrieben. Anschließend werden die in der Untersuchung herangezogenen Einflussfaktoren abgeleitet. In dem darauffolgenden Kapitel wird abschließend die Zielgröße, der Credit Spread, bestimmt.

\subsubsection{Aufbau und Struktur des Datenbestandes}

Der Datenbestand setzt sich schwerpunktmäßig aus zwei Datenbankabfragen zusammen. Zunächst wurden die Schlusskurse von gehandelten amerikanischen Industrieanleihen jeweils zum 15. April der Jahre 1995 bis 1999 ermittelt. ${ }^{660}$ Es wurde der 15. April gewählt, damit die bis zu diesem Zeitpunkt veröffentlichten relevanten Bilanzinformationen der Anleihenemittenten gemäß der unterstellten Effizienzhypothese in dem Marktpreis der Anleihe verarbeitet waren (mittel-strenge Effizienz $z^{661}$ ). Bei den ermittelten Kursen der Industrieanleihen handelt es sich um den Ask Price. ${ }^{662}$ Bei den untersuchten Wertpapieren handelt es sich um Titel, die weder eine Garantie $e^{663}$ noch ein Kündigungsrecht besitzen und bei denen alle Zahlungen in US-Dollar vereinbart sind.

658 An dieser Stelle dankt der Verfasser der Reuters GmbH zur Bereitstellung der Daten.

659 Bei der folgenden Datenbeschreibung und der folgenden Darstellung der Ergebnisse der KNN-Analyse wurde die Anwendung SPSS ${ }^{\circledR}$ in der Version 10.0 verwendet.

660 Falls der 15. April kein Börsentag war, wurde der nächstfolgende Börsentag gewählt.

661 Vgl. Kap. 4.2.

662 Der Unterschied zwischen Kauf- und Verkaufskurs ist als Händlerspanne zu deuten. Alle im weiteren Verlauf folgenden Preisgrößen beziehen sich auf den Ask Price (Kaufkurs).

663 Garantien werden bei Anleihen von Dritten ausgesprochen und stellen somit eine Art der Besicherung dar. Vgl. zu den verschiedenen Besicherungsformen Perridon, Steiner (1997), S. $301 \mathrm{ff}$. Keine Anleihe in der Untersuchung wies eine Besicherungsform auf, die über die gesetzlichen Mindestbesicherungen hinausgeht. 
Marktorientierte Kreditrisikobewertung - Empirische Untersuchung 150 zur marktorientierten Bewertung des Kreditrisikos mittels Künstlicher Neuronaler Netze

Folgende Wertpapierinformationen wurden ausgewiesen:

- Ticker (Kürzel des Emittenten),

- Committee on Uniform Securities Identification Procedures (CUSIP, Wertpapierspezifische Identifkationsnummer),

- Ask Price bzw. Yield (effektive Verzinsung der Anleihe), ${ }^{664}$

- Cupon (Nominalzins),

- Maturity (Fälligkeitstermin), Restlaufzeit (Fälligkeitstermin - Stichtag),

- Emissionsvolumen der Anleihe und

- Branchenzuordnung.

Wegen deutlicher Unterschiede in den Bilanzierungsvorschriften sowohl innerhalb der Vorschriften des US-GAAP als auch des HGBs, werden die Anleihen von Unternehmen aus dem Finanzdienstleitungssektor von der Untersuchung ausgeklammert. ${ }^{665} \mathrm{Im}$ Datenhaushalt der REUTERS GMBH werden lediglich die Bilanzdaten von aktiennotierten Unternehmen abgespeichert, sodass alle in die Untersuchung einfließenden Unternehmen aktiennotiert sind. ${ }^{666}$ Alle Bilanzen sind nach den US-GAAP Vorschriften aufgestellt. Folgende Informationen konnten ermittelt werden:

- Allgemeine Unternehmensdaten: Ticker, Organisation Full Name, Record Date, Pay Date, Announcement Date, Short Name of Organisation, Description of Company Activities, First Line of Address, Second Line of Address, Third Line of Address, Accounts Format ID, Period End Date, Report Period Year.

- Bilanzzahlen und weitere Größen aus dem Jahresabschluss: Revenue, Other Income, Operating Costs, Depreciation (Profit and Loss), Operating Result (Profit and Loss), Net Interest, Pretax Profit or Loss, Tax, Net Profit or Loss, Preference Dividends, Average Shares, Equity Issued, Total Current Assets, Cash and Equivalents, Receivables, Inventories, Fixed Assets, Total Assets, Total Liabilities, Current Liabilities, Long Term Debt, Deferred Liabilities, Shareholder's Equity, Preferred Stock, Net Cash Operating, Other Income, Exchange Rate Effect, Net Income or Loss, Depreciation and Amortisation, Price Earnings Ratio, Price to Book Ratio, Dividend Yield, Net Cash Investing.

Der Autor vermutet, dass Reuters die marktübliche ISMA-Methodik (vgl. Nöth, (1995), S. 541f.) anwendet. Ein entsprechender Hinweis findet sich bei der Produktdokumentation Reuters (1999), S. 32.

Diese exogen bestimmte Einschränkung der Datengrundlage ist als besonders kritisch anzusehen, falls die Tatsache der Börsennotierung einen eigenständiger Einflussfaktor auf den Credit Spread darstellt. 
Marktorientierte Kreditrisikobewertung - Empirische Untersuchung zur marktorientierten Bewertung des Kreditrisikos mittels Künstlicher Neuronaler Netze

Insgesamt konnte in 1666 Fällen ein Datensatz bestehend aus Bilanzinformationen und dem Anleihenkurs zum 15. April des entsprechenden Jahres generiert werden. Die zu den Kursen korrespondierenden Bilanzdaten beziehen sich jeweils auf das Vorjahr, d.h. einem Anleihenkurs vom 15. April 1995 wurden die Bilanzdaten des Geschäftsjahres 1994 zugeordnet.

Es lassen sich einzelne Unternehmen identifizieren, die über den gesamten Untersuchungszeitraum nur zu bestimmten Stichtagen Anleihen auf dem Markt platziert haben, und andere wiederum, welche über den gesamten Zeitraum zu jedem Zeitpunkt mit mehreren verschiedenen Anleihen auf dem Markt präsent waren. Diese Informationen sind bei Stichprobenziehung (vgl. Kapitel 5.4.2) von besonderer Relevanz. Insgesamt handelt es sich um 136 verschiedene Unternehmen bzw. um 345 verschiedene Unternehmensbilanzen. Die Abbildung 39 zeigt eine Verteilung der untersuchten Unternehmen hinsichtlich der Branchenzugehörigkeit. Unternehmen aus der Chemiebranche sind am hăufigsten, unterproportional sind Unternehmen aus den Bereichen der Schifffahrt, Textilien und elektronischen Ausstattung vertreten. ${ }^{667}$

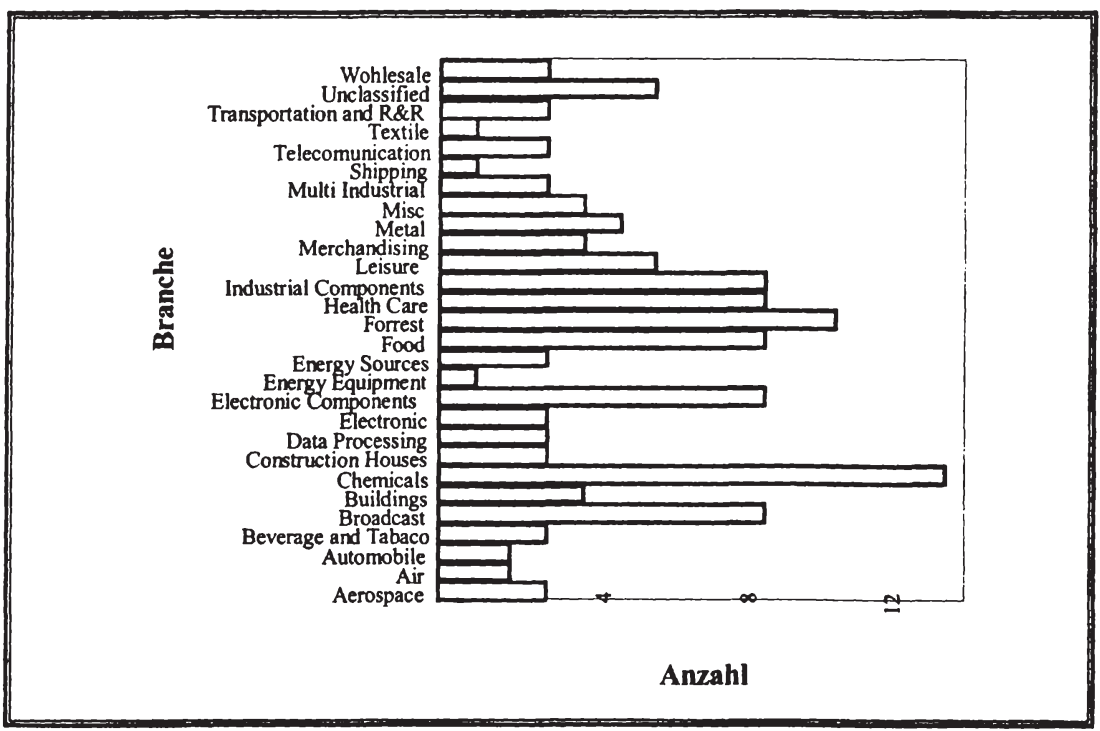

Abbildung 39: Branchenverteilung der untersuchten Unternehmen

Innerhalb der analysierten Unternehmen überwiegen große Firmen, was durch eine durchschnittliche Bilanzsumme von ca. 16,615 Milliarden US-Dollar verdeutlicht

667 Die Brancheneinteilung ist von der Reuters $\mathrm{GmbH}$ vorgenommen worden. Eine detaillierte Darstellung findet sich im Anhang. 
Marktorientierte Kreditrisikobewertung - Empirische Untersuchung 152 zur marktorientierten Bewertung des Kreditrisikos mittels Künstlicher Neuronaler Netze

wird. Dies wird darin bestätigt, dass 75 Prozent der Unternehmen eine Bilanzsumme größer 12,141 Milliarden US-Dollar aufweisen (vgl. Tabelle 13).

\begin{tabular}{|c|c|c|}
\hline & & Angaben in US Dollar \\
\hline \multirow{6}{*}{$\begin{array}{l}\text { Bilanzsumme: Es sind Unter- } \\
\text { nehmen aus allen Branchen } \\
\text { untersucht worden (außer } \\
\text { Versicherungen und Banken). }\end{array}$} & Mittelwert & $16.615 .838 .168,60$ \\
\hline & Median & $5.445 .000 .000,00$ \\
\hline & Standardabweichung & $37.592 .634 .352,85$ \\
\hline & Minimum & $18.375 .000,00$ \\
\hline & Maximum & $279.097 .000 .000,00$ \\
\hline & Interquartilbereich & $12.141 .975 .000,00$ \\
\hline
\end{tabular}

Tabelle 13: Deskriptive Kennzahlen der Untersuchungsmenge hinsichtlich des Merkmales „Bilanzsumme"

\subsubsection{Ableitung von Bilanzkennzahlen und weiterer Inputfaktoren}

Wie in der unter Kapitel 4.4 dargestellten Konzeption eines Prognosemodells dargestellt wurde, werden sowohl unternehmensinterne als auch externe Faktoren zur Bestimmung des Credit Spread benötigt. Die Bilanzkennzahlen bilden die unternehmensinternen Kennzahlen. Es wird nicht als Ziel angesehen, neue Kennzahlen zu generieren, sondern aus den bereits heute in der Praxis verwendeten eine Auswahl unter zu Hilfenahme des KNN zu treffen. Somit ist es notwendig, auf Basis der Inputdaten (zunächst die reinen Bilanzkennzahlen) die in Kapitel 3 dargestellten und in Tabelle 14 zusammengefassten Kennzahlen zu bilden. Hierbei stellt der Detaillierungsgrad der von REUTERS bereitgestellten Daten eine entscheidende Restriktion bei der Kennzahlenbildung dar. Ein weiteres Problem besteht in den (teilweise) unterschiedlichen Rechnungslegungsvorschriften. Dieses Problem lässt sich ebenfalls aufgrund des Detaillierungsgrades der Ausgangsdaten nicht in allen Fällen lösen.

Insgesamt werden 52 Bilanzkennzahlen ausgewiesen, die entweder analog zu einem der oben genannten Analyseinstrumente berechnet oder in Anlehnung an eine der Vorgehensweisen (aufgrund von Informationsdefiziten) gebildet wurden. AnschlieBend wird eine partielle Korrelationsanalyse durchgeführt, um die Inputfaktoren um bestimmte Eingangsinformationen zu reduzieren. Diese Inputfaktorenreduktion greift auf die Problematik der zu großen Anzahl von Eingangsneuronen innerhalb der Bildung von Neuronalen Netzen zurück. Falls in einem Datensatz zwei Eingangsneuronen dieselben Informationen enthalten, d.h. es liegt eine vollständige Korrelation 
Marktorientierte Kreditrisikobewertung - Empirische Untersuchung zur marktorientierten Bewertung des Kreditrisikos mittels Künstlicher Neuronaler Netze 153

\begin{tabular}{||l|l|l||}
\hline \multicolumn{1}{|c|}{ Bezeichnung } & $\begin{array}{c}\text { Beschreibung } \\
\text { im Kapitel }\end{array}$ & $\begin{array}{c}\text { Darstellung in Tabelle, } \\
\text { Abbildung oder Gleichung }\end{array}$ \\
\hline Allgemeine Kennzahlen $\left(\mathrm{K}_{\mathrm{I}}\right.$ bis $\left.\mathrm{K}_{70}\right)$ & Kap. 3.3.1 & Tabelle 6 \\
\hline Rentabilitäts-Liquiditätssystem & Kap. 3.3.2 & Abbildung 20 \\
\hline Backpropagation-14/ Baetge-Bilanz-Rating-14 & Kap. 3.4.2 & Tabelle 9 \\
\hline Z''-Score & Kap. 3.4.1 & Tabelle 8, Gleichung 23 \\
\hline DuPont-Kennzahlensystem / ROI-System & Kap. 3.3.2 & Abbildung 19 \\
\hline Risiko-Sicherheit-Wachstum-Verfahren & Kap. 3.3.3 & Tabelle 7 \\
\hline Saarbrücker-Modell & Kap. 3.3.3 & Abbildung 21 \\
\hline
\end{tabular}

Tabelle 14: Ableitung der untersuchten Kennzahlen

zwischen den korrespondierenden Eingangsinformationen vor, dann besitzt dieser zweite Faktor keine neue Information für das $\mathrm{KNN}$ und er ist für die KNN-Analyse unnötig. Diese unnötigen Faktoren verlangsamen die Lernzeit der KNN und können gleichzeitig nicht dazu beitragen, eine bessere Lösung zu finden. Im Gegenteil besteht sogar die Gefahr, dass das KNN nicht die wahren Strukturen aufgrund von zu vielen unnötigen Informationen erlernen kann. ${ }^{668}$

Als Schwellenwert für die Definition als „gleiche Information“ fungiert ein Korrelationskoeffizient nach PEARSON ${ }^{669}$ zwischen zwei Eingangsinformationen von 0,95. Der Grund liegt darin, dass durch die hohe Korrelation die Informationen des einen Inputfaktors auch innerhalb eines zweiten enthalten sind. Bei Paaren mit dieser hohen Korrelation wird einer der beiden Inputfaktoren (zufallsbedingt) ausgewählt und von der weiteren Analyse ausgegrenzt. ${ }^{670}$ Ein Beispiel furr eine deutliche Korrelation ist die Kennzahl Eigenkapitalrentabilität vor und nach Steuern. Hier liegt eine partielle Korrelation von 0,974 mit einer zweiseitigen Signifikanz auf einem Niveau von 0,01 vor. Nach der Reduktion werden die folgenden Kennzahlen $\left(B_{1}-B_{26}\right)$ in die weitere Untersuchung einbezogen:

668 Vgl. bspw. Glormann (2001), S. 246f.

${ }_{669}$ Vgl. Hartung, Elpelt (1989), S. 145ff.; oder auch Bamberg, Baur (1996), S. $36 \mathrm{ff}$.

670 Unterstellt wird hierbei, dass beide Faktoren eine gleich große Anzahl von fehlenden Datensätzen aufweisen (vgl. Kapitel 5.3.4). Falls dies nicht der Fall ist, wird der Faktor mit der höheren Anzahl ausgeklammert. 
Marktorientierte Kreditrisikobewertung - Empirische Untersuchung 154 zur marktorientierten Bewertung des Kreditrisikos mittels Künstlicher Neuronaler Netze

\begin{tabular}{|c|c|}
\hline$B_{n}$ & Berechnungsvorschrift \\
\hline$B_{1}$ & Revenue / Inventories \\
\hline$B_{2}$ & Revenue / Total Assets \\
\hline$B_{3}$ & Current Liabilities / Shareholder's Equity \\
\hline$B_{4}$ & Current Liabilities / Total Assets \\
\hline$B_{5}$ & Shareholder's Equity / Equity Issued \\
\hline$B_{6}$ & Net Cash Operating / (Shareholder's Equity + Long Term Debt) \\
\hline $\mathrm{B}_{7}$ & Current Liabilities / Revenue \\
\hline $\mathrm{B}_{8}$ & Operating Result / Income bzw. Loss Before Tax \\
\hline $\mathrm{B}_{9}$ & Operating Result / Revenue \\
\hline $\mathrm{B}_{10}$ & Income bzw. Loss Before Tax / Equity Issued \\
\hline $\mathrm{B}_{11}$ & Net Cash Operating / Equity Issued \\
\hline $\mathrm{B}_{12}$ & Cash And Equivalents \\
\hline $\mathrm{B}_{13}$ & Net Cash Operating / Current Liabilities \\
\hline $\mathrm{B}_{14}$ & Revenue / Shareholder's Equity \\
\hline $\mathrm{B}_{13}$ & (Total Current Assets - Current Liabilities)/ Total Assets \\
\hline $\mathrm{B}_{16}$ & (Income bzw. Loss Before Tax + Interest) / Total Assets \\
\hline $\mathrm{B}_{17}$ & Income bzw. Loss Before Tax / Shareholder's Equity \\
\hline $\mathrm{B}_{18}$ & Income bzw. Loss Before Tax / Revenue \\
\hline $\mathrm{B}_{19}$ & Net Cash Operating Before Tax \\
\hline $\mathrm{B}_{20}$ & Total Liabilities / Net Cash Operating \\
\hline $\mathrm{B}_{21}$ & Inventories - Current Liabilities \\
\hline $\mathrm{B}_{22}$ & Cash And Equivalents / Current Liabilities \\
\hline $\mathrm{B}_{23}$ & Shareholder's Equity / Total Assets \\
\hline $\mathrm{B}_{24}$ & Net Cash Operating Before Tax / Revenue \\
\hline $\mathrm{B}_{25}$ & Net Cash Operating Before Tax / Total Assets \\
\hline $\mathrm{B}_{26}$ & (Long Term Debt + Shareholder's Equitiy) / Fixed Assets \\
\hline
\end{tabular}

Tabelle 15: In der Untersuchung berücksichtigte Bilanzkennzahlen ${ }^{671}$

671 Die Position „Revenues and expenses“ ist die vergleichbare Position der betriebsbedingte Erträge und Aufwendungen i.e.S.; „Inventories“ ist eine Bilanzposition für die Vorräte, die als Umlaufvermögen gewertet werden und somit der Position „current assets“ zugeordnet werden; die Position „current liabilities“ gibt die kurzfristigen Verbindlichkeiten wieder, die innerhalb des nächsten Wirtschaftsjahres fällig werden; „shareholders' equity" ist die Summe aus dem gezeichneten Kapital, den Kapitalrücklagen und den Gewinnrücklagen; die Position ,net cash operating“" entspricht den Einzahlungen resultierend aus der gewöhnlichen Geschäftstätigkeit; die Position „operating result" entspricht dem betriebsbedingten Ergebnis; die Position „cash and equivalents“ bildet Geldmittel und geldmittelähnliche Zahlungsgrößen ab; die Position „long term debt" entspricht einer Sammelposition für Verbindlichkeiten, die nicht im kommenden Wirtschaftsjahr fällig sind. Vgl. für die Definitionen Gräfer (1992), S. 17ff. und Glormann (2000), S. 69ff. 
Marktorientierte Kreditrisikobewertung - Empirische Untersuchung zur marktorientierten Bewertung des Kreditrisikos mittels Künstlicher Neuronaler Netze

Neben den unternehmensinternen Faktoren wurden bei der Darstellung des Prognosemodells weitere Einflussfaktoren bestimmt (vgl. Abbildung 28). Diese Faktoren kann man in die Ebenen makroökonomische und titelspezifische Kennzahlen einteilen (vgl. auch Kap. 4.3.3.3). Makroökonomisch bedeutet, dass dieser Einflussfaktor sich auf die Bewertung aller Anleihen auswirkt, titelspezifische Faktoren wirken sich lediglich bei der Bewertung auf die mit der Kennzahl korrespondierende Anleihe aus. In Anlehnung an die in Kap. 4.3.3.3 dargestellten kapitalmarkttheoretischen, empirischen Untersuchungen werden die folgenden Messgrößen gewählt: ${ }^{672 / 673}$

\begin{tabular}{||l|l|l|l|}
\hline \multicolumn{1}{||c|}{ Einflussfaktor } & \multicolumn{1}{c|}{ Abk. } & \multicolumn{1}{c|}{ Makrovariable } \\
\hline \multicolumn{3}{|c|}{ Messgröße } \\
\hline Zinsvolatilität des Marktes & $\mathbf{M}_{\mathbf{1}}$ & 10-Tages-Standardabweichung des risikolosen Zinsniveaus. ${ }^{674}$ \\
\hline Marktzinsniveau & $\mathbf{M}_{2}$ & $\begin{array}{l}\text { Individuelles, d.h. kupon- und laufzeitorientiertes, risikoloses } \\
\text { Zinsniveau. }\end{array}$ \\
\hline Zinserwartung & $\mathbf{M}_{3}$ & $\begin{array}{l}\text { Kursdifferenz aus einer risikolosen Anleihe mit 10,5 und 0,5 } \\
\text { Jahren Restlaufzeit, wobei der Nominalzins konstant bei 6\% liegt. }\end{array}$ \\
\hline \multicolumn{3}{|c|}{ Titelspezifische Kennzeichen } \\
\hline Marktliquidităt des Papiers & $\mathbf{T}_{1}$ & Emissionsvolumen der Anleihe in Mio. US\$. \\
\hline Restlaufzeit der Anleihe & $\mathbf{T}_{2}$ & $\begin{array}{l}\text { Differenz zwischen Stichtag und Fälligkeitstag der Anleihe in } \\
\text { Jahren. }\end{array}$ \\
\hline Nominalverzinsung & $\mathbf{T}_{3}$ & Nominalverzinsung in Prozent vom Nominalwert der Anleihe. \\
\hline
\end{tabular}

Tabelle 16: Zusammenfassung der bilanzunabhăngigen, unternehmensexternen Kennzahlen

672 Die Bonitätsprämie wird annahmegemäß nicht über den Faktor Zeit erklărt, sodass Autokorrelationen zwischen einzelnen Faktoren und dem Credit Spread auszuschließen sind. Falls die Einflussfaktoren nicht unabhängig voneinander sind, werden bei der $\mathrm{KNN}$ Analyse keine Verzerrungen erzeugt, da ein großer Vorteil der KNN in der Lösung von den restriktiven Voraussetzungen der linearen Separabilität und der Unabhängigkeit der Inputfaktoren liegt. Vgl. Bröker (2000), S. 62.

673 Als Indexrendite zur Berechnung der risikolosen Rendite wird auf die Indexbildung im Rahmen der später folgenden Credit Spread Bestimmung zurückgegriffen.

$674 \mathrm{Zu}$ dieser Vorgehensweise vgl. Thompson, Vaz (1990), S. 460; Heinke (1998), S. 323.

Die Standardabweichung berechnet sich als: $s_{t}=\left[\frac{1}{9} \cdot \sum_{j=t-10}^{t-1}\left(r_{j}(T, c)-\frac{\sum_{j=t-10}^{t-1} r_{j}(T, c)}{10}\right)^{2}\right.$,

mit $s_{t}=10$-Tages-Standardabweichung täglicher Differenzen der Indexrenditen in Prozent. Die Herleitung der Indexrendite wird im Rahmen der Bestimmung des Credit Spread erklärt. 
Marktorientierte Kreditrisikobewertung - Empirische Untersuchung 156 zur marktorientierten Bewertung des Kreditrisikos mittels Künstlicher Neuronaler Netze

Somit ergibt sich folgende Struktur für die Inputfaktoren:

- $\mathrm{B}_{1}$ bis $\mathrm{B}_{26}$ : Unternehmensindividuelle Bilanzkennzahlen,

- $\mathbf{M}_{1}$ bis $\mathbf{M}_{3}$ : Makroökonomische Kennzahlen und somit stichtagsabhängig,

- $\mathrm{T}_{1}$ bis $\mathrm{T}_{3}$ : Titelspezifische Kennzahlen und somit anleiheabhängig.

\subsubsection{Ermittlung des Credit Spread}

Ziel der Arbeit ist die Untersuchung des Zusammenhangs zwischen den verschiedenen Inputfaktoren und der Risikoprämie. Während die Inputfaktoren zunächst abgeleitet wurden, wird nun die quantifizierte Risikoprämie ermittelt.

Es besteht die Notwendigkeit der Isolierung des Credit Spread aus der effektiven Rendite. Diese wurde als Rendite nach der ISMA-Methode bestimmt. Im Kap. 4.3.3.2 wurden verschiedene Verfahren zur Berechnung des Credit Spread vorgestellt. Eine Möglichkeit besteht in der Bestimmung eines Indexes, welcher zu jedem KuponRestlaufzeit-Paar eine entsprechende Rendite ausweist. Der Index leitet sich aus einer Menge an risikolosen Wertpapierrenditen ab, indem eine Regressionsfunktion gebildet wird. Auch in der vorliegenden Analyse wird eine Bestimmung des Credit Spread unter zu Hilfenahme einer Menge an risikolosen Wertpapieren gebildet. Hierzu wurden die Wertpapierrenditen von US-Staatsanleihen zu jedem der untersuchten Stichtage ermittelt. ${ }^{675}$

In der vorliegenden Arbeit wird ein Index gemäß des REX ${ }^{\circledast}$-Rentenindexes gebildet: ${ }^{676}$ Gleichung 30

$\mathrm{r}_{\mathrm{B}, \mathrm{t}}(\mathrm{J}, \mathrm{C})=\beta_{1}+\beta_{2} \cdot \mathrm{J}+\beta_{3} \cdot \mathrm{J}^{2}+\beta_{4} \cdot \mathrm{J}^{3}+\beta_{5} \cdot \ln (\mathrm{J})+\beta_{6} \cdot \mathrm{C}+\beta_{7} \cdot \mathrm{C}^{2}$

mit $\quad r_{B, t}(J, C)=$ Bonitätsrisikolose Rendite in $t$ für eine Restlaufzeit von $J$ Jahren und einem Kupon von C Prozent,

$\beta_{i}=\quad$ Regressionskoeffizienten, mit $\mathrm{i}=[1 ; 7]{ }^{677}$

Diese Regressionsgleichung bildet den Zusammenhang zwischen Restlaufzeit, Kupon und effektiver Rendite ab. Die Rendite $r_{\mathrm{B}, \mathrm{t}}$ ist lediglich eine „virtuelle“ oder ,synthetische" Rendite, da kein faktisches Wertpapier diese Rendite ausweist, sondern diese aus der Menge von verschiedenen Staatsanleihen gebildet wird. In der vorliegenden Arbeit wird diese Funktion des REX $^{\circledR}$-Rentenindexes verwendet, da sie einen deutlich niedri-

675 Eine Auflistung der US-Staatsanleihen findet sich im Anhang.

$676 \mathrm{Zu}$ den unterschiedlichen Vorgehensweisen der Credit Spread Isolation vgl. Kap. 4.3.3.2.

677 Vgl. Deutsche Börse AG (1997), S. 13; vgl. auch Heinke (1998), S. $318 f$. 
Marktorientierte Kreditrisikobewertung - Empirische Untersuchung zur marktorientierten Bewertung des Kreditrisikos mittels Künstlicher Neuronaler Netze 157

geren Fehlerwert aufweist als alternative Gleichungen (bspw. einer linearen Regression). Im Anhang sind die Fehlerwerte der entsprechenden Jahre aufgezeigt. ${ }^{678}$

Zur Ermittlung der Indexes wurde zu jedem der untersuchten Stichtage eine Abfrage der entsprechenden US-Staatsanleihen durchgeführt, um die zugehörigen Regressionskoeffizienten $\beta_{\mathrm{i}}(\mathrm{i}=[1 ; 7])$ zu erhalten. Durch Einsetzen (Gleichung 30$)$ gelangt man zum Credit Spread als Differenz aus der risikobehafteten Rendite der Unternehmensanleihe i und der bonitätsrisikolosen Rendite der virtuellen Staatsanleihe B:

Gleichung 31

$e_{i, t}(J, C)=r_{i, t}(J, C)-r_{B, t}(J, C)$

mit $r_{i, t}(J, C)=$ Effektive Rendite der zu untersuchenden Anleihe i zum Zeitpunkt $t$, $\mathrm{r}_{\mathrm{B}, \mathrm{t}}(\mathrm{J}, \mathrm{C})=$ Effektive Rendite der bonitätsrisikolosen Anleihe B zum Zeitpunkt $\mathrm{t}$, $\mathrm{e}_{\mathrm{i}, \mathrm{t}}(\mathrm{J}, \mathrm{C})=$ Credit Spread Anleihe i zum Zeitpunkt $\mathrm{t}$.

Unabhängig davon, welche Vorgehensweise man zur Bildung des Credit Spread heranzieht, kommt es zu einer Fehlerimplikation, da es sich entweder um eine geschätzte Größe handelt, oder eine exakt vergleichbare Anleihe nicht vorhanden ist. ${ }^{679}$ Die Autoren ZIEBART/REITER setzen in ihrer Untersuchung nicht die Inputfaktoren zum Credit Spread, sondern zur effektiven Verzinsung der Unternehmensanleihe in Beziehung. ${ }^{680}$ Dementsprechend verzichten die Autoren darauf, den Credit Spread zu isolieren, sondern untersuchen die Einflussfaktoren der gesamten Anleihenrendite.

Diesem Vorgehen kann man nach Meinung des Verfassers nur dann zustimmen, falls sichergestellt werden kann, dass die Informationen der Zinsstrukturkurve, welche bei der Berechnung des Credit Spread benötigt werden, als (weiterer) Inputfaktor in das System eingesetzt werden. $\mathrm{Da}$ in der vorliegenden Arbeit als Inputfaktor für das allgemeine Zinsniveau der Effektivzins der entsprechenden virtuellen Staatsanleihe mit gleicher Laufzeit (abgelesen aus der Regressionsgleichung) integriert wird ${ }^{681}$, kann in einer Modellmodifikation der Fehler, welcher durch die Credit Spread Bildung ent-

678 Die Summe der korrigierten, quadrierten Abweichungen werden regelmäßig nicht in Untersuchungen veröffentlicht. Die in der vorliegenden Arbeit erzielten Werte sind im Vergleich zu anderen Studien „als durchschnittlich“ zu bewerten. Die aus einer falschen Berechnung des Credit Spread resultierende Ungenauigkeit wird jedoch durch eine Modellmodifikation (Kapitel 5.5.1) behoben.

679 Vgl. Kap. 4.3.3.2.

680 Vgl. Ziebart, Reiter (1992).

681 Vgl. Heinke (1998), S. 322f. 
Marktorientierte Kreditrisikobewertung - Empirische Untersuchung 158 zur marktorientierten Bewertung des Kreditrisikos mittels Künstlicher Neuronaler Netze

steht ${ }^{682}$, rückgängig gemacht und der Zusammenhang zwischen den Inputfaktoren und der effektiven Rendite untersucht werden. ${ }^{63}$

\subsubsection{Behandlung von fehlenden Daten und Extremwerten}

Neuronale Netze - insbesondere die Konzeption von Multi-Layer-Perceptronen in Verbindung mit dem Backpropagation-Lernalgorithmus - verlangen eine lückenlose Datenbasis. ${ }^{684}$ Dies bedeutet, dass im Inputdatensatz keine Datenlücken (missing values) vorhanden sein dürfen. Grundsätzlich gibt es bei der Verwendung eines MLP zwei Möglichkeiten, mit fehlenden Daten umzugehen: ${ }^{685}$ Entweder werden sie ersetzt, oder der Datensatz wird von der Untersuchung ausgeschlossen. In der vorliegenden Arbeit wurde aufgrund des geringen Anteils von fehlenden Daten an der gesamten Datenmenge (Grundmodell 1,4\% bzw. 13er-Modell unter einem Prozent) ${ }^{686}$ der pragmatische Weg gewählt, der durch die angewendete Software Neural Connections $2.0^{\circledR}$ unterstützt wird. ${ }^{687}$ Fehlende Informationen werden durch den Median innerhalb dieses Inputfaktors geschätzt und ersetzt ${ }^{688} \mathrm{Die}$ fehlenden Werte sind deutlich über die Untersuchungsmenge verstreut, und es lassen sich keine eindeutigen Schwerpunkte finden.

Ausreißer oder Extremwerte, d.h. extrem hohe oder niedrige Inputfaktoren, können die Mittelwerte und Streuungen der Inputfaktoren stark beinträchtigen. ${ }^{69} \mathrm{Da}$ die Inputfaktoren vor dem Import in das Neuronale Netz normalisiert werden (damit Unterschiede in der Dimension nicht Verzerrungen bei den Gewichten bedingen), führen Extremwerte zu deutlichen Veränderungen innerhalb der normierten Inputfaktoren. Es findet hierdurch ein deutlicher Anstieg der Häufung um den Nullwert statt, sodass durch die Existenz von Extremwerten die übrigen Inputfaktoren zu Konstanten gestaucht werden. Somit wird, falls die Datengrundlage dies zulässt, eine Korrektur der Extremwerte vorgenommen. Als Extremwerte werden in der vorliegenden Arbeit ausschließlich hohe bzw. niedrige Credit Spreads bezeichnet. Negative Credit Spreads sind von der Definition her nicht möglich und können lediglich durch die Berechnungsvorschrift erklärt werden. Insbesondere in den Bereichen von extrem kurzen oder extrem langen

682

683

684

685

686

687

688

689

Bspw. werden - wie im weiteren Analyseverlauf gezeigt wird - aufgrund der Berechnung des Credit Spread unter Zuhilfenahme einer Indexrendite negative Werte ausgewiesen.

Vgl. Kap. 5.5.1.

Vgl. Glormann (2001), S. 182.

Vgl. hier und im Folgenden Glormann (2001), S. 180ff.

Vgl. zum Grundmodell Kap. 5.4.3 und zum 13er-Modell Kap. 5.4.5.

Vgl. zur Beschreibung der Software Kap. 5.4.1.

Die Möglichkeit des Datenausschlusses würde zu einer Kürzung der Datensätze von über 500 oder über $50 \%$ der Inputfaktoren führen, da die missing values über die gesamte Untersuchungsmenge und alle Inputfaktoren verstreut sind.

Vgl. hier und im Folgenden Glormann (2001), S. 185f. 
Marktorientierte Kreditrisikobewertung - Empirische Untersuchung zur marktorientierten Bewertung des Kreditrisikos mittels Künstlicher Neuronaler Netze

Restlaufzeiten werden Schwächen in der Berechnung deutlich. ${ }^{690}$ Der Datenbestand wird um die 12 höchsten und 13 niedrigsten Credit Spreads (als Extremwerte) gekürzt. Diese 25 Werte entsprechen ca. 1,5\% des gesamten Datenbestands. Somit wird ein Kompromiss zwischen der vollständigen Erfassung aller Datensätze und der Verzerrung der Inputfaktoren durch Extremwerte gefunden. Die weiteren Ausführungen und Untersuchungen beziehen sich auf den folgenden Datensatz:

Nach der Reduktion um 25 Datensätze besteht die in die KNN-Analyse einfließende Untersuchungsmenge insgesamt aus 1641 Wertpapierrenditen, ausgedrückt durch den Credit Spread. ${ }^{691}$ Die Tabelle 17 gibt die deskriptiven Kennzahlen zur Beschreibung der Datenmenge hinsichtlich der Merkmale „Credit Spread“, „Restlaufzeit“, und „Nominalzins" wieder. Auffallend ist der minimale negative Credit Spread in Höhe von -191 Basispunkten. Der Mittelwert für das Kriterium Credit Spread in Höhe von 0,7674 liegt in einem mit anderen Studien vergleichbaren Bereich. ${ }^{692}$ Die Streuung der Risikoprämie deutet bereits auf die marktübliche Häufung von Anleihen mit guter Bonität hin. Sowohl Restlaufzeit als auch Nominalzins bilden im Mittel markttypische Werte ab. Das Minimum beim Nominalzins in Höhe von „0" lässt sich mit Nullkuponanleihen erklären, die ebenfalls in die Untersuchung miteinbezogen wurden.

\begin{tabular}{|l|l|l|l|l|l|l|l|l|}
\hline Merkmal & Dimension & Anzahl N & Spannweite & Minimum & Maximum & Mittelwert & $\begin{array}{l}\text { Standard- } \\
\text { abwelchung }\end{array}$ & \begin{tabular}{l} 
Varlanz \\
\hline $\begin{array}{l}\text { Credit } \\
\text { Spread }\end{array}$
\end{tabular} \\
$\begin{array}{l}\text { Prozent- } \\
\text { punkte }\end{array}$ & 1641 & 4,81 & $-1,91^{*}$ & 2,90 & 0,7674 & 0,5071 & 0,257 \\
\hline $\begin{array}{l}\text { Restlauf- } \\
\text { zeit }\end{array}$ & Jahre & 1641 & 34,88 & 0,51 & 35,39 & 8,1732 & 7,3719 & 54,346 \\
\hline $\begin{array}{l}\text { Nominal- } \\
\text { zins }\end{array}$ & $\begin{array}{l}\text { Prozent- } \\
\text { punkte }\end{array}$ & 1641 & 13,50 & 0,00 & 13,50 & 7,7162 & 1,2686 & 1,609 \\
\hline $\begin{array}{l}\text { Gultige } \\
\text { Werte }\end{array}$ & - & 1641 & - & - & - & - & - & - \\
\hline
\end{tabular}

Tabelle 17 Deskriptive Kennzahlen der Untersuchungsmenge hinsichtlich der Merkmale „Credit Spread“, „Restlaufzeit" und Nominalzins"

- = Ein negativer Credit Spread ist von der Definition her ausgeschlossen und kann lediglich durch die Berechnungsvorschrift erklärt werden.

s90 Dies zeigt sich auch bei der Anwendung des REX in der Praxis. Vgl. Deutsche Börse AG (1997). Darüber hinaus muss bei jeder Indexbildung eine Gewichtung der Staatsanleihen hinsichtlich der einzelnen Restlaufzeiten und Kupons vorgenommen werden. Vgl. Deutsche Börse (2001), S. 5. Diese Tatsache könnte zu einer Verzerrung bei der Abbildung der Zinsstrukturkurve führen.

691 Die durchschnittliche Bilanzsumme (gemessen als Median) liegt bei 5.445 Mio. US\$.

Vgl. hier und im Folgenden Kap. 5.6.2. 
Marktorientierte Kreditrisikobewertung - Empirische Untersuchung 160 zur marktorientierten Bewertung des Kreditrisikos mittels Künstlicher Neuronaler Netze

\subsection{Die Datenanalyse mit dem Backpropagation-Netz}

\subsubsection{Einzustellende Parameter innerhalb der Software Neural Connection ${ }^{\otimes}$}

Während in Kap. 5.1 und Kap. 5.2 ein konkretes Netz für die Analyse des in Kap. 5.3 dargestellten Datenbestands ausgewählt und beschrieben wurde, wird im Weiteren mittels einer Software dieser Datenbestand hinsichtlich der in Kap. 4.4 dargestellten Zielsetzung des Prognosemodells analysiert. Eine hierzu geeignete Software ist Neural Connection 2.1 ${ }^{\circledast}$, die in Deutschland von der Firma SPSS ${ }^{\circledR}$ vertrieben wird. ${ }^{693}$ Neben dem Netzwerktyp „Multi-Layer-Perceptron“ verfügt die Software auch über weitere bekannte Neuronale Netze wie „Kohonen-“ oder „Bayesian-Netze“. Die bereits in den vorangegangenen Kapiteln dargestellten vom Netzwerkarchitekten zu treffenden Entscheidungen, wie die Aufteilung der Datenmenge in die Bereiche Trainings-, Validierungs- und Generalisierungsdaten oder die Festlegung des Momentums oder der Aktivierungsfunktion, werden auch von dem Einsatz einer Software mitbestimmt. Bspw. ist es nicht möglich, Aktivierungsfunktionen außerhalb der in der Software bereitgestellten Typen zu wählen, obwohl theoretisch auch andere Funktionstypen eingesetzt werden könnten. Im Weiteren werden nun die softwarespezifischen Besonderheiten für die einzelnen Parameter vorgestellt. ${ }^{694}$

Zunächst wird von der Software ein Grundschema vorgeschlagen. Dieses Grundschema gibt bspw. die Anzahl an Neuronen in der versteckten Schicht vor. Der Aufbau des Grundschemas ist abhängig vom Untersuchungsdesign (bspw. von der Anzahl an Eingangsneuronen) ${ }^{695}$

Die Datenaufteilung zwischen den o.g. drei Datenbereichen kann individuell variiert werden. Die Aktivierungsfunktion kann entweder „Linear“, „Tanh“ oder „Sigmoid“ sein. Weitere Funktionstypen können nicht integriert werden. Wahlweise kann die versteckte Schicht ein oder zwei Unterschichten (layers) beinhalten. Falls zwei Unterschichten gewählt werden, können je layer unterschiedliche Aktivierungsfunktionen herangezogen werden. Die Anzahl der Neuronen in den einzelnen layers kann individuell gebildet werden. Wie bereits oben beschrieben wurde, müssen in der ersten Propagationsphase zufallsbedingte Startgewichte gewählt werden. Diese Startgewichte werden in Anlehnung an eine Verteilungsfunktion bestimmt. Hierzu bietet die Soft-

693 Vgl. hier und im Folgenden www.spss.com/Germany.

694 Vgl. hier und im Folgenden SPSS (1997).

695 Auf Basis des Softwarehandbuches ist nicht eindeutig nachzuvollziehen, wie die Software zum Grundschema gelangt (vgl. SPSS (1997)). Auf Nachfragen des Verfassers wurde der Vorgang der Konzeption des Grundschemas als Betriebsgeheimnis deklariert. Dementsprechend ist eine detaillierte Beschreibung nicht möglich. 
Marktorientierte Kreditrisikobewertung - Empirische Untersuchung zur marktorientierten Bewertung des Kreditrisikos mittels Künstlicher Neuronaler Netze

ware zwei Verteilungsfunktionen (,uniform“ und ,gaussian“) an. ${ }^{696}$ Über den Parameter ,range" kann in der Software für die verwendete Verteilungsfunktion eine Bandbreite für die Startgewichte bestimmt werden. ${ }^{697}$ Eine Besonderheit der Software besteht in der Möglichkeit der Bildung von Lerngruppen. ${ }^{698}$ Eine Lerngruppe wird definiert durch eine festgelegte Anzahl von Lernzyklen bzw. Lemschritten. Falls bspw. eine Lerngruppe aus 100 Lernschritten besteht, ist sie beendet, sobald 100 Lemzyklen, d.h. 100 Backpropagation-Schritte ausgeführt wurden. Für jede Lerngruppe kann die Anzahl an Lerndaten individuell definiert werden. Bspw. können in der ersten Lerngruppe 250 und in der zweiten 500 Datensätze vorhanden sein. Hierdurch wird ein (zeitlich) schnelles Lernen mit wenigen Daten in den ersten Lerngruppen ermöglicht. Grundsätzlich sollte die Anzahl an Daten in den einzelnen Lerngruppen ansteigen. Die gesamte Anzahl an Lernschritten ist unbegrenzt. Die Werte für die Parameter „Momentum“ und „Lernkoeffizient“ können individuell eingestellt werden. Es können für die unterschiedlichen Lerngruppen unterschiedliche Werte angesetzt werden, sodass man durchaus auch steigende bzw. fallende Werte innerhalb eines KNN für diese beiden Parameter verwenden kann.

Tabelle 18 fasst die einzustellenden Parameter zusammen. Die Spalte „Mögliche Ausprägungen" zeigt das Spektrum an Ausprägungen auf. Sie ergibt sich aus den in den vorangegangenen Kapiteln aufgezeigten Möglichkeiten der Netzwerkarchitektur. Während einzelne Einstellungen wie die Aktivierungsfunktion grundsätzlich nicht eingeschränkt werden können, weisen andere wie das „Momentum“ bestimmte sinnvolle Ausprägungsintervalle auf. Die Spalte „Softwarespezifische Einstellungen“ geht auf die verwendete Software Neural Connection $2.1^{\text {B }}$ und die damit verbundenen Möglichkeiten ein.

696 „Uniform” („gaussian“) bedeutet eine konstante Gleichverteilung (normalverteilte Verteilung) der Gewichte in dem Intervall der Bandbreite („Range“).

697 Vgl. SPSS (1997), S. 134f. „The Multi-Layer Perceptron begins training with random node input weights. However, as mentioned above, the choice of these weights can have a large effect on the performance of your network. Neural Connection therefore allows you to change the distribution pattern of the weight values, and the range within which the values are chosen. It also allows you to change the seed for the random number generation." Auszug aus der Online-Hilfe, Neural Connection 2.1.

Zu der Bedeutung der Lemgruppe vgl. SPSS (1997), S. 128f. „When Neural Connection trains a Multi-Layer Perceptron, it uses an incremental learning technique, where the Multi-Layer Perceptron is trained in stages. In the first stage a sample of examples from the training set is used to train the Multi-Layer Perceptron. The best network produced at this stage is then passed on to the second stage, and is used as a starting point for training. (...) In the second stage a larger sample of the data is used to train the network, and again the best network is passed on to the next stage. This procedure continues for four stages." Auszug aus der Online-Hilfe, Neural Connection 2.1. 
Marktorientierte Kreditrisikobewertung - Empirische Untersuchung 162 zur marktorientierten Bewertung des Kreditrisikos mittels Künstlicher Neuronaler Netze

\begin{tabular}{|c|c|c|}
\hline Parameter & Mögliche Ausprägungen & $\begin{array}{c}\text { Softwarespezifische Einstel- } \\
\text { lungen }\end{array}$ \\
\hline Datenaufteilung & $\begin{array}{l}\text { Alle Variationen furr die prozentuale Auftei- } \\
\text { lung in die drei Mengen. }\end{array}$ & $\begin{array}{l}\text { Grundschema: } 80 \% \text { Trainings-, } 10 \% \\
\text { Validierungs- und } 10 \% \text { Generalisie- } \\
\text { rungsdaten; jedoch alle Variationen } \\
\text { moglich. }\end{array}$ \\
\hline $\begin{array}{l}\text { Variation der } \\
\text { Aktivierungsfunk- } \\
\text { tionen }\end{array}$ & $\begin{array}{l}\text { Grundsätzlich alle mathematischen Funktionen } \\
\text { denkbar, in der Regel: lineare Funktion, } \\
\text { Schwellenwertfunktion, Sigmoid-Funktion }{ }^{699} \\
\text { und Tanh-Funktion. }\end{array}$ & Linear, Tanh- und Sigmoid-Funktion. \\
\hline Anzahl der layer & Theoretisch unbegrenzt. & $\begin{array}{l}\text { Wahlweise ein oder zwei versteckte } \\
\text { Schichten. }\end{array}$ \\
\hline $\begin{array}{l}\text { Anzahl der } \\
\text { Neuronen je } \\
\text { Schicht }\end{array}$ & $\begin{array}{l}\text { Theoretisch unbegrenzt, jedoch gibt es eine } \\
\text { Vielzahl von verschiedenen heuristischen } \\
\text { Regeln (vgl. auch die folgenden Kapitel). }\end{array}$ & Theoretisch unbegrenzt \\
\hline Startgewichte & $\begin{array}{l}\text { Jede rationale Zahl, u.U. gekoppelt an eine } \\
\text { Verteilungsfunktion. }\end{array}$ & $\begin{array}{l}\text { Als Verteilungsfunktionen werden die } \\
\text { Typen „uniform“ und „gaussian“ } \\
\text { vorgeschlagen; der Parameter ,range“ } \\
\text { gibt Anwendern die Möglichkeit, die } \\
\text { Verteilungsfunktionen zu beschreiben. }\end{array}$ \\
\hline $\begin{array}{l}\text { Anzahl der Lern- } \\
\text { schritte }\end{array}$ & $\begin{array}{l}\text { Theoretisch unbegrenzt, jedoch Problem des } \\
\text { overlearnings und der Performance. }\end{array}$ & $\begin{array}{l}\text { In bis zu vier Lerngruppen konnen } \\
\text { theoretisch unendlich viele Lernzyklen } \\
\text { durchlaufen werden. }\end{array}$ \\
\hline Momentum & $\begin{array}{l}\text { FÛSER schlägt einen Wert von } 0,9 \text { vor, } \\
\text { jedoch können keine allgemeinen Vorgaben } \\
\text { abgeleitet werden. }{ }^{701}\end{array}$ & $\begin{array}{l}\text { Manuelle Eingabe möglich; Momen- } \\
\text { tum kann sich in Abhängigkeit der } \\
\text { Lerngruppen (im Lernverlauf) verän- } \\
\text { dern. }\end{array}$ \\
\hline Lernkoeffizient & $\begin{array}{l}\text { Sollte in dem Intervall von }[0 ; 1] \text { liegen, häufig } \\
\text { liegt er in dem Intervall }[0,7 ; 0,9]{ }^{702}\end{array}$ & $\begin{array}{l}\text { Manuelle Eingabe möglich; Lernkoef- } \\
\text { fizient kann sich in Abhängigkeit der } \\
\text { Lerngruppen (im Lernverlauf) verăn- } \\
\text { dern. }\end{array}$ \\
\hline
\end{tabular}

Tabelle 18: Einzustellende Parameter für das KNN unter der Beachtung der softwarespezifischen Moglichkeiten

\subsubsection{Ziehung der Stichproben für die Künstliche Neuronale Netzanalyse}

Bevor mit Hilfe der KNN-Analyse der in Kap. 5.3 beschriebene Datenbestand untersucht werden kann, muss dieser in die Mengen der Trainings-, Validierungs- und Generalisierungsdaten eingeteilt werden. ${ }^{703}$ Die Ziehung der drei Stichproben sollte folgende Voraussetzungen erfüllen: ${ }^{704}$

Vgl. Füser (1995), S. 27.

Vgl. Füser (1995), S. 71; vgl. zur Darstellung der Sigmoid-Funktion die Ausführungen im Anhang.

Vgl. Schiffmann (1999), S. 48f.

Vgl. Füser (1995), S. 61.

Vgl. Krause (1993), 124ff.

Vgl. hier und im Folgenden Glormann (2001), S. 149ff. 
Marktorientierte Kreditrisikobewertung - Empirische Untersuchung zur marktorientierten Bewertung des Kreditrisikos mittels Künstlicher Neuronaler Netze 163

- Bei einem Klassifikationsproblem sollten die verschiedenen Klassen innerhalb der Trainingsmengen gleichverteilt sein,

- jede Gruppe sollte möglichst repräsentativ für den gesamten Datenbestand sein.

Bei Klassifizierungsproblemen sollte darauf geachtet werden, dass die verschiedenen Klassenstärken innerhalb der Trainingsmenge gleich groß sind, damit nicht eine von beiden Klassen beim Lernprozess dominiert wird. Beispielsweise sollten bei der Untersuchung von Kreditausfallwahrscheinlichkeiten in den Trainingsdaten solvente und insolvenzgefährdete Unternehmen gleich stark vertreten sein. Das vorliegende Problem stellt jedoch ein Prognoseproblem dar. Dementsprechend liegen keine einzelnen Klassen vor.

Innerhalb der im weiteren Verlauf beschriebenen Szenarienbildung wird die gesamte Datenmenge in Gruppen separiert. Folglich lassen sich in Abhängigkeit der Gruppendefinition verschiedene Aufteilungen (Szenarien) finden. In Szenario 1 wird unterstellt, dass ein Unternehmen bereits verschiedene Anleihen begeben haben kann. Das KNN soll folglich lernen, die Credit Spreads von Anleihen zu bestimmten, deren Schuldner bereits auf dem Kapitalmarkt als Emittenten weiterer Anleihen agieren. Es findet eine vollkommen zufällige Stichprobenziehung statt und jede Anleihe stellt eine einzelne Gruppe dar, sodass es sich um 1641 Gruppen handelt. Über alle Gruppen hinweg lag eine Standardabweichung des Credit Spread von 0,7669 vor. Die Standardabweichung drückt aus, wie stark der Wert des Credit Spread zwischen den Anleihen schwankt.

Falls man unterstellt, dass ein Unternehmen (unabhängig von der wertpapierspezifischen Ausstattung) mit derselben Kreditprämie bewertet wird, muss innerhalb der Datenaufteilung keine zufällige Stichprobe verfolgt, sondern vielmehr eine teilweise gesteuerte Unternehmensauswahl getroffen werden.

Falls man unterstellt, dass ein Unternehmen bereits in der Vergangenheit eine Anleihe emittiert hat, jedoch augenblicklich kein Wertpapier von diesem Unternehmen gehandelt werden soll, bildet die zugehörige Bilanz das Kriterium zur Gruppeneinteilung. Dementsprechend wird sichergestellt, dass zu einem Stichtag Anleihen bzw. die zugehörigen Credit Spreads desselben Unternehmens in eine Gruppe eingeteilt werden, jedoch gleichzeitig Anleihen bzw. die zugehörigen Credit Spreads der vergangenen Jahre (mit entsprechend unterschiedlichen Bilanzkennzahlen) einer anderen Gruppe zugeordnet werden. Diese Annahme wird als Szenario 2 bezeichnet. Bei dieser Einteilung entstehen 345 Gruppen. Innerhalb einer Gruppe beträgt die Standardabweichung zwischen dem Gruppenmittelwert des Credit Spread (arithmetisches Mittel) und den einzelnen Ausprägungen im Durchschnitt 0,5035. Dieser Schnitt gewichtet jede Gruppe gleich und zeigt, dass es auch innerhalb der einzelnen Gruppen zu deutlichen Unterschieden zwischen den Credit Spreads kommt.

Im Szenario 3 bildet ein Unternehmen eine Gruppe. Dies unterstellt, dass Anleihen bzw. die zugehörigen Credit Spreads eines Unternehmens in einer Gruppe zu erfassen 
Marktorientierte Kreditrisikobewertung - Empirische Untersuchung 164 zur marktorientierten Bewertung des Kreditrisikos mittels Künstlicher Neuronaler Netze

sind. Dementsprechend handelt es sich um 136 Gruppen. Innerhalb einer Gruppe beträgt die Standardabweichung zwischen dem Klassenmittelwert des Credit Spread (arithmetisches Mittel) und den einzelnen Ausprägungen im Durchschnitt 0,5741. ${ }^{705}$ An dieser hohen durchschnittlichen Abweichung wird deutlich, dass einem Unternehmen sehr unterschiedliche Credit Spreads zugeordnet sind. Die Tabelle 19 fasst die gebildeten Szenarien zusammen. Dementsprechend kann die KNN-Analyse anhand von drei Szenarien geführt werden. Während in dem folgenden Kap. 5.4.3.1 zunächst für das Grundmodell die Parameter konfiguriert werden, wird im Kap. 5.4.3.2 der Datenbestand jeweils getrennt für die drei Szenarien ausgewertet. Bei der Herleitung der Parameterkonfiguration wird jeweils das Szenario 1 herangezogen, da die Software Neural Connections ${ }^{\circledast}$ keine automatisierte Gruppenbildung bei der Aufteilung in die Lern- und Testmenge unterstützt. Im Rahmen der Parameterkonfiguration ist es somit nicht möglich, für jedes Szenario getrennt eine Netzstruktur abzuleiten.

\begin{tabular}{|l|l|l|l|}
\hline Szenario & $\begin{array}{l}\text { Gruppeneinteilung } \\
\text { nach dem Kriterium }\end{array}$ & Gruppenanzahl & $\begin{array}{l}\text { Standardabweichung des Credit } \\
\text { Spread vom Mittelwert der jeweiligen } \\
\text { Klasse als Mittelwert uber alle Grup- } \\
\text { pen }\end{array}$ \\
\hline 1 & Anleihe & 1641 & 0,7669 \\
\hline 2 & Bilanzen & 345 & 0,5035 \\
\hline 3 & Unternehmen & 136 & 0,5741 \\
\hline
\end{tabular}

Tabelle 19: Standardabweichungen des Credit Spread innerhalb verschiedener Szenarien

\subsubsection{Das Grundmodell}

\subsubsection{Parameterkonfiguration}

Für die Analyse des in Kap. 5.3 dargestellten Datenbestands mit Hilfe des MLP ist wie in dem vorangegangenen Kapitel bereits dargestellt wurde, eine Vielfalt von einzustellenden Parametern vorhanden, die teilweise von der herangezogenen Software bedingt werden. Bei der Ableitung der optimalen Netzstruktur ist fraglich, in welcher Reihenfolge die Parameter variiert werden sollen und wie stark eine Variation ausfallen soll. An dem Beispiel von zwei Einstellungsparametern soll diese verdeutlicht werden.

Zum einen muss eine Entscheidung bezüglich der Anzahl der layers getroffen und zum anderen die Einteilung der Daten in die Gruppen Test-, Trainings- und Validierungsdaten vorgenommen werden. Während die layers als Anzahl bestimmt werden, ist die Dimension der Mengeneinteilung häufig prozentual anzugeben. Falls man nun von einem Startnetz ausgeht, besteht die Möglichkeit, entweder die Anzahl an layer oder

Dieser Schnitt gewichtet wiederum jede Klasse gleich. 
Marktorientierte Kreditrisikobewertung - Empirische Untersuchung zur marktorientierten Bewertung des Kreditrisikos mittels Künstlicher Neuronaler Netze

die Datenaufteilung zu variieren. ${ }^{706}$ Hieraus resultieren zwei Fragen: Welche Anpassung soll man zunächst vornehmen und wie stark soll diese ausfallen? Zur Beantwortung dieser Fragen ist es notwendig, die im Analyseverlauf verwendete Software heranzuziehen und gleichzeitig auf Erfahrungswerten bei anderen Untersuchungen aufzubauen.

Die unter Kapitel 5.4.1 dargestellten Parameter lassen sich in Abhängigkeit von der benutzten Software Neural Connections $2.0^{\circledR}$ nach dynamischen und starren Einstellungen unterscheiden. ${ }^{707}$ Wie bereits o.g., sind die Parameter „Lernkoeffizient“ und „Momentum“ durch die Spezifika der Software während des Lernprozesses veränderbar. Aufgrund der individuellen Einstellungsmöglichkeiten durch den Anwender kann der Lernkoeffizient z.B. im Trainingsverlauf ab- und das Momentum zunehmend sein. Somit sind diese Parameter als dynamische Einstellungen zu charakterisieren. Für diese dynamischen Parameter sind keine eigenständigen Netzmodifikationen notwendig, da diese Einstellungen innerhalb eines Netzes variieren können. Die übrigen starren Einstellungen müssen jeweils durch die Konzeption eigener Netzstrukturen verändert werden.

Die Veränderung der Parametereinstellungen in der vorliegenden Arbeit ist an die Vorgehensweise der Software Neuronaler-Netz-Simulator SNNS ${ }^{\circledast}$ (Stuttgarter Neuronaler Netz Simulator) angelehnt: ${ }^{708}$

- Ausgehend von einem Grundschema (Standardeinstellungen in der Software Neural Connections ${ }^{\circledR}$ ) wird

- zunächst die Aktivierungsfunktion variiert.

- Anschließend wird die Netzgröße verändert, indem

706 Bezugsbasis für Angaben der prozentualen Datenaufteilung ist immer die gesamte Datenmenge.

707 Es wurde ein PC mit folgenden Eigenschaften eingesetzt: $800 \mathrm{MHZ}$, Pentium III ${ }^{\$}, 256$ MB RAM.

708 Vgl. Lackes, Mack (2000), S. 239. 
Marktorientierte Kreditrisikobewertung - Empirische Untersuchung 166 zur marktorientierten Bewertung des Kreditrisikos mittels Künstlicher Neuronaler Netze

o erstens die Anzahl an Knotenpunkten (Neuronen in der versteckten Schicht) und

- zweitens die Anzahl der layers in der versteckten Schicht variiert werden.

- Im folgenden Schritt wird die prozentuale Aufteilung der Datenmengen in die Trainings-, Validierungs- und Testdaten variiert.

- Aufbauend auf diesen Ergebnissen werden verschiedene Verteilungsfunktionen für die Startgewichte konfiguriert und

- abschließend die Anzahl an Lernschritten variiert. ${ }^{709}$

Im Weiteren werden nun (ausgehend von dem Grundschema) die Parametervariationen durchgeführt. Hinzugezogen wird hierbei das Szenario 1, d.h. die Aufteilung in Trainings-, Validierungs- und Testdaten ist vollkommen zufallsverteilt. Dabei wird jede einzelne Parameterkonfiguration anhand des Fehlerwertes des Netzes beurteilt. Eine Parameterkonfiguration wird dann übernommen, wenn sie im Vergleich zu den Alternativen den niedrigsten Fehlerwert aufweisen kann. Es besteht somit die Frage, wie die Netzgüte bestimmt werden kann.

Unabhängig von der konkreten Parameterbelegung besitzt jedes Netz 32 Eingangsneuronen, da neben 3 makroökonomischen Variablen $\left(\mathrm{M}_{1}-\mathrm{M}_{3}\right), 3$ titelspezifische Größen $\left(\mathrm{T}_{1}-\mathrm{T}_{3}\right)$ und 26 Bilanzkennzahlen $\left(\mathrm{B}_{1}-\mathrm{B}_{26}\right)$ herangezogen werden. Neben diesen 32 Inputneuronen besitzt jedes Netz genau ein Outputneuron, welches den Wert des Credit Spread angibt. Während die Inputneuronen beobachtete Größen sind, wird das Outputneuron bzw. der Wert dessen vom KNN bestimmt. Somit handelt es sich um den prognostizierten Credit Spread. Bei einem perfekten Netz würde der Wert des prognostizierten Credit Spread für eine Anleihe i ( $\left.\hat{e}_{i}\right)$ dem Wert des am Markt beobachtbaren $\left(e_{i}\right)$ entsprechen. Aus dieser Grundüberlegung heraus wird die Modellgüte eines Netzes als die Höhe der durchschnittlichen Abweichungen zwischen dem prognostizierten und dem beobachteten Credit Spread gemessen an den Generalisierungsdaten definiert. Im Weiteren wird diese durchschnittliche Abweichung als Fehlerwert des Netzes bzw. Fehlerwert der Testmenge $\left(\mathrm{s}_{\mathrm{F}}\right)$ bezeichnet und berechnet sich als:

Lernkoeffizienten bzw. Momentum werden im Lernverlauf dynamisch verändert. Der Lernkoeffizient startet bei 0,9 und endet bei 0,4 . Das Momentum startet bei 0,1 und endet bei 0,6 . Die Veränderung findet in vier Teilschritten statt, wobei jeder Teilschritt $1 / 4$ der gesamten Lernschritte umfasst. 
Marktorientierte Kreditrisikobewertung - Empirische Untersuchung zur marktorientierten Bewertung des Kreditrisikos mittels Künstlicher Neuronaler Netze 167

Gleichung 32

$$
S_{F}=\sqrt{\frac{1}{P_{T}} \cdot \sum_{i=1}^{P_{T}^{T}}\left|e_{i}-\hat{e}_{i}\right|^{2}},
$$

mit: $\quad \mathrm{S}_{\mathrm{F}}=$ Fehlerwert eines Netzes, Fehlerwert der Testmenge

$\mathrm{P}_{\mathrm{T}}=$ Menge der Testdatensätze,

$e_{i}=$ gemessene Bonitätsprämie (Credit Spread) des Datensatzes $i$,

$\hat{\mathrm{e}}_{\mathrm{i}=}$ prognostizierte Bonitätsprämie (Credit Spread) des Datensatzes i,

$e_{i}-\hat{e}_{i}=$ Differenz eines Datensatzes i.

Ausgangspunkt für die Parametervariationen ist die softwarespezifische Grundeinstellung (Grundschema):

- Tanh-Aktivierungsfunktion,

- Netzgröße,

- 16 Knotenpunkte,

- 1 versteckte Schicht,

- 80-10-10 Aufteilung der Datenmenge,

- Startgewichte: 0,1; Verteilungsform: uniform,

- 400 Lernschritte in vier Gruppen („Lernstufe“). Nach Beendigung einer jeden Gruppe wird der Lernkoeffizient gesenkt bzw. das Momentum erhöht.

Zunächst wird die Aktivierungsfunktion variiert (vgl. Tabelle 20). Durch die softwarespezifischen Ausgestaltungsmerkmale werden drei Typen vorgegeben:

\begin{tabular}{|l|l|l|l|}
\hline \multicolumn{1}{|c|}{$\begin{array}{c}\text { Aktivierungs- } \\
\text { funktion }\end{array}$} & \multicolumn{1}{|c|}{ Tanh } & \multicolumn{1}{|c|}{ Sigmoid } & \multicolumn{1}{c|}{ Linear } \\
\hline $\begin{array}{l}\text { Fehlerwert der Test- } \\
\text { menge }\end{array}$ & 0,2523 & $\mathbf{0 , 2 3 3 5 1}$ & 0,3600 \\
\hline
\end{tabular}

Tabelle 20: Fehlerwertes $S_{\mathrm{F}}$ in Abhăngigkeit der Aktivierungsfunktion fur das Grundmodell

Da die erzielten Ergebnisse mit der Sigmoid-Aktivierungsfunktion im direkten Vergleich die Besten sind, wird diese furr die weiteren Untersuchungen verwendet. Zunächst werden die Knotenpunkte (Neuronen in der versteckten Schicht) variiert. $\mathrm{Zu}$ dieser Parametereinstellung gibt es sehr unterschiedliche Empfehlungen. Häufig werden „Faustregeln“ angewendet, die sich auf die Anzahl der Datensätze und / oder die 
Marktorientierte Kreditrisikobewertung - Empirische Untersuchung 168 zur marktorientierten Bewertung des Kreditrisikos mittels Künstlicher Neuronaler Netze

Anzahl der Eingangsneuronen beziehen. Beispielsweise wird von UHLIG vorgeschlagen: ${ }^{710}$

- $2 \cdot \sqrt{I+K}$, wobei $I+K=$ Summe aus Ausgabeneuronen und Eingabeneuronen; hier ca. 11 Knotenpunkte;

- Anzahl der Neuronen liegt zwischen der Hälfte und dem Zehnfachen der Anzahl der Eingabeneuronen (hier [16;320]).

Es wird deutlich, dass die Schwankungsbreite der Empfehlungen sehr groß ist. Die folgende Abbildung 40 zeigt die Abhängigkeit des Fehlerwertes von der Anzahl der Neuronen in der versteckten Schicht. Die weiteren Parametereinstellungen sind gemäß der vorangegangenen Untersuchung gleich der softwarespezifischen Grundeinstellung, wobei als Aktivierungsfunktion die Sigmoid-Funktion beibehalten wird.

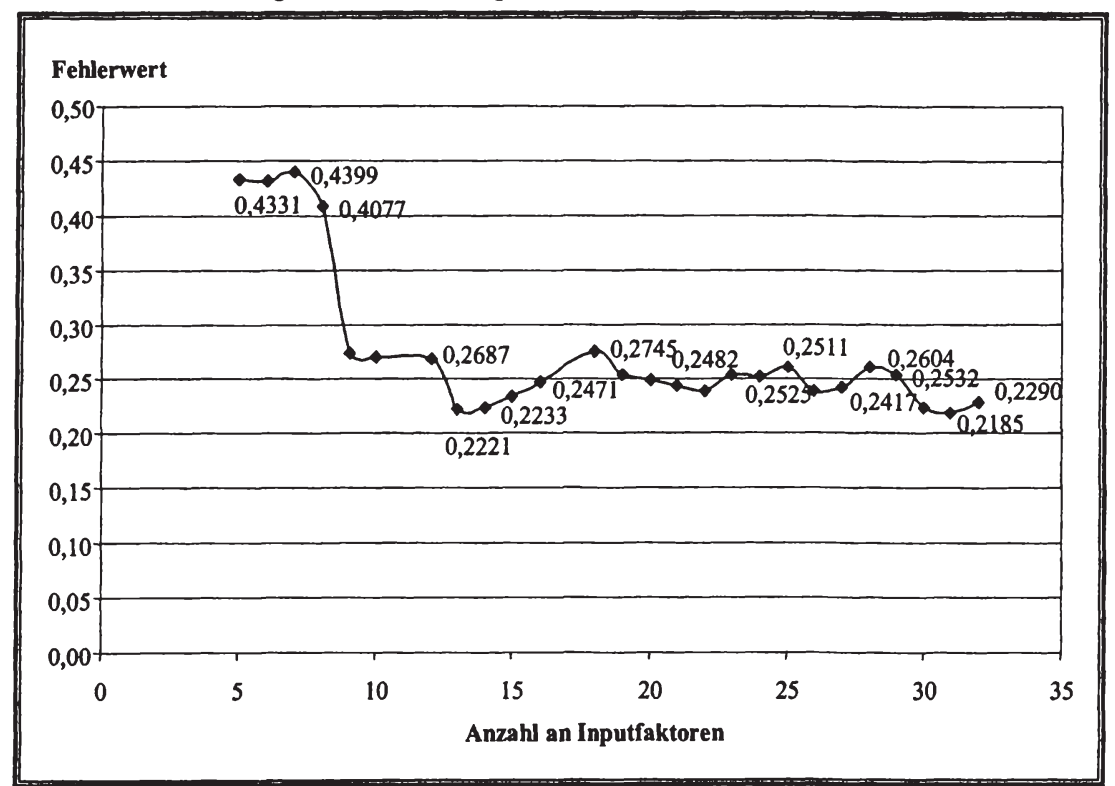

Abbildung 40: Fehlerwert $\mathrm{s}_{\mathrm{F}}$ in Abhängigkeit von der Anzahl der Neuronen in der versteckten Schicht for das Grundmodell

Obwohl das Ergebnis $(0,23243)$ bei $n=15$ Knotenpunkten von der Einstellung mit $\mathrm{n}=27$ dominiert wird (0,22799), wird für die weiteren Versuche die Einstellung mit

710 Vgl. Uhlig (1995), S. 48; Brown (1993), S. 50ff.; Katz (1992), S. 58ff.; Shih (1991), S. $62 \mathrm{ff}$. 
Marktorientierte Kreditrisikobewertung - Empirische Untersuchung zur marktorientierten Bewertung des Kreditrisikos mittels Künstlicher Neuronaler Netze

15 Knotenpunkten verwendet, da hier eine wesentlich günstigere Lernzeit zu messen ist. $^{.11}$

Eine Netzvergrößerung kann neben der Erweiterung der Knotenanzahl in der ersten Schicht durch die Aufnahme einer zweiten (oder weiteren) Schicht erfolgen. Dies hat den Vorteil, in der zweiten (oder weiteren Schicht) eine andere Aktivierungsfunktion benutzen zu können. Das oben gefundene Netż mit 15 Neuronen wird im Weiteren um eine zweite Schicht ergänzt. Es werden weiterhin 15 Neuronen in der versteckten Schicht benutzt, bzw. bei den Variationen 10-12 werden insgesamt 20 Neuronen in der versteckten Schicht verwendet. Aufgrund der bereits gewonnenen Ergebnisse wird mindestens in einem layer eine Sigmoid-Funktion als Aktivierungsfunktion benutzt. Die Tabelle 21 zeigt einen Überblick hinsichtlich der gemessenen Fehlerwerte.

\begin{tabular}{|c|c|}
\hline Aktivierungsfunktion (zwei Schichten) & Fehlerwert \\
\hline (Ausgangsnetz) 1 Schicht, 15 Knoten; sigmoid-Funktion & 0,23243 \\
\hline (Variation 1) 2 Schichten, $8+7$ Knoten; sigmoid / tanh-Funktion & 0,26818 \\
\hline (Variation 2) 2 Schichten, $8+7$ Knoten, tanh / sigmoid-Funktion & 0,28603 \\
\hline (Variation 3) 2 Schichten, $8+7$ Knoten; sigmoid / sigmoid-Funktion & 0,42300 \\
\hline (Variation 4) 2 Schichten, $10+5$ Knoten; sigmoid / tanh-Funktion & 0,42024 \\
\hline (Variation 5) 2 Schichten, $10+5$ Knoten; tanh / sigmoid-Funktion & 0,33276 \\
\hline (Variation 6) 2 Schichten, $10+5$ Knoten; sigmoid / sigmoid-Funktion & 0,30414 \\
\hline (Variation 7) 2 Schichten, $5+10$ Knoten; sigmoid / tanh-Funktion & 0,26573 \\
\hline (Variation 8) 2 Schichten, $5+10$ Knoten; tanh / sigmoid-Funktion & 0,32847 \\
\hline (Variation 9) 2 Schichten, $5+10$ Knoten; sigmoid / sigmoid-Funktion & 0,28034 \\
\hline (Variation 10) 2 Schichten, $10+10$ Knoten; sigmoid / tanh-Funktion & 0,27513 \\
\hline (Variation 11) 2 Schichten, $10+10$ Knoten; tanh / sigmoid-Funktion & 0,30024 \\
\hline (Variation 12) 2 Schichten, $10+10$ Knoten; sigmoid / sigmoid-Funktion & 0,28948 \\
\hline
\end{tabular}

Tabelle 21: Fehlerwert $s_{\mathrm{F}}$ in Abhängigkeit von der Anzahl der layer, der Anzahl der Neuronen in der versteckten Schicht und der Aktivierungsfunktion für das Grundmodell

Die Variationen durch die Hinzunahme einer zweiten Schicht führen nicht zu einer Verringerung des Fehlerwertes. ${ }^{712}$ Negativ anzumerken ist darüber hinaus, dass sich die Performance durch eine zweite Schicht verschlechtert.

Der nächste Schritt zur Parametervariation ist die Veränderung der prozentualen Aufteilung der gesamten Datenmenge. Es stellt sich die Frage, wie man bei einem erfolg-

711 Insbesondere bei der späteren Erhöhung der Lernzyklen ist eine schnelle Bearbeitung notwendig. Mitunter dauert bei Lernzyklen von 10.000 Einheiten die Bearbeitung 24 Stunden. Somit kann man bei einem nahezu gleichwertigen Fehlerwert die Bearbeitungszeit deutlich (über 50 Prozent) reduzieren.

712 Diese Feststellung deckt sich mit der Mehrzahl der KNN-Analysen aus dem finanzwissenschaftlichen Bereich, vgl. Fadlalla, Lin (2001), S. 117. 
Marktorientierte Kreditrisikobewertung - Empirische Untersuchung 170 zur marktorientierten Bewertung des Kreditrisikos mittels Künstlicher Neuronaler Netze

reichen Lernverhalten (große Trainingsmenge) eine Generalisierung (Testmenge) sicherstellen kann. Bei UHLIG findet sich ein übertragbarer Hinweis, und zwar: ${ }^{713}$

- Die Anzahl der Datensätze in der Trainingsmenge soll zwanzigmal so groß sein, wie die Anzahl der Eingabeneuronen (hier: $32 \cdot 20=640$ ), und

- es sollen viermal mehr Datensätze in der Trainingsmenge als Verbindungsgewichte (hier: $32 \cdot 15 \cdot 4=1920$ ) vorhanden sein.

Im Folgenden werden die Ergebnisse verschiedener Aufteilungen in die Trainings-, Validierungs- und Testmenge tabellarisch aufgezeigt (Tabelle 22). Die oben bereits optimierten Einstellungen (Aktivierungsfunktion: sigmoid, 15 Neuronen, 1 Schicht) werden beibehalten.

\begin{tabular}{||c|c|c|c||}
\hline $\begin{array}{c}\text { Datenaufteilung in Trai- } \\
\text { nings- / Validierungs- } \\
\text { Testmengen [Angaben in } \\
\text { Prozent] }\end{array}$ & $\begin{array}{c}\text { Fehlerwert } \\
\text { gemessen an } \\
\text { der Testmen- } \\
\text { ge }\end{array}$ & $\begin{array}{c}\text { Datenaufteilung in Trai- } \\
\text { nings- / Validierungs- } \\
\text { Testmengen [Angaben in } \\
\text { Prozent] }\end{array}$ & $\begin{array}{c}\text { Fehlerwert } \\
\text { gemessen an } \\
\text { der Test- } \\
\text { menge }\end{array}$ \\
\hline $95 / 2,5 / 2,5$ & 0,27831 & $60 / 10 / 30$ & 0,27745 \\
\hline $90 / 5 / 5$ & 0,27712 & $50 / 10 / 40$ & 0,28382 \\
\hline $85 / 5 / 10$ & 0,28678 & $33 / 33 / 34$ & 0,31985 \\
\hline $\mathbf{8 0 / 1 0 / 1 0}$ & $\mathbf{0 , 2 3 2 4 3}$ & $20 / 20 / 60$ & 0,34620 \\
\hline $70 / 10 / 20$ & 0,23705 & $10 / 20 / 70$ & 0,39151 \\
\hline
\end{tabular}

Tabelle 22: Fehlerwert $s_{\mathrm{p}}$ in Abhăngigkeit von der Aufteilung der Datenmenge für das Grundmodell

Die Aufteilung 80/10/10 stellt die beste Lösung dar, die eine gute Kombination zwischen Lernverhalten und Generalisierungsfähigkeit aufweist.

Der Parameter „Startgewichte“ ist, wie bereits oben erläutert wurde, zum einen durch die Einstellung einer „range“ und zum anderen durch eine Verteilungsform (uniform oder gaussian) bestimmt. ${ }^{714}$ Somit werden Veränderungen sowohl des Wertebereiches (der Startgewichte) als auch der Verteilung hinsichtlich der Fehlerbeeinträchtigung analysiert (vgl. Tabelle 23). ${ }^{715}$ Die beste Lösung liegt bei einer range von 0,3 und einer Gaussian-Verteilung der Startgewichte.

713 Vgl. Uhlig (1995), S. 36; Cassetti (1993), S. 78ff.; Kean (1992), S. 58ff.; Kean (1993), S. $78 \mathrm{ff}$.

714 Für genauere Informationen siehe SPSS (1997), S. 134f.

715 Es wurden weitere Einstellungen für den Parameter „Range“ gewählt (bspw. auch Werte größer 0,3), dabei wurden jedoch keine deutlichen Unterschiede sichtbar. 
Marktorientierte Kreditrisikobewertung - Empirische Untersuchung zur marktorientierten Bewertung des Kreditrisikos mittels Künstlicher Neuronaler Netze

\begin{tabular}{|l|l|l|}
\hline Range / Verteilung & Gaussian & Uniform \\
\hline 0,05 & 0,25121 & 0,26882 \\
\hline 0,1 & 0,23243 & $\mathbf{0 , 2 3 2 4 1}$ \\
\hline 0,3 & 0,22907 & 0,24372 \\
\hline
\end{tabular}

Tabelle 23: Fehlerwert $s_{F}$ in Abhångigkeit von der Verteilung der Startgewichte für das Grundmodell

Als weitere Möglichkeit, den Lemprozess zu verändern, kann die Anzahl an Lernschritten durch den Netzarchitekten beeinflusst werden. Darüber hinaus besteht bei Neural Connection ${ }^{\circledR}$, wie bereits o.g., die Möglichkeit der Einteilung von vier Lerngruppen. Die Lernschritte der einzelnen Lernstufen werden jeweils in gleich große Werte eingeteilt. Die Abbildung 41 zeigt die Entwicklung des Fehlerwertes in Abhängigkeit von den Lernschritten. Die untersuchten Lernschritte sind 50, 100, 200, 400, $800,1.200,1.600,2.000,2.400,2.800,3.200,3.600,4.000,5.000,6.000,7.000,8.000$, 9.000 und 10.000. Der geringste Fehlerwert liegt bei 800 Lernschritten vor (Fehlerwert: 0,2291). Strenggenommen dürfte die Kurve sich ab einer gewissen Anzahl an Lernschritten nicht weiter verändern. Die Abbildung lässt vermuten, dass dies ab ca. 1200 Lernschritten der Fall ist. Lernwiederholungen darüber hinaus lassen ein „overlearning" stattfinden, somit ist ab diesem Punkt zu erwarten, dass der Fehlerwert nicht durch weitere Lernschritte gesenkt werden kann. Der (rückwirkende) Abbruchalgorithmus - niedrigster Fehlerwert der Validierungsmenge, „early-stopping-Zeitpunkt“ stoppt das Netz jeweils an derselben Stelle. Die Veränderungen bei 6.000 und 8.000 Lernschritten lassen sich nicht direkt erklären. Es ist zu vermuten, dass durch die programmspezifische Aufteilung in vier Lerngruppen der Lemprozess sich bei höheren Lernschritten leicht verändern kann. ${ }^{716}$ Die Differenz dieser Unterschiede ist jedoch sehr gering. Insgesamt wird deutlich, dass keine Lemerfolge durch eine besonders große Anzahl von Lernschritten erzielt werden können. Da ein Übertrainieren durch die Validierungsmenge ausgeschlossen wird und das ausgewiesene Netz nicht durch den geringsten Fehlerwert der Trainings-, sondern den geringsten Fehlerwert der Validierungsmenge bestimmt wird, kann die Anzahl der Lernschritte nicht zu groß werden. Gleichzeitig wird jedoch die Bearbeitungszeit deutlich gesteigert. Als Indiz des „overlearning“ ab 800 Lernwiederholungen lässt sich folgender Test anführen:

Falls man keinen ,early-stopping-Zeitpunkt" misst, den Validierungsfehler als Abbruchkriterium also ausklammert, und eine Lernwiederholungsrate von 10.000 vorgibt, lässt sich ein Fehlerwert für die Trainingsmenge von unter 0,094673 messen. Gleich-

716 Diese Vermutung wird durch die Beobachtung gestützt, dass es bei Deaktivierung des Lernstufenmodus zu keiner Veränderung des Fehlerwertes bei hohen Lernwiederholungen kommt. 
Marktorientierte Kreditrisikobewertung - Empirische Untersuchung 172 zur marktorientierten Bewertung des Kreditrisikos mittels Künstlicher Neuronaler Netze

zeitig weist die Testmenge einen Fehlerwert von 0,328575 auf. Durch das oben genannte Abbruchkriterium, dem ,early-stopping-Zeitpunkt“", ist somit die Generalisierungsfähigkeit des Netzes gesichert. Ein reines „Auswendiglernen“ der Muster innerhalb der Trainingsdaten ist nicht möglich.

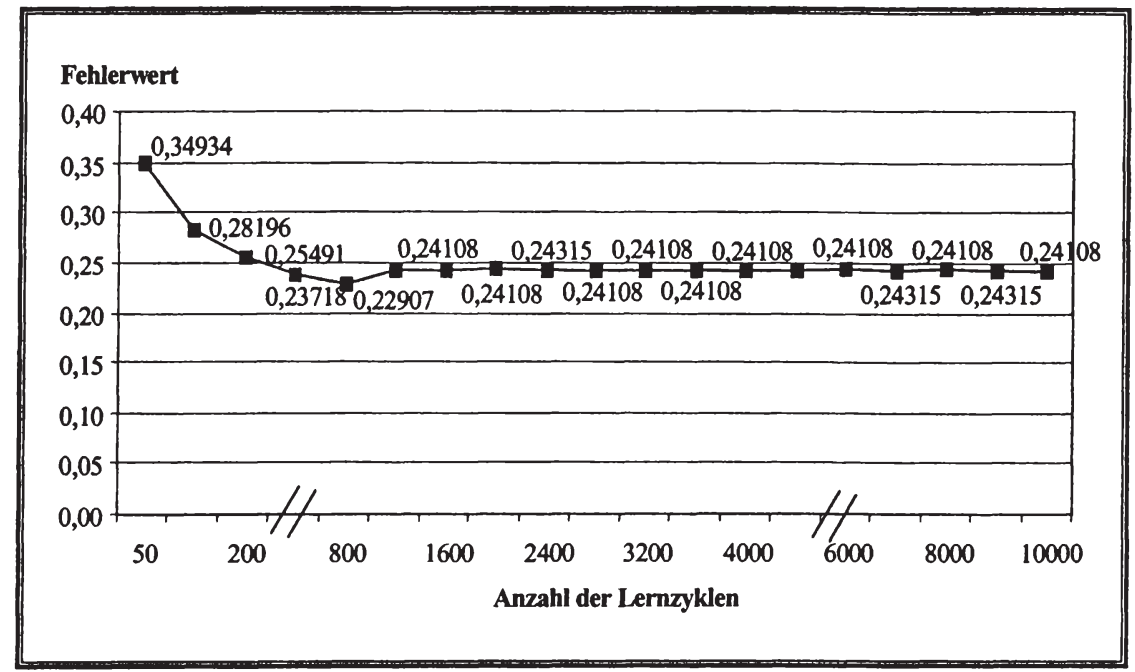

Abbildung 41: Fehlerwert $s_{\mathrm{F}}$ in Abhăngigkeit von der Anzahl der Lernschritte für das Grundmodell

Es lässt sich konstatieren, dass die Ergebnisse, gemessen durch den Fehlerwert der Testmenge, in einem recht engen Intervall liegen. Die meisten Einstellungen führen zu einem Fehlerwert, der in dem Intervall von 0,24 - 0,25 liegt. Dies bedeutet eine relativ große Ergebnisrobustheit gegenüber Veränderungen in den verschiedenen Netzeinstellungen und der damit verbundenen Subjektivität durch den Netzwerkarchitekten. 
Marktorientierte Kreditrisikobewertung - Empirische Untersuchung zur marktorientierten Bewertung des Kreditrisikos mittels Künstlicher Neuronaler Netze 173

\subsubsection{Ergebnisdarstellung}

Während in dem vorangegangenen Kapitel 5.4.3.1 die Parameterkonfiguration des Grundmodells abgeleitet wurde, wird im Weiteren sowohl die Netzwerkstruktur als auch der ausgewiesene Fehlerwert beschrieben. Das Grundmodell weist folgende Parametereinstellungen auf:

- Sigmoid-Aktivienungsfunktion

- Netzgröße

- 20 Neuronen in der versteckten Schicht

- 1 versteckte Schicht

- 80-10-10 Aufteilung der Datenmenge (Trainings- / Validierungs- / Generalisierungsdaten)

- Verteilungsfunktion der Startgewichte: gaussian ${ }^{717}$

- 800 Lernschritte.

Das Grundmodell besitzt den in Abbildung 42 dargestellten Aufbau. Insgesamt nehmen 32 Eingangsneuronen die Informationen auf, wobei sich drei auf die makroökonomischen Daten, drei auf die titelspezifischen Daten und 26 auf die Bilanzkennzahlen beziehen. Diese 32 Neuronen leiten die (normierten) Werte an 20 Neuronen in der versteckten Schicht weiter. Es handelt sich hierbei um einen layer, d.h. eine Unterschicht in der versteckten Schicht. Die im Rahmen der Informationsverarbeitung verwendete Aktivierungsfunktion ist die Sigmoid-Funktion. Die 20 Neuronen in der versteckten Schicht leiten ihre Werte an ein Ausgangsneuron weiter. Dieses gibt den prognostizierten Credit Spread wieder.

717 Softwarespezifische Einstellung, Range 0,3 (vgl. SPSS (1997), S. 134f.). 
Marktorientierte Kreditrisikobewertung - Empirische Untersuchung 174 zur marktorientierten Bewertung des Kreditrisikos mittels Künstlicher Neuronaler Netze

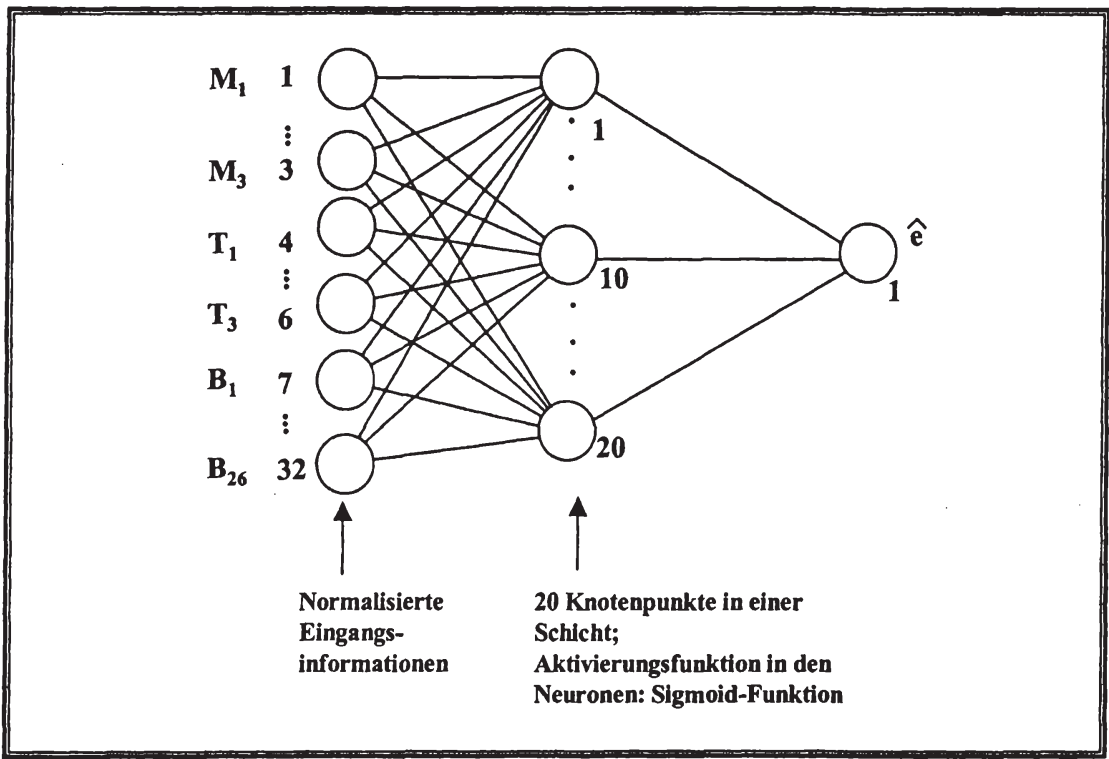

Abbildung 42: Schematischer Aufbau des Grundmodells

Zur Messung der Netzgüte wurde der Fehlerwert $\mathrm{s}_{\mathrm{F}}$ bestimmt. Dieser berechnet sich aus den durchschnittlichen Abweichungen zwischen dem prognostizierten und dem beobachteten Credit Spread. Falls man nun die Differenz der einzelnen Datensätze bildet, d.h. je Anleihe $i$ in der Testmenge die Differenz aus $e_{i}$ und $\hat{e}_{i}$ bildet und diese gemäß einer Häufigkeitsverteilung abbildet, gelangt man zu der Fehlerverteilungsfunktion. Die Fehlerverteilungsfunktion ist die Häufigkeitsfunktion der Differenz von Prognose- und Istwert. Es sollte ein Mittelwert der Verteilungsfunktion nahe bei Null zu beobachten sein. Die Standardabweichung dieser Funktion entspricht dem Fehlerwert $\mathrm{S}_{\mathrm{F}}$. Darüber hinaus wird innerhalb der Beschreibung der Häufigkeiten der Abweichungen zwischen beobachteten und gemessenen Credit Spread eine Ausweitung des Intervalls über die Standardabweichung hinaus gewählt. Das 90-Prozent-Intervall bezieht sich auf die in Absolutbeträgen gemessene Abweichung. Für das Szenario 1 bedeutet ein Wert von 0,3478 , dass 90 Prozent der Abweichungen geringer sind als dieser Wert. Somit lassen sich Aussagen bezüglich der prozentualen Häufigkeit von Abweichungsgrößen innerhalb der Testmenge treffen. 
Marktorientierte Kreditrisikobewertung - Empirische Untersuchung zur marktorientierten Bewertung des Kreditrisikos mittels Künstlicher Neuronaler Netze

Für das Grundmodell sind folgende Ergebnisse erzielt worden (vgl. Tabelle 24):

\begin{tabular}{|l|l|l|l|}
\hline & $\begin{array}{l}\text { Datenaufteilung in Prozent (Trai- } \\
\text { ning / Validierung / Test); dle } \\
\text { Anzahl an Făllen fur die Testmenge } \\
\text { ist Jeweils 164 }\end{array}$ & $\begin{array}{l}\text { Standardabweichung } \\
\text { der Fehlerverteilungs- } \\
\text { funktion (Testmenge) }\end{array}$ & $\begin{array}{l}\text { 90 Prozent Inter- } \\
\text { vall (Testmenge) }\end{array}$ \\
\hline Szenario 1 & $80 / 10 / 10$ & 0,2294 & 0,3478 \\
\hline Szenario 2 & $80 / 10 / 10$ & 0,2437 & 0,4302 \\
\hline Szenario 3 & $80 / 10 / 10$ & 0,2942 & 0,5359 \\
\hline
\end{tabular}

Tabelle 24: Standardabweichungen und 90-Prozent-Intervall-Fehlerwert der Fehlerverteilungsfunktion des Grundmodells

Das Szenario 1 entspricht einer vollkommen zufälligen Datenaufteilung. Dies bedeutet, dass bei Unternehmen mit mehreren Wertpapieren auf dem Markt ein Wertpapier in der Test- und eines in der Trainingsmenge vorhanden sein kann. Für die Übertragung auf die Kreditvergabe würde dies bedeuten, die Kreditprämie eines bekannten Kunden zu berechnen, der bereits einen laufenden Kredit (mit anderen Ausstattungsmerkmalen) erhalten hat. Für das Szenario 1 wurde bei einer Standardabweichung von 0,2294 das beste Ergebnis erzielt. Dies kann damit begründet werden, dass die Credit Spread Bestimmung für eine zweite Anleihe bzw. einen zweiten Kredit mit einer höheren Genauigkeit durchgefuhrt werden kann, da bereits kundenspezifische Informationen vorliegen. Die Fehlerverteilungsfunktion (Abbildung 43) zeigt eine deutlich Häufung um den Nullpunkt. Diese Beobachtung wird auch durch den Mittelwert in Höhe von 0,01299 Prozentpunkten und einer Varianz von 0,05262 (Tabelle 25) bestätigt. Diese hohe Prognosefähigkeit kommt auch in dem Wert des 90-Prozent-Intervalls zum Ausdruck, da der Credit Spread von 90 Prozent der 164 untersuchten Anleihen von dem KNN mit einer Ungenauigkeit von maximal 0,3478 Prozentpunkten prognostiziert werden kann.

\begin{tabular}{|c|c|}
\hline $\begin{array}{l}\text { Deskriptive Kennzahlen Szenario 1, } \\
\text { Grundmodell }\end{array}$ & $\mathbf{e}_{\mathrm{i}}-\hat{\mathrm{e}}_{\mathrm{i}}$ \\
\hline $\mathrm{N}$ & 164 \\
\hline Median & 0,09397 \\
\hline Mittelwert & 0,01299 \\
\hline Standardabweichung $\left(=\mathrm{s}_{\mathrm{F}}\right)$ & 0,2294 \\
\hline Varianz & 0,05262 \\
\hline Spannweite & 1,92 \\
\hline Minimum & $-0,57$ \\
\hline Maximum & 1,35 \\
\hline
\end{tabular}

Tabelle 25: Deskriptive Kennzahlen der Testmenge, Szenario 1 im Grundmodell 
Marktorientierte Kreditrisikobewertung - Empirische Untersuchung 176 zur marktorientierten Bewertung des Kreditrisikos mittels Künstlicher Neuronaler Netze

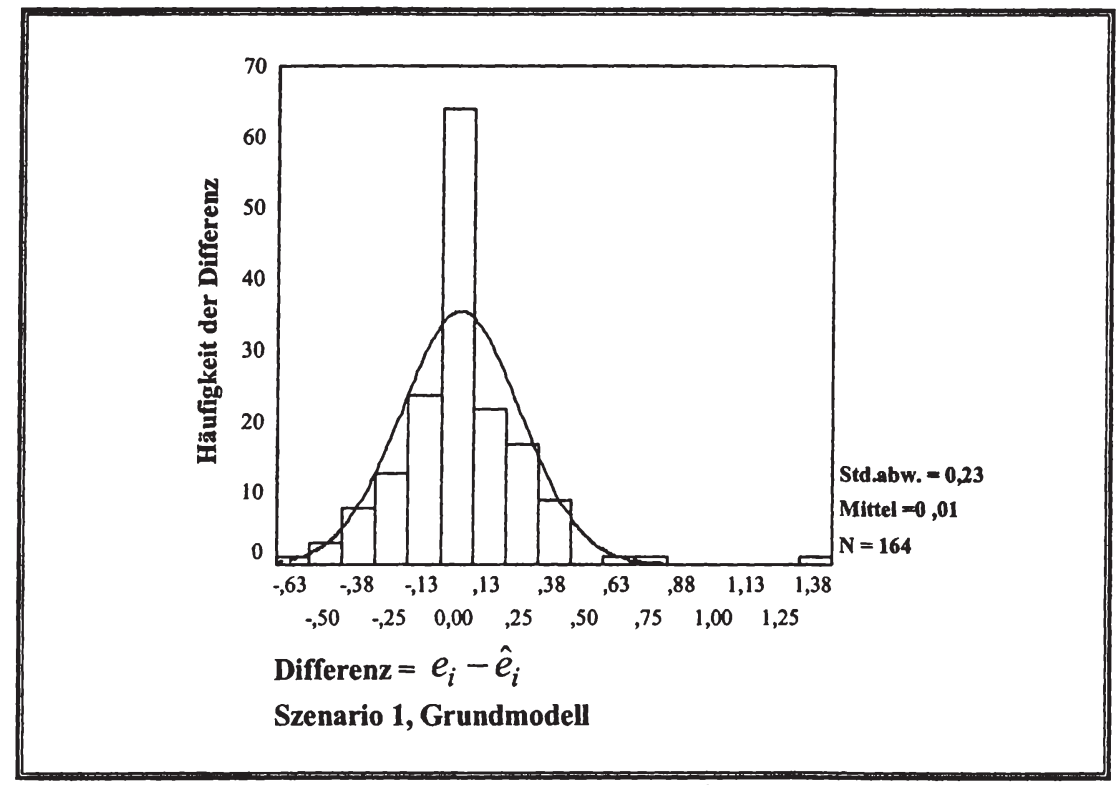

Abbildung 43: Hăufigkeitsfunktion der Differenzen, Szenario 1, Grundmodell

Szenario 2 berücksichtigt, dass ein Unternehmen zu einem Stichtag nicht ein Wertpapier in der Trainings- und ein anderes in der Testmenge haben kann. Man kann dies interpretieren als ein Unternehmen, welches zwar bereits Kunde einer Bank ist, jedoch zu dem aktuellen Zeitpunkt keinen laufenden Kredit erhalten hat. Der Fehlerwert für das Szenario 2 ist günstiger als für das Szenario 3 jedoch schlechter als für das Szenario 1. Ebenfalls lässt sich eine Häufung der Fehlerverteilungsfunktion um den Nullwert beobachten (Abbildung 44), sodass auch der Mittelwert nahe bei Null liegt (Tabelle 26).

\begin{tabular}{||c|c|}
\hline $\begin{array}{l}\text { Deskriptive Kennzahlen Szenario 2, } \\
\text { Grundmodell }\end{array}$ & $\mathbf{e}_{\mathbf{i}}-\widehat{\mathrm{e}}_{\mathbf{i}}$ \\
\hline $\mathbf{N}$ & 164 \\
\hline Median & $-0,084955$ \\
\hline Mittelwert & $-0,069203$ \\
\hline Standardabweichung $\left(=\mathbf{s}_{\mathrm{s}}\right)$ & 0,2437 \\
\hline Varianz & 0,05940 \\
\hline Spannweite & 1,03 \\
\hline Minimum & $-0,55$ \\
\hline Maximum & 0,48 \\
\hline
\end{tabular}

Tabelle 26: Deskriptive Kennzahlen der Testmenge, Szenario 2 im Grundmodell 
Marktorientierte Kreditrisikobewertung - Empirische Untersuchung zur marktorientierten Bewertung des Kreditrisikos mittels Künstlicher Neuronaler Netze 177

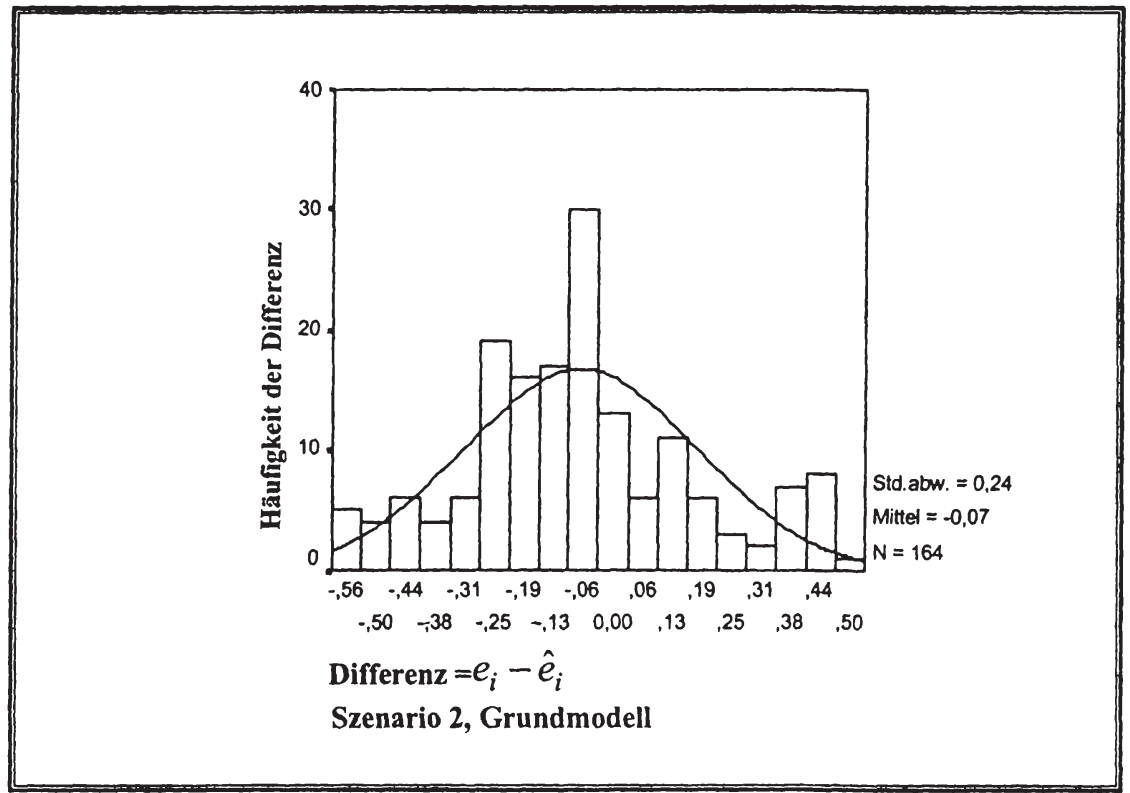

Abbildung 44: Häufigkeitsfunktion der Differenzen, Szenario 2, Grundmodell

Szenario 3 stellt sicher, dass ein Unternehmen nicht gleichzeitig in die Lern- und in die Testmenge eingeteilt werden kann. Dies bedeutet, dass ein Unternehmen noch kein Kunde der Bank ist. Es ist auffällig, dass im Szenario 3 die höchste Standardabweichung vorliegt (Tabelle 27) und es bei Betrachtung der Fehlerverteilungsfunktion zu keiner Häufung um den Nullwert kommt (Abbildung 45).

Die im Vergleich zu Szenario 1 schlechten Ergebnisse der Szenarien 2 und insbesondere 3 können damit begründet werden, dass das Grundmodell auf Basis des Szenarios 1 abgeleitet wurde. Es kann vermutet werden, dass die Fehlerwerte der beiden anderen Szenarien sich reduzieren lassen, falls eigene Parameterkonfigurationen dafür abgeleitet würden.

\begin{tabular}{|c|c|}
\hline \begin{tabular}{|c|} 
Deskriptive Kennzahlen Szenario 3, \\
Grundmodell
\end{tabular} & $\mathrm{e}_{\mathrm{i}}-\hat{\mathrm{e}}_{\mathrm{i}}$ \\
\hline $\mathrm{N}$ & 164 \\
\hline Median & 0,1277 \\
\hline Mittelwert & 0,1005 \\
\hline Standardabweichung $\left(=\mathrm{s}_{\mathrm{f}}\right)$ & 0,2942 \\
\hline Varianz & 0,08653 \\
\hline Spannweite & 1,15 \\
\hline Minimum & $-0,41$ \\
\hline Maximum & 0,74 \\
\hline
\end{tabular}

Tabelle 27: Deskriptive Kennzahlen der Testmenge, Szenario 3 im Grundmodell 
Marktorientierte Kreditrisikobewertung - Empirische Untersuchung 178 zur marktorientierten Bewertung des Kreditrisikos mittels Künstlicher Neuronaler Netze

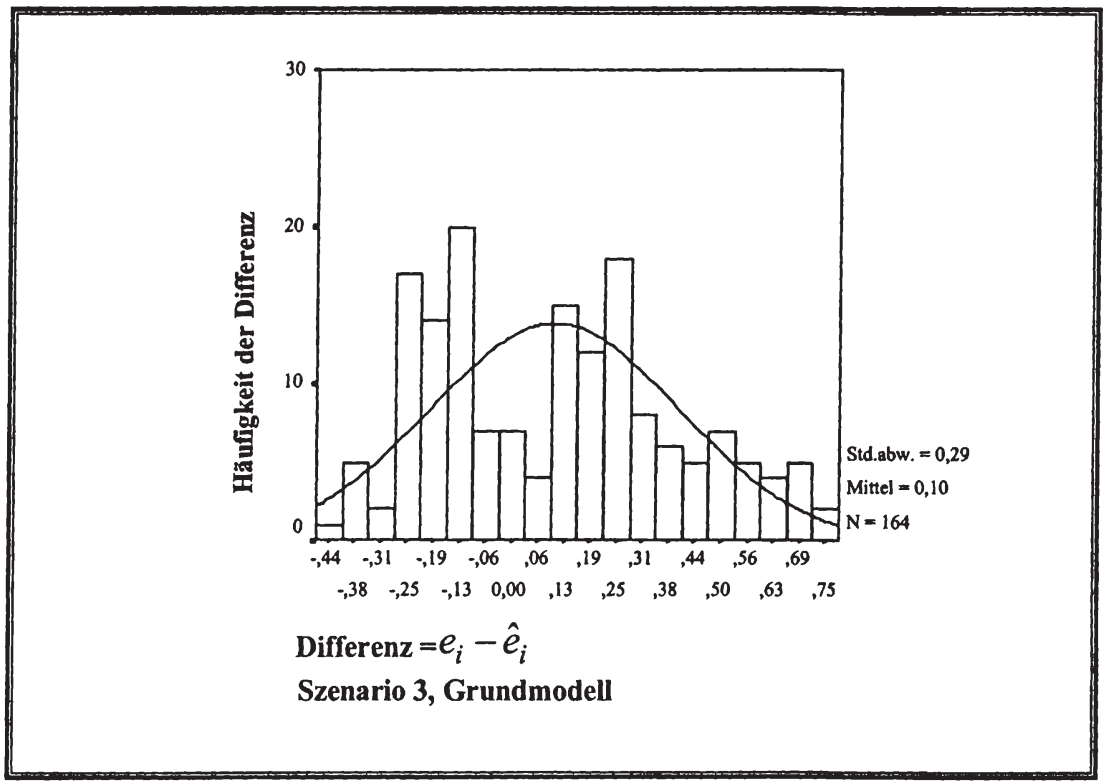

Abbildung 45: Hăufigkeitsfunktion der Differenzen, Szenario 3, Grundmodell

\subsubsection{Sensitivitätsanalyse als Ausgangspunkt einer Faktorenreduktion}

Während sich die im Kapitel 5.4.3.1 dargestellte Parametervariation auf die Veränderung der Netzwerkeinstellungen und die damit verbundenen Ergebnisveränderungen, gemessen durch den Fehlerwert $\mathrm{s}_{\mathrm{F}}$ der Testmenge, bezogen hat, werden im Folgenden die Inputfaktoren und deren Bedeutung für das Ergebnis untersucht. Grundsätzlich wird im Umgang mit Neuronalen Netzen versucht, die Anzahl der Inputfaktoren auf ein Minimum zu reduzieren. ${ }^{718}$ Ausgangspunkt ist zunächst ein Netz mit einem möglichst umfassenden Katalog an Kennzahlen, d.h. Eingangsinformationen, aus denen dann die für die Mustererkennung relevanten Informationen bestimmt werden. Nichtrelevante werden "abgeschnitten“, sodass der Begriff „Pruning“ (to prune $=$ abschneiden) für diese Technik verwendet wird.

Das Pruning könnte zum einen damit begründet werden, dass ein Neuronales Netz so einfach wie möglich gestaltet sein sollte, um die Anwendbarkeit zu stützen. ${ }^{71}$ Eine Funktion mit wenigen Inputfaktoren ist ,überschaubarer" und lässt sich von Anwendern bspw. bei Kreditentscheidungen leichter nachvollziehen. Außerdem wird die

718 Vgl. bspw. hier und im Folgenden Glormann (2001), S. 247ff.

719 Vgl. zu der Begründung des Pruning hier und im Folgeden Karnin (1990), S. 239. 
Marktorientierte Kreditrisikobewertung - Empirische Untersuchung zur marktorientierten Bewertung des Kreditrisikos mittels Künstlicher Neuronaler Netze

Berechnungszeit und somit die Performance durch eine geringere Anzahl an Inputfaktoren positiv beeinflusst. Zum anderen - und dies ist der wichtigere Grund - wird die Reduktion der Inputfaktoren mit dem „Verrauschen“ von Datensätzen begründet. Durch die Reduktion um nicht gebrauchte Informationen soll nämlich der Fehlerwert verringert werden. Eine Information wird dann nicht gebraucht, wenn sie furr die Mustererkennung nicht notwendig ist. Beispielsweise könnte man für die Prognose des Credit Spread auch die „Tagestemperatur“ als weiteren Inputfaktor aufnehmen. Es ist zu erwarten, dass diese Eingangsinformationen keinen Einfluss auf den Credit Spread ausübt. Dementsprechend müsste in einem optimalen Netz die Information „Tagestemperatur" mit einem Gewichtungsfaktor von „Null“ bewertet werden. Eine andere Möglichkeit besteht darin den Eingangswert „Tagestemperatur“ nicht als Inputfaktor aufzunehmen. Da man nicht von vorneherein die Relevanz oder Irrelevanz der Eingangsinformationen erkennen kann, ergibt sich zunächst die Möglichkeit, alle Kombinationen, die die Eingangsinformationen bilden, als Netzstrukturen anzulegen, und die entsprechenden Fehlerwerte abzulesen. Das Netz mit dem geringsten Fehlerwert beinhaltet die relevanten Eingangsinformationen. Bei 32 Inputfaktoren - wie bei der hier geführten Untersuchungen - müssten somit $2^{32}-1=4.294 .967 .295$ Kombinationen untersucht werden. Aufgrund der großen Zahl kann jedoch nicht jede Kombination getestet werden. Somit ist es wichtig, Kenntnis über die Relevanz (oder anders ausgedrückt Sensitivität) der Eingangsinformationen auf den Zielwert zu erlangen.

Da durch die Software Neural Connections ${ }^{\circledast}$ keine direkten Pruningverfahren zur Verfügung gestellt werden, wird im Folgenden das Pruning auf der Grundlage einer Sensitivitätsanalyse durchgefuhrt. ${ }^{720}$ Hierzu wird zunächst der Zielwert bei einem Datensatz ermittelt, bei dem alle Inputfaktoren gleich dem jeweiligen Median ihrer Verteilungsfunktion sind. Für diesen „virtuellen“ Datensatz wird der Credit Spread mittels KNN bestimmt $\left(\hat{\mathrm{e}}_{\mathrm{M}}\right)$. Anschließend wird jeweils ein Inputfaktor sowohl auf das 1. als auch auf das 3. Quartil seiner eigenen Verteilung gesetzt. Falls man bspw. die Sensitivität für die Eingangsinformation $M_{1}$ berechnen will, wird diese (und nur diese) Information gleich dem Wert des 1. Quartils (3. Quartils) gesetzt und der Credit

720 Ein Überblick zu verschiedenen Pruningverfahren findet sich bei Baun, S. (1994), S. 163; Miller (1994), S. 125ff.; Benenati (1998), S. $51 \mathrm{ff}$. 
Marktorientierte Kreditrisikobewertung - Empirische Untersuchung 180 zur marktorientierten Bewertung des Kreditrisikos mittels Künstlicher Neuronaler Netze

Spread mittels des KNN bestimmt ( $\hat{\mathrm{e}}^{\mathrm{x}_{1} \text {. Quaril }}$ bzw. $\left.\hat{\mathrm{e}}_{M_{1}}^{\mathrm{x}_{3} \text {. puaril }}\right)$. Die Differenz der beiden Credit Spreads $\left(\left|\hat{e}_{M_{1}}^{x_{1 . \text { Quaril }}}-\hat{e}_{M}\right|\right.$ bzw. $\left.\left|\hat{e}_{M_{1}}^{x_{1}}{ }_{M_{1} \text { Quartil }}-\hat{e}_{M}\right|\right)$ beschreibt die Sensitivität des Netzes in Bezug auf die Veränderungen des Inputfaktors $M_{1}$ (vgl. Abbildung 46). ${ }^{721}$

Es werden anschließend Rankings der Sensitivitäten in Bezug auf Änderungen hinsichtlich des 1. und des 3. Quartils aufgestellt, indem für alle Eingangsinformationen die Sensitivitäten einzeln bestimmt werden. Ein Inputfaktor kann in einem Quartil einen sehr starken Einfluss, in dem anderen jedoch nur eine geringere Bedeutung auf die Veränderung des Credit Spread besitzen. Bspw. könnte der Inputfaktor $\mathbf{M}_{1}$ für das 1. Quartil einen 3. Platz und für das 3. Quartil einen 7. Platz ausweisen. Die Einzelrankings je Inputfaktor für das 1 . und das 3. Quartil werden anschließend durch eine einfache Summierung zusammengezogen (für den Inputfaktor $\mathbf{M}_{1}$ wäre dies ein Wert von 10) und mit Hilfe der Summe der Einzelrankings wird ein neues Gesamtranking ausgewiesen. Dem Inputfaktor mit der niedrigsten Summe aus den Einzelrankings wird der Platz 1 und dem Inputfaktor mit der höchsten Summe wird Platz 32 zugeordnet. Nachdem nun ein Ranking der Sensitivitäten aufgestellt wurde, wird der Inputfaktor mit dem tiefsten Rang gestrichen und von der weiteren Untersuchung ausgeklammert. Die Anzahl der Inputfaktoren des neuen Netzes reduziert sich somit um „1“. Es wird wiederum für das neue Netz der Fehlerwert gemessen und im Anschluss an die Sensitivitätsanalyse ein neues Ranking als Ausgangspunkt für die Reduktion um einen Inputfaktor gebildet. Die Abbildung 47 zeigt den Verlauf des Fehlerwertes $S_{F}$ in Abhängigkeit der Folge der Verringerung der Inputfaktoren.

721 Bei dieser Vorgehensweise wird unterstellt, dass alle Inputfaktoren voneinander unabhängig sind. Diese Annahme konnte nicht durch eine Korrelationsanalyse belegt werden. Trotz der Verletzung der Annahme wird die Sensitivitätsanalyse herangezogen, um die Einflussstärke einzelner Faktoren zu bestimmen. Vgl. auch zur Vorgehensweise der „globalen Sensitivitätsanalyse“ bspw. Baetge (1998), S. 600f.; Glormann (2001), S. $280 \mathrm{ff}$. Da im weiteren Analyseverlauf nach jeder auf Basis der Sensitivitätsanalyse bestimmten Faktorenreduktion der Fehlerwert gemessen wird, kann zumindest ausgeschlossen werden, dass relevante Informationen vernachlässigt werden. Es kann jedoch nicht widerlegt werden, dass andere Faktorenreduktionen (Pruningverfahren) zu besseren Netzstrukturen führen könnten. Vgl. auch das in Kap. 5.2.2 dargelegte Verständnis einer "optimalen" Struktur. 


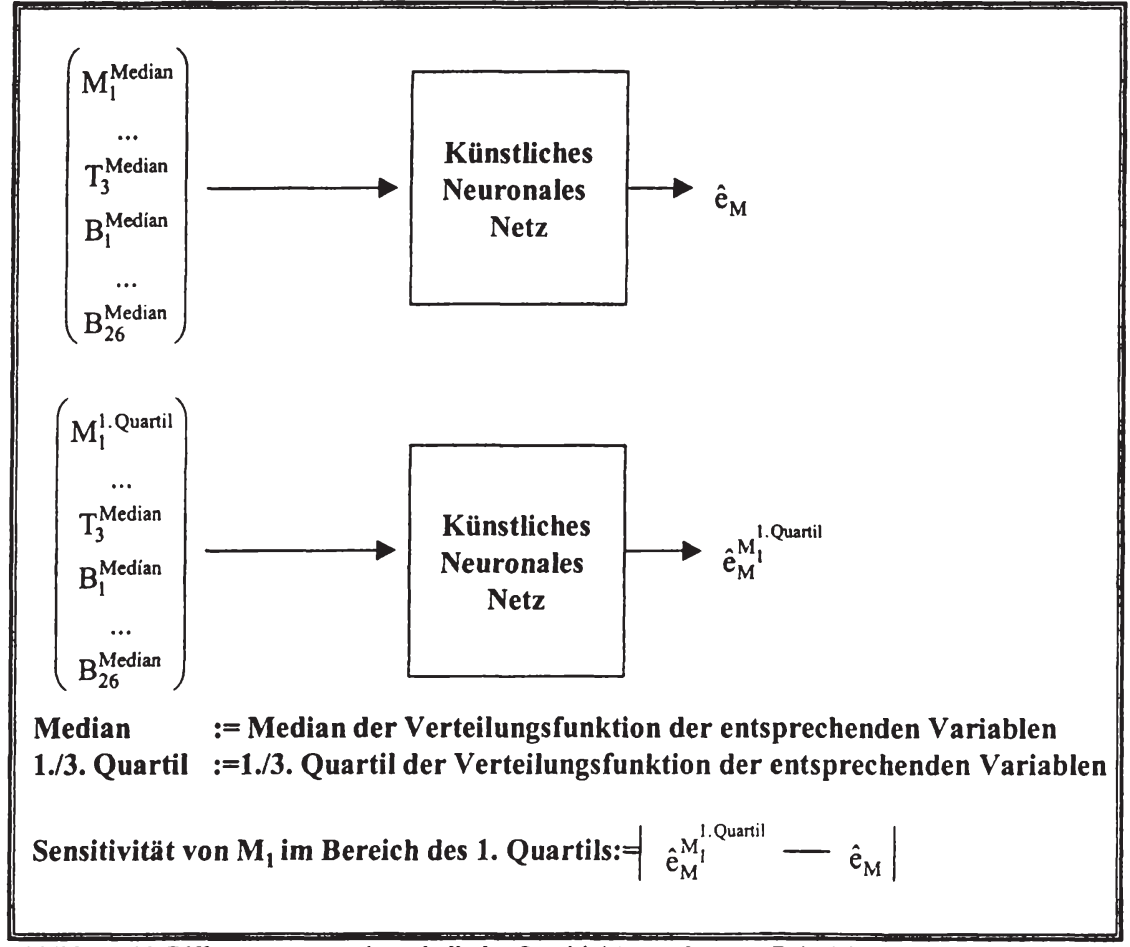

Abbildung 46: Differenzmessung innerhalb der Sensitivitätsanalyse am Beispiel des Inputfaktors $M_{1}$ und im Bereich des 1. Quartils

Ein Abbruchkriterium für die Inputreduktion wird durch den starken Anstieg von 0,2221 auf 0,2687 bei der Reduktion von 13 auf 12 Inputfaktoren aufgezeigt. Hier ist sowohl ein sehr guter Fehlerwert als auch eine sehr gute Performancezeit zu finden. Durch die starke Reduktion der Inputfaktoren liegt die Bearbeitungszeit unter $60 \mathrm{Mi}$ nuten. ${ }^{722}$ Folgende 13 Inputfaktoren wurden vom $\mathrm{KNN}$ als relevante Informationen charakterisiert: $\mathrm{M}_{1}, \mathrm{M}_{2}, \mathrm{M}_{3}, \mathrm{~T}_{2}, \mathrm{~T}_{3}, \mathrm{~B}_{2}, \mathrm{~B}_{4}, \mathrm{~B}_{6}, \mathrm{~B}_{13}, \mathrm{~B}_{16}, \mathrm{~B}_{18}, \mathrm{~B}_{22}$ und $\mathrm{B}_{24}{ }^{723}$ 
Marktorientierte Kreditrisikobewertung - Empirische Untersuchung 182 zur marktorientierten Bewertung des Kreditrisikos mittels Künstlicher Neuronaler Netze

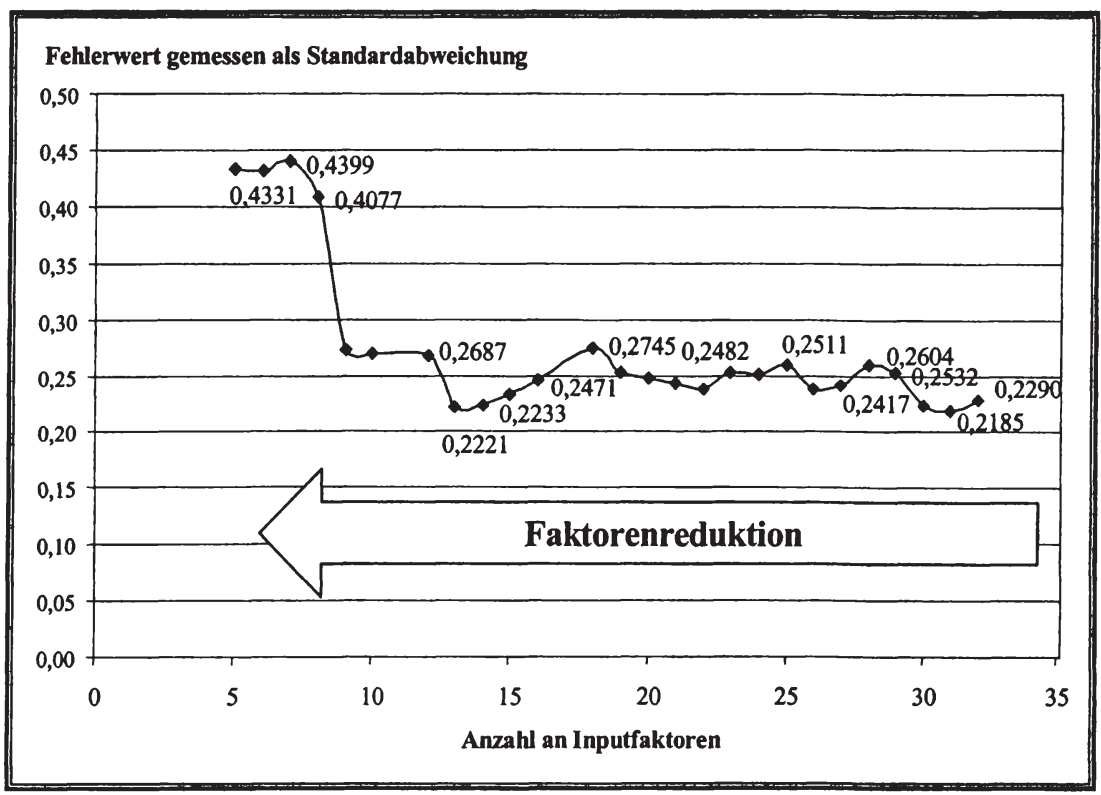

Abbildung 47: Fehlerwert $s_{F}$ in Abhängigkeit von der Anzahl der Inputfaktoren

Man kann konstatierten, dass ein Netz mit einem Inputfaktorensatz von 13 verschiedenen Kennzahlen in sehr kurzer Zeit sehr gute Lernerfolge erzielen kann. Das Ziel der Sensitivitätsanalyse bestand zum einen in der Reduktion der Bearbeitungszeit (Performance) und zum anderen in der Reduktion des Fehlerwertes. Beide Ziele wurden erreicht. 
Marktorientierte Kreditrisikobewertung - Empirische Untersuchung

\subsubsection{Das 13er-Modell}

\subsubsection{Parameterkonfiguration}

Parallel zu der Vorgehensweise in Kapitel 5.4.3.1 werden die Einstellungsparameter variiert. Das Grundschema ist hierbei das aus der Sensitivitätsanalyse abgeleitete Modell mit 13 Inputfaktoren. Unterstellt wird wiederum das Szenario 1. Folgende Einstellungen liegen auf Grund des Grundschemas der Software Neural Connection ${ }^{\circledast}$ vor:

- Sigmoid-Aktivierungsfunktion,

- Netzgröße:

○ 9 Knotenpunkte,

- 1 versteckte Schicht,

- 80-10-10 Aufteilung der Datenmenge,

- Range: 0,3; Distribution: gaussian und

- 800 Lernschritte in vier Gruppen („Lernstufen“). Nach Beendigung einer jeden Gruppe wird der Lernkoeffizient gesenkt bzw. das Momentum erhöht.

Der Fehlerwert $s_{\mathrm{F}}$ des Grundschemas beträgt 0,2221. Analog zu der Herleitung des Grundmodells wird auch in dem 13er-Modell der Fehlerwert nach jeder Parametervariation gemessen und die Struktur wird als Ausgangsbasis für die nächste Variation herangezogen, die den niedrigsten Fehlerwert aufweist. ${ }^{724}$

Zunächst wird die Aktivierungsfunktion variiert (vgl. Tabelle 28). Durch die softwarespezifischen Ausgestaltungsmerkmale sind drei Typen vorgegeben.

\begin{tabular}{|l|l|l|l|}
\hline Aktivierungsfunktion & Tanh & sigmoid & linear \\
\hline Fehlerwert der Testmenge & 0,3062 & $\mathbf{0 , 2 2 2 1}$ & 0,3378 \\
\hline
\end{tabular}

Tabelle 28: Fehlerwert $s_{\mathrm{F}}$ in Abhăngigkeit von der Aktivierungsfunktion für das 13er-Modell

Anschließend wird bei Verwendung der Sigmoid-Aktivierungsfunktion die Anzahl der Neuronen in der versteckten Schicht verändert (erster Schritt) Netzgrößenvariation). Für das Netz, basierend auf 11 Knotenpunkten, liegt der niedrigste Fehlerwert mit 0,2217 vor (vgl. Abbildung 48).

724 Abweichend kann fallbezogen ein Netz mit einem sehr niedrigen Fehlerwert und gleichzeitig besten Performance gewählt werden. 
Marktorientierte Kreditrisikobewertung - Empirische Untersuchung 184 zur marktorientierten Bewertung des Kreditrisikos mittels Künstlicher Neuronaler Netze

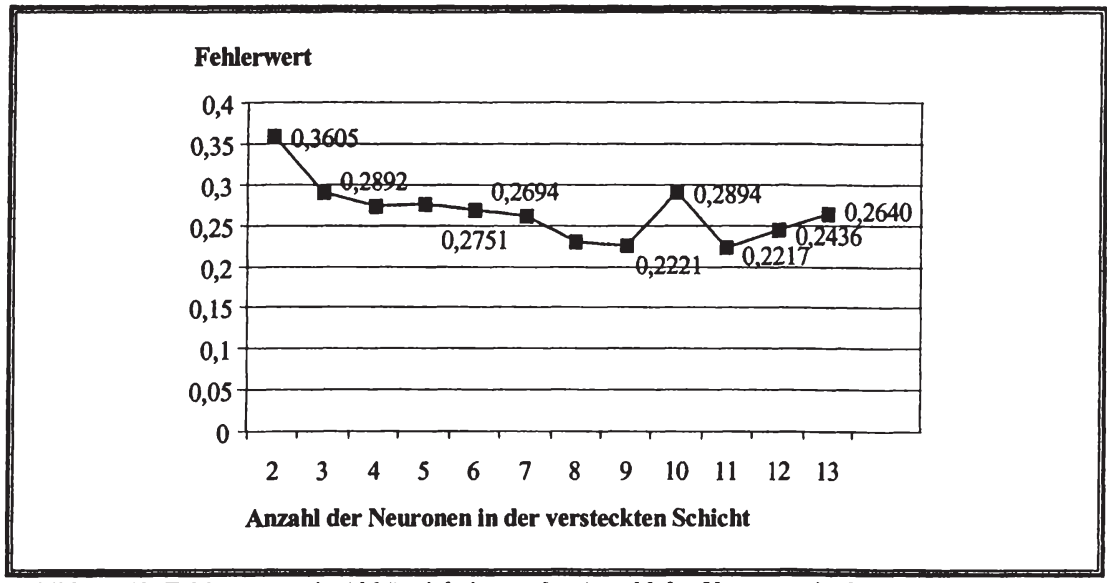

Abbildung 48: Fehlerwert $\mathrm{s}_{\mathrm{F}}$ in Abhängigkeit von der Anzahl der Neuronen in der versteckten Schicht fur das 13er-Modell

Für die weiteren Parametervariationen werden 11 Neuronen in der versteckten Schicht beibehalten. In dem zweiten Schritt der Netzgrößenvariation wird versucht, durch die Aufnahme eines zweiten layers den Fehlerwert zu verringern. Analog zu Kapitel 5.4.3.1 wird mindestens in einem layer eine Sigmoid-Aktivierungsfunktion verwendet. Die Tabelle 29 zeigt einen Überblick hinsichtlich der gemessenen Fehlerwerte.

\begin{tabular}{|l|l|}
\hline Aktivierungsfunktion (zwei Schichten) & Fehlerwert \\
\hline (Ausgangsnetz) Schicht, 11 Knoten, sigmoid-Funktion & $\mathbf{0 , 2 2 1 7}$ \\
\hline (Variation 1) 2 Schichten, 5 + 6 Knoten, sigmoid / tanh-Funktion & 0,2900 \\
\hline (Variation 3) 2 Schichten, 5 + 6 Knoten, tanh / sigmoid-Funktion & 0,2822 \\
\hline (Variation 2) 2 Schichten, 5 + 6 Knoten, sigmoid / sigmoid-Funktion & 0,2868 \\
\hline
\end{tabular}

Tabelle 29: Fehlerwert $s_{\mathrm{F}}$ in Abhängigkeit von der Anzahl der Schichten der Knotenpunkte und der Aktivierungsfunktion für das 13er-Modell

Die bereits im Rahmen des Grundmodells gefundenen Ergebnisse zur Netzgrößenvariation durch Aufnahme einer zweiten Schicht werden auch beim 13er-Modell bestätigt. Die Lerndauer nimmt zu, während gleichzeitig der Lernerfolg - gemessen als Fehlerwert der Testmenge - deutlich abnimmt. Somit werden hier keine weiteren Schichten in die Untersuchung aufgenommen. Im Weiteren wird die Aufteilung der Datenmenge variiert. Tabelle 30 zeigt die Ergebnisse. 
Marktorientierte Kreditrisikobewertung - Empirische Untersuchung zur marktorientierten Bewertung des Kreditrisikos mittels Künstlicher Neuronaler Netze 185

\begin{tabular}{|l|l|l|l|}
\hline $\begin{array}{l}\text { Datenaufteilung in } \\
\text { Trainings- / Validie- } \\
\text { rungs- / Testmengen } \\
\text { [Angaben in Prozent] }\end{array}$ & $\begin{array}{l}\text { Fehlerwert, } \\
\text { gemessen an der } \\
\text { Testmenge }\end{array}$ & $\begin{array}{l}\text { Datenaufteilung in } \\
\text { Trainings- / Validie- } \\
\text { rungs- / Testmengen } \\
\text { [Angaben in Prozent] }\end{array}$ & $\begin{array}{l}\text { Fehlerwert, } \\
\text { gemessen an } \\
\text { der Test- } \\
\text { menge }\end{array}$ \\
\hline $95 / 2,5 / 2,5$ & 0,2235 & $60 / 10 / 30$ & 0,2522 \\
\hline $90 / 5 / 5$ & 0,2663 & $50 / 10 / 40$ & 0,2805 \\
\hline $85 / 5 / 10$ & 0,2960 & $33 / 33 / 34$ & 0,2937 \\
\hline $\mathbf{8 0 / 1 0 / 1 0}$ & $\mathbf{0 , 2 2 1 7}$ & $20 / 20 / 60$ & 0,2911 \\
\hline $70 / 10 / 20$ & 0,2306 & $10 / 20 / 70$ & 0,3701 \\
\hline
\end{tabular}

Tabelle 30: Fehlerwert $s_{\mathrm{F}}$ in Abhängigkeit von der Aufteilung der Datenmenge für das 13er-Modell

Für den Bereich 80/10/10 liegt im direkten Vergleich die beste Lösung vor, die eine gute Kombination zwischen Lernverhalten (große Lernmenge) und Generalisierungsfähigkeit (große Testmenge) aufzeigt. Somit können die Datenaufteilungen des Grundschemas übernommen werden.

Die Startgewichte wurden nun hinsichtlich der Verteilung und des Wertebereiches verändert. Tabelle 31 zeigt einen Ergebnisüberblick:

\begin{tabular}{|l|l|l|}
\hline Range / Verteilung & Gaussian & Uniform \\
\hline 0,3 & 0,2217 & 0,2468 \\
\hline 0,05 & 0,2373 & 0,2394 \\
\hline 0,03 & $\mathbf{0 , 2 2 0 5}$ & 0,2379 \\
\hline
\end{tabular}

Tabelle 31: Fehlerwert $s_{\mathrm{F}}$ in Abhängigkeit der von Verteilung der Startgewichte für das 13er-Modell

In einer abschließenden Variation werden die Lernschritte verändert. Es wird analog zu der Herleitung des Grundmodells eine Lernschrittvariation vorgenommen. Das bedeutet jeweils, für die unterschiedlichen Anzahlen an Lernzyklen (50, 100, 200, 400, $800,1.200,1.600,2.000,2.400,2.800,3.200,3.600,4.000,5.000,6.000,7.000,8.000$, 9.000 und 10.000), eine Netzstruktur zu entwickeln. Parallel zu den o.g. Beobachtungen sollte eine Fixierung des Fehlerwertes ab einer bestimmten Anzahl an Lernschritten zu beobachten sein. Wie Abbildung 49 zeigt, ist dies jedoch nicht zu beobachten. Der Verfasser ist der Meinung, dass dies wiederum ein programmspezifisches Verhalten ist. Durch die Aufteilung in vier Lernstufen ist es scheinbar möglich, auch bei hohen Lernzyklen zu neuen Netzstrukturen zu gelangen. Hieraus resultieren unterschiedliche Fehlerwerte bei der Erhöhung der Anzahl an Lernschritten. ${ }^{725}$

725 Diese Vermutung wird wiederum durch die Beobachtung gestützt, dass es bei der Deaktivierung des Lernstufenmodus zu keiner Veränderung des Fehlerwertes bei hohen Lernwiederholungen kommt. 
Marktorientierte Kreditrisikobewertung - Empirische Untersuchung 186 zur marktorientierten Bewertung des Kreditrisikos mittels Künstlicher Neuronaler Netze

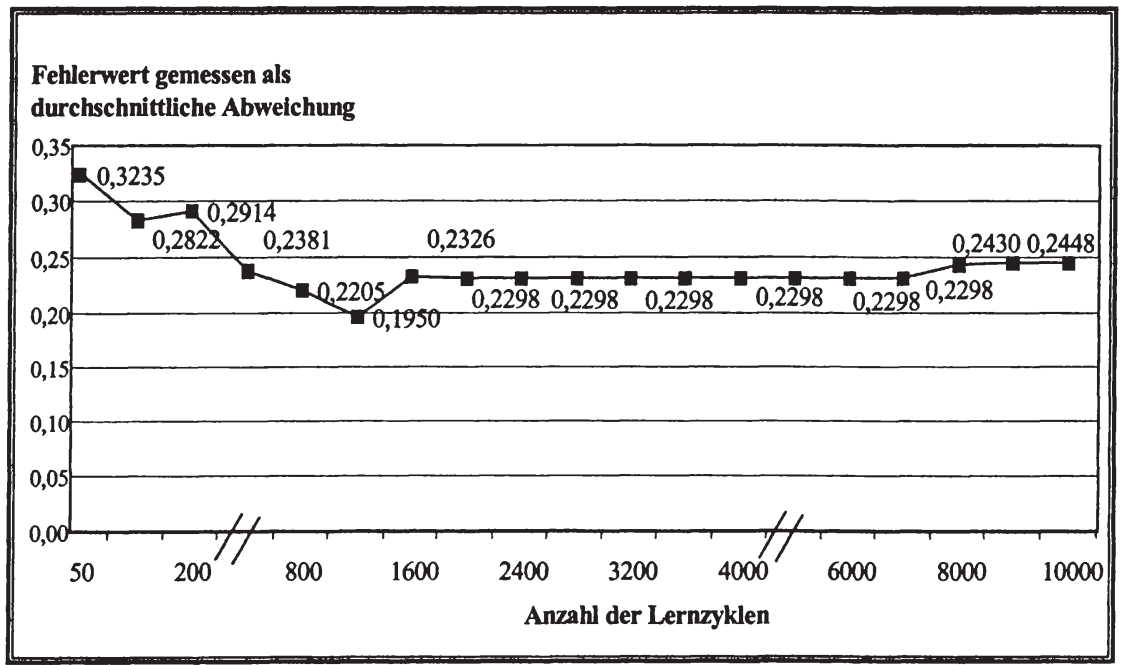

Abbildung 49: Fehlerwert $s_{\mathrm{F}}$ in Abhängigkeit von der Anzahl an Lernschritten für das 13er-Modell

Ein ähnlicher Kurvenverlauf wie bei Abbildung 41 ist zu beobachten: Ein zunächst sehr stark fallender Fehlerwert, der sich nach einem (lokalen) Minimum nicht weiter positiv verändert. Auffallend sind in der Abbildung 49 die bereits oben genannten Veränderungen des Fehlerwertes bei sehr hohen Lernwiederholungsraten. Das lokale Minimum liegt bei 1200 Lernschritten und einem Fehlerwert von 0,1950.

Zusammenfassend kann man - ähnlich wie beim Grundmodell - festhalten, dass die Ergebnisse recht stabil sind. Sie liegen wiederum in einem engen Ergebnisintervall und belegen die Unabhängigkeit von den verschiedenen Netzeinstellungen. Der geringste Fehlerwert lag bei 0,1950 Prozentpunkten.

\subsubsection{Ergebnisdarstellung}

Auf Basis der Grundmodells wurde eine Inputfaktorenreduktion auf Basis einer Sensitivitätsanalyse durchgeführt. Im Ergebnis wurden 13 Inputfaktoren bestimmt, die (bzw. die dahinter liegenden Informationen) im Rahmen der KNN-Analyse als die wichtigsten Eingangsinformationen analysiert wurden. Ohne diese 13 Inputfaktoren konnte das KNN den Credit Spread vergleichbar schlechter prognostizieren. Folglich wurden diese 13 Inputfaktoren herangezogen, um ein zweites Netz zu bestimmen: Das 13erModell. Analog zu der Herleitung des Grundmodells wurden auch bei dem 13erModell Parametervariationen durchgeführt, um einen möglichst geringen Fehlerwert zu ermöglichen. Die beste Parameterkonstellation innerhalb der bestmöglichen Netzstruktur wies einen Fehlerwert in Höhe von 0,1950 Prozentpunkten auf. Das 13erModell hat folgende Parametereinstellungen: 
Marktorientierte Kreditrisikobewertung - Empirische Untersuchung zur marktorientierten Bewertung des Kreditrisikos mittels Künstlicher Neuronaler Netze 187

- Sigmoid-Aktivierungsfunktion,

- Netzgröße:

- 11 Knotenpunkte,

- 1 versteckte Schicht,

- 80-10-10 Aufteilung der Datenmenge,

- Verteilung der Startgewichte: range 0,03 und gaussian,

- 1.200 Lernschritte.

Wie in Abbildung 50 dargestellt, leiten 13 Inputfaktoren $\left(M_{1}, M_{2}, M_{3}, T_{2}, T_{3}, B_{2}, B_{4}\right.$, $B_{6}, B_{13}, B_{16}, B_{18}, B_{22}$ und $\left.B_{24}\right)^{726}$ in der Eingabeschicht die (normierten) Informationen in die versteckte Schicht. Diese besteht aus einem layer mit 11 Neuronen. Die mit Hilfe der Sigmoid-Aktivierungsfunktion verarbeiteten Informationen werden an ein Neuron in der Ausgabeschicht weitergeleitet. Dieses gibt den prognostizierten Credit Spread $\hat{e}_{i}$ für die Anleihe i wieder.

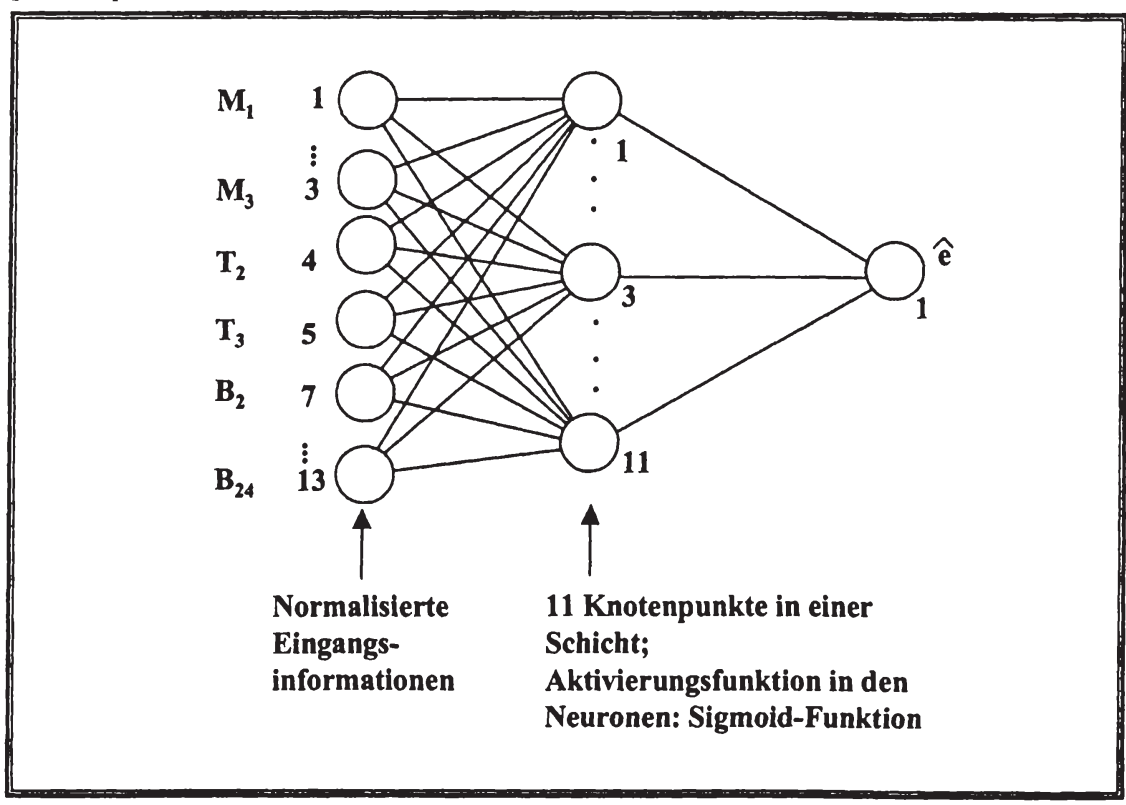

Abbildung 50: Schematischer Aufbau des 13er-Modells

Analog zu der Vorgehensweise beim Grundmodell wird das 13er-Modell nicht nur für das Szenario 1, sondern ebenfalls für die Szenarien 2 und 3 verwendet. Die Fehlerver-

Zur Beschreibung und Interpretation vgl. Kap. 5.4.6. 
Marktorientierte Kreditrisikobewertung - Empirische Untersuchung 188 zur marktorientierten Bewertung des Kreditrisikos mittels Künstlicher Neuronaler Netze

teilungsfunktion resultiert wiederum aus der Häufigkeitsverteilungsfunktion der einzelnen Differenzen. Die Standardabweichung der Fehlerverteilungsfunktion entspricht wiederum dem Fehlerwert des entsprechenden Netzes. Wie auch bei dem Grundmodell weist das Szenario 1 den geringsten Fehlerwert auf. Im Vergleich zwischen dem Grundmodell und dem 13er-Modell konnte sowohl die Standardabweichung (von 0,2294 auf 0,1950) als auch das 90 Prozent Intervall (von 0,3478 auf 0,3234) gesenkt werden. Dies bedeutet, dass bei der Prognose des Credit Spread einer Anleihe das 13er-Modell in 90 Prozent der untersuchten Fälle eine maximale Differenz zum gemessenen Credit Spread in Höhe von 0,3234 Prozentpunkten hat. Auch für die Szenarien 2 und 3 konnten die Fehlerwerte deutlich gesenkt werden, so wies bspw. das 90 Prozent Intervall für das Szenario 3 beim Grundmodell noch eine Abweichung von 0,5359 auf. Das 13er-Modell hat demgegenüber eine geringere Abweichung in Höhe von 0,4044 Prozentpunkten. Die Tabelle 32 fasst die Ergebnisse der unterschiedlichen Szenarien zusammen.

\begin{tabular}{|l|l|l|l|}
\hline & $\begin{array}{l}\text { Datenaufteilung in Prozent (Trai- } \\
\text { ning / Validierung / Test); die } \\
\text { Anzahl an Fällen für die Test- } \\
\text { menge ist jeweils 162 }\end{array}$ & $\begin{array}{l}\text { Standardabweichung } \\
\text { der Fehlervertei- } \\
\text { lungsfunktion (Test- } \\
\text { menge) }\end{array}$ & $\begin{array}{l}\text { 90 Prozent } \\
\text { Intervall } \\
\text { (Testmenge) }\end{array}$ \\
\hline Szenario 1 & $80 / 10 / 10$ & 0,1950 & 0,3234 \\
\hline Szenario 2 & $80 / 10 / 10$ & 0,2247 & 0,4632 \\
\hline Szenario 3 & $80 / 10 / 10$ & 0,2589 & 0,4044 \\
\hline
\end{tabular}

Tabelle 32: Standardabweichungen und 90-Prozent-Intervall-Fehlerwert der Fehlerverteilungsfunktion des 13er-Modells

Für das Szenario 1 konnte wie auch beim Grundmodell der mit 0,1950 geringste Fehlerwert ermittelt werden (vgl. auch Tabelle 33). Dementsprechend konnten Anleihen von Emittenten, von denen zeitgleich weitere Anleihen gehandelt werden, von dem $\mathrm{KNN}$ am treffendsten abgebildet werden. Die Fehlerverteilungsfunktion besitzt wiederum eine Häufung um den Nullpunkt (vgl. Abbildung 51), sodass auch der Mittelwert mit $-0,000251$ sehr nahe an Null liegt.

Die Testmenge ( $10 \%$ der Datengrundlage) besteht aus exakt 9,872\% oder 162 Fällen. Diese Rundungsdifferenzen führen dazu, dass, obwohl sowohl im Grundmodell als auch im 13er-Modell $10 \%$ der Testmenge gewählt wurden, die Anzahl der Fälle sich leicht unterscheidet. 
Marktorientierte Kreditrisikobewertung - Empirische Untersuchung

\begin{tabular}{|c|c|}
\hline $\begin{array}{l}\text { Deskriptive Kennzahlen Szenario 1, } \\
\text { 13er-Modell }\end{array}$ & $\mathrm{e}_{\mathrm{i}}-\widehat{\mathrm{e}}_{\mathrm{i}}$ \\
\hline $\mathrm{N}$ & 162 \\
\hline Median & $-0,00518$ \\
\hline Mittelwert & $-0,000251$ \\
\hline Standardabweichung $\left(=\mathrm{s}_{\mathrm{F}}\right)$ & 0,1950 \\
\hline Varianz & 0,03802 \\
\hline Spannweite & 1,03 \\
\hline Minimum & $-0,27$ \\
\hline Maximum & 0,76 \\
\hline
\end{tabular}

Tabelle 33: Deskriptive Kennzahlen der Testmenge, Szenario $1 \mathrm{im}$ 13er-Modell

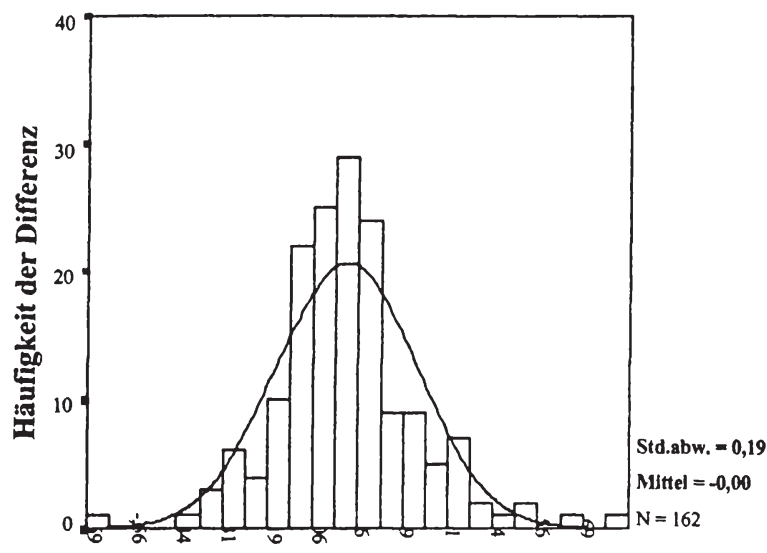

Differenz $=e_{i}-\hat{e}_{i}$

Szenario 1, 13er Modell

\section{Abbildung 51: Hăufigkeitsfunktion der Differenzen, Szenario 1, 13er-Modell}

Für das Szenario 2, was unterstellt, dass vom Emittent der zu bewertenden Anleihe zeitgleich keine weitere Anleihe auf dem Kapitalmarkt gehandelt wird, kann zwar im Vergleich zum Grundmodell eine günstigere Standardabweichung gemessen werden, jedoch ist der Fehlerwert für das 90 Prozent Intervall größer. In Abbildung 52 ist zu erkennen, dass in dem Bereich der Differenzen vom $-0,56$ bis $-0,31$ es zu einer relativ großen Häufung kommt. Aufgrund dieser deutlichen Differenzen resultiert ein relativ schlechter 90 Prozent Intervall Wert. Der Mittelwert in Höhe von 0,06092 (vgl. Tabelle 34) ist (erwartungsgemäß) nahe Null. 
Marktorientierte Kreditrisikobewertung - Empirische Untersuchung 190 zur marktorientierten Bewertung des Kreditrisikos mittels Künstlicher Neuronaler Netze

\begin{tabular}{|c|c|}
\hline \begin{tabular}{|c|} 
Deskriptive Kennzahlen Szenario 2, \\
13er-Modell
\end{tabular} & $\mathbf{e}_{\mathbf{i}}-\mathbf{e}_{\mathbf{i}}$ \\
\hline $\mathbf{N}$ & 162 \\
\hline Median & 0,06329 \\
\hline Mittelwert & 0,06092 \\
\hline Standardabweichung $\left(=\mathrm{s}_{\mathrm{F}}\right)$ & 0,2247 \\
\hline Varianz & 0,05050 \\
\hline Spannweite & 1,16 \\
\hline Minimum & $-0,57$ \\
\hline Maximum & 0,59 \\
\hline
\end{tabular}

Tabelle 34: Deskriptive Kennzahlen der Testmenge, Szenario 2 im 13er-Modell

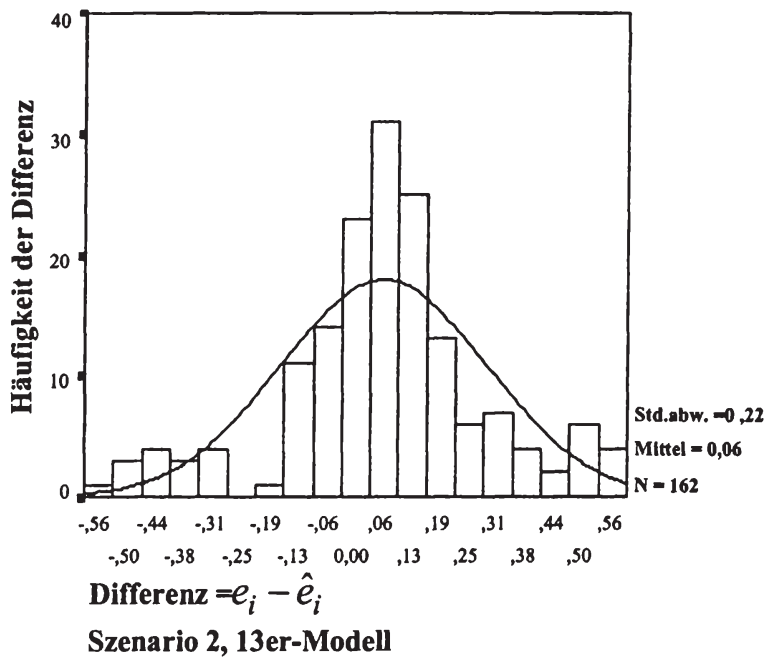

Abbildung 52: Hăufigkeitsfunktion der Differenzen, Szenario 2, 13er-Modell

Insbesondere der niedrige Mittelwert in Höhe von $-0,0343$ (vgl. Tabelle 35) und der im Vergleich zum Grundmodell deutlich niedrigere 90 Prozent Intervall Wert dokumentieren die positiven Auswirkungen der Inputfaktorenreduktion für das Szenario 3. Trotzdem wird in Abbildung 53 deutlich, dass es sowohl im Bereich der positiven als auch negativen Differenzen zwischen dem prognostizierten und dem beobachteten Credit Spread zu Häufungen außerhalb des Nullwertbereiches kommt. Dementsprechend liegt eine relativ hohe Varianz mit 0,06701 vor.

Wie auch bei der Ergebnisdarstellung des Grundmodells können die schlechten Ergebnisse der Szenarien 2 und 3 damit begründet werden, dass das 13er-Modell auf Basis 
Marktorientierte Kreditrisikobewertung - Empirische Untersuchung

des Szenarios 1 abgeleitet wurde. Es kann vermutet werden, dass die Fehlerwerte der beiden anderen Szenarien sich reduzieren lassen, falls eigene Parameterkonfigurationen dafür abgeleitet werden.

\begin{tabular}{|c|c|}
\hline \begin{tabular}{|c|} 
Deskriptive Kennzahlen Szenario 3, \\
13er-Modell
\end{tabular} & $\mathbf{e}_{\mathrm{i}}-\widehat{\mathrm{e}}_{\mathrm{i}}$ \\
\hline $\mathrm{N}$ & 162 \\
\hline Median & $-0,0282$ \\
\hline Mittelwert & $-0,0343$ \\
\hline Standardabweichung $\left(=\mathrm{s}_{\mathrm{F}}\right)$ & 0,2589 \\
\hline Varianz & 0,06701 \\
\hline Spannweite & 0,99 \\
\hline Minimum & $-0,51$ \\
\hline Maximum & 0,48 \\
\hline
\end{tabular}

Tabelle 35: Deskriptive Kennzahlen der Testmenge, Szenario $3 \mathrm{Im}$ 13er-Modell

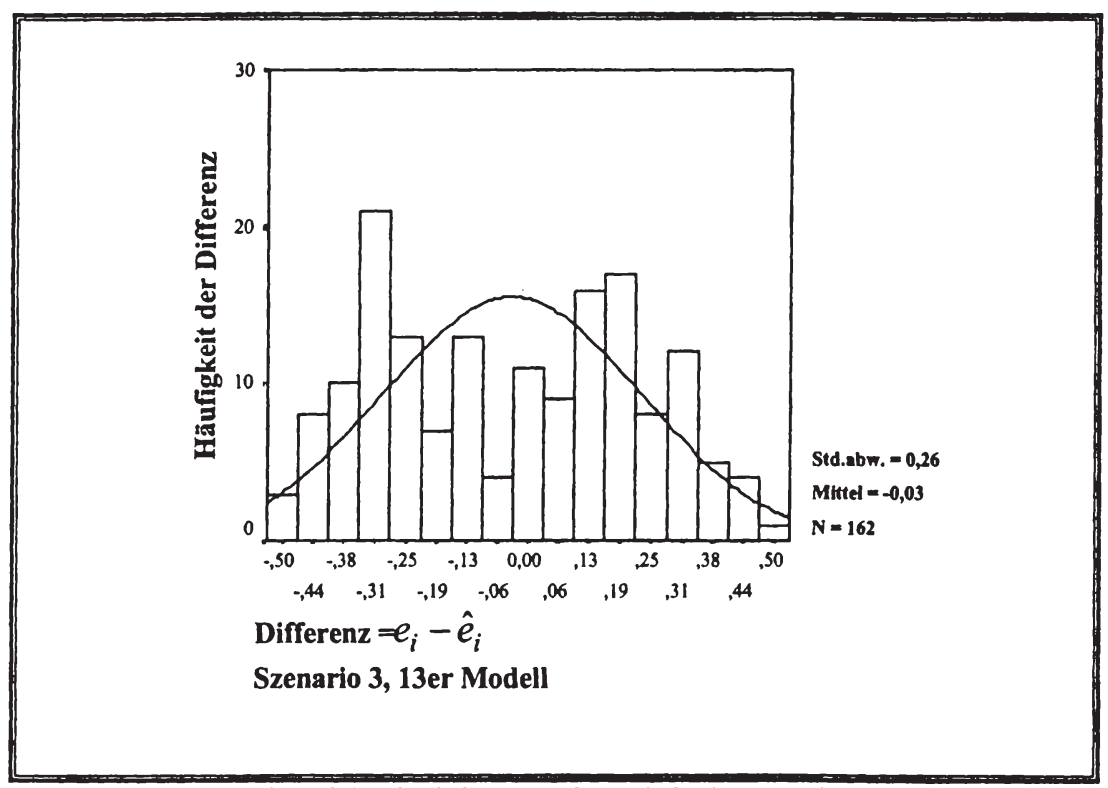

Abbildung 53: Hăufigkeitsfunktion der Differenzen, Szenario 3, 13er-Modell

\subsubsection{Einordnung des 13er-Modells in die Bilanzanalyse und in die modelltheoretischen Verfahren der Kreditzinskalkulation}

Während in den vorangegangenen Kapiteln ein Neuronales Netz entwickelt und die für dieses Netz relevanten Eingangsinformationen abgeleitet wurden, werden diese Erkenntnisse im weiteren Analyseverlauf zu den in den Kap. 2 und Kap. 3 gewonnenen Erkenntnissen der Kreditzinskalkulationsverfahren und der Bilanzanalyse in Beziehung gesetzt. Die Auswahl von 13 Faktoren verdeutlicht die Fähigkeit des Netzes, 
Marktorientierte Kreditrisikobewertung - Empirische Untersuchung 192 zur marktorientierten Bewertung des Kreditrisikos mittels Künstlicher Neuronaler Netze

anhand von wenigen Inputfaktoren die Erwartungsbildung des Kapital- bzw. Kreditmarktes bezüglich des Ausfallrisikos des Kreditnehmers zufriedenstellend abzubilden. Diese Inputfaktorenauswahl wird im Folgenden näher untersucht. Hierzu soll die „Netzauswahl" mit den im Kapitel 3 dargestellten traditionellen und modernen Kennzahlen bzw. Kennzahlensystemen verglichen werden. Dieser Vergleich sieht von der Bilanzierungsproblematik (US-GAAP vs. HGB) ab.

In Tabelle 36 werden die gefundenen Inputfaktoren genannt und (falls möglich) dem Informationsbereich der Bilanz (Ertrags-, Finanz-, Vermögenslage) zugeordnet. Gleichzeitig werden diese Kennzahlen in die bekannten Bilanzanalyseverfahren aus Kapitel 3 eingeordnet. Eine eindeutige Einordnung ist nicht möglich, da die untersuchten Kennzahlen auf aggregierten Informationsebenen in die empirische Analyse eingeflossen sind. Neben der Einordnung in die Bilanzanalyseverfahren ist auch die Zuordnung zu den im Kapitel 2.3.2 vorgestellten modelltheoretischen Konzepten der Kreditzinskalkulation ersichtlich.

In Kap. 4.4 wurde ein Konzept für ein Prognosemodell vorgestellt, das den Bewertungsprozess des Kreditmarktes heranzieht, um den Credit Spread für eine einzelne Anleihe bzw. Kredit zu bestimmten. Hierzu war es notwendig ein Analyseinstrument zu integrieren, das in der Lage ist, die am Markt beobachtbaren Zusammenhänge (Muster) zwischen dem Credit Spread und den unterschiedlichen Inputfaktoren abzubilden. Ein Instrument, das hierzu in der Lage ist, sind die Künstlichen Neuronalen Netze. Diese können den Bewertungsprozess des Kreditmarktes abbilden. Das 13er-Modell macht deutlich, dass der Bewertungsprozess des Kreditmarktes eine Kombination aus den bekannten Bilanzanalyseverfahren darstellt und gleichzeitig die Bilanzanalyse um Faktoren erweitert.

Die acht Kennzahlen $\left(\mathrm{B}_{2}, \mathrm{~B}_{4}, \mathrm{~B}_{6}, \mathrm{~B}_{13}, \mathrm{~B}_{16}, \mathrm{~B}_{18}, \mathrm{~B}_{22}\right.$ und $\left.\mathrm{B}_{24}\right)$ beziehen sich auf alle drei Bereiche (Ertrags-, Vermögens- und Finanzlage) des Jahresabschlusses. Das geforderte Ganzheitlichkeitsprinzip wird folgerichtig bei der Kennzahlenauswahl vom KNN beachtet. Die im Rahmen der Kreditzinskalkulation geforderten Determinanten Zinsvolatilität $\left(M_{1}\right)$, allgemeines Marktzinsniveau $\left(M_{2}\right)$, Zinserwartungen $\left(M_{3}\right)$, Restlaufzeit des Kredits $\left(T_{2}\right)$ und Nominalverzinsung $\left(T_{3}\right)$ werden ebenfalls in das Prognosemodell integriert. Sie finden sich auch in den im Kap. 2.3.2 dargestellten Modellen wieder. Man kann das für die Bilanzanalyse geforderte Ganzheitlichkeitsprinzip um Komponenten für die Kreditzinskalkulation erweitern, da, modelltheoretisch belegt und durch empirische Studien gestützt, ein Zusammenhang zwischen weiteren Faktoren und dem Kreditzins besteht. Makroökonomische und titelspezifische Faktoren müssen bei Einhaltung des Ganzheitlichkeitsprinzips in ein Kreditzinskalkulationsverfahren integriert werden. 
Marktorientierte Kreditrisikobewertung - Empirische Untersuchung zur marktorientierten Bewertung des Kreditrisikos mittels Künstlicher Neuronaler Netze

\begin{tabular}{|c|c|c|c|c|}
\hline & Definition & $\begin{array}{c}\text { Informations- } \\
\text { bereich der } \\
\text { Bilanz }\end{array}$ & $\begin{array}{c}\text { Parallelen zu Bilanzanalyse- } \\
\text { verfahren }\end{array}$ & $\begin{array}{l}\text { Parallelen zum sto- } \\
\text { chastischen (st) oder } \\
\text { optionspreistheoreti- } \\
\text { schen (op) Modell }\end{array}$ \\
\hline $\mathrm{M}_{1}$ & Zinsvolalitat des Marktes & Keine & Keine & op: direkt $t^{729}$ \\
\hline $\mathrm{M}_{2}$ & Marktzinsniveau & Keine & Keine & st: direkt, op: direkt \\
\hline $\mathrm{M}_{3}$ & \begin{tabular}{|l|} 
Zinserwartung \\
\end{tabular} & Keine & Keine & st: direkt \\
\hline $\mathrm{T}_{2}$ & Restlaufzeit der Anleihe & Keine & Keine & st: direkt, op: direkt \\
\hline$T_{1}$ & Nominalverzinsung & Keine & Keine & st: direkt, op: indirekt $t^{730}$ \\
\hline $\mathrm{B}_{2}$ & Revenue / Total Assets & Ertragslage & $\begin{array}{l}\text { Bspw. RL-System (Kapitalum- } \\
\text { schlagshäufigkeit) }\end{array}$ & \multirow{8}{*}{$\begin{array}{l}\text { st: indirekt in der Ausfallwahr- } \\
\text { scheinlichkeit bzw. der } \\
\text { Rackzahlungsquote; op: } \\
\text { indirekt im Untemehmens- } \\
\text { wert, die Kennzah! „Quasi- } \\
\text { Verschuldungsgrad“ zeigt } \\
\text { diesen Einfluss exemplarisch } \\
\text { auf }\end{array}$} \\
\hline $\mathrm{B}_{1}$ & Current Liabilities / Total Assets & Vermögenslage & Bspw. BP-14 (Fremdkapitalquote) & \\
\hline$B_{6}$ & $\begin{array}{l}\text { Net Cash Operating (Share- } \\
\text { holder's Equity + Long Term } \\
\text { Debt) }\end{array}$ & Finanzlage & $\begin{array}{l}\text { Bspw. RL-System (dynamischer } \\
\text { Verschuldungsgrad) }\end{array}$ & \\
\hline$B_{13}$ & $\begin{array}{l}\begin{array}{l}\text { Net Cash Operating / Current } \\
\text { Liabilities }\end{array} \\
\end{array}$ & Finanzlage & Bspw. BP-14 (Finanzkraft2) & \\
\hline$B_{16}$ & $\begin{array}{l}\text { (Income before Tax + Inter- } \\
\text { est)/ Total Assets }\end{array}$ & Ertragslage & Bspw. Z"'Score $\left(\mathrm{X}_{3}\right)$ & \\
\hline$B_{18}$ & Income Before Tax / Revenue & Ertragslage & $\begin{array}{l}\text { Bspw. ROI (Periodener- } \\
\text { folg / Umsatz) }\end{array}$ & \\
\hline $\mathrm{B}_{22}$ & $\begin{array}{l}\text { Cash and Equivalents / Current } \\
\text { Liabilities }\end{array}$ & Finanzlage & $\begin{array}{l}\text { Bspw. RL-System (Liquiditatskoeffi- } \\
\text { zient) }\end{array}$ & \\
\hline$B_{24}$ & $\begin{array}{l}\text { Shareholder's Equity / Total } \\
\text { Assets }\end{array}$ & Vermögenslage & $\begin{array}{l}\text { Bspw. Saarbrilckener Modell } \\
\text { (Eigenkapitalquote) }\end{array}$ & \\
\hline
\end{tabular}

Tabelle 36: Darstellung der erklärenden Kennzahlen des 13er-Modells und ihre Einordnung in den Informationsbereich des Jahresabschlusses, hinsichtlich der bekannten Bllanzanalyseverfahren und der modelltheoretischen Ansătze zur Bepreisung des Bonitătsrisikos

\subsection{Modellmodifikationen}

Im Folgenden werden einige Modellmodifikationen behandelt. Das Ausgangsmodell ist hierbei das unter Kapitel 5.4.5 herausgearbeitete 13er-Modell. Es wird darauf verzichtet, die oben genannten Parametervariationen in den Modellmodifikationen durchzuführen, da es nicht Ziel ist, die Ergebnisgüte signifikant zu verbessern, sondern vielmehr sollen die Effekte modifizierter im Folgenden erläuterter Fragestellungen untersucht werden.

728 Es kann lediglich von Parallelen gesprochen werden. Bspw. bildet $\mathrm{B}_{13}$ den Quotienten aus „Current Liabilities“ und „Net Cash Operating“. Die Größe Finanzkraft2 setzt kurzfristige Verbindlichkeiten ins Verhältnis zum ertragswirtschaftlichen Cash Flow. Somit lassen sich Parallelen im Bezug auf die Fristigkeit der Verbindlichkeiten sehen. Die Aggregationsebene der bereitgestellten Daten verhindert einen detaillierten Vergleich.

729 Neuere Ansätze bspw. Shimko, Tejima, van Deventer (1993) oder Longstaff, Schwartz (1995) nehmen die Zinsvolatilität mit auf.

730 Bei einer Modellerweiterung erhält die Nominalverzinsung einen Einfluss, da die verbundenen Zahlungen in Cash Flows separiert werden. Vgl. Kirmße (1996), S. 148ff. 
Marktorientierte Kreditrisikobewertung - Empirische Untersuchung 194 zur marktorientierten Bewertung des Kreditrisikos mittels Künstlicher Neuronaler Netze

\subsubsection{Effektive Rendite vs. Credit Spread}

Die Bildung des Credit Spread ist - wie im Kapitel 4.3.3 dargestellt - in der Praxis immer mit Fehlerwerten behaftet. Somit könnte man davon absehen, den Credit Spread zu ermitteln. Alternativ kann man ein Prognosemodell zur Herleitung der effektiven Rendite konzipieren. ${ }^{731}$ Dadurch ist die Zielvariable in dem KNN nicht die Differenz aus effektiver Rendite und dem berechneten sicheren Zins, sondern lediglich der effektive Zins. Fragwürdig ist dieses Vorgehen, falls man dem System die Informationen der Indexkurve vorenthält. $\mathrm{Da}$ jedoch bei dem Inputfaktor „Zinsniveau“ parallel zu der Vorgehensweise von HEINKE ${ }^{732}$ hier der ,analoge ${ }^{[/ 733}$ Kreditmarktzins gewählt wurde, ist dem System explizit die Information aus der Regressionsgleichung bekannt. Somit wird keine Zusatzinformation durch die Veränderung des Zielwertes erreicht. Das o.g. 13er-Modell mit einem Fehlerwert von 0,1950 wird im Weiteren modifiziert, indem die Zielvariable, der Credit Spread, durch die effektive Rendite ersetzt wird. ${ }^{734}$

Wie für die hier geführte Untersuchung zu erwarten ist, bleibt der Fehlerwert nahezu gleich und liegt bei 0,1951. Dies ist nicht verwunderlich, da es eine rein mathematische Umformung darstellt, welche durch das Netz "nachgebildet" wurde. Es ist zu erwarten, dass man nach einer Netzmodifikation denselben Fehlerwert erzielen kann. Dies wiederum unterstellt jedoch, dass die Netzstruktur die Umformung nachbilden kann. Somit muss der Anwender Strukturwissen besitzen, um den (fast) identischen Fehlerwert zu erzielen. ${ }^{735}$ In diesem Fall würde das zweite Netz eine lineare Verknüpfung besitzen.

Ausgangssituation ist die Berechnung der Bonitätsprämie nach der Gleichung 25 $e_{i}=r_{i}\left(M, W_{i}, B_{i}\right)-r_{B}\left(M, W_{B}, 0\right)$.

Somit kann die effektive Rendite eines Wertpapiers bestimmt werden als:

Gleichung 33

$r_{i}\left(M, W_{i}, B_{i}\right)=e_{i}+r_{B}\left(M, W_{B}, 0\right)$.

731 Vgl. Ziebart, Reiter (1992), S. 252ff.

732 Vgl. Heinke (1998), S. 309ff.

733 Analog bedeutet, es wurde die fiktive effektive Rendite einer laufzeit- und kupongleichen Anleihe benutzt.

734 Falls man den nominalen Zins nicht anhand der Indexkurve bzw. den bei der Credit Spread Bestimmung verwendeten sicheren Zins $\left(r_{B}\right)$ bildet, wird dem System eine Information vorenthalten, so das unterschiedliche Ergebnisse bei der Prognose der effektiven Rendite im Vergleich zum Credit Spread zu erwarten sind. Vgl. bspw. Thompson, Vaz (1990), S. 460; Billingsley, Lamy, Mare (1985), S. 62 und Hsueh, Kidwell (1988), S. 49. Vgl. Adam, Best, Dehnen, Steffens (2000), S. 20 f. 
Marktorientierte Kreditrisikobewertung - Empirische Untersuchung zur marktorientierten Bewertung des Kreditrisikos mittels Künstlicher Neuronaler Netze 195

Ein entsprechendes Netz zur Prognose der effektiven Rendite könnte somit die in Abbildung 54 dargestellte Struktur aufweisen.

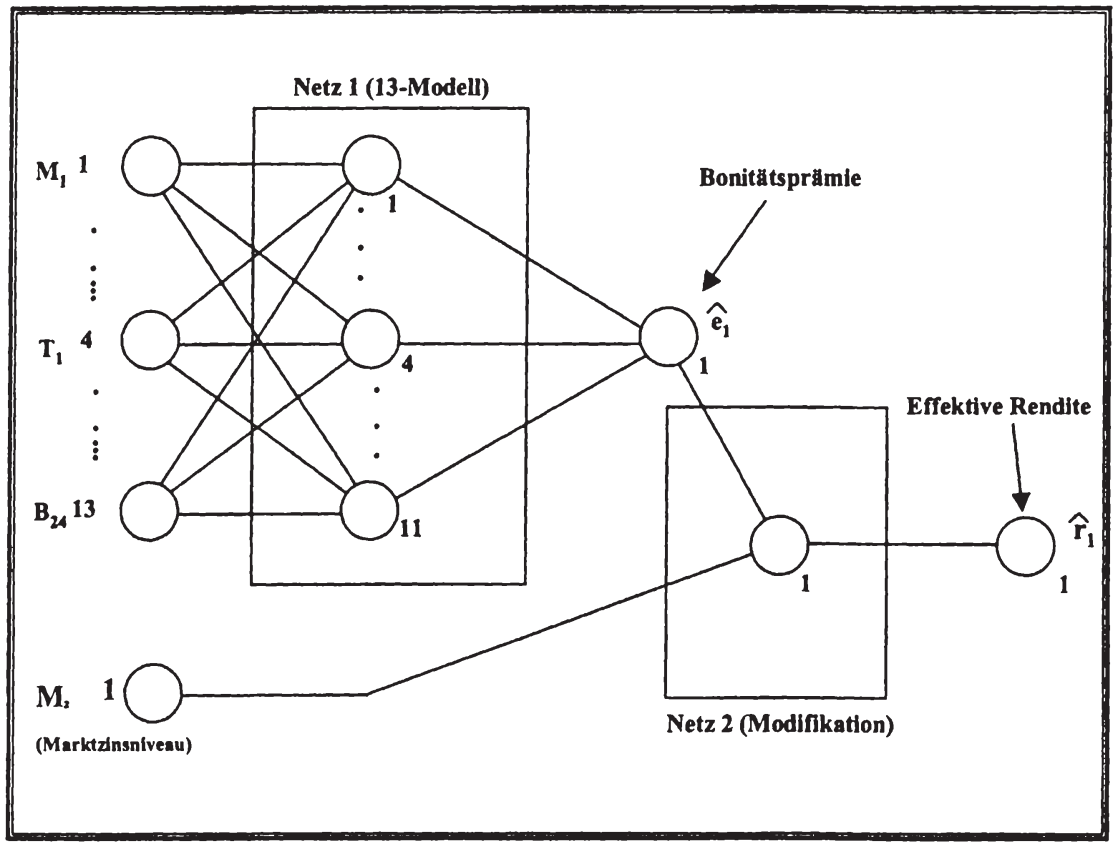

Abbildung 54: Schematischer Aufbau eines KNN zur Prognose der effektiven Rendite

Während die Standardabweichung von 0,1951 im Vergleich zum Mittelwert der Testmenge einen Anteil von 0,2186 besitzt (d.h. eine prozentuale Standardabweichung um den Mittelwert von 21,86\%), ist bei der effektiven Rendite dieser Wert weitaus geringer. Da der Mittelwert der effektiven Rendite 6,4238 Prozentpunkte beträgt, ergibt sich eine relative Abweichung von 3,0371\%.

\subsubsection{Untersuchungen innerhalb einzelner Risikoklassen}

Im Rahmen von Klassifizierungsproblemen wird regelmäßig darauf geachtet, dass der Anteil der verschiedenen Klassen innerhalb der Lernmenge gleich groß ist. ${ }^{736}$ Diese Gleichverteilung bezieht sich in erster Linie auf die Trainingsdaten. Bei einem Prognoseproblem ist diese Gleichverteilung nicht durch die Häufigkeit innerhalb der einzelnen Mengen (Lern- und Testmenge) direkt messbar, da keine Klassen vorliegen. Der Grund, warum man eine Gleichverteilung anstrebt, liegt in dem Bemühen, das Netz

Vgl. hier und im Folgenden bspw. Glormann (2001) S. 148ff. 
Marktorientierte Kreditrisikobewertung - Empirische Untersuchung 196 zur marktorientierten Bewertung des Kreditrisikos mittels Künstlicher Neuronaler Netze

gleich stark auf alle potenziellen Datensätze zu trainieren. Für das vorliegende Problem bedeutet dies, dass es nicht zu einer überproportional hohen Anzahl von weniger stark ausfallgefährdeten Unternehmen in der Trainingsmenge kommen darf. Falls man jedoch die Verteilung der Credit Spreads untersucht (vgl. Abbildung 55), kann man erkennen, dass es sehr wohl zu einer Anhäufung kommt. Diese ist damit zu erklären, dass es auf dem Kreditmarkt für besonders sichere Unternehmen tendenziell einfacher ist, sich zu refinanzieren, als für bonitätsmäßig schlechter gestellte Unternehmen. Folglich ist auch die Gleichverteilung in der Trainingsmenge nicht gewährleistet.

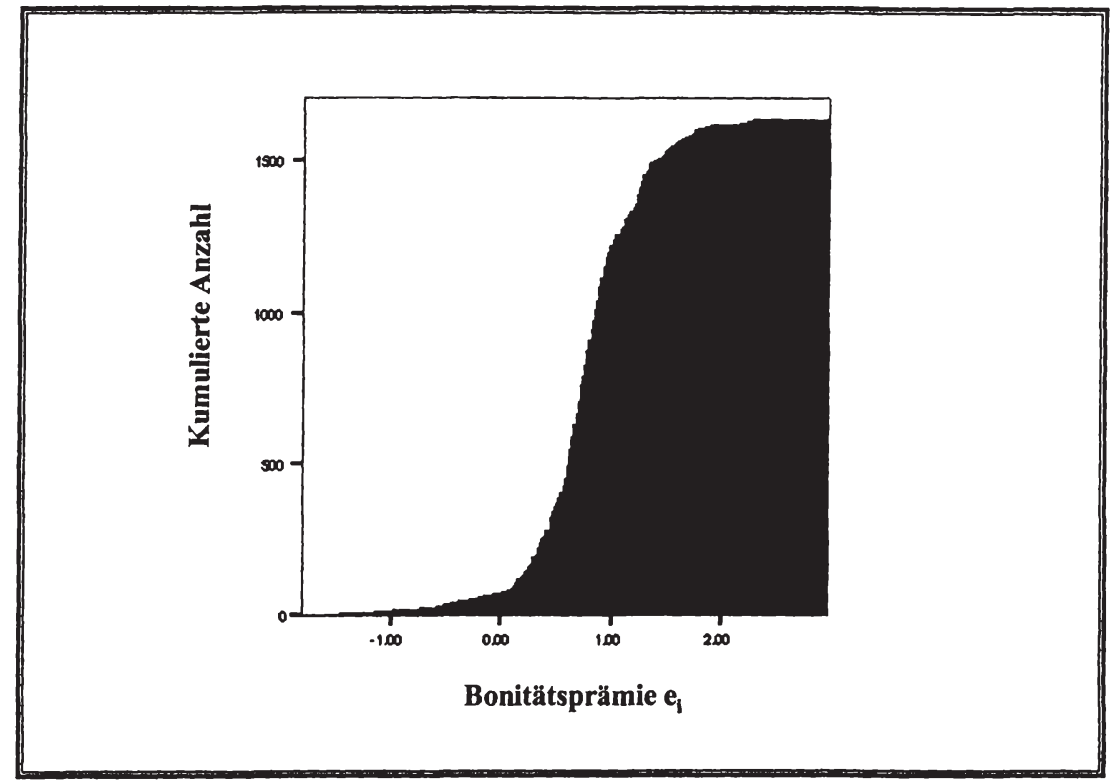

Abbildung 55: Häufigkeitsprofil der Untersuchungsdaten hinsichtlich der Bonitätspramie

Somit muss der Gefahr vorgebeugt werden, dass es zu einem „overlearning“ innerhalb der bonitätsstärkeren Unternehmen kommt. Dies kann man in zwei Schritten sicherstellen. Zunächst kann der Fehlerwert der verschiedenen Gruppen gegen die Bonität abgetragen werden. Anschließend kann man die Inputdatensätze so „manipulieren“, dass es zu einer Gleichverteilung kommt. Dies gelingt, indem man zunächst „künstliche Klassen" bildet und anschließend entweder nur eine bestimmte Anzahl an Fällen pro Klasse zulässt oder die weniger stark besetzten Klassen durch eine einfache Vervielfältigung der einzelnen Fälle künstlich vergrößert.

Zunächst wird der Fehlerwert der Testmenge gegen den Credit Spread abgetragen. Abbildung 56 verdeutlicht die Ergebnisse. Auf der x-Achse wird der Credit Spread abgetragen und auf der y-Achse der Fehlerwert (dargestellt als Differenz aus Zielwert und Prognosewert). Es wird deutlich, dass ein leichter Anstieg des Fehlerwertes in 
Marktorientierte Kreditrisikobewertung - Empirische Untersuchung zur marktorientierten Bewertung des Kreditrisikos mittels Künstlicher Neuronaler Netze

Abhängigkeit von der Risikohöhe zu beobachten ist. Falls man eine lineare Regression unterlegt, gelangt man zu einem korrigierten $R^{2}$ von 0,114 . Es liegt eine äußerst schwache (lineare) Korrelation vor. Eine Residuenüberprüfung (vgl. Abbildung 57) lässt keine starken systematischen Bewegungen erkennen. Es besteht eine äußerst geringe Gefahr, dass sich das System auf bonitätsstarke Unternehmen spezialisiert hat.

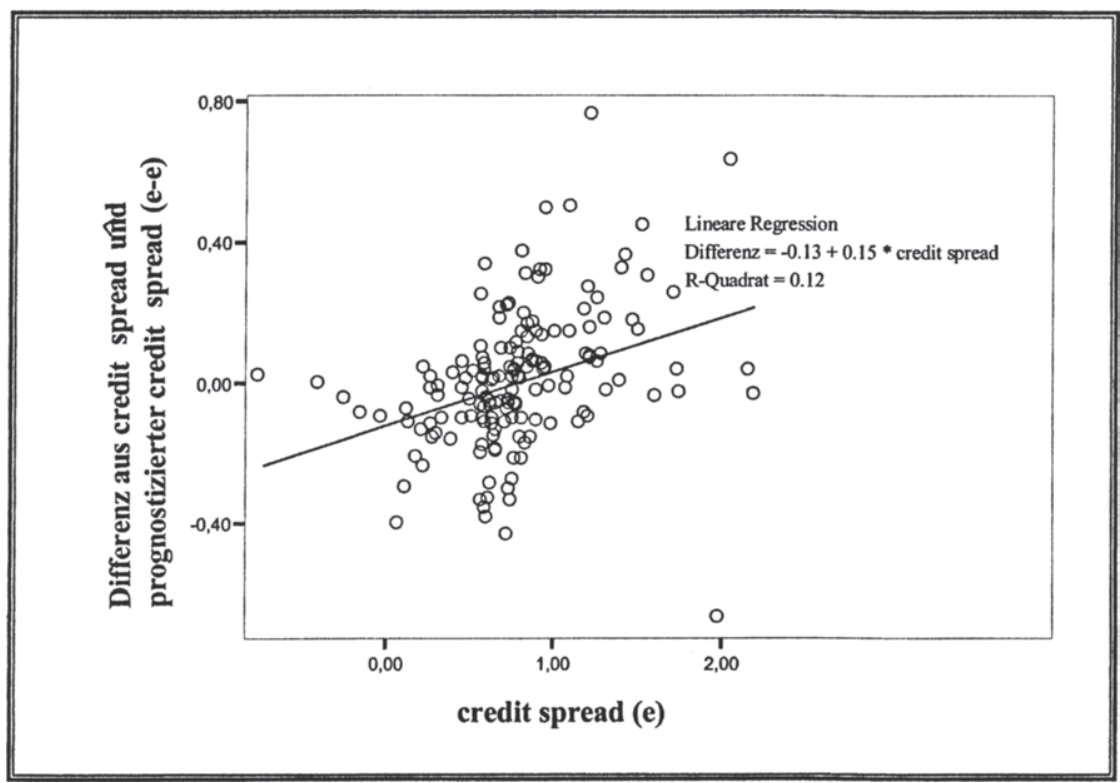

Abbildung 56: Lineare Regressionsanalyse von Credit Spread und der Differenz von dem prognostizierten Credit Spread und dem Credit Spread 
Marktorientierte Kreditrisikobewertung - Empirische Untersuchung 198 zur marktorientierten Bewertung des Kreditrisikos mittels Künstlicher Neuronaler Netze

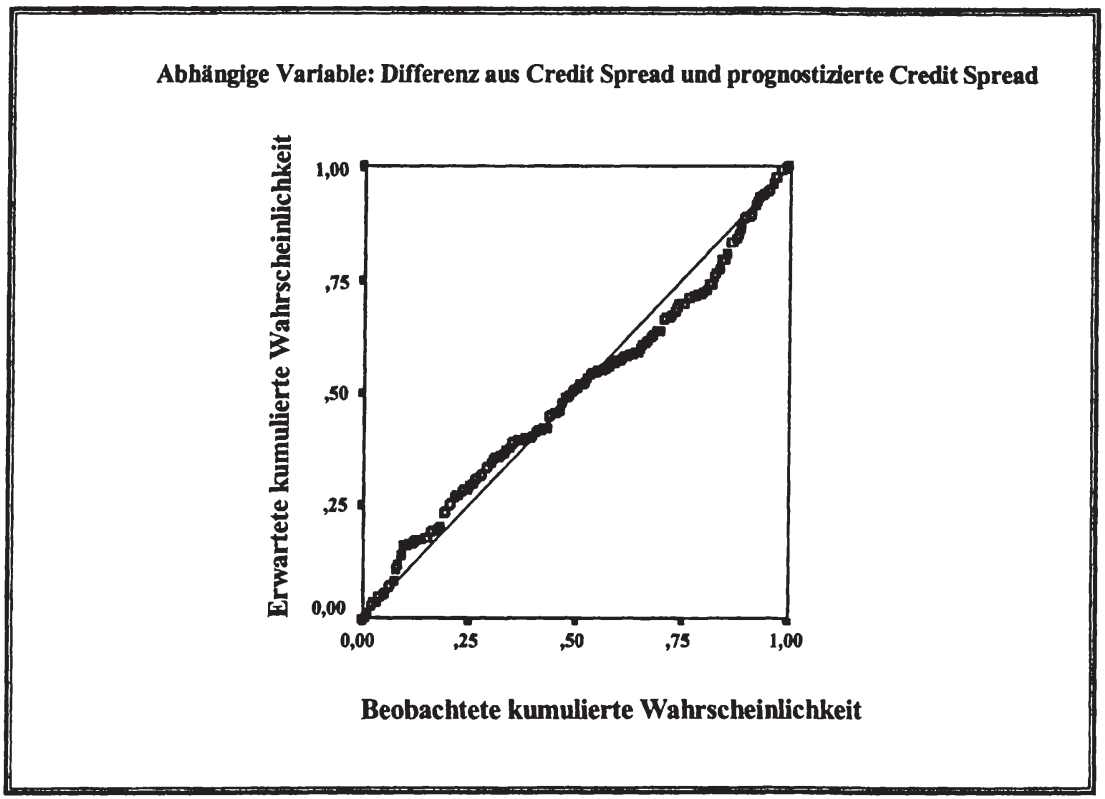

Abbildung 57: P-P-Diagramm der standardisierten Residuen der gefuhrten Regressionsanalyse

Obwohl keine deutliche „Spezialisierung“ aufgrund der durchgeführten Regressionsanalyse erkennbar ist, wird im Folgenden eine Möglichkeit überprüft, diesen wenn auch geringen Effekt zu vermeiden. Zunächst wurde der Inputdatensatz - lediglich die Lernmenge - in folgende Klassen (in Abhängigkeit vom Credit Spread) eingeteilt (vgl. Tabelle 37):

\begin{tabular}{||l|l|l|l|}
\hline \multirow{3}{*}{ Klasse } & \multicolumn{2}{|c|}{ Credit Spread (e) } & \multirow{2}{*}{ Klassengröße } \\
\cline { 2 - 4 } & $\begin{array}{c}\text { von [Prozentpunkte] } \\
\text { (ausschließlich) }\end{array}$ & $\begin{array}{c}\text { bis [Prozentpunkte], } \\
\text { (einschließlich) }\end{array}$ & \\
\hline 1 & Offen & 0 & 68 \\
\hline 2 & 0 & 0,25 & 70 \\
\hline 3 & 0,25 & 0,50 & 186 \\
\hline 4 & 0,50 & 0,75 & 379 \\
\hline 5 & 0,75 & 1,00 & 383 \\
\hline 6 & 1,00 & 1,25 & 161 \\
\hline 7 & 1,25 & 1,50 & 120 \\
\hline 8 & 1,50 & 1,75 & 57 \\
\hline 9 & 1.75 & 2,00 & 19 \\
\hline 10 & 2,00 & Offen & 18 \\
\hline
\end{tabular}

Tabelle 37: Klassenaufteilung der Credit Spreads

Somit ergibt sich folgendes Klassenhäufigkeitsprofil (Abbildung 58): 
Marktorientierte Kreditrisikobewertung - Empirische Untersuchung zur marktorientierten Bewertung des Kreditrisikos mittels Künstlicher Neuronaler Netze 199

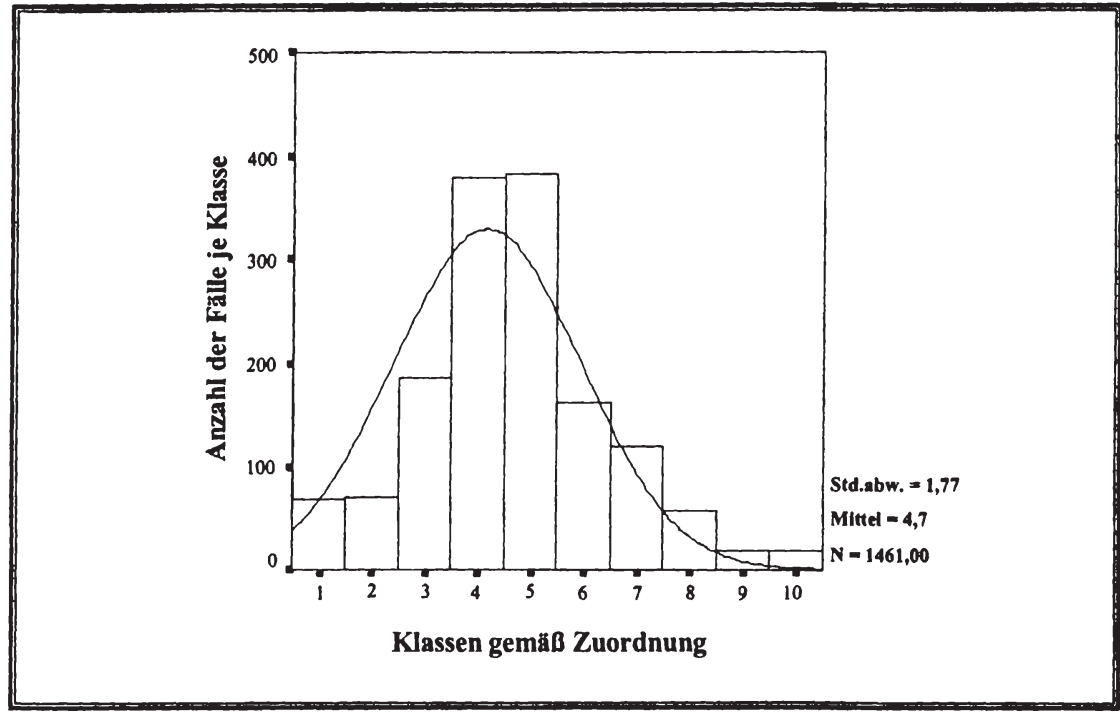

Abbildung 58: Klassenhäufigkeiten der Credit Spreads

Die größte Häufigkeit (Klassen 4 und 5) wird als Referenzpunkt für die Multiplikation gewählt. Dementsprechend werden die anderen Klassen bzw. die einzelnen Datensätze durch einen entsprechenden Multiplikationsfaktor vervielfacht. Tabelle 38 zeigt die unterschiedlichen Sätze auf:

\begin{tabular}{||l|l|l|l|l|}
\hline \multirow{2}{*}{ Klasse } & \multicolumn{2}{|c|}{ Credit Spread (e) } & Gewichtungsfaktor & $\begin{array}{c}\text { Korrigierte } \\
\text { Klassengröße }\end{array}$ \\
\cline { 2 - 5 } & $\begin{array}{c}\text { von [Prozent- } \\
\text { punkte], (aus- } \\
\text { schließlich) }\end{array}$ & $\begin{array}{c}\text { bis [Prozent- } \\
\text { punkte] } \\
\text { (einschließlich) }\end{array}$ & 6 & \\
\hline 1 & Offen & 0 & 6 & 408 \\
\hline 2 & 0 & 0,25 & 5 & 350 \\
\hline 3 & 0,25 & 0,50 & 2 & 372 \\
\hline 4 & 0,50 & 0,75 & 1 & 379 \\
\hline 5 & 0,75 & 1,00 & 1 & 383 \\
\hline 6 & 1,00 & 1,25 & 2 & 322 \\
\hline 7 & 1,25 & 1,50 & 3 & 360 \\
\hline 8 & 1,50 & 1,75 & 7 & 399 \\
\hline 9 & 1.75 & 2,00 & 21 & 399 \\
\hline 10 & 2,00 & Offen & 22 & 396 \\
\hline
\end{tabular}

Tabelle 38: Korrigierte Klassenaufteilung der Credit Spreads

Der veränderte Inputdatensatz besitzt somit folgendes Klassenhäufigkeitsprofil hinsichtlich der gebildeten Klassen (vgl. Abbildung 59): 
Marktorientierte Kreditrisikobewertung - Empirische Untersuchung 200 zur marktorientierten Bewertung des Kreditrisikos mittels Künstlicher Neuronaler Netze

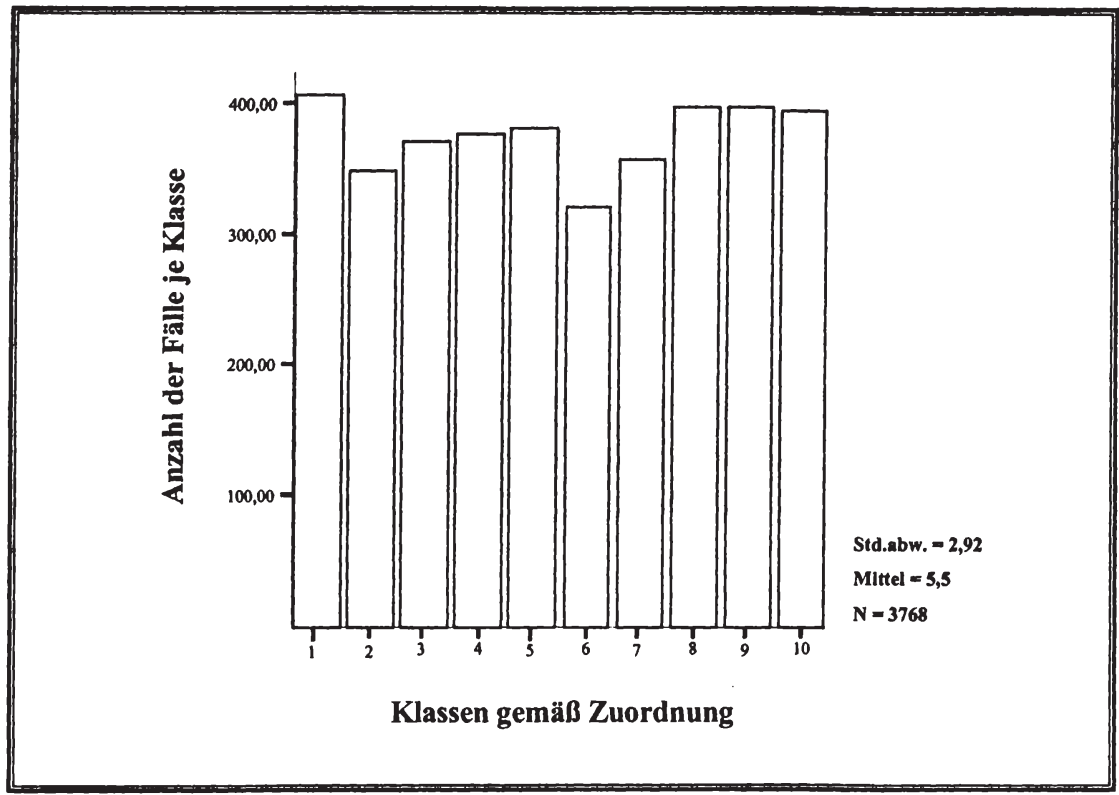

Abbildung 59: Korrigierte Klassenhäufigkeiten der Credit Spreads

Folglich hat sich auch der Datenumfang auf 3.768 vergrößert. Die modifizierte Datenmenge wird anschließend als Lernmenge für das 13er-Modell-Netz benutzt. Der gemessene Fehlerwert von 0,2235 liegt innerhalb des in den Parametervariationen gefundenen Intervalls (vgl. Kap. 5.4.5). Es ist zwar möglich, dass durch weitere Netzoptimierungsprozesse der Fehlerwert reduziert werden kann. Fraglich ist jedoch, ob dadurch ein günstigerer Fehlerwert als 0,1950 erreicht werden kann.

Durch die „künstliche“ Vergrößerung der Lernmenge wurden dem KNN für die Analyse keine neuen, weiteren Informationen gegeben. Es wurde lediglich eine Gleichverteilung von Informationen von bonitätsstärkeren und bonitätsschwächeren Unternehmen erreicht. Dementsprechend wurde der berechnete gesamte Netzfehler nicht von den Fehlerwerten bonitätsstärkerer Unternehmen überproportional beeinflusst. Die auf Basis des berechneten Gesamtfehlers durchgeführte Gewichtsanpassungsphase (ErrorBackpropagation) wurde (aufgrund der ,künstlichen Vergrößerung) nicht in die Richtung einer verstärkten Ausprägung (im Sinne einer Gewichtsanpassung) auf bonitätsstärkere Unternehmen gelenkt. Alternativ hätte man auch bei der Bildung der Lernmenge - ähnlich wie bei der Vorgehensweise bei Klassifizierungsproblemen ${ }^{737}$ - einen

Vgl. hier und im Folgenden bspw. Glormann (2001), S. $150 \mathrm{f}$. 
Marktorientierte Kreditrisikobewertung - Empirische Untersuchung zur marktorientierten Bewertung des Kreditrisikos mittels Künstlicher Neuronaler Netze

hohen Credit Spread (bspw. Klasse 10), stellvertretend für ein bonitätsschwaches Unternehmen, heranziehen können, um für die anderen Klassen eine Umfangsbegrenzung vorzugeben. Folglich hätte man jedoch lediglich $10 \times 18=180$ Anleihen in die Lernmenge aufgenommen. Dies entspricht ca. 10 Prozent der gesamten Untersuchungsdaten. In Anlehnung an die erzielten Ergebnisse zu den unterschiedlichen Variationen der Datenaufteilung (vgl. Kap. 5.4.3.1 und Kap. 5.4.5) wurde vom Verfasser die (potenzielle) Möglichkeit einer Fehlerwertreduktion mittels dieser alternativen Vorgehensweise als äußerst gering eingestuft.

Insgesamt lässt sich konstatieren, dass die Gefahr einer Überbetonung von bonitätsstarken Unternehmen nicht ausgeschlossen werden kann, die Modellmodifikation mit einer „künstlich“" vergrößerten Menge an Trainingsdaten jedoch keinen Hinweis auf diese Verzerrung geben. Im weiteren Analyseverlauf wird das 13er-Modell hinsichtlich der Prognosegüte ausgedrückt im Fehlerwert $\mathrm{s}_{\mathrm{F}}$ bewertet.

\subsection{Ergebnisbewertung}

\subsubsection{Definition eines Vergleichsobjektes}

Die Ergebnisse der hier geführten Untersuchung mittels Neuronaler Netze können durch den Vergleich mit den Ergebnissen anderer Studien gewürdigt werden. Ein Vergleichsobjekt sollte folgende Eigenschaften haben:

- Lösung einer gleichen Problemstellung,

- ein vergleichbares Gütemaß ausweisen und

- gleiche Rahmenbedingungen besitzen.

Die Einschätzung der Güte des Neuronalen Netzes bzw. des 13er-Modells kann durch zwei Ansätze erreicht werden: Zum einen können die Ergebnisse anderer wissenschaftlicher Untersuchungen zum Credit Spread verwendet werden und so direkt die Fehlerfunktionswerte miteinander verglichen werden, zum anderen können die von anderen Quellen gelieferten Einschätzungen zum Ausfallrisiko eines Unternehmens betrachtet werden.

\subsubsection{Vergleich der Testergebnisse}

Als erste Möglichkeit wird ein Vergleich zwischen publizierten Untersuchungen über die Einflussfaktoren des Credit Spread geführt. Wie bereits im Kapitel 4.3.3.3 dargelegt wurde, gibt es bereits eine Fülle von Untersuchungen, insbesondere aus dem amerikanischen Raum, die sich mit dem Credit Spread auseinander gesetzt haben. Ein Vergleich mit diesen Vorgehensweisen widerspricht dem letzten Punkt der o.g. Kriterien für ein Vergleichsobjekt. Die Rahmenbedingungen, in diesem Fall die Datengrundlage, sind nicht deckungsgleich. Dies ist darin begründet, dass der Untersuchungszeitraum regelmäßig nicht gleich ist. Darüber hinaus sollten dieselben Anleihen analysiert werden. Ein weiteres Problem ist die Vergleichbarkeit der Inputfaktoren. 
Marktorientierte Kreditrisikobewertung - Empirische Untersuchung 202 zur marktorientierten Bewertung des Kreditrisikos mittels Künstlicher Neuronaler Netze

Die veröffentlichten Studien beziehen sich regelmäßig auf Schwankungen des Credit Spread innerhalb bestimmter Ratingklassen. ${ }^{738}$ Eine Arbeit, welche die Abweichung zwischen einem modelltheoretisch bestimmten Credit Spread und dem am Kreditmarkt beobachteten Credit Spread ausweist, ist die Arbeit von LAMY/THOMPSON (1988).

Die Autoren benutzen das Rating neben dem Marktzinsniveau, der Volatilităt und dem Emissionsvolumen ${ }^{739}$ um den Credit Spread zu erklären. Von ihrer Untersuchung werden „unusual" ${ }^{\text {"70 }}$ Anleihen, wie Nullkuponanleihen oder Anleihen mit variabler Verzinsung, ausgeklammert. Der Untersuchungszeitraum geht von Januar 1977 bis Juni 1983 und schließt eine Menge von 392 Unternehmen mit „investment grade“ ein. ${ }^{741}$ Ihre Analyse (multivariate Regressionsanalyse) führt zu einem adjustieren $R^{2}$ von ca. $0,44{ }^{742}$ Der Fehlerwert bezieht sich auf die gesamte Untersuchungsmasse. Dies bedeutet, es wurde nicht in verschiedene Untergruppen (wie unterschiedliche Ratingklassen) unterschieden. Nicht zuletzt hieraus wird deutlich, dass, obwohl die Grundfragestellung beider Untersuchungen äußerst ähnlich ist, ein Vergleich nur sehr eingeschränkt möglich ist. ${ }^{743}$ Falls man hiervon abstrahiert, können die Ergebnisse des Neuronalen Netzes als treffender bezeichnet werden, da sich (für die Testmenge des 13er-Modells im Szenario 1) ein Wert, ermittelt nach der Methodik des $R^{2}$ Bestimmtheitsmaßes, von 0,89 bei der Credit-Spread-Prognose ergibt (bzw. nach der Methodik des adjustierten $\mathrm{R}^{2}$-Bestimmtheitsmaßes ein Wert von 0,88 ). Die analoge Berechnung der Werte für die Prognose der effektiven Rendite liegt bei 0,94 (gemäß des adjustierten $\mathrm{R}^{2}$-Bestimmtheitsmaßes ist ebenfalls ein Wert von 0,94 zu beobachten)..$^{74 / 745}$

Eine zweite Vergleichsmöglichkeit bietet die veröffentlichte Einschätzung des Ausfallrisikos von Ratingagenturen. Hier sei bereits angemerkt, dass ein Rating eine Klassifizierung darstellt. Somit ist die Zielsetzung nicht mit dem Informationsgehalt des Credit

Vgl. bspw. Heinke (1998); Hsueh, Kidwell (1988); Johnson (1967).

Neben diesen Einflussfaktoren ziehen die Autoren weitere Variablen heran, um Spezifika der Datenmenge, wie Kündigungsrechte, abzudecken. Vgl. Lamy, Thompson (1988), S. 593.

Lamy, Thompson (1988), S. 594.

Zur Definition von ,investement grade“ vgl. Kapitel 2.3.3.

Vgl. Lamy, Thompson (1988) S. 597.

Diese Einschränkung bezieht sich ebenfalls auf die Interpretationsmöglichkeiten der Größe R².

Vgl. zu der Definition des adjustierten Bestimmtheitsmaßes Hartung, Elpelt (1989), S. 87.

Bei der Berechnung des Bestimmtheitsmaßes wurden die Differenzen zwischen dem prognostizierten und dem gemessenen Credit Spread herangezogen. Diese Differenzen sind analog einer Regressionsanalyse als quadrierte Abweichungen bewertet worden, die Inputfaktoren wurden als Einflussfaktoren gedeutet. 
Marktorientierte Kreditrisikobewertung - Empirische Untersuchung zur marktorientierten Bewertung des Kreditrisikos mittels Künstlicher Neuronaler Netze

Spread deckungsgleich. Aufgrund der Geheimhaltung des Ratingprozesses ist es nicht möglich, eine Prognose bzgl. eines Unternehmens bzw. einer Anleihe abzuleiten. Diese Tatsache steht somit im Widerspruch zu der genannten Voraussetzung, dass die Fragestellung der zu vergleichenden Studien deckungsgleich ist. ${ }^{746} \mathrm{Da}$ jedoch das Rating als Instrument zur Einschätzung bzw. Quantifizierung des Kreditrisikos von Banken verwendet wird, ${ }^{747}$ kann ein Vergleich zwischen der Aussagefähigkeit der veröffentlichten Ratingeinstufungen und den berechneten Credit Spreads gezogen werden. ${ }^{748}$ Es wird ein Vergleich zwischen den Modellergebnissen und der Streuung des Credit Spread innerhalb von verschiedenen Ratingklassen geführt. Falls somit das Rating für eine Anleihe bekannt ist und dieser Anleihe daraufhin der durchschnittliche, für diese Ratingklasse gemessene Credit Spread, zugeordnet wird, ist eine Differenz zwischen dem zugeordneten und dem gemessenen Credit Spread zu beobachten. Diese Differenzen können als Häufigkeitsfunktion dargestellt werden. Die Standardabweichung dieser Funktion drückt die Unsicherheit in der Prognose aus. Es wird die Unsicherheit in der Prognose des richtigen Credit Spread mittels eines Rating simuliert. Es werden verschiedene Untersuchungen zitiert, um zum einen die Datenmenge und den Zeitraum zu variieren, und zum anderen verschiedene Methoden zur Berechnung des Credit Spread heranzuziehen.

Zunächst werden die von LONGSTAFF/SCHWARTZ veröffentlichten Daten genutzt. ${ }^{749} \mathrm{Im}$ Zeitraum von 1977-1992 haben die Autoren für verschiedene Unternehmen die Ratingklassen nach MOODY'S (aufgeteilt nach Utilities / Industrials / Railroads und jeweils Aaa - Baa) und die jeweils zugehörigen durchschnittlichen Standardabweichungen der Credit Spreads um den Ratingklassenmittelwert als arithmetisches Mittel berechnet. ${ }^{750}$ Die Standardabweichung drückt den Fehlerwert aus, dem man bei Heranziehung der Ratingeinstufung als Prognosemodell für den Credit Spread durchschnittlich unterliegt. Dieser wird mittels einer berechneten laufzeitgleichen Staatsanleihe ermittelt, welche wiederum aus einer Durchschnittsberechnung einer 10- und einer 30-jährigen Anleihe stammt. Die Tabelle 39 zeigt die Ergebnisse, die in Abbildung 60 veranschaulicht werden.

746 Darüber hinaus besteht die Problematik der absoluten Vergleichbarkeit der Untersuchungsdaten (bspw. hinsichtlich einer zeitlichen Deckung).

747 Vgl. Kapitel 2.3.3.

748 Die Prognosefähigkeit von Ratingagenturen kann nicht verglichen werden, da lediglich das veröffentlichte Rating als Vergleichsmaßstab verwendet werden kann. Folglich wird die Prognosefähigkeit mittels veröffentlichter Rating untersucht.

749 Vgl. hier und im Folgenden Longstaff, Schwartz (1995).

750 Vgl. Longstaff, Schwartz (1995), S. 808; risikoreichere (,speculativ grades“) Unternehmen werden bei der Untersuchung ausgeklammert. 
Marktorientierte Kreditrisikobewertung - Empirische Untersuchung 204 zur marktorientierten Bewertung des Kreditrisikos mittels Künstlicher Neuronaler Netze

\begin{tabular}{|l|l|l|l|}
\hline Rating / \\
Branche & $\begin{array}{l}\text { Mittelwert (des } \\
\text { Credit Spread in } \\
\text { Prozentpunkte) }\end{array}$ & $\begin{array}{l}\text { Standardabweichung } \\
\text { (des Credit Spread in } \\
\text { Prozentpunkte) }\end{array}$ & $\begin{array}{l}\text { Häufigkeit } \\
\text { (Anzahl) }\end{array}$ \\
\hline Aa Railroads & 0,191 & 0,869 & 149 \\
\hline Aaa Industrials & 0,481 & 0,373 & 190 \\
\hline A Railroads & 0,794 & 0,770 & 149 \\
\hline Aa Industrials & 0,809 & 0,452 & 190 \\
\hline Aaa Utility & 0,930 & 0,349 & 180 \\
\hline A Industrials & 1,231 & 0,580 & 190 \\
\hline Baa Railroad & 1,240 & 0,821 & 149 \\
\hline Aa Utility & 1,276 & 0,431 & 190 \\
\hline A Utility & 1,660 & 0,667 & 190 \\
\hline Baa Industrials & 1,835 & 0,654 & 190 \\
\hline Baa Utility & 2,077 & 0,758 & 190 \\
\hline
\end{tabular}

Tabelle 39: Numerische Darstellung der Untersuchungsergebnisse von Longstaff/Schwartz (1995) bezuglich der Standardabweichung des Credit Spread innerhalb einzelner RatingkJassen

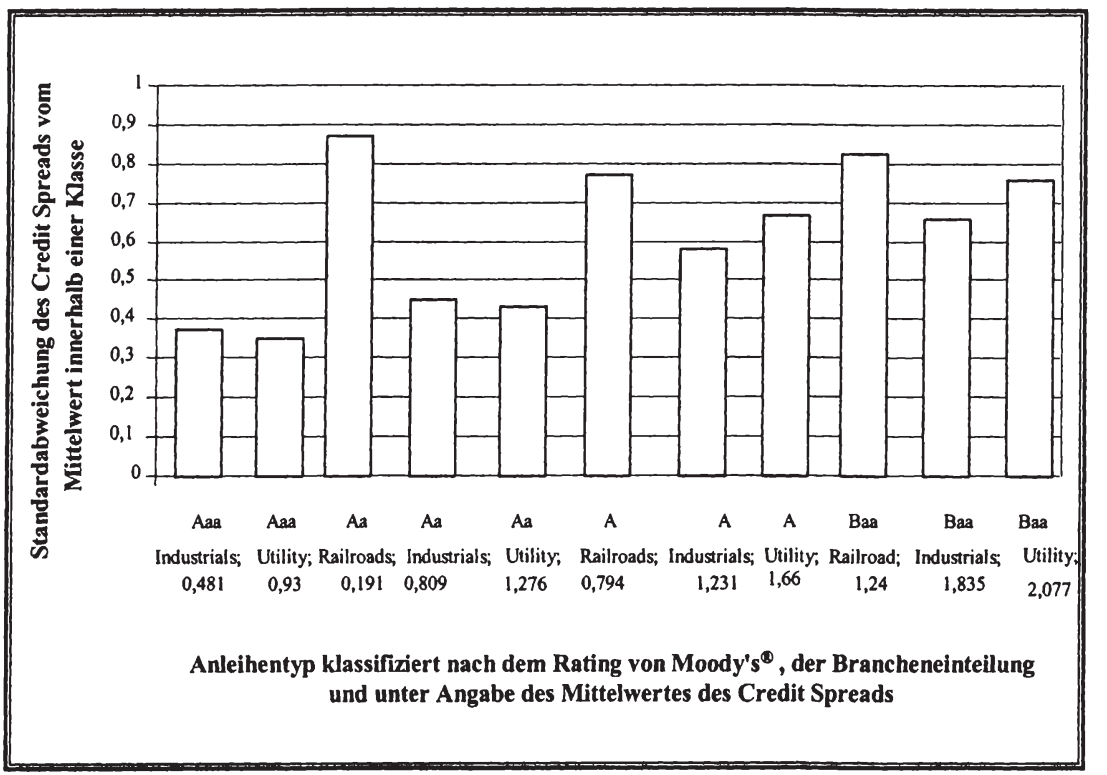

Abbildung 60: Standardabweichung des Credit Spread innerhalb einzelner Ratingklassen bei der Untersuchung von Longstaff/Schwartz (1995)

Falls man eine Rangfolge (x-Achse) in Abhängigkeit des Rating bildet, sieht man einen leichten Anstieg der Standardabweichung bei risikoreicheren Unternehmen. 
Marktorientierte Kreditrisikobewertung - Empirische Untersuchung zur marktorientierten Bewertung des Kreditrisikos mittels Künstlicher Neuronaler Netze 205

Alternativ kann man auch die Ergebnisse von HEINKE heranziehen. ${ }^{751 / 752}$ Diese Untersuchung bezieht sich auf einen Zeitraum von 1988 bis 1997 und umfasst 868 Wertpapiere. In dieser Untersuchung werden spekulative Anleihen nicht von der Untersuchung ausgeschlossen. Gleichzeitig werden jedoch von allen Branchen und Emittenten Anleihen berücksichtigt. Somit werden auch supranationale Organisationen, Staaten, lokale Gebietskörperschaften, Finanzierungsgesellschaften und Banken einbezogen. Lediglich ein Anteil von 21,2 Prozent der untersuchten Anleihen bezieht sich auf Industrieanleihen. Der Credit Spread wird analog zu der vorliegenden Untersuchung mittels Index-Bildung durch die oben verwendete Regressionsgleichung (vgl. Gleichung 30 und Gleichung 31) berechnet. Tabelle 40 zeigt die Ergebnisse numerisch und Abbildung 61 grafisch auf.

\begin{tabular}{||l|l|l|l|}
\hline Rating & $\begin{array}{l}\text { Mittelwert (des Credit } \\
\text { Spread in Prozent- } \\
\text { punkte) }\end{array}$ & $\begin{array}{l}\text { Standardabweichung (des } \\
\text { Credit Spread in Prozent- } \\
\text { punkte) }\end{array}$ & $\begin{array}{l}\text { Häufigkeit } \\
\text { (Anzahl) }\end{array}$ \\
\hline Aaa & 0,076 & 0,190 & 253 \\
\hline Aa1 & 0,121 & 0,164 & 53 \\
\hline $\mathrm{Aa} 2$ & 0,220 & 0,183 & 43 \\
\hline $\mathrm{Aa} 3$ & 0,242 & 0,162 & 43 \\
\hline $\mathrm{A} 1$ & 0,346 & 0,157 & 31 \\
\hline $\mathrm{A} 2$ & 0,425 & 0,168 & 20 \\
\hline $\mathrm{A} 3$ & 0,571 & 0,248 & 9 \\
\hline $\mathrm{Baa} 1$ & 0,619 & 0,549 & 6 \\
\hline $\mathrm{Ba} 2$ & 0,750 & 0,214 & 5 \\
\hline $\mathrm{Baa} 3$ & 1,390 & 0,721 & 14 \\
\hline $\mathrm{Ba} 1$ & 2,476 & 0,630 & 12 \\
\hline $\mathrm{Ba} 2$ & 4,056 & 1,393 & 8 \\
\hline $\mathrm{Ba} 3$ & 2,926 & 0,425 & 7 \\
\hline $\mathrm{B} 1$ & 3,990 & 1,159 & 18 \\
\hline
\end{tabular}

Tabelle 40: Numerische Darstellung der Untersuchungsergebnisse von Heinke (1998) bezüglich der Standardabweichung des Credit Spread innerhalb einzelner Ratingklassen

751 Vgl. hier und im Folgenden Heinke (1998).

752 Die Untersuchung bezieht sich auf den EURO-Kapitalmarkt. Somit ist die Bedingung für ein Vergleichsobjekt hinsichtlich der Übereinstimmung der Datengrundlage verletzt. Trotz dieser Einschränkung ist nach Meinung des Verfassers ein Vergleich lohnenswert, da somit ein Eindruck bzgl. der Prognosefähigkeit von Rating für den europäischen Markt gegeben werden kann. 
Marktorientierte Kreditrisikobewertung - Empirische Untersuchung 206 zur marktorientierten Bewertung des Kreditrisikos mittels Künstlicher Neuronaler Netze

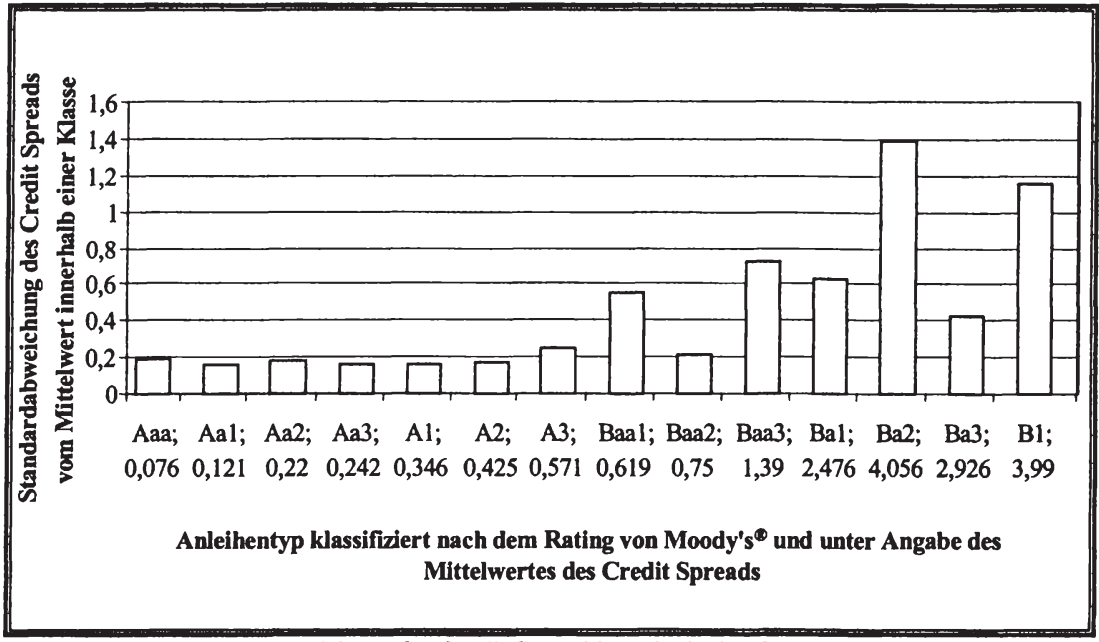
Abbildung 61: Standardabweichung des Credit Spread innerhalb einzelner Ratingklassen bei der
Untersuchung von Heinke (1998)

Es ist auffällig, dass es zu einem deutlichen Anstieg der Unsicherheit der Prognose innerhalb der risikoreicheren, niedrigen Ratingklassen kommt.

Abschließend wird die Untersuchung der Autoren BRISTER/KENNEDY/LIU aus dem Jahre 1994 herangezogen. ${ }^{733}$ Die Autoren schlossen 498 US-amerikanische Neuemissionen aus dem Zeitraum 1982-1987 in die Untersuchung ein. Es wurden alle Risikoklassen und sämtliche Industrieanleihen (nicht nach Branchen getrennt) untersucht. Als Rating wurde die Einschätzung von Standard and Poor's gewählt. Der Credit Spread wurde mittels einer durationsgleichen Staatsanleihe gebildet. Existierte keine durationsgleiche Staatsanleihe, wurden zwei benachbarte Anleihen zu diesem Zweck als Portfolio betrachtet und mittels linearer Interpolation der exakte Punkt bestimmt. Tabelle 41 und Abbildung 62 fassen die Zusammenhänge numerisch und grafisch zusammen, wobei wiederum eine steigende Unsicherheit bei risikoreicheren Ratingklassen $\mathrm{zu}$ beobachten ist.

Vgl. hier und im Folgenden Brister, Kennedy, Liu (1994). 
Marktorientierte Kreditrisikobewertung - Empirische Untersuchung zur marktorientierten Bewertung des Kreditrisikos mittels Künstlicher Neuronaler Netze 207

\begin{tabular}{|l|l|l|l|}
\hline Rating & $\begin{array}{l}\text { Mittelwert (des Credit } \\
\text { Spread in Prozent- } \\
\text { punkte) }\end{array}$ & $\begin{array}{l}\text { Standardabweichung (des } \\
\text { Credit Spread in Prozent- } \\
\text { punkte) }\end{array}$ & $\begin{array}{l}\text { Häufigkeit } \\
\text { (Anzahl) }\end{array}$ \\
\hline AAA & 0,351 & 0,2617 & 14 \\
\hline AA & 0,701 & 0,4629 & 102 \\
\hline A & 0,966 & 0,5556 & 195 \\
\hline BBB & 1,406 & 0,7668 & 89 \\
\hline BB & 3,336 & 1,0329 & 27 \\
\hline B & 4,203 & 1,2678 & 63 \\
\hline CCC & 5,222 & 1,1782 & 8 \\
\hline
\end{tabular}

Tabelle 41: Numerische Darstellung der Untersuchungsergebnisse von Brister/Kennedy/Liu (1994) bezüglich der Standardabweichung des Credit Spread innerhalb einzelner Ratingklassen

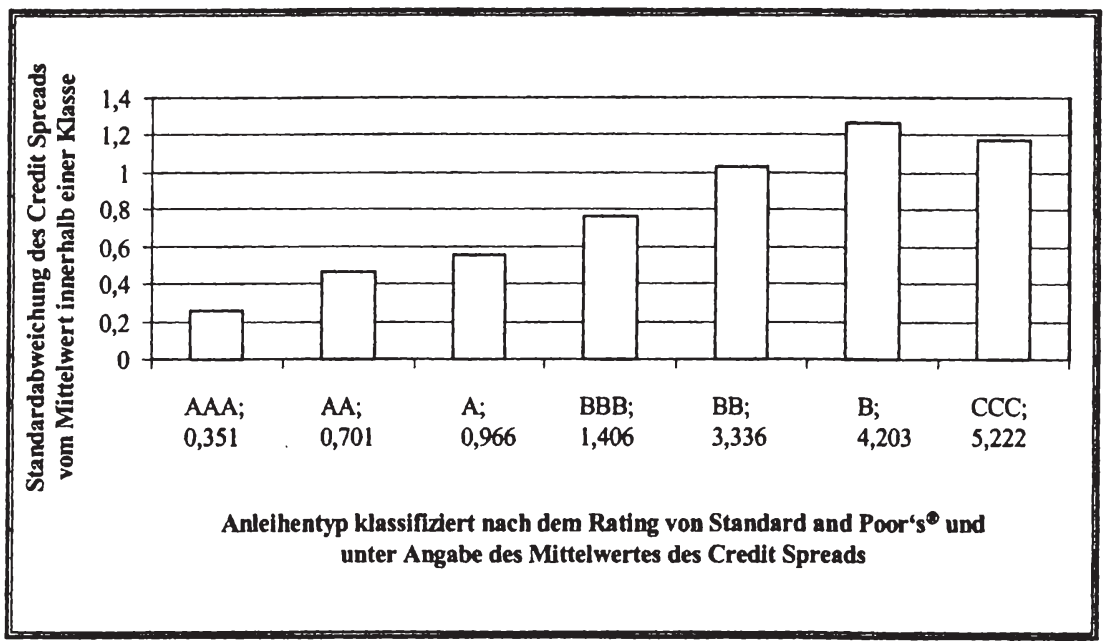

Abbildung 62: Standardabwelchung des Credit Spread innerhalb einzelner Ratingklassen bei der Untersuchung von Brister/Kennedy/Liu (1994)

Um die durch das 13er-Modell gewonnenen Erkenntnisse nun mit den innerhalb der Ratingklassen erkannten Standardabweichungen zu vergleichen, werden die Fehlerwerte in Abhängigkeit von der Höhe des Credit Spread ausgewiesen. Da keine Klassen vorliegen, werden „Punktwolken“ mit den einzelnen Werten erzeugt. Auf der x-Achse wird dabei das Risikopotenzial und auf der y-Achse die Abweichung zwischen dem beobachteten und dem prognostizierten Credit Spread abgetragen. Hierbei werden sowohl die Werte der Test- als auch die der Lern- und Validierungsmengen in dem Koordinatensystem abgetragen (vgl. Abbildung 63). Für den Vergleich der KNNAnalyse mit den zitierten Arbeiten zur Regressionsanalyse wird neben der Testmenge 
Marktorientierte Kreditrisikobewertung - Empirische Untersuchung 208 zur marktorientierten Bewertung des Kreditrisikos mittels Künstlicher Neuronaler Netze

auch die Lern- bzw. Validierungsmenge herangezogen, da auch bei den zitierten Regressionsanalysen die gesamte Datenmenge betrachtet wurden, ${ }^{754}$ obwohl auch bei einer Regressionsanalyse eine Trennung in Trainings- und Generalisierungsdaten möglich wäre. Die Testmenge der KNN-Analyse wird auch bei dem im Weiteren beschriebenen Vergleich der Untersuchungen gesondert ausgewiesen.

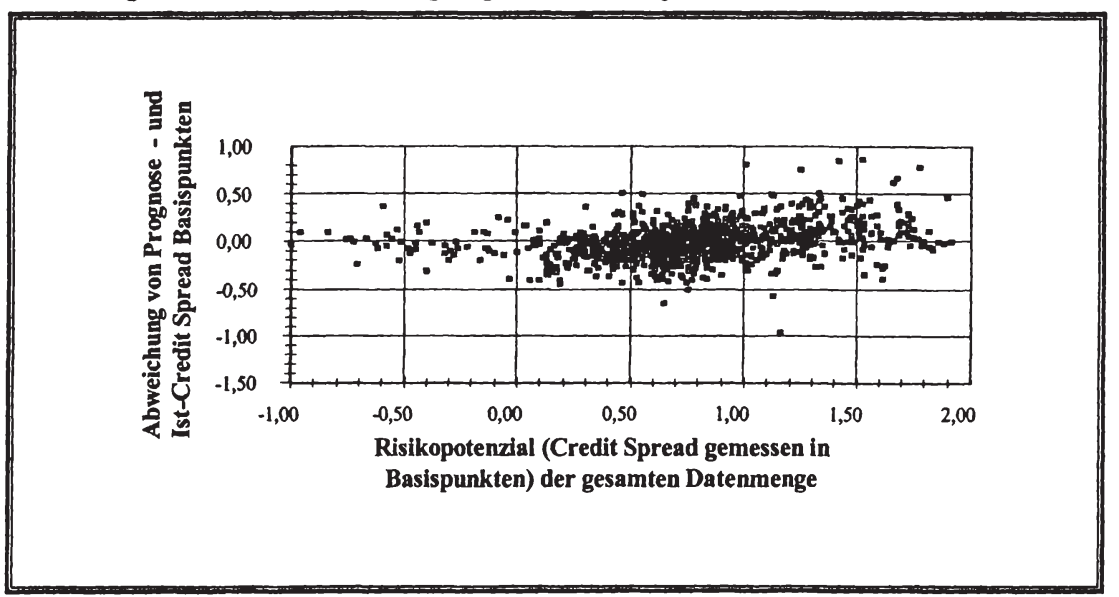

Abbildung 63: Abweichung der Prognose des Credit Spread in Abhängigkeit des Risikopotenzials für die Lern- und Validierungsmenge

Wie man erkennen kann, findet eine Häufung der Abweichungen bei $x=[0,5 ; 1]$ statt, ohne dass man eine Tendenz bei den Ausreißern erkennen könnte. Dies bestätigt die Tatsache, dass ein Großteil der untersuchten Anleihen einen Credit Spread zwischen 0,5 und 1 Prozentpunkten besitzt. Die Abweichungen zwischen dem prognostizierten und dem beobachteten (Ist) Credit Spread sind tendenziell bei einem höheren Risikopotenzial (gemessen an dem Ist Credit Spread) im Vergleich zu Anleihen mit einem niedrigen Risikopotenzial höher. Diese Beobachtungen decken sich mit der in Abbildung 56 für die Testmenge durchgeführten Regressionsanalyse.

Um eine Vergleichbarkeit der Untersuchungen zu ermöglichen, werden die Daten der Test- und der gesamten Datenmenge in neun Klassen eingeteilt. Die Einteilung geschieht in Anlehnung an die von Ratingagenturen ausgewiesenen Gruppen. Die o.g. drei Untersuchungen dienen hierzu als Beispiel für die quantifizierten Klassengrenzen. Bei Anleihen mit einem niedrigen Credit Spread (unter einem Prozentpunkt) wurden kleinere Intervalle ( 0,25 Prozentpunkte) als bei Anleihen mit einem höheren Credit

Vgl. Brister, Kennedy, Liu (1994); Heinke (1998); Longstaff, Schwartz (1995). 
Marktorientierte Kreditrisikobewertung - Empirische Untersuchung zur marktorientierten Bewertung des Kreditrisikos mittels Künstlicher Neuronaler Netze 209

Spread ( 0,50 Prozentpunke) gewählt. Somit gelangt man zu der in Tabelle 42 dargestellten Klasseneinteilung.

Für einen direkten Vergleich werden die Ergebnisse des 13er-Modells und der genannten drei Untersuchungen gemeinsam in einem Koordinatensystem abgetragen. Auf der $\mathrm{x}$-Achse wird in Abhängigkeit vom Mittelwert des Credit Spread die Ratingklasse abgetragen. Die y-Achse weist die entsprechende Standardabweichung innerhalb der einzelnen Klassen aus.

\begin{tabular}{|c|c|c|c|c|c|c|c|c|}
\hline 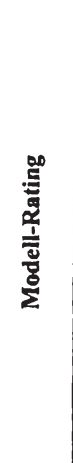 & 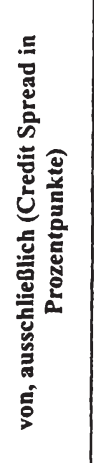 & 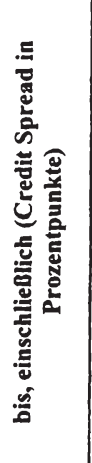 & 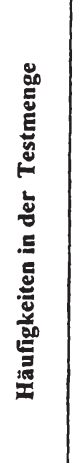 & 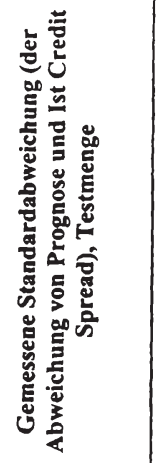 & 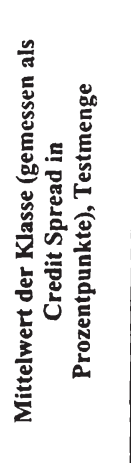 & 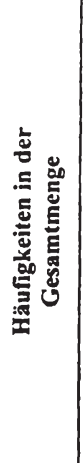 & 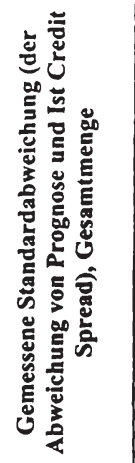 & 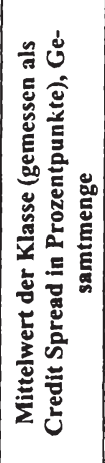 \\
\hline 1 & offen & 0,00 & 5 & 0,05 & $-0,29$ & 73 & 0,13 & $-0,60$ \\
\hline 2 & 0,00 & 0,25 & 6 & 0,12 & 0,17 & 73 & 0,15 & 0,16 \\
\hline 3 & 0,25 & 0,50 & 15 & 0,09 & 0,36 & 201 & 0,13 & 0,39 \\
\hline 4 & 0,50 & 0,75 & 48 & 0,16 & 0,64 & 450 & 0,14 & 0,64 \\
\hline 5 & 0,75 & 1,00 & 50 & 0,17 & 0,86 & 419 & 0,15 & 0,86 \\
\hline 6 & 1,00 & 1,50 & 27 & 0,20 & 1,24 & 316 & 0,19 & 1,22 \\
\hline 7 & 1,50 & 2,00 & 7 & 0,18 & 1,66 & 86 & 0,22 & 1,67 \\
\hline 8 & 2,00 & 2,50 & 4 & 0,53 & 2,13 & 22 & 0,34 & 2,19 \\
\hline 9 & 2,50 & offen & 0 & 0 & 0 & & 0 & 2,90 \\
\hline
\end{tabular}

Tabelle 42: Klasseneinteilung für die Untersuchungsmenge

Sowohl bei der KNN-Analyse als auch bei Rating nimmt die Prognosefähigkeit mit steigendem Risiko ab. Dies kann bei dem KNN mit der unterproportionalen Vertretung von entsprechenden Unternehmen in der Lernmasse begründet werden (vgl. Kapitel 5.5.2). Der Anstieg der Standardabweichung des Credit Spread bei höherem Rating ist bereits in anderen wissenschaftlichen Studien herausgestellt worden und lässt sich bei der Prognose mittels des 13er-Modells bestätigen.

Falls man sowohl die absoluten Fehlerwerte als auch den Anstieg direkt vergleicht, kann das $13 e r$-Modell bessere Ergebnisse als die zur Kreditzinskalkulation verwendeten Ratingeinschätzungen erzielen. Diese Feststellung abstrahiert von den o.g. Problemen bzgl. eines Vergleiches. In Abbildung 64 wurde sowohl die Testmenge als auch 
Marktorientierte Kreditrisikobewertung - Empirische Untersuchung 210 zur marktorientierten Bewertung des Kreditrisikos mittels Künstlicher Neuronaler Netze

die vollständige Datenmenge (Lern- Validierungs- und Generalisierungsdaten) verwendet. Beide Gruppen sind dunkler gehalten. Die helleren Balken beziehen sich auf die o.g. Untersuchungen.

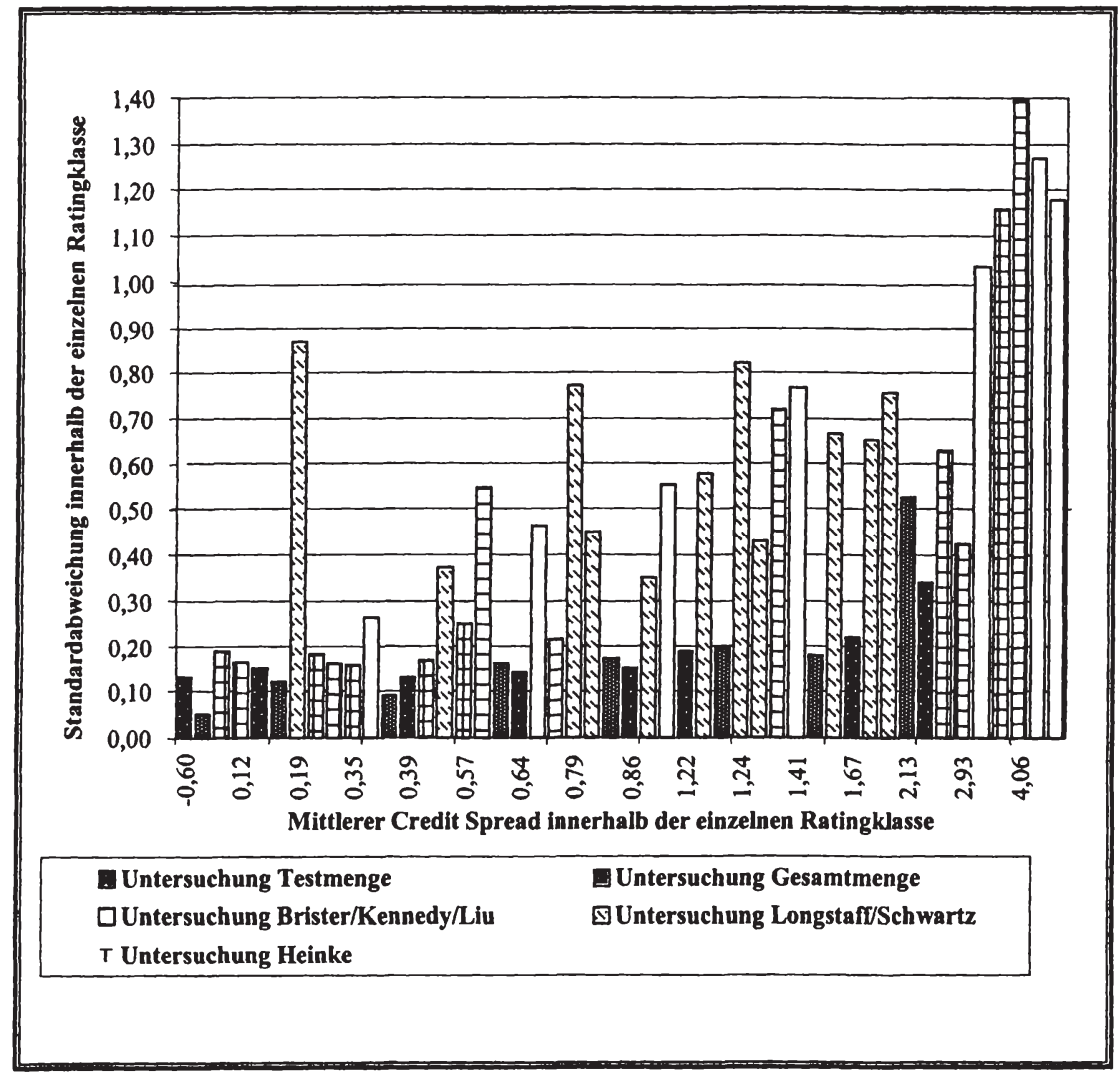

Abbildung 64: Gegenüberstellung der Prognosefähigkeit des 13er-Modells und der Prognose von Ratings 
Marktorientierte Kreditrisikobewertung - Empirische Untersuchung zur marktorientierten Bewertung des Kreditrisikos mittels Künstlicher Neuronaler Netze 211

\subsection{Ergebniszusammenfassung}

Die Untersuchungen mittels Neuronaler Netze konnten zeigen, dass es einen Zusammenhang zwischen den gefundenen Inputfaktoren und dem vom Kreditmarkt bewerteten Risiko gibt. Die gestellten Fragen unter Kapitel 4.1 können insoweit beantwortet werden.

Kreditgeber verwenden die Bilanzanalyse im Rahmen ihrer Dispositionsentscheidung. Es findet jedoch keine isolierte Betrachtung statt, sondern vielmehr werden weitere Kriterien, wie makroökonomische oder titelspezifische Faktoren, zur Bestimmung des Kreditrisikos verwendet. Diese Faktoren finden sich auch in empirischen Studien und modelltheoretischen Ansätzen wieder. Es kann ein Set von Kennzahlen benannt werden, das den Entscheidungsprozess besonders gut nachbilden kann. Dieses System besitzt jedoch nicht den Anspruch der Ausschließlichkeit. Auch andere Kombinationen von Kennzahlen führen zu ähnlich guten Ergebnissen. Mit Hilfe der getroffenen Kennzahlenauswahl im 13er-Modell ist eine Prognose des Credit Spread und damit des bewerteten Kreditrisikos möglich. Auf Basis eines Vergleichs mit drei Studien zur Prognosefähigkeit von Ratings kann die Schlussfolgerung gezogen werden, dass die mit der Prognose des 13er-Modells verbundene Unsicherheit geringer ist, als die bei der Prognose mittels externer Ratingeinschätzungen.

Als Prognosemodell wurde ein Neuronales Netz eingesetzt. Im Rahmen dieser Anwendung sind subjektive Parametereinstellungen vorzunehmen. Eine Vielzahl von Modifikationen des Grundmodells zeigten eine gute Ergebnisstabilität, sodass die gefundene Lösung zum einen eine stabile, gute Strukturwahl hat. Die im Fehlerwert gemessene Lösungsgüte variiert in einem recht engen Intervall. Zum anderen erhält der Vorgang der subjektiven Parametereinstellung durch eine möglichst große Variation und eine gleichzeitig argumentative Begründung eine Objektivierung.

Die Ergebnisse geben Anlass, das Instrument der Neuronalen Netze weiter zu untersuchen, da deutliche Hinweise auf deren Eignung als Prognoseinstrument innerhalb der Kreditbewertung gefunden wurden. Wie bereits im Kapitel 4.5 dargestellt, erfüllt das Prognosemodell die geforderten Kriterien für ein Kalkulationsverfahren. Darüber hinaus konnte die empirische Studie durch die Modellgüte die Eignung als Kreditzinskalkulationsverfahren herausstellen und diese im exemplarisch geführten Vergleich zu drei Studien zur Prognosefähigkeit von Ratings als besser einstufen. Die Kennzahlenauswahl des 13er-Modells erfuillt die Anforderungen des Ganzheitlichkeitskriteriums, da das Unternehmen mittels der Vermögens-, Ertrags- und Finanzlage bewertet wird. ${ }^{755}$ Die Erfuillung des Neutralitätskriteriums kann nicht direkt nachgewiesen werden. Entweder unterstellt man dem Mechanismus des Kreditmarktes, wie es COENENBERG

Vgl. Tabelle 36. 
aus der Annahme eines effizienten Kapitalmarktes schlussfolgert, ${ }^{756}$ dieses Prinzip oder es gilt, in einem weiteren Schritt die Einhaltung des Neutralitätskriteriums zu prüfen. Dies könnte beispielsweise durch „eigenhändig“ durchgeführte Bilanzmanipulationen geschehen, indem zunächst durch Ausnutzung bilanzieller Spielräume einzelne Positionen verändert werden und anschließend der auf Basis der veränderten Bilanz vom $\mathrm{KNN}$ ausgewiesene Kreditzins mit dem ursprünglichen verglichen wird. Falls das Neutralitätsprinzip erfüllt ist, darf keine Veränderung des berechneten Kreditzinses zu beobachten sein.

Vgl. Coenenberg (2000), S. 1124. 
Marktorientierte Kreditrisikobewertung - Bewertung von Unternehmenskrediten und Unternehmensanleihen mittels Künstlicher Neuronaler Netze

\section{Bewertung von Unternehmenskrediten und Unternehmensanleihen mittels Künstlicher Neuronaler Netze}

\subsection{Anwendungsgebiete der Forschungsergebnisse}

Die gefundenen Ergebnisse der Untersuchung führen zu der Frage, inwieweit sich diese Erkenntnisse für die praktische Nutzung eignen. Denkbar ist die Anwendung grundsätzlich in allen Bereichen, in denen Kreditrisiken zu bewerten sind. Im Weiteren wird geprüft, welche Möglichkeiten zum Einsatz des dargestellten Verfahrens zum einen im Rahmen der Kreditbewertung durch Kreditinstitute (Kapitel 6.1.1) und zum anderen für die Bewertung von Anleihen (Kapitel 6.1.2) bestehen.

\subsubsection{Kreditvergabe}

Die Übertragung der Untersuchungsergebnisse vom Anwendungskontext „Kreditmarkt" auf die Vergabe von Bankenkrediten bedingt, dass sich diese beiden Fremdkapitaltitel nicht fundamental unterscheiden. Obwohl es diese Unterschiede gibt, ${ }^{757}$ ist dem Autor keine wissenschaftliche Arbeit bekannt, die wertmäßige Konsequenzen der Unterschiede zwischen Bankenkrediten und Unternehmensanleihen thematisiert. ${ }^{758}$ Beispielsweise wird das aus der Kapitalmarkttheorie abgeleitete Optionspreismodell ebenfalls auf Bankenkredite übertragen. ${ }^{759}$

Die Kreditvergabe an Firmenkunden ist an die aus der amerikanischen Bankenpraxis bekannten sechs „Cs of Credit“ geknüpft. Innerhalb der Kreditwürdigkeitsprüfung werden Informationen hinsichtlich der folgenden Bereiche erhoben: ${ }^{760}$

- Character (Unternehmerpersönlichkeit und -integrität),

- Capacity (sachliches und personelles Leistungsvermögen des Unternehmens),

- Capital (Vermögenslage des Kreditnehmers)

- Collaterals (Sicherheitensituation),

- Conditions (technologische und konjunkturelle Bedingungen) und

- Coverage (Wirkungskreis und Aktionsradius des Unternehmens).

Insbesondere bei der Vergabe von Krediten an kleine und mittlere Unternehmen wird der erste Punkt (Unternehmerpersönlichkeit und -integrität) in das Entscheidungskal-

757 Ein Vergleich der beiden Instrumente (Bankenkredite vs. Anleihen) findet sich bspw. bei Sommerfeld (2001), S. 1 ff.

758 Vgl. Sommerfeld (2001), S. 297.

759 Vgl. Kap. 2.3.2.2, ein weiteres Beispiel ist die Übertragung des CAPM. Vgl. Bröker (2000), S. 52.

760 Vgl. hier und im Folgenden Hartmann-Wendels, Pfingsten, Weber (2000), S. 161; Sinkey (1992), S. 528; Priewasser (1992), S. 362. 

und Unternehmensanleihen mittels Künstlicher Neuronaler Netze

kül des Kreditsachbearbeiters einbezogen. Insbesondere die subjektive Beurteilung qualitativer Merkmale wie die Bewertung der Unternehmerpersönlichkeit führen zu einer mangelnden Transparenz der Kreditentscheidung. ${ }^{761}$ Die getroffenen Entscheidungen können nicht von einem Dritten vollständig nachgebildet werden und führen insbesondere bei unerfahrenen Kreditentscheidern zu vermehrten Fehlbeurteilungen.

Die Vorgehensweise des oben vorgestellten 13er-Modells abstrahiert von der Beurteilung durch den Einzelnen. Es wird eine marktliche und somit objektive und nachvollziehbare Bewertung des Kreditrisikos vorgenommen. ${ }^{762}$ Unter zu Hilfenahme der KNN-Analyse könnten die relevanten Informationen hinsichtlich der Vermögens-, Ertrags- und Finanzlage, der allgemeinen Marktsituation und der gesamtwirtschaftlichen Erwartungen abgedeckt, analysiert und sichtbar gemacht werden. In der im Kap. 5.3 beschriebenen Untersuchungsmenge, die mittels des Instrumentes der KNN analysiert wurde, waren vorrangig große Unternehmen vertreten. Fraglich ist somit ob im Rahmen von mittleren oder kleineren Unternehmen dieselben Kennzahlen entscheidend sind.

Relative Kennzahlen, wie sie auch im vorgestellten 13er-Modell verwendet werden, erfassen Verzerrungen aufgrund der Größe mathematisch. ${ }^{763}$ Beispielsweise setzt die Kennzahl $\mathrm{B}_{2}$ den Umsatz („,Revenue“) in Beziehung zum Gesamtkapital („Total Assets"). Folglich muss ein größeres Unternehmen einen entsprechend höheren Umsatz ausweisen, um denselben Kennzahlenwert wie ein kleines Unternehmen zu erreichen. Neben dieser Eigenschaft von relativen Kennzahlen, Unternehmensgrößen zu berücksichtigen, weisen die untersuchten Unternehmen eine gewisse Varianz in der Unternehmensgröße auf. ${ }^{764}$ Das kleinste untersuchte Unternehmen hat eine Bilanzsumme von 18,375 Millionen US-\$, das größte von 279.097,000 Millionen US-\$. Falls sich durch die Unternehmensgröße die Relevanz von Kennzahlen ändert, würde das Netz dies für die untersuchten Objekte beachten. Für Unternehmen, die eine geringere Größe als die untersuchten aufweisen, kann vermutet werden, dass dieselben oder ähnliche Kennzahlen in das Entscheidungskalkül einfließen würden. KreditScoring-Modelle greifen regelmäßig auf „bekannte“ Kennzahlen zurück, die auch in dieser vorliegenden Untersuchung beachtet wurden, wie bspw. die bereits o.g. Kenn-

Vgl. hier und im Folgenden Glormann (2001), S. $307 f$.

Vgl. Kap. 4.4.

Dieser Aussage stehen die Ergebnisse von Jerschensky und von Küting, Weber gegenüber. Die Autoren weisen auf die Bedeutung von Größenunterschieden auch bei relativen Kennzahlen hin. Vgl. Jerschensky (1998), S. 231 ff.; Küting, Weber (2000), S. $25 \mathrm{ff}$.

Vgl. Tabelle 13. 
Marktorientierte Kreditrisikobewertung - Bewertung von Unternehmenskrediten und Unternehmensanleihen mittels Künstlicher Neuronaler Netze

zahl Umsatz / Gesamtkapital. ${ }^{765}$ In anderen Scoring-Modellen ${ }^{766}$ wird ebenfalls nicht explizit auf die Unternehmensgröße eingegangen.

Die bei der Kreditvergabe geprüfte Frage nach Sicherheiten des Kreditnehmers wird nicht im 13er-Modell abgebildet. Es wird eine Anleihe ohne Sonderrechte innerhalb der Besicherung unterlegt, sodass weitere Vereinbarungen zu einer tendenziellen Reduktion des Credit Spread bzw. Kreditzinses führen sollten. Jedoch weisen MüLLER/MüLLER darauf hin, dass die Vergabe von Sicherheiten ,im allgemeinen nur eine sekundäre Rolle spielen [darf], da sie letztlich nur die Funktion einer Notbremse erfüllen (...). Banken legen im Allgemeinen Kredite nicht mit der Absicht heraus, diese im Rahmen von Zwangsmaßnahmen durch Sicherheitsverwertung zurückzuführen, sondern im Rahmen einer ordnungsgemäßen Bedienung über die Laufzeit" ${ }^{\text {"767 }}$.

Besonders für die Kalkulation eines im obigen Sinne objektiven Kreditzinses eignen sich die Ergebnisse des Neuronalen Netzes. Das 13er-Modell ist in der Lage, einen marktlichen, objektiven Wert für das Kreditrisiko zu bestimmen. Da nicht alle von Kreditinstituten bei der Kreditvergabe verlangten Kriterien in die Bewertung einbezogen werden, kann man - falls man an der gängigen Bankpraxis bei der Vergabe von Krediten, insbesondere an mittelständische Untemehmen, festhalten möchte - das bepreiste Ausfallrisiko als Intervall in das Entscheidungskalkül für die Kreditentscheidung einbeziehen. Falls bspw. ein Unternehmen einen Kredit beantragt, kann auf Basis der eingereichten Bilanz, der aktuellen Marktsituation (bspw. das allgemeine Zinsniveau) und der Kreditmerkmale (bspw. Kreditvolumen oder Kreditlaufzeit) das 13erModell einen Kreditzins berechnen. Dieser könnte beispielsweise bei $6 \%$ p.a. liegen. Dieser Zinssatz drückt den Preis aus, der sich gemäß der Prognose des Modells für diesen Kredit am Kapitalmarkt bilden würde. Die Prognose selber ist mit einer Unsicherheit behaftet. Diese lässt sich bspw. anhand des Fehlerwertes $\mathrm{s}_{\mathrm{F}}$ operationalisieren. Ein Wert $s_{F}$ in Höhe von 0,195 bedeutet, dass in $66 \%$ der untersuchten Fälle die Differenz aus dem prognostizierten Kreditzinssatz und dem am Markt beobachtbaren Satz eine geringere Abweichung als 0,1950 Prozentpunkte aufweist. Falls man eine Aussage über die Abweichung für $90 \%$ der untersuchten Fälle treffen möchte, kann man den Wert ,90-Prozent-Intervall“" $(=0,3234)$ heranziehen. ${ }^{768}$ Für die Prognose des o.g. Beispiels bedeutet dies, dass man einen Kreditzins von $6 \%$ prognostiziert und ein entsprechendes „66-Prozent-Intervall“ ([5,805;6,195]) bzw. ein „90-Prozent-Intervall“ $([5,6766 ; 6,3234])$ bilden kann. Diese unterstellten Intervalle berechnen sich durch die

765 Vgl. Altman, Saunders (1997).

766 Vgl. Altman (1993), S. 186.

767 Müller, Müller (1998), S. 3; (die mit [ ] gekennzeichneten Inhalte wurden vom Verfasser hinzugefügt). Die Autoren weisen jedoch daraufhin, dass die Praxis das Gegenteil als den gängigen Fall aufzeigt.

768

Vgl. Tabelle 32. 
Marktorientierte Kreditrisikobewertung - Bewertung von Unternehmenskrediten und Unternehmensanleihen mittels Künstlicher Neuronaler Netze

Annahme eines Sicherheitsniveaus (66\% bzw. $90 \%$ ). Der Kreditentscheider kann innerhalb des Intervalls den Kreditzins festlegen. ${ }^{769770}$ Dies kombiniert die Ergebnisse der KNN mit dem Erfahrungswissen des Kreditentscheiders. ${ }^{711}$ Darüber hinaus kann das Intervall für die Berücksichtigung von Sicherheiten benutzt werden.

Der nach dem Prognosemodell berechnete Kreditzins stellt die Prämie für einen unbesicherten $^{772}$ Kredit dar. Falls man wiederum ein Intervall auf Basis eines Sicherheitsniveaus um den prognostizierten Zins berechnet, kann die Sicherheit in Form eines Zinsabschlages bedacht werden. Der bestehende Prozess der Kreditwürdigkeitsprüfung kann um die Erkenntnisse des Prognosemodells erweitert werden.

Während die Kreditzinskalkulation die Bepreisung des Ausfallrisikos zur Zielsetzung hat, wird im Rahmen eines (internen) Rating der Schuldner einer Klasse zugeteilt. ${ }^{773}$ Annahmegemäß unterliegen die Schuldner in einer Klasse demselben Ausfallrisiko. Gemäß dem Baseler Abkommen werden Kreditinstitute in Zukunft für die Berechnung der Risikoaktiva einen Gewichtungsfaktor heranziehen. ${ }^{774}$ Dieser soll sich individuell je Schuldner(klasse) aus der Bonität ableiten. Somit handelt es sich um einen risikoadäquaten Bonitätsgewichtungsfaktor. „Zur Festlegung des zu verwendenden Gewichtungsfaktors sind objektiv nachvollziehbare Kriterien heranzuziehen, wie sie z.B. von

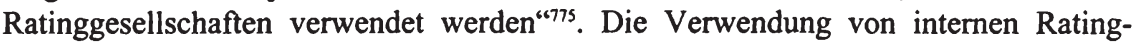
systemen unterliegt der Zustimmungspflicht durch die Bankenaufsicht. Für die Aner-

Es wird in der Diskussion von einem weiteren Problemkreis abstrahiert. Sowohl Ratingagenturen als auch der Kapitalmarkt haben im Gegensatz zu Kreditinstituten einen geringeren Erfahrungswert bei der Vergabe von Krediten an den Mittelstand als Kreditinstitute, sodass eine externe Ratingeinstufung u.U. nicht einer internen vorzuziehen ist.

Durch die unterschiedlichen Rahmenbedingungen des Kreditmarktes im weiteren und im engeren Sinne können sich die Bewertungsmechanismen unterscheiden. Bspw. sind die Transaktionskosten bei einer gehandelten Anleihe und einem Investitionskredit sicherlich unterschiedlich, sodass ein Kreditzinskalkulationsverfahren diese Unterschiede aufnehmen sollte. Das Optionspreismodell geht bspw. nicht auf diese Besonderheiten ein. Dem Verfasser ist kein Kalkulationsverfahren bekannt, das explizit diese Unterschiede aufgreift und den Bewertungsprozess daraufhin anpasst.

771 Dieser Vorschlag ähnelt der Vorgehensweise, die Ergebnisse des KNN und das Expertenwissen hinsichtlich der Jahresabschlussanalyse zu kombinieren, (,mit Hilfe von KNN und Experten-(Erfahrungs)wissen noch bessere Ergebnisse der Jahresabschlussanalyse zur Beurteilung der wirtschaftlichen Lage von Unternehmen erzielbar"), Enders (2001), S. 372).

Es handelt sich um eine über die rechtliche nicht hinausgehende Besicherung.

Vgl. Kap. 2.3.3.

Vgl. hier und im Folgenden Schulte-Mattler (1999), S. 530ff.

Vgl. Schulte-Mattler (1999), S. 531. 
Marktorientierte Kreditrisikobewertung - Bewertung von Unternehmenskrediten und Unternehmensanleihen mittels Künstlicher Neuronaler Netze

kennung interner Ratings hat der Baseler Ausschuss eine Reihe von Kriterien definiert: ${ }^{776}$

- Vollständigkeit, Transparenz, hinreichende Klarheit und Nachvollziehbarkeit der im Rating berücksichtigten Kriterien,

- statistischer Nachweis der Güte (Trennfähigkeit) sowie der Sensitivität der Einstufung bei im Zeitablauf sich verändernder Bonität,

- eine ausreichende Differenzierung der Ratingklassen,

- die Möglichkeit der Ableitung von empirischen Ausfallraten, Rückzahlungsquoten etc. aus einer eigenen Datenbasis,

- die Möglichkeit des Backtestings von erwarteten und realisierten Kreditnehmerausfällen und erwarteten Verlusten,

- Sicherstellung der organisatorischen Maßnahmen zur unabhängigen Kontrolle der Ratings durch Mitarbeiter, die nicht direkt im Kreditentscheidungsprozess (bspw. bei der Konditionengestaltung) involviert sind.

Während die konzeptionellen Ansprüche der Ermittlung einer objektiven Risikobewertung durch die kreditmarktorientierte Vorgehensweise gedeckt sind, stellt sich die Frage, inwieweit sich das Prognosemodell eignet, Unternehmen bzw. Kredite in einzelne Ratingklassen einzustufen. Folglich erweist sich eine Differenzierung in Ratingklassen als Modifikationsbedarf des vorgestellten Modells. Darüber hinaus ist die Datenmenge in der Untersuchung aufgrund des Umfangs, der Stichprobenauswahl und des Bezugs zum amerikanischen Kapitalmarkt kritisch zu bewerten. Sicherlich geben jedoch die guten Ergebnisse Anlass, weitere Untersuchungen vorzunehmen, um eine vollständige Deckung der Anforderungen zu gewährleisten.

\subsubsection{Einsatzmöglichkeiten beim Primär- und Sekundärhandel von Anleihen}

Grundsätzlich stehen Emittenten von Unternehmensanleihen zwei Wege zur Abwicklung der Primärtransaktion, d.h. der Erstemission von Finanztiteln, zur Verfügung. ${ }^{\text {"n }}$ Entweder wählen Gesellschaften einen direkten Absatz ohne Einschaltung einer Bank oder aber sie entscheiden sich unter Einschaltung eines Kreditinstitutes zu einem indirekten Vertriebsweg. Der erste Fall wird als Eigenemission, der zweite als Fremdemission bezeichnet. ${ }^{778}$ Der entscheidende Unterschied liegt in der Übernahme des vorhandenen Risikos der Nichtplatzierung einzelner Titel. Während bei der Eigenemission

\footnotetext{
776 Vgl. Jansen (2001b), S. 99f.

777 Vgl. hier und im Folgenden Jacob, Klein (1996), S. 107, hinsichtlich der unterschiedlichen Risikoprofile bei der Emission vgl. Jacob, Klein (1996), S. $120 \mathrm{ff}$.

778 Vgl. Rudolph (1981), S. 61.
} 
Marktorientierte Kreditrisikobewertung - Bewertung von Unternehmenskrediten und Unternehmensanleihen mittels Künstlicher Neuronaler Netze

dieses Risiko beim Emittenten verbleibt, übernimmt bei der Fremdemission die Emissionsbank unter Zahlung einer Prämie dieses Risiko. ${ }^{79}$

Somit besteht bei der Emission von Fremdkapitaltiteln das Problem der Preisfindung. Als Preis wird die Emissionsrendite verstanden, die der Effektivverzinsung und somit dem Zins nach der aus der Investitionstheorie bekannten internen Zinsfußmethode gleichkommt. ${ }^{780}$ Hierzu eignen sich zwei Verfahren: das konkurrenzorientierte und das investorenorientierte Verfahren. Im Rahmen des konkurrenzorientierten Verfahrens sucht die Bank eine Vergleichsanleihe mit möglichst gleichen Ausstattungsmerkmalen. Neben instrumentellen Bestandteilen wie Laufzeit und Zins ist es besonders wichtig, einen Vergleich mit einem Emittenten gleicher Bonität durchzuführen. ${ }^{781}$ „Um die Vergleichbarkeit zwischen emittierender und Referenzanleihe hinsichtlich des Bonitätsrisikos zu ermitteln, wird dabei - wenn existent - auf das Rating der Fremdkapitaltitel zurückgegriffen." ${ }^{\text {"782 }}$ Die Emissionsbank kann somit den Kurs der zu emittierenden Anleihe an die Referenzanleihe anlehnen. Da dieses Vorgehen zunächst die Existenz eines Rating und einer Referenzanleihe voraussetzt, sind die in den Kapiteln 2.3.3 und 5.6 angebrachten Kritikpunkte zu beachten. Bei der zweiten Verfahrensweise, dem investorenorientierten Preisfindungsverfahren, wird im Rahmen einer auktionsähnlichen Vorgehensweise von Käufern ein Gebot abgegeben. ${ }^{783}$ Dieses bezieht sich auf einen Aufschlag, zu dem der potenzielle Käufer bereit ist, die Unternehmensanleihe zu erwerben. Marktzinsänderungen während der Gebotsphase werden somit aus dem Verfahren eliminiert. ${ }^{784}$ Dieses Vorgehen - das „,fixed price reoffer“-Verfahren - wird in den USA und Großbritannien praktiziert. Im direkten Vergleich zu dem konkurrenzorientierten Verfahren liegt der Vorteil des investorenorientierten Verfahrens in dem Verzicht auf eine Referenzanleihe. Es muss somit keine Anleihe gefunden werden, die ähnliche Ausstattungsmerkmale besitzt.

Die oben mittels des KNN identifizierten Zusammenhänge zwischen der effektiven Verzinsung und verschiedenen Inputfaktoren innerhalb des Prognosemodells spiegeln sich sowohl im konkurrenzorientierten als auch im investorenorientierten Verfahren wider, da im Rahmen beider Methoden eine Einschätzung des Risikos und des daraus resultierenden Preises vorgenommen werden muss. Während bei dem konkurrenzorientierten Verfahren ein Emittenten-Rating bzw. eine Referenzanleihe bekannt sein

Die Möglichkeit des „private placements“ auf dem US-Markt stellt eine hier nicht thematisierte Zwischenform dar. Vgl. Fabozzi, Modigliani (1992), 73f. oder auch Jacob, Klein (1996), S. 108.

780 Vgl. hier und im Folgenden Jacob, Klein (1996), S. 138.

781 Vgl. Fabozzi (1995), S. 51.

782 Jacob, Klein (1996), S. 138.

783 Vgl. hier und im Folgenden Jacob, Klein (1996), S. 138f.

784 Vgl. hierzu Schmitz (1995), Sp. 519. 
Marktorientierte Kreditrisikobewertung - Bewertung von Unternehmenskrediten und Unternehmensanleihen mittels Künstlicher Neuronaler Netze

muss, ist das oben beschriebene 13er-Modell in der Lage, einen Preis für eine Anleihe zu bilden, ohne diese spezifischen Kenntnisse zu haben. Im Rahmen des investorenorientierten Verfahrens kann das 13er-Modell genutzt werden, um das Gebot des potenziellen Käufers zu würdigen.

Während sich diese beiden Verfahren der Preisfindung und die Möglichkeiten der Nutzung des hier vorgestellten 13er-Modells auf den Primärmarkt stützen, zeichnet sich der Kapitalmarkt durch den Handel von emittierten Papieren auf dem Sekundärmarkt aus. Es findet ein kontinuierlicher $\mathrm{Handel}^{785}$ mit der Anleihe und dadurch eine kontinuierliche Bewertung des Risikos statt. Marktteilnehmer, insbesondere Händler, werden in die Lage versetzt, ihren aktuellen und potenziellen Wertpapierbestand ständig zu überprüfen und (für Unternehmensanleihen) das Kreditrisiko des Emittenten und das Marktzinsrisiko einzuschätzen, um zu einer Kauf- bzw. Verkaufsentscheidung zu gelangen. Dieser Bewertungsprozess führt zu der in Kap. 4.2 erläuterten Effizienz des Kapitalmarktes. Für einen ertragreichen Handel müssen die Märkte aber vorübergehend ineffizient sein. ${ }^{786}$ Händler analysieren und bestimmen Wertpapiere, die überoder unterbewertet sind. Das heißt, der Marktpreis entspricht nicht dem „Wert". Durch den entsprechenden Wertpapierkauf bzw. -verkauf bewegt sich dann der Marktpreis in Richtung „Wert", sodass sich lediglich vorubergehende Ungleichheiten, also Ineffizienzen, bilden können. Zu untersuchen ist, wie Händler an diese Informationen gelangen.

Um die permanente Überprüfung einer Vielzahl von potenziellen Einflussfaktoren der Wertpapierkurse zu erleichtern, können Händler auf unterstützende Handelssysteme zurückgreifen, welche permanent Kennzahlen und Indikatoren berechnen und ausweisen. Durch die Definition von Schwellenwerten (bzw. Kombinationen von Schwellenwerten) macht das System auf einzelne Anleihen aufmerksam. Während diese Handelssysteme sehr häufig auf markttechnischen Indikatoren aufbauen, wird im Rahmen einer Fundamentalanalyse, aufbauend auf Kennzahlen (bspw. aus der Bilanzanalyse

785 Auf den Unterschied zwischen stetigen und kontinuierlichen Preisfindungsmechanismen soll nicht eingegangen werden. An dieser Stelle ist mit dem Begriff ,kontinuierlicher Handel" ein dauerhafter, permanenter Handel gemeint, wobei einzelne Preisfindungen bspw. über einen Handelstag verteilt sein können. Somit handelt es sich um einen diskreten Handelsverlauf. Im Vergleich zu einem Bankenkredit soll jedoch der kontinuierliche Charakter herausgestellt werden.

$786 \mathrm{Vgl}$. zu den Effizienzformen und dem Informationsparadoxon Kap. 4.2. 
Marktorientierte Kreditrisikobewertung - Bewertung von Unternehmenskrediten und Unternehmensanleihen mittels Künstlicher Neuronaler Netze

oder einem Branchenvergleich), eine Einschätzung des Unternehmens vorgenommen. ${ }^{787}$

Die Erkenntnisse des hier vorgestellten Prognosemodells unterstützen den Händler bei der Überwachung des Wertpapierportfolios. Aufbauend auf fundamentalen Daten des Unternehmens - ergänzt um täglich schwankende Größen (wie die Zinserwartung) kann eine Einschätzung des Risikos des Unternehmens und des Kurses der Anleihe durch das Prognosemodell vorgenommen werden. Wie bereits in Kap. 6.1.1 dargelegt, bestimmt das Prognosemodell den objektiven Wert einer Anleihe, indem ein Effektivzins ausgewiesen wird. Diese Kurs- oder Preisinformation kann mittels eines Intervalls konkretisiert werden. Gehandelte Kurse, die über oder unter dem objektiven Wert liegen bzw. sich bspw. außerhalb eines 90-Prozent-Intervalls befinden, geben deutliche Handlungsempfehlungen. Es kann somit ein bestehendes Handelssystem oder auch eine Fundamentalanalyse durch die Integration des hier vorgestellten Prognosemodells erweitert werden.

\subsection{Anwendungsvoraussetzungen}

Während Kapitel 6.1 exemplarisch einige mögliche Anwendungsfelder der Untersuchungsergebnisse aufzeigt, besteht bei einer Übertragung der Erkenntnisse auf den deutschen Markt weiterer Untersuchungsbedarf. Zum einen wurde die Analyse an der US-amerikanischen Börse durchgeführt und zum anderen wurden die in die Studie einfließenden Bilanzkennzahlen aus nach US-GAAP bilanzierten Jahresabschlüssen entnommen. Somit kann geprüft werden, ob sich die funktionalen Zusammenhänge übertragen lassen und ob die Inputfaktoren systemabhängig sind. Dieses würde bedeuten, dass bei der Eingabe von zwei Bilanzen desselben Unternehmens - wobei eine nach US-GAAP und eine nach dem HGB aufgestellt wurde - zwei deutlich abweichende Ergebnisse durch das dargestellte Prognosemodell generiert würden. Das folgende Kapitel 6.2.1 überprüft, ob die unterschiedlichen Rahmenbedingungen gegen eine Übertragung sprechen. Kap. 6.2.2 erläutert, inwieweit sich die vom KNN identifizierten Zusammenhänge, die sich auf das Bilanzkennzahlen nach dem US-GAAP beziehen, auf Bilanzkennzahlen nach dem HGB überleiten lassen.

787 Vgl. zu Handelsstrategien und Handelssystemen auch Zapotocky (2000), S. 1082. Der Autor nennt auch exemplarisch ein Handelssystem, das auf einem Neuronalen Netz basiert. 
Marktorientierte Kreditrisikobewertung - Bewertung von Unternehmenskrediten und Unternehmensanleihen mittels Künstlicher Neuronaler Netze

\subsubsection{Vergleich der institutionellen Rahmenbedingungen}

Die Ergebnisse der Untersuchung basieren auf dem Bewertungsmechanismus der USamerikanischen Börse. Es gilt somit zu prüfen, inwieweit eine Bewertung börsen- bzw. länderspezifisch ist. Anders formuliert wird untersucht, ob ein Wertpapier an der USamerikanischen Börse anders bewertet werden würde, als an einer deutschen Börse. Die Effizienzannahme schließt eine unterschiedliche Bewertung aus. ${ }^{788}$ Die restriktiven Modellannahmen sind jedoch regelmäßig nicht erfüllt (bspw. keine Transaktionskosten), sodass eine Untersuchung den Einfluss der Rahmenbedingungen analysieren muss. Man könnte einen derartigen Vergleich beispielsweise anhand der Kriterien Börsenaufsicht, Marktstrukturen und gesetzliche Steuernormen führen. ${ }^{789}$

Offensichtlich sind die Ausprägungen der genannten Kriterien an den Börsen unterschiedlich. Die im Jahre 1933 in den Vereinigten Staaten eingerichtete Wertpapierüberwachungsbehörde „Securities and Exchange Commission“ (SEC) ${ }^{790}$ bedingt im Vergleich zum „Bundesaufsichtsamt für den Wertpapierhandel" in Frankfurt (BAWe) eine geringere Regulierung der nationalen Finanzmärkte. ${ }^{791}$ Ebenfalls uneinheitlich sind die Marktstrukturen. Grundsätzlich liegt in beiden Systemen eine dezentrale Marktstruktur vor. ${ }^{72}$ Im US-amerikanischen System wird besonders stark um die Investorenaufträge konkurriert, sodass sich heterogene Strukturen bilden. In Deutschland jedoch sind diese Marktstrukturen nicht durch eine auffallende Heterogenität gekennzeichnet. Erst die Einführung des Xetra ${ }^{\circledR}$-Handels zwingt die Regionalbörsen dazu, Innovationen zu realisieren, die letztendlich zu heterogenen Strukturen führen. ${ }^{793}$ Neben abwicklungstechnischen Unterschieden sind die Steuersysteme in den beiden Staaten unterschiedlich. Bei Kauf- bzw. Verkaufsentscheidungen von Investoren, die

788 Vgl. zu dieser Schlussfolgerung die in Kap. 4.2 getätigte Definition des effizienten Marktes.

789 Zur detaillierten Beschreibung der amerikanischen aufsichtsrechtlichen Gesetzgebung mit besonderem Bezug zum Rechnungslegungssystem vgl. Glormann (2001), S. $11 \mathrm{ff}$.

790 „Ihre Aufgabe ist es seitdem, die Einhaltung der bestehenden Wertpapiergesetze zu überprüfen, um so den Anleger und die Öffentlichkeit vor Mißbräuchen zu schützen“, Puckler (1986), S. 45.

791 Vgl. Bohl, Alexander (2000), S. 469.

792 Vgl. hier und im Folgenden Kirchner (1999), S. 22.

793

Vgl. Kirchner (1999), FN 12, S. 22f. 

und Unternehmensanleihen mittels Künstlicher Neuronaler Netze

letztendlich die Preise determinieren, sind steuerliche Aspekte zu beachten. Beispielsweise sind in Deutschland für den privaten Anleger realisierte Kursgewinne durch den An- und Verkauf desselben Wertpapiers nicht steuerpflichtig. ${ }^{794}$ Für den USamerikanischen Anleger jedoch sind sowohl im privaten wie im erwerbswirtschaftlichen Bereich, unabhängig von einer Haltefrist oder der Höhe der Beteiligung bzw. der Kursgewinne, Veräußerungsgewinne $\mathrm{zu}$ versteuern. ${ }^{795}$ Somit kann aus steuerlichen Aspekten heraus der Anleger dem Verkauf einer Anleihe in Deutschland zu einem bestimmten Kurs zustimmen, während unter sonst gleichen Bedingungen der Anleger in den USA davon absieht. Der Grund für diesen Einzelfall liegt in der unterschiedlichen Rendite nach Steuern.

Daraus folgt, dass unterschiedliche Rahmenbedingungen an US-amerikanischen und deutschen Börsen grundsätzlich zu einer unterschiedlichen Preisfindung und somit zu einem nicht gleichen Bewertungsmechanismus führen können. Falls diese Annahme unterstellt wird, muss geprüft werden, inwieweit die gefundenen Strukturen innerhalb des dargestellten Prognosemodells von diesen Unterschieden betroffen sind. Die Relevanz für das 13er-Modell könnte untersucht werden, indem weitere Analysen sowohl Daten vom deutschen als auch vom US-amerikanischen Finanzmarkt berücksichtigen. Falls die Information (deutscher bzw. amerikanischer) „Finanzmarkt“ für die Prognose eine Relevanz besitzt, würde die Gewichtung der entsprechenden Information „Finanzmarkt" bzw. des korrespondierenden Eingangsneurons ungleich Null sein oder der Fehlerwert der Netzstruktur deutlich steigen, falls man dem KNN diese Eingangsinformation vorenthält (Pruning-Verfahren).

\subsubsection{Transformationsmöglichkeiten der Ergebnisse auf Bilanzen nach dem HGB}

Da die oben präsentierten Ergebnisse sich auf den Zusammenhang zwischen der Risikoprämie und nach US-GAAP bilanzierten Unternehmenskennzahlen beziehen, ist fraglich, inwieweit sich diese Erkenntnisse auf die Ermittlung von Risikoprämien mittels nach HGB bilanzierten Kennzahlen übertragen lassen. Drei Möglichkeiten bieten sich, diesem Problem zu begegnen.

794 Eine Ausnahme stellten die Spekulationssteuer dar. Als Spekulationssteuer sind Steuern auf realisierte Kursgewinne abzuführen, falls diese durch ein Geschäft innerhalb von 12 Monaten zustande kamen. Falls innerhalb von einem Steuerjahr die Summe der Kursgewinne 1.000,00 DM übersteigt, unterliegt der Gesamtbetrag der Einkommensteuer. Vgl. ESTG $2001 \S 23$.

795 Vgl. American Chamber of Commerce in Germany e.V. (2001), S. 60. 
Marktorientierte Kreditrisikobewertung - Bewertung von Unternehmenskrediten und Unternehmensanleihen mittels Künstlicher Neuronaler Netze

Erstens ist es möglich, aufbauend auf den gefundenen Strukturen, ein neues Netz zu entwerfen. ${ }^{796}$ Dieses würde nicht mit US-GAAP, sondern mit HGB-Zahlen trainiert werden oder aber es würde sowohl mit US-GAAP als auch HGB-Zahlen (optional ergänzt um IAS-Bilanzen) trainiert. Die in die Untersuchung einfließenden Datensätze würden durch einen entsprechenden Inputfaktor „Bilanzierungstyp“ gekennzeichnet. Das Netz erkennt Unterschiede durch entsprechende Datenkonstellationen. Im Ergebnis hat man somit ein bilanzierungssystemneutrales Netz. Dies setzt voraus, dass eine entsprechend große Zahl an Unternehmen nach HGB bilanzieren und von diesen Unternehmen Anleihen gehandelt werden. Dies kann augenblicklich nicht beobachtet werden. Es kann jedoch vermutet werden, dass durch die Ausdehnung des EUROKapitalmarktes und die expansiven Tendenzen auf dem Markt für Unternehmensanleihen sich zukünftig eine entsprechende Untersuchungsmasse bilden wird. ${ }^{797}$

Zweitens kann geprüft werden, inwieweit sich das gefundene Netz neutral gegenüber dem zugrunde gelegten Bilanzierungssystem verhält. ${ }^{798}$ Hierzu ist es notwendig, Unternehmen zu identifizieren, die zu einem Stichtag zwei Bilanzen veröffentlicht haben. Vorraussetzung hierfür ist, dass eine Bilanz gemäß US-GAAP und eine gemäß HGB aufgestellt wurden. Somit kann man direkt ablesen, inwieweit sich die mittels des dargestellten Prognosemodells bestimmten Risikoprämien auf Basis der beiden Bilanzen unterscheiden oder ob ein neutrales Verhalten des Prognosemodells beobachtet werden kann.

Als dritte Reaktionsmöglichkeit auf das Problem der unterschiedlichen Bilanzierungsvorschriften kann die aktuelle Entwicklung zu einem einheitlichen Bilanzierungssystem gedeutet werden. Bereits heute bilanzieren viele deutsche Großunternehmen nicht

796 Glormann konnte in einer aktuellen Untersuchung nachweisen, dass mittels einer ähnlichen Vorgehensweise wie bei der Herleitung des BP-14, jedoch auf Basis von nach USGAAP bilanzierten Jahresabschlüssen, ein ähnlicher Fehlerwert ermittelt werden kann. Vgl. Glormann (2001), S. 123ff. Dies gibt Anlass, auch eine erfolgreiche Übertragung des 13er Prognosemodells zu erwarten.

797 Kritisch an dieser Vorgehensweise ist, dass Unternehmen, die Anleihen emittieren, nach Meinung des Verfassers nicht nach dem HGB, sondern eher nach dem US-GAAP oder dem IAS bilanzieren. Tendenziell werden große Unternehmen die Finanzierungsform „Anleihe" wählen und es ist zu beobachten, dass große deutsche Unternehmen bereits heute nach US-GAAP bzw. IAS bilanzieren. Vgl Schaudwet (2001). 
Marktorientierte Kreditrisikobewertung - Bewertung von Unternehmenskrediten und Unternehmensanleihen mittels Künstlicher Neuronaler Netze

mehr nach dem HGB. Im Jahre 2001 benutzte von den 30 DAX Unternehmen lediglich das Unternehmen Karstadt/Quelle das HGB als Rechnungslegungssystem. ${ }^{799}$ Für börsennotierte Unternehmen ist absehbar, dass eine einheitliche Bilanzierung verpflichtend wird. ${ }^{800}$ Folglich werden die für die Berechnung der Risikoprämie erforderlichen Inputfaktoren (Kennzahlen nach dem US-GAAP) auch bei deutschen Unternehmen ableitbar sein. ${ }^{801}$ Abbildung 65 fasst die drei Möglichkeiten zusammen.

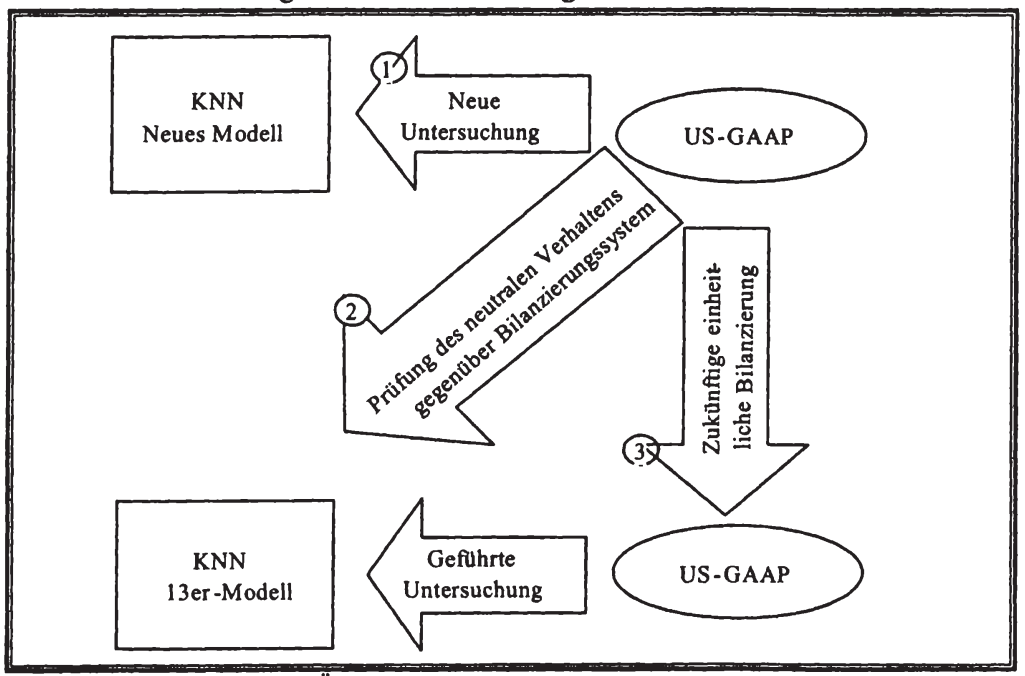

Abbildung 65: Möglichkeiten zur Übertragung der Forschungsergebnisse auf Bilanzen nach dem HGB

Vgl. Schaudwet (2001). Im Jahre 2002 wird sich auch die Karstadt Quelle AG von der HGB-Bilanzierung lösen.

800 Vgl. Goergen (2001), S. $92 \mathrm{ff}$.

801 Unterstellt wird hierbei, dass das US-GAAP als einheitliches Bilanzierungssystem herangezogen wird. Falls sich bspw. das IAS als System durchsetzt (vgl. Goergen (2001), S. 92ff.), muss die Gültigkeit des 13er-Modells gesondert untersucht werden. 


\section{Schlussbetrachtung}

Gegenstand der vorliegenden Arbeit ist die Untersuchung der Problemstellungen bei der Quantifizierung des Kreditrisikos. In diesem Sinne wird die Konzeption eines innovativen, heuristischen Verfahrens zur marktorientierten Kreditrisikobewertung entwickelt.

Hierzu sind zunächst modelltheoretische und heuristische Ansätze für die Kreditzinskalkulation analysiert worden. Kapitel 2 und 3 belegen, dass ein abschließendes Kreditzinskalkulationsverfahren nicht gefunden werden konnte, obwohl die Notwendigkeit zur Quantifizierung von Kreditrisiken, wie in Kap. 1 beschrieben, aufgrund von marktlichen Entwicklungen und neuen aufsichtsrechtlichen Verpflichtungen entscheidend zugenommen hat.

Modelltheoretische Konzepte, wie das Optionspreismodell, können zwar eine theoretisch objektive Kalkulation sicherstellen, doch kann bei der praktischen Handhabung eine subjektive Komponente nicht ausgeschlossen werden. Selbst die Bestimmung von scheinbar objektiv ermittelbaren Größen, wie dem sicheren Zins, erlauben dem Anwender einen großen subjektiven Entscheidungsspielraum.

Heuristische Verfahren verstoßen i.d.R. gegen den Anspruch der individuellen Kreditbeurteilung, da sie eine Segmentierung vornehmen. Im Fall der Vergabe eines (externen) Rating kann die objektive qualitative Risikobeurteilung nicht nachvollzogen werden. Es besteht darüber hinaus die Problematik der Quantifizierung des Risikogehalts.

Kreditinstitute setzten regelmäßig die Bilanzanalyse im Rahmen der Firmenkreditvergabe ein, um eine objektive Kreditrisikoeinschätzung zu gewährleisten. Folglich wurde untersucht, inwieweit die Bilanzanalyse dem Anspruch eines objektiven Bewertungsvorganges standhalten kann. Insbesondere Verfahren der klassischen Bilanzanalyse, wie das ROI-System, widersprechen diesem Anspruch. Neuere Instrumente, wie Verfahrensweisen der Diskriminanzanalyse, können eine objektive qualitative Aussage anhand eines statistischen Verfahrens ermitteln. Diese Techniken können jedoch nicht kritiklos auf die Kreditzinskalkulation übertragen werden. Mit der notwendigen Quantifizierung des Kreditrisikos stößt die Bilanzanalyse an ihre Grenzen. Zwar berücksichtigen einige Ansätze die wichtige Anforderung einer individuellen Kalkulation, doch scheitern die Verfahren an dem Anspruch einer objektiven Bepreisung des Risikos.

Insgesamt erscheint somit das Problem einer objektiven, individuellen Kreditzinskalkulation in Literatur und Praxis nicht abschließend gelöst. Auf Basis dieser Erkenntnisse und der Annahme effizienter Kapitalmärkte konnte im Kapitel 4 ein Prognosemodell abgeleitet werden. Hierzu wurde zunächst das Untersuchungsobjekt „Unternehmensanleihe" gewählt, um das in der Anleihenrendite enthaltene Kreditrisiko isolieren zu können. Das daraus entwickelte Prognosemodell erfüllt die Anforderungen an ein Kreditzinskalkulationsmodell. Dieses Verfahren erlaubt eine marktgerechte Beprei- 
sung des Kreditrisikos und baut auf den bereits gewonnenen Erkenntnissen der empirischen Kapitalmarktforschung, den finanzmarkttheoretischen Modellen und der Bilanzanalyse auf, indem bspw. das allgemeine Marktzinsniveau oder Zinserwartungen in den Preismechanismus einbezogen werden.

Das Prognosemodell benötigt die Integration eines Instrumentes, das die Ansprüche, die insbesondere aus den Aspekten der Nicht-Linearität und der Multidimensionalität resultieren, erfüllen kann. Künstliche Neuronale Netze erfüllen diese Voraussetzungen, sodass sie als zentrales Werkzeug der empirischen Untersuchung im Kapitel 5 gewählt wurden. Hier wurde mittels eines Künstlichen Neuronalen Netzes eine empirische Untersuchung durchgeführt.

Ein zentrales Ergebnis der Untersuchung ist die Abbildung der Erwartungsbildung von Kreditmärkten mittels eines Künstlichen Neuronalen Netzes. Damit lässt sich die Marktbewertung des Kreditrisikos simulieren.

Aus der Fülle von Bilanzkennzahlen konnten marktrelevante Größen extrahiert werden. Diese, erweitert um makroökonomische Einflussfaktoren, stellen ein System der kreditmarktorientierten Bilanzanalyse dar.

Innerhalb der $\mathrm{KNN}$-Analyse wurde eine Struktur durch die bestmöglichen Parametereinstellungen bzw. den resultierenden Gewichtungsfaktoren bestimmt. Hierzu wurde die Prognosegüte, gemessen als Fehlerwert $s_{\mathrm{F}}$, der verschiedenen Parametereinstellungen dokumentiert. Die Prognosegüte des Künstlichen Neuronalen Netzes liegt auch bei leicht modifizierten Netzstrukturen aufgrund unterschiedlicher Parametereinstellungen in einem engen Intervall, wodurch einem Kritikpunkt bezüglich Neuronaler Netze widersprochen werden kann. Die subjektiven Parametereinstellungen haben keinen signifikanten Einfluss auf die Modellgüte, da bei einer hinreichend großen Untersuchung verschiedener Strukturen ähnliche Ergebnisse generiert werden. Trotzdem kann die gefundene Netzstruktur des 13er-Modells nicht als optimal beschrieben werden, da weitere Parametervariationen u.U. zu besseren Ergebnissen führen können. Ein absolutes Fehlerminimum kann nicht garantiert werden. Bei den gefundenen Netzstrukturen handelt es sich um eine (heuristisch) gute Lösung.

Um die Modellgüte des Künstlichen Neuronalen Netzes zu würdigen, kann man die Ratingeinschätzungen von Agenturen wie STANDARD \& POOR's vergleichend hinzuziehen. Falls man unterstellt, dass diese in ein Kreditzinskalkulationsverfahren integriert werden und mittels der vom Kapitalmarkt verlangten Prämien (Credit Spread) eine Quantifizierung des Kreditrisikos vorgenommen wird, ist - insbesondere bei risikoreicheren Unternehmen - die Prognosegüte des Künstlichen Neuronalen Netzes, wie der geführte Vergleich darlegen konnte, überlegen. 
Die Anwendung des Prognosemodells für die Kreditzinskalkulation benötigt Bilanzkennzahlen, ausgehend von einem nach den US-GAAP bilanzierten Jahresabschluss. Somit ist die Frage offen, wie man mit Bilanzen nach dem HGB oder auch IAS umgehen soll. Es sind alternative Verfahrensweisen denkbar, wobei die internationalen Anpassungstendenzen die Erkenntnisse des Prognosemodells, basierend auf einer US-amerikanischen Bilanz, begünstigen.

Diese Erkenntnisse geben Anlass, die gefundenen Zusammenhänge innerhalb des heuristischen Kalkulationsverfahren erweiternd zu analysieren, indem bspw. auf Basis einer größeren Datenmenge die Strukturen weitergehend untersucht werden.

Die Untersuchungsergebnisse eignen sich grundsätzlich für den primären und den sekundären Wertpapierhandel. Bei der Übertragung des Modells auf die Kreditzinskalkulation von Firmenkrediten, insbesondere an mittelständische Unternehmen, ist ein weiterer Forschungsbedarf erkennbar.

Das Prognosemodell unterstellt ein Marktkreditportfolio. Dies bedeutet, der einzelne Kredit ist Teil eines Marktportfolios. Da ein Einzelrisiko nicht isoliert vom Markt bewertet wird, sondern im Zusammenhang zu anderen im Marktportfolio vorhandenen Risiken steht, findet eine Risikobewertung auf Basis des Marktkreditportfolios statt. Es besteht die Notwendigkeit, das bankspezifische, individuelle Kreditportfolio zu bewerten. Eine Überführung des Prognosemodells in ein Modell zur Bestimmung des Kreditportfoliorisikos steht noch aus. Die reine Risikoquantifizierung als Informationserstellung muss darüber hinaus in das Risikomanagement integriert werden.

Zusammenfassend kann man das Prognosemodell als eine Erweiterung der bisherigen Instrumente zur Bepreisung von Ausfallrisiken beschreiben. Dem Problem der Quantifizierung von Kreditrisiken wurde mittels eines heuristischen Lösungsverfahrens unter dem Fokus einer Marktorientierung begegnet. 
Andreas Siemes - 978-3-631-75322-4

Downloaded from PubFactory at 01/11/2019 06:12:16AM

via free access 


\section{Anhang}

Regressionsergebnisse der Indexbildung über den Untersuchungszeitraum. Bei allen Darstellungen sind die Werte $K_{1}$ bis $K_{7}$ gleich $\beta_{1}$ bis $\beta_{7}$ zu setzen.

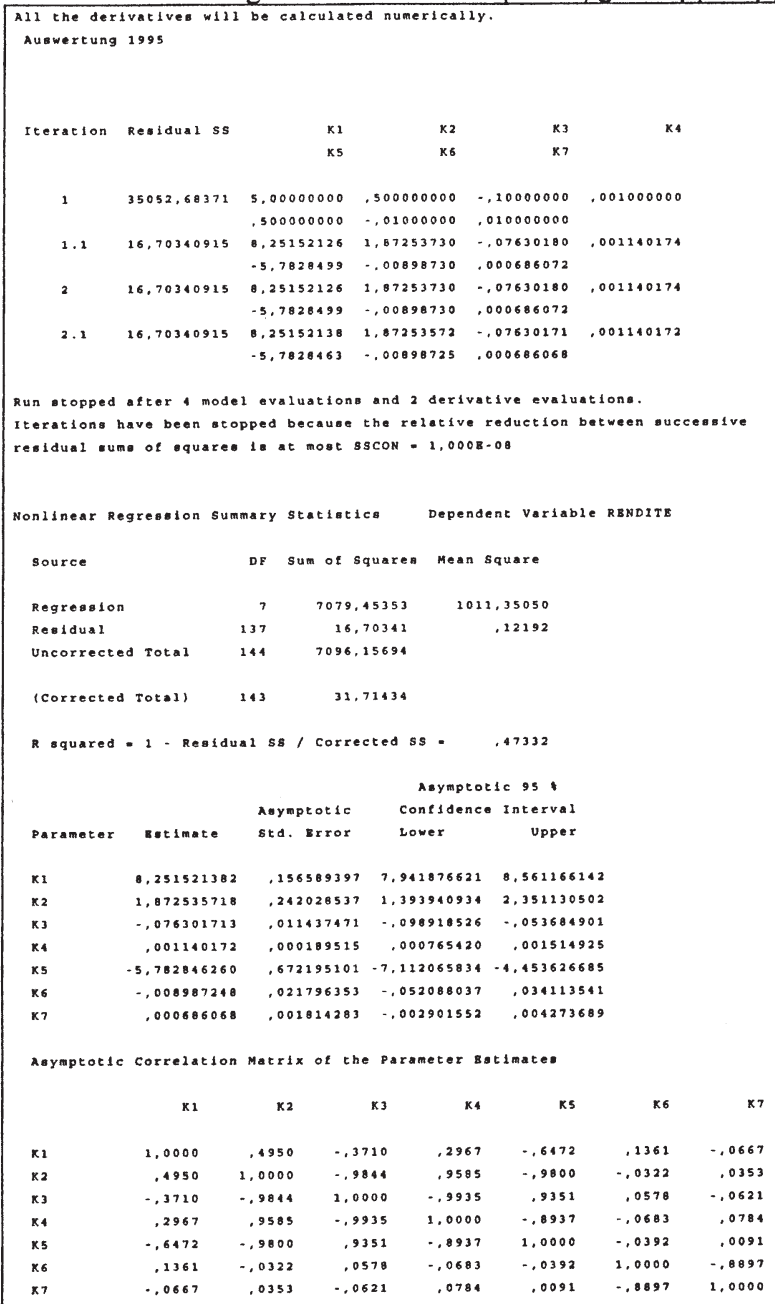

Tabelle 43: Ergebnisse der Regressionsbildung für das Jahr 1995 


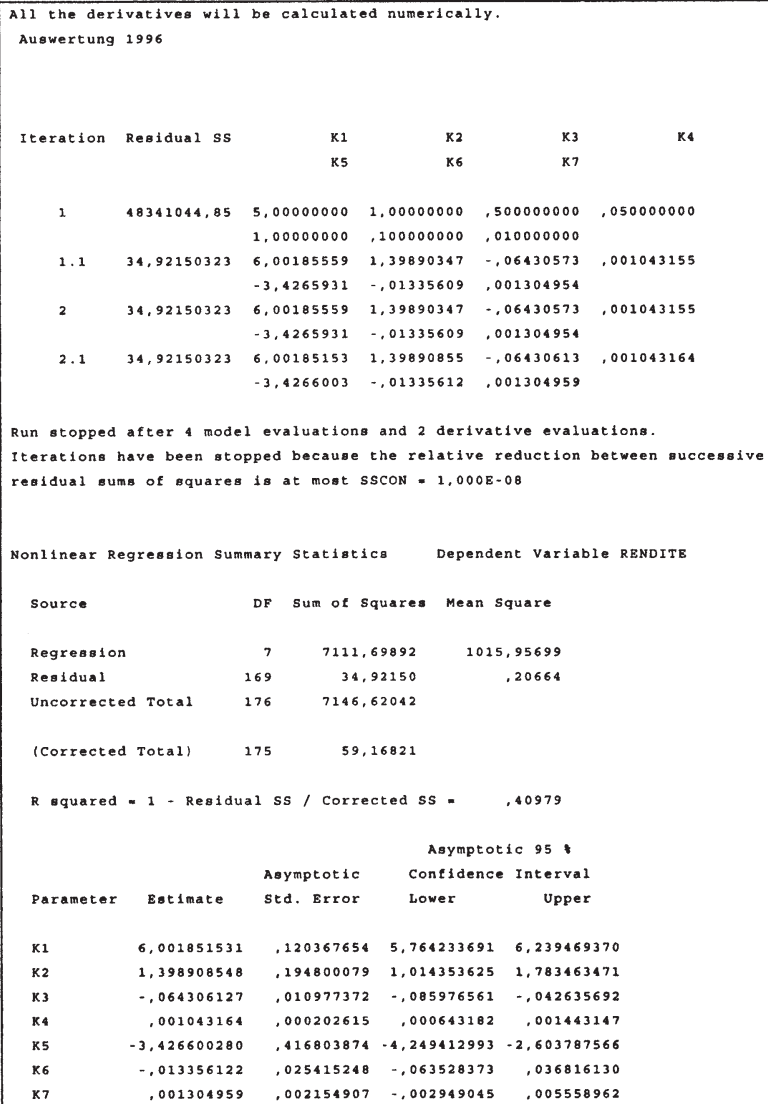

Asymptotic Correlation Matrix of the Parameter Eatimates

\begin{tabular}{|c|c|c|c|c|c|c|c|}
\hline & $\mathrm{KI}$ & $x_{2}$ & $\mathrm{~K}_{3}$ & K4 & K5 & K6 & $K 7$ \\
\hline K1 & 1,0000 & -.7116 & .7755 &,- 7886 & .5539 & .0447 & -.0462 \\
\hline K2 &,- 7116 & 2,0000 &,- 9784 & .9471 &.,- 9671 &,- 1043 & .0913 \\
\hline $\mathrm{K} 3$ & .7755 &,- 9784 & 1,0000 & -.9926 &, 9051 & .1361 &,- 1342 \\
\hline$x_{4}$ & -.7886 & .9471 & -.9926 & 1,0000 & -.8558 &,- 1473 & .1569 \\
\hline$\times 5$ & .5539 & -.9671 & .9051 &,- 8558 & 1,0000 & .0034 & -.0233 \\
\hline K6 & .0447 & -.1043 & .1361 &,- 1473 & .0034 & 1,0000 & -8874 \\
\hline K7 &,- 0462 & .0913 &,- 1342 & .1569 &,- 0233 &,- 8874 & 1,0000 \\
\hline
\end{tabular}

Tabelle 44: Ergebnisse der Regressionsbildung für das Jahr 1996 


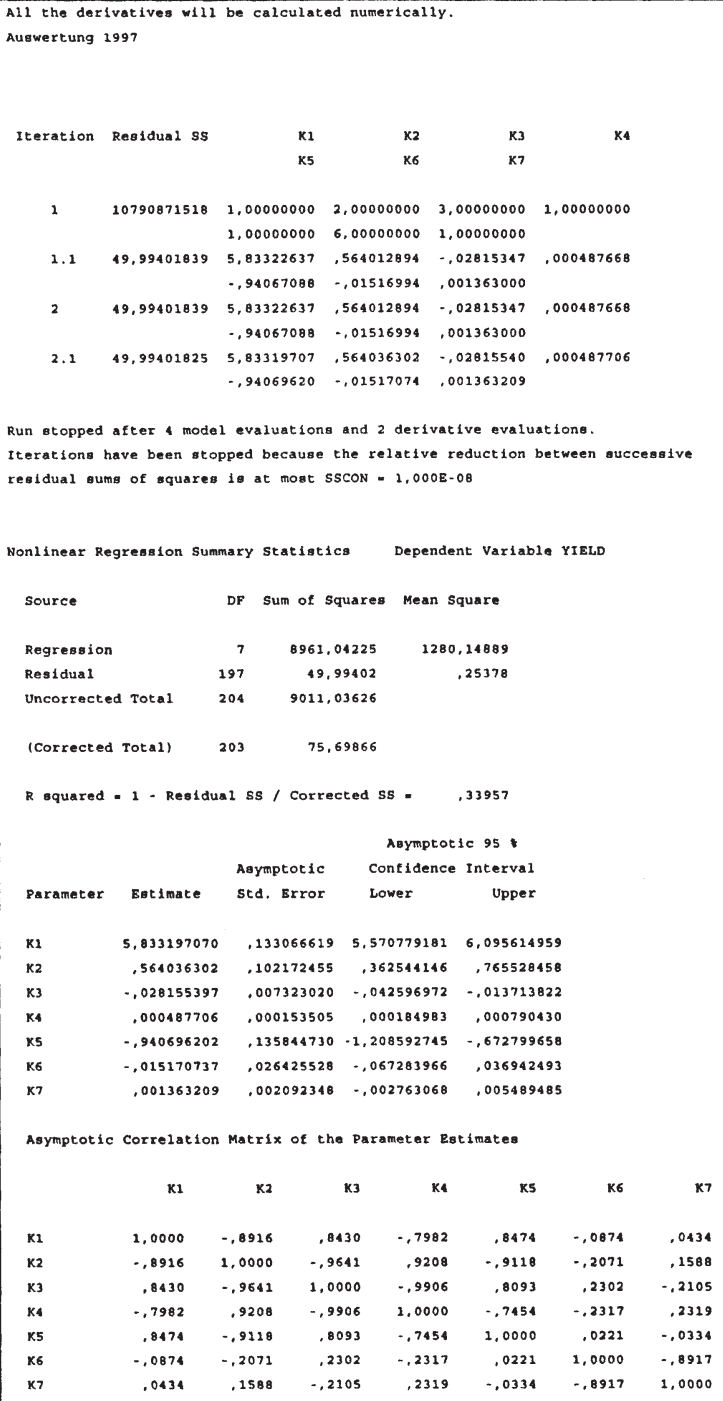

Asymptotic Correlation Matrix of the Parameter Estimates

\begin{tabular}{|c|c|c|c|c|c|c|c|}
\hline & $\mathrm{K}_{1}$ & $\mathrm{x} 2$ & K3 & K4 & K5 & K6 & K7 \\
\hline $\mathbf{K}_{\mathbf{1}}$ & 1,0000 & -.8916 &, 8430 &,- 7982 & .8474 & -.0874 & .0434 \\
\hline $\mathrm{K} 2$ & -8916 & 1,0000 & -9641 &, 9208 &,- 9118 &,- 2071 &, 1588 \\
\hline K3 & .8430 &,- 9641 & 1,0000 & -9906 & .8093 & .2302 & -2105 \\
\hline K4 & -7982 & .9208 & - 9906 & 1.0000 & -.7454 & -.2317 & .2319 \\
\hline K5 & .8474 & -9118 & .8093 & -.7454 & 1.0000 & .0221 & -.0334 \\
\hline $\mathrm{x} 6$ &,- 0874 & -.2071 & .2302 & -.2317 & .0221 & 1,0000 &,- 8917 \\
\hline K7 & .0434 &, 1588 &,- 2105 &, 2319 & -.0334 & -.8917 & 1,0000 \\
\hline
\end{tabular}

Tabelle 45: Ergebnisse der Regressionsbildung für das Jahr 1997 


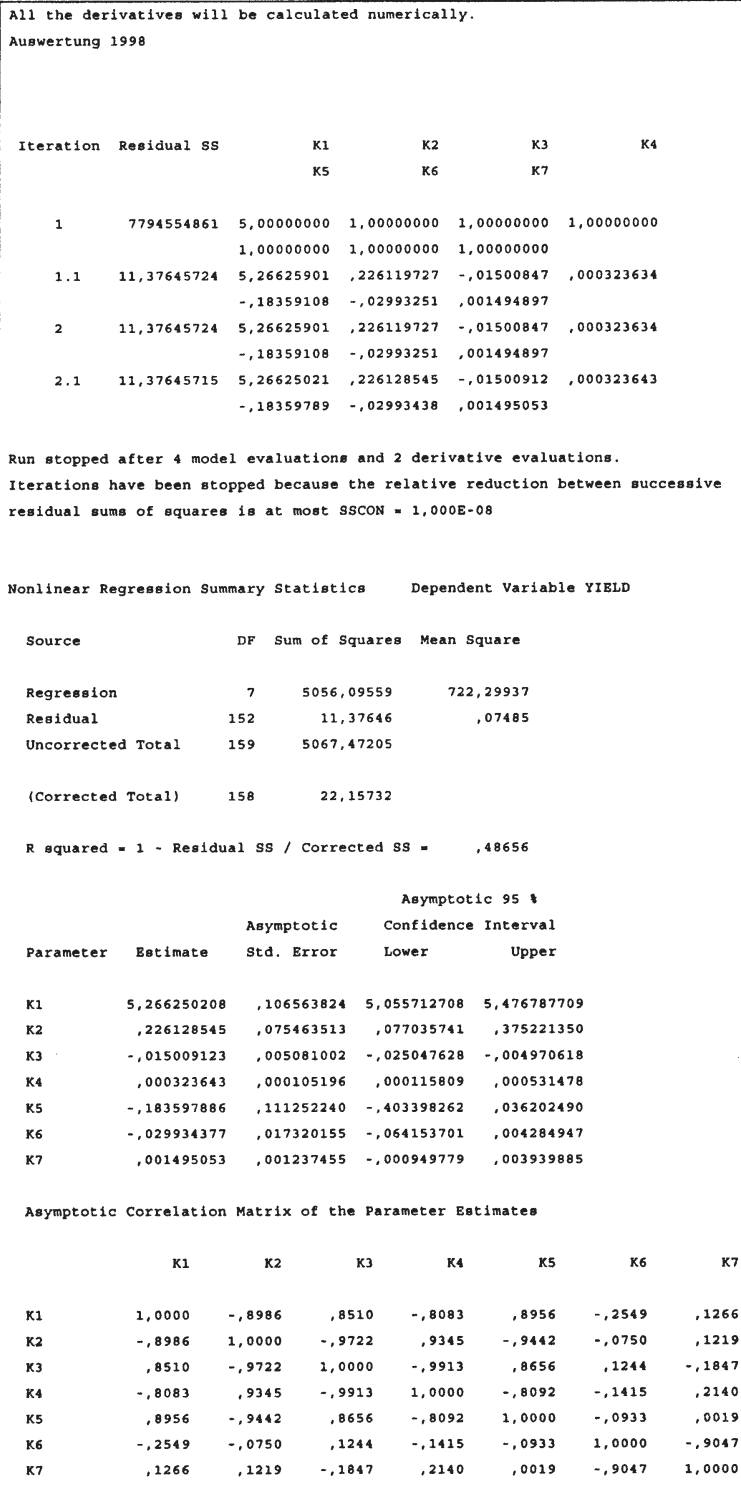

Tabelle 46: Ergebnisse der Regressionsbildung für das Jahr 1998 


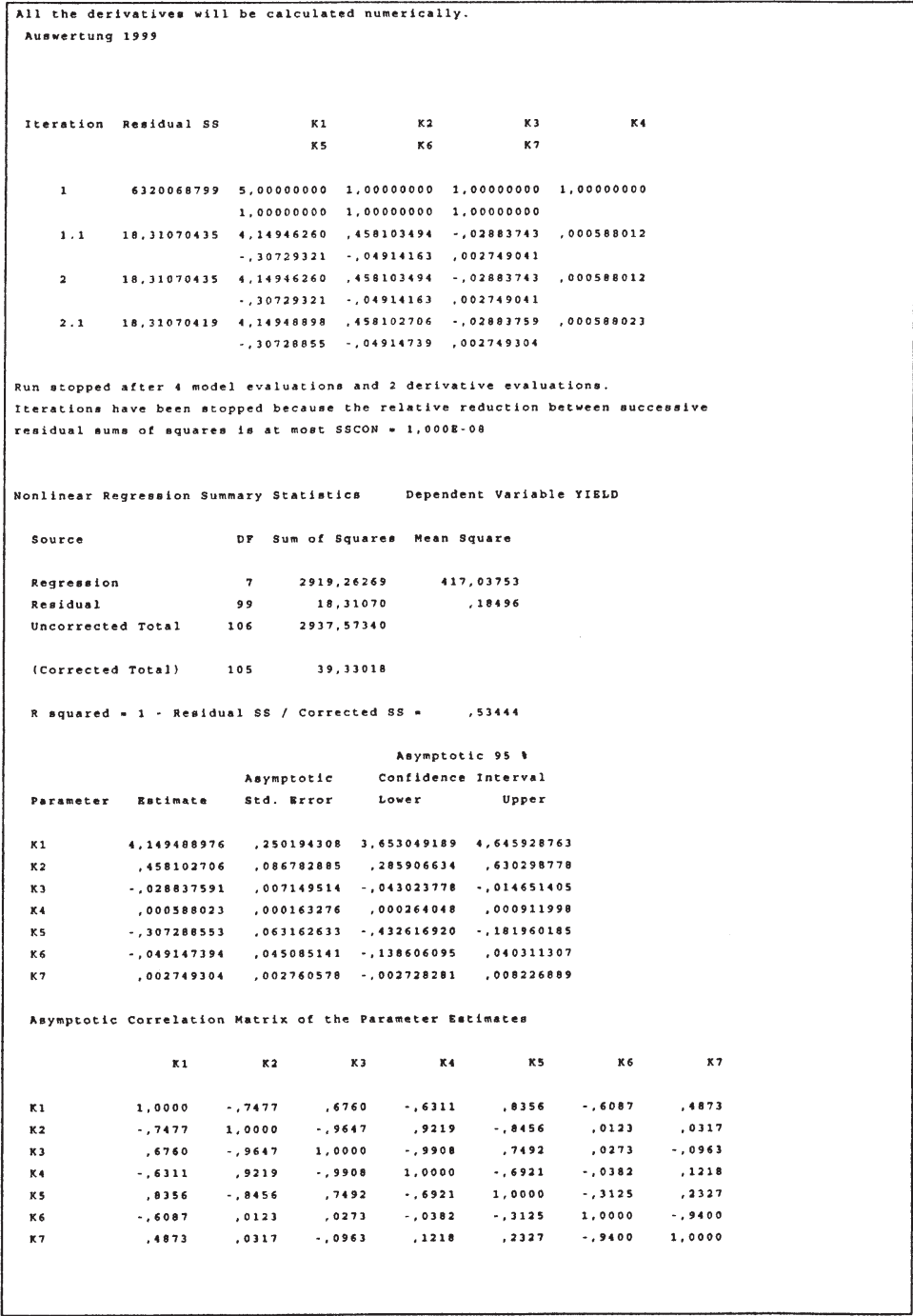

Tabelle 47: Ergebnisse der Regressionsbildung für das Jahr 1999 


\begin{tabular}{|c|c|c|}
\hline Lfd. Nummer & Unternehmen & Ticker \\
\hline 1 & ABBOTT LABORATORIES & ABT \\
\hline 2 & ANALOG DEVICES INC & $\mathrm{ADI}$ \\
\hline 3 & ATLANTIC RICHFIELD CO & ARC \\
\hline 4 & AVERY DENNISON CORP & AVY \\
\hline 5 & AVNET INC & AVT \\
\hline 6 & BF GOODRICH CO & $\mathrm{GR}$ \\
\hline 7 & BORG-WARNER AUTO INC & BWA \\
\hline 8 & CABOT CORP & CBT \\
\hline 9 & CARNIVAL CORP & $\mathrm{CCL}$ \\
\hline 10 & CARPENTER TECHNOLOGY CORP & CRS \\
\hline 11 & CBS INC & CBS \\
\hline 12 & CENTURY TELEPHONE ENTERPRISES INC & CTL \\
\hline 13 & COASTAL CORP & CGP \\
\hline 14 & COMDISCO INC & $\mathrm{CDO}$ \\
\hline 15 & DIAL CORP & $\mathrm{DL}$ \\
\hline 16 & DILLARD DEPARTMENT STORES INC & $\mathrm{DDS}$ \\
\hline 17 & DONNELLEY RR AND SONS CO & DNY \\
\hline 18 & DOW CHEMICAL CO & DOW \\
\hline 19 & DOW JONES \& COMPANY INC & DJ \\
\hline 20 & EASTMAN CHEMICAL CO & EMN \\
\hline 21 & EATON CORP & ETN \\
\hline 22 & EI DU PONT DE NEMOURS AND CO & DD \\
\hline 23 & ELI LILLY AND CO & LLY \\
\hline 24 & EMERSON ELECTRIC CO & EMR \\
\hline 25 & EQUIFAX INC & EFX \\
\hline 26 & FEDERAL-MOGUL CORP & FMO \\
\hline 27 & FERRO CORP & FOE \\
\hline 28 & FMC CORP & FMC \\
\hline 29 & FORD MOTOR CO & F \\
\hline 30 & FORT JAMES CORP & FJ \\
\hline 31 & FOSTER WHEELER CORP & FWC \\
\hline 32 & GANNETT CO INC & GCI \\
\hline 33 & GENERAL ELECTRIC CO & GE \\
\hline 34 & GENERAL MILLS INC & GIS \\
\hline 35 & GENERAL MOTORS CORP & $\mathrm{GM}$ \\
\hline 36 & GEON CO & GON \\
\hline 37 & GEORGIA-PACIFIC CORP & GP \\
\hline 38 & GILLETTE CO & G \\
\hline 39 & GLATFELTER PH CO & GLT \\
\hline 40 & GTE CORP & GTE \\
\hline 41 & GUIDANT CORP & GDT \\
\hline 42 & HALLIBURTON CO & HAL \\
\hline 43 & HANNA MA CO & MAH \\
\hline 44 & HARCOURT GENERAL INC & $\mathrm{H}$ \\
\hline 45 & HARRIS CORP & HRS \\
\hline 46 & HARSCO CORP & HSC \\
\hline 47 & HERCULES INC & HPC \\
\hline 48 & HERSHEY FOODS CORP & HSY \\
\hline 49 & HILTON HOTELS CORP & HLT \\
\hline 50 & HJ HEINZ CO & $\mathrm{HNZ}$ \\
\hline 51 & HONEYWELL INC & $\mathrm{HON}$ \\
\hline 52 & HOUGHTON MIFFLIN CO & HTN \\
\hline 53 & IBP INC & IBP \\
\hline 54 & ILLINOIS TOOL WORKS INC & ITW \\
\hline 55 & INGERSOLL-RAND CO & IR \\
\hline 56 & INTERNATIONAL BUSINESS MACHINES CORP (IBM) & IBM \\
\hline 57 & INTERNATIONAL MULTIFOODS CORP & IMC \\
\hline 58 & INTERNATIONAL PAPER CO & IP \\
\hline
\end{tabular}




\begin{tabular}{|c|c|c|}
\hline Lfd. Nummer & Unternehmen & Ticker \\
\hline 59 & JC PENNEY COMPANY INC & $\mathrm{JCP}$ \\
\hline 60 & JOHNSON \& JOHNSON INC & JNJ \\
\hline 61 & KANSAS CITY SOUTHERN INDUSTRIES INC & KSU \\
\hline 62 & KAUFMAN \& BROAD HOME CORP & KBH \\
\hline 63 & KELLOGG CO & $\mathbf{K}$ \\
\hline 64 & KIMBERLY-CLARK CORP & $\mathrm{KMB}$ \\
\hline 65 & KINARK CORP & KIN \\
\hline 66 & KNIGHT-RIDDER INC & KRI \\
\hline 67 & LAFARGE CORP & LAF \\
\hline 68 & LITTON INDUSTRIES INC & LIT \\
\hline 69 & LORAL CORP & LOR \\
\hline 70 & LYONDELL PETROCHEMICAL CO & LYO \\
\hline 71 & MAGNETEK INC & MAG \\
\hline 72 & MALLINCKRODT INC & MKG \\
\hline 73 & MARRIOTT INTERNATIONAL INC & MAR \\
\hline 74 & MASCO CORP & MAS \\
\hline 75 & MCDONALD'S CORP & $\mathrm{MCD}$ \\
\hline 76 & MCGRAW-HILL INC & MHP \\
\hline 77 & MEAD CORP & MEA \\
\hline 78 & MERCK \& CO INC & MRK \\
\hline 79 & MILLIPORE CORP & MIL \\
\hline 80 & MONSANTO CO & MTC \\
\hline 81 & MOTOROLA INC & MOT \\
\hline 82 & NEW YORK TELEPHONE CO & NYT \\
\hline 83 & NEWELL CO & NWL \\
\hline 84 & NORFOLK SOUTHERN CORP & NSC \\
\hline 85 & NORTHROP GRUMMAN CORP & NOC \\
\hline 86 & OLIN CORP & OLN \\
\hline 87 & PARKER HANNIFIN CORP & $\mathrm{PH}$ \\
\hline 88 & PEP BOYS-MANNY MOE \& JACK INC & PBY \\
\hline 89 & PEPSICO INC & PEP \\
\hline 90 & PHELPS DODGE CORP & PD \\
\hline 91 & PHILIP MORRIS COMPANIES INC & MO \\
\hline 92 & POTLATCH CORP & $\mathrm{PCH}$ \\
\hline 93 & PPG INDUSTRIES INC & PPG \\
\hline 94 & PRAXAIR INC & PX \\
\hline 95 & PROCTER \& GAMBLE CO, THE & PG \\
\hline 96 & PULTE CORP & PHM \\
\hline 97 & QUAKER OATS CO & OAT \\
\hline 98 & RALSTON PURINA CO & RAL \\
\hline 99 & RAYONIER INC & RYN \\
\hline 100 & REYNOLDS METALS CO & RLM \\
\hline 101 & ROCKWELL INTERNATIONAL CORP & ROK \\
\hline 102 & ROHM \& HAAS CO & $\mathrm{ROH}$ \\
\hline 103 & SARA LEE CORP & SLE \\
\hline 104 & SEARS ROEBUCK AND CO & $\mathbf{S}$ \\
\hline 105 & SHERWIN-WILLIAMS CO & SHW \\
\hline 106 & SOLUTIA INC & SOI \\
\hline 107 & SOUTHWEST AIRLINES INC & LUV \\
\hline 108 & TEMPLE-INLAND INC & TIN \\
\hline 109 & TEXAS INSTRUMENTS INC & $\mathrm{TXN}$ \\
\hline 110 & THOMAS \& BETTS CORP & TNB \\
\hline 111 & TIME WARNER INC & TWX \\
\hline 112 & TIMES MIRROR CO & TMC \\
\hline 113 & TIMKEN CO & TKR \\
\hline 114 & TRIBUNE CO & TRB \\
\hline 115 & TRW INC & TRW \\
\hline 116 & UNION PACIFIC CORP & UNP \\
\hline
\end{tabular}




\begin{tabular}{|c|c|c|}
\hline Lfd. Nummer & Unternehmen & Ticker \\
\hline 117 & UNISYS CORP & UIS \\
\hline 118 & UNITED TECHNOLOGIES CORP & UTX \\
\hline 119 & UNIVERSAL CORP & UFC \\
\hline 120 & UNIVERSAL FOODS CORP & UH \\
\hline 121 & US HOME CORP & U \\
\hline 122 & USAIR GROUP INC & USG \\
\hline 123 & USG CORP & VFC \\
\hline 124 & VF CORP & VIA \\
\hline 125 & VIACOM INC & VMC \\
\hline 126 & VULCAN MATERIALS CO & WMT \\
\hline 127 & WAL-MART STORES INC & DIS \\
\hline 128 & WALT DISNEY CO, THE & WLA \\
\hline 129 & WARNER-LAMBERT CO & WS \\
\hline 130 & WEIRTON STEEL CORP & WEN \\
\hline 131 & WENDY'S INTERNATIONAL INC & W \\
\hline 132 & WESTVACO CORP & WY \\
\hline 133 & WEYERHAEUSER CO & WH \\
\hline 134 & WHITMAN CORP & GRA \\
\hline 135 & WR GRACE AND CO & XRX \\
\hline 136 & XEROX CORP & \\
\hline
\end{tabular}

Tabelle 48: Untersuchte Menge an Unternehmen mit dem zugehorigen Tickersymbol

\begin{tabular}{|c|c|c|c|c|c|c|c|}
\hline Lfd. Nr. & Ticker & CUSIP & Ask Kurs in Dollar & ISMA Rendite in \% & Kupon in \% & Falligkeit & Restlaufzeit [Jahren] \\
\hline 1 & ABT & $002824 \mathrm{AH} 3$ & 93,36 & 7,38 & 6,40 & 01.12 .06 & 9,77 \\
\hline 2 & $\mathrm{ADI}$ & 032654AA3 & 98,66 & 7,15 & 6,63 & 01.03 .00 & 2,92 \\
\hline 3 & ARC & $048825 \mathrm{AN} 3$ & 121,66 & 7,32 & 10,88 & 15.07 .05 & 8,37 \\
\hline 4 & ARC & $048825 \mathrm{AU} 7$ & 124,00 & 7,48 & 9,88 & 01.03 .16 & 19,15 \\
\hline 5 & ARC & 048825AV5 & 111,02 & 7,89 & 9,13 & 01.03 .11 & 16,10 \\
\hline 6 & ARC & 048825AV5 & 116,43 & 7,29 & 9,13 & 01.03 .11 & 15,09 \\
\hline 7 & ARC & 048825AV5 & 113,25 & 7,56 & 9,13 & 01.03 .11 & 14,08 \\
\hline 8 & ARC & 048825AV5 & 120,75 & 6,69 & 9,13 & 01.03 .11 & 13,06 \\
\hline 9 & ARC & $048825 \mathrm{AW} 3$ & 113,22 & 7,77 & 9,00 & 01.04 .21 & 24,31 \\
\hline 10 & ARC & 048825AY9 & 111,29 & 8,03 & 9,00 & 01.05 .31 & 34,54 \\
\hline 11 & ARC & $048825 \mathrm{AZ6}$ & 112,76 & 8,03 & 9,13 & 01.08 .31 & 34,79 \\
\hline 12 & ARC & $048825 \mathrm{BA} 0$ & 102,68 & 8,00 & 8,25 & 01.02 .22 & 25,16 \\
\hline 13 & ARC & 048825BB8 & 108,40 & 8,03 & 8,75 & 01.03 .32 & 35,39 \\
\hline 14 & ARC & $048825 \mathrm{BC} 6$ & 105,19 & 7,94 & 8,50 & 01.04 .12 & 17,20 \\
\hline 15 & ARC & $048825 \mathrm{BC} 6$ & 110,79 & 7,34 & 8,50 & 01.04 .12 & 16,19 \\
\hline 16 & ARC & 048825BC6 & 107,68 & 7,63 & 8,50 & 01.04 .12 & 15,18 \\
\hline 17 & ARC & 04882PAY5 & 108,14 & 4,80 & 8,54 & 15.08 .01 & 2,37 \\
\hline 18 & ARC & 04882PAZ2 & 108,20 & 4,80 & 8,55 & 20.08 .01 & 2,39 \\
\hline 19 & ARC & 04882PBB4 & 107,93 & 4,80 & 8,42 & 23.08 .01 & 2,39 \\
\hline 20 & ARC & 04882PBY4 & 119,49 & 6,21 & 8,44 & 21.02 .12 & 13,04 \\
\hline 21 & ARC & 04882PCAS & 118,92 & 6,21 & 8,38 & 21.02 .12 & 13,04 \\
\hline 22 & ARC & $04882 \mathrm{PCCl}$ & 119,59 & 6,21 & 8,45 & 22.02 .12 & 13,05 \\
\hline 23 & ARC & 04882PCE7 & 120,29 & 6,21 & 8,53 & 21.02 .12 & 13,04 \\
\hline 24 & ARC & 04882PCH0 & 120,49 & 6,21 & 8,55 & 01.03 .12 & 13,07 \\
\hline 25 & ARC & $04882 \mathrm{PCJ} 6$ & 121,14 & 6,21 & 8,62 & 12.03 .12 & 13,10 \\
\hline 26 & ARC & $04882 \mathrm{PCLI}$ & 121,09 & 6,22 & 8,60 & 15.05 .12 & 13,28 \\
\hline 27 & ARC & 04882PCN7 & 108,55 & 5,02 & 8,18 & 01.04 .02 & 3,01 \\
\hline 28 & ARC & 04882PCN9 & 108,55 & 5,02 & 8,18 & 01.04 .02 & 3,01 \\
\hline 29 & AVY & 05361HAY0 & 103,61 & 5,45 & 6,47 & 21.04 .03 & 4,08 \\
\hline 30 & AVY & $05361 \mathrm{HBB} 9$ & 103,71 & 5,46 & 6,50 & 22.04 .03 & 4,08 \\
\hline 31 & AVY & 05361HBDS & 103,05 & 5,75 & 6,50 & 15.01 .04 & 4,83 \\
\hline 32 & AVY & 05361HBG8 & 97,38 & 6,97 & 6,50 & 15.05 .03 & 7,18 \\
\hline 33 & AVY & $05361 \mathrm{HBW} 3$ & 99,12 & 7,68 & 7,55 & 10.06 .04 & 9,28 \\
\hline
\end{tabular}




\begin{tabular}{|c|c|c|c|c|c|c|c|}
\hline Lfd. Nr. & Ticker & CUSIP & Ask Kurs in Dollar & ISMA Rendite in \% & Kupon in \% & Falligkeit & Restlaufzeit [Jahren] \\
\hline 34 & AVY & 05361HBW3 & 103,12 & 7,04 & 7,55 & 10.06 .04 & 8,27 \\
\hline 35 & AVY & 05361 HCE2 & 97,65 & 7,76 & 7,56 & 23.05 .25 & 29,53 \\
\hline 36 & AVT & $053807 \mathrm{AC7}$ & 97,36 & 7,37 & 6,88 & 15.03 .04 & 7,02 \\
\hline 37 & BWA & 099724AA4 & 94,91 & 7,76 & 7,00 & 01.11 .06 & 9,69 \\
\hline 38 & PX & $124800 \mathrm{AC7}$ & 96,11 & 7,45 & 6,63 & 15.03 .03 & 6,00 \\
\hline 39 & PX & $124800 \mathrm{ADS}$ & 97,47 & 7,14 & 6,25 & 30.06 .00 & 3,26 \\
\hline 40 & CBS & 124845AD0 & 98,69 & 7,96 & 7,63 & 01.01 .02 & 4,78 \\
\hline 41 & CBT & 12705QAF2 & 112,04 & 6,83 & 8,22 & 06.08 .12 & 13,51 \\
\hline 42 & CBT & 12705QAL9 & 112,48 & 6,83 & 8,27 & 14.08 .12 & 13,53 \\
\hline 43 & $\mathrm{CCL}$ & $14365 \mathrm{HAAO}$ & 100,35 & 6,81 & 7,00 & 05.05 .99 & 2,08 \\
\hline 44 & CRS & 14428TAA9 & 101,06 & 6,92 & 7,43 & 16.08 .99 & 2,37 \\
\hline 45 & CRS & 14428TAB7 & 101,08 & 6.92 & 7,44 & 16.08 .99 & 2,37 \\
\hline 46 & CRS & 14428TAEI & 102,31 & 7,18 & 7,80 & 19.09 .01 & 4,49 \\
\hline 47 & CRS & 14428TAF8 & 102,28 & 7.18 & 7,79 & 19.09 .01 & 4,49 \\
\hline 48 & CRS & 14428TBG5 & 100,06 & 7,02 & 7,03 & 22.05 .18 & 19,38 \\
\hline 49 & CTL & $156686 \mathrm{AF} 4$ & 100,90 & 7,58 & 7,75 & 01.05 .04 & 7,15 \\
\hline 50 & CTL & $156686 \mathrm{AG2}$ & 92,86 & 7,70 & 6,55 & 01.12 .05 & 8,76 \\
\hline 51 & CGP & $190441 \mathrm{AH} 8$ & 115,16 & 7,56 & 10,25 & 15.10 .04 & 7,61 \\
\hline 52 & CGP & 190441 AJ4 & 109,46 & 7,23 & 10,38 & 01.10 .00 & 3,51 \\
\hline 53 & CGP & $190441 \mathrm{AKI}$ & 123,78 & 7,85 & 10,75 & 01.10 .10 & 13,66 \\
\hline 54 & CGP & 190441AL9 & 108,89 & 7,27 & 10,00 & 01.02 .01 & 3,86 \\
\hline 55 & CGP & 190441AM7 & 111,05 & 7,51 & 9,75 & 01.08 .03 & 6,39 \\
\hline 56 & CGP & 190441 AN5 & 103,33 & 6,99 & 8,75 & 15.05 .99 & 2,11 \\
\hline 57 & CGP & $190441 \mathrm{APO}$ & 114,85 & 7,92 & 9,63 & 15.05 .12 & 15,30 \\
\hline 58 & CGP & $190441 \mathrm{AQ} 8$ & 102,92 & 7.46 & 8,13 & 15.09 .02 & 5,50 \\
\hline 59 & $\mathrm{CDO}$ & 20033RDH9 & 100,02 & 6,02 & 6,05 & 31.10 .97 & 0,55 \\
\hline 60 & $\mathrm{DL}$ & 25247QAM4 & 94,66 & 7,09 & 5,90 & 25.10 .01 & 5,61 \\
\hline 61 & DDS & 254063AJ9 & 105,46 & 7,10 & 9,63 & 15.09 .97 & 2,44 \\
\hline 62 & DDS & 254063AJ9 & 104,65 & 6,12 & 9,63 & 15.09 .97 & 1,44 \\
\hline 63 & DIS & 25468PAQ9 & 100,23 & 5,26 & 5.60 & 13.01 .00 & 0,76 \\
\hline 64 & DIS & 25468PAU0 & 100,30 & 5,28 & 5,60 & 17.04 .00 & 1,03 \\
\hline 65 & DIS & 25468PAV8 & 100,59 & 5.23 & 5,83 & 05.05 .00 & 1,08 \\
\hline 66 & DIS & $25468 \mathrm{PAX} 4$ & 98,23 & 5.69 & 5,25 & 10.11 .03 & 4,64 \\
\hline 67 & DIS & 25468PAZ9 & 98,11 & 5,72 & 5,25 & 01.12 .03 & 4,70 \\
\hline 68 & DIS & $25468 \mathrm{PBB} 1$ & 100,68 & 4,74 & 5,14 & 08.02 .01 & 1,85 \\
\hline 69 & DIS & 25468PBC9 & 107,14 & 5,19 & 13,50 & 10.03 .00 & 0,92 \\
\hline 70 & DIS & $25469 \mathrm{HBD} 4$ & 87,53 & 7,34 & 5,80 & 27.10 .08 & 12,71 \\
\hline 71 & DIS & 25469HBD4 & 86,14 & 7,63 & 5,80 & 27.10 .08 & 11,70 \\
\hline 72 & DNY & $257867 \mathrm{AB} 7$ & 108,30 & 7,29 & 9,13 & 01.12 .00 & 5,70 \\
\hline 73 & $\mathrm{DNY}$ & $257867 \mathrm{AB} 7$ & 109,38 & 6,72 & 9,13 & 01.12 .00 & 4,69 \\
\hline 74 & DOW & 260543ATO & 106,71 & 7,70 & 8,63 & 01.04 .06 & 11,11 \\
\hline 75 & DOW & 260543AT0 & 109,64 & 7,25 & 8,63 & 01.04 .06 & 10,10 \\
\hline 76 & DOW & 260543BC6 & 108,02 & 8,11 & 8,85 & 15.09 .21 & 26,79 \\
\hline 77 & DOW & $260543 \mathrm{BC} 6$ & 111,76 & 7,78 & 8,85 & 15.09 .21 & 25,79 \\
\hline 78 & DOW & 260543BE2 & 91,70 & 8,13 & 7,38 & 01.03 .23 & 28,27 \\
\hline 79 & Dow & 260543BE2 & 94,78 & 7,84 & 7,38 & 01.03 .23 & 27,26 \\
\hline 80 & DOW & 260543BF9 & 88,00 & 8,12 & 6,85 & 15.08 .13 & 18,59 \\
\hline 81 & DOW & 260543BF9 & 93,13 & 7,57 & 6,85 & 15.08 .13 & 17,58 \\
\hline 82 & DOW & 26054EAD1 & 103,11 & 5,24 & 9,04 & 25.02 .00 & 0,88 \\
\hline 83 & DOW & 26054EAN9 & 117,43 & 5,86 & 9,35 & 20.04 .05 & 6,11 \\
\hline 84 & DOW & 26054EAR0 & 116,96 & 5,88 & 9,20 & 21.06 .05 & 6,28 \\
\hline 85 & DOW & 26054EAT6 & 96,09 & 8,11 & 7,75 & 15.09 .20 & 25,78 \\
\hline 86 & DOW & 26054EAT6 & 99,73 & 7,77 & 7,75 & 15.09 .20 & 24,77 \\
\hline 87 & DJ & $260561 \mathrm{AD} 7$ & 96,39 & 6,67 & 5,75 & 01.12 .00 & 4,69 \\
\hline 88 & $\mathrm{DD}$ & 263534AN9 & 102,27 & 6,80 & 8,45 & 15.10 .96 & 1,51 \\
\hline 89 & $\mathrm{DD}$ & 263534AN9 & 101,35 & 5,62 & 8,45 & 15.10 .96 & 0,51 \\
\hline 90 & $\mathrm{DD}$ & 263534 AR0 & 107,87 & 7,24 & 9,15 & 15.04 .00 & 5,06 \\
\hline 91 & $\mathrm{DD}$ & 263534 AR0 & 108,90 & 6,58 & 9,15 & 15.04 .00 & 4,06 \\
\hline
\end{tabular}




\begin{tabular}{|c|c|c|c|c|c|c|c|}
\hline Lfd. Nr. & Ticker & CUSIP & Ask Kurs in Dollar & ISMA Rendite in \% & Kupon in \% & Falligkeit & Restlaufzeit [Jahren] \\
\hline 92 & DD & 263534AS8 & 103,84 & 7,01 & 8,65 & 01.12 .97 & 2,66 \\
\hline 93 & $\mathrm{DD}$ & 263534AS8 & 103,72 & 6,19 & 8,65 & 01.12 .97 & 1,65 \\
\hline 94 & $\mathrm{DD}$ & 263534 AW9 & 104,65 & 7,63 & 8,25 & 15.09 .06 & 11,58 \\
\hline 95 & DD & 263534AW9 & 108,93 & 7,02 & 8,25 & 15.09 .06 & 10,57 \\
\hline 96 & DD & 26353LDJ7 & 102,14 & 5,07 & 9,00 & 10.11 .99 & 0,58 \\
\hline 97 & DD & 26353LDQ1 & 106,31 & 4,81 & 8,98 & 21.11 .00 & 1,63 \\
\hline 98 & DD & 26353LED9 & 106,44 & 4,78 & 8,93 & 06.12 .00 & 1,67 \\
\hline 99 & $\mathrm{DD}$ & 26353LEN7 & 103,76 & 5,09 & 8,60 & 01.06 .00 & 1,15 \\
\hline 100 & $\mathrm{DD}$ & 26353LER8 & 106,89 & 4,70 & 8,80 & 25.01 .01 & 1,81 \\
\hline 101 & DD & 26353LEXS & 106,87 & 4,68 & 8,70 & 07.02 .01 & 1,85 \\
\hline 102 & EMN & 277432AA8 & 90,08 & 7,97 & 6,38 & 15.01 .04 & 8,87 \\
\hline 103 & EMN & 277432AA8 & 95,99 & 7,05 & 6,38 & 15.01 .04 & 7,86 \\
\hline 104 & EMN & 277432AB6 & 83,29 & 8,86 & 7,25 & 15.01 .24 & 29,16 \\
\hline 105 & EMN & $277432 \mathrm{AB} 6$ & 93,55 & 7,82 & 7,25 & 15.01 .24 & 28,15 \\
\hline 106 & ETN & 278058AL6 & 108,12 & 7,80 & 8,90 & 15.08 .06 & 11,49 \\
\hline 107 & ETN & 278058AL6 & 112,40 & 7,18 & 8,90 & 15.08 .06 & 10,48 \\
\hline 108 & ETN & 278058AM4 & 104,47 & 7,70 & 8,10 & 15.08 .22 & 26,71 \\
\hline 109 & ETN & 278058AN2 & 97,06 & 7,24 & 6,38 & 01.04 .99 & 4,01 \\
\hline 110 & ETN & 278058AN2 & 99,51 & 6,56 & 6,38 & 01.04 .99 & 3,00 \\
\hline 111 & ETN & 278058AP7 & 93,78 & 8,19 & 7,63 & 01.04 .24 & 29,38 \\
\hline 112 & ETN & 278058AP7 & 98,66 & 7,74 & 7,63 & 01.04 .24 & 28,37 \\
\hline 113 & \begin{tabular}{|l|} 
EMR \\
\end{tabular} & 291011 AE4 & 95,27 & 6,99 & 6,30 & 01.11 .05 & 9,68 \\
\hline 114 & EMR & 291011AF1 & 97,32 & 5,87 & 5,50 & 15.09 .08 & 9,56 \\
\hline 115 & EMR & 291011AG9 & 93,65 & 5,88 & 5,00 & 15.10 .08 & 9,64 \\
\hline 116 & EMR & 291011 AH7 & 99,71 & 5,89 & 5,85 & 15.03 .09 & 10,06 \\
\hline 117 & EFX & 294429AA3 & 92,83 & 7,70 & 6,50 & 15.06 .03 & 8,28 \\
\hline 118 & EFX & $294429 \mathrm{AA3}$ & 96,87 & 7,06 & 6,50 & 15.06 .03 & 7,27 \\
\hline 119 & FMC & 302491AJ4 & 104,71 & 7,35 & 8,75 & 01.04 .99 & 4,01 \\
\hline 120 & FMC & $302491 \mathrm{AJ} 4$ & 105,33 & 6,72 & 8,75 & 01.04 .99 & 3,00 \\
\hline 121 & FMC & 302491AK1 & 90,62 & 7,93 & 6,38 & 01.09 .03 & 8,49 \\
\hline 122 & FMC & $302491 \mathrm{AK} 1$ & 95,28 & 7,21 & 6,38 & 01.09 .03 & 7,48 \\
\hline 123 & FMC & 30249QAD2 & 100,95 & 6,56 & 6,75 & 05.05 .05 & 6,15 \\
\hline 124 & FMC & 30249QAE0 & 101,26 & 6,81 & 7,00 & 15.05 .08 & 9,22 \\
\hline 125 & FMC & 30249QAF7 & 103,74 & 5,95 & 7,13 & 25.11 .02 & 3,67 \\
\hline 126 & FMC & 30249QAG5 & 102,35 & 5,32 & 6,60 & 30.03 .01 & 1,99 \\
\hline 127 & FMC & $30249 \mathrm{QAH} 3$ & 100,51 & 6,40 & 6,53 & 21.12 .03 & 4,76 \\
\hline 128 & FMO & 313906AA7 & 101,48 & 8,03 & 8,33 & 15.11 .01 & 6,67 \\
\hline 129 & FMO & 313906AA7 & 104,83 & 7,26 & 8,33 & 15.11 .01 & 5,66 \\
\hline 130 & FMO & $313906 \mathrm{ABS}$ & 101,14 & 7,75 & 8,06 & 20.10 .99 & 4,57 \\
\hline 131 & FMO & 313906AB5 & 103,29 & 6,98 & 8,06 & 20.10 .99 & 3,56 \\
\hline 132 & FMO & $313906 \mathrm{AC} 3$ & 101,80 & 8,13 & 8,46 & 26.10 .02 & 7,63 \\
\hline 133 & FMO & $313906 \mathrm{AC} 3$ & 105,78 & 7,33 & 8,46 & 26.10 .02 & 6,62 \\
\hline 134 & FMO & 313906AN9 & 99,13 & 8,38 & 8,25 & 03.03 .05 & 10,02 \\
\hline 135 & FMO & 313906AN9 & 104,73 & 7,51 & 8,25 & 03.03 .05 & 9,01 \\
\hline 136 & FMO & 313906AN9 & 104,73 & 7,51 & 8,25 & 03.03 .05 & 9,01 \\
\hline 137 & FMO & 313906AP4 & 99,93 & 8,18 & 8,16 & 06.03 .03 & 7,99 \\
\hline 138 & FMO & 313906AP4 & 104,30 & 7,35 & 8,16 & 06.03 .03 & 6,99 \\
\hline 139 & FMO & $313906 \mathrm{AQ2}$ & 99,62 & 8,17 & 8,12 & 06.03 .03 & 7,99 \\
\hline 140 & FMO & $313906 \mathrm{AQ} 2$ & 104,08 & 7,35 & 8,12 & 06.03 .03 & 6,99 \\
\hline 141 & FOE & 315405AE0 & 91,36 & 8,58 & 7,63 & 01.05 .13 & 18,30 \\
\hline 142 & FOE & 315405AE0 & 101,48 & 7,47 & 7,63 & 01.05 .13 & 17,29 \\
\hline 143 & FOE & 315405AF7 & 98,22 & 7,55 & 7,38 & 01.11 .15 & 19,83 \\
\hline 144 & $\mathbf{F}$ & 345370AP5 & 107,89 & 7,79 & 8,88 & 01.04 .06 & 11,11 \\
\hline 145 & $F$ & $345370 \mathrm{APS}$ & 112,22 & 7,14 & 8,88 & 01.04 .06 & 10,10 \\
\hline 146 & $\mathbf{F}$ & 345370 AY6 & 107,52 & 7,50 & 9,00 & 15.09 .01 & 6,50 \\
\hline 147 & $\mathbf{F}$ & $345370 \mathrm{AY} 6$ & 109,65 & 6,83 & 9,00 & 15.09 .01 & 5,49 \\
\hline 148 & $F$ & $345370 \mathrm{AZ3}$ & 113,47 & 8,01 & 9,50 & 15.09 .11 & 16,65 \\
\hline 149 & $\mathbf{F}$ & $345370 \mathrm{AZ3}$ & 119,19 & 7,39 & 9,50 & 15.09 .11 & 15,64 \\
\hline
\end{tabular}




\begin{tabular}{|c|c|c|c|c|c|c|c|}
\hline Lfd. Nr. & Ticker & CUSIP & Ask Kurs in Dollar & ISMA Rendite in \% & Kupon in \% & Falligkeit & Restlaufzeit [Jahren] \\
\hline 150 & $\mathbf{F}$ & 345370BA7 & 101,40 & 6,86 & 7,88 & 15.10 .96 & 1,51 \\
\hline 151 & $\mathbf{F}$ & $345370 \mathrm{BA} 7$ & 101,07 & 5,63 & 7,88 & 15.10 .96 & 0,51 \\
\hline 152 & $\mathbf{F}$ & $345370 \mathrm{BJ} 8$ & 107,55 & 8,17 & 8,88 & 15.01 .22 & 27,13 \\
\hline 153 & $F$ & $345370 \mathrm{BJ} 8$ & 113,29 & 7,68 & 8,88 & 15.01 .22 & 26,13 \\
\hline 154 & $\mathbf{F}$ & 345370BK5 & 100,67 & 7,32 & 7,50 & 15.11 .99 & 4,64 \\
\hline 155 & $F$ & 345370BK5 & 102,81 & 6,60 & 7,50 & 15.11 .99 & 3,63 \\
\hline 156 & $F$ & $345370 \mathrm{BN} 9$ & 92,28 & 7,80 & 7,13 & 15.11 .25 & 30,01 \\
\hline 157 & $F$ & 345370 BT6 & 99,70 & 6,65 & 6,63 & 15.02 .28 & 30,27 \\
\hline 158 & $F$ & $345370 \mathrm{BT} 6$ & 99,25 & 6,68 & 6,63 & 15.02 .28 & 29,26 \\
\hline 159 & $F$ & $345370 \mathrm{BX7}$ & 100,90 & 6,42 & 6,50 & 01.08 .18 & 19,58 \\
\hline 160 & FJ & 347471AS3 & 101,24 & 6,17 & 6,50 & 15.09 .02 & 4,48 \\
\hline 161 & FJ & $347471 \mathrm{AS} 3$ & 101,89 & 5,88 & 6,50 & 15.09 .02 & 3,47 \\
\hline 162 & FWC & 350244AA7 & 96,87 & 7,21 & 6,75 & 15.11 .05 & 9,72 \\
\hline 163 & GTE & $362320 \mathrm{AQ} 6$ & 110,38 & 6,72 & 9,38 & 01.12 .00 & 4,69 \\
\hline 164 & GTE & 362320AR4 & 104,13 & 7,21 & 8,85 & 01.03 .98 & 2,91 \\
\hline 165 & GTE & 362320AR4 & 104,46 & 6,27 & 8,85 & 01.03 .98 & 1,90 \\
\hline 166 & GTE & $362320 \mathrm{AS2}$ & 107,48 & 7,83 & 9,10 & 01.06 .03 & 8,24 \\
\hline 167 & GTE & 362320 AS2 & 112,03 & 6,93 & 9,10 & 01.06 .03 & 7,23 \\
\hline 168 & GTE & 362320AT0 & 99,35 & 8,81 & 8,75 & 01.11 .21 & 26,93 \\
\hline 169 & GTE & $362320 \mathrm{ATO}$ & 112,31 & 7,65 & 8,75 & 01.11 .21 & 25,92 \\
\hline 170 & GTE & $362320 \mathrm{AXI}$ & 99,29 & 6,49 & 6,36 & 15.04 .06 & 7,11 \\
\hline 171 & GTE & $362320 \mathrm{AY9}$ & 98,67 & 6,66 & 6,46 & 15.04 .08 & 9,14 \\
\hline 172 & GTE & $362320 \mathrm{AZ6}$ & 98,56 & 6,98 & 6.84 & 15.04 .18 & 19,28 \\
\hline 173 & GTE & $362320 \mathrm{BAO}$ & 96,33 & 7,24 & 6,94 & 15.04 .28 & 29,43 \\
\hline 174 & GTE & $36232 \mathrm{HACO}$ & 100,72 & 6,46 & 6,60 & 22.09 .05 & 6,54 \\
\hline 175 & $\mathrm{GCI}$ & $364730 \mathrm{AF} 8$ & 95,47 & 7,03 & 5,25 & 01.03 .98 & 2,91 \\
\hline 176 & $\mathrm{GCl}$ & 364730AF8 & 98,11 & 6,33 & 5,25 & 01.03 .98 & 1,90 \\
\hline 177 & $\mathrm{GCl}$ & 364730AG6 & 94,19 & 7,25 & 5,85 & 01.05 .00 & 5,11 \\
\hline 178 & $\mathrm{GCl}$ & 364730AG6 & 97,75 & 6,49 & 5,85 & 01.05 .00 & 4,10 \\
\hline 179 & GCI & 364730 AG6 & 99,25 & 6,25 & 5,85 & 01.05 .00 & 2,08 \\
\hline 180 & GE & 369604AT0 & 101,26 & 6,57 & 7,88 & 01.05 .96 & 1,05 \\
\hline 181 & GE & 369604AT0 & 100,08 & 5,24 & 7,88 & 01.05 .96 & 0,04 \\
\hline 182 & GE & 369604AVS & 102,67 & 6,97 & 7,88 & 15.09 .98 & 3,46 \\
\hline 183 & GE & 369604AVS & 103,68 & 6,20 & 7,88 & 15.09 .98 & 2,45 \\
\hline 184 & GIS & 37033LAA4 & 100,91 & 4,69 & 9,00 & 06.07 .99 & 0,23 \\
\hline 185 & GIS & 37033LAC0 & 101,00 & 4,75 & 8,85 & 19.07 .99 & 0,27 \\
\hline 186 & GIS & 37033LAS5 & 106,59 & 4,81 & 9,00 & 15.12 .00 & 1,70 \\
\hline 187 & GIS & 37033LAU0 & 102,41 & 5,17 & 8.95 & 15.12 .99 & 0,68 \\
\hline 188 & GIS & 37033LBP0 & 117,43 & 5,93 & 9,14 & 15.12 .05 & 6,77 \\
\hline 189 & GIS & $37033 \mathrm{LBQ} 8$ & 106,67 & 4,81 & 9,05 & 15.12 .00 & 1,70 \\
\hline 190 & GIS & 37033 LBR6 & 106,34 & 4,81 & 8,82 & 18.12 .00 & 1,71 \\
\hline 191 & GIS & 37033LBS4 & 106,55 & 4,81 & 8,95 & 18.12 .00 & 1,71 \\
\hline 192 & GIS & 37033 LBT2 & 112,18 & 5,30 & 9,00 & 20.12 .02 & 3,74 \\
\hline 193 & GIS & 37033LBU9 & 109,99 & 4,96 & 8,85 & 01.02 .02 & 2,84 \\
\hline 194 & GIS & 37033 LBV7 & 116,43 & 5,96 & 8,90 & 15.03 .06 & 7,02 \\
\hline 195 & GIS & $37033 \mathrm{LBX} 3$ & 112,37 & 5,69 & 8,75 & 15.12 .03 & 4,74 \\
\hline 196 & GIS & 37033 LBZ8 & 112,46 & 5,75 & 8,75 & 12.02 .04 & 4,90 \\
\hline 197 & GIS & $37033 \mathrm{LCA} 2$ & 116,81 & 5,98 & 8,90 & 15.06 .06 & 7,28 \\
\hline 198 & GIS & 37033 LCB0 & 107,42 & 4,67 & 8,58 & 23.04 .01 & 2,06 \\
\hline 199 & GIS & 37033 LCB0 & 107,42 & 4,67 & 8,58 & 23.04 .01 & 2,06 \\
\hline 200 & GIS & 37033 LCC8 & 109,62 & 5,67 & 8,08 & 18.11 .03 & 4,66 \\
\hline 201 & GIS & 37033 LCD6 & 110,72 & 5,83 & 8,11 & 18.11 .04 & 5,68 \\
\hline 202 & GIS & 37033LCE4 & 112,76 & 6,01 & 8,13 & 20.11 .06 & 7,71 \\
\hline 203 & GIS & $37033 \mathrm{LCF} 1$ & 112,70 & 6,01 & 8,12 & 20.11 .06 & 7,71 \\
\hline 204 & GIS & $37033 \mathrm{LCN} 4$ & 107,63 & 5,10 & 7,77 & 05.06 .02 & 3,19 \\
\hline 205 & GIS & 37033 LCP9 & 107,63 & 5,10 & 7,77 & 03.06 .02 & 3,18 \\
\hline 206 & GIS & $37033 \mathrm{LCQ7}$ & 102,43 & 5,16 & 7,42 & 01.06 .00 & 1,15 \\
\hline 207 & GIS & 37033 LCRS & 102,55 & 5,15 & 7,50 & 05.06 .00 & 1,16 \\
\hline
\end{tabular}




\begin{tabular}{|c|c|c|c|c|c|c|c|}
\hline Lfd. Nr. & Ticker & CUSIP & Ask Kurs in Dollar & ISMA Rendite in $\%$ & Kupon in \% & Falligkeit & Restlaufzeit [Jahren] \\
\hline 208 & GIS & 37033LDAI & 100,48 & 4,78 & 7,27 & 30.06 .99 & 0,21 \\
\hline 209 & GIS & 37033LDB9 & 100,44 & 4,77 & 7,26 & 24.06 .99 & 0,20 \\
\hline 210 & GIS & 37033LDK9 & 100,89 & 5,15 & 6,89 & 28.10 .99 & 0,55 \\
\hline 211 & GIS & 37033LDL7 & 100,89 & 5,15 & 6,88 & 28.10 .99 & 0,55 \\
\hline 212 & GIS & 37033LDMS & 100,84 & 5,15 & 6,80 & 28.10 .99 & 0,55 \\
\hline 213 & GIS & 37033LDN3 & 100,86 & 5,15 & 6,83 & 29.10 .99 & 0,55 \\
\hline 214 & GIS & 37033LDP8 & 100,86 & 5,15 & 6,82 & 29.10 .99 & 0,55 \\
\hline 215 & GIS & 37033LDY9 & 95,11 & 7,11 & 5,38 & 08.07 .98 & 3,27 \\
\hline 216 & GIS & 37033LDY9 & 97,92 & 6,40 & 5,38 & 08.07 .98 & 2,26 \\
\hline 217 & GIS & 37033LDZ6 & 92,80 & 7,45 & 5,98 & 09.07 .01 & 6,31 \\
\hline 218 & GIS & $37033 \mathrm{LDZ} 6$ & 96,38 & 6,82 & 5,98 & 09.07 .01 & 5,31 \\
\hline 219 & GIS & 37033 LFA9 & 96,44 & 5,66 & 4,75 & 08.10 .03 & 4,55 \\
\hline 220 & GIS & 37033 LFC5 & 95,11 & 6,15 & 5,47 & 03.12 .08 & 9,78 \\
\hline 221 & GIS & 37033LFD3 & 94,58 & 6,15 & 5,40 & 08.12 .08 & 9,79 \\
\hline 222 & GIS & 37033LFE1 & 95,24 & 6,16 & 5,50 & 12.01 .09 & 9,89 \\
\hline 223 & GIS & 37033LFF8 & 98,23 & 6,21 & 6,00 & 22.01 .11 & 11,95 \\
\hline 224 & GIS & 37033LFG6 & 94,42 & 6,16 & 5,39 & 20.01 .09 & 9,91 \\
\hline 225 & GM & $370442 \mathrm{AH} 10$ & 110,86 & 6,84 & 9,63 & 01.12 .00 & 4,69 \\
\hline 226 & $\mathrm{GM}$ & $370442 \mathrm{AH} 8$ & 109,17 & 7,58 & 9,63 & 01.12 .00 & 5,70 \\
\hline 227 & GM & 370442AH8 & 110,86 & 6,84 & 9,63 & 01.12 .00 & 4,69 \\
\hline 228 & $\mathrm{GM}$ & 370442AM7 & 107,08 & 7,67 & 9,13 & 15.07 .01 & 6,33 \\
\hline 229 & GM & 370442AM7 & 109,55 & 6,92 & 9,13 & 15.07 .01 & 5,32 \\
\hline 230 & GM & 370442AP0 & 101,01 & 7,01 & 7,63 & 15.02 .97 & 1,86 \\
\hline 231 & GM & 370442AP0 & 101,37 & 5,88 & 7,63 & 15.02 .97 & 0,85 \\
\hline 232 & $\mathrm{GM}$ & $370442 \mathrm{AQ} 8$ & 94,67 & 7,90 & 7,00 & 15.06 .03 & 8,28 \\
\hline 233 & $\mathrm{GM}$ & $370442 \mathrm{AQ8}$ & 99,68 & 7,06 & 7,00 & 15.06 .03 & 7,27 \\
\hline 234 & GM & 370442AR6 & 94,44 & 7,89 & 7,40 & 01.09 .25 & 29,81 \\
\hline 235 & GM & 370442AS4 & 99,10 & 7,23 & 7,10 & 15.03 .06 & 10,06 \\
\hline 236 & GM & 370442AU9 & 100,51 & 7,65 & 7,70 & 15.04 .16 & 20,29 \\
\hline 237 & GM & 37045EAC2 & 107,46 & 5,28 & 9,02 & 07.06 .01 & 2,18 \\
\hline 238 & GM & 37045EAD0 & 107,19 & 5,28 & 8,88 & 11.06 .01 & 2,19 \\
\hline 239 & GM & 37045EAF5 & 108,00 & 5,30 & 9,20 & 02.07 .01 & 2,25 \\
\hline 240 & GM & 37045EAHI & 108,16 & 5,32 & 9,22 & 18.07.01 & 2,29 \\
\hline 241 & GM & 37045EAN8 & 110,28 & 6,15 & 8,89 & 18.08 .03 & 4,41 \\
\hline 242 & GM & 37045EAP3 & 107,55 & 5,35 & 8,83 & 20.08.01 & 2,39 \\
\hline 243 & GM & 37045EAS7 & 122,20 & 6,79 & 9,45 & 01.11 .11 & 12,73 \\
\hline 244 & GON & 37246WAA3 & 102,82 & 6,40 & 6,88 & 15.12 .05 & 7,78 \\
\hline 245 & GON & 37246 WAA3 & 101,42 & 6,61 & 6,88 & 15.12 .05 & 6,77 \\
\hline 246 & GON & 37246 WAC9 & 107,02 & 6,81 & 7,50 & 15.12 .15 & 17,93 \\
\hline 247 & GON & 37246WAC9 & 103,93 & 7,09 & 7,50 & 15.12 .15 & 16,91 \\
\hline 248 & GP & 373298BE7 & 104,58 & 8,96 & 9,50 & 01.12 .11 & 16,86 \\
\hline 249 & GP & 373298BE7 & 114,41 & 7,88 & 9,50 & 01.12 .11 & 15,85 \\
\hline 250 & GP & 373298BM9 & 96,59 & 8,05 & 7,70 & 15.06 .15 & 19,44 \\
\hline 251 & GP & $373298 \mathrm{BN} 7$ & 90,04 & 8,28 & 7,38 & 01.12 .25 & 30,06 \\
\hline 252 & $\mathbf{G}$ & 375766AB8 & 99,74 & 5,52 & 4,75 & 15.08 .96 & 0,34 \\
\hline 253 & G & $375766 \mathrm{AC6}$ & 96,63 & 6,84 & 6,25 & 15.08 .03 & 7,44 \\
\hline 254 & $\mathbf{G}$ & 375766AD4 & 91,50 & 6,99 & 5,75 & 15.10 .05 & 9,64 \\
\hline 255 & GLT & $377316 \mathrm{AA2}$ & 96,59 & 7,21 & 5,88 & 01.03 .98 & 2,91 \\
\hline 256 & GLT & $377316 \mathrm{AA2}$ & 99,05 & 6,42 & 5,88 & 01.03 .98 & 1,90 \\
\hline 257 & GR & 382388 AH9 & 109,52 & 7,66 & 9,63 & 01.07 .01 & 6,29 \\
\hline 258 & GR & 382388AH9 & 112,60 & 6,71 & 9,63 & 01.07 .01 & 5,28 \\
\hline 259 & GR & 38238QAA4 & 111,28 & 7,67 & 8,65 & 15.04 .25 & 29,42 \\
\hline 260 & GR & 38238QAB2 & 111,28 & 7,67 & 8,65 & 14.03.25 & 29,33 \\
\hline 261 & GR & $38238 \mathrm{QACO}$ & 110,93 & 7,67 & 8,62 & 15.04 .25 & 29,42 \\
\hline 262 & GR & 38238QAD8 & 110,81 & 7,67 & 8,61 & 15.04 .25 & 29,42 \\
\hline 263 & GR & 38238QAE6 & 110,81 & 7,67 & 8,61 & 14.03 .25 & 29,33 \\
\hline 264 & GR & 38238QAF3 & 100,72 & 7,69 & 7,75 & 19.05 .25 & 29,51 \\
\hline 265 & GRA & 383883AG0 & 97,89 & 8,13 & 7,75 & 01.10 .02 & 7,56 \\
\hline
\end{tabular}




\begin{tabular}{|c|c|c|c|c|c|c|c|}
\hline Lfd. Nr. & Ticker & CUSIP & Ask Kurs in Dollar & ISMA Rendite in $\%$ & Kupon in \% & Falligkeit & Restlaufzeit [Jahren] \\
\hline 266 & GRA & 383883AG0 & 103,11 & 7,14 & 7,75 & 01.10 .02 & 6,55 \\
\hline 267 & GRA & 383883AG0 & 101,47 & 7,42 & 7,75 & 01.10 .02 & 5,54 \\
\hline 268 & GRA & $383909 \mathrm{AB} 4$ & 98,42 & 7,80 & 7,40 & 01.02 .00 & 4,86 \\
\hline 269 & GRA & 383909AB4 & 101,84 & 6.83 & 7,40 & 01.02 .00 & 3,85 \\
\hline 270 & GRA & 383909AB4 & 100,74 & 7,10 & 7,40 & 01.02 .00 & 2,84 \\
\hline 271 & GRA & 383909AC2 & 97,85 & 8,33 & 8,00 & 15.08 .04 & 9,46 \\
\hline 272 & GRA & $383909 \mathrm{AC2}$ & 104,45 & 7,27 & 8,00 & 15.08 .04 & 8,45 \\
\hline 273 & GRA & $383909 \mathrm{AC2}$ & 102,61 & 7,53 & 8,00 & 15.08 .04 & 7,44 \\
\hline 274 & GDT & 401698AA3 & 97,66 & 6,58 & 6,15 & 15.02 .06 & 6,94 \\
\hline 275 & $\mathrm{HAL}$ & 406216AH4 & 101,45 & 8,61 & 8,75 & 15.02 .21 & 26,21 \\
\hline 276 & HAL & $406216 \mathrm{AH} 4$ & 112,97 & 7,58 & 8,75 & 15.02 .21 & 25,20 \\
\hline 277 & $\mathrm{MAH}$ & 41051PAE6 & 100,96 & 6,55 & 6,74 & 22.09 .05 & 6,54 \\
\hline 278 & $\mathrm{MAH}$ & 41051 PAF3 & 100,82 & 6,77 & 6,89 & 22.09 .08 & 9,58 \\
\hline 279 & $\mathrm{MAH}$ & 41051PAGI & 100,63 & 6,82 & 6,91 & 06.10 .09 & 10,63 \\
\hline 280 & $\mathrm{MAH}$ & 41051PAH9 & 97,56 & 6,84 & 6,52 & 23.02 .10 & 11,02 \\
\hline 281 & MAH & 41051PAJ5 & 97,65 & 6,87 & 6,58 & 23.02 .11 & 12,04 \\
\hline 282 & $\mathrm{MAH}$ & 410522AE6 & 106.89 & 8,22 & 9,38 & 15.09 .03 & 8,53 \\
\hline 283 & $\mathrm{MAH}$ & $410522 \mathrm{AE} 6$ & 112,36 & 7,19 & 9,38 & 15.09 .03 & 7,52 \\
\hline 284 & $\mathrm{H}$ & $41163 \mathrm{GAC5}$ & 102,55 & 7,77 & 8,25 & 01.06 .02 & 7,22 \\
\hline 285 & $\mathrm{H}$ & $41163 \mathrm{GAC} 5$ & 106,18 & 6,99 & 8,25 & 01.06 .02 & 6,21 \\
\hline 286 & $\mathrm{H}$ & $41163 \mathrm{GAD} 3$ & 100,90 & 8,79 & 8,88 & 01.06 .22 & 27,51 \\
\hline 287 & $\mathrm{H}$ & $41163 \mathrm{GAD} 3$ & 111.07 & 7,87 & 8,88 & 01.06 .22 & 26,51 \\
\hline 288 & HRS & 413875AE5 & 86,37 & 8,23 & 7,00 & 15.01 .26 & 30,18 \\
\hline 289 & HSC & 415864AE7 & 102,01 & 6,75 & 8,75 & 15.05 .96 & 1,09 \\
\hline 290 & HSC & 415864AE7 & 100,24 & 5,20 & 8,75 & 15.05 .96 & 0,08 \\
\hline 291 & $\mathrm{HSC}$ & $415864 \mathrm{AG2}$ & 88,27 & 7,94 & 6,00 & 15.09 .03 & 8,53 \\
\hline 292 & HSC & 415864 AG2 & 94,77 & 6,91 & 6,00 & 15.09 .03 & 7,52 \\
\hline 293 & HNZ & 423074AG8 & 99,27 & 6,10 & 6,00 & 15.03 .08 & 10,06 \\
\hline 294 & HNZ & 423074AG8 & 99.41 & 6,09 & 6,00 & 15.03 .08 & 9,05 \\
\hline 295 & HPC & 427056AM8 & 94,58 & 7,53 & 6,63 & 01.06 .03 & 8,24 \\
\hline 296 & HPC & 427056AM8 & 97,58 & 7,06 & 6,63 & 01.06 .03 & 7,23 \\
\hline 297 & HPC & 427056AN6 & 101,41 & 5,88 & 7,85 & 15.01 .00 & 0,77 \\
\hline 298 & HSY & 427866AE8 & 108,94 & 7,98 & 8,80 & 15.02 .21 & 26,21 \\
\hline 299 & HSY & 427866AE8 & 113,16 & 7,61 & 8,80 & 15.02 .21 & 25,20 \\
\hline 300 & HSY & 427866AFS & 97,27 & 7,10 & 6,70 & 01.10 .05 & 9,60 \\
\hline 301 & HLT & 432848 AF6 & 100,81 & 7,55 & 7,70 & 15.07 .02 & 7,34 \\
\hline 302 & HLT & $432848 \mathrm{AF} 6$ & 103,57 & 6,98 & 7,70 & 15.07 .02 & 6.34 \\
\hline 303 & HLT & 432848AR0 & 104,57 & 6.63 & 7.20 & 15.12 .09 & 11,84 \\
\hline 304 & HLT & 432848AR0 & 102,78 & 6,83 & 7,20 & 15.12 .09 & 10,83 \\
\hline 305 & HLT & 432848AS8 & 107,50 & 6,80 & 7,50 & 15.12 .17 & 19,96 \\
\hline 306 & HLT & 432848AS8 & 104,36 & 7,07 & 7.50 & 15.12 .17 & 18,94 \\
\hline 307 & HLT & 43284QAA7 & 101,17 & 6,09 & 7,02 & 17.08 .97 & 1,36 \\
\hline 308 & HLT & 43284QABS & 102,35 & 6,60 & 7,40 & 17.08 .99 & 3,38 \\
\hline 309 & HLT & 43284QAC3 & 103,48 & 6,86 & 7,65 & 17.08 .01 & 5,41 \\
\hline 310 & HLT & 43284QAC4 & 103,48 & 6,86 & 7,65 & 17.08 .01 & 5,41 \\
\hline 311 & HLT & 43284QADI & 103,44 & 6,87 & 7,65 & 20.08 .01 & 5,42 \\
\hline 312 & HLT & 43284QAE9 & 102,49 & 6,61 & 7,45 & 19.08 .99 & 3,39 \\
\hline 313 & HLT & 43284QAH2 & 99,65 & 7,17 & 7,05 & 24.08 .98 & 3,40 \\
\hline 314 & HLT & $43284 \mathrm{QAH2}$ & 101,25 & 6,47 & 7,05 & 24.08 .98 & 2,39 \\
\hline 315 & HLT & 43284QAJ8 & 100,08 & 7,17 & 7,20 & 30.08 .98 & 3,41 \\
\hline 316 & HLT & 43284QAJ8 & 101,58 & 6,47 & 7,20 & 30.08 .98 & 2,41 \\
\hline 317 & HLT & 43284QAK5 & 100,21 & 7,29 & 7,35 & 30.08 .99 & 4,43 \\
\hline 318 & HLT & 43284QAKS & 102,22 & 6,60 & 7.35 & 30.08 .99 & 3,42 \\
\hline 319 & HON & $438506 \mathrm{AHO}$ & 105,20 & 7,91 & 8,63 & 15.04 .06 & 11,15 \\
\hline 320 & HON & $438506 \mathrm{AHO}$ & 109,89 & 7,22 & 8.63 & 15.04 .06 & 10,14 \\
\hline 321 & HON & $438506 \mathrm{AHO}$ & 105,20 & 7,91 & 8,63 & 15.04 .06 & 11,15 \\
\hline 322 & HON & 438506AS6 & 99,13 & 6,69 & 6,63 & 15.06 .28 & 29,60 \\
\hline 323 & $\mathrm{HON}$ & 43850QAAI & 102,34 & 6,66 & 7,46 & 16.08 .99 & 3,38 \\
\hline
\end{tabular}




\begin{tabular}{|c|c|c|c|c|c|c|c|}
\hline Lfd. Nr. & Ticker & CUSIP & Ask Kurs in Dollar & ISMA Rendite in $\%$ & Kupon in \% & Falligkeit & Restlaufzeit [Jahren] \\
\hline 324 & HON & 43850QAB9 & 102,06 & 6,67 & 7,36 & 31.08 .99 & 3,42 \\
\hline 325 & HON & 43850QAC7 & 100,01 & 7,24 & 7,25 & 23.03 .98 & 2,97 \\
\hline 326 & HON & 43850QAC7 & 101,50 & 6,41 & 7,25 & 23.03 .98 & 1,96 \\
\hline 327 & HON & 43850QADS & 101,18 & 7,25 & 7,70 & 01.04 .98 & 2,99 \\
\hline 328 & HON & 43850QAD5 & 102,32 & 6,42 & 7,70 & 01.04 .98 & 1,99 \\
\hline 329 & HON & 43850QAE3 & 101,21 & 7,26 & 7,71 & 07.05 .98 & 3,09 \\
\hline 330 & HON & 43850QAE3 & 102,38 & 6,45 & 7,71 & 07.05 .98 & 2,09 \\
\hline 331 & $\mathrm{HON}$ & 43850QAFO & 99,40 & 7,50 & 7,35 & 15.05 .00 & 5,15 \\
\hline 332 & HON & 43850QAF0 & 102,08 & 6,75 & 7,35 & 15.05 .00 & 4,14 \\
\hline 333 & HON & 43850QAG8 & 99,37 & 7,50 & 7,35 & 01.06 .00 & 5,19 \\
\hline 334 & HON & 43850QAG8 & 102,08 & 6,76 & 7,35 & 01.06 .00 & 4,19 \\
\hline 335 & HON & 43850QAH6 & 99,38 & 7,59 & 7,45 & 11.04 .01 & 6,07 \\
\hline 336 & HON & 43850QAH6 & 102,37 & 6,88 & 7,45 & 11.04 .01 & 5,06 \\
\hline 337 & HON & 43850 QAJ2 & 99,10 & 7,65 & 7,48 & 11.04 .02 & 7,08 \\
\hline 338 & HON & 43850QAJ2 & 102,47 & 6,97 & 7,48 & 11.04 .02 & 6,07 \\
\hline 339 & HON & 43850QAK9 & 99,75 & 7,25 & 7,15 & 15.04 .98 & 3,03 \\
\hline 340 & HON & 43850QAK9 & 101,32 & 6,43 & 7,15 & 15.04 .98 & 2,03 \\
\hline 341 & HTN & $441560 \mathrm{AA} 7$ & 95,75 & 7,79 & 7,13 & 01.04 .04 & 9,08 \\
\hline 342 & HTN & $441560 \mathrm{AA} 7$ & 98,12 & 7,44 & 7,13 & 01.04 .04 & 8,08 \\
\hline 343 & HTN & 441560AB5 & 95,85 & 7,60 & 7,00 & 01.03 .06 & 10,02 \\
\hline 344 & IBP & $449223 \mathrm{AB2}$ & 92,33 & 7,23 & 6,13 & 01.02 .06 & 9,94 \\
\hline 345 & IBP & 44922 XAA8 & 97,98 & 6,24 & 6,00 & 15.01 .11 & 11,93 \\
\hline 346 & ITW & 452308AB5 & 101,26 & 7,09 & 7,50 & 01.12 .98 & 3,67 \\
\hline 347 & ITW & 452308ABS & 102,49 & 6,45 & 7,50 & 01.12 .98 & 2,66 \\
\hline 348 & ITW & $452308 \mathrm{ADI}$ & 94,52 & 7,23 & 5,88 & 01.03 .00 & 4,94 \\
\hline 349 & ITW & 452308AD1 & 97,64 & 6,57 & 5,88 & 01.03 .00 & 3,93 \\
\hline 350 & IR & 456866AF9 & 101,87 & 6,93 & 8,25 & 01.11 .96 & 1,56 \\
\hline 351 & IR & 456866AF9 & 101,31 & 5,71 & 8,25 & 01.11 .96 & 0,55 \\
\hline 352 & IR & 456866AG7 & 110,04 & 8,07 & 9,00 & 15.08 .21 & 26,71 \\
\hline 353 & IR & 456866AG7 & 116,52 & 7,53 & 9,00 & 15.08 .21 & 25,70 \\
\hline 354 & IR & 456866 AH5 & 95,26 & 7,69 & 6,88 & 01.02 .03 & 7,90 \\
\hline 355 & IR & $456866 \mathrm{AHS}$ & 99,06 & 7,05 & 6,88 & 01.02 .03 & 6,89 \\
\hline 356 & IR & 456866AN2 & 100,77 & 5,95 & 6,26 & 15.02 .01 & 2,88 \\
\hline 357 & IR & 456866AN2 & 102,55 & 4,77 & 6,26 & 15.02 .01 & 1,87 \\
\hline 358 & IR & 45686XAA1 & 101,76 & 5,11 & 6,56 & 28.07 .00 & 1,31 \\
\hline 359 & IR & $45686 \times 1 \mathrm{AB} 9$ & 101,81 & 5,11 & 6,60 & 28.07 .00 & 1,31 \\
\hline 360 & IR & 45686XAF0 & 100,43 & 5,02 & 6,57 & 02.08 .99 & 0,31 \\
\hline 361 & IR & $45686 \times \mathrm{XAH} 6$ & 101,83 & 5,10 & 6,60 & 01.08 .00 & 1,32 \\
\hline 362 & IR & 45686 XAL7 & 104,89 & 5,22 & 6,86 & 05.08 .02 & 3,36 \\
\hline 363 & IR & 45686XAM5 & 104,80 & 5,22 & 6,83 & 05.08 .02 & 3,36 \\
\hline 364 & IR & 45686XAN3 & 104,68 & 5,23 & 6,79 & 07.08 .02 & 3,36 \\
\hline 365 & IR & 45686XAP8 & 104,65 & 5,23 & 6,78 & 07.08 .02 & 3,36 \\
\hline 366 & IR & $45686 \times \mathrm{AQ} 6$ & 101,79 & 5,10 & 6,54 & 07.08 .00 & 1,34 \\
\hline 367 & IR & 45686XAR4 & 101,80 & 5,10 & 6,55 & 07.08 .00 & 1,34 \\
\hline 368 & IR & 45686XAS2 & 101,83 & 5,10 & 6,57 & 07.08 .00 & 1,34 \\
\hline 369 & IR & 45686XAT0 & 101,84 & 5,10 & 6,58 & 07.08 .00 & 1,34 \\
\hline 370 & IR & 45686 XAU7 & 104,56 & 5,23 & 6,75 & 07.08 .02 & 3,36 \\
\hline 371 & IR & 45686XAV5 & 100,43 & 5,05 & 6,51 & 09.08 .99 & 0,33 \\
\hline 372 & IR & 45686XAW3 & 100,43 & 5,05 & 6,50 & 09.08 .99 & 0,33 \\
\hline 373 & IR & 45686XAX1 & 104,82 & 5,87 & 6,94 & 09.08 .04 & 5,40 \\
\hline 374 & IR & 45686XAY9 & 100,44 & 5,05 & 6,55 & 09.08 .99 & 0,33 \\
\hline 375 & IR & $45686 \times \mathrm{AZ} 6$ & 100,44 & 5,05 & 6,53 & 09.08 .99 & 0,33 \\
\hline 376 & IR & $45686 \times \mathrm{BAA0}$ & 100,45 & 5,05 & 6,54 & 10.08 .99 & 0,33 \\
\hline 377 & IR & $45686 \times \mathrm{XBB} 8$ & 105,01 & 5,87 & 6,98 & 10.08 .04 & 5,40 \\
\hline 378 & IR & $45686 \times \mathrm{XC} 6$ & 104,96 & 5,87 & 6,97 & 11.08 .04 & 5,41 \\
\hline 379 & IR & 45686XBD4 & 104,92 & 5,87 & 6,96 & 10.08 .04 & 5,40 \\
\hline 380 & IR & $45686 \times \mathrm{XEE} 2$ & 104,65 & 5,23 & 6,78 & 12.08 .02 & 3,38 \\
\hline 381 & IR & $45686 \times$ BF9 & 104,62 & 5,23 & 6,77 & 12.08 .02 & 3,38 \\
\hline
\end{tabular}




\begin{tabular}{|c|c|c|c|c|c|c|c|}
\hline Lfd. Nr. & Ticker & CUSIP & Ask Kurs in Dollar & ISMA Rendite in \% & Kupon in \% & Falligkeit & Restlaufzeit [Jahren] \\
\hline 382 & IR & 45686XBG7 & 101,80 & 5,09 & 6,53 & 11.08 .00 & 1,35 \\
\hline 383 & IR & $45686 \times B \times 0$ & 100,54 & 5,10 & 6,62 & 30.08 .99 & 0,38 \\
\hline 384 & IR & 45686XBY8 & 102,11 & 5,06 & 6,69 & 28.08 .00 & 1,39 \\
\hline 385 & IR & $45686 \times \mathrm{BZZ}$ & 100,53 & 5,25 & 6,20 & 15.11 .99 & 0,60 \\
\hline 386 & IR & $45686 \times \mathrm{XA} 9$ & 100,51 & 5,25 & 6,17 & 17.11 .99 & 0,60 \\
\hline 387 & IR & $45686 \times \mathrm{CB} 7$ & 103,42 & 4,95 & 6,38 & 19.11 .01 & 2,64 \\
\hline 388 & $\mathbf{I R}$ & $45686 \times \mathrm{XCD} 3$ & 102,86 & 5,74 & 6,46 & 19.11 .03 & 4,67 \\
\hline 389 & IR & $45686 \mathrm{XCG} 6$ & 102,87 & 5,90 & 6,51 & 01.12 .04 & 5,72 \\
\hline 390 & IR & $45686 \mathrm{XCH} 4$ & 103,01 & 6,11 & 6,57 & 03.12 .07 & 8,76 \\
\hline 391 & IR & $45686 \mathrm{XCJ} 0$ & 100,51 & 5,27 & 6,12 & 01.12 .99 & 0,64 \\
\hline 392 & IR & 45686XCK7 & 103,34 & 4,97 & 6,34 & 03.12 .01 & 2,68 \\
\hline 393 & IR & 45686XCLS & 103,15 & 6,00 & 6,58 & 05.12 .05 & 6,74 \\
\hline 394 & IBM & 459200AG6 & 102,64 & 8,12 & 8,38 & 01.11 .19 & 24,89 \\
\hline 395 & IBM & $459200 \mathrm{AG} 6$ & 109,45 & 7,51 & 8,38 & 01.11 .19 & 23,89 \\
\hline 396 & IBM & $459200 \mathrm{AG} 6$ & 119,63 & 6,65 & 8,38 & 01.11 .19 & 21,86 \\
\hline 397 & IBM & 459200AG6 & 115,13 & 6,98 & 8,38 & 01.11 .19 & 20,85 \\
\hline 398 & IBM & $459200 \mathrm{AH} 4$ & 98,75 & 6,92 & 6,38 & 01.11 .97 & 2,58 \\
\hline 399 & IBM & 459200AH4 & 100,43 & 6,07 & 6,38 & 01.11 .97 & 1,57 \\
\hline 400 & IBM & 459200AJO & 99,25 & 7,38 & 7,25 & 01.11 .02 & 7,65 \\
\hline 401 & IBM & $459200 \mathrm{AJO}$ & 102,20 & 6,83 & 7,25 & 01.11 .02 & 6,64 \\
\hline 402 & IBM & 459200A A & 105,00 & 5,97 & 7,25 & 01.11 .02 & 4,61 \\
\hline 403 & IBM & 459200AJ0 & 105,00 & 5,67 & 7,25 & 01.11 .02 & 3,60 \\
\hline 404 & IBM & 459200AK7 & 96,38 & 7,23 & 6,38 & 15.06 .00 & 5,23 \\
\hline 405 & IBM & 459200AK7 & 99,63 & 6,48 & 6,38 & 15.06 .00 & 4,23 \\
\hline 406 & $1 \mathrm{BM}$ & 459200AK7 & 101,00 & 5,87 & 6,38 & 15.06 .00 & 2,20 \\
\hline 407 & IBM & 459200AK7 & 101,13 & 5,35 & 6,38 & 15.06 .00 & 1,19 \\
\hline 408 & IBM & 459200AL5 & 95,38 & 7,99 & 7,50 & 15.06 .13 & 18,42 \\
\hline 409 & IBM & 459200AL5 & 101,31 & 7,36 & 7,50 & 15.06 .13 & 17,41 \\
\hline 410 & IBM & 459200AL5 & 108,13 & 6,64 & 7,50 & 15.06 .13 & 15,39 \\
\hline 411 & IBM & 459200AL5 & 111,63 & 6,25 & 7,50 & 15.06 .13 & 14,38 \\
\hline 412 & IBM & 45920QARS & 101,28 & 5,00 & 6,04 & 07.08 .00 & 1,34 \\
\hline 413 & IBM & 45920QAU8 & 101.10 & 5,01 & 5,85 & 05.09 .00 & 1,42 \\
\hline 414 & IBM & 459200AV6 & 102,40 & 4,71 & 5,95 & 14.05 .01 & 2,11 \\
\hline 415 & IBM & 459200AX2 & 101,61 & 5,51 & 5,95 & 02.06 .03 & 4,19 \\
\hline 416 & IBM & 45920QBAI & 102,36 & 4,76 & 5,91 & 29.06 .01 & 2,24 \\
\hline 417 & IBM & 459200BB9 & 100,76 & 5,10 & 5,76 & 10.07 .00 & 1,26 \\
\hline 418 & IBM & 45920QBDS & 102,12 & 4,71 & 5,80 & 15.05 .01 & 2,12 \\
\hline 419 & $1 \mathrm{BM}$ & $459200 \mathrm{QBL} 7$ & 98,96 & 5,64 & 5,37 & 22.09 .03 & 4,51 \\
\hline 420 & IBM & 459200BM5 & 94,73 & 6,14 & 5,40 & 01.10 .08 & 9,61 \\
\hline 421 & IBM & 45920QBR4 & 97,63 & 5,70 & 5,10 & 10.11 .03 & 4,64 \\
\hline 422 & IBM & 45920QBS2 & 98,31 & 5,67 & 5,25 & 01.12 .03 & 4,70 \\
\hline 423 & IBM & 459200BT0 & 94,73 & 6,13 & 5,40 & 01.12 .08 & 9,78 \\
\hline 424 & IBM & $459200 \mathrm{BX} 1$ & 95,37 & 6,14 & 5,50 & 15.01 .09 & 9,90 \\
\hline 425 & IBM & 459200BY9 & 94,54 & 6,14 & 5,39 & 22.01 .09 & 9,92 \\
\hline 426 & IBM & $459200 \mathrm{BZ} 6$ & 94,55 & 6,14 & 5,40 & 26.02 .09 & 10,02 \\
\hline 427 & IBM & $459200 \mathrm{QAA} 0$ & 100,75 & 4,68 & 5,13 & 12.02 .01 & 1,86 \\
\hline 428 & IBM & 459200CB8 & 99,82 & 5,27 & 5,07 & 22.03 .00 & 0,95 \\
\hline 429 & IBM & $45920 \mathrm{QCC} 6$ & 99,82 & 5,27 & 5,08 & 03.04 .00 & 0,99 \\
\hline 430 & IBM & $459200 \mathrm{CD} 4$ & 101,63 & 4,64 & 5,56 & 08.03 .01 & 1,93 \\
\hline 431 & IBM & 459200CE2 & 101,25 & 4,62 & 5,30 & 26.03 .01 & 1,98 \\
\hline 432 & IBM & $459200 \mathrm{QCG} 7$ & 99,46 & 5,75 & 5,63 & 12.04 .04 & 5,07 \\
\hline 433 & IMC & 46004QAF8 & 100,39 & 6,89 & 7,00 & 23.05 .00 & 4,16 \\
\hline 434 & IMC & 46004QAH4 & 100,43 & 6,76 & 6,91 & 24.05 .99 & 3,15 \\
\hline 435 & IMC & 46004QAK7 & 98,19 & 6,90 & 6,39 & 09.06 .00 & 4,21 \\
\hline 436 & IP & $460146 \mathrm{AJ} 2$ & 108,10 & 7,68 & 9,70 & 15.03 .00 & 4,98 \\
\hline 437 & IP & $460146 \mathrm{AJ} 2$ & 110,00 & 6,74 & 9,70 & 15.03 .00 & 3,97 \\
\hline 438 & IP & 460146AK9 & 108,07 & 7,89 & 9,40 & 01.06 .02 & 7,22 \\
\hline 439 & IP & 460146AK9 & 111,82 & 6,99 & 9,40 & 01.06 .02 & 6,21 \\
\hline
\end{tabular}




\begin{tabular}{|c|c|c|c|c|c|c|c|}
\hline Lfd. Nr. & Ticker & CUSIP & Ask Kurs in Dollar & ISMA Rendite in $\%$ & Kupon in \% & Falligkeit & Restlaufzeit [Jahren] \\
\hline 440 & IP & 460146AK9 & 111,82 & 6,99 & 9,40 & 01.06 .02 & 6,21 \\
\hline 441 & IP & 460146AM5 & 99,26 & 7,72 & 7,63 & 15.01 .07 & 11,91 \\
\hline 442 & IP & 460146AMS & 102,83 & 7,24 & 7,63 & 15.01 .07 & 10,91 \\
\hline 443 & IP & 460146AMS & 102,28 & 7,12 & 7,50 & 15.05 .04 & 8,20 \\
\hline 444 & IP & $460146 \mathrm{AQ} 6$ & 96,44 & 8,06 & 7,50 & 15.05 .04 & 9,21 \\
\hline 445 & IP & 460146AQ6 & 102,28 & 7,12 & 7,50 & 15.05 .04 & 8,20 \\
\hline 446 & IP & 460146AR4 & 88,43 & 8,03 & 6,13 & 01.11 .03 & 8,66 \\
\hline 447 & IP & 460146AR4 & 94,41 & 7,10 & 6,13 & 01.11 .03 & 7,65 \\
\hline 448 & IP & 460146ATO & 101,36 & 7,69 & 7,88 & 01.08 .06 & 11,45 \\
\hline 449 & IP & 460I46AT0 & 104,76 & 7,21 & 7,88 & 01.08 .06 & 10,44 \\
\hline 450 & IP & 460146AU7 & 100,46 & 7,55 & 7,63 & 01.08 .04 & 9,42 \\
\hline 451 & IP & 460146AU7 & 102,99 & 7,14 & 7,63 & 01.08 .04 & 8,41 \\
\hline 452 & IP & 46014HABS & 106,40 & 5,27 & 9,05 & 08.02 .01 & 1,85 \\
\hline 453 & IP & 46014HAG4 & 110,39 & 5,98 & 8,99 & 21.03 .03 & 3,99 \\
\hline 454 & IP & 46014HAL3 & 107,29 & 5,36 & 8,69 & 30.08 .01 & 2,41 \\
\hline 455 & IP & 46014HAMI & 107,30 & 5,36 & 8,70 & 27.08 .01 & 2,41 \\
\hline 456 & IP & 46014HAN9 & 107,23 & 5,36 & 8,67 & 27.08 .01 & 2,41 \\
\hline 457 & IP & 46014HAP4 & 107,19 & 5,36 & 8,65 & 28.08 .01 & 2,41 \\
\hline 458 & IP & $46014 \mathrm{HAQ} 2$ & 112,33 & 6,58 & 8,72 & 29.08 .06 & 7,48 \\
\hline 459 & IP & 46014HAR0 & 107,38 & 5,37 & 8,70 & 11.09 .01 & 2,45 \\
\hline 460 & IP & 46014HAS8 & 107,45 & 5,37 & 8,72 & 14.09 .01 & 2,46 \\
\hline 461 & IP & 46014HAW9 & 107,36 & 5,37 & 8,68 & 14.09 .01 & 2,46 \\
\hline 462 & IP & $46014 \mathrm{HAX7}$ & 105,51 & 5,49 & 7,72 & 03.01 .02 & 2,76 \\
\hline 463 & IP & $46014 \mathrm{HBA} 6$ & 106,92 & 5,57 & 8,17 & 18.03 .02 & 2,97 \\
\hline 464 & IP & $46014 \mathrm{HBB} 4$ & 107,70 & 6,37 & 8,22 & 18.03 .04 & 5,00 \\
\hline 465 & IP & 46014HBE8 & 107,56 & 5,58 & 8,39 & 01.04 .02 & 3,01 \\
\hline 466 & IP & 46014HBF5 & 107,48 & 5,58 & 8,36 & 01.04 .02 & 3,01 \\
\hline 467 & IP & $46014 \mathrm{HBJ} 7$ & 107,45 & 5,58 & 8,35 & 01.04 .02 & 3,01 \\
\hline 468 & IP & $46014 \mathrm{HBL} 2$ & 108,52 & 6,37 & 8,41 & 25.03 .04 & 5,02 \\
\hline 469 & IP & 46014HBM0 & 107,45 & 5,57 & 8,36 & 25.03 .02 & 2,99 \\
\hline 470 & IP & $46014 \mathrm{HBN} 8$ & 106,18 & 5,64 & 7,85 & 21.05 .02 & 3,15 \\
\hline 471 & IP & 46014HBS7 & 105,65 & 7,23 & 8,00 & 15.11 .06 & 10,74 \\
\hline 472 & IP & 46014HBTS & 105,63 & 7,35 & 8,03 & 15.05 .09 & 13,27 \\
\hline 473 & IP & $46014 \mathrm{HBU} 2$ & 104,41 & 7,12 & 7,85 & 20.05 .04 & 8,21 \\
\hline 474 & IP & $46014 \mathrm{HBVO}$ & 106,22 & 7,35 & 8,10 & 20.05 .09 & 13,28 \\
\hline 475 & IP & $46014 \mathrm{HBW} 8$ & 101,96 & 6,94 & 7,39 & 10.08 .01 & 5,39 \\
\hline 476 & IP & $46014 \mathrm{HBX} 6$ & 102,00 & 6,94 & 7,40 & 10.08 .01 & 5,39 \\
\hline 477 & IP & $46014 \mathrm{HBY} 4$ & 100,11 & 7,85 & 7,88 & 03.10 .01 & 6,55 \\
\hline 478 & IP & $46014 \mathrm{HBY} 4$ & 104,13 & 6,95 & 7,88 & 03.10 .01 & 5,54 \\
\hline 479 & JNJ & $478160 \mathrm{AE} 4$ & 100,61 & 7,26 & 7,38 & 29.06 .02 & 7,30 \\
\hline 480 & NJ & $478160 \mathrm{AE} 4$ & 103,59 & 6,66 & 7,38 & 29.06 .02 & 6,29 \\
\hline 481 & JNJ & 478160AF1 & 87,20 & 7,86 & 6,73 & 15.11 .23 & 28,99 \\
\hline 482 & JNJ & $478160 \mathrm{AFl}$ & 92,63 & 7,36 & 6,73 & 15.11 .23 & 27,98 \\
\hline 483 & $\mathrm{KSU}$ & $485170 \mathrm{AB} 0$ & 100,47 & 7,79 & 7,88 & 01.07 .02 & 7,31 \\
\hline 484 & KSU & $485170 \mathrm{ABO}$ & 103,69 & 7,12 & 7,88 & 01.07 .02 & 6,30 \\
\hline 485 & $\mathrm{KSU}$ & 485170AD6 & 95,39 & 7,34 & 6,63 & 01.03 .05 & 9,00 \\
\hline 486 & $\mathrm{KSU}$ & 485170AE4 & 95,76 & 7,25 & 5,75 & 08.07 .98 & 3,27 \\
\hline 487 & $\mathrm{KSU}$ & 485170AE4 & 98,23 & 6,61 & 5,75 & 08.07 .98 & 2,26 \\
\hline 488 & $\mathrm{KBH}$ & 486168AE7 & 100,00 & 7,75 & 7,75 & 15.10 .04 & 6,60 \\
\hline 489 & $\mathrm{KBH}$ & 486168AE7 & 101,25 & 7,47 & 7,75 & 15.10 .04 & 5,59 \\
\hline 490 & $\mathbf{K}$ & 487836AK4 & 95,08 & 5,80 & 4,88 & 15.10 .05 & 6,60 \\
\hline 491 & KMB & $494368 \mathrm{AG} 8$ & 104,27 & 6,90 & 9,13 & 01.06 .97 & 2,15 \\
\hline 492 & KMB & 494368AG8 & 103,38 & 5,94 & 9,13 & 01.06 .97 & 1,14 \\
\hline 493 & KMB & 494368AL7 & 107,65 & 7,22 & 9,00 & 01.08 .00 & 5,36 \\
\hline 494 & $\mathrm{KMB}$ & 494368AL7 & 108,76 & 6,61 & 9,00 & 01.08 .00 & 4,36 \\
\hline 495 & $\mathrm{KMB}$ & $494368 \mathrm{AN} 3$ & 106,45 & 7,28 & 8,63 & 01.05 .01 & 6,12 \\
\hline 496 & KMB & 494368AN3 & 108,11 & 6,70 & 8,63 & 01.05 .01 & 5,11 \\
\hline 497 & KMB & $494368 \mathrm{AQ6}$ & 90,20 & 7,88 & 6,88 & 15.02 .14 & 19,10 \\
\hline
\end{tabular}




\begin{tabular}{|c|c|c|c|c|c|c|c|}
\hline Lfd. Nr. & Ticker & CUSIP & Ask Kurs in Dollar & ISMA Rendite in $\%$ & Kupon in \% & Falligkeit & Restlaufzeit [Jahren] \\
\hline 498 & KMB & $494368 \mathrm{AQ6}$ & 95,25 & 7,36 & 6,88 & 15.02 .14 & 18,09 \\
\hline 499 & KIN & 495836AA6 & 98.50 & 7.10 & 6,00 & 01.10 .97 & 1,48 \\
\hline 500 & KRI & 499040ADS & 116,80 & 7,87 & 9,88 & 15.04 .09 & 14,19 \\
\hline 501 & $\mathrm{KRI}$ & 499040ADS & 121,70 & 7,27 & 9,88 & 15.04 .09 & 13,19 \\
\hline 502 & LAF & 50586FAM5 & 103,57 & 5,82 & 9,75 & 30.03 .00 & 0,98 \\
\hline 503 & LAF & 50586FAQ6 & 104,33 & 5,67 & 9,67 & 08.06 .00 & 1,17 \\
\hline 504 & LAF & 50586FAR4 & 104,32 & 5,67 & 9,65 & 09.06 .00 & 1,17 \\
\hline 505 & LAF & $50586 \mathrm{FAXI}$ & 106,58 & 5,39 & 9,70 & 01.12 .00 & 1,66 \\
\hline 506 & LAF & 50586FBA0 & 107,52 & 5,28 & 9,75 & 02.02 .01 & 1,83 \\
\hline 507 & LAF & 50586FBE2 & 116,79 & 6,55 & 9,58 & 18.04 .06 & 7,11 \\
\hline 508 & LAF & 50586FBF9 & 107,73 & 5,22 & 9,32 & 24.04 .01 & 2,06 \\
\hline 509 & LAF & 50586FBG7 & 108,31 & 5,24 & 9,57 & 07.05 .01 & 2,09 \\
\hline 510 & LAF & 50586FBH5 & 108,12 & 5,24 & 9,46 & 10.05 .01 & 2,10 \\
\hline 511 & LAF & $50586 \mathrm{FBL} 6$ & 108,72 & 5,28 & 9,63 & 15.06 .01 & 2,20 \\
\hline 512 & LAF & 50586FBM4 & 110,29 & 5,50 & 9,60 & 15.01 .02 & 2,80 \\
\hline 513 & LAF & 50586FBN2 & 108,49 & 5,27 & 9,57 & 30.05 .01 & 2,16 \\
\hline 514 & LAF & 50586FBQ5 & 106,06 & 5,42 & 9,50 & 15.11 .00 & 1,61 \\
\hline 515 & LAF & $50586 \mathrm{FBSI}$ & 104,82 & 5,56 & 9,40 & 15.08 .00 & 1,36 \\
\hline 516 & LAF & 50586FBT9 & 100,62 & 5,17 & 9,38 & 15.06 .99 & 0,17 \\
\hline 517 & LLY & 532457AHI & 98,27 & 7,20 & 6,75 & 15.11 .99 & 4,64 \\
\hline 518 & LLY & $532457 \mathrm{AHI}$ & 100,66 & 6,54 & 6,75 & 15.11 .99 & 3,63 \\
\hline 519 & LLY & 532457AJ7 & 92,99 & 7,44 & 6,25 & 15.03 .03 & 8,02 \\
\hline 520 & LLY & 532457AJ7 & 96.90 & 6,82 & 6,25 & 15.03 .03 & 7,01 \\
\hline 521 & LLY & $532457 \mathrm{AK} 4$ & 104,25 & 7,30 & 8,13 & 01.12 .01 & 6,72 \\
\hline 522 & LLY & $532457 \mathrm{AK} 4$ & 106,33 & 6,75 & 8,13 & 01.12 .01 & 5,71 \\
\hline 523 & LLY & 532457AK4 & 106,75 & 6,02 & 8,13 & 01.12 .01 & 3,68 \\
\hline 524 & LLY & $532457 \mathrm{AK} 4$ & 106,00 & 5,62 & 8,13 & 01.12 .01 & 2.67 \\
\hline 525 & LLY & 532457AL2 & 105,59 & 7,64 & 8,38 & 01.12 .06 & 11,79 \\
\hline 526 & LLY & $532457 \mathrm{AL} 2$ & 111,00 & 6,90 & 8,38 & 01.12 .06 & 10,78 \\
\hline 527 & LLY & $532457 \mathrm{AL2}$ & 113,00 & 6,39 & 8,38 & 01.12 .06 & 8,76 \\
\hline 528 & \begin{tabular}{|l|} 
LLY \\
\end{tabular} & 532457AL2 & 106,25 & 7,29 & 8,38 & 01.12 .06 & 7,74 \\
\hline 529 & LLY & 532457AM0 & 90,50 & 7,97 & 7,13 & 01.06 .25 & 29,55 \\
\hline 530 & LLY & 532457AM0 & 102,13 & 6,95 & 7,13 & 01.06 .25 & 27,53 \\
\hline 531 & LLY & 532457AM0 & 105,00 & 6,72 & 7,13 & 01.06 .25 & 26.51 \\
\hline 532 & LIT & 538021AJ5 & 99,63 & 6,14 & 6,05 & 15.04 .03 & 5.07 \\
\hline 533 & LIT & 538021AJS & 99,92 & 6,07 & 6.05 & 15.04 .03 & 4,06 \\
\hline 534 & LOR & 543859AE2 & 103,59 & 8,77 & 9,13 & 01.02 .22 & 27,18 \\
\hline 535 & LOR & 543859AE2 & 112,33 & 7,99 & 9,13 & 01.02 .22 & 26,17 \\
\hline 536 & LOR & 543859 AG7 & 95,49 & 8,81 & 8,38 & 15.01 .23 & 28,15 \\
\hline 537 & LOR & 543859AG7 & 103.97 & 8,01 & 8.38 & 15.01 .23 & 27,14 \\
\hline 538 & LOR & 543859AH5 & 80,77 & 8,86 & 7,00 & 15.09 .23 & 28,82 \\
\hline 539 & LOR & 543859AH5 & 88.55 & 8,04 & 7.00 & 15.09 .23 & 27,81 \\
\hline 540 & LOR & $543859 \mathrm{AJI}$ & 97,58 & 8,00 & 7,63 & 15.06 .04 & 9,29 \\
\hline 541 & LOR & $543859 \mathrm{AJ1}$ & 102,15 & 7,27 & 7,63 & 15.06 .04 & 8.28 \\
\hline 542 & LYO & 552078AA5 & 102,97 & 7,09 & 9,95 & 01.06 .96 & 1,14 \\
\hline 543 & LYO & 552078AA5 & 100,48 & 5,56 & 9,95 & 01.06 .96 & 0,13 \\
\hline 544 & LYO & $552078 \mathrm{AB} 3$ & 107,99 & 7,69 & 10,00 & 01.06 .99 & 4,18 \\
\hline 545 & LYO & 552078AB3 & 109,06 & 6,72 & 10,00 & 01.06 .99 & 3,17 \\
\hline 546 & LYO & $552078 \mathrm{ACl}$ & 101,55 & 7,35 & 8,25 & 15.03 .97 & 1,93 \\
\hline 547 & LYO & $552078 \mathrm{ACl}$ & 101,96 & 5,99 & 8,25 & 15.03 .97 & 0,93 \\
\hline 548 & LYO & 552078AD9 & 105,52 & 8,06 & 9,13 & 15.03 .02 & 7,01 \\
\hline 549 & LYO & S52078AD9 & 109,77 & 7,07 & 9,13 & 15.03 .02 & 6,00 \\
\hline 550 & LYO & 552078AE7 & 94,09 & 7,35 & 6,50 & 15.02 .06 & 9,98 \\
\hline 551 & LYO & 552078AF4 & 95,96 & 7,90 & 7,55 & 15.02 .26 & 30,27 \\
\hline 552 & MAG & $559424 \mathrm{AE} 6$ & 103,00 & 9,73 & 10,75 & 15.11 .98 & 3.63 \\
\hline 553 & MAG & $559424 \mathrm{AE} 6$ & 100,75 & 10,40 & 10,75 & 15.11 .98 & 2,62 \\
\hline 554 & MKG & 561226AA9 & 96,37 & 7,29 & 6,75 & 15.09 .05 & 9,55 \\
\hline 555 & MKG & $561226 \mathrm{AB} 7$ & 92,85 & 7,43 & 6,50 & 15.11 .07 & 11,75 \\
\hline
\end{tabular}




\begin{tabular}{|c|c|c|c|c|c|c|c|}
\hline Lfd. Nr. & Ticker & CUSIP & Ask Kurs in Dollar & ISMA Rendite in \% & Kupon in \% & Falligkeit & Restlaufzeit [Jahren] \\
\hline 556 & MAR & 571900AB5 & 98,61 & 8,08 & 7,88 & 15.04 .05 & 10,14 \\
\hline 557 & MAR & $571900 \mathrm{AB} 5$ & 104,34 & 7,21 & 7,88 & 15.04 .05 & 9,13 \\
\hline 558 & MAR & $571900 \mathrm{AC3}$ & 98,07 & 7,38 & 7,13 & 01.06 .07 & 11,29 \\
\hline 559 & MAR & 571900AD1 & 93,34 & 7,54 & 6,75 & 01.12 .09 & 13,83 \\
\hline 560 & MAS & 574599AK2 & 106,52 & 7,69 & 9,00 & 01.10 .01 & 6,55 \\
\hline 561 & MAS & 574599AK2 & 109,25 & 6,93 & 9,00 & 01.10 .01 & 5,54 \\
\hline 562 & MAS & 574599AM8 & 97,04 & 7,43 & 6,63 & 15.09 .99 & 4,47 \\
\hline 563 & MAS & 574599AM8 & 99,88 & 6,66 & 6,63 & 15.09 .99 & 3,46 \\
\hline 564 & MAS & 574599AN6 & 86,50 & 8,60 & 7,13 & 15.08 .13 & 18,59 \\
\hline 565 & MAS & 574599AN6 & 94,62 & 7,69 & 7,13 & 15.08 .13 & 17,58 \\
\hline 566 & MAS & 574599AP1 & 89,04 & 7,94 & 6,13 & 15.09 .03 & 8,53 \\
\hline 567 & MAS & 574599API & 94,50 & 7,09 & 6,13 & 15.09 .03 & 7,52 \\
\hline 568 & MAS & 574599AR7 & 101,95 & 6,45 & 6,63 & 15.04 .18 & 19,28 \\
\hline 569 & MAS & 574599AS5 & 97,02 & 6,17 & 5,75 & 15.10 .08 & 9,64 \\
\hline 570 & $\mathrm{MCD}$ & 580135BF7 & 110,02 & 7,77 & 8,88 & 01.04 .11 & 16,18 \\
\hline 571 & MCD & 580135BF7 & 114,83 & 7,23 & 8,88 & 01.04 .11 & 15,18 \\
\hline 572 & $\mathrm{MCD}$ & 580135BK6 & 102,08 & 7,49 & 7,80 & 01.10 .04 & 9,59 \\
\hline 573 & MCD & 580135BK6 & 105,67 & 6,90 & 7,80 & 01.10 .04 & 8,58 \\
\hline 574 & $\mathrm{MCD}$ & 580135BR1 & 101,72 & 5,03 & 8,38 & 29.10 .99 & 0,55 \\
\hline 575 & MHP & $580645 \mathrm{AC} 3$ & 109,02 & 7,36 & 9,43 & 01.09 .00 & 5,45 \\
\hline 576 & MHP & $580645 \mathrm{AC} 3$ & 110,22 & 6,69 & 9,43 & 01.09 .00 & 4,44 \\
\hline 577 & MEA & 582834ALI & 97,26 & 7,27 & 6,60 & 01.03 .02 & 4,95 \\
\hline 578 & MEA & 582834AM9 & 95,11 & 7,84 & 7,35 & 01.03 .17 & 20,17 \\
\hline 579 & MEA & $58283 \mathrm{KAB} 7$ & 121,76 & 6,48 & 8,39 & 15.03 .20 & 21,23 \\
\hline 580 & MEA & 58283KAC5 & 107,57 & 5,21 & 7,75 & 02.08 .02 & 3,35 \\
\hline 581 & MEA & 58283KAD3 & 107,42 & 5,21 & 7,70 & 03.08 .02 & 3,35 \\
\hline 582 & MEA & 58283KAE1 & 108,70 & 5,85 & 7,79 & 06.08 .04 & 5,39 \\
\hline 583 & MEA & 58283KAF8 & 103,06 & 5,07 & 7,45 & 23.08 .00 & 1,38 \\
\hline 584 & MEA & 58283KAG6 & 107,53 & 5,24 & 7,72 & 23.08 .02 & 3,41 \\
\hline 585 & MEA & $58283 \mathrm{KAH} 4$ & 106,30 & 5,28 & 7,30 & 30.09 .02 & 3,51 \\
\hline 586 & MRK & 589331AC1 & 86,97 & 7,39 & 6,30 & 01.01 .26 & 30,14 \\
\hline 587 & MRK & $589331 \mathrm{ACl}$ & 84,46 & 7,64 & 6,30 & 01.01 .26 & 29,13 \\
\hline 588 & MIL & $601073 \mathrm{AA} 7$ & 99,31 & 7,37 & 7,20 & 01.04 .02 & 5,03 \\
\hline 589 & MIL & $601073 \mathrm{AB} 5$ & 98,58 & 7,71 & 7,50 & 01.04 .07 & 10,11 \\
\hline 590 & MTC & $611662 \mathrm{AJ} 6$ & 108,56 & 7,88 & 8,88 & 15.12 .09 & 14,87 \\
\hline 591 & MTC & $611662 \mathrm{AJ} 6$ & 112,14 & 7,44 & 8,88 & 15.12 .09 & 13,86 \\
\hline 592 & MTC & $611662 \mathrm{AJ} 6$ & 109,88 & 7,64 & 8,88 & 15.12 .09 & 12,85 \\
\hline 593 & MTC & 611662AM9 & 106,38 & 8,11 & 8,70 & 15.10 .21 & 26,88 \\
\hline 594 & MTC & 611662AM9 & 110,07 & 7,79 & 8,70 & 15.10 .21 & 25,87 \\
\hline 595 & MTC & 611662AM9 & 108,76 & 7,89 & 8,70 & 15.10 .21 & 24,86 \\
\hline 596 & MTC & 611662AN7 & 93,74 & 7,47 & 6,00 & 01.07 .00 & 5,28 \\
\hline 597 & MTC & $611662 \mathrm{AN7}$ & 97,13 & 6,79 & 6,00 & 01.07 .00 & 4,27 \\
\hline 598 & MTC & $611662 \mathrm{AN} 7$ & 97,06 & 7,04 & 6,00 & 01.07 .00 & 3,26 \\
\hline 599 & MTC & $611662 \mathrm{AS6}$ & 101,37 & 6,64 & 6,75 & 15.12 .27 & 30,10 \\
\hline 600 & MTC & 611662 AS6 & 100,94 & 6,68 & 6,75 & 15.12 .27 & 29,09 \\
\hline 601 & MTC & 611662AV9 & 101,10 & 4,92 & 5,38 & 01.12 .01 & 2,67 \\
\hline 602 & MTC & 61167HAS2 & 107,30 & 4,73 & 9,00 & 07.02 .01 & 1,85 \\
\hline 603 & MTC & 61167HAT0 & 112,24 & 5,36 & 8,95 & 10.02 .03 & 3,88 \\
\hline 604 & MTC & 61167HAU7 & 114,40 & 5,85 & 8,85 & 15.02 .05 & 5,93 \\
\hline 605 & MTC & 61167HAV5 & 111,79 & 5,81 & 8,85 & 17.02 .04 & 4,92 \\
\hline 606 & MTC & 61167HAW3 & 109,96 & 4,98 & 8,80 & 15.02 .02 & 2,88 \\
\hline 607 & MTC & $61167 \mathrm{HAZ6}$ & 107,59 & 4,68 & 8,95 & 05.03 .01 & 1,92 \\
\hline 608 & MTC & $61167 \mathrm{HBE} 2$ & 107,76 & 4,65 & 8,85 & 02.04 .01 & 2,00 \\
\hline 609 & MTC & 61167HBG7 & 107,92 & 4,70 & 8,75 & 15.05 .01 & 2,12 \\
\hline 610 & MTC & $61167 \mathrm{HBH} 5$ & 101,12 & 5,88 & 6,11 & 03.02 .05 & 5,89 \\
\hline 611 & MTC & $61167 \mathrm{HBJ}$ & 100,76 & 6,10 & 6,21 & 05.02 .08 & 8,94 \\
\hline 612 & MTC & $61167 \mathrm{HBK} 8$ & 100,71 & 6,40 & 6,47 & 26.02 .18 & 19,15 \\
\hline 613 & MTC & $61167 \mathrm{HBL} 6$ & 100,40 & 6,10 & 6,16 & 27.02 .08 & 9,00 \\
\hline
\end{tabular}




\begin{tabular}{|c|c|c|c|c|c|c|c|}
\hline Lfd. Nr. & Ticker & CUSIP & Ask Kurs in Dollar & ISMA Rendite in $\%$ & Kupon in \% & Fălligkeit & Restlaufzeit [Jahren] \\
\hline 614 & MOT & 620076AF6 & 99,60 & 7,65 & 7,60 & 01.01 .07 & 11,88 \\
\hline 615 & MOT & 620076AF6 & 104,08 & 7,05 & 7,60 & 01.01 .07 & 10,87 \\
\hline 616 & MOT & 620076AF6 & 101,40 & 7,39 & 7,60 & 01.01 .07 & 9,86 \\
\hline 617 & MOT & 620076AG4 & 90,18 & 7,72 & 6,50 & 01.03 .08 & 13,06 \\
\hline 618 & MOT & 620076AG4 & 95.01 & 7,13 & 6,50 & 01.03 .08 & 12,05 \\
\hline 619 & MOT & $620076 \mathrm{AG4}$ & 92,95 & 7,46 & 6,50 & 01.03 .08 & 11,04 \\
\hline 620 & MOT & 620076AH2 & 97,70 & 7,70 & 7,50 & 15.05 .25 & 28,49 \\
\hline 621 & MOT & 620076AN9 & 97,73 & 6,12 & 5,80 & 15.10 .08 & 9,64 \\
\hline 622 & NYT & 650094BL8 & 105,60 & 7,99 & 8,63 & 15.11 .10 & 15,80 \\
\hline 623 & NYT & $650094 \mathrm{BLB}$ & 110,99 & 7,38 & 8,63 & 15.11 .10 & 14,79 \\
\hline 624 & NYT & 650094BL8 & 111,75 & 7,19 & 8,63 & 15.11 .10 & 12,77 \\
\hline 625 & \begin{tabular}{|l|} 
NYT \\
\end{tabular} & 650094BS3 & 91,51 & 7,75 & 6,50 & 01.03 .05 & 10,01 \\
\hline 626 & NYT & 650094BS3 & 96,08 & 7,10 & 6,50 & 01.03 .05 & 9,00 \\
\hline 627 & NYT & 650094BS3 & 101,63 & 6,20 & 6,50 & 01.03 .05 & 6,98 \\
\hline 628 & NYT & $650094 \mathrm{BS} 3$ & 101,25 & 6,24 & 6,50 & 01.03 .05 & 5,97 \\
\hline 629 & NYT & $650094 \mathrm{BTI}$ & 89,76 & 8,09 & 7,00 & 01.05 .13 & 18,30 \\
\hline 630 & NYT & $650094 \mathrm{BTI}$ & 95,38 & 7,48 & 7,00 & 01.05 .13 & 17,29 \\
\hline 631 & NYT & $650094 \mathrm{BTI}$ & 101,13 & 6,87 & 7,00 & 01.05 .13 & 14,25 \\
\hline 632 & NYT & 650094 BV6 & 91,00 & 7,94 & 7,00 & 15.06 .13 & 18,42 \\
\hline 633 & NYT & $650094 \mathrm{BV} 6$ & 97,25 & 7,28 & 7,00 & 15.06 .13 & 17,41 \\
\hline 634 & NYT & 650094BV6 & 100,13 & 6,99 & 7,00 & 15.06 .13 & 15,39 \\
\hline 635 & NYT & 650094BV6 & 101,00 & 6,89 & 7,00 & 15.06 .13 & 14,38 \\
\hline 636 & NYT & $650094 \mathrm{BX} 2$ & 94,34 & 7,18 & 5,25 & 01.09 .98 & 3,42 \\
\hline 637 & NYT & $650094 \mathrm{BX2}$ & 97,34 & 6,48 & 5,25 & 01.09 .98 & 2,41 \\
\hline 638 & NYT & $650094 \mathrm{BX2}$ & 99,53 & 6.52 & 5,25 & 01.09 .98 & 0,39 \\
\hline 639 & NYT & $650094 \mathrm{BYO} 0$ & 89,23 & 7,64 & 5,88 & 01.09 .03 & 8,49 \\
\hline 640 & NYT & $650094 \mathrm{BY} 0$ & 93,61 & 7,00 & 5,88 & 01.09 .03 & 7,48 \\
\hline 641 & NYT & 650094BY0 & 98,75 & 6,15 & 5,88 & 01.09 .03 & 5,46 \\
\hline 642 & NYT & $650094 \mathrm{BZ7}$ & 87,46 & 7,66 & 5,63 & 01.11 .03 & 8,66 \\
\hline 643 & NYT & $650094 \mathrm{BZ7}$ & 92,25 & 6,96 & 5,63 & 01.11 .03 & 7,65 \\
\hline 644 & NYT & $650094 \mathrm{BZ7}$ & 99,38 & 5,78 & 5,63 & 01.11 .03 & 4,62 \\
\hline 645 & NYT & $650094 \mathrm{CDS}$ & 98,50 & 6,31 & 6,13 & 15.01 .10 & 11,93 \\
\hline 646 & NYT & 650094CDS & 100,38 & 6,08 & 6,13 & 15.01 .10 & 10,91 \\
\hline 647 & NYT & 650094CE3 & 91,75 & 7,55 & 6,25 & 15.02 .04 & 8,96 \\
\hline 648 & NYT & 650094 CE 3 & 95,38 & 7,03 & 6,25 & 15.02 .04 & 7,95 \\
\hline 649 & NYT & 650094CE3 & 101,00 & 6,04 & 6,25 & 15.02 .04 & 5,92 \\
\hline 650 & NYT & 650094CE3 & 101,38 & 5,92 & 6,25 & 15.02 .04 & 491 \\
\hline 651 & NYT & $650094 \mathrm{CH} 6$ & 98,37 & 6,22 & 6,00 & 15.04 .08 & 10,15 \\
\hline 652 & NYT & $650094 \mathrm{CH} 6$ & 99,25 & 6,11 & 6,00 & 15.04 .08 & 9,14 \\
\hline 653 & NYT & $650094 \mathrm{CJ} 2$ & 98,69 & 6,60 & 6,50 & 15.04 .28 & 30,44 \\
\hline 654 & NYT & $650094 \mathrm{CJ} 2$ & 98,81 & 6,59 & 6,50 & 15.04 .28 & 29,43 \\
\hline 655 & NWL & 65119QAJ2 & 100,23 & 4,46 & 7,83 & 14.05 .99 & 0,08 \\
\hline 656 & NWL & 651190AW3 & 94,76 & 7,62 & 6,75 & 08.06 .05 & 8,27 \\
\hline 657 & NWL & $65119 \mathrm{QAW} 3$ & 94,76 & 7,62 & 6,75 & 08.06 .05 & 8,27 \\
\hline 658 & NWL & 65119QAXI & 95,43 & 7,41 & 6,33 & 10.06 .02 & 5,23 \\
\hline 659 & NWL & 65119QAY9 & 95,73 & 7,41 & 6,40 & 10.06 .02 & 5,23 \\
\hline 660 & NWL & 651190AZ6 & 97,18 & 7,17 & 6,18 & 11.07 .00 & 3,29 \\
\hline 661 & NWL & $651190 \mathrm{QBB} 8$ & 96,80 & 7,20 & 6,15 & 18.10 .00 & 3,56 \\
\hline 662 & NWL & 65119QBD4 & 96,22 & 7,21 & 6,00 & 22.11 .00 & 3,66 \\
\hline 663 & NWL & $65119 \mathrm{QBE} 2$ & 95,50 & 6,70 & 6,35 & 15.07 .28 & 29,68 \\
\hline 664 & NWL & $651190 \mathrm{QBG} 7$ & 100,25 & 5,63 & 5,70 & 22.09 .03 & 4,51 \\
\hline 665 & NWL & $65119 \mathrm{QBHS}$ & 99,02 & 5,67 & 5,42 & 21.10 .03 & 4,59 \\
\hline 666 & NWL & $65119 \mathrm{QBJ1}$ & 98,99 & 5,97 & 5,78 & 17.11 .05 & 6,69 \\
\hline 667 & NSC & 655844AA6 & 111,15 & 7,97 & 9,00 & 01.03 .21 & 26,24 \\
\hline 668 & NSC & $655844 \mathrm{AA} 6$ & 117,26 & 7,46 & 9,00 & 01.03 .21 & 25,24 \\
\hline 669 & NSC & $655844 \mathrm{AA} 6$ & 112,30 & 7,85 & 9,00 & 01.03 .21 & 24,23 \\
\hline 670 & NSC & $655844 \mathrm{AB} 4$ & 102,45 & 7,49 & 7,88 & 15.02 .04 & 8,96 \\
\hline 671 & NSC & $655844 \mathrm{AB} 4$ & 106,05 & 6,86 & 7,88 & 15.02 .04 & 7,95 \\
\hline
\end{tabular}




\begin{tabular}{|c|c|c|c|c|c|c|c|}
\hline Lfd. Nr. & Ticker & CUSIP & Ask Kurs in Dollar & ISMA Rendite in $\%$ & Kupon in \% & Falligkeit & Restlaufzeit [Jahren] \\
\hline 672 & NSC & $655844 \mathrm{AB} 4$ & 102,88 & 7,33 & 7,88 & 15.02 .04 & 6,94 \\
\hline 673 & NSC & 655855BT0 & 102,85 & 5,95 & 6,50 & 01.08 .04 & $\dot{0}, 39$ \\
\hline 674 & NSC & 655855BT0 & 103,35 & 5,75 & 6,50 & 01.08 .04 & 5,38 \\
\hline 675 & NSC & 655855 CT9 & 102,86 & 5,88 & 7,18 & 15.09 .00 & 2,46 \\
\hline 676 & NSC & 655855CT9 & 103,00 & 4,94 & 7,18 & 15.09 .00 & 1,44 \\
\hline 677 & NSC & $655855 \mathrm{CU} 6$ & 104,36 & 5,89 & 7,32 & 15.09 .01 & 3,47 \\
\hline 678 & NSC & 655855CU6 & 105,71 & 4,78 & 7,32 & 15.09 .01 & 2,46 \\
\hline 679 & NSC & $655855 \mathrm{CV} 4$ & 106,00 & 5,88 & 7,45 & 15.09 .02 & 4,48 \\
\hline 680 & NSC & $655855 \mathrm{CV} 4$ & 107,06 & 5,16 & 7,45 & 15.09 .02 & 3,47 \\
\hline 681 & NSC & $655855 \mathrm{CW} 2$ & 107,43 & 5,90 & 7,53 & 15.09 .03 & 5,50 \\
\hline 682 & NSC & $655855 \mathrm{CW} 2$ & 107,62 & 5,56 & 7,53 & 15.09 .03 & 4,49 \\
\hline 683 & NSC & $655855 \mathrm{CX} 0$ & 108,41 & 5,95 & 7,55 & 15.09 .04 & 6,51 \\
\hline 684 & NSC & $655855 \mathrm{CX} 0$ & 108,27 & 5,75 & 7,55 & 15.09 .04 & 5,50 \\
\hline 685 & NSC & $655855 \mathrm{CY} 8$ & 109,49 & 5,99 & 7,60 & 15.09 .05 & 7,53 \\
\hline 686 & NSC & $655855 \mathrm{CY} 8$ & 109,56 & 5,79 & 7,60 & 15.09 .05 & 6,52 \\
\hline 687 & NSC & $655855 \mathrm{CZ5}$ & 110,54 & 6,03 & 7,65 & 15.09 .06 & 8,54 \\
\hline 688 & NSC & $655855 \mathrm{CZ5}$ & 110,80 & 5,83 & 7,65 & 15.09 .06 & 7,53 \\
\hline 689 & NSC & $655855 \mathrm{DA} 9$ & 111,54 & 6,07 & 7,70 & 15.09 .07 & 9,56 \\
\hline 690 & NSC & $655855 \mathrm{DA} 9$ & 112,02 & 5,87 & 7,70 & 15.09 .07 & 8,54 \\
\hline 691 & NSC & $655855 \mathrm{DB} 7$ & 112,42 & 6,10 & 7,73 & 15.09 .08 & 10,57 \\
\hline 692 & NSC & $655855 \mathrm{DB} 7$ & 113,04 & 5,90 & 7,73 & 15.09 .08 & 9,56 \\
\hline 693 & NSC & $655855 \mathrm{DB} 7$ & 112,42 & 6,10 & 7,73 & 15.09 .08 & 10,57 \\
\hline 694 & NSC & $655855 \mathrm{DC} 5$ & 113,23 & 6,12 & 7,75 & 15.09 .09 & 11,59 \\
\hline 695 & NSC & $655855 \mathrm{DC} 5$ & 113,92 & 5,94 & 7,75 & 15.09 .09 & 10,58 \\
\hline 696 & NOC & $666807 \mathrm{AD} 4$ & 101,82 & 8,34 & 8,63 & 15.10 .04 & 9,63 \\
\hline 697 & NOC & $666807 \mathrm{AD} 4$ & 107,13 & 7,47 & 8,63 & 15.10 .04 & 8,62 \\
\hline 698 & NOC & 666807AD4 & 105,70 & 7,61 & 8,63 & 15.10 .04 & 7,61 \\
\hline 699 & NOC & 666807AP7 & 109,61 & 6,81 & 7,75 & 01.03 .16 & 18,14 \\
\hline 700 & NOC & 666807AP7 & 106,37 & 7,10 & 7,75 & 01.03 .16 & 17,13 \\
\hline 701 & OLN & $680665 \mathrm{AB} 2$ & 104,06 & 7,41 & 9,50 & 15.06 .97 & 2,19 \\
\hline 702 & OLN & $680665 \mathrm{AB2}$ & 103,67 & 6,14 & 9,50 & 15.06 .97 & 1,18 \\
\hline 703 & OLN & $680665 \mathrm{AB} 2$ & 100,54 & 5,86 & 9,50 & 15.06 .97 & 0,17 \\
\hline 704 & OLN & $680665 \mathrm{ACO}$ & 101,18 & 7,78 & 8,00 & 15.06 .02 & 7,26 \\
\hline 705 & OLN & $680665 \mathrm{ACO}$ & 105,27 & 6,93 & 8,00 & 15.06 .02 & 6,25 \\
\hline 706 & OLN & $680665 \mathrm{ACO}$ & 102,65 & 7,37 & 8,00 & 15.06 .02 & 5,24 \\
\hline 707 & OLN & $68066 \times \mathrm{XAA}^{3}$ & 102,91 & 6,52 & 7,11 & 01.06 .05 & 6,22 \\
\hline 708 & PPG & $693506 \mathrm{AQ0}$ & 109,65 & 8,10 & 9,00 & 01.05 .21 & 26,41 \\
\hline 709 & PPG & $693506 \mathrm{AQ0}$ & 113,49 & 7,77 & 9,00 & 01.05 .21 & 25,41 \\
\hline 710 & PPG & $693506 \mathrm{AQ0}$ & 111,99 & 7,88 & 9,00 & 01.05 .21 & 24,39 \\
\hline 711 & PPG & 693506AR8 & 97,69 & 7,22 & 6,88 & 01.08 .05 & 9,43 \\
\hline 712 & PPG & 693506AR8 & 96,54 & 7,44 & 6,88 & 01.08 .05 & 8,42 \\
\hline 713 & PPG & 693506AT4 & 96,07 & 7,23 & 6,25 & 15.02 .02 & 4,91 \\
\hline 714 & PH & $701094 \mathrm{AFI}$ & 96,52 & 7,71 & 7,30 & 15.05 .11 & 14,29 \\
\hline 715 & PH & 70109 HAD7 & 102,52 & 5,72 & 6,35 & 16.12 .03 & 4,74 \\
\hline 716 & PH & $70109 \mathrm{HAF} 2$ & 100,08 & 5,63 & 5,65 & 15.09 .03 & 4,49 \\
\hline 717 & JCP & $708160 \mathrm{AY} 2$ & 106,56 & 7,06 & 10,00 & 15.10 .97 & 2,53 \\
\hline 718 & JCP & $708160 \mathrm{AY} 2$ & 105,44 & 6,12 & 10,00 & 15.10 .97 & 1,52 \\
\hline 719 & JCP & $708160 \mathrm{BF} 2$ & 98,62 & 7,26 & 6,88 & 15.06 .99 & 4,22 \\
\hline 720 & $\mathrm{JCP}$ & $708160 \mathrm{BF} 2$ & 100,93 & 6,54 & 6,88 & 15.06 .99 & 3,21 \\
\hline 721 & PBY & $713278 \mathrm{ADI}$ & 97,65 & 7,36 & 7,00 & 01.06 .05 & 9,26 \\
\hline 722 & PBY & $713278 \mathrm{AD} 1$ & 95,90 & 7,69 & 7,00 & 01.06 .05 & 8,25 \\
\hline 723 & PBY & 713278 AE9 & 92,54 & 7,89 & 6,63 & 15.05 .03 & 8,19 \\
\hline 724 & PBY & 713278 AE 9 & 96,90 & 7,19 & 6,63 & 15.05 .03 & 7,18 \\
\hline 725 & PBY & 713278 AE9 & 95,41 & 7,58 & 6,63 & 15.05 .03 & 6,17 \\
\hline 726 & PBY & 713278 AE9 & 95,41 & 7,58 & 6,63 & 15.05 .03 & 6,17 \\
\hline 727 & PBY & $713281 \mathrm{AC7}$ & 100,89 & 6,51 & 6,71 & 03.11 .04 & 5,64 \\
\hline 728 & PBY & $713281 \mathrm{ADS}$ & 100,71 & 6,52 & 6,67 & 05.11 .04 & 5,64 \\
\hline 729 & PBY & $713281 \mathrm{AE} 3$ & 100,76 & 6,46 & 6,65 & 03.03 .04 & 4,96 \\
\hline
\end{tabular}




\begin{tabular}{|c|c|c|c|c|c|c|c|}
\hline Lfd. Nr. & Ticker & CUSIP & Ask Kurs in Dollar & ISMA Rendite in $\%$ & Kupon in \% & Falligkeit & Restlaufzeit [Jahren] \\
\hline 730 & PBY & 713281AF0 & 101,38 & 6,62 & 6,88 & 06.03 .06 & 6,99 \\
\hline 731 & PBY & $713281 \mathrm{AG} 8$ & 100,66 & 6.63 & 6,75 & 10.03 .06 & 7,01 \\
\hline 732 & PBY & $713281 \mathrm{AH} 6$ & 101,49 & 6,65 & 6,92 & 07.07 .06 & 7,34 \\
\hline 733 & PEP & 713448AE8 & 101,48 & 6,66 & 7,63 & 18.12 .98 & 1,70 \\
\hline 734 & PEP & 713448AU2 & 101,64 & 7,20 & 7,75 & 01.10 .98 & 3,50 \\
\hline 735 & PEP & $713448 \mathrm{AU} 2$ & 102,80 & 6,49 & 7,75 & 01.10 .98 & 2,49 \\
\hline 736 & PEP & 713448AU2 & 101,62 & 6,56 & 7,75 & 01.10 .98 & 1,48 \\
\hline 737 & PEP & $713448 \mathrm{AVO}$ & 101,47 & 6,60 & 7,63 & 01.11 .98 & 1,57 \\
\hline 738 & PEP & $713448 \mathrm{AW} 8$ & 100,14 & 6,90 & 7,00 & 15.11 .96 & 1,60 \\
\hline 739 & PEP & $713448 \mathrm{AW} 8$ & 100,72 & 5,70 & 7,00 & 15.11 .96 & 0,59 \\
\hline 740 & PEP & $713448 \mathrm{AZI}$ & 99.71 & 7,03 & 6,88 & 15.05 .97 & 2,10 \\
\hline 741 & PEP & $713448 \mathrm{AZ1}$ & 100,95 & 5,94 & 6,88 & 15.05 .97 & 1,09 \\
\hline 742 & PEP & $713448 \mathrm{AZI}$ & 100,07 & 5,74 & 6,88 & 15.05 .97 & 0,08 \\
\hline 743 & PEP & $713448 \mathrm{BB} 3$ & 96,07 & 7,32 & 6,25 & 01.09 .99 & 4,43 \\
\hline 744 & PEP & $713448 \mathrm{BB} 3$ & 98,89 & 6,62 & 6,25 & 01.09 .99 & 3,43 \\
\hline 745 & PEP & $713448 \mathrm{BB} 3$ & 98,58 & 6,90 & 6,25 & 01.09 .99 & 2,41 \\
\hline 746 & PEP & $713448 \mathrm{BCl}$ & 97,55 & 7,12 & 6,13 & 15.01 .98 & 2,78 \\
\hline 747 & PEP & $713448 \mathrm{BCl}$ & 99,73 & 6,29 & 6,13 & 15.01 .98 & 1,78 \\
\hline 748 & PEP & $713448 \mathrm{BCl}$ & 100,02 & 6,09 & 6,13 & 15.01 .98 & 0,76 \\
\hline 749 & PEP & 71345 LBP2 & 99,78 & 5,66 & 4,60 & 30.06 .96 & 0,21 \\
\hline 750 & PD & 717265 AF9 & 101,20 & 7.52 & 7,75 & 01.01 .02 & 6,80 \\
\hline 751 & $\mathrm{PD}$ & 717265AF9 & 104,00 & 6,89 & 7,75 & 01.01 .02 & 5,79 \\
\hline 752 & $\mathrm{PD}$ & 717265 AF9 & 102,14 & 7,20 & 7,75 & 01.01 .02 & 4,78 \\
\hline 753 & PD & $717265 \mathrm{AHS}$ & 102,11 & 5,98 & 6,38 & 01.11 .04 & 6,64 \\
\hline 754 & $\mathrm{PD}$ & $717265 \mathrm{AHS}$ & 102,23 & 5,90 & 6,38 & 01.11 .04 & 5,63 \\
\hline 755 & $\mathrm{MO}$ & $718154 \mathrm{AY} 3$ & 102,27 & 6,83 & 8,88 & 01.07 .96 & 1,22 \\
\hline 756 & MO & $718154 \mathrm{AY} 3$ & 100,63 & 5,53 & 8,88 & 01.07 .96 & 0,21 \\
\hline 757 & $\mathrm{MO}$ & $718154 \mathrm{BA} 4$ & 102,60 & 7,00 & 8,75 & 01.12 .96 & 1,64 \\
\hline 758 & $\mathrm{MO}$ & $718154 \mathrm{BA4}$ & 101,80 & 5,71 & 8,75 & 01.12 .96 & 0,64 \\
\hline 759 & MO & $718154 \mathrm{BD} 8$ & 104,87 & 7,12 & 9,75 & 01.05 .97 & 2,06 \\
\hline 760 & $\mathrm{MO}$ & $718154 \mathrm{BD} 8$ & 103,78 & 5,92 & 9,75 & 01.05 .97 & 1,06 \\
\hline 761 & MO & $718154 \mathrm{BD} 8$ & 100,14 & 5,73 & 9,75 & 01.05 .97 & 0,04 \\
\hline 762 & MO & $718154 \mathrm{BH} 9$ & 104,74 & 7,21 & 9,25 & 01.12 .97 & 2,66 \\
\hline 763 & $\mathrm{MO}$ & $718154 \mathrm{BH} 9$ & 104,58 & 6,22 & 9,25 & 01.12 .97 & 1,65 \\
\hline 764 & $\mathrm{MO}$ & $718154 \mathrm{BH} 9$ & 101,92 & 6,02 & 9,25 & 01.12 .97 & 0,64 \\
\hline 765 & $\mathrm{MO}$ & $718154 \mathrm{BLO}$ & 104,12 & 7,37 & 8,63 & 01.03 .99 & 3,92 \\
\hline 766 & $\mathrm{MO}$ & $718154 \mathrm{BLO}$ & 105,35 & 6,54 & 8,63 & 01.03 .99 & 2,91 \\
\hline 767 & $\mathrm{MO}$ & $718154 \mathrm{BLO}$ & 103,24 & 6,74 & 8,63 & 01.03 .99 & 1,90 \\
\hline 768 & $\mathrm{MO}$ & $718154 \mathrm{BN} 6$ & 103,13 & 7,14 & 8,75 & 15.06 .97 & 2,19 \\
\hline 769 & $\mathrm{MO}$ & $718154 \mathrm{BN} 6$ & 103,03 & 5,98 & 8,75 & 15.06 .97 & 1,18 \\
\hline 770 & MO & $718154 \mathrm{BN} 6$ & 100,44 & 5,77 & 8,75 & 15.06 .97 & 0,17 \\
\hline 771 & $\mathrm{MO}$ & 718154BR7 & 100,01 & 7,37 & 7,38 & 15.02 .99 & 3,88 \\
\hline 772 & $\mathrm{MO}$ & $718154 \mathrm{BR} 7$ & 102,11 & 6,54 & 7,38 & 15.02 .99 & 2,88 \\
\hline 773 & MO & 718154BR7 & 101,08 & 6,73 & 7,38 & 15.02 .99 & 1,86 \\
\hline 774 & MO & 718154BS5 & 100,71 & 7,09 & 7,50 & 15.03 .97 & 1,93 \\
\hline 775 & MO & $718154 \mathrm{BSS}$ & 101,43 & 5,85 & 7,50 & 15.03 .97 & 0,93 \\
\hline 776 & $\mathrm{MO}$ & $718154 \mathrm{BT} 3$ & 101,21 & 7,40 & 7,75 & 01.05 .99 & 4,09 \\
\hline 777 & MO & $718154 \mathrm{BT} 3$ & 103,21 & 6,56 & 7,75 & 01.05 .99 & 3,08 \\
\hline 778 & MO & $718154 \mathrm{BT} 3$ & 101,77 & 6,80 & 7,75 & 01.05 .99 & 2,07 \\
\hline 779 & $\mathrm{MO}$ & $718154 \mathrm{BT3}$ & 103,21 & 6,56 & 7,75 & 01.05 .99 & 3,08 \\
\hline 780 & MO & $718156 \mathrm{LL} 4$ & 99,72 & 6,32 & 6,15 & 11.02 .98 & 1,85 \\
\hline 781 & MO & $718156 \mathrm{LL} 4$ & 100,01 & 6,14 & 6,15 & 11.02 .98 & 0,84 \\
\hline 782 & $\mathrm{MO}$ & $718156 \mathrm{LM} 2$ & 99,70 & 6,33 & 6,15 & 18.02 .98 & 1,87 \\
\hline 783 & $\mathrm{MO}$ & $718156 \mathrm{LM} 2$ & 100,00 & 6,15 & 6,15 & 18.02 .98 & 0,86 \\
\hline 784 & $\mathrm{PCH}$ & $737628 \mathrm{ACl}$ & 104,70 & 8,55 & 9,13 & 01.12 .09 & 14,83 \\
\hline 785 & $\mathrm{PCH}$ & $737628 \mathrm{ACl}$ & 114,00 & 7,47 & 9,13 & 01.12 .09 & 13,83 \\
\hline 786 & $\mathrm{PCH}$ & $737628 \mathrm{ACl}$ & 110,23 & 7,83 & 9,13 & 01.12 .09 & 12,81 \\
\hline 787 & $\mathrm{PCH}$ & $737628 \mathrm{AF} 4$ & 91,78 & 7,77 & 6,95 & 15.12 .15 & 19,95 \\
\hline
\end{tabular}




\begin{tabular}{|c|c|c|c|c|c|c|c|}
\hline Lfd. Nr. & Ticker & CUSIP & Ask Kurs in Dollar & ISMA Rendite in $\%$ & Kupon in \% & Falligkeit & Restlaufzeit [Jahren] \\
\hline 788 & $\mathrm{PCH}$ & 737628AF4 & 89,22 & 8,08 & 6,95 & 15.12 .15 & 18,94 \\
\hline 789 & $\mathrm{PCH}$ & 73763HAF3 & 110,45 & 5,57 & 9,46 & 02.04 .02 & 3,01 \\
\hline 790 & $\mathrm{PCH}$ & 73763HAGI & 111,94 & 5,99 & 9,42 & 04.04 .03 & 4,03 \\
\hline 791 & $\mathrm{PCH}$ & 73763 HAH 9 & 115,48 & 6,55 & 9,35 & 12.04 .06 & 7,10 \\
\hline 792 & $\mathrm{PCH}$ & 73763HAM8 & 119,44 & 6,97 & 8,88 & 01.06 .17 & 18,40 \\
\hline 793 & $\mathrm{PCH}$ & 73763HAN6 & 115,03 & 6,77 & 8,65 & 17.01 .11 & 11,93 \\
\hline 794 & $\mathrm{PCH}$ & 73763HAPI & 118,34 & 7,11 & 8,75 & 14.01 .22 & 23,09 \\
\hline 795 & $\mathrm{PCH}$ & $73763 \mathrm{HAQ} 9$ & 116,41 & 6,80 & 8,75 & 23.01 .12 & 12,96 \\
\hline 796 & $\mathrm{PCH}$ & 73763HAR7 & 116,43 & 6,81 & 8,75 & 01.02 .12 & 12,99 \\
\hline 797 & $\mathrm{PCH}$ & 73763HAS5 & 118,39 & 6,93 & 8,80 & 01.02 .16 & 17,05 \\
\hline 798 & $\mathrm{PCH}$ & 73763HAV8 & 119,71 & 6,99 & 8,89 & 30.01 .18 & 19,07 \\
\hline 799 & $\mathrm{PCH}$ & $73763 \mathrm{HAY} 2$ & 112,79 & 7,13 & 8,27 & 01.08 .22 & 23,64 \\
\hline 800 & PX & 74005 PAB 0 & 98,71 & 7,04 & 6,85 & 15.06 .05 & 9,30 \\
\hline 801 & PX & $74005 \mathrm{PAB} 0$ & 95,84 & 7,54 & 6,85 & 15.06 .05 & 8,29 \\
\hline 802 & PX & 74005 PAC 8 & 93,53 & 7,87 & 6,75 & 01.03 .03 & 7,98 \\
\hline 803 & PX & 74005 PAC 8 & 99,40 & 6,86 & 6,75 & 01.03 .03 & 6,97 \\
\hline 804 & PX & 74005 PAC 8 & 97,12 & 7,36 & 6,75 & 01.03 .03 & 5,96 \\
\hline 805 & $\mathrm{PX}$ & $74005 \mathrm{PAC} 8$ & 99,40 & 6,86 & 6,75 & 01.03 .03 & 6,97 \\
\hline 806 & $\mathrm{PX}$ & 74005 PAD6 & 99,99 & 6,70 & 6,70 & 15.04 .01 & 5,07 \\
\hline 807 & PX & 74005PAD6 & 98,27 & 7,21 & 6,70 & 15.04 .01 & 4,06 \\
\hline 808 & PX & 74005 PAE 4 & 95,06 & 7,64 & 6,90 & 01.11 .06 & 9,69 \\
\hline 809 & PX & 74005PAG9 & 100,27 & 6,09 & 6,15 & 15.04 .03 & 5,07 \\
\hline 810 & PX & 74005PAG9 & 100,41 & 6,03 & 6,15 & 15.04 .03 & 4,06 \\
\hline 811 & PG & $742718 \mathrm{AU} 3$ & 106,71 & 7,71 & 8,50 & 10.08 .09 & 14,52 \\
\hline 812 & $P G$ & $742718 \mathrm{AU} 3$ & 111,23 & 7,17 & 8,50 & 10.08 .09 & 13,51 \\
\hline 813 & PG & $742718 \mathrm{AU3}$ & 108,26 & 7,46 & 8,50 & 10.08 .09 & 12,50 \\
\hline 814 & PG & $742718 \mathrm{AYS}$ & 106,94 & 7,30 & 8,70 & 01.08 .01 & 6,38 \\
\hline 815 & PG & 742718AY5 & 108,66 & 6,72 & 8,70 & 01.08 .01 & 5,37 \\
\hline 816 & PG & 742718AYS & 106,02 & 7,04 & 8,70 & 01.08 .01 & 4,36 \\
\hline 817 & PG & $742718 \mathrm{BA} 6$ & 103,56 & 7,43 & 8,00 & 15.11 .03 & 8,70 \\
\hline 818 & PG & $742718 \mathrm{BA} 6$ & 106,87 & 6,82 & 8,00 & 15.11 .03 & 7,69 \\
\hline 819 & PG & $742718 \mathrm{BA6}$ & 104,00 & 7,22 & 8,00 & 15.11 .03 & 6,68 \\
\hline 820 & PG & $742718 \mathrm{BA} 6$ & 106,87 & 6,82 & 8,00 & 15.11 .03 & 7,69 \\
\hline 821 & PG & $742718 \mathrm{BC} 2$ & 99,89 & 6,90 & 6,85 & 01.06 .97 & 2,15 \\
\hline 822 & PG & $742718 \mathrm{BC} 2$ & 100,95 & 5,95 & 6,85 & 01.06 .97 & 1,14 \\
\hline 823 & PG & $742718 \mathrm{BC} 2$ & 100,12 & 5,68 & 6,85 & 01.06 .97 & 0,13 \\
\hline 824 & PG & $742718 \mathrm{BK} 4$ & 98,83 & 5,55 & 5,25 & 15.09 .03 & 4,49 \\
\hline 825 & PHM & 745867AD3 & 108,30 & 6,85 & 7,63 & 15.10 .17 & 19,79 \\
\hline 826 & PHM & 745867AD3 & 104,85 & 7,15 & 7,63 & 15.10 .17 & 18,78 \\
\hline 827 & OAT & 747402AD7 & 94,85 & 7,93 & 7,47 & 14.03 .24 & 27,31 \\
\hline 828 & OAT & $747402 \mathrm{AJ} 4$ & 102,93 & 7,24 & 7,49 & 02.06 .25 & 26,52 \\
\hline 829 & OAT & 74740FAE6 & 111,30 & 5,20 & 9,00 & 29.07 .02 & 3,34 \\
\hline 830 & OAT & $74740 \mathrm{FAF} 3$ & 100,93 & 5,41 & 8,95 & 27.07 .99 & 0,29 \\
\hline 831 & OAT & $74740 \mathrm{FAH} 9$ & 110,43 & 5,00 & 9,13 & 15.01 .02 & 2,80 \\
\hline 832 & OAT & $74740 \mathrm{FAJ} 5$ & 101,10 & 4,88 & 9,00 & 28.07 .99 & 0,29 \\
\hline 833 & OAT & 74740 FAK2 & 101,12 & 4,89 & 9,00 & 30.07 .99 & 0,30 \\
\hline 834 & \begin{tabular}{|l|} 
OAT \\
\end{tabular} & $74740 \mathrm{FAL} 0$ & 101,14 & 4,90 & 9,00 & 02.08 .99 & 0,31 \\
\hline 835 & OAT & 74740FAM 8 & 100,89 & 4,79 & 8,65 & 15.07 .99 & 0,26 \\
\hline 836 & OAT & 74740FAR7 & 111,53 & 5,22 & 9,05 & 14.08 .02 & 3,38 \\
\hline 837 & OAT & 74740FAV8 & 102,91 & 5,27 & 9,13 & 31.01 .00 & 0,81 \\
\hline 838 & OAT & $74740 \mathrm{FAZ9}$ & 101,46 & 5,01 & 9,10 & 31.08 .99 & 0,39 \\
\hline 839 & OAT & $74740 \mathrm{FBC} 9$ & 101,46 & 5,01 & 9,10 & 01.09 .99 & 0,39 \\
\hline 840 & OAT & $74740 \mathrm{FBJ} 4$ & 115,34 & 5,85 & 9,20 & 15.09 .04 & 5,50 \\
\hline 841 & OAT & $74740 \mathrm{FBN5}$ & 101,77 & 5,09 & 9,13 & 01.10 .99 & 0,47 \\
\hline 842 & OAT & 74740FBR6 & 101,81 & 5,09 & 9,20 & 01.10 .99 & 0,47 \\
\hline 843 & OAT & $74740 \mathrm{FBT} 2$ & 110,09 & 4,90 & 9,25 & 15.10 .01 & 2,54 \\
\hline 844 & OAT & $74740 \mathrm{FBV} 7$ & 101,95 & 5,11 & 9,20 & 15.10 .99 & 0,51 \\
\hline 845 & OAT & 74740 FBWS & 101,87 & 5,12 & 9,05 & 15.10 .99 & 0,51 \\
\hline
\end{tabular}




\begin{tabular}{|c|c|c|c|c|c|c|c|}
\hline Lfd. Nr. & Ticker & CUSIP & Ask Kurs in Dollar & ISMA Rendite in $\%$ & Kupon in \% & Falligkeit & Restlaufzeit [Jahren] \\
\hline 846 & OAT & $74740 \mathrm{FBX} 3$ & 115,74 & 5,86 & 9,25 & 15.10 .04 & 5,59 \\
\hline 847 & OAT & $74740 \mathrm{FCA} 2$ & 104,63 & 5,10 & 9,00 & 17.07 .00 & 1,28 \\
\hline 848 & OAT & $74740 \mathrm{FCB} 0$ & 109,73 & 4,91 & 9,05 & 30.10 .01 & 2,58 \\
\hline 849 & OAT & $74740 \mathrm{FCC} 8$ & 115,33 & 5,86 & 9,15 & 28.10 .04 & 5,62 \\
\hline 850 & OAT & 74740 FCE 4 & 115,59 & 5,86 & 9,20 & 01.11 .04 & 5,63 \\
\hline 851 & OAT & $74740 \mathrm{FCFI}$ & 112,33 & 5,31 & 9,15 & 15.11 .02 & 3,64 \\
\hline 852 & OAT & $74740 \mathrm{FCG} 9$ & 113,31 & 5,58 & 9,15 & 15.07 .03 & 4,31 \\
\hline 853 & OAT & $74740 \mathrm{FCJ} 3$ & 115,80 & 5,86 & 9,23 & 15.11 .04 & 5,67 \\
\hline 854 & OAT & $74740 \mathrm{FCN} 4$ & 111,51 & 5,19 & 9,10 & 15.07 .02 & 3,30 \\
\hline 855 & OAT & $74740 \mathrm{FCP} 9$ & 123.25 & 6,17 & 9,20 & 16.11 .09 & 10,75 \\
\hline 856 & OAT & $74740 \mathrm{FCQ} 7$ & 109,95 & 4,93 & 9,10 & 15.11 .01 & 2,63 \\
\hline 857 & OAT & $74740 \mathrm{FCR} 5$ & 106,10 & 4,92 & 9,00 & 15.11 .00 & 1,61 \\
\hline 858 & OAT & $74740 \mathrm{FCS} 3$ & 115,66 & 5,86 & 9,20 & 15.11 .04 & 5,67 \\
\hline 859 & OAT & $74740 \mathrm{FCTI}$ & 115,26 & 5,87 & 9,10 & 01.12 .04 & 5,72 \\
\hline 860 & OAT & $74740 \mathrm{FCU} 8$ & 102,07 & 5,19 & 8,80 & 22.11 .99 & 0.62 \\
\hline 861 & OAT & $74740 \mathrm{FCV} 6$ & 120,97 & 6,08 & 9,25 & 27.11 .07 & 8,75 \\
\hline 862 & OAT & $74740 \mathrm{FCY} 0$ & 115,38 & 5,87 & 9,13 & 01.12 .04 & 5,72 \\
\hline 863 & OAT & $74740 \mathrm{FCZ7}$ & 123,67 & 6,17 & 9,25 & 25.11 .09 & 10,77 \\
\hline 864 & OAT & 74740 FDB 9 & 123,27 & 6,17 & 9,20 & 23.11 .09 & 10,77 \\
\hline 865 & OAT & $74740 \mathrm{FDC7}$ & 111,89 & 5,33 & 9,00 & 25.11 .02 & 3,67 \\
\hline 866 & OAT & 74740 FDF0 & 109,80 & 4,95 & 9,00 & 28.11 .01 & 2,66 \\
\hline 867 & OAT & 74740 FDG8 & 113,83 & 5,77 & 9,15 & 15.01 .04 & 4,83 \\
\hline 868 & OAT & 74740 FDH 6 & 109,71 & 4,95 & 8,95 & 03.12 .01 & 2,68 \\
\hline 869 & OAT & 74740 FDJ2 & 109,72 & 4,96 & 8,95 & 05.12 .01 & 2,68 \\
\hline 870 & OAT & 74740 FDK 9 & 109,85 & 4,96 & 9,00 & 05.12 .01 & 2,68 \\
\hline 871 & OAT & 74740 FDL 7 & 109,86 & 4,96 & 9,00 & 07.12 .01 & 2.69 \\
\hline 872 & OAT & 74740 FDM5 & 128,85 & 6,32 & 9,25 & 15.12 .14 & 15,90 \\
\hline 873 & OAT & 74740 FDN3 & 113,36 & 5,74 & 9,05 & 15.12 .03 & 4,74 \\
\hline 874 & OAT & 74740 FDQ 6 & 123,84 & 6,18 & 9,25 & 15.01 .10 & 10,91 \\
\hline 875 & OAT & 74740FDR4 & 109,88 & 4,96 & 9,00 & 10.12 .01 & 2,70 \\
\hline 876 & OAT & 74740 FDS2 & 123,95 & 6,17 & 9,28 & 08.12 .09 & 10,81 \\
\hline 877 & OAT & 74740 FDU7 & 114,66 & 5,83 & 9,13 & 15.07 .04 & 5,33 \\
\hline 878 & OAT & 74740 FDW 3 & 132,62 & 6,46 & 9,34 & 12.12 .19 & 20,96 \\
\hline 879 & OAT & 74740 FDX 1 & 109,89 & 4,96 & 9,00 & 12.12 .01 & 2,70 \\
\hline 880 & OAT & $74740 \mathrm{FDZ6}$ & 129,35 & 6,32 & 9,30 & 15.12 .14 & 15,90 \\
\hline 881 & OAT & $74740 \mathrm{FEAO}$ & 123,74 & 6,17 & 9,25 & 15.12 .09 & 10,83 \\
\hline 882 & OAT & 74740FED4 & 109,93 & 4,97 & 9,00 & 17.12 .01 & 2,72 \\
\hline 883 & OAT & $74740 \mathrm{FEE} 2$ & 113,56 & 5,74 & 9,10 & 15.12 .03 & 4,74 \\
\hline 884 & OAT & 74740FEL6 & 104,40 & 5,11 & 8,84 & 14.07 .00 & 1,27 \\
\hline 885 & OAT & $74740 \mathrm{FEM} 4$ & 131,59 & 6,46 & 9,25 & 16.12 .19 & 20,98 \\
\hline 886 & OAT & $74740 \mathrm{FEN} 2$ & 122,78 & 6,18 & 9,13 & 21.12 .09 & 10,84 \\
\hline 887 & OAT & 74740FEP7 & 131,59 & 6,46 & 9,25 & 20.12 .19 & 20,99 \\
\hline 888 & OAT & $74740 \mathrm{FEQS}$ & 109,95 & 4,97 & 9,00 & 20.12 .01 & 2,73 \\
\hline 889 & OAT & $74740 \mathrm{FESI}$ & 122,79 & 6,18 & 9,13 & 22.12 .09 & 10,85 \\
\hline 890 & OAT & 74740FEU6 & 104,27 & 5,11 & 8,70 & 17.07 .00 & 1,28 \\
\hline 891 & OAT & $74740 \mathrm{FEV} 4$ & 87,38 & 7,94 & 6,79 & 01.09 .23 & 26,76 \\
\hline 892 & OAT & $74740 \mathrm{FEW} 2$ & 87,49 & 7,94 & 6,80 & 01.09 .23 & 26,76 \\
\hline 893 & OAT & $74740 \mathrm{FEXO}$ & 87,60 & 7,94 & 6,81 & 01.09 .23 & 26,76 \\
\hline 894 & OAT & 74740FEZS & 89,39 & 7,79 & 6,63 & 09.09 .13 & 16,64 \\
\hline 895 & OAT & 74740 FFA 9 & 90,65 & 7,70 & 6,57 & 09.09 .10 & 13,60 \\
\hline 896 & OAT & $74740 \mathrm{FFB} 7$ & 90,17 & 7,69 & 6,50 & 15.07 .10 & 13,44 \\
\hline 897 & OAT & $74740 \mathrm{FFD} 3$ & 89,18 & 7,94 & 6,96 & 18.01 .24 & 27,15 \\
\hline 898 & OAT & 74740 FFF8 & 88,39 & 7,94 & 6,89 & 08.02 .24 & 27,21 \\
\hline 899 & \begin{tabular}{|l|} 
OAT \\
\end{tabular} & 74740 FFJO & 100,46 & 7,42 & 7,50 & 02.05 .05 & 8,16 \\
\hline 900 & OAT & $74740 \mathrm{FFJ} 8$ & 88,39 & 7,94 & 6,89 & 08.02 .24 & 27,21 \\
\hline 901 & OAT & 74740FFK7 & 105,05 & 6,48 & 7,51 & 02.05 .05 & 6,14 \\
\hline 902 & OAT & 74740 FFL5 & 100,76 & 6,32 & 7,07 & 11.05 .98 & 1,09 \\
\hline 903 & OAT & 74740 FFM 3 & 100,77 & 6,32 & 7,08 & 11.05 .98 & 1,09 \\
\hline
\end{tabular}




\begin{tabular}{|c|c|c|c|c|c|c|c|}
\hline Lfd. Nr. & Ticker & CUSIP & Ask Kurs in Dollar & ISMA Rendite in $\%$ & Kupon in \% & Fălligkeit & Restlaufzeit [Jahren] \\
\hline 904 & OAT & 74740FFNI & 100,11 & 7,74 & 7,75 & 15.07 .21 & 25,61 \\
\hline 905 & OAT & $74740 \mathrm{FFN} 1$ & 98,35 & 7,90 & 7,75 & 15.07 .21 & 24,60 \\
\hline 906 & OAT & 74740FFP6 & 98,16 & 7,93 & 7,77 & 12.05 .25 & 28,48 \\
\hline 907 & OAT & $74740 \mathrm{FFQ} 4$ & 97,94 & 7,33 & 6,91 & 19.05 .03 & 6,18 \\
\hline 908 & OAT & 74740FFR2 & 99,49 & 7,03 & 6,94 & 19.05 .03 & 7,19 \\
\hline 909 & OAT & 74740 FFR2 & 98,09 & 7,33 & 6,94 & 19.05 .03 & 6,18 \\
\hline 910 & OAT & 74740FFS0 & 95,66 & 7,34 & 6,45 & 09.06 .03 & 6,24 \\
\hline 911 & OAT & 74740FFT8 & 100,66 & 6,51 & 6,64 & 13.06 .05 & 6,26 \\
\hline 912 & OAT & 74740FFUS & 96,60 & 7,43 & 6,87 & 14.06 .05 & 8,28 \\
\hline 913 & OAT & 74740FFV3 & 101,73 & 6,51 & 6,85 & 16.06 .05 & 6,26 \\
\hline 914 & OAT & 74740FFWI & 101,57 & 6,51 & 6,82 & 16.06 .05 & 6,26 \\
\hline 915 & OAT & $74740 \mathrm{FFX} 9$ & 98,42 & 7,04 & 6,47 & 21.06 .00 & 3,23 \\
\hline 916 & OAT & $74740 \mathrm{FFY} 7$ & 98,44 & 7,04 & 6,48 & 21.06 .00 & 3,23 \\
\hline 917 & OAT & $74740 \mathrm{FFY} 7$ & 98,44 & 7,04 & 6,48 & 21.06 .00 & 3,23 \\
\hline 918 & OAT & $74740 \mathrm{FGA} 8$ & 95,56 & 7,44 & 6,71 & 13.07 .05 & 8,36 \\
\hline 919 & OAT & 74740 FGB 6 & 99,30 & 7,29 & 7,13 & 14.08 .02 & 5,41 \\
\hline 920 & OAT & $74740 \mathrm{FGB} 6$ & 99,30 & 7,29 & 7,13 & 14.08 .02 & 5,41 \\
\hline 921 & OAT & $74740 \mathrm{FGC4}$ & 100,57 & 7,71 & 7,78 & 15.06 .11 & 14,37 \\
\hline 922 & OAT & $74740 \mathrm{FGD} 2$ & 99,11 & 7,44 & 7,30 & 29.08 .05 & 8,49 \\
\hline 923 & OAT & $74740 \mathrm{FGD} 7$ & 98,44 & 7,04 & 6,48 & 21.06 .00 & 3,23 \\
\hline 924 & OAT & 74740 FGE 0 & 98,99 & 7,44 & 7,28 & 29.08 .05 & 8,49 \\
\hline 925 & OAT & 74740 FGF7 & 96,75 & 7,75 & 7,38 & 15.06 .12 & 15,39 \\
\hline 926 & OAT & 74740 FGG5 & 94,73 & 7,82 & 7,28 & 16.10 .15 & 18,77 \\
\hline 927 & OAT & $74740 \mathrm{FGH} 3$ & 94,86 & 7,46 & 6,63 & 18.10 .05 & 8,63 \\
\hline 928 & OAT & 74740 FGJ9 & 93,57 & 7,49 & 6,48 & 28.02 .06 & 9,00 \\
\hline 929 & OAT & 74740 FGK6 & 93,95 & 7,48 & 6,54 & 28.02 .06 & 9,00 \\
\hline 930 & OAT & $74740 \mathrm{FGL} 4$ & 94,28 & 7,95 & 7,44 & 02.03 .26 & 29,30 \\
\hline 931 & OAT & 74740 FGM2 & 94,39 & 7,95 & 7,45 & 02.03 .26 & 29,30 \\
\hline 932 & OAT & $74740 \mathrm{FGNO}$ & 97,27 & 7,83 & 7,55 & 22.03 .16 & 19,21 \\
\hline 933 & RAL & $751277 \mathrm{AJ3}$ & 102,38 & 6,78 & 9,00 & 15.06 .96 & 1,18 \\
\hline 934 & RAL & $751277 \mathrm{AJ} 3$ & 100,52 & 5,46 & 9,00 & 15.06 .96 & 0,17 \\
\hline 935 & RAL & 751277AM6 & 107,27 & 8,37 & 9,25 & 15.10 .09 & 14,70 \\
\hline 936 & $\mathrm{RAL}$ & 751277AM6 & 115,50 & 7,41 & 9,25 & 15.10 .09 & 13,69 \\
\hline 937 & RAL & 751277AM6 & 111,38 & 7,81 & 9,25 & 15.10 .09 & 12,68 \\
\hline 938 & RAL & 751277AM6 & 118,25 & 6,79 & 9,25 & 15.10 .09 & 10,66 \\
\hline 939 & RAL & 751277AN4 & 111,35 & 8,21 & 9,30 & 01.05 .21 & 24,39 \\
\hline 940 & RAL & $751277 \mathrm{AQ} 7$ & 98,35 & 8,78 & 8,63 & 15.02 .22 & 27,22 \\
\hline 941 & RAL & $751277 \mathrm{AQ7}$ & 108,29 & 7,87 & 8,63 & 15.02 .22 & 26,21 \\
\hline 942 & RAL & $751277 \mathrm{AQ} 7$ & 105,75 & 8,08 & 8,63 & 15.02 .22 & 25,20 \\
\hline 943 & RAL & $751277 \mathrm{AQ} 7$ & 115,50 & 7,23 & 8,63 & 15.02 .22 & 23,18 \\
\hline 944 & RAL & 751277AR5 & 92,85 & 8,82 & 8,13 & 01.02 .23 & 28,19 \\
\hline 945 & RAL & 751277ARS & 102,61 & 7,89 & 8,13 & 01.02 .23 & 27,19 \\
\hline 946 & $\mathrm{RAL}$ & 751277AR5 & 98,82 & 8,23 & 8,13 & 01.02 .23 & 26,18 \\
\hline 947 & RAL & 751277AS3 & 99,68 & 7,90 & 7,88 & 15.06 .25 & 29,59 \\
\hline 948 & RAL & 751277AS3 & 95,81 & 8,26 & 7,88 & 15.06 .25 & 28,58 \\
\hline 949 & $\mathrm{RAL}$ & 751277AU8 & 101,00 & 7,65 & 7,75 & 01.10 .15 & 19,74 \\
\hline 950 & RAL & 751277AU8 & 96,36 & 8,13 & 7,75 & 01.10 .15 & 18,73 \\
\hline 951 & RAL & 75127 MAA9 & 104,49 & 5,73 & 10,18 & 08.05 .00 & 1,08 \\
\hline 952 & RAL & 75127MAE1 & 123,23 & 6,74 & 9,74 & 11.06 .10 & 11,32 \\
\hline 953 & RAL & 75127MAF8 & 122,26 & 6,71 & 9,76 & 30.06 .09 & 10,36 \\
\hline 954 & RAL & $75127 \mathrm{MAH} 4$ & 100,69 & 5,27 & 8,77 & 01.07 .99 & 0,22 \\
\hline 955 & RAL & 75127MALS & 100,67 & 5,27 & 8,66 & 01.07 .99 & 0,22 \\
\hline 956 & RYN & 75507QAK8 & 101,18 & 5,32 & 6,00 & 23.02 .01 & 1,89 \\
\hline 957 & RYN & 75507QAL6 & 98,95 & 6,41 & 6,15 & 02.02 .04 & 4,88 \\
\hline 958 & RLM & 761763AD3 & 107,00 & 7,38 & 9,38 & 15.06 .99 & 4,22 \\
\hline 959 & RLM & $761763 \mathrm{AD} 3$ & 107,71 & 6,62 & 9,38 & 15.06 .99 & 3,21 \\
\hline 960 & RLM & 761763AD3 & 104,77 & 6,95 & 9,38 & 15.06 .99 & 2,20 \\
\hline 961 & RLM & 761763AF8 & 106,62 & 7,89 & 9,00 & 15.08 .03 & 8,44 \\
\hline
\end{tabular}




\begin{tabular}{|c|c|c|c|c|c|c|c|}
\hline Lfd. Nr. & Ticker & CUSIP & Ask Kurs in Dollar & ISMA Rendite in $\%$ & Kupon in \% & Fulligkeit & Restlaufzeit [Jahren] \\
\hline 962 & RLM & 761763AF8 & 110,91 & 7,06 & 9,00 & 15.08 .03 & 7,44 \\
\hline 963 & RLM & $761763 \mathrm{AF} 8$ & 107,45 & 7,50 & 9.00 & 15.08 .03 & 6,43 \\
\hline 964 & $R L M$ & 76176LAA9 & 108,02 & 5,34 & 9,30 & 21.06 .01 & 2,22 \\
\hline 965 & RLM & $76176 \mathrm{LAB} 7$ & 108,04 & 5,34 & 9,31 & 21.06 .01 & 2,22 \\
\hline 966 & RLM & 76176LAD3 & 100,93 & 5,42 & 9,15 & 20.07 .99 & 0,27 \\
\hline 967 & RLM & 76176LAEI & 104,18 & 5,66 & 9,15 & 21.07 .00 & 1,29 \\
\hline 968 & RLM & 76176LAF8 & 112,24 & 6.42 & 9,20 & 21.07 .04 & 5,35 \\
\hline 969 & RLM & $76176 \mathrm{LAG6}$ & 112,07 & 6,42 & 9,15 & 03.08 .04 & 5,38 \\
\hline 970 & RLM & $76176 \mathrm{LAH} 4$ & 104,21 & 5,63 & 9,03 & 07.08 .00 & 1,34 \\
\hline 971 & RLM & $76176 \mathrm{LAH} 4$ & 104,21 & 5,63 & 9,03 & 07.08 .00 & 1,34 \\
\hline 972 & $R L M$ & $76176 \mathrm{LAJ} 0$ & 102,50 & 5,85 & 8,99 & 15.02 .00 & 0,85 \\
\hline 973 & RLM & 76176LALS & 105,46 & 5,47 & 9,15 & 15.11 .00 & 1,61 \\
\hline 974 & RLM & $76176 \mathrm{LAM} 3$ & 105,71 & 5,44 & 9,15 & 05.12 .00 & 1,67 \\
\hline 975 & $R L M$ & 76176LAN1 & 107,65 & 5,30 & 9,25 & 15.05 .01 & 2,12 \\
\hline 976 & $R L M$ & $76176 \mathrm{LAP6}$ & 109,27 & 5,52 & 9,31 & 18.12 .01 & 2,72 \\
\hline 977 & RLM & $76176 \mathrm{LAQ} 4$ & 109,36 & 5,54 & 9,31 & 03.01 .02 & 2,76 \\
\hline 978 & RLM & 76176LAR2 & 110,16 & 5,58 & 9,50 & 21.02 .02 & 2,90 \\
\hline 979 & RLM & 76176 LAS0 & 110.09 & 5,67 & 9,30 & 15.05 .02 & 3,13 \\
\hline 980 & $\mathrm{RLM}$ & 76176LAUS & 106,92 & 5,37 & 9,62 & 10.01 .01 & 1,77 \\
\hline 981 & RLM & $76176 \mathrm{LAV} 3$ & 113,05 & 6,17 & 9,67 & 06.08 .03 & 4,38 \\
\hline 982 & RLM & 76176LAWI & 109,00 & 5,39 & 9,60 & 06.08 .01 & 2,35 \\
\hline 983 & RLM & $76176 \mathrm{LAX9}$ & 113,05 & 6,21 & 9,64 & 15.09 .03 & 4,49 \\
\hline 984 & RLM & $76176 \mathrm{LAY7}$ & 111,42 & 5,77 & 9,60 & 15.08 .02 & 3,39 \\
\hline 985 & RLM & 76176LAZ4 & 111,32 & 5,77 & 9,58 & 07.08 .02 & 3,36 \\
\hline 986 & $R L M$ & $76176 \mathrm{LBA} 8$ & 112,90 & 6,17 & 9,63 & 07.08 .03 & 4,38 \\
\hline 987 & RLM & 76176 LBB 6 & 112.81 & 6,18 & 9,60 & 15.08 .03 & 4,40 \\
\hline 988 & RLM & $76176 \mathrm{LBB} 6$ & 112,81 & 6,18 & 9,60 & 15.08 .03 & 4,40 \\
\hline 989 & RLM & $76176 \mathrm{LBC} 4$ & 115,83 & 6.51 & 9,61 & 08.08 .05 & 6,41 \\
\hline 990 & $R L M$ & 76176 LBD2 & 115,75 & 6,51 & 9,59 & 15.08 .05 & 6,43 \\
\hline 991 & RLM & 76176 LBE0 & 113,59 & 6,42 & 9,48 & 11.08 .04 & 5,41 \\
\hline 992 & RLM & 76176LBX8 & 106,74 & 5,42 & 9,75 & 12.12 .00 & 1,69 \\
\hline 993 & $R L M$ & $761761 . B Y 6$ & 107,16 & 5,37 & 9,75 & 12.01 .01 & 1,78 \\
\hline 994 & $R L M$ & $76176 \mathrm{LBZ3}$ & 106,47 & 5,41 & 9,55 & 15.12 .00 & 1,70 \\
\hline 995 & $R L M$ & $76176 \mathrm{LCA} 7$ & 106,45 & 5,41 & 9,50 & 20.12 .00 & 1,71 \\
\hline 996 & RLM & 76176 LCBS & 112,00 & 5,92 & 9.60 & 20.12 .02 & 3,74 \\
\hline 997 & RLM & $76176 \mathrm{LCC} 3$ & 112,62 & 5,96 & 9,73 & 03.02 .03 & 3,86 \\
\hline 998 & RLM & $76176 \mathrm{LCDI}$ & 113,31 & 6,32 & 9,65 & 26.12 .03 & 4,77 \\
\hline 999 & RLM & 76176 LCE9 & 109,92 & 5,57 & 9,45 & 04.02 .02 & 2,85 \\
\hline 1000 & RLM & 76176 LCF6 & 109,89 & 5,57 & 9,43 & 08.02 .02 & 2,86 \\
\hline 1001 & RLM & $76176 \mathrm{LCG} 4$ & 109,84 & 5,57 & 9,41 & 08.02 .02 & 2,86 \\
\hline 1002 & RLM & $76176 \mathrm{LCH} 2$ & 110,00 & 5,60 & 9,40 & 08.03 .02 & 2,94 \\
\hline 1003 & RLM & 76176LCJ8 & 111,53 & 5,97 & 9,40 & 11.02 .03 & 3,89 \\
\hline 1004 & RLM & $76176 \mathrm{LCK5}$ & 114,46 & 6,47 & 9,49 & 11.02 .05 & 5,92 \\
\hline 1005 & $R L M$ & $76176 \mathrm{LCL} 3$ & 112,49 & 6,38 & 9,43 & 12.02 .04 & 4,90 \\
\hline 1006 & RLM & $76176 \mathrm{LCM1}$ & 112.45 & 6,38 & 9,42 & 12.02 .04 & 4,90 \\
\hline 1007 & RLM & 76176LCN9 & 112,32 & 6,38 & 9,39 & 12.02 .04 & 4,90 \\
\hline 1008 & RLM & $76176 \mathrm{LCP} 4$ & 114,43 & 6,47 & 9,48 & 14.02 .05 & 5.93 \\
\hline 1009 & RLM & $76176 \mathrm{LCQ} 2$ & 114,04 & 6,47 & 9,40 & 15.02 .05 & 5,93 \\
\hline 1010 & RLM & 76176LCRO & 113,99 & 6.47 & 9,39 & 15.02 .05 & 5,93 \\
\hline 1011 & RLM & $76176 \mathrm{LCS} 8$ & 116,10 & 6,56 & 9,50 & 15.03 .06 & 7,02 \\
\hline 1012 & RLM & $76176 \mathrm{LCVI}$ & 111,69 & 6,01 & 9,41 & 17.03 .03 & 3,98 \\
\hline 1013 & RLM & $76176 \mathrm{LCW} 9$ & 114,12 & 6,48 & 9,39 & 15.03 .05 & 6,01 \\
\hline 1014 & RLM & $76176 \mathrm{LCYS}$ & 113,76 & 6,48 & 9,31 & 23.03 .05 & 6,03 \\
\hline 1015 & RLM & $76176 \mathrm{LCZ2}$ & 113,78 & 6,48 & 9,30 & 11.04 .05 & 6,08 \\
\hline 1016 & RLM & 76176 LDA6 & 114,99 & 6,57 & 9,28 & 20.04 .06 & 7,12 \\
\hline 1017 & RLM & $76176 \mathrm{LDB} 4$ & 113,33 & 6,49 & 9,20 & 25.04 .05 & 6,12 \\
\hline 1018 & RLM & $76176 \mathrm{LDB} 4$ & 113,33 & 6,49 & 9,20 & 25.04 .05 & 6,12 \\
\hline 1019 & RLM & $76176 \mathrm{LDC} 2$ & 114,55 & 6,58 & 9,20 & 24.04 .06 & 7,13 \\
\hline
\end{tabular}




\begin{tabular}{|c|c|c|c|c|c|c|c|}
\hline Lfd. Nr. & Ticker & CUSIP & Ask Kurs in Dollar & ISMA Rendite in $\%$ & Kupon in \% & Falligkeit & Restlaufzeit [Jahren] \\
\hline 1020 & RLM & 76176LDD0 & 111,16 & 6,11 & 9,20 & 10.06 .03 & 4,22 \\
\hline 1021 & RLM & 76176LDE8 & 111,10 & 6,18 & 9,15 & 11.08 .03 & 4,39 \\
\hline 1022 & RLM & 76176LDF5 & 111,06 & 6,18 & 9,14 & 11.08 .03 & 4,39 \\
\hline 1023 & RLM & 76176 LDG3 & 107,77 & 5,40 & 9,00 & 15.08 .01 & 2,37 \\
\hline 1024 & RLM & $76176 \mathrm{LDHI}$ & 114,63 & 6,60 & 9,15 & 15.08 .06 & 7,44 \\
\hline 1025 & RLM & 76176LDJ7 & 107,74 & 5,40 & 8,99 & 14.08 .01 & 2,37 \\
\hline 1026 & RLM & $76176 \mathrm{LDK} 4$ & 107,72 & 5,41 & 8,92 & 04.09 .01 & 2,43 \\
\hline 1027 & RLM & $76176 \mathrm{LDL} 2$ & 114,12 & 6,60 & 9,05 & 06.09 .06 & 7,51 \\
\hline 1028 & RLM & 76176LDM0 & 114,43 & 6,60 & 9,10 & 15.09 .06 & 7,53 \\
\hline 1029 & $\mathrm{RLM}$ & $76176 \mathrm{LDN} 8$ & 113,28 & 6,61 & 8,90 & 20.09 .06 & 7,54 \\
\hline 1030 & RLM & $76176 \mathrm{LDP} 3$ & 113,39 & 6,61 & 8,92 & 20.09 .06 & 7,54 \\
\hline 1031 & RLM & $76176 \mathrm{LDQ1}$ & 113,01 & 6,61 & 8,85 & 02.10 .06 & 7,58 \\
\hline 1032 & RLM & 76176LDR9 & 112,52 & 6,61 & 8,76 & 16.10 .06 & 7,62 \\
\hline 1033 & RLM & 76176LDU2 & 109,64 & 6,64 & 8,25 & 16.01 .07 & 7,87 \\
\hline 1034 & RLM & 76176LDV0 & 109,58 & 6,64 & 8,24 & 16.01 .07 & 7,87 \\
\hline 1035 & $R L M$ & 76176LDW8 & 106,93 & 6,42 & 8,04 & 17.05 .04 & 5,17 \\
\hline 1036 & RLM & $76176 \mathrm{LDX} 6$ & 107,35 & 6,44 & 8,09 & 16.08 .04 & 5,42 \\
\hline 1037 & RLM & $76176 \mathrm{LDY} 4$ & 109,22 & 6,64 & 8,18 & 18.01 .07 & 7,88 \\
\hline 1038 & RLM & $76176 \mathrm{LDZl}$ & 108,07 & 6,44 & 8,25 & 16.08 .04 & 5,42 \\
\hline 1039 & RLM & 76176LEB3 & 109,19 & 6,40 & 8,59 & 05.04 .04 & 5,05 \\
\hline 1040 & RLM & $76176 \mathrm{LECl}$ & 109,07 & 6,40 & 8,56 & 08.04 .04 & 5,06 \\
\hline 1041 & $R L M$ & 76176LED9 & 108,67 & 6,40 & 8,46 & 16.04 .04 & 5,08 \\
\hline 1042 & RLM & 76176LEE7 & 111,07 & 6,65 & 8,46 & 16.04 .07 & 8,12 \\
\hline 1043 & $\mathrm{RLM}$ & 76176LEF4 & 108,97 & 6,41 & 8,52 & 03.05 .04 & 5,13 \\
\hline 1044 & RLM & 76176LEG2 & 112,10 & 6,66 & 8,62 & 14.05 .07 & 8,20 \\
\hline 1045 & RLM & 76176LEH0 & 111,61 & 6,66 & 8,54 & 15.05 .07 & 8,20 \\
\hline 1046 & RLM & 76176LEJ6 & 111,23 & 6,66 & 8,48 & 15.05 .07 & 8,20 \\
\hline 1047 & RLM & 76176 LEK3 & 110,37 & 6,66 & 8,34 & 22.05 .07 & 8,22 \\
\hline 1048 & RLM & $76176 \mathrm{LELI}$ & 109,63 & 6,66 & 8,22 & 30.05 .07 & 8,24 \\
\hline 1049 & RLM & 76176LEM9 & 105,06 & 6,38 & 7,63 & 26.01 .04 & 4,86 \\
\hline 1050 & RLM & 76176LEN7 & 105,68 & 6,49 & 7,68 & 28.01 .05 & 5,88 \\
\hline 1051 & RLM & 76176LEP2 & 106,12 & 6,72 & 7,65 & 04.02 .08 & 8,94 \\
\hline 1052 & RLM & 76176LER8 & 101,91 & 6,42 & 6,88 & 17.03 .04 & 5,00 \\
\hline 1053 & RLM & 76176LES6 & 102,05 & 6,51 & 6,93 & 18.03 .05 & 6,01 \\
\hline 1054 & RLM & 76176LET4 & 101,75 & 6,74 & 7,00 & 25.03 .08 & 9,08 \\
\hline 1055 & RLM & 76176LEU1 & 102,47 & 6,52 & 7,02 & 05.05 .05 & 6,15 \\
\hline 1056 & RLM & 76176LEW7 & 100,06 & 5,94 & 6,07 & 19.09 .97 & 0,44 \\
\hline 1057 & RLM & 76176LEZ0 & 100,02 & 5,99 & 6,03 & 14.10 .97 & 0,51 \\
\hline 1058 & ROK & $774347 \mathrm{AK} 4$ & 106,29 & 7,18 & 8,88 & 15.09 .99 & 4,47 \\
\hline 1059 & ROK & $774347 \mathrm{AK} 4$ & 107,09 & 6,52 & 8,88 & 15.09 .99 & 3,46 \\
\hline 1060 & ROK & 774347AK4 & 104,37 & 6,87 & 8,88 & 15.09 .99 & 2,45 \\
\hline 1061 & ROK & 774347AL2 & 104,95 & 7,31 & 8,38 & 15.02 .01 & 5,91 \\
\hline 1062 & ROK & 774347AL2 & 106,88 & 6,68 & 8,38 & 15.02 .01 & 4,91 \\
\hline 1063 & ROK & 774347 AL2 & 104,30 & 7,07 & 8,38 & 15.02 .01 & 3,89 \\
\hline 1064 & ROK & 774347AM0 & 96,30 & 7,41 & 6,75 & 15.09 .02 & 7,52 \\
\hline 1065 & ROK & 774347AM0 & 99,76 & 6,80 & 6,75 & 15.09 .02 & 6,51 \\
\hline 1066 & ROK & 774347AM0 & 97,77 & 7,25 & 6,75 & 15.09 .02 & 5,50 \\
\hline 1067 & ROK & 774347AN8 & 101,49 & 7,03 & 7,63 & 17.02 .98 & 2,88 \\
\hline 1068 & ROK & 774347AN8 & 102,29 & 6,27 & 7,63 & 17.02 .98 & 1,87 \\
\hline 1069 & ROK & 774347AN8 & 101,19 & 6,12 & 7,63 & 17.02 .98 & 0,86 \\
\hline 1070 & ROK & 774347AP3 & 97,78 & 6,96 & 6,63 & 01.06 .05 & 9,26 \\
\hline 1071 & ROK & $774347 \mathrm{AP3}$ & 95,33 & 7,40 & 6,63 & 01.06 .03 & 8,25 \\
\hline 1072 & ROK & $774347 \mathrm{AQ1}$ & 102,20 & 7,55 & 7,88 & 15.02 .05 & 9,97 \\
\hline 1073 & ROK & $774347 \mathrm{AQ1}$ & 106,16 & 6,93 & 7,88 & 15.02 .05 & 8,96 \\
\hline 1074 & ROK & $774347 \mathrm{AQ1}$ & 102,92 & 7,37 & 7,88 & 15.02 .05 & 7,95 \\
\hline 1075 & $\mathrm{ROH}$ & $775371 \mathrm{AHO}$ & 108,35 & 7,04 & 9,88 & 01.09 .00 & 3,43 \\
\hline 1076 & SLE & 80311 TAF9 & 100,84 & 5,04 & 5,75 & 14.07 .00 & 1,27 \\
\hline 1077 & SLE & 80311 TAG7 & 96,26 & 7,01 & 5,70 & 14.07 .00 & 3,29 \\
\hline
\end{tabular}




\begin{tabular}{|c|c|c|c|c|c|c|c|}
\hline Lfd. $\mathrm{Nr}$. & Ticker & CUSIP & Ask Kurs in Dollar & ISMA Rendite in \% & Kupon in \% & Falligkeit & Restlaufzeit [Jahren] \\
\hline 1078 & SLE & 80311TAH5 & 88,72 & 7,60 & 5,75 & 03.09 .03 & 8,50 \\
\hline 1079 & SLE & 80311TAHS & 93,12 & 6,96 & 5,75 & 03.09 .03 & 7,49 \\
\hline 1080 & SLE & 80311TAHS & 92,09 & 7,32 & 5,75 & 03.09 .03 & 6,48 \\
\hline 1081 & SLE & 80311 TAJI & 84,77 & 7,75 & 5,60 & 12.10 .05 & 10,64 \\
\hline 1082 & SLE & $80311 \mathrm{TAJ}$ & 89,68 & 7,12 & 5,60 & 12.10 .05 & 9,63 \\
\hline 1083 & SLE & $80311 \mathrm{TAJ}$ & 88,63 & 7,43 & 5,60 & 12.10 .05 & 8,62 \\
\hline 1084 & SLE & 80311 TAK8 & 94,34 & 6,73 & 5,25 & 20.10 .00 & 4,58 \\
\hline 1085 & SLE & 80311TAK8 & 94,51 & 7,04 & 5,25 & 20.10 .00 & 3,57 \\
\hline 1086 & SLE & 80311TAL6 & 97,44 & 6,56 & 4,75 & 22.10 .98 & 1,54 \\
\hline 1087 & SLE & 80311TAN2 & 97,33 & 6,49 & 5,38 & 16.12 .98 & 2,71 \\
\hline 1088 & SLE & 80311 TAN2 & 98,07 & 6,63 & 5,38 & 16.12 .98 & 1,69 \\
\hline 1089 & SLE & 80311TAP7 & 97,71 & 6,63 & 5,15 & 16.12 .98 & 1,69 \\
\hline 1090 & SLE & 80311 TASI & 96,43 & 6,89 & 4,65 & 14.01 .97 & 1,77 \\
\hline 1091 & SLE & 80311 TASI & 99,23 & 5,74 & 4,65 & 14.01 .97 & 0,76 \\
\hline 1092 & SLE & 80311 TAT9 & 95,15 & 7,04 & 4,90 & 30.10 .97 & 2,57 \\
\hline 1093 & SLE & 80311TAT9 & 98,20 & 6,15 & 4,90 & 30.10 .97 & 1,56 \\
\hline 1094 & SLE & 80311 TAT9 & 99,49 & 5,89 & 4,90 & 30.10 .97 & 0,55 \\
\hline 1095 & SLE & 80311 TAV4 & 91,41 & 7,63 & 6,28 & 23.02 .04 & 8,98 \\
\hline 1096 & SLE & 80311 TAV4 & 95,75 & 6,99 & 6,28 & 23.02 .04 & 7,97 \\
\hline 1097 & SLE & 80311 TAV4 & 94,34 & 7,34 & 6,28 & 23.02 .04 & 6,96 \\
\hline 1098 & SLE & 80311 TAV4 & 95,75 & 6,99 & 6,28 & 23.02 .04 & 7,97 \\
\hline 1099 & SLE & 80311 TAX0 & 98,37 & 6,98 & 6,15 & 16.06 .97 & 2,19 \\
\hline 1100 & SLE & 80311 TAX0 & 100,23 & 5,95 & 6,15 & 16.06 .97 & 1,18 \\
\hline 1101 & SLE & 8031ITAX0 & 100,13 & 5,32 & 6,15 & 16.06 .97 & 0,17 \\
\hline 1102 & SLE & 80311 TAY8 & 102,41 & 6,91 & 7,95 & 09.11 .99 & 2.61 \\
\hline 1103 & SLE & 80311 TBB7 & 101,78 & 7,30 & 7,75 & 03.02 .00 & 4,86 \\
\hline 1104 & SLE & 80311 TBB7 & 103,76 & 6,61 & 7,75 & 03.02 .00 & 3,86 \\
\hline 1105 & SLE & 80311 TBB7 & 102,01 & 6,95 & 7,75 & 03.02 .00 & 2,84 \\
\hline 1106 & SLE & $803111 \mathrm{TBB} 7$ & 101,78 & 7,30 & 7,75 & 03.02 .00 & 4,86 \\
\hline 1107 & SLE & 80311 TBG6 & 93,95 & 7,40 & 6,40 & 09.06 .05 & 8,27 \\
\hline 1108 & SLE & 80311 TBH 4 & 95,40 & 7,40 & 6,64 & 16.06 .05 & 8,29 \\
\hline 1109 & SLE & 80311 TBR2 & 93,79 & 7,29 & 6,30 & 07.11 .05 & 8,69 \\
\hline 1110 & SLE & 80311 TBS0 & 91,81 & 7,29 & 6,00 & 05.12 .05 & 8,77 \\
\hline 1111 & SLE & 80311 TBUS & 91,39 & 7,30 & 5,95 & 09.01 .06 & 8,86 \\
\hline 1112 & SLE & 80311 TBV3 & 89,10 & 7,31 & 5,60 & 23.01 .06 & 8,90 \\
\hline 1113 & SLE & $803111 \mathrm{TCD} 2$ & 100,53 & 5,84 & 5,95 & 20.01 .05 & 5,86 \\
\hline 1114 & SLE & 80311 TCEO & 99,73 & 6,09 & 6,05 & 14.04 .08 & 9,13 \\
\hline 1115 & SLE & 80311TCF7 & 100,36 & 6,10 & 6,15 & 19.06 .08 & 9,32 \\
\hline 1116 & $\mathrm{~s}$ & $81238 \times B W 0$ & 101,49 & 5,14 & 8,27 & 15.10 .99 & 0,51 \\
\hline 1117 & $s$ & $81238 \times \mathrm{XPP} 3$ & 101,06 & 5,00 & 8,15 & 23.08 .99 & 0,36 \\
\hline 1118 & $\mathrm{~s}$ & $81238 \times$ XJF9 & 100,07 & 0,05 & 7,99 & 22.04 .99 & 0,02 \\
\hline 1119 & $\mathrm{~s}$ & $81238 \times N N 2$ & 100,12 & 2,99 & 8,24 & 27.04 .99 & 0,04 \\
\hline 1120 & $\mathbf{S}$ & $81238 \times J P 7$ & 100,12 & 3,22 & 8,22 & 28.04 .99 & 0,04 \\
\hline 1121 & $\mathrm{~s}$ & $81238 \mathrm{XJS1}$ & 100,13 & 3,40 & 8,22 & 29.04 .99 & 0,04 \\
\hline 1122 & $\mathrm{~s}$ & 81238 XJT9 & 100,13 & 3,39 & 8,23 & 29.04 .99 & 0,04 \\
\hline 1123 & $\mathrm{~s}$ & $81238 \times \mathrm{XJV} 4$ & 100,14 & 3,54 & 8,23 & 30.04 .99 & 0,04 \\
\hline 1124 & $\mathrm{~s}$ & $81238 \times \mathrm{XKO}$ & 96,20 & 7,25 & 5,90 & 08.07 .98 & 3,27 \\
\hline 1125 & $\mathrm{~s}$ & $81238 \times \mathrm{XQK0}$ & 98,56 & 6,61 & 5,90 & 08.07 .98 & 2,26 \\
\hline 1126 & $\mathrm{~s}$ & $81238 \times Q K 0$ & 99,39 & 6,43 & 5,90 & 08.07 .98 & 1,25 \\
\hline 1127 & $\mathrm{~S}$ & $81238 \times Q L 8$ & 96,05 & 7,25 & 5,85 & 10.07 .98 & 3,27 \\
\hline 1128 & $\mathrm{~s}$ & $81238 \times Q L 8$ & 98,45 & 6.61 & 5,85 & 10.07 .98 & 2,26 \\
\hline 1129 & S & $81238 \times$ XLL 8 & 99,32 & 6,44 & 5,85 & 10.07 .98 & 1,25 \\
\hline 1130 & $\mathrm{~S}$ & 81238XQR5 & 95,70 & 7,26 & 5,74 & 21.07 .98 & 3,30 \\
\hline 1131 & $\mathrm{~S}$ & $81238 \times$ QRS & 98,18 & 6,62 & 5,74 & 21.07 .98 & 2,29 \\
\hline 1132 & $\mathrm{~s}$ & $81238 \times Q R 5$ & 99,15 & 6,46 & 5,74 & 21.07 .98 & 1,28 \\
\hline 1133 & $\mathrm{~s}$ & $81238 \times R Q 6$ & 95,63 & 7,27 & 5,77 & 25.08 .98 & 3,40 \\
\hline 1134 & $\mathrm{~S}$ & $81238 \times R Q 6$ & 98,13 & 6,64 & 5,77 & 25.08 .98 & 2,39 \\
\hline 1135 & $\mathrm{~S}$ & $81238 \times R Q 6$ & 99,06 & 6,51 & 5,77 & 25.08 .98 & 1,38 \\
\hline
\end{tabular}




\begin{tabular}{|c|c|c|c|c|c|c|c|}
\hline Lfd. Nr. & Ticker & CUSIP & Ask Kurs in Dollar & ISMA Rendite in $\%$ & Kupon in \% & Falligkeit & Restlaufzeit [Jahren] \\
\hline 1136 & $\mathbf{S}$ & $81238 \times$ TG6 & 100,53 & 7,12 & 7,37 & 20.08 .97 & 2,37 \\
\hline 1137 & $\mathbf{S}$ & $81238 \times$ TG6 & 101,42 & 6,25 & 7,37 & 20.08 .97 & 1,36 \\
\hline 1138 & $\mathrm{~S}$ & 81238 XTG6 & 100,52 & 5,79 & 7,37 & 20.08 .97 & 0,35 \\
\hline 1139 & $\mathrm{~S}$ & $81238 \times$ TK7 & 100,51 & 7,12 & 7,36 & 15.08 .97 & 2,36 \\
\hline 1140 & $S$ & $81238 \times T K 7$ & 101,40 & 6,24 & 7,36 & 15.08 .97 & 1,35 \\
\hline 1141 & $\mathbf{S}$ & $81238 \times$ TK7 & 100,50 & 5,78 & 7,36 & 15.08 .97 & 0,34 \\
\hline 1142 & $\mathbf{S}$ & $81238 \times$ TP6 & 101,21 & 7,44 & 7,76 & 25.10 .99 & 4,58 \\
\hline 1143 & $\mathbf{S}$ & 81238 XTP6 & 102,96 & 6,80 & 7,76 & 25.10 .99 & 3,58 \\
\hline 1144 & $\mathbf{S}$ & $81238 \times$ TP6 & 101,86 & 6,94 & 7,76 & 25.10 .99 & 2,56 \\
\hline 1145 & $\mathrm{~S}$ & $81238 \times T Q 4$ & 101,17 & 7,44 & 7,75 & 25.10 .99 & 4,58 \\
\hline 1146 & $\mathbf{S}$ & $81238 \times$ TQ $^{4}$ & 102,93 & 6,80 & 7,75 & 25.10 .99 & 3,58 \\
\hline 1147 & $\mathbf{S}$ & $81238 \times$ TQ $^{4}$ & 101,83 & 6,94 & 7,75 & 25.10 .99 & 2,56 \\
\hline 1148 & $\mathrm{~S}$ & 81238 XTT8 & 100,91 & 7,12 & 7,55 & 15.08 .97 & 2,36 \\
\hline 1149 & $\mathrm{~S}$ & $81238 \times T T 8$ & 101,64 & 6,24 & 7,55 & 15.08 .97 & 1,35 \\
\hline 1150 & $\mathrm{~S}$ & $81238 \times T T 8$ & 100,56 & 5,78 & 7,55 & 15.08 .97 & 0,34 \\
\hline 1151 & $\mathrm{~S}$ & 81238 XTU5 & 101,06 & 7,15 & 7,62 & 03.11 .97 & 2,58 \\
\hline 1152 & $S$ & 81238 XTUS & 101,83 & 6,35 & 7,62 & 03.11 .97 & 1,57 \\
\hline 1153 & $\mathbf{S}$ & 81238 XTU5 & 100,89 & 5,94 & 7,62 & 03.11 .97 & 0,56 \\
\hline 1154 & $\mathrm{~S}$ & 81238 XUC3 & 101,42 & 6,11 & 7,94 & 06.02 .98 & 0,83 \\
\hline 1155 & $\mathrm{~S}$ & 81238 XUE9 & 101,94 & 7,19 & 7,97 & 06.02 .98 & 2,84 \\
\hline 1156 & $\mathrm{~S}$ & 81238 XUE9 & 102,52 & 6,47 & 7,97 & 06.02 .98 & 1,84 \\
\hline 1157 & $\mathrm{~S}$ & 81238 XUE9 & 101,42 & 6,14 & 7,97 & 06.02 .98 & 0,83 \\
\hline 1158 & $\mathrm{~S}$ & 81238 XUM1 & 100,45 & 7,20 & 7,38 & 06.03 .98 & 2,92 \\
\hline 1159 & $S$ & $81238 X U M 1$ & 101,51 & 6,51 & 7,38 & 06.03 .98 & 1,91 \\
\hline 1160 & $\mathrm{~S}$ & 81238 XUM! & 101,04 & 6,15 & 7,38 & 06.03 .98 & 0,90 \\
\hline 1161 & $\mathrm{~S}$ & $81238 \times$ UN9 & 100,49 & 7,21 & 7,40 & 16.03 .98 & 2,95 \\
\hline 1162 & $\mathrm{~S}$ & 81238 XUN9 & 101,57 & 6,51 & 7,40 & 16.03 .98 & 1,94 \\
\hline 1163 & $\mathbf{S}$ & 81238 XUN9 & 101,03 & 6,22 & 7,40 & 16.03 .98 & 0,93 \\
\hline 1164 & $\mathrm{~S}$ & $81238 \times \mathrm{UQ}^{2}$ & 100,46 & 7,21 & 7,39 & 17.03 .98 & 2,95 \\
\hline 1165 & $\mathrm{~S}$ & $81238 \times$ UQ2 & 101,55 & 6,51 & 7,39 & 17.03 .98 & 1,94 \\
\hline 1166 & $\mathrm{~S}$ & $81238 \times U Q 2$ & 101,02 & 6,23 & 7,39 & 17.03 .98 & 0,93 \\
\hline 1167 & $\mathrm{~S}$ & 81238 XUS8 & 100,28 & 7,21 & 7,32 & 20.03 .98 & 2,96 \\
\hline 1168 & $\mathrm{~S}$ & 81238 XUS8 & 101,42 & 6,52 & 7,32 & 20.03 .98 & 1,95 \\
\hline 1169 & $\mathrm{~S}$ & 81238 XUS 8 & 100,96 & 6,23 & 7,32 & 20.03 .98 & 0,94 \\
\hline 1170 & SHW & 824348AH9 & 98,18 & 6,98 & 6,25 & 01.02 .00 & 2,84 \\
\hline 1171 & SHW & 824348 AJ5 & 97,13 & 7,22 & 6,50 & 01.02 .02 & 4,87 \\
\hline 1172 & SOI & 834376AA3 & 101,46 & 6,12 & 6,50 & 15.10 .02 & 4,57 \\
\hline 1173 & SOI & 834376 AA3 & 102,00 & 5,86 & 6,50 & 15.10 .02 & 3,56 \\
\hline 1174 & LUV & $844741 \mathrm{AC2}$ & 105,13 & 7,19 & 9,25 & 15.02 .98 & 2,87 \\
\hline 1175 & LUV & $844741 \mathrm{AC2}$ & 104,91 & 6,35 & 9,25 & 15.02 .98 & 1,86 \\
\hline 1176 & LUV & $844741 \mathrm{AC2}$ & 102,41 & 6,18 & 9,25 & 15.02 .98 & 0,85 \\
\hline 1177 & LUV & 844741 AD0 & 108,42 & 7,66 & 9,40 & 01.07 .01 & 6,29 \\
\hline 1178 & LUV & 844741 AD0 & 110,74 & 6,90 & 9,40 & 01.07 .01 & 5,28 \\
\hline 1179 & LUV & 844741 AD0 & 108,05 & 7,14 & 9,40 & 01.07 .01 & 4,27 \\
\hline 1180 & LUV & 844741 AE 8 & 105,08 & 7,92 & 8,75 & 15.10 .03 & 8,61 \\
\hline 1181 & LUV & 844741 AE8 & 109,59 & 7,08 & 8,75 & 15.10 .03 & 7,61 \\
\hline 1182 & LUV & $844741 \mathrm{AE} 8$ & 107,15 & 7,35 & 8,75 & 15.10 .03 & 6,59 \\
\hline 1183 & LUV & 844741 AF5 & 97,00 & 8,27 & 7,88 & 01.09 .07 & 12,55 \\
\hline 1184 & LUV & 844741 AF5 & 103,69 & 7,39 & 7,88 & 01.09 .07 & 11,54 \\
\hline 1185 & LUV & 844741 AFS & 102,23 & 7,56 & 7,88 & 01.09 .07 & 10,53 \\
\hline 1186 & LUV & $844741 \mathrm{AHI}$ & 99,54 & 8,07 & 8,00 & 01.03 .05 & 10,01 \\
\hline 1187 & LUV & $844741 \mathrm{AHI}$ & 105,18 & 7,20 & 8,00 & 01.03 .05 & 9,00 \\
\hline 1188 & LUV & $844741 \mathrm{AHI}$ & 103,45 & 7,41 & 8,00 & 01.03 .05 & 7,99 \\
\hline 1189 & TRW & $87265 \mathrm{CAA} 8$ & 102,16 & 5,80 & 9,00 & 30.12 .99 & 0,72 \\
\hline 1190 & TRW & $87265 \mathrm{CAC} 4$ & 122,97 & 7,11 & 9,25 & 12.01 .20 & 21,05 \\
\hline 1191 & TRW & 87265 CAD2 & 119,97 & 6,87 & 9,25 & 30.12 .11 & 12,90 \\
\hline 1192 & TRW & 87265 CAPS & 106,24 & 5,32 & 9,00 & 09.02 .01 & 1,85 \\
\hline 1193 & TRW & 87265 CAS9 & 106,39 & 5,30 & 9,00 & 22.02 .01 & 1,89 \\
\hline
\end{tabular}




\begin{tabular}{|c|c|c|c|c|c|c|c|}
\hline Lfd. Nr. & Ticker & CUSIP & Ask Kurs in Dollar & ISMA Rendite in $\%$ & Kupon in \% & Falligkeit & Restlaufzeit [Jahren] \\
\hline 1194 & TRW & 87265 CAT7 & 106,44 & 5,30 & 9,00 & 26.02 .01 & 1,90 \\
\hline 1195 & TRW & $87265 \mathrm{CAV}$ & 98,21 & 6,68 & 6,16 & 27.03 .00 & 4,00 \\
\hline 1196 & TRW & 87265 CAV2 & 92,83 & 7,73 & 6.50 & 17.03 .03 & 8,03 \\
\hline 1197 & TRW & 87265CAV2 & 97,47 & 6,97 & 6,50 & 17.03 .03 & 7,02 \\
\hline 1198 & TRW & $87265 \mathrm{CAV} 2$ & 96,11 & 7,32 & 6,50 & 17.03 .03 & 6,01 \\
\hline 1199 & TRW & 87265CAW0 & 93,29 & 7,72 & 6,57 & 17.03 .03 & 8,03 \\
\hline 1200 & TRW & 87265CAWO & 97,86 & 6,97 & 6,57 & 17.03 .03 & 7,02 \\
\hline 1201 & TRW & 87265CAW0 & 96,44 & 7,32 & 6,57 & 17.03 .03 & 6,01 \\
\hline 1202 & TRW & 87265CAX8 & 93.46 & 7,72 & 6,60 & 18.03 .03 & 8,03 \\
\hline 1203 & TRW & 87265 CAX8 & 98,01 & 6,97 & 6.60 & 18.03 .03 & 7,02 \\
\hline 1204 & TRW & $87265 \mathrm{CAX8}$ & 96,56 & 7,33 & 6,60 & 18.03 .03 & 6,01 \\
\hline 1205 & TRW & 87265 CAY' $6^{\prime}$ & 94,61 & 7.49 & 6,16 & 27.03 .00 & 5,01 \\
\hline 1206 & TRW & $87265 \mathrm{CAY} 6$ & 98,21 & 6.68 & 6.16 & 27.03 .00 & 4,00 \\
\hline 1207 & TRW & $87265 \mathrm{CAZ3}$ & 101,90 & 6,05 & 6,60 & 31.03 .03 & 4,02 \\
\hline 1208 & TRW & $87265 \mathrm{CBA} 7$ & 92,89 & 7,73 & 6.53 & 13.05 .03 & 8,18 \\
\hline 1209 & TRW & $87265 \mathrm{CBA7}$ & 97,54 & 6,98 & 6,53 & 13.05 .03 & 7,18 \\
\hline 1210 & TRW & $87265 \mathrm{CBA} 7$ & 96,11 & 7,34 & 6,53 & 13.05 .03 & 6,16 \\
\hline 1211 & TRW & $87265 \mathrm{CBA} 7$ & 92,89 & 7,73 & 6,53 & 13.05 .03 & 8,18 \\
\hline 1212 & TRW & 87265 CBB5 & 94,01 & 7,50 & 6,06 & 15.05 .00 & 5,15 \\
\hline 1213 & TRW & 87265 CBB5 & 97,74 & 6,71 & 6,06 & 15.05 .00 & 4,14 \\
\hline 1214 & TRW & 87265 CBBS & 97,37 & 7,03 & 6,06 & 15.05 .00 & 3,13 \\
\hline 1215 & TRW & $87265 \mathrm{CBC} 3$ & 92.94 & 7,73 & 6,54 & 19.05 .03 & 8,20 \\
\hline 1216 & TRW & $87265 \mathrm{CBC} 3$ & 97,58 & 6,98 & 6,54 & 19.05 .03 & 7,19 \\
\hline 1217 & TRW & $87265 \mathrm{CBC} 3$ & 96,15 & 7,34 & 6,54 & 19.05 .03 & 6,18 \\
\hline 1218 & TRW & 87265 CBD1 & 100,09 & 5,86 & 5,98 & 31.12 .99 & 0,73 \\
\hline 1219 & TRW & $87265 \mathrm{CBD} 1$ & 100,09 & 5,86 & 5,98 & 31.12 .99 & 0,73 \\
\hline 1220 & TRW & 87265 CBE 9 & 93.08 & 7,74 & 6,58 & 23.06 .03 & 8,30 \\
\hline 1221 & TRW & 87265 CBE9 & 97,74 & 6,99 & 6,58 & 23.06 .03 & 7.29 \\
\hline 1222 & TRIV & 87265 CBE9 & 96,25 & 7,35 & 6,58 & 23.06 .03 & 6,28 \\
\hline 1223 & TRW & $87265 \mathrm{CBF} 6$ & 92,90 & 7,74 & 6,55 & 23.06 .03 & 8,30 \\
\hline 1224 & TRW & 87265 CBF 6 & 97,57 & 6,99 & 6,55 & 23.06 .03 & 7,29 \\
\hline 1225 & \begin{tabular}{|l|} 
TRW \\
\end{tabular} & $87265 \mathrm{CBF} 6$ & 96,11 & 7,35 & 6,55 & 23.06 .03 & 6,28 \\
\hline 1226 & TRW & 87265 CBG 4 & 91,90 & 7,74 & 6,39 & 07.07 .03 & 8,34 \\
\hline 1227 & TRW & $87265 \mathrm{CBG} 4$ & 96,65 & 6,99 & 6,39 & 07.07 .03 & 7,33 \\
\hline 1228 & TRW & $87265 \mathrm{CBG} 4$ & 95,28 & 7,35 & 6,39 & 07.07 .03 & 6,32 \\
\hline 1229 & TRW & $8726.5 \mathrm{CBH} 2$ & 100,78 & 6,17 & 6,38 & 07.07 .03 & 4,29 \\
\hline 1230 & TRW & $87265 \mathrm{CBMI}$ & 94,57 & 7,79 & 6,94 & 30.03 .04 & 9.08 \\
\hline 1231 & TRW & $87265 \mathrm{CBM}$ & 99,45 & 7,03 & 6,94 & 30.03 .04 & 8,07 \\
\hline 1232 & TRW & $87265 \mathrm{CBM} 1$ & 97,69 & 7,37 & 6,94 & 30.03 .04 & 7,06 \\
\hline 1233 & TRW & $87265 \mathrm{CBP} 4$ & 104,31 & 6,67 & 7,37 & 18.04 .07 & 8,13 \\
\hline 1234 & \begin{tabular}{|l|} 
TRW \\
\end{tabular} & 87265CBR0 & 97,49 & 6,75 & 6,38 & 19.05 .08 & 9,23 \\
\hline 1235 & TRW & 87265 CBS8 & 97,07 & 6.76 & 6,32 & 27.05 .08 & 9,25 \\
\hline 1236 & TRW & 87265 CBT6 & 97,00 & 6,76 & 6,31 & 27.05 .08 & 9,25 \\
\hline 1237 & TRW & $87265 \mathrm{CBU} 3$ & 96,94 & 6,76 & 6,30 & 15.05 .08 & 9,22 \\
\hline 1238 & TIN & 879868AB3 & 106,21 & 7,69 & 9,00 & 01.05 .01 & 6,12 \\
\hline 1239 & TIN & $879868 \mathrm{AB3}$ & 109,06 & 6,84 & 9.00 & 01.05 .01 & 5,11 \\
\hline 1240 & TIN & 879868AB3 & 106,34 & 7.16 & 9,00 & 01.05 .01 & 4,10 \\
\hline 1241 & TIN & 879868AD9 & 98,67 & 7,45 & 7,25 & 15.09 .04 & 9,55 \\
\hline 1242 & TIN & 879868AD9 & 101,36 & 7,03 & 7,25 & 15.09 .04 & 8,54 \\
\hline 1243 & TIN & 879868AD9 & 98,92 & 7,44 & 7,25 & 15.09 .04 & 7,53 \\
\hline 1244 & TIN & $87987 \mathrm{HBES}$ & 109,54 & 6,63 & 8,24 & 20.12 .06 & 7,80 \\
\hline 1245 & TIN & $87987 \mathrm{HBF} 2$ & 99,61 & 6.80 & 6,75 & 01.03 .09 & 10,03 \\
\hline 1246 & TXN & 882508 AE4 & 106.67 & 7,57 & 9,00 & 15.03 .01 & 5.99 \\
\hline 1247 & TXN & 882508 AE 4 & 108,76 & 6,86 & 9,00 & 15.03 .01 & 4,98 \\
\hline 1248 & TXN & $882508 A E 4$ & 106,34 & 7,11 & 9,00 & 15.03 .01 & 3,97 \\
\hline 1249 & \begin{tabular}{|l|} 
TXN \\
\end{tabular} & $882508 \mathrm{AFI}$ & 109,11 & 7,72 & 9,25 & 15.06 .03 & 8,28 \\
\hline 1250 & TXN & $882508 \mathrm{AFI}$ & 112,35 & 7,02 & 9,25 & 15.06 .03 & 7,27 \\
\hline 1251 & TXN & $882508 \mathrm{AFI}$ & 109,40 & 7,32 & 9,25 & 15.06 .03 & 6,26 \\
\hline
\end{tabular}




\begin{tabular}{|c|c|c|c|c|c|c|c|}
\hline Lfd. Nr. & Ticker & CUSIP & Ask Kurs in Dollar & ISMA Rendite in $\%$ & Kupon in \% & Fălligkeit & Restlaufzeit [Jahren] \\
\hline 1252 & TXN & 882508 AG9 & 106,06 & 7,95 & 8,75 & 01.04 .07 & 12,13 \\
\hline 1253 & TXN & 882508 AG9 & 111,00 & 7,28 & 8,75 & 01.04 .07 & 11,12 \\
\hline 1254 & \begin{tabular}{|l|} 
TXN \\
\end{tabular} & $882508 \mathrm{AG} 9$ & 108,43 & 7,53 & 8,75 & 01.04 .07 & 10,11 \\
\hline 1255 & TXN & $882508 \mathrm{AH} 7$ & 92,33 & 7,23 & 6,13 & 01.02 .06 & 9,94 \\
\hline 1256 & TXN & $882508 \mathrm{AH7}$ & 91,38 & 7,48 & 6,13 & 01.02 .06 & 8,93 \\
\hline 1257 & TXN & $882508 \mathrm{AH} 7$ & 91,38 & 7,48 & 6,13 & 01.02 .06 & 8,93 \\
\hline 1258 & TXN & $882508 \mathrm{AJ} 3$ & 99,74 & 6,87 & 6,75 & 15.07 .99 & 2,28 \\
\hline 1259 & TXN & $882508 \mathrm{AKO}$ & 99,51 & 7,04 & 6,88 & 15.07 .00 & 3,30 \\
\hline 1260 & TNB & $884315 \mathrm{AA0}$ & 99,87 & 8,27 & 8,25 & 15.01 .04 & 8,87 \\
\hline 1261 & TNB & $884315 \mathrm{AA0}$ & 105,96 & 7,23 & 8,25 & 15.01 .04 & 7,86 \\
\hline 1262 & TNB & $884315 \mathrm{AA0}$ & 103,58 & 7,56 & 8,25 & 15.01 .04 & 6,85 \\
\hline 1263 & TNB & 88431QAA4 & 100,95 & 6,00 & 6,29 & 13.02 .03 & 3,89 \\
\hline 1264 & TNB & $88431 \mathrm{QAB2}$ & 99,17 & 6,75 & 6,63 & 07.05 .08 & 9,20 \\
\hline 1265 & TNB & $88431 \mathrm{QAC} 0$ & 97,06 & 6,81 & 6,39 & 10.02 .09 & 9,97 \\
\hline 1266 & \begin{tabular}{|l|} 
TWX \\
\end{tabular} & $887315 \mathrm{AJ} 8$ & 99,75 & 7,54 & 7,45 & 01.02 .98 & 2,83 \\
\hline 1267 & TWX & 887315 AJ8 & 101,13 & 6,76 & 7,45 & 01.02 .98 & 1,82 \\
\hline 1268 & \begin{tabular}{|l|} 
TWX \\
\end{tabular} & 887315 AJ8 & 100,63 & 6,60 & 7,45 & 01.02 .98 & 0,81 \\
\hline 1269 & TWX & 887315 AKS & 97,63 & 9,40 & 9,13 & 15.01 .13 & 18,00 \\
\hline 1270 & TWX & 887315 AK5 & 106,75 & 8,37 & 9,13 & 15.01 .13 & 16,99 \\
\hline 1271 & TWX & 887315AK5 & 105,38 & 8,50 & 9,13 & 15.01 .13 & 15,98 \\
\hline 1272 & TWX & 887315 AK5 & 118,63 & 7,07 & 9,13 & 15.01 .13 & 14,97 \\
\hline 1273 & TWX & 887315 AKS & 122,63 & 6,59 & 9,13 & 15.01 .13 & 13,96 \\
\hline 1274 & TWX & 887315 AL3 & 100,00 & 7,95 & 7,95 & 01.02 .00 & 4,86 \\
\hline 1275 & TWX & $887315 \mathrm{AL} 3$ & 103,00 & 7,03 & 7,95 & 01.02 .00 & 3,85 \\
\hline 1276 & TWX & $887315 \mathrm{AL} 3$ & 101,38 & 7,39 & 7,95 & 01.02 .00 & 2,84 \\
\hline 1277 & TWX & $887315 A L 3$ & 101,44 & 6,02 & 7,95 & 01.02 .00 & 0,81 \\
\hline 1278 & TWX & $887315 \mathrm{AMI}$ & 96,00 & 9,56 & 9,15 & 01.02 .23 & 28,19 \\
\hline 1279 & TWX & $887315 \mathrm{AMI}$ & 106,50 & 8,53 & 9,15 & 01.02 .23 & 27,19 \\
\hline 1280 & TWX & $887315 \mathrm{AMI}$ & 104,63 & 8,70 & 9,15 & 01.02 .23 & 26,18 \\
\hline 1281 & TWX & 887315AMI & 123,00 & 7,15 & 9,15 & 01.02 .23 & 25,16 \\
\hline 1282 & TWX & $887315 \mathrm{AMI}$ & 125,50 & 6,94 & 9,15 & 01.02 .23 & 24,15 \\
\hline 1283 & TWX & 887315AN9 & 99,75 & 7,79 & 7,75 & 15.06 .05 & 9,30 \\
\hline 1284 & TWX & $887315 \mathrm{AN} 9$ & 98,38 & 8,02 & 7,75 & 15.06 .05 & 8,29 \\
\hline 1285 & TWX & 887315 AN9 & 106,50 & 6,59 & 7,75 & 15.06 .05 & 7,27 \\
\hline 1286 & TWX & 887315AN9 & 108,00 & 6,17 & 7,75 & 15.06 .05 & 6,26 \\
\hline 1287 & TWX & 887315 AT6 & 95,63 & 8,09 & 7,48 & 15.01 .08 & 10,91 \\
\hline 1288 & TWX & 887315 AT6 & 105,50 & 6,70 & 7,48 & 15.01 .08 & 9,89 \\
\hline 1289 & TWX & 887315AT6 & 106,00 & 6,56 & 7,48 & 15.01 .08 & 8,88 \\
\hline 1290 & TWX & $887315 \mathrm{AVI}$ & 100,13 & 7,95 & 7,98 & 15.08 .04 & 7,44 \\
\hline 1291 & TWX & $887315 \mathrm{AVI}$ & 107,00 & 6,60 & 7,98 & 15.08 .04 & 6,43 \\
\hline 1292 & TWX & $887315 \mathrm{AVI}$ & 108,25 & 6,13 & 7,98 & 15.08 .04 & 5,42 \\
\hline 1293 & TWX & 887315AW9 & 100,50 & 8,03 & 8,11 & 15.08 .06 & 9,47 \\
\hline 1294 & TWX & 887315AW9 & 109,25 & 6,64 & 8,11 & 15.08 .06 & 8,46 \\
\hline 1295 & TWX & 887315 AW9 & 108,75 & 6,58 & 8,11 & 15.08 .06 & 7,44 \\
\hline 1296 & TWX & $887315 \mathrm{AX7}$ & 100,88 & 8,05 & 8,18 & 15.08 .07 & 10,48 \\
\hline 1297 & TWX & $887315 \mathrm{AX7}$ & 110,00 & 6,72 & 8,18 & 15.08 .07 & 9,47 \\
\hline 1298 & TWX & $887315 \mathrm{AX7}$ & 111,13 & 6,43 & 8,18 & 15.08 .07 & 8,46 \\
\hline 1299 & TWX & 887315AY5 & 96,82 & 7,18 & 6,88 & 15.06 .18 & 19,45 \\
\hline 1300 & TWX & $887315 \mathrm{BA} 6$ & 113,00 & 6,74 & 8,05 & 15.01 .16 & 17,00 \\
\hline 1301 & TMC & $887360 \mathrm{AQ} 8$ & 107,64 & 6,99 & 8,88 & 01.03 .01 & 4,94 \\
\hline 1302 & TMC & $887360 \mathrm{AQ} 8$ & 105,33 & 7,27 & 8,88 & 01.03 .01 & 3,93 \\
\hline 1303 & TMC & 887364AAS & 92,21 & 8,08 & 7,25 & 01.03 .13 & 18,13 \\
\hline 1304 & TMC & 887364AAS & 98,24 & 7,43 & 7,25 & 01.03 .13 & 17,12 \\
\hline 1305 & TMC & 887364AA5 & 95,64 & 7,73 & 7,25 & 01.03 .13 & 16,11 \\
\hline 1306 & TMC & $887364 \mathrm{AB} 3$ & 92,46 & 8,19 & 7,50 & 01.07 .23 & 28,61 \\
\hline 1307 & TMC & $887364 \mathrm{AB} 3$ & 97,85 & 7,69 & 7,50 & 01.07 .23 & 27,60 \\
\hline 1308 & TMC & $887364 \mathrm{AB} 3$ & 95,68 & 7,89 & 7,50 & 01.07 .23 & 26,59 \\
\hline 1309 & TMC & 88736HAF5 & 102,51 & 5,88 & 8,62 & 03.04 .00 & 0,99 \\
\hline
\end{tabular}




\begin{tabular}{|c|c|c|c|c|c|c|c|}
\hline Lfd. Nr. & Ticker & CUSIP & Ask Kurs in Dollar & ISMA Rendite in \% & Kupon in \% & Falligkeit & Restlaufzeit [Jahren] \\
\hline 1310 & TKR & $887389 \mathrm{AA} 2$ & 105,93 & 5,24 & 7,20 & 20.08 .02 & 3,40 \\
\hline 1311 & TKR & 887389AB0 & 106,08 & 5,24 & 7,25 & 20.08 .02 & 3,40 \\
\hline 1312 & TKR & $887389 B^{*} 4$ & 106,29 & 5,25 & 7,31 & 04.09 .02 & 3,44 \\
\hline 1313 & TKR & 887389D\#8 & 106,22 & 5,23 & 7,30 & 13.08 .02 & 3,38 \\
\hline 1314 & TKR & $887389 \mathrm{D} * 2$ & 101,38 & 5,26 & 7,38 & 21.12 .99 & 0,70 \\
\hline 1315 & TKR & 887389D@0 & 106,30 & 5,26 & 7,31 & 10.09 .02 & 3,46 \\
\hline 1316 & TKR & 88738TAA8 & 101,78 & 6,73 & 6,88 & 08.05 .28 & 29,49 \\
\hline 1317 & TKR & 88739 LAN6 & 98,82 & 7,80 & 7,68 & 05.07 .16 & 19,50 \\
\hline 1318 & TKR & 88739 LAP1 & 97,29 & 8,00 & 7,76 & 06.07 .26 & 29,65 \\
\hline 1319 & TKR & 88739LAQ9 & 96,92 & 7,80 & 7,49 & 08.08 .16 & 19,60 \\
\hline 1320 & TKR & 88739LAU0 & 104,34 & 6,06 & 6,78 & 13.12 .06 & 7,78 \\
\hline 1321 & TKR & 88739LAV8 & 105,65 & 6,71 & 7,16 & 03.11 .27 & 28,97 \\
\hline 1322 & TKR & 88739LAW6 & 106,25 & 6,43 & 7,01 & 06.11 .17 & 18,84 \\
\hline 1323 & TKR & 88739LAX4 & 102,35 & 5.90 & 6,40 & 12.11 .04 & 5,66 \\
\hline 1324 & TKR & 88739LAY2 & 104,86 & 6,71 & 7,10 & 17.11 .27 & 29,01 \\
\hline 1325 & TKR & 88739LBB 1 & 100,49 & 6,13 & 6,20 & 15.01 .08 & 8,88 \\
\hline 1326 & TRB & 896047AD9 & 95,65 & 7,52 & 6,88 & 01.11 .06 & 9,69 \\
\hline 1327 & TRB & $89604 \mathrm{KAA} 6$ & 101,71 & 6,10 & 6,35 & 01.02 .08 & 8,93 \\
\hline 1328 & TRB & $89604 \mathrm{KAB} 4$ & 96,41 & 5,96 & 5,25 & 11.10 .05 & 6,59 \\
\hline 1329 & TRB & $89604 \mathrm{KAC2}$ & 98,35 & 5,67 & 5,25 & 20.10 .03 & 4,58 \\
\hline 1330 & TRB & $89604 \mathrm{KAG} 3$ & 95,44 & 6,14 & 5,50 & 06.10 .08 & 9,62 \\
\hline 1331 & TRB & 89604 KAN8 & 96,55 & 6,15 & 5,67 & 08.12 .08 & 9,79 \\
\hline 1332 & TRB & $89605 \mathrm{HBAI}$ & 107,88 & 4,84 & 8,30 & 27.09 .01 & 2,49 \\
\hline 1333 & TRB & $89605 \mathrm{HBC7}$ & 88,68 & 7,60 & 5,75 & 15.09 .03 & 8,53 \\
\hline 1334 & TRB & $89605 \mathrm{HBC7}$ & 93,09 & 6,96 & 5,75 & 15.09 .03 & 7,52 \\
\hline 1335 & TRB & $89605 \mathrm{HBC} 7$ & 91,92 & 7,35 & 5,75 & 15.09 .03 & 6,51 \\
\hline 1336 & TRB & 8960SHBDS & 91,81 & 7,35 & 5,73 & 15.09 .03 & 6,51 \\
\hline 1337 & TRB & 8960 SHBE3 & 95,44 & 7,01 & 5,30 & 17.04 .00 & 3,05 \\
\hline 1338 & TRB & 89605 HBF0 & 88,68 & 7,60 & 5,75 & 16.09 .03 & 8,53 \\
\hline 1339 & TRB & 8960 SHBFO & 93,09 & 6,96 & 5,75 & 16.09 .03 & 7,53 \\
\hline 1340 & TRB & $89605 \mathrm{HBF} 0$ & 91,92 & 7,35 & 5,75 & 16.09 .03 & 6,51 \\
\hline 1341 & TRB & 89605 HBG 8 & 97,42 & 7,26 & 6,65 & 03.06 .02 & 5,21 \\
\hline 1342 & TRB & $89605 \mathrm{HBH} 6$ & 95,88 & 7,43 & 6,75 & 06.06 .05 & 8,26 \\
\hline 1343 & TRB & $89605 \mathrm{HBH} 8$ & 97,42 & 7,26 & 6,65 & 03.06 .02 & 5,21 \\
\hline 1344 & TRB & $89605 \mathrm{HBJ} 2$ & 95,57 & 7,43 & 6,70 & 08.06 .05 & 8,27 \\
\hline 1345 & TRB & 8960 SHBK 9 & 95,45 & 7,43 & 6,68 & 08.06 .05 & 8,27 \\
\hline 1346 & TRB & $89605 \mathrm{HBL} 7$ & 95,15 & 7,43 & 6,63 & 08.06 .05 & 8,27 \\
\hline 1347 & TRB & 89605 HBMS & 97,13 & 7,03 & 6,00 & 09.06 .00 & 3,20 \\
\hline 1348 & TRB & 8960 SHBN3 & 93,40 & 7,46 & 6,40 & 25.10 .05 & 8,65 \\
\hline 1349 & TRB & $89605 \mathrm{HBP} 8$ & 93,05 & 7,46 & 6,35 & 07.11 .05 & 8,69 \\
\hline 1350 & TRB & $89605 \mathrm{HBQ6}$ & 93,79 & 7,31 & 5,95 & 10.12 .02 & 5,74 \\
\hline 1351 & TRB & 89605 HBR4 & 90,34 & 7,48 & 5,95 & 09.01 .06 & 8,86 \\
\hline 1352 & TRB & 89605 HBS2 & 90,31 & 7,48 & 5,95 & 17.01 .06 & 8,89 \\
\hline 1353 & TRB & $89605 \mathrm{HBTO}$ & 90,02 & 7,48 & 5,90 & 09.01 .06 & 8,86 \\
\hline 1354 & TRB & 89605 HBU7 & 89,97 & 7,48 & 5,90 & 24.01 .06 & 8,91 \\
\hline 1355 & TRB & 89605 HBVS & 92,45 & 7,38 & 5,95 & 17.02 .04 & 6,94 \\
\hline 1356 & TRB & $89605 \mathrm{HBWW} 3$ & 98,77 & 7,05 & 6,63 & 15.08 .00 & 3,38 \\
\hline 1357 & TRB & $89605 \mathrm{HBXI}$ & 99,17 & 6,90 & 6,50 & 13.08 .99 & 2,36 \\
\hline 1358 & USG & $903293 \mathrm{AAC} 6$ & 98,50 & 8,99 & 8,00 & 15.12 .96 & 1,68 \\
\hline 1359 & USG & 903293AA6 & 100,63 & 6,98 & 8,00 & 15.12 .96 & 0,68 \\
\hline 1360 & USG & 903293AD0 & 98,13 & 9,09 & 8,00 & 15.03 .97 & 1,93 \\
\hline 1361 & USG & 903293AD0 & 100,50 & 7,41 & 8,00 & 15.03 .97 & 0,93 \\
\hline 1362 & USG & 903293AN8 & 105,00 & 8,09 & 9,25 & 15.09 .01 & 5,49 \\
\hline 1363 & USG & 903293AN8 & 105,50 & 7,75 & 9,25 & 15.09 .01 & 4,48 \\
\hline 1364 & USG & $903293 \mathrm{AP3}$ & 102,00 & 8,19 & 8,50 & 01.08 .05 & 9,43 \\
\hline 1365 & USG & 903293AP3 & 100,75 & 8,37 & 8,50 & 01.08 .05 & 8,42 \\
\hline 1366 & USG & $903293 \mathrm{AP3}$ & 106,00 & 7,42 & 8,50 & 01.08 .05 & 7,40 \\
\hline 1367 & UNP & 907818AL2 & 113,81 & 6,99 & 9,63 & 15.12 .02 & 6,76 \\
\hline
\end{tabular}




\begin{tabular}{|c|c|c|c|c|c|c|c|}
\hline Lfd. Nr. & Ticker & CUSIP & Ask Kurs in Dollar & ISMA Rendite in $\%$ & Kupon in \% & Fălligkeit & Restlaufzeit [Jahren] \\
\hline 1368 & UNP & 907818AL2 & 109,76 & 7,47 & 9,63 & 15.12 .02 & 5,75 \\
\hline 1369 & UNP & 907818AM0 & 104,33 & 6,95 & 7,88 & 15.02 .02 & 5,92 \\
\hline 1370 & UNP & 907818AM0 & 101,90 & 7,40 & 7,88 & 15.02 .02 & 4,91 \\
\hline 1371 & UNP & 907818AR9 & 78,10 & 6,74 & 0,00 & 11.01 .00 & 3,79 \\
\hline 1372 & UNP & 907818AR9 & 82,54 & 7,15 & 0,00 & 11.01 .00 & 2,78 \\
\hline 1373 & UNP & 907818AU2 & 97,85 & 7,51 & 7,00 & 15.06 .00 & 5,23 \\
\hline 1374 & UNP & 907818AU2 & 100,81 & 6,77 & 7,00 & 15.06 .00 & 4,23 \\
\hline 1375 & UNP & 907818AU2 & 99,47 & 7,19 & 7,00 & 15.06 .00 & 3,21 \\
\hline 1376 & UNP & 907818AV0 & 102,86 & 7,16 & 7,60 & 01.05 .05 & 9,17 \\
\hline 1377 & UNP & 907818AV0 & 99,74 & 7,64 & 7,60 & 01.05 .05 & 8,16 \\
\hline 1378 & UNP & 907818AX6 & 102,03 & 6,89 & 7,38 & 15.05 .01 & 5,15 \\
\hline 1379 & UNP & $907818 \mathrm{AX6}$ & 100,25 & 7,30 & 7,38 & 15.05 .01 & 4,14 \\
\hline 1380 & UNP & 907818AY4 & 94,25 & 7,23 & 6,40 & 01.02 .06 & 9,94 \\
\hline 1381 & UNP & 907818AY4 & 91,74 & 7,71 & 6,40 & 01.02 .06 & 8,93 \\
\hline 1382 & UNP & $907818 \mathrm{AZ1}$ & 93,75 & 7,62 & 7,00 & 01.02 .16 & 20,08 \\
\hline 1383 & UNP & 907818BAS & 95,19 & 7,89 & 7,25 & 01.11 .08 & 11,72 \\
\hline 1384 & UNP & $907818 \mathrm{BX} 5$ & 99,95 & 6,63 & 6,63 & 01.02 .08 & 9,94 \\
\hline 1385 & UNP & $907818 \mathrm{~B} \times 5$ & 98,90 & 6,79 & 6,63 & 01.02 .08 & 8,93 \\
\hline 1386 & UNP & $907818 \mathrm{BY} 3$ & 100,58 & 7,08 & 7,13 & 01.02 .28 & 30,23 \\
\hline 1387 & UNP & $907818 \mathrm{BY} 3$ & 95,75 & 7,49 & 7,13 & 01.02 .28 & 29,22 \\
\hline 1388 & UNP & $907818 \mathrm{CE} 6$ & 100,61 & 5,51 & 5,78 & 15.10 .01 & 2,54 \\
\hline 1389 & UNP & $907821 L 67$ & 102,02 & 5,84 & 7,06 & 01.02 .00 & 1,83 \\
\hline 1390 & UNP & $907821 \mathrm{~L} 67$ & 101,40 & 5,20 & 7,06 & 01.02 .00 & 0,81 \\
\hline 1391 & UNP & $907821 \mathrm{L75}$ & 103,29 & 5,89 & 7,20 & 01.02 .01 & 2,84 \\
\hline 1392 & UNP & $907821 \mathrm{~L} 75$ & 104,23 & 4,69 & 7,20 & 01.02 .01 & 1,83 \\
\hline 1393 & UNP & $907821 \mathrm{~L} 83$ & 104,79 & 5,88 & 7,32 & 01.02 .02 & 3,86 \\
\hline 1394 & UNP & $907821 \mathrm{~L} 83$ & 106,15 & 4,92 & 7,32 & 01.02 .02 & 2,84 \\
\hline 1395 & UNP & 907821 L91 & 105,91 & 5,88 & 7,32 & 01.02 .03 & 4,87 \\
\hline 1396 & UNP & $907821 \mathrm{~L} 91$ & 106,78 & 5,31 & 7,32 & 01.02 .03 & 3,86 \\
\hline 1397 & UNP & $907821 \mathrm{M} 25$ & 106,90 & 5,92 & 7,35 & 01.02 .04 & 5,88 \\
\hline 1398 & UNP & $907821 \mathrm{M} 25$ & 106,78 & 5,71 & 7,35 & 01.02 .04 & 4,87 \\
\hline 1399 & UNP & $907821 \mathrm{M} 33$ & 107,71 & 5,97 & 7,37 & 01.02 .05 & 6,90 \\
\hline 1400 & UNP & $907821 \mathrm{M} 33$ & 107,48 & 5,83 & 7,37 & 01.02 .05 & 5,89 \\
\hline 1401 & UNP & $907821 \mathrm{M} 41$ & 108,47 & 6,01 & 7,39 & 01.02 .06 & 7,91 \\
\hline 1402 & UNP & $907821 \mathrm{M} 41$ & 107,96 & 5,94 & 7,39 & 01.02 .06 & 6,90 \\
\hline 1403 & UNP & 907821M58 & 109,10 & 6,05 & 7,40 & 01.02 .07 & 8,93 \\
\hline 1404 & UNP & $907821 \mathrm{M} 58$ & 108,53 & 6,01 & 7,40 & 01.02 .07 & 7,92 \\
\hline 1405 & UNP & $907821 \mathrm{M} 66$ & 110,51 & 6,09 & 7,53 & 01.02 .08 & 9,94 \\
\hline 1406 & UNP & $907821 \mathrm{M} 66$ & 109,89 & 6,06 & 7,53 & 01.02 .08 & 8,93 \\
\hline 1407 & UNP & $907821 \mathrm{M} 74$ & 112,55 & 6,11 & 7,72 & 01.02 .09 & 10,96 \\
\hline 1408 & UNP & $907821 \mathrm{M} 74$ & 111,72 & 6,11 & 7,72 & 01.02 .09 & 9,95 \\
\hline 1409 & UNP & $907821 \mathrm{M} 82$ & 114,20 & 6,14 & 7,85 & 01.02 .10 & 11,97 \\
\hline 1410 & UNP & $907821 \mathrm{M} 82$ & 113,33 & 6,14 & 7,85 & 01.02 .10 & 10,96 \\
\hline 1411 & UNP & $907821 \mathrm{M} 90$ & 115,81 & 6,16 & 7,97 & 01.02 .11 & 12,99 \\
\hline 1412 & UNP & $907821 \mathrm{M} 90$ & 114,94 & 6,17 & 7,97 & 01.02 .11 & 11,98 \\
\hline 1413 & UNP & $907821 \mathrm{~N} 24$ & 117,18 & 6,19 & 8,06 & 01.02 .12 & 14,00 \\
\hline 1414 & UNP & $907821 \mathrm{~N} 24$ & 116,32 & 6,19 & 8,06 & 01.02 .12 & 12,99 \\
\hline 1415 & UNP & 90782EAFI & 110,51 & 5,87 & 9,29 & 26.09 .02 & 3,50 \\
\hline 1416 & UNP & 90782EAU8 & 112,80 & 6,50 & 9,20 & 01.02 .05 & 5,89 \\
\hline 1417 & UNP & $90782 \mathrm{EBH} 6$ & 120,25 & 7,03 & 9,20 & 03.11 .14 & 15,78 \\
\hline 1418 & UNP & 90782EBP8 & 104,09 & 5,69 & 9,05 & 01.08 .00 & 1,32 \\
\hline 1419 & UNP & 90782EBS2 & 101,93 & 5,81 & 9,08 & 29.11 .99 & 0,64 \\
\hline 1420 & UNP & 90782EBVS & 101,97 & 5,82 & 9,05 & 06.12 .99 & 0,66 \\
\hline 1421 & UNP & 90782EBXI & 105,56 & 5,47 & 9,06 & 11.12 .00 & 1,69 \\
\hline 1422 & UNP & 90782EBY9 & 101,99 & 5,82 & 9,05 & 08.12 .99 & 0,66 \\
\hline 1423 & UNP & $90782 \mathrm{EBZ6}$ & 101,99 & 5,83 & 9,00 & 13.12 .99 & 0,68 \\
\hline 1424 & UNP & 90782ECK8 & 102,46 & 5,86 & 9,30 & 17.01 .00 & 0,77 \\
\hline 1425 & UNP & 90782ECM4 & 102,45 & 5,88 & 9,15 & 31.01 .00 & 0,81 \\
\hline
\end{tabular}




\begin{tabular}{|c|c|c|c|c|c|c|c|}
\hline Lfd. Nr. & Ticker & CUSIP & Ask Kurs in Dollar & ISMA Rendite in \% & Kupon in \% & Falligkeit & Restlaufzeit [Jahren] \\
\hline 1426 & UNP & $90782 \mathrm{ECN} 2$ & 102,49 & 5,88 & 9,20 & 01.02 .00 & 0,81 \\
\hline 1427 & UNP & $90782 \mathrm{ECP} 7$ & 110,19 & 5,62 & 9,60 & 11.02 .02 & 2,87 \\
\hline 1428 & UNP & $90782 \mathrm{ECQ} 5$ & 110,22 & 5,63 & 9,60 & 15.02 .02 & 2,88 \\
\hline 1429 & UNP & 90782ECT9 & 103,56 & 5,90 & 9,72 & 10.04 .00 & 1,01 \\
\hline 1430 & UNP & 90782ECU6 & 130,18 & 7,19 & 10,00 & 10.04 .20 & 21,30 \\
\hline 1431 & UNP & 90782ECU6 & 130,18 & 7,19 & 10,00 & 10.04 .20 & 21,30 \\
\hline 1432 & UNP & $90782 \mathrm{ECY} 8$ & 103,55 & 5,90 & 9,68 & 12.04 .00 & 1,01 \\
\hline 1433 & UNP & 90782EDA9 & 124,05 & 7,10 & 9,50 & 15.03 .17 & 18,18 \\
\hline 1434 & UNP & 90782EDCS & 103,58 & 5,89 & 9,65 & 17.04 .00 & 1.03 \\
\hline 1435 & UNP & 90782EDD3 & 116,00 & 6,52 & 9,78 & 20.04 .05 & 6.11 \\
\hline 1436 & UNP & 90782EDE 1 & 129.51 & 7,19 & 9,94 & 01.04 .20 & 21,27 \\
\hline 1437 & UNP & 90782EDX9 & 128,57 & 7,13 & 9.90 & 15.05 .18 & 19,36 \\
\hline 1438 & UNP & 90782EDZ4 & 123,15 & 6,89 & 9.90 & 01.06 .10 & 11,29 \\
\hline 1439 & UNP & $90782 \mathrm{EDZ4}$ & 123,15 & 6,89 & 9,90 & 01.06 .10 & 11.29 \\
\hline 1440 & UNP & 90782EED2 & 104,12 & 5,79 & 9,65 & 01.06 .00 & 1,15 \\
\hline 1441 & UNP & 90782EEE0 & 104,17 & 5,78 & 9,65 & 05.06 .00 & 1,16 \\
\hline 1442 & UNP & 90782EEF7 & 104,10 & 5,78 & 9,55 & 08.06 .00 & 1,17 \\
\hline 1443 & UNP & $90782 \mathrm{EEQ} 3$ & 106,39 & 5,55 & 9,95 & 01.11 .00 & 1,58 \\
\hline 1444 & UNP & 90782EES9 & 118,06 & 6,56 & 10,00 & 02.11 .05 & 6,65 \\
\hline 1445 & UNP & 90782EEY6 & 106,45 & 5,52 & 9,87 & 14.11 .00 & 1,61 \\
\hline 1446 & UNP & 90782EEZ3 & 106,44 & 5,52 & 9,85 & 15.11 .00 & 1,61 \\
\hline 1447 & UNP & 90782EEZ6 & 106,45 & 5,52 & 9,87 & 14.11 .00 & 1,61 \\
\hline 1448 & UNP & 90782EFA7 & 106,45 & 5,51 & 9,81 & 20.11 .00 & 1,63 \\
\hline 1449 & UNP & 90782EFDI & 106,06 & 5,55 & 9,73 & 01.11 .00 & 1,58 \\
\hline 1450 & UNP & $90782 \mathrm{EFJ} 8$ & 106,43 & 5,49 & 9,70 & 01.12 .00 & 1,66 \\
\hline 1451 & \begin{tabular}{|l|} 
UNP \\
\end{tabular} & $90782 \mathrm{EFL} 3$ & 106,37 & 5,49 & 9,67 & 30.11 .00 & 1,66 \\
\hline 1452 & UNP & 90782EFMI & 106,51 & 5,49 & 9,75 & 01.12 .00 & 1,66 \\
\hline 1453 & UNP & 90782EFP4 & 106,43 & 5,47 & 9,60 & 12.12 .00 & 1,69 \\
\hline 1454 & UNP & 90782EFQ2 & 109,92 & 5,63 & 9,48 & 15.02 .02 & 2,88 \\
\hline 1455 & UNP & 90782EFR0 & 111,50 & 5,99 & 9,47 & 15.01 .03 & 3,81 \\
\hline 1456 & UNP & 90782EFS8 & 106,53 & 5,42 & 9,42 & 12.01 .01 & 1,78 \\
\hline 1457 & UNP & 90782EFT6 & 106,74 & 5,41 & 9,51 & 17.01 .01 & 1,79 \\
\hline 1458 & UNP & 90782EFU3 & 106,73 & 5,40 & 9,44 & 25.01 .01 & 1,81 \\
\hline 1459 & UNP & 90782EFU6 & 106.74 & 5,41 & 9,51 & 17.01 .01 & 1,79 \\
\hline 1460 & UNP & 90782EFV1 & 106,79 & 5,39 & 9,44 & 30.01 .01 & 1,83 \\
\hline 1461 & UNP & 907S2EFW9 & 106,81 & 5,39 & 9,44 & 01.02 .01 & 1,83 \\
\hline 1462 & UNP & 90782 EFY 5 & 106,77 & 5,39 & 9,42 & 01.02 .01 & 1,83 \\
\hline 1463 & UNP & 90782EFZ2 & 106,71 & 5,39 & 9,38 & 01.02 .01 & 1,83 \\
\hline 1464 & UNP & 90782EGB4 & 106,79 & 5,39 & 9,42 & 02.02 .01 & 1,83 \\
\hline 1465 & UNP & 90782 EGC2 & 106.74 & 5,39 & 9,40 & 01.02 .01 & 1,83 \\
\hline 1466 & UNP & 90782 EGDO & 106,83 & 5,36 & 9,35 & 15.02 .01 & 1,87 \\
\hline 1467 & UNP & 90782EGD0 & 106,83 & 5,36 & 9,35 & 15.02 .01 & 1,87 \\
\hline 1468 & UNP & 90782EGE8 & 106,76 & 5,38 & 9,37 & 07.02 .01 & 1,85 \\
\hline 1469 & UNP & 90782EGF5 & 106,52 & 5,38 & 9,22 & 08.02 .01 & 1,85 \\
\hline 1470 & UNP & 90782EGG3 & 106,57 & 5,38 & 9,25 & 08.02 .01 & 1,85 \\
\hline 1471 & UNP & 90782EGHI & 106,31 & 5,37 & 9,05 & 15.02 .01 & 1,87 \\
\hline 1472 & UNP & 90782EGJ7 & 110,35 & 6,03 & 9,10 & 14.02 .03 & 3,89 \\
\hline 1473 & UNP & $90782 \mathrm{EGK} 4$ & 106,20 & 5,37 & 8.99 & 14.02 .01 & 1,87 \\
\hline 1474 & UNP & 90782EGL2 & 106,20 & 5,36 & 8.96 & 19.02 .01 & 1,88 \\
\hline 1475 & UNP & 90782EGM0 & 110,02 & 6.03 & 9,00 & 20.02 .03 & 3,91 \\
\hline 1476 & UNP & 90782EGM0 & 110,02 & 6,03 & 9.00 & 20.02 .03 & 3,91 \\
\hline 1477 & UNP & 90782EGN'8 & 106,34 & 5,35 & 8,99 & 26.02 .01 & 1,90 \\
\hline 1478 & UNP & 90782EGP3 & 114,04 & 6,60 & 9,17 & 07.03 .06 & 7,00 \\
\hline 1479 & UNP & 90782 EGQ1 & 119,73 & 6,92 & 9,38 & 07.03 .11 & 12,07 \\
\hline 1480 & UNP & 90782EGR9 & 106,79 & 5,33 & 9,18 & 06.03 .01 & 1,92 \\
\hline 1481 & UNP & 90782EGS7 & 106,65 & 5,34 & 9.11 & 05.03 .01 & 1,92 \\
\hline 1482 & UNP & 90782EGU2 & 99,41 & 6,52 & 6,39 & 01.11 .04 & 5,63 \\
\hline 1483 & UNP & $90782 \mathrm{EGV} 0$ & 100,13 & 6,77 & 6,79 & 09.11 .07 & 8,70 \\
\hline
\end{tabular}




\begin{tabular}{|c|c|c|c|c|c|c|c|}
\hline Lfd. Nr. & Ticker & CUSIP & Ask Kurs in Dollar & ISMA Rendite in $\%$ & Kupon in \% & Falligkeit & Restlaufzeit [Jahren] \\
\hline 1484 & UNP & 90782EGW8 & 99.87 & 6,37 & 6,34 & 25.11 .03 & 4,68 \\
\hline 1485 & UNP & 90782EGW8 & 99,87 & 6,37 & 6,34 & 25.11 .03 & 4,68 \\
\hline 1486 & UIS & $909214 \mathrm{AYY} 4$ & 102,13 & 8,09 & 9,75 & 15.09 .96 & 1,43 \\
\hline 1487 & UIS & $909214 \mathrm{AYY} 4$ & 100,63 & 8,08 & 9,75 & 15.09 .96 & 0,42 \\
\hline 1488 & $\mathrm{U}$ & $911546 \mathrm{VV} 8$ & 77,50 & 15,68 & 9,63 & 01.02 .01 & 5,88 \\
\hline 1489 & $\mathrm{U}$ & $911546 \mathrm{VV} 8$ & 90,88 & 12,19 & 9,63 & 01.02 .01 & 4,87 \\
\hline 1490 & $\mathrm{U}$ & $911546 \mathrm{VV} 8$ & 99,50 & 9,78 & 9,63 & 01.02 .01 & 3,86 \\
\hline 1491 & $\mathrm{UH}$ & 911920AD8 & 97,50 & 8,72 & 7,95 & 01.03 .01 & 3,93 \\
\hline 1492 & UTX & 913017AR0 & 111,12 & 7,84 & 8,88 & 15.11 .19 & 22,91 \\
\hline 1493 & UTX & 913017AS8 & 113,70 & 7,52 & 8,75 & 01.03 .21 & 25,24 \\
\hline 1494 & UTX & 913017AS8 & 109,83 & 7,83 & 8,75 & 01.03 .21 & 24,23 \\
\hline 1495 & UTX & $91302 \mathrm{LAH} 0$ & 126,21 & 6,17 & 9,34 & 01.02 .11 & 11,98 \\
\hline 1496 & UVV & $913456 \mathrm{ABS}$ & 94,50 & 7,29 & 6,50 & 15.02 .06 & 9,98 \\
\hline 1497 & UVV & $913456 \mathrm{AB} 5$ & 92,53 & 7,68 & 6,50 & 15.02 .06 & 8,97 \\
\hline 1498 & UFC & $913538 \mathrm{FA} 7$ & 97,76 & 6,81 & 6,50 & 01.04 .09 & 10,11 \\
\hline 1499 & VFC & 918204AE8 & 111,00 & 6,88 & 9,50 & 01.05 .01 & 5,11 \\
\hline 1500 & VFC & 918204AE8 & 108,21 & 7,12 & 9,50 & 01.05 .01 & 4,10 \\
\hline 1501 & VFC & $918204 \mathrm{AJ} 7$ & 97,17 & 7,18 & 6,75 & 01.06 .05 & 9,26 \\
\hline 1502 & VFC & 918204AJ7 & 95,88 & 7,43 & 6,75 & 01.06 .05 & 8,25 \\
\hline 1503 & VIA & $925524 \mathrm{AD} 2$ & 98,00 & 8,06 & 7,75 & 01.06 .05 & 9,26 \\
\hline 1504 & VIA & 925524AD2 & 95,50 & 8,53 & 7,75 & 01.06 .05 & 8,25 \\
\hline 1505 & VIA & 925524AD2 & 102,50 & 7,29 & 7,75 & 01.06 .05 & 7,23 \\
\hline 1506 & VIA & 925524AE0 & 93,38 & 8,22 & 6,75 & 15.01 .03 & 5,84 \\
\hline 1507 & VIA & 925524AEO & 100,25 & 6,67 & 6,75 & 15.01 .03 & 3,81 \\
\hline 1508 & VIA & 925524AF7 & 92,00 & 8,46 & 7,63 & 15.01 .16 & 20,04 \\
\hline 1509 & VIA & $925524 \mathrm{AF} 7$ & 102,00 & 7,42 & 7,63 & 15.01 .16 & 18,01 \\
\hline 1510 & \begin{tabular}{|l|}
$\mathrm{VMC}$ \\
\end{tabular} & $929160 \mathrm{AB} 5$ & 98,97 & 6,14 & 6,00 & 01.04 .09 & 10,11 \\
\hline 1511 & VMC & $929160 \mathrm{AC} 3$ & 100,01 & 5,75 & 5,75 & 01.04 .04 & 5,04 \\
\hline 1512 & VMC & $929160 \mathrm{AC} 3$ & 100,01 & 5,75 & 5,75 & 01.04 .04 & 5,04 \\
\hline 1513 & $\mathrm{VMC}$ & 92916HAE2 & 108,22 & 4,67 & 8,80 & 29.05 .01 & 2,16 \\
\hline 1514 & VMC & 92916HAF9 & 103,90 & 5,09 & 8,74 & 30.05 .00 & 1,14 \\
\hline 1515 & VMC & 92916HAHS & 101,25 & 4,96 & 8,28 & 07.09 .99 & 0,41 \\
\hline 1516 & VMC & $92916 \mathrm{HAJI}$ & 110,35 & 5,15 & 8,51 & 11.09 .02 & 3,46 \\
\hline 1517 & VMC & $92916 \mathrm{HAJ} 4$ & 115,22 & 5,97 & 8,55 & 11.09 .06 & 7,52 \\
\hline 1518 & VMC & 92916 HAK8 & 111,52 & 5,54 & 8,53 & 11.09 .03 & 4,48 \\
\hline 1519 & VMC & 92916HAL6 & 112,78 & 5,76 & 8,55 & 15.09 .04 & 5,50 \\
\hline 1520 & VMC & 92916HAM4 & 115,22 & 5,97 & 8,55 & 11.09 .06 & 7,52 \\
\hline 1521 & VMC & 92916HAN2 & 115,35 & 5,97 & 8,57 & 12.09 .06 & 7,52 \\
\hline 1522 & VMC & 92916 HAP7 & 128,52 & 6,43 & 8,85 & 01.10 .21 & 22,79 \\
\hline 1523 & VMC & 92916HAP7 & 128,52 & 6,43 & 8,85 & 01.10 .21 & 22,79 \\
\hline 1524 & VMC & $92916 \mathrm{HAQS}$ & 112,16 & 5,90 & 8,18 & 17.10 .05 & 6,61 \\
\hline 1525 & VMC & 92916HAR3 & 111,64 & 5,91 & 8,07 & 25.11 .05 & 6,71 \\
\hline 1526 & VMC & 92916HASI & 122,72 & 6,18 & 8,80 & 28.11 .11 & 12,81 \\
\hline 1527 & VMC & 92916HAT9 & 122,79 & 6,21 & 8,70 & 15.01 .13 & 13,96 \\
\hline 1528 & WMT & $931142 \mathrm{AL7}$ & 100,08 & 5,40 & 8,00 & 01.05 .96 & 0,04 \\
\hline 1529 & WMT & 931142AM5 & 99,20 & 6,10 & 5,50 & 15.09 .97 & 1,44 \\
\hline 1530 & WMT & 931142AM5 & 99,87 & 5,80 & 5,50 & 15.09 .97 & 0,43 \\
\hline 1531 & WMT & $931142 \mathrm{AN} 3$ & 96,77 & 6,92 & 6,13 & 01.10 .99 & 4,52 \\
\hline 1532 & \begin{tabular}{|l|} 
WMT \\
\end{tabular} & $931142 \mathrm{AN} 3$ & 98,99 & 6,38 & 6,13 & 01.10 .99 & 3,51 \\
\hline 1533 & WMT & 931142AN3 & 98,05 & 6,88 & 6,13 & 01.10 .99 & 2,50 \\
\hline 1534 & WMT & $931142 \mathrm{AP} 8$ & 98,69 & 6,25 & 5,50 & 01.03 .98 & 1,90 \\
\hline 1535 & WMT & 931142AP8 & 99,48 & 6,12 & 5,50 & 01.03 .98 & 0,89 \\
\hline 1536 & WLA & $934488 \mathrm{AG2}$ & 103,43 & 6,41 & 8,00 & 01.09 .98 & 2,41 \\
\hline 1537 & WLA & $934488 \mathrm{AG} 2$ & 101,95 & 6,48 & 8,00 & 01.09 .98 & 1,40 \\
\hline 1538 & WLA & $934488 \mathrm{AHO}$ & 99,12 & 6,80 & 6,63 & 15.09 .02 & 6,51 \\
\hline 1539 & WLA & $934488 \mathrm{AHO}$ & 97,22 & 7,25 & 6,63 & 15.09 .02 & 5,50 \\
\hline 1540 & WLA & $934488 \mathrm{AK} 3$ & 99,44 & 5,89 & 5,75 & 15.01 .03 & 4,82 \\
\hline 1541 & WLA & 934488AK3 & 101,47 & 5,31 & 5,75 & 15.01 .03 & 3,81 \\
\hline
\end{tabular}




\begin{tabular}{|c|c|c|c|c|c|c|c|}
\hline Lfd. Nr. & Ticker & CUSIP & Ask Kurs in Dollar & ISMA Rendite in $\%$ & Kupon in \% & Falligkeit & Restlaufzeit [Jahren] \\
\hline 1542 & WLA & $934488 \mathrm{ALI}$ & 99,31 & 6,09 & 6,00 & 15.01 .08 & 9,89 \\
\hline 1543 & WLA & $934488 \mathrm{ALI}$ & 99,47 & 6,08 & 6,00 & 15.01 .08 & 8,88 \\
\hline 1544 & WS & 948774AA2 & 98,88 & 11,20 & 10,88 & 15.10 .99 & 4,56 \\
\hline 1545 & WS & $948774 \mathrm{AA2}$ & 101,50 & 10,35 & 10,88 & 15.10 .99 & 3,55 \\
\hline 1546 & WS & 948774AA2 & 102,00 & 9,95 & 10,88 & 15.10 .99 & 2,54 \\
\hline 1547 & WS & $948774 \mathrm{AA2}$ & 103,25 & 8,50 & 10,88 & 15.10 .99 & 1,52 \\
\hline 1548 & WS & $948774 \mathrm{AA2}$ & 100,22 & 10,39 & 10,88 & 15.10 .99 & 0,51 \\
\hline 1549 & WS & $948774 \mathrm{ABO}$ & 101,25 & 10,96 & 11,50 & 01.03 .98 & 2,91 \\
\hline 1550 & wS & $948774 \mathrm{ABO}$ & 103,25 & 9,54 & 11,50 & 01.03 .98 & 1,90 \\
\hline 1551 & ws & $948774 \mathrm{AB} 0$ & 101,75 & 9,33 & 11,50 & 01.03 .98 & 0,89 \\
\hline 1552 & WEN & 950590ADI & 93,61 & 7,28 & 6,35 & 15.12 .05 & 9,81 \\
\hline 1553 & WEN & $950590 \mathrm{ADI}$ & 91,76 & 7,67 & 6,35 & 15.12 .05 & 8,79 \\
\hline 1554 & WEN & $950590 \mathrm{AG} 4$ & 89,55 & 7,92 & 7,00 & 15.12 .25 & 30,10 \\
\hline 1555 & WEN & $950590 \mathrm{AG4}$ & 86,07 & 8,28 & 7,00 & 15.12 .25 & 29,09 \\
\hline 1556 & $W$ & $961548 \mathrm{AKO}$ & 113,00 & 6,91 & 9,65 & 01.03 .02 & 5,96 \\
\hline 1557 & $w$ & $961548 \mathrm{AKO}$ & 109,68 & 7,25 & 9,65 & 01.03 .02 & 4,95 \\
\hline 1558 & $\mathrm{~W}$ & 961548AL8 & 124,14 & 7,56 & 9,75 & 15.06 .20 & 24,52 \\
\hline 1559 & $w$ & 961548AL8 & 119,90 & 7,87 & 9,75 & 15.06 .20 & 23,51 \\
\hline 1560 & WY & $962166 \mathrm{AKO}$ & 108,90 & 7,18 & 8,38 & 15.02 .07 & 10,99 \\
\hline 1561 & WY & $962166 \mathrm{AKO}$ & 106,22 & 7,47 & 8,38 & 15.02 .07 & 9,98 \\
\hline 1562 & WY & 962166AP9 & 111,08 & 6,97 & 9,05 & 01.02 .03 & 6,89 \\
\hline 1563 & WY & $962166 \mathrm{AP} 9$ & 107,94 & 7,34 & 9,05 & 01.02 .03 & 5,88 \\
\hline 1564 & WY & $962166 \mathrm{AQ7}$ & 96,22 & 7,90 & 7,50 & 01.03 .13 & 18,13 \\
\hline 1565 & WY & $962166 \mathrm{AQ} 7$ & 100,73 & 7,42 & 7,50 & 01.03 .13 & 17,12 \\
\hline 1566 & WY & $962166 \mathrm{AQ7}$ & 97,90 & 7,73 & 7,50 & 01.03 .13 & 16,11 \\
\hline 1567 & $\mathrm{WY}$ & 962166AR5 & 93,63 & 7,91 & 7,25 & 01.07 .13 & 18,47 \\
\hline 1568 & WY & 962166ARS & 98,21 & 7,43 & 7,25 & 01.07 .13 & 17,46 \\
\hline 1569 & WY & 962166ARS & 95,48 & 7,74 & 7,25 & 01.07 .13 & 16,45 \\
\hline 1570 & WY & $962166 \mathrm{AS3}$ & 87,39 & 8,29 & 7,13 & 15.07 .23 & 28,65 \\
\hline 1571 & WY & $962166 \mathrm{AS} 3$ & 93,84 & 7,67 & 7,13 & 15.07 .23 & 27,64 \\
\hline 1572 & WY & $962166 \mathrm{AS3}$ & 91,01 & 7,94 & 7,13 & 15.07 .23 & 26,63 \\
\hline 1573 & WY & $962166 \mathrm{ATI}$ & 106,18 & 7,94 & 8,50 & 15.01 .25 & 28,16 \\
\hline 1574 & WY & 962166AU8 & 99,99 & 7,95 & 7,95 & 15.03 .25 & 28,32 \\
\hline 1575 & WY & $962166 \mathrm{AW} 4$ & 104,06 & 6,63 & 6,95 & 01.10 .27 & 29,89 \\
\hline 1576 & WY & 962166AlV4 & 103,61 & 6,66 & 6,95 & 01.10 .27 & 28,88 \\
\hline 1577 & WY & $96216 \mathrm{HAC4}$ & 107,66 & 4,68 & 8,98 & 07.03 .01 & 1,93 \\
\hline 1578 & WY & $96216 \mathrm{HAF} 7$ & 103,42 & 5,25 & 8,90 & 10.04 .00 & 1,01 \\
\hline 1579 & WY & $96216 \mathrm{HAG} 5$ & 107,98 & 4,66 & 8,94 & 09.04 .01 & 2,02 \\
\hline 1580 & WY & $96216 \mathrm{HAH} 3$ & 103,39 & 5,25 & 8,85 & 12.04 .00 & 1,01 \\
\hline 1581 & WY & 96216HAK6 & 107,90 & 4,66 & 8,88 & 12.04 .01 & 2,03 \\
\hline 1582 & WY & $96216 \mathrm{HAL} 4$ & 103,46 & 5,24 & 8,86 & 17.04 .00 & 1,03 \\
\hline 1583 & WY & $96216 \mathrm{HAM} 2$ & 107,97 & 4,66 & 8,90 & 16.04 .01 & 2,04 \\
\hline 1584 & WY & 96216HANO & 108,03 & 4,67 & 8,92 & 19.04 .01 & 2,04 \\
\hline 1585 & WY & $96216 \mathrm{HAQ} 3$ & 103,53 & 5,23 & 8,90 & 19.04 .00 & 1,03 \\
\hline 1586 & WY & $96216 \mathrm{HAR}$ & 107,99 & 4,67 & 8,90 & 19.04 .01 & 2,04 \\
\hline 1587 & WY & $96216 \mathrm{HAS} 9$ & 103,49 & 5,22 & 8,80 & 24.04 .00 & 1,04 \\
\hline 1588 & WY & $96216 \mathrm{HAU} 4$ & 103,54 & 5,22 & 8,85 & 24.04 .00 & 1,04 \\
\hline 1589 & WY & $96216 \mathrm{HAU} 4$ & 103,54 & 5,22 & 8,85 & 24.04 .00 & 1,04 \\
\hline 1590 & WY & $96216 \mathrm{HAZ3}$ & 108,11 & 4,69 & 8,88 & 08.05 .01 & 2.10 \\
\hline 1591 & WY & $96216 \mathrm{HBA7}$ & 103,89 & 5,17 & 8,92 & 17.05 .00 & 1,11 \\
\hline 1592 & WY & $96216 \mathrm{HBD} 1$ & 108,36 & 4,72 & 8,90 & 04.06 .01 & 2,17 \\
\hline 1593 & WY & $96216 \mathrm{HBF} 6$ & 108,40 & 4,73 & 8,88 & 15.06 .01 & 2,20 \\
\hline 1594 & WY & $96216 \mathrm{HBG} 1$ & 108,36 & 4,72 & 8,90 & 04.06 .01 & 2,17 \\
\hline 1595 & WY & $96216 \mathrm{HBG} 4$ & 100,61 & 4,57 & 8,85 & 11.06 .99 & 0,16 \\
\hline 1596 & WY & $96216 \mathrm{HBG} 4$ & 100,61 & 4,57 & 8,85 & 11.06 .99 & 0,16 \\
\hline 1597 & WY & $96216 \mathrm{HBH} 2$ & 101,12 & 4,83 & 8,85 & 02.08 .99 & 0,31 \\
\hline 1598 & WY & $96216 \mathrm{HBKS}$ & 104,79 & 5,02 & 8,85 & 10.08 .00 & 1,34 \\
\hline 1599 & $W Y$ & $96216 \mathrm{HBL} 3$ & 110,78 & 5,17 & 8,75 & 13.08 .02 & 3,38 \\
\hline
\end{tabular}




\begin{tabular}{|c|c|c|c|c|c|c|c|}
\hline Lfd. Nr. & Ticker & CUSIP & Ask Kurs in Dollar & ISMA Rendite in \% & Kupon in \% & Fălligkeit & Restlaufzeit [Jahren] \\
\hline 1600 & WY & $96216 \mathrm{HBMl}$ & 102,61 & 5,92 & 6,43 & 12.07 .05 & 6,34 \\
\hline 1601 & WH & 96647KAA0 & 97,97 & 7,85 & 7,50 & 01.02 .03 & 7,90 \\
\hline 1602 & WH & $96647 \mathrm{KAAO}$ & 101,76 & 7,16 & 7,50 & 01.02 .03 & 6,89 \\
\hline 1603 & WH & $96647 \mathrm{KAA} 0$ & 99,98 & 7,50 & 7,50 & 01.02 .03 & 5,88 \\
\hline 1604 & WH & 96647KAB8 & 88,15 & 8,17 & 6,50 & 01.02 .06 & 10,95 \\
\hline 1605 & WH & 96647KAB8 & 93,69 & 7,42 & 6,50 & 01.02 .06 & 9,94 \\
\hline 1606 & $\mathrm{WH}$ & $96647 \mathrm{KAB} 8$ & 92,37 & 7,71 & 6,50 & 01.02 .06 & 8,93 \\
\hline 1607 & WH & 96647KAC6 & 99,05 & 7,69 & 7,50 & 15.08 .01 & 6,42 \\
\hline 1608 & WH & $96647 \mathrm{KAC} 6$ & 101,93 & 7,05 & 7,50 & 15.08 .01 & 5,41 \\
\hline 1609 & WH & 96647KAC6 & 100,60 & 7,33 & 7,50 & 15.08 .01 & 4,40 \\
\hline 1610 & WH & 96647KAD4 & 100,20 & 8,22 & 8,25 & 15.02 .07 & 12,00 \\
\hline 1611 & WH & 96647KAD4 & 105,68 & 7,47 & 8,25 & 15.02 .07 & 10,99 \\
\hline 1612 & WH & 96647KAD4 & 103,22 & 7,77 & 8,25 & 15.02 .07 & 9,98 \\
\hline 1613 & WH & 96647KAE2 & 97,63 & 7,87 & 7,63 & 15.06 .15 & 19,44 \\
\hline 1614 & WH & 96647KAE2 & 94,98 & 8,16 & 7,63 & 15.06 .15 & 18,43 \\
\hline 1615 & WH & 96647TAD5 & 91,18 & 8,10 & 6,80 & 02.05 .05 & 10,18 \\
\hline 1616 & WH & 96647TADS & 96,39 & 7,35 & 6,80 & 02.05 .05 & 9,18 \\
\hline 1617 & WH & 96647TADS & 94,96 & 7,65 & 6,80 & 02.05 .05 & 8,16 \\
\hline 1618 & WH & 96647TAH6 & 101,81 & 7,00 & 8,12 & 27.01 .97 & 1,80 \\
\hline 1619 & WH & 96647TAH6 & 101,62 & 5,93 & 8,12 & 27.01 .97 & 0,79 \\
\hline 1620 & WH & 96647TAH6 & 101,81 & 7,00 & 8,12 & 27.01 .97 & 1,80 \\
\hline 1621 & $\mathrm{XRX}$ & $984121 \mathrm{AQ} 6$ & 110,06 & 6,77 & 9,75 & 15.03 .00 & 3,97 \\
\hline 1622 & $\mathrm{XRX}$ & $984121 \mathrm{AQ} 6$ & 106,83 & 7,11 & 9,75 & 15.03 .00 & 2,96 \\
\hline 1623 & XRX & 984121AR4 & 104,51 & 6,13 & 9,63 & 01.09 .97 & 1,40 \\
\hline 1624 & \begin{tabular}{|l|}
$\mathrm{XRX}$ \\
\end{tabular} & 984121 AR4 & 101,31 & 5,94 & 9,63 & 01.09 .97 & 0,39 \\
\hline 1625 & \begin{tabular}{|l|}
$\mathrm{XRX}$ \\
\end{tabular} & $984121 \mathrm{ATO}$ & 106,48 & 6,79 & 8,13 & 15.04 .02 & 6,08 \\
\hline 1626 & \begin{tabular}{|l}
$\mathrm{XRX}$ \\
\end{tabular} & 984121 ATO & 104,18 & 7,12 & 8,13 & 15.04 .02 & 5,07 \\
\hline 1627 & $\mathrm{XRX}$ & 984121 AU7 & 101,31 & 6,94 & 7,15 & 01.08 .04 & 8,41 \\
\hline 1628 & XRX & 984121 AU7 & 99,31 & 7,27 & 7,15 & 01.08 .04 & 7,40 \\
\hline 1629 & XRX & 984121 AU7 & 101,31 & 6,94 & 7,15 & 01.08 .04 & 8,41 \\
\hline 1630 & $\mathrm{XRX}$ & $984121 \mathrm{AW} 3$ & 99,21 & 5,70 & 5,50 & 15.11 .03 & 4,66 \\
\hline 1631 & $\mathrm{XRX}$ & 98412JAD6 & 99,10 & 7,41 & 7,14 & 31.03 .99 & 4,01 \\
\hline 1632 & $\mathrm{XRX}$ & 98412JAD6 & 101,31 & 6,64 & 7,14 & 31.03 .99 & 3,00 \\
\hline 1633 & $\mathrm{XRX}$ & 98412JAD6 & 100,44 & 6,90 & 7,14 & 31.03 .99 & 1,99 \\
\hline 1634 & $\mathrm{XRX}$ & 98412JAD6 & 99,10 & 7,41 & 7,14 & 31.03 .99 & 4,01 \\
\hline 1635 & $\mathrm{XRX}$ & 98412JAE4 & 100,39 & 6,92 & 7,13 & 30.04 .99 & 2,07 \\
\hline 1636 & $\mathrm{XRX}$ & 984I2JAFI & 100,56 & 7,30 & 7,41 & 15.05 .01 & 6,16 \\
\hline 1637 & $\mathrm{XRX}$ & 98412JAFI & 102,94 & 6,72 & 7,41 & 15.05 .01 & 5,15 \\
\hline 1638 & $\mathrm{XRX}$ & 98412JAFI & 101,41 & 7,01 & 7,41 & 15.05 .01 & 4,14 \\
\hline 1639 & $\mathrm{XRX}$ & 98412JAG9 & $102,4 !$ & 6,73 & 7,29 & 31.05 .01 & 5,20 \\
\hline 1640 & $\mathrm{XRX}$ & 98412JAG9 & 100,96 & 7,02 & 7,29 & 31.05 .01 & 4,19 \\
\hline 1641 & $\mathrm{XRX}$ & $98412 \mathrm{JAH} 7$ & 98,70 & 7,42 & 7,04 & 30.04 .99 & 4,09 \\
\hline 1642 & $\mathrm{XRX}$ & 98412JAH7 & 101,05 & 6,65 & 7,04 & 30.04 .99 & 3,08 \\
\hline 1643 & $\mathrm{XRX}$ & 984I2JAH7 & 100,23 & 6,92 & 7,04 & 30.04 .99 & 2,07 \\
\hline 1644 & $\mathrm{XRX}$ & $98412 \mathrm{JAJ} 3$ & 98,60 & 7,42 & 7,01 & 30.04 .99 & 4,09 \\
\hline 1645 & $\mathrm{XRX}$ & $98412 \mathrm{JAJ} 3$ & 100,97 & 6,65 & 7,01 & 30.04 .99 & 3,08 \\
\hline 1646 & $\mathrm{XRX}$ & $984 ! 2 \mathrm{JAJ} 3$ & 100,17 & 6,92 & 7,01 & 30.04 .99 & 2,07 \\
\hline 1647 & $\mathrm{XRX}$ & 98412JAK0 & 100,26 & 7,30 & 7,35 & 15.05 .01 & 6,16 \\
\hline 1648 & $\mathrm{XRX}$ & 98412JAK0 & 102,68 & 6,72 & 7,35 & 15.05 .01 & 5,15 \\
\hline 1649 & $\mathrm{XRX}$ & $98412 \mathrm{JAK} 0$ & 101,20 & 7,01 & 7,35 & 15.05 .01 & 4,14 \\
\hline 1650 & $\mathrm{XRX}$ & 98412JAL8 & 98,87 & 7,42 & 7,09 & 30.04 .99 & 4,09 \\
\hline 1651 & $\mathrm{XRX}$ & 98412JAL8 & 101,19 & 6,65 & 7,09 & 30.04 .99 & 3,08 \\
\hline 1652 & $\mathrm{XRX}$ & 98412JAL8 & 100,32 & 6,92 & 7,09 & 30.04 .99 & 2,07 \\
\hline 1653 & $\mathrm{XRX}$ & 98412JAM6 & 100,31 & 7,30 & 7,36 & 15.05 .01 & 6,16 \\
\hline 1654 & $\mathrm{XRX}$ & 98412JAM6 & 102,73 & 6,72 & 7,36 & 15.05 .01 & 5,15 \\
\hline 1655 & $\mathrm{XRX}$ & 98412JAM6 & 101,23 & 7,01 & 7,36 & 15.05 .01 & 4,14 \\
\hline 1656 & XRX & 98412JAM6 & 102,73 & 6,72 & 7,36 & 15.05 .01 & 5,15 \\
\hline 1657 & XRX & 98412JAN4 & 101,10 & 6,66 & 7,06 & 05.05 .99 & 3,09 \\
\hline
\end{tabular}




\begin{tabular}{|c|c|c|c|c|c|c|c|}
\hline Lfd. Nr. & Ticker & CUSIP & Ask Kurs in Dollar & ISMA Rendite in \% & Kupon in \% & Falligkeit & Restlaufzeit [Jahren] \\
\hline 1658 & XRX & $98412 \mathrm{JAN} 4$ & 100,25 & 6,93 & 7,06 & 05.05 .99 & 2,08 \\
\hline 1659 & XRX & $98412 \mathrm{JAN} 4$ & 101,10 & 6.66 & 7,06 & 05.05 .99 & 3,09 \\
\hline 1660 & XRX & $98412 \mathrm{JAP} 9$ & 101,34 & 6,66 & 7,15 & 05.05 .99 & 3,09 \\
\hline 1661 & XRX & $98412 \mathrm{JAP} 9$ & 100,42 & 6,93 & 7,15 & 05.05 .99 & 2,08 \\
\hline 1662 & XRX & $98412 \mathrm{JAS} 3$ & 103,14 & 6,72 & 7,46 & 07.05 .01 & 5,13 \\
\hline 1663 & XRX & $98412 \mathrm{JAS} 3$ & 101,57 & 7,01 & 7,46 & 07.05 .01 & 4,12 \\
\hline 1664 & XRX & $98412 \mathrm{JBAl}$ & 94,47 & 7,76 & 7,20 & 01.04 .16 & 19,24 \\
\hline 1665 & XRX & $98412 \mathrm{JBB} 9$ & 103,67 & 4,67 & 6,63 & 16.04 .01 & 2,04 \\
\hline 1666 & XRX & $98412 \mathrm{JBNW} 3$ & 98,03 & 5,74 & 5,25 & 15.12 .03 & 4,74 \\
\hline
\end{tabular}

\section{Tabelle 49: Untersuchte Menge an Unternehmensanleihen}

\begin{tabular}{|c|c|c|c|c|}
\hline Ldf. Nummer & CUSIP & Ask-Kurs in Dollar & Fylligkeit & Kupon in \% \\
\hline 1 & $912810 \mathrm{EQ}^{7}$ & 86,11 & 15.02 .10 & 11,75 \\
\hline 2 & $912827 \mathrm{~L} 83$ & 91,98 & 30.04 .99 & 6,38 \\
\hline 3 & $912827 \mathrm{~N} 81$ & 92,34 & 06.05 .99 & 0,00 \\
\hline 4 & $912810 \mathrm{BG} 2$ & 92,91 & 18.12 .97 & 0,00 \\
\hline 5 & $912827 \mathrm{M} 66$ & 94,01 & 17.09 .98 & 0,00 \\
\hline 6 & $912827 \mathrm{M} 41$ & 94,22 & 10.09 .98 & 0,00 \\
\hline 7 & 912827 N65 & 94,40 & 22.10 .98 & 0,00 \\
\hline 8 & $912827 \mathrm{M} 25$ & 94,50 & 03.09 .98 & 0,00 \\
\hline 9 & $912827 \mathrm{~K} 43$ & 94,75 & 31.12 .98 & 5,13 \\
\hline 10 & $912827 \mathrm{~N} 40$ & 94,95 & 08.10 .98 & 0,00 \\
\hline 11 & $912827 N 24$ & 95,06 & 01.10 .98 & 0,00 \\
\hline 12 & $912827 J 78$ & 95,55 & 15.08 .05 & 10,75 \\
\hline 13 & $912827 \mathrm{~L} 42$ & 95,88 & 27.08 .98 & 0,00 \\
\hline 14 & $912827 L 67$ & 95,93 & 31.08 .98 & 4,75 \\
\hline 15 & $912827 \mathrm{~K} 68$ & 95,96 & 15.08 .98 & 9,25 \\
\hline 16 & $912827 \mathrm{P} 22$ & 96,02 & 31.10 .98 & 5,88 \\
\hline 17 & $912827 \mathrm{~K} 35$ & 96,12 & 06.08 .98 & 0,00 \\
\hline 18 & $912827 \mathrm{J94}$ & 96,22 & 31.07 .98 & 6,25 \\
\hline 19 & 912810 EP9 & 96,28 & 15.11 .09 & 10,38 \\
\hline 20 & $912827 \mathrm{~L} 26$ & 96,52 & 20.08 .98 & 0,00 \\
\hline 21 & $912827 \mathrm{G} 55$ & 96,67 & 15.05 .05 & 8,25 \\
\hline 22 & 912827 N73 & 97,09 & 31.05 .98 & 6,00 \\
\hline 23 & $912827 \mathrm{P} 48$ & 97,15 & 05.11 .98 & 0,00 \\
\hline 24 & $912827 \mathrm{H} 21$ & 97,33 & 27.11 .98 & 0,00 \\
\hline 25 & $912827 \mathrm{J45}$ & 97,57 & 31.07 .98 & 5,25 \\
\hline 26 & 912810 EM6 & 97,67 & 15.11 .08 & 4,75 \\
\hline 27 & $912827 \mathrm{G} 97$ & 97,70 & 25.06 .98 & 0,00 \\
\hline 28 & 912810DWs & 97,77 & 15.02 .08 & 5,50 \\
\hline 29 & $912827 \mathrm{G} 30$ & 97,94 & 11.06 .98 & 0,00 \\
\hline 30 & $912827 \mathrm{G} 71$ & 98,03 & 18.06 .98 & 0,00 \\
\hline 31 & $912827 \mathrm{H} 47$ & 98,14 & 30.06 .98 & 6,25 \\
\hline 32 & $912827 \mathrm{~J} 37$ & 98,34 & 15.07 .02 & 3,63 \\
\hline 33 & $912827 J 29$ & 98,63 & 23.07 .98 & 0,00 \\
\hline 34 & $912827 \mathrm{H} 88$ & 98,63 & 15.07 .98 & 8,25 \\
\hline 35 & $912827 \mathrm{~F} 98$ & 98,78 & 31.12 .01 & 6,13 \\
\hline 36 & $912827 D 74$ & 99,16 & 15.08 .01 & 7,88 \\
\hline 37 & $912827 P 63$ & 99,20 & 30.09 .01 & 6,38 \\
\hline 38 & 912827D90 & 99,71 & 31.10 .00 & 4,00 \\
\hline 39 & $912827 \mathrm{~F} 80$ & 99,84 & 31.01 .01 & 5,25 \\
\hline 40 & $912827 Q 70$ & 99,95 & 15.02 .01 & 11,75 \\
\hline 41 & 912827047 & 99,99 & 30.11 .01 & 5,88 \\
\hline 42 & $912827 \mathrm{Q} 21$ & 100,00 & 15.11 .01 & 15,75 \\
\hline 43 & 912827P71 & 100,00 & 07.12 .00 & 0,00 \\
\hline 44 & $912827 \mathrm{~T} 36$ & 100.29 & 15.11 .00 & 5.75 \\
\hline 45 & $912827 \mathrm{~T} 44$ & 100,34 & 30.09 .02 & 5,88 \\
\hline 46 & $912810 \mathrm{DX} 3$ & 100,36 & 15.05 .08 & 5,63 \\
\hline 47 & $912827 R 20$ & 100,41 & 28.02 .02 & 6,25 \\
\hline 48 & $912827 \mathrm{Q} 62$ & 100,41 & 31.01 .02 & 6.25 \\
\hline 49 & $912827 F 64$ & 100,48 & 31.12 .00 & 4,63 \\
\hline
\end{tabular}




\begin{tabular}{|c|c|c|c|c|}
\hline Ldf. Nummer & CUSIP & Ask-Kurs in Dollar & Falligkeit & Kupon in \% \\
\hline 50 & 912827E57 & 100,55 & 31.10 .00 & 5,75 \\
\hline 51 & $912810 \mathrm{BV} 9$ & 100,66 & 21.08 .97 & 0,00 \\
\hline 52 & $912827 S 94$ & 100,72 & 09.11 .00 & 0,00 \\
\hline 53 & 912827 F23 & 100,76 & 30.11 .00 & 4,63 \\
\hline 54 & 912827E73 & 100,77 & 15.11 .00 & 8,50 \\
\hline 55 & $912827 \mathrm{E} 81$ & 101,02 & 15.08 .01 & 13,38 \\
\hline 56 & $912810 \mathrm{ES} 3$ & 101,13 & 15.05 .10 & 10,00 \\
\hline 57 & 912827088 & 101,27 & 15.11 .05 & 5,88 \\
\hline 58 & $912827 \mathrm{P} 89$ & 101,28 & 15.08 .05 & 6,50 \\
\hline 59 & $912827 \mathrm{~T} 28$ & 101,30 & 31.08 .02 & 6,25 \\
\hline 60 & $912827 R 46$ & 101,34 & 31.03 .02 & 6,63 \\
\hline 61 & $912810 \mathrm{BX} 5$ & 101,63 & 15.05 .07 & 6,63 \\
\hline 62 & 912827C67 & 101,69 & 31.07 .01 & 6,63 \\
\hline 63 & $912810 \mathrm{BW} 7$ & 101,71 & 15.08 .03 & 5,25 \\
\hline 64 & $912827 S 78$ & 101,72 & 28.02 .01 & 5,63 \\
\hline 65 & $912827 \mathrm{~S} 52$ & 101,74 & 12.10 .00 & 0,00 \\
\hline 66 & 912827R79 & 101,85 & 15.02 .01 & 5,38 \\
\hline 67 & $912810 \mathrm{EN} 4$ & 102,19 & 15.05 .09 & 9,13 \\
\hline 68 & 912827R61 & 102,78 & 30.04 .02 & 6,63 \\
\hline 69 & $912827 \mathrm{D} 25$ & 103,08 & 15.02 .04 & 5,88 \\
\hline 70 & $912810 \mathrm{ET} 1$ & 103,13 & 15.11 .10 & 12,75 \\
\hline 71 & $912827 \mathrm{ZT2}$ & 103,21 & 15.02 .01 & 7,75 \\
\hline 72 & $912827 S 86$ & 103,25 & 15.02 .06 & 5,63 \\
\hline 73 & $912827 F 49$ & 103,27 & 15.11 .04 & 7,88 \\
\hline 74 & 912827A44 & 103,42 & 30.04 .01 & 6,25 \\
\hline 75 & $912827 S 29$ & 103,76 & 31.05 .02 & 6,50 \\
\hline 76 & $912827 S 45$ & 103,79 & 30.06 .02 & 6,25 \\
\hline 77 & $912827 \mathrm{~S} 60$ & 103,80 & 31.07 .02 & 6,00 \\
\hline 78 & $912810 \mathrm{BZO}$ & 103,83 & 15.01 .08 & 3,63 \\
\hline 79 & 912827VW9 & 103,93 & 31.07 .97 & 0,00 \\
\hline 80 & 912827YE6 & 104,26 & 15.05 .02 & 7,50 \\
\hline 81 & $912827 \mathrm{ZX} 3$ & 104,29 & 15.02 .03 & 6,25 \\
\hline 82 & 912810 EH7 & 104,30 & 15.08 .08 & 8,38 \\
\hline 83 & $912827 \times W 7$ & 104,64 & 15.02 .02 & 14,25 \\
\hline 84 & 912827B50 & 104,65 & 31.07 .97 & 5,88 \\
\hline 85 & $912810 \mathrm{BU}$ & 104,65 & 15.09 .97 & 0,00 \\
\hline 86 & $912827 \mathrm{~B} 92$ & 104,98 & 15.08 .03 & 11,13 \\
\hline 87 & 912827 VN9 & 105,38 & 24.07 .97 & 0,00 \\
\hline 88 & 912827R87 & 105,63 & 15.02 .06 & 9,38 \\
\hline 89 & 912827A85 & 105,67 & 30.04 .03 & 5,75 \\
\hline 90 & 912810 EL8 & 106,02 & 15.11 .08 & 8,75 \\
\hline 91 & 912827WE8 & 106,55 & 31.07 .97 & 0,00 \\
\hline 92 & $912827 W W 8$ & 106,86 & 07.08 .97 & 0,00 \\
\hline 93 & 912810 ED6 & 106,94 & 06.11 .97 & 0,00 \\
\hline 94 & 912827 YN6 & 106,96 & 15.08 .97 & 8,63 \\
\hline 95 & $912810 \mathrm{CC} 0$ & 107,13 & 25.09 .97 & 0,00 \\
\hline 96 & $912827 \times E 7$ & 107,21 & 07.08 .97 & 0,00 \\
\hline 97 & $912810 \mathrm{EJ} 3$ & 107,28 & 15.11 .97 & 7,38 \\
\hline 98 & $912810 \mathrm{EKO}$ & 107,30 & 20.11 .97 & 0,00 \\
\hline 99 & $912827 \mathrm{WN} 8$ & 107,66 & 31.07 .97 & 5,50 \\
\hline 100 & $912827 \mathrm{ZNS}$ & 107,91 & 28.08 .97 & 0,00 \\
\hline 101 & $912827 \mathrm{XN7}$ & 108,44 & 14.08 .97 & 0,00 \\
\hline 102 & 912827ZE5 & 108,55 & 21.08 .97 & 0,00 \\
\hline 103 & $912827 \mathrm{YW} 6$ & 108,95 & 15.08 .97 & 6,50 \\
\hline 104 & $912810 \mathrm{CE} 6$ & 109,63 & 25.09 .97 & 0,00 \\
\hline 105 & $912810 \mathrm{EE} 4$ & 111,19 & 13.11 .97 & 0,00 \\
\hline 106 & $912810 \mathrm{CG} 1$ & 112,38 & 30.09 .97 & 5,50 \\
\hline 107 & 912810DY1 & 113,47 & 30.10 .97 & 0,00 \\
\hline 108 & $912810 \mathrm{EF} 1$ & 114,06 & 13.11 .97 & 0,00 \\
\hline 109 & 912810EG9 & 114,11 & 15.11 .97 & 8,88 \\
\hline 110 & $912810 \mathrm{DZ} 8$ & 114,86 & 30.10 .97 & 0,00 \\
\hline 111 & $912810 \mathrm{EC} 8$ & 115,17 & 06.11 .97 & 0,00 \\
\hline 112 & $912810 \mathrm{~EB} 0$ & 116,52 & 31.10 .97 & 5,63 \\
\hline
\end{tabular}




\begin{tabular}{|c|c|c|c|c|}
\hline Ldf. Nummer & CUSIP & Ask-Kurs in Dollar & Falligkeit & Kupon in \% \\
\hline 113 & 912810 DU9 & 116,81 & 18.09 .97 & 0,00 \\
\hline 114 & $912810 \mathrm{EA2}$ & 117,80 & 31.10 .97 & 5,75 \\
\hline 115 & $912810 \mathrm{DV} 7$ & 118,58 & 23.10 .97 & 0,00 \\
\hline 116 & $912810 \mathrm{CP} 1$ & 119,39 & 02.10 .97 & 0,00 \\
\hline 117 & $912810 \mathrm{CK} 2$ & 121,44 & 30.09 .97 & 5,75 \\
\hline 118 & $912810 \mathrm{DC} 9$ & 122,02 & 31.08 .97 & 5,63 \\
\hline 119 & $912810 \mathrm{DD} 7$ & 122,47 & 04.09 .97 & 0,00 \\
\hline 120 & $912810 \mathrm{CT} 3$ & 123,59 & 28.08 .97 & 0,00 \\
\hline 121 & $912810 \mathrm{DB} 1$ & 124,80 & 09.10 .97 & 0,00 \\
\hline 122 & $9 ! 2810 \mathrm{DT} 2$ & 125,17 & 23.10 .97 & 0,00 \\
\hline 123 & 912810DE5 & 125,23 & 04.09 .97 & 0,00 \\
\hline 124 & 912810 DR6 & 125,97 & 18.09 .97 & 0,00 \\
\hline 125 & $912810 \mathrm{DA} 3$ & 126,73 & 31.08 .97 & 6,00 \\
\hline 126 & $912810 \mathrm{DG} 0$ & 130,42 & 11.09 .97 & 0,00 \\
\hline 127 & $912810 \mathrm{CUO}$ & 130,54 & 15.02 .25 & 7,63 \\
\hline 128 & $912810 \mathrm{DM} 7$ & 131,04 & 11.09 .97 & 0,00 \\
\hline 129 & $912810 \mathrm{CM} 8$ & 131,83 & 02.10 .97 & 0,00 \\
\hline 130 & $912810 \mathrm{CW6}$ & 132,69 & 15.08 .25 & 6,88 \\
\hline 131 & $912810 \mathrm{DS} 4$ & 132,91 & 16.10 .97 & 0,00 \\
\hline 132 & $912810 \mathrm{DQ} 8$ & 134,53 & 15.09 .97 & 0,00 \\
\hline 133 & $912810 \mathrm{DH} 8$ & 134,80 & 15.11 .26 & 6,50 \\
\hline 134 & $912810 \mathrm{DN} 5$ & 138,80 & 15.10 .97 & 8,75 \\
\hline 135 & $912810 \mathrm{DPO}$ & 139,09 & 16.10 .97 & 0,00 \\
\hline 136 & $912810 \mathrm{DF} 2$ & 139,22 & 09.10 .97 & 0,00 \\
\hline 137 & $912810 \mathrm{CZ} 9$ & 139,44 & 15.08 .26 & 6,75 \\
\hline 138 & $912810 \mathrm{CSS}$ & 140,28 & 15.08 .27 & 6,38 \\
\hline 139 & $912810 \mathrm{DK} 1$ & 144,80 & 15.02 .27 & 6,63 \\
\hline 140 & 912810 DL9 & 144,98 & 15.11 .28 & 5,25 \\
\hline 141 & $912810 \mathrm{CX} 4$ & 146,06 & 15.02 .26 & 6,00 \\
\hline 142 & $912810 \mathrm{CV} 8$ & 149,91 & 15.11 .27 & 6,13 \\
\hline 143 & $9128100 \mathrm{J4}$ & 151,30 & 15.08 .28 & 5,50 \\
\hline 144 & 912810 CY2 & 152,31 & 15.04 .28 & 3,63 \\
\hline 145 & $912810 \mathrm{EW} 4$ & 90,05 & 27.05 .99 & 0,00 \\
\hline 146 & $912810 \mathrm{EQ7}$ & 91,37 & 15.02 .10 & 11,75 \\
\hline 147 & $912827 \mathrm{~W} 81$ & 93,95 & 15.05 .99 & 9,13 \\
\hline 148 & $912827 \mathrm{~V} 82$ & 95,77 & 13.05 .99 & 0,00 \\
\hline 149 & $912827 \mathrm{~L} 83$ & 95,81 & 30.04 .99 & 6,38 \\
\hline 150 & $912827 W 65$ & 96,13 & 28.02 .99 & 5,88 \\
\hline 151 & $912827 \mathrm{~N} 81$ & 96,56 & 06.05 .99 & 0,00 \\
\hline 152 & $912827 \mathrm{~W} 40$ & 96,92 & 18.02 .99 & 0,00 \\
\hline 153 & $912827 \mathrm{M} 66$ & 97,05 & 17.09 .98 & 0,00 \\
\hline 154 & $912827 \mathrm{M} 41$ & 97,20 & 10.09 .98 & 0,00 \\
\hline 155 & $912827 \mathrm{M} 25$ & 97,29 & 03.09 .98 & 0,00 \\
\hline 156 & $912827 W 73$ & 97,31 & 29.10 .98 & 0,00 \\
\hline 157 & 912827 N65 & 97,31 & 22.10 .98 & 0,00 \\
\hline 158 & $912827 \times 23$ & 97,39 & 04.03 .99 & 0,00 \\
\hline 159 & $912827 W 24$ & 97,45 & 15.02 .99 & 8,88 \\
\hline 160 & $912827 \mathrm{~K} 43$ & 97,67 & 31.12 .98 & 5,13 \\
\hline 161 & $912827 \mathrm{~N} 40$ & 97,76 & 08.10 .98 & 0,00 \\
\hline 162 & 912827 N24 & 97,81 & 01.10 .98 & 0,00 \\
\hline 163 & $912827 \mathrm{~V} 66$ & 98,00 & 04.02 .99 & 0,00 \\
\hline 164 & $912827 \mathrm{~L} 42$ & 98,30 & 27.08 .98 & 0,00 \\
\hline 165 & 912827 L67 & 98,45 & 31.08 .98 & 4,75 \\
\hline 166 & $912827 \mathrm{P} 22$ & 98,50 & 31.10 .98 & 5,88 \\
\hline 167 & $912827 \mathrm{~K} 68$ & 98,50 & 15.08 .98 & 9.25 \\
\hline 168 & 912827 W57 & 98,50 & 30.07 .98 & 0,00 \\
\hline 169 & $912827 \mathrm{~K} 35$ & 98,57 & 06.08 .98 & 0,00 \\
\hline 170 & $912827 J 94$ & 98,66 & 31.07 .98 & 6,25 \\
\hline 171 & $912827 \cup 42$ & 98,67 & 15.01 .99 & 6,38 \\
\hline 172 & $912827 \mathrm{~V} 74$ & 98,72 & 30.09 .98 & 4,75 \\
\hline 173 & 912827 L26 & 98,86 & 20.08 .98 & 0,00 \\
\hline 174 & $912827 \mathrm{~J} 78$ & 98,97 & 15.08 .05 & 10,75 \\
\hline 175 & $912827 \mathrm{~W} 32$ & 99,03 & 16.07 .98 & 0,00 \\
\hline
\end{tabular}




\begin{tabular}{|c|c|c|c|c|}
\hline Ldf. Nummer & CUSIP & Ask-Kurs in Dollar & Falligkeit & Kupon in $\%$ \\
\hline 176 & $912827 \mathrm{~V} 90$ & 99,26 & 09.07 .98 & 0,00 \\
\hline 177 & $912827 N 73$ & 99,38 & 31.05 .98 & 6,00 \\
\hline 178 & $912810 \mathrm{BG} 2$ & 99,41 & 18.12 .97 & 0,00 \\
\hline 179 & $912827 \mathrm{P} 48$ & 99,47 & 05.11 .98 & 0,00 \\
\hline 180 & $912827 \mathrm{~V} 41$ & 99,48 & 15.02 .03 & 10,75 \\
\hline 181 & $912827 \mathrm{~J} 45$ & 99,52 & 31.07 .98 & 5,25 \\
\hline 182 & 912827 U67 & 99,53 & 31.12 .02 & 5,63 \\
\hline 183 & $912827 \mathrm{H} 21$ & 99,60 & 27.11 .98 & 0,00 \\
\hline 184 & $912827 \mathrm{~T} 85$ & 99,63 & 15.05 .06 & 6,88 \\
\hline 185 & 912827 U83 & 99,63 & 15.07 .06 & 7,00 \\
\hline 186 & $912827 \mathrm{G} 97$ & 99,64 & 25.06 .98 & 0,00 \\
\hline 187 & $912827 \mathrm{G} 30$ & 99,75 & 11.06 .98 & 0,00 \\
\hline 188 & $912827 \mathrm{U} 75$ & 99,77 & 31.08 .98 & 6,13 \\
\hline 189 & $912827 \mathrm{G} 71$ & 99,81 & 18.06 .98 & 0,00 \\
\hline 190 & 912827 V 58 & 99,91 & 02.07 .98 & 0,00 \\
\hline 191 & $912827 G 55$ & 99,92 & 15.05 .05 & 8,25 \\
\hline 192 & $912827 \mathrm{H} 47$ & 99,94 & 30.06 .98 & 6,25 \\
\hline 193 & $912827 \mathrm{U} 34$ & 99,94 & 31.05 .98 & 5,38 \\
\hline 194 & $912827 \mathrm{~V} 33$ & 99,95 & 30.06 .98 & 5,13 \\
\hline 195 & $912827 \mathrm{~V} 25$ & 99,97 & 31.01 .03 & 5,50 \\
\hline 196 & $912827 \mathrm{U} 26$ & 100,05 & 30.11 .02 & 5,75 \\
\hline 197 & 912827U59 & 100,16 & 04.06 .98 & 0,00 \\
\hline 198 & $912827 J 29$ & 100,22 & 23.07 .98 & 0,00 \\
\hline 199 & $912827 \mathrm{H} 88$ & 100,25 & 15.07 .98 & 8,25 \\
\hline 200 & 912827 U91 & 100,27 & 15.06 .98 & 0,00 \\
\hline 201 & $912810 \mathrm{EV} 6$ & 100,34 & 15.05 .11 & 13,88 \\
\hline 202 & $912827 T 77$ & 100,34 & 15.05 .01 & 8,00 \\
\hline 203 & $912827 \times 31$ & 100,41 & 31.03 .01 & 6,38 \\
\hline 204 & $912827 \times 49$ & 100,44 & 28.02 .03 & 5,50 \\
\hline 205 & 912827193 & 100,50 & 31.12 .00 & 5,50 \\
\hline 206 & $912827 \mathrm{D} 90$ & 100,61 & 31.10 .00 & 4,00 \\
\hline 207 & $912827 \mathrm{~J} 37$ & 100,63 & 15.07 .02 & 3,63 \\
\hline 208 & $912827 \mathrm{F98}$ & 100,71 & 31.12 .01 & 6,13 \\
\hline 209 & $912827 \mathrm{~F} 80$ & 100,78 & 31.01 .01 & 5,25 \\
\hline 210 & 912827 D74 & 100,78 & 15.08 .01 & 7,88 \\
\hline 211 & $912827 \mathrm{P} 71$ & 100,89 & 07.12 .00 & 0,00 \\
\hline 212 & 912827T51 & 100,89 & 30.11 .00 & 5,63 \\
\hline 213 & $912810 \mathrm{BW7}$ & 100,91 & 15.08 .03 & 5,25 \\
\hline 214 & 912827070 & 100,95 & 15.02 .01 & 11,75 \\
\hline 215 & $912827 \mathrm{~T} 36$ & 100,97 & 15.11 .00 & 5,75 \\
\hline 216 & 912827E57 & 101,02 & 31.10 .00 & 5,75 \\
\hline 217 & 912827594 & 101,13 & 09.11 .00 & 0,00 \\
\hline 218 & $912827 F 64$ & 101,14 & 31.12 .00 & 4,63 \\
\hline 219 & 912827 P63 & 101,16 & 30.09 .01 & 6,38 \\
\hline 220 & $912827 \mathrm{E} 73$ & 101,20 & 15.11 .00 & 8,50 \\
\hline 221 & 912827 F23 & 101,28 & 30.11 .00 & 4,63 \\
\hline 222 & $912827 \mathrm{~S} 52$ & 101,53 & 12.10 .00 & 0,00 \\
\hline 223 & $912827 \mathrm{Q} 21$ & 101,75 & 15.11 .01 & 15,75 \\
\hline 224 & 912827047 & 101,80 & 30.11 .01 & 5,88 \\
\hline 225 & $912827 \mathrm{~T} 69$ & 101,81 & 31.10 .02 & 5,75 \\
\hline 226 & 912810 EP9 & 101,91 & 15.11 .09 & 10,38 \\
\hline 227 & $912827 Q 62$ & 102,16 & 31.01 .02 & 6,25 \\
\hline 228 & 912827R20 & 102,16 & 28.02 .02 & 6,25 \\
\hline 229 & $912827 S 78$ & 102,26 & 28.02 .01 & 5,63 \\
\hline 230 & $912827 \mathrm{~T} 44$ & 102,27 & 30.09 .02 & 5,88 \\
\hline 231 & 912827R79 & 102,27 & 15.02 .01 & 5,38 \\
\hline 232 & $912827 \mathrm{E} 81$ & 102,44 & 15.08 .01 & 13,38 \\
\hline 233 & $912827 \mathrm{C} 67$ & 102,55 & 31.07 .01 & 6,63 \\
\hline 234 & $912827 \mathrm{R} 46$ & 102,95 & 31.03 .02 & 6,63 \\
\hline 235 & $912827 T 28$ & 103,09 & 31.08 .02 & 6,25 \\
\hline 236 & 912810DWs & 103,19 & 15.02 .08 & 5,50 \\
\hline 237 & $912810 \mathrm{EM} 6$ & 103,24 & 15.11 .08 & 4,75 \\
\hline 238 & $912827 Z \mathrm{ZT2}$ & 103,28 & 15.02 .01 & 7,75 \\
\hline
\end{tabular}




\begin{tabular}{|c|c|c|c|c|}
\hline Ldf. Nummer & CUSIP & Ask-Kurs in Dollar & Falligkeit & Kupon in \% \\
\hline 239 & $912827 \mathrm{~A} 44$ & 103,63 & 30.04 .01 & 6,25 \\
\hline 240 & $912827 \mathrm{VW9}$ & 103,78 & 31.07 .97 & 0,00 \\
\hline 241 & 912827R61 & 104,11 & 30.04 .02 & 6,63 \\
\hline 242 & $912827 P 89$ & 104,47 & 15.08 .05 & 6,50 \\
\hline 243 & 912827088 & 104,50 & 15.11 .05 & 5,88 \\
\hline 244 & 912827VN9 & 104,53 & 24.07 .97 & 0,00 \\
\hline 245 & $912827 \mathrm{BSO}$ & 104,68 & 31.07 .97 & 5,88 \\
\hline 246 & $912827 S 29$ & 105,00 & 31.05 .02 & 6,50 \\
\hline 247 & $912827 S 45$ & 105,09 & 30.06 .02 & 6,25 \\
\hline 248 & 912827560 & 105,13 & 31.07 .02 & 6,00 \\
\hline 249 & $912810 \mathrm{BXS}$ & 105,24 & 15.05 .07 & 6,63 \\
\hline 250 & $912827 \mathrm{YE} 6$ & 105,33 & 15.05 .02 & 7,50 \\
\hline 251 & $912827 \mathrm{D} 25$ & 105,36 & 15.02 .04 & 5,88 \\
\hline 252 & $912827 \times W 7$ & 105,48 & 15.02 .02 & 14,25 \\
\hline 253 & $912827 \mathrm{~F} 49$ & 105,69 & 15.11 .04 & 7,88 \\
\hline 254 & 912827WVE8 & 105,87 & 31.07 .97 & 0,00 \\
\hline 255 & $912810 \mathrm{DX} 3$ & 105,91 & 15.05 .08 & 5,63 \\
\hline 256 & $9128272 \times 3$ & 106,06 & 15.02 .03 & 6,25 \\
\hline 257 & $912827 S 86$ & 106,25 & 15.02 .06 & 5,63 \\
\hline 258 & $912827 W W 8$ & 106,70 & 07.08 .97 & 0,00 \\
\hline 259 & $912810 \mathrm{BUI}$ & 106,71 & 15.09 .97 & 0,00 \\
\hline 260 & $912810 \mathrm{ES} 3$ & 106,92 & 15.05 .10 & 10,00 \\
\hline 261 & $912827 B 92$ & 106,92 & 15.08 .03 & 11,13 \\
\hline 262 & $912827 \mathrm{WN8}$ & 106,95 & 31.07 .97 & 5,50 \\
\hline 263 & 912827XE7 & 107,16 & 07.08 .97 & 0,00 \\
\hline 264 & 912827A85 & 107,30 & 30.04 .03 & 5,75 \\
\hline 265 & $912810 \mathrm{BZO}$ & 107,44 & 15.01 .08 & 3.63 \\
\hline 266 & $912827 \mathrm{YN} 6$ & 107,73 & 15.08 .97 & 8,63 \\
\hline 267 & $912810 \mathrm{EN} 4$ & 107,84 & 15.05 .09 & 9,13 \\
\hline 268 & $912827 \times \mathrm{N} 7$ & 108,31 & 14.08 .97 & 0,00 \\
\hline 269 & $912827 \mathrm{R} 87$ & 108,59 & 15.02 .06 & 9,38 \\
\hline 270 & $912810 \mathrm{ET} 1$ & 108,66 & 15.11 .10 & 12,75 \\
\hline 271 & $9128272 \mathrm{~N} 5$ & 108,80 & 28.08 .97 & 0,00 \\
\hline 272 & 912827ZES & 109,38 & 21.08 .97 & 0,00 \\
\hline 273 & $912827 \mathrm{YW} 6$ & 109,47 & 15.08 .97 & 6,50 \\
\hline 274 & $912810 \mathrm{EH} 7$ & 110,42 & 15.08 .08 & 8,38 \\
\hline 275 & $912810 \mathrm{CCO}$ & 110,52 & 25.09 .97 & 0,00 \\
\hline 276 & 912810 EL8 & 112,08 & 15.11 .08 & 8,75 \\
\hline 277 & 912810 CE6 & 112,69 & 25.09 .97 & 0,00 \\
\hline 278 & 912810 ED6 & 113,01 & 06.11 .97 & 0,00 \\
\hline 279 & 912810 EKO & 113,41 & 20.11 .97 & 0,00 \\
\hline 280 & $912810 \mathrm{EJ} 3$ & 113,41 & 15.11 .97 & 7,38 \\
\hline 281 & $912810 \mathrm{CGl}$ & 115,31 & 30.09 .97 & 5,50 \\
\hline 282 & $912810 \mathrm{EE} 4$ & 117,46 & 13.11 .97 & 0,00 \\
\hline 283 & $912810 \mathrm{DY} 1$ & 119,53 & 30.10 .97 & 0,00 \\
\hline 284 & $912810 \mathrm{EF} 1$ & 120,45 & 13.11 .97 & 0,00 \\
\hline 285 & 912810 EG9 & 120,52 & 15.11 .97 & 8,88 \\
\hline 286 & 912810 DU9 & 120,68 & 18.09 .97 & 0,00 \\
\hline 287 & $912810 \mathrm{DZ} 8$ & 120,98 & 30.10 .97 & 0,00 \\
\hline 288 & $912810 \mathrm{EC} 8$ & 121,46 & 06.11 .97 & 0,00 \\
\hline 289 & $912810 \mathrm{CPI}$ & 122,36 & 02.10 .97 & 0,00 \\
\hline 290 & $912810 \mathrm{CT} 3$ & 122,63 & 28.08 .97 & 0,00 \\
\hline 291 & $912810 \mathrm{~EB} 0$ & 122,81 & 31.10 .97 & 5,63 \\
\hline 292 & $912810 \mathrm{DC} 9$ & 123,44 & 31.08 .97 & 5,63 \\
\hline 293 & $912810 \mathrm{DD} 7$ & 124,02 & 04.09 .97 & 0,00 \\
\hline 294 & $912810 \mathrm{CK} 2$ & 124,04 & 30.09 .97 & 5,75 \\
\hline 295 & $912810 \mathrm{EA2}$ & 124,08 & 31.10 .97 & 5,75 \\
\hline 296 & $912810 \mathrm{DV} 7$ & 124,66 & 23.10 .97 & 0,00 \\
\hline 297 & $9128100 E 5$ & 126,70 & 04.09 .97 & 0,00 \\
\hline 298 & $912810 \mathrm{DA}^{3}$ & 127,66 & 31.08 .97 & 6,00 \\
\hline 299 & $912810 \mathrm{DB} 1$ & 128,52 & 09.10 .97 & 0,00 \\
\hline 300 & 912810DR6 & 128,96 & 18.09 .97 & 0.00 \\
\hline 301 & $912810 \mathrm{CU} 0$ & 129,34 & 15.02 .25 & 7,63 \\
\hline
\end{tabular}




\begin{tabular}{|c|c|c|c|c|}
\hline Ldf. Nummer & CUSIP & Ask-Kurs in Dollar & Falligkeit & Kupon in $\%$ \\
\hline 302 & 912810DT2 & 131,28 & 23.10 .97 & 0,00 \\
\hline 303 & $912810 \mathrm{DGO}$ & 131,70 & 11.09 .97 & 0,00 \\
\hline 304 & $912810 \mathrm{CW6}$ & 131,75 & 15.08 .25 & 6,88 \\
\hline 305 & $912810 \mathrm{DM} 7$ & 133,35 & 11.09 .97 & 0,00 \\
\hline 306 & $912810 \mathrm{CM} 8$ & 133,47 & 02.10 .97 & 0,00 \\
\hline 307 & $912810 \mathrm{DH} 8$ & 136,68 & 15.11 .26 & 6,50 \\
\hline 308 & $912810 \mathrm{DQ} 8$ & 137,08 & 15.09 .97 & 0,00 \\
\hline 309 & $912810 \mathrm{CZ9}$ & 138,55 & 15.08 .26 & 6,75 \\
\hline 310 & $912810 \mathrm{DS} 4$ & 139,17 & 16.10 .97 & 0,00 \\
\hline 311 & $912810 \mathrm{CS} 5$ & 142,64 & 15.08 .27 & 6,38 \\
\hline 312 & $912810 \mathrm{DF} 2$ & 142,94 & 09.10 .97 & 0,00 \\
\hline 313 & $912810 \mathrm{DN} 5$ & 143,37 & 15.10 .97 & 8,75 \\
\hline 314 & $912810 \mathrm{CX} 4$ & 144,41 & 15.02 .26 & 6,00 \\
\hline 315 & 912810DP0 & 145,43 & 16.10 .97 & 0,00 \\
\hline 316 & $912810 \mathrm{DK} 1$ & 145,72 & 15.02 .27 & 6,63 \\
\hline 317 & 912810DL9 & 149,25 & 15.11 .28 & 5,25 \\
\hline 318 & $912810 \mathrm{CV} 8$ & 152,20 & 15.11 .27 & 6,13 \\
\hline 319 & $912810 \mathrm{CY} 2$ & 154,92 & 15.04 .28 & 3,63 \\
\hline 320 & $912810 \mathrm{DJ} 4$ & 155,16 & 15.08 .28 & 5,50 \\
\hline 321 & $912827 \times 56$ & 100,03 & 13.08 .98 & 0,00 \\
\hline 322 & $912827 \mathrm{~K} 68$ & 100,00 & 15.08 .98 & 9,25 \\
\hline 323 & $912827 \times 98$ & 100,07 & 15.08 .98 & 5,88 \\
\hline 324 & $912827 \mathrm{~L} 26$ & 100,06 & 20.08 .98 & 0,00 \\
\hline 325 & $912827 L 42$ & 100,03 & 27.08 .98 & 0,00 \\
\hline 326 & $912827 \mathrm{~L} 67$ & 99,99 & 31.08 .98 & 4,75 \\
\hline 327 & 912827 U75 & 100,16 & 31.08 .98 & 6,13 \\
\hline 328 & $912827 \mathrm{M} 25$ & 99,77 & 03.09 .98 & 0,00 \\
\hline 329 & $912827 \mathrm{M} 41$ & 99,75 & 10.09 .98 & 0,00 \\
\hline 330 & 912827247 & 100,28 & 15.09 .98 & 0,00 \\
\hline 331 & $912827 \mathrm{M} 66$ & 99,61 & 17.09 .98 & 0,00 \\
\hline 332 & 912827270 & 100,20 & 24.09 .98 & 0,00 \\
\hline 333 & $912827 \mathrm{~V} 74$ & 100,01 & 30.09 .98 & 4,75 \\
\hline 334 & $9128272 \mathrm{~B} 7$ & 100,12 & 30.09 .98 & 6,00 \\
\hline 335 & 912827 N24 & 99,80 & 01.10 .98 & 0,00 \\
\hline 336 & $912827 \mathrm{~N} 40$ & 99,77 & 08.10 .98 & 0,00 \\
\hline 337 & $9128272 \mathrm{D} 3$ & 100,23 & 15.10 .98 & 0,00 \\
\hline 338 & $9128272 \mathrm{~F} 8$ & 100,28 & 15.10 .98 & 7,13 \\
\hline 339 & $912827 \mathrm{~N} 65$ & 99,61 & 22.10 .98 & 0,00 \\
\hline 340 & $912827 W 73$ & 99,59 & 29.10 .98 & 0,00 \\
\hline 341 & $912827 \mathrm{P} 22$ & 100,01 & 31.10 .98 & 5,88 \\
\hline 342 & $9128272 \mathrm{~K} 7$ & 100,33 & 31.10 .98 & 4,75 \\
\hline 343 & $912827 \mathrm{P} 48$ & 100,38 & 05.11 .98 & 0,00 \\
\hline 344 & $9128272 \times 9$ & 100,55 & 12.11 .98 & 0,00 \\
\hline 345 & $9128273 \mathrm{~B} 6$ & 100,38 & 15.11 .98 & 8,88 \\
\hline 346 & $9128273 F 7$ & 100,42 & 15.11 .98 & 5,50 \\
\hline 347 & $912827 Y 89$ & 100,56 & 15.11 .98 & 3,50 \\
\hline 348 & $9128273 \mathrm{H} 3$ & 100,27 & 19.11 .98 & 0,00 \\
\hline 349 & $912827 \mathrm{H} 21$ & 100,63 & 27.11 .98 & 0,00 \\
\hline 350 & $9128273 \mathrm{~K} 6$ & 100,07 & 30.11 .98 & 5,63 \\
\hline 351 & 912827296 & 100,46 & 30.11 .98 & 5,13 \\
\hline 352 & 9128273P5 & 100,09 & 03.12 .98 & 0,00 \\
\hline 353 & 9128273RI & 100,13 & 10.12 .98 & 0,00 \\
\hline 354 & $9128273 \cup 4$ & 99,70 & 15.12 .98 & 0,00 \\
\hline 355 & $9128272 \mathrm{H} 4$ & 100,56 & 17.12 .98 & 0,00 \\
\hline 356 & $9128273 Y 6$ & 99,91 & 24.12 .98 & 0,00 \\
\hline 357 & $912827 \mathrm{~K} 43$ & 99,92 & 31.12 .98 & 5,13 \\
\hline 358 & $9128274 \mathrm{~A} 7$ & 99,94 & 31.12 .98 & 0,00 \\
\hline 359 & $912827 \mathrm{U} 42$ & 100,66 & 15.01 .99 & 6,38 \\
\hline 360 & $9128273 \mathrm{D} 2$ & 100,97 & 28.01 .99 & 0,00 \\
\hline 361 & $912827 \mathrm{~V} 66$ & 100,47 & 04.02 .99 & 0,00 \\
\hline 362 & $9128273 \mathrm{M} 2$ & 100,45 & 11.02 .99 & 0,00 \\
\hline 363 & $912827 \mathrm{~W} 24$ & 100,16 & 15.02 .99 & 8,88 \\
\hline 364 & $912827 \mathrm{~W} 40$ & 99,85 & 18.02 .99 & 0,00 \\
\hline
\end{tabular}




\begin{tabular}{|c|c|c|c|c|}
\hline Ldf. Nummer & CUSIP & Ask-Kurs in Dollar & Falligkeit & Kupon in \% \\
\hline 365 & $912827 W 65$ & 99,22 & 28.02 .99 & 5,88 \\
\hline 366 & $9128273 \mathrm{WO}$ & 99,56 & 28.02 .99 & 5,50 \\
\hline 367 & $912827 \times 23$ & 100,13 & 04.03 .99 & 0,00 \\
\hline 368 & $9128272 \mathrm{C5}$ & 100,90 & 15.03 .99 & 0,00 \\
\hline 369 & $9128273 \mathrm{C} 4$ & 101,63 & 18.03 .99 & 0,00 \\
\hline 370 & $9128273 \mathrm{~J} 9$ & 101,07 & 25.03 .99 & 0,00 \\
\hline 371 & $9128273 Q^{3}$ & 100,59 & 31.03 .99 & 6,25 \\
\hline 372 & $9128273 \mathrm{~L} 4$ & 100,59 & 31.03 .99 & 5,88 \\
\hline 373 & 912827359 & 100,13 & 01.04 .99 & 0,00 \\
\hline 374 & $9128273 \mathrm{~V} 2$ & 99,61 & 08.04 .99 & 0,00 \\
\hline 375 & 912827323 & 99,69 & 15.04 .99 & 0,00 \\
\hline 376 & $9128274 \mathrm{BS}$ & 99,69 & 15.04 .99 & 7,00 \\
\hline 377 & $912827 L 83$ & 100,69 & 30.04 .99 & 6,38 \\
\hline 378 & $912827 N 81$ & 101,53 & 06.05 .99 & 0,00 \\
\hline 379 & $912827 \mathrm{~V} 82$ & 101,30 & 13.05 .99 & 0,00 \\
\hline 380 & $9128273 \times 8$ & 99,28 & 15.05 .99 & 8,50 \\
\hline 381 & $912827 \mathrm{~W} 81$ & 99,73 & 15.05 .99 & 9,13 \\
\hline 382 & $912810 \mathrm{EW} 4$ & 100,73 & 27.05 .99 & 0,00 \\
\hline 383 & 912827177 & 100,06 & 15.05 .01 & 8,00 \\
\hline 384 & $912827 Y 30$ & 100,22 & 15.05 .01 & 13,13 \\
\hline 385 & $912827 Y 63$ & 100,28 & 31.05 .01 & 6,50 \\
\hline 386 & 912827221 & 100,27 & 30.06 .01 & 6,63 \\
\hline 387 & $912827 \mathrm{C} 67$ & 100,88 & 31.07 .01 & 6,63 \\
\hline 388 & 912827 D74 & 100,66 & 15.08 .01 & 7,88 \\
\hline 389 & $9128272 \mathrm{N1}$ & 100,73 & 15.08 .01 & 8,00 \\
\hline 390 & $912827 \mathrm{E} 81$ & 101,47 & 15.08 .01 & 13,38 \\
\hline 391 & 9128272R2 & 100,86 & 31.08 .01 & 6,50 \\
\hline 392 & $912827 \mathrm{P} 63$ & 101,00 & 30.09 .01 & 6,38 \\
\hline 393 & $912827 \times 72$ & 100,84 & 31.10 .01 & 6,25 \\
\hline 394 & $9128272 \mathrm{~V} 3$ & 100,76 & 15.11 .01 & 7,50 \\
\hline 395 & $912827 \mathrm{Q} 21$ & 101,30 & 15.11 .01 & 15,75 \\
\hline 396 & $912827 Q 47$ & 101,41 & 30.11 .01 & 5,88 \\
\hline 397 & $912827 \mathrm{~F} 98$ & 100,99 & 31.12 .01 & 6,13 \\
\hline 398 & $912827 Q 62$ & 101,63 & 31.01 .02 & 6,25 \\
\hline 399 & $912827 \times W 7$ & 103,13 & 15.02 .02 & 14,25 \\
\hline 400 & $912827 \mathrm{R} 20$ & 101,73 & 28.02 .02 & 6,25 \\
\hline 401 & $912827 \mathrm{R} 46$ & 102,17 & 31.03 .02 & 6,63 \\
\hline 402 & 912827R61 & 102,80 & 30.04 .02 & 6,63 \\
\hline 403 & 912827 YE6 & 103,45 & 15.05 .02 & 7,50 \\
\hline 404 & $912827 \$ 29$ & 103,33 & 31.05 .02 & 6,50 \\
\hline 405 & $912827 S 45$ & 103,52 & 30.06 .02 & 6,25 \\
\hline 406 & $912827 \mathrm{~J} 37$ & 101,34 & 15.07 .02 & 3,63 \\
\hline 407 & 912827560 & 103,67 & 31.07 .02 & 6,00 \\
\hline 408 & $912827 \mathrm{~T} 28$ & 102,75 & 31.08 .02 & 6,25 \\
\hline 409 & $912827 \mathrm{~T} 44$ & 102,41 & 30.09 .02 & 5,88 \\
\hline 410 & $912827 T 69$ & 102,29 & 31.10 .02 & 5,75 \\
\hline 411 & $9128272 \mathrm{~T} 8$ & 101,61 & 15.11 .02 & 11,63 \\
\hline 412 & $912827 \cup 26$ & 101,36 & 30.11 .02 & 5,75 \\
\hline 413 & 912827 U67 & 101,22 & 31.12 .02 & 5,63 \\
\hline 414 & $912827 \mathrm{~V} 25$ & 101,52 & 31.01 .03 & 5,50 \\
\hline 415 & $912827 \mathrm{~V} 41$ & 101,30 & 15.02 .03 & 10,75 \\
\hline 416 & $9128272 \times 3$ & 105,64 & 15.02 .03 & 6,25 \\
\hline 417 & $912827 \times 49$ & 102,16 & 28.02 .03 & 5,50 \\
\hline 418 & $912827 \times 64$ & 101,86 & 31.03 .03 & 5,50 \\
\hline 419 & 912827A85 & 106,88 & 30.04 .03 & 5,75 \\
\hline 420 & $912827 Y 22$ & 102,61 & 15.05 .03 & 10,75 \\
\hline 421 & $912827 Y 48$ & 103,00 & 31.05 .03 & 5,50 \\
\hline 422 & $912827 \mathrm{Y71}$ & 103,09 & 30.06 .03 & 5,38 \\
\hline 423 & 912827239 & 102,76 & 15.08 .03 & 5,75 \\
\hline 424 & $912827 \mathrm{~B} 92$ & 106,94 & 15.08 .03 & 11,13 \\
\hline 425 & 912827288 & 102,19 & 15.11 .03 & 4,25 \\
\hline 426 & 912827254 & 102,41 & 15.11 .03 & 11,88 \\
\hline 427 & $912827 \mathrm{D} 25$ & 106,09 & 15.02 .04 & 5,88 \\
\hline
\end{tabular}




\begin{tabular}{|c|c|c|c|c|}
\hline Ldf. Nummer & CUSIP & Ask-Kurs in Dollar & Falligkeit & Kupon in \% \\
\hline 428 & $9128272 \mathrm{E} 1$ & 101,74 & 15.05 .04 & 7,25 \\
\hline 429 & $9128272 \mathrm{G} 6$ & 102,19 & 15.05 .04 & 12,38 \\
\hline 430 & $9128272 \mathrm{LS}$ & 102,23 & 15.08 .04 & 7,25 \\
\hline 431 & $9128272 \mathrm{P} 6$ & 103,59 & 15.08 .04 & 13,75 \\
\hline 432 & $9128272 \mathrm{SO}$ & 103,65 & 15.11 .04 & 11,63 \\
\hline 433 & $912827 \mathrm{~F} 49$ & 107,00 & 15.11 .04 & 7,88 \\
\hline 434 & $9128272 \mathrm{Wl}$ & 103,26 & 15.02 .05 & 7,50 \\
\hline 435 & $9128273 \mathrm{GS}$ & 102,44 & 15.05 .05 & 6,50 \\
\hline 436 & $9128272 \mathrm{Y} 7$ & 102,44 & 15.05 .05 & 12,00 \\
\hline 437 & 912827G55 & 103,00 & 15.05 .05 & 8,25 \\
\hline 438 & $912827 \mathrm{~J} 78$ & 102,84 & 15.08 .05 & 10,75 \\
\hline 439 & $912827 P 89$ & 108,30 & 15.08 .05 & 6,50 \\
\hline 440 & 912827088 & 108,55 & 15.11 .05 & 5,88 \\
\hline 441 & $912827 \mathrm{~S} 86$ & 110,36 & 15.02 .06 & 5,63 \\
\hline 442 & 912827R87 & 112,09 & 15.02 .06 & 9,38 \\
\hline 443 & $912827 \mathrm{~T} 85$ & 104,89 & 15.05 .06 & 6,88 \\
\hline 444 & 912827U83 & 104,98 & 15.07 .06 & 7,00 \\
\hline 445 & $912827 \times 80$ & 107,75 & 15.10 .06 & 6,50 \\
\hline 446 & 912827 Y55 & 108,58 & 15.01 .07 & 3,38 \\
\hline 447 & $9128272 \mathrm{~J} 0$ & 104,00 & 15.02 .07 & 7,63 \\
\hline 448 & 912827262 & 105,42 & 15.02 .07 & 6,25 \\
\hline 449 & $912810 \mathrm{BXS}$ & 106,35 & 15.05 .07 & 6,63 \\
\hline 450 & $9128272 \mathrm{US}$ & 106,66 & 15.08 .07 & 6,13 \\
\hline 451 & $9128273 \mathrm{E} 0$ & 103,33 & 15.11 .07 & 7,88 \\
\hline 452 & $912810 \mathrm{BZO}$ & 109,09 & 15.01 .08 & 3,63 \\
\hline 453 & $912810 \mathrm{DW} 5$ & 114,52 & 15.02 .08 & 5,50 \\
\hline 454 & $912810 \mathrm{DX} 3$ & 117,44 & 15.05 .08 & 5,63 \\
\hline 455 & $912810 \mathrm{EH} 7$ & 123,66 & 15.08 .08 & 8,38 \\
\hline 456 & $912810 \mathrm{EM} 6$ & 116,34 & 15.11 .08 & 4,75 \\
\hline 457 & 912810EL8 & 125,54 & 15.11 .08 & 8,75 \\
\hline 458 & $912810 \mathrm{EN} 4$ & 121,22 & 15.05 .09 & 9,13 \\
\hline 459 & 912810 EP9 & 114,94 & 15.11 .09 & 10,38 \\
\hline 460 & $912810 \mathrm{EQ} 7$ & 103,87 & 15.02 .10 & 11,75 \\
\hline 461 & $912810 \mathrm{ES} 3$ & 120,47 & 15.05 .10 & 10,00 \\
\hline 462 & $912810 \mathrm{ET} 1$ & 122,23 & 15.11 .10 & 12,75 \\
\hline 463 & $912810 \mathrm{EV} 6$ & 112,38 & 15.05 .11 & 13,88 \\
\hline 464 & $912810 \mathrm{EX} 2$ & 110,95 & 15.11 .11 & 14,00 \\
\hline 465 & $912810 \mathrm{EYO}$ & 107,57 & 15.11 .12 & 10,38 \\
\hline 466 & $912810 \mathrm{EZ7}$ & 109,42 & 15.08 .13 & 12,00 \\
\hline 467 & $912810 \mathrm{FAl}$ & 106,51 & 15.05 .14 & 13,25 \\
\hline 468 & $912810 \mathrm{FB} 9$ & 103,42 & 15.08 .14 & 12,50 \\
\hline 469 & $912810 \mathrm{CU} 0$ & 121,41 & 15.02 .25 & 7,63 \\
\hline 470 & $912810 \mathrm{CW6}$ & 123,53 & 15.08 .25 & 6,88 \\
\hline 471 & $912810 \mathrm{CX} 4$ & 132,81 & 15.02 .26 & 6,00 \\
\hline 472 & $912810 \mathrm{CZ9}$ & 129,69 & 15.08 .26 & 6,75 \\
\hline 473 & $912810 \mathrm{DH} 8$ & 134,34 & 15.11 .26 & 6,50 \\
\hline 474 & $912810 \mathrm{DK} 1$ & 142,75 & 15.02 .27 & 6,63 \\
\hline 475 & $912810 \mathrm{CS} 5$ & 142,47 & 15.08.27 & 6,38 \\
\hline 476 & $912810 \mathrm{CV} 8$ & 151,78 & 15.11 .27 & 6,13 \\
\hline 477 & 912810 CY2 & 155,08 & 15.04 .28 & 3,63 \\
\hline 478 & $912810 \mathrm{DJ} 4$ & 159,89 & 15.08 .28 & 5,50 \\
\hline 479 & 912810 DL9 & 154,59 & 15.11 .28 & 5,25 \\
\hline 480 & $9128273 \mathrm{Z3}$ & 101,43 & 15.04 .99 & 0,00 \\
\hline 481 & $9128274 \mathrm{BS}$ & 101,47 & 15.04 .99 & 7,00 \\
\hline 482 & $9128274 \mathrm{DI}$ & 102,47 & 19.04 .99 & 0,00 \\
\hline 483 & $9128274 \mathrm{H} 2$ & 101,48 & 22.04 .99 & 0,00 \\
\hline 484 & $9128274 \mathrm{KS}$ & 101,32 & 23.04 .99 & 0,00 \\
\hline 485 & $9128274 \mathrm{N9}$ & 100,61 & 29.04 .99 & 0,00 \\
\hline 486 & $9128274 \mathrm{U} 3$ & 96,66 & 30.04 .99 & 6,50 \\
\hline 487 & $912827 \mathrm{~L} 83$ & 102,51 & 30.04 .99 & 6,38 \\
\hline 488 & $912827 \mathrm{~N} 81$ & 103,44 & 06.05 .99 & 0,00 \\
\hline 489 & $912827 \mathrm{~V} 82$ & 103,72 & 13.05 .99 & 0,00 \\
\hline 490 & $9128273 \times 8$ & 102,16 & 15.05 .99 & 8,50 \\
\hline
\end{tabular}




\begin{tabular}{|c|c|c|c|c|}
\hline Ldf. Nummer & CUSIP & Ask-Kurs in Dollar & Falligkeit & Kupon in \% \\
\hline 491 & $912827 W 81$ & 102,36 & 15.05 .99 & 9,13 \\
\hline 492 & $9128274 \mathrm{~F} 6$ & 103,24 & 15.05 .99 & 6,38 \\
\hline 493 & $9128274 \mathrm{VI}$ & 97,16 & 20.05 .99 & 0,00 \\
\hline 494 & $912810 \mathrm{EW} 4$ & 103,63 & 27.05 .99 & 0,00 \\
\hline 495 & $912810 \mathrm{FFO}$ & 94,91 & 31.05 .99 & 6,75 \\
\hline 496 & $912810 \mathrm{FE} 3$ & 97,52 & 31.05 .99 & 6,25 \\
\hline 497 & $9128272 \mathrm{R2}$ & 100,07 & 31.08 .01 & 6,50 \\
\hline 498 & $912827 \mathrm{P} 63$ & 100,08 & 30.09 .01 & 6,38 \\
\hline 499 & $912827 \times 72$ & 100,13 & 31.10 .01 & 6,25 \\
\hline 500 & $9128272 \mathrm{V3}$ & 100,20 & 15.11 .01 & 7,50 \\
\hline 501 & $912827 \mathrm{Q} 21$ & 100,26 & 15.11 .01 & 15,75 \\
\hline 502 & $912827 Q 47$ & 100,47 & 30.11 .01 & 5,88 \\
\hline 503 & $912827 F 98$ & 100,48 & 31.12 .01 & 6,13 \\
\hline 504 & $912827 Q 62$ & 100,68 & 31.01 .02 & 6,25 \\
\hline 505 & $912827 \mathrm{XW7}$ & 101,14 & 15.02 .02 & 14.25 \\
\hline 506 & $912827 R 20$ & 100,84 & 28.02 .02 & 6,25 \\
\hline 507 & $912827 R 46$ & 101,14 & 31.03 .02 & 6,63 \\
\hline 508 & $912827 \mathrm{R} 61$ & 101,48 & 30.04 .02 & 6,63 \\
\hline 509 & 912827 YE6 & 101,91 & 15.05 .02 & 7,50 \\
\hline 510 & $912827 S 29$ & 101,90 & 31.05 .02 & 6,50 \\
\hline 511 & $912827 S 45$ & 102,12 & 30.06 .02 & 6,25 \\
\hline 512 & $912827 J 37$ & 101,15 & 15.07 .02 & 3,63 \\
\hline 513 & $912827 S 60$ & 102,28 & 31.07 .02 & 6,00 \\
\hline 514 & $912827 T 28$ & 101,96 & 31.08 .02 & 6,25 \\
\hline 515 & $912827 \mathrm{~T} 44$ & 101,91 & 30.09 .02 & 5,88 \\
\hline 516 & $912827 T 69$ & 102,00 & 31.10 .02 & 5,75 \\
\hline 517 & $9128272 \mathrm{~T} 8$ & 101,57 & 15.11 .02 & 11,63 \\
\hline 518 & $912827 \cup 26$ & 101,48 & 30.11 .02 & 5,75 \\
\hline 519 & $912827 \mathrm{U} 67$ & 101,58 & 31.12 .02 & 5,63 \\
\hline 520 & $912827 \mathrm{~V} 25$ & 101,74 & 31.01 .03 & 5,50 \\
\hline 521 & $912827 \mathrm{~V} 41$ & 101,63 & 15.02 .03 & 10,75 \\
\hline 522 & $9128272 \times 3$ & 104,84 & 15.02 .03 & 6,25 \\
\hline 523 & $912827 \times 49$ & 102,63 & 28.02 .03 & 5,50 \\
\hline 524 & $912827 \times 64$ & 102,49 & 31.03 .03 & 5,50 \\
\hline 525 & $912827 \mathrm{~A} 85$ & 105,92 & 30.04 .03 & 5,75 \\
\hline 526 & $912827 Y 22$ & 103,05 & 15.05 .03 & 10,75 \\
\hline 527 & $912827 Y 48$ & 103,39 & 31.05 .03 & 5,50 \\
\hline 528 & $912827 Y 71$ & 103,55 & 30.06 .03 & 5,38 \\
\hline 529 & 912827239 & 103,36 & 15.08 .03 & 5,75 \\
\hline 530 & $912827 \mathrm{~B} 92$ & 106,26 & 15.08 .03 & 11,13 \\
\hline $53 !$ & 912827288 & 102,98 & 15.11 .03 & 4,25 \\
\hline 532 & 912827254 & 103,17 & 15.11 .03 & 11,88 \\
\hline 533 & $912827 \mathrm{D} 25$ & 105,96 & 15.02 .04 & 5,88 \\
\hline 534 & $9128272 \mathrm{El}$ & 102,80 & 15.05 .04 & 7,25 \\
\hline 535 & $9128272 \mathrm{G} 6$ & 103,20 & 15.05 .04 & 12,38 \\
\hline 536 & 9128272L5 & 103,26 & 15.08 .04 & 7,25 \\
\hline 537 & $9128272 \mathrm{P} 6$ & 104,34 & 15.08 .04 & 13,75 \\
\hline 538 & 9128272 SO & 104,41 & 15.11 .04 & 11,63 \\
\hline 539 & $912827 \mathrm{~F} 49$ & 106,95 & 15.11 .04 & 7,88 \\
\hline 540 & $9128272 \mathrm{WV} 1$ & 104,16 & 15.02 .05 & 7,50 \\
\hline 541 & $9128272 Y 7$ & 103,56 & 15.05 .05 & 12,00 \\
\hline 542 & $9128273 \mathrm{GS}$ & 103,75 & 15.05 .05 & 6,50 \\
\hline 543 & $912827 \mathrm{GS5}$ & 104,09 & 15.05 .05 & 8,25 \\
\hline 544 & $912827 J 78$ & 104,02 & 15.08 .05 & 10,75 \\
\hline 545 & $912827 P 89$ & 109,51 & 15.08 .05 & 6,50 \\
\hline 546 & 912827088 & 109,78 & 15.11 .05 & 5,88 \\
\hline 547 & 912827586 & 111,62 & 15.02 .06 & 5,63 \\
\hline 548 & $912827 \mathrm{R} 87$ & 113,05 & 15.02 .06 & 9,38 \\
\hline 549 & 912827185 & 106,97 & 15.05 .06 & 6,88 \\
\hline 550 & $912827 \mathrm{U} 83$ & 107,09 & 15.07 .06 & 7,00 \\
\hline 551 & $912827 \times 80$ & 109,66 & 15.10 .06 & 6,50 \\
\hline 552 & 912827 Y55 & 110,41 & 15.01 .07 & 3,38 \\
\hline 553 & $9128272 \mathrm{J0}$ & 106,38 & 15.02 .07 & 7,63 \\
\hline
\end{tabular}




\begin{tabular}{|c|c|c|c|c|}
\hline Ldf. Nummer & CUSIP & Ask-Kurs in Dollar & Falligkeit & Kupon in \% \\
\hline 554 & 912827262 & 107,60 & 15.02 .07 & 6,25 \\
\hline 555 & $912810 \mathrm{BX} 5$ & 106,20 & 15.05 .07 & 6,63 \\
\hline 556 & 9128272U5 & 108,94 & 15.08 .07 & 6,13 \\
\hline 557 & 9128273E0 & 105,81 & 15.11 .07 & 7,88 \\
\hline 558 & $912810 \mathrm{BZO}$ & 108,47 & 15.01 .08 & 3,63 \\
\hline 559 & $912810 \mathrm{DW} 5$ & 116,44 & 15.02 .08 & 5,50 \\
\hline 560 & $912810 \mathrm{DX} 3$ & 119,26 & 15.05 .08 & 5,63 \\
\hline 561 & $912810 \mathrm{EH} 7$ & 125,80 & 15.08 .08 & 8,38 \\
\hline 562 & $912810 \mathrm{EM} 6$ & 118,81 & 15.11 .08 & 4,75 \\
\hline 563 & 912810EL8 & 127,97 & 15.11 .08 & 8,75 \\
\hline 564 & $912810 \mathrm{EN} 4$ & 123,63 & 15.05 .09 & 9,13 \\
\hline 565 & 912810EP9 & 117,44 & 15.11 .09 & 10,38 \\
\hline 566 & $912810 \mathrm{EQ7}$ & 106,33 & 15.02 .10 & 11,75 \\
\hline 567 & $912810 \mathrm{ES} 3$ & 123,16 & 15.05 .10 & 10,00 \\
\hline 568 & $912810 \mathrm{ETI}$ & 125,05 & 15.11 .10 & 12,75 \\
\hline 569 & $912810 \mathrm{EV} 6$ & 115,16 & 15.05 .11 & 13,88 \\
\hline 570 & $912810 \mathrm{E} \times 2$ & 113,80 & 15.11 .11 & 14,00 \\
\hline 571 & $912810 \mathrm{EYO}$ & 110,50 & 15.11 .12 & 10,38 \\
\hline 572 & $912810 \mathrm{EZ7}$ & 112,34 & 15.08 .13 & 12,00 \\
\hline 573 & $912810 \mathrm{FAl}$ & 109,06 & 15.05 .14 & 13,25 \\
\hline 574 & $912810 \mathrm{FB} 9$ & 105,75 & 15.08 .14 & 12,50 \\
\hline 575 & $912810 \mathrm{CUO}$ & 115,89 & 15.02 .25 & 7,63 \\
\hline 576 & $912810 \mathrm{CW6}$ & 118,22 & 15.08 .25 & 6,88 \\
\hline 577 & $912810 \mathrm{CX} 4$ & 125,66 & 15.02 .26 & 6,00 \\
\hline 578 & $912810 \mathrm{CZ9}$ & 124,09 & 15.08 .26 & 6,75 \\
\hline 579 & $912810 \mathrm{DH} 8$ & 131,63 & 15.11 .26 & 6,50 \\
\hline 580 & $912810 \mathrm{DK} 1$ & 139,24 & 15.02 .27 & 6,63 \\
\hline 581 & $912810 \mathrm{CS} 5$ & 140,48 & 15.08 .27 & 6,38 \\
\hline 582 & $912810 \mathrm{CV} 8$ & 149,44 & 15.11 .27 & 6,13 \\
\hline 583 & $912810 \mathrm{CY} 2$ & 152,91 & 15.04 .28 & 3,63 \\
\hline 584 & $912810 \mathrm{DJ} 4$ & 159,44 & 15.08 .28 & 5,50 \\
\hline 585 & $912810 \mathrm{DL} 9$ & 154,59 & 15.11 .28 & 5,25 \\
\hline 586 & 912810DL9 & 143,81 & 15.11 .28 & 5,25 \\
\hline 587 & $912810 \mathrm{DJ} 4$ & 149,36 & 15.08 .28 & 5,50 \\
\hline 588 & $912810 \mathrm{CY} 2$ & 148,50 & 15.04 .28 & 3,63 \\
\hline 589 & 912810 CV8 & 145,84 & 15.11 .27 & 6,13 \\
\hline 590 & $912810 \mathrm{CSS}$ & 136,91 & 15.08 .27 & 6,38 \\
\hline 591 & $912810 \mathrm{DK} 1$ & 139,22 & 15.02 .27 & 6,63 \\
\hline 592 & $912810 \mathrm{DH} 8$ & 130,48 & 15.11 .26 & 6,50 \\
\hline 593 & $912810 \mathrm{CZ9}$ & 130,63 & 15.08 .26 & 6,75 \\
\hline 594 & $912810 \mathrm{CX} 4$ & 135,13 & 15.02 .26 & 6,00 \\
\hline 595 & $912810 \mathrm{CW} 6$ & 124,78 & 15.08 .25 & 6,88 \\
\hline 596 & $912810 \mathrm{CUO}$ & 122,72 & 15.02 .25 & 7,63 \\
\hline 597 & $912810 \mathrm{EZ7}$ & 94,27 & 15.08 .13 & 12,00 \\
\hline 598 & $912810 E Y 0$ & 92,30 & 15.11 .12 & 10,38 \\
\hline 599 & $912810 \mathrm{EX} 2$ & 95,11 & 15.11 .11 & 14,00 \\
\hline 600 & 912810 EV6 & 96,48 & 15.05 .11 & 13,88 \\
\hline 601 & 912810ET1 & 105,39 & 15.11 .10 & 12,75 \\
\hline 602 & $912810 \mathrm{ES3}$ & 103,83 & 15.05 .10 & 10,00 \\
\hline 603 & $912810 \mathrm{EQ7}$ & 89,05 & 15.02 .10 & 11,75 \\
\hline 604 & 912810 EP9 & 99,22 & 15.11 .09 & 10,38 \\
\hline 605 & 912810 EN4 & 104,91 & 15.05 .09 & 9,13 \\
\hline 606 & 912810 EM6 & 100,52 & 15.11 .08 & 4,75 \\
\hline 607 & 912810 EL8 & 109,03 & 15.11 .08 & 8,75 \\
\hline 608 & $912810 \mathrm{EH} 7$ & 107,50 & 15.08 .08 & 8,38 \\
\hline 609 & $912810 \mathrm{DX} 3$ & 103,19 & 15.05 .08 & 5,63 \\
\hline 610 & $912810 \mathrm{DW} 5$ & 100,72 & 15.02 .08 & 5,50 \\
\hline 611 & $912810 \mathrm{BZO}$ & 105,09 & 15.01 .08 & 3,63 \\
\hline 612 & $912810 \mathrm{~B} \times 5$ & 102,72 & 15.05 .07 & 6,63 \\
\hline 613 & $9128272 \mathrm{~J} 0$ & 95,63 & 15.02 .07 & 7,63 \\
\hline 614 & $912827 Z 62$ & 97,25 & 15.02 .07 & 6,25 \\
\hline 615 & $912827 Y 55$ & 100,61 & 15.01 .07 & 3,38 \\
\hline 616 & $912827 \times 80$ & 99,75 & 15.10 .06 & 6,50 \\
\hline
\end{tabular}




\begin{tabular}{|c|c|c|c|c|}
\hline Ldf. Nummer & CUSIP & Ask-Kurs in Dollar & Falligkeit & Kupon in \% \\
\hline 617 & 912827 U83 & 97,52 & 15.07 .06 & 7,00 \\
\hline 618 & $912827 \mathrm{~T} 85$ & 97,77 & 15.05 .06 & 6,88 \\
\hline 619 & $912827 \mathrm{~S} 86$ & 103,72 & 15.02 .06 & 5,63 \\
\hline 620 & $912827 \mathrm{R} 87$ & 105,84 & 15.02 .06 & 9,38 \\
\hline 621 & $912827 Q 88$ & 102,25 & 15.11 .05 & 5,88 \\
\hline 622 & $912827 P 89$ & 102,20 & 15.08 .05 & 6,50 \\
\hline 623 & 912827178 & 97,53 & 15.08 .05 & 10,75 \\
\hline 624 & $912827 \mathrm{GSS}$ & 98,37 & 15.05 .05 & 8,25 \\
\hline 625 & $912827 \mathrm{~F} 49$ & 103,18 & 15.11 .04 & 7,88 \\
\hline 626 & $9128272 \mathrm{P} 6$ & 99,45 & 15.08 .04 & 13,75 \\
\hline 627 & $9128272 \mathrm{LS}$ & 97,94 & 15.08.04 & 7,25 \\
\hline 628 & $9128272 \mathrm{G} 6$ & 97,97 & 15.05 .04 & 12,38 \\
\hline 629 & $9128272 \mathrm{E} 1$ & 97,56 & 15.05 .04 & 7,25 \\
\hline 630 & $912827 \mathrm{D} 25$ & 102,88 & 15.02 .04 & 5,88 \\
\hline 631 & 912827288 & 98,06 & 15.11 .03 & 4,25 \\
\hline 632 & $912827 Z 54$ & 98,59 & 15.11 .03 & 11,88 \\
\hline 633 & 912827239 & 99,13 & 15.08 .03 & 5,75 \\
\hline 634 & $912827 \mathrm{B92}$ & 104,25 & 15.08 .03 & 11,13 \\
\hline 635 & $912827 \times 71$ & 99,56 & 30.06 .03 & 5,38 \\
\hline 636 & $912827 Y 48$ & 99,55 & 31.05 .03 & 5,50 \\
\hline 637 & $912827 \mathrm{Y} 22$ & 99,16 & 15.05 .03 & 10,75 \\
\hline 638 & $912827 \mathrm{~A} 85$ & 104,92 & 30.04 .03 & 5,75 \\
\hline 639 & $912827 \times 64$ & 98,36 & 31.03 .03 & 5,50 \\
\hline 640 & $912827 \times 49$ & 98,80 & 28.02 .03 & 5,50 \\
\hline 641 & $9128272 \times 3$ & 103,56 & 15.02 .03 & 6,25 \\
\hline 642 & $912827 \mathrm{~V} 41$ & 98,44 & 15.02 .03 & 10,75 \\
\hline 643 & $912827 \mathrm{~V} 25$ & 98,78 & 31.01 .03 & 5,50 \\
\hline 644 & 912827 U67 & 98,47 & 31.12 .02 & 5,63 \\
\hline 645 & $912827 \cup 26$ & 98,93 & 30.11 .02 & 5,75 \\
\hline 646 & $912827 \mathrm{~T} 69$ & 100,31 & 31.10 .02 & 5,75 \\
\hline 647 & $912827 T 44$ & 100,67 & 30.09 .02 & 5,88 \\
\hline 648 & $912827 T 28$ & 101,30 & 31.08 .02 & 6,25 \\
\hline 649 & $912827 S 60$ & 102,88 & 31.07 .02 & 6,00 \\
\hline 650 & 912827337 & 99,59 & 15.07 .02 & 3,63 \\
\hline 651 & 912827545 & 102,91 & 30.06 .02 & 6,25 \\
\hline 652 & 912827529 & 102,73 & 31.05 .02 & 6,50 \\
\hline 653 & 912827 YE6 & 103,04 & 15.05 .02 & 7,50 \\
\hline 654 & 912827R61 & 102,22 & 30.04 .02 & 6,63 \\
\hline 655 & 912827R46 & 101,31 & 31.03 .02 & 6,63 \\
\hline 656 & $912827 R 20$ & 100,70 & 28.02 .02 & 6,25 \\
\hline 657 & $912827 \times W 7$ & 103,13 & 15.02 .02 & 14,25 \\
\hline 658 & $912827 Q 62$ & 100,75 & 31.01 .02 & 6,25 \\
\hline 659 & 912827 F98 & 99,77 & 31.12 .01 & 6,13 \\
\hline 660 & 912827047 & 100,50 & 30.11 .01 & 5,88 \\
\hline 661 & $912827 Q 21$ & 100,50 & 15.11 .01 & 15,75 \\
\hline 662 & $912827 \times 72$ & 99,75 & 31.10 .01 & 6,25 \\
\hline 663 & $912827 \mathrm{P} 63$ & 100,08 & 30.09 .01 & 6,38 \\
\hline 664 & $912827 \mathrm{E} 81$ & 101,00 & 15.08 .01 & 13,38 \\
\hline 665 & $9128272 \mathrm{~N} 1$ & 99,66 & 15.08 .01 & 8,00 \\
\hline 666 & 912827074 & 100,00 & 15.08 .01 & 7,88 \\
\hline 667 & $912827 C 67$ & 101,19 & 31.07 .01 & 6,63 \\
\hline 668 & 912827221 & 99,83 & 30.06 .01 & 6,63 \\
\hline 669 & $912827 Y 63$ & 100,04 & 31.05 .01 & 6,50 \\
\hline 670 & $912827 Y 30$ & 100,11 & 15.05 .01 & 13,13 \\
\hline 671 & $912827 T 77$ & 100,00 & 15.05 .01 & 8,00 \\
\hline 672 & $912827 \mathrm{~A} 44$ & 101,72 & 30.04 .01 & 6,25 \\
\hline 673 & $912827 \times 31$ & 100,09 & 31.03 .01 & 6,38 \\
\hline 674 & 912827578 & 100,97 & 28.02 .01 & 5,63 \\
\hline 675 & $9128272 T 2$ & 101,38 & 15.02 .01 & 7,75 \\
\hline 676 & $912827 R 79$ & 100,82 & 15.02 .01 & 5,38 \\
\hline 677 & $912827 Q 70$ & 100,31 & 15.02 .01 & 11,75 \\
\hline 678 & $912827 \mathrm{~F} 80$ & 100,22 & 31.01 .01 & 5,25 \\
\hline 679 & $912827 \mathrm{~F} 64$ & 100,16 & 31.12 .00 & 4,63 \\
\hline
\end{tabular}




\begin{tabular}{|c|c|c|c|c|}
\hline Ldf. Nummer & CUSIP & Ask-Kurs in Dollar & Falligkeit & Kupon in \% \\
\hline 680 & $912827 \mathrm{~T} 93$ & 100,07 & 31.12 .00 & 5,50 \\
\hline 681 & $912827 \mathrm{P} 71$ & 100,09 & 07.12 .00 & 0,00 \\
\hline 682 & $912827 \mathrm{~F} 23$ & 100,07 & 30.11 .00 & 4,63 \\
\hline 683 & $91282 \pi \mathrm{T} 51$ & 100,04 & 30.11 .00 & 5,63 \\
\hline 684 & $912810 \mathrm{EW} 4$ & 85,97 & 27.05 .99 & 0,00 \\
\hline 685 & $912827 W 81$ & 91,66 & 15.05 .99 & 9,13 \\
\hline 686 & $912827 \mathrm{~V} 82$ & 93,42 & 13.05 .99 & 0,00 \\
\hline 687 & $912827 N 81$ & 94,81 & 06.05 .99 & 0,00 \\
\hline 688 & 912827L83 & 94,60 & 30.04 .99 & 6,38 \\
\hline 689 & $9128272 \mathrm{C5}$ & 96,63 & 15.03 .99 & 0,00 \\
\hline 690 & $912827 \times 23$ & 96,37 & 04.03 .99 & 0,00 \\
\hline 691 & $912827 W 65$ & 95,34 & 28.02 .99 & 5,88 \\
\hline 692 & $912827 W 40$ & 96,09 & 18.02 .99 & 0,00 \\
\hline 693 & $912827 W 24$ & 96,63 & 15.02 .99 & 8,88 \\
\hline 694 & 912827 V 66 & 97,22 & 04.02 .99 & 0,00 \\
\hline 695 & $912827 \mathrm{U} 42$ & 97,85 & 15.01 .99 & 6,38 \\
\hline 696 & $912827 \mathrm{~K} 43$ & 97,02 & 31.12 .98 & 5,13 \\
\hline 697 & $9128272 \mathrm{H} 4$ & 98,17 & 17.12 .98 & 0,00 \\
\hline 698 & 912827296 & 98,38 & 30.11 .98 & 5,13 \\
\hline 699 & $912827 \mathrm{H} 21$ & 98,83 & 27.11 .98 & 0,00 \\
\hline 700 & $912827 Y 89$ & 98,88 & 15.11 .98 & 3,50 \\
\hline 701 & 912827P48 & 98,98 & 05.11 .98 & 0,00 \\
\hline 702 & $912827 \mathrm{P} 22$ & 98,37 & 31.10 .98 & 5,88 \\
\hline 703 & $9128272 \mathrm{~K} 7$ & 99,03 & 31.10 .98 & 4,75 \\
\hline 704 & $912827 W 73$ & 97,56 & 29.10 .98 & 0,00 \\
\hline 705 & $912827 \mathrm{~N} 65$ & 97,63 & 22.10 .98 & 0,00 \\
\hline 706 & $9128272 \mathrm{~F} 8$ & 99,09 & 15.10 .98 & 7,13 \\
\hline 707 & 9128272D3 & 99,09 & 15.10 .98 & 0,00 \\
\hline 708 & $912827 \mathrm{~N} 40$ & 98,03 & 08.10 .98 & 0,00 \\
\hline 709 & $912827 N 24$ & 98,16 & 01.10 .98 & 0,00 \\
\hline 710 & $9128272 \mathrm{~B} 7$ & 99,00 & 30.09 .98 & 6,00 \\
\hline 711 & $912827 \mathrm{~V} 74$ & 98,73 & 30.09 .98 & 4,75 \\
\hline 712 & 912827270 & 99,31 & 24.09 .98 & 0,00 \\
\hline 713 & $912827 \mathrm{M} 66$ & 97,73 & 17.09 .98 & 0,00 \\
\hline 714 & 912827247 & 99,61 & 15.09 .98 & 0,00 \\
\hline 715 & $912827 \mathrm{M} 41$ & 97,91 & 10.09 .98 & 0,00 \\
\hline 716 & $912827 \mathrm{M} 25$ & 98,04 & 03.09 .98 & 0,00 \\
\hline 717 & $912827 \mathrm{U} 75$ & 99,51 & 31.08 .98 & 6,13 \\
\hline 718 & 912827L67 & 98,81 & 31.08 .98 & 4,75 \\
\hline 719 & $912827 \mathrm{LA2}$ & 98,83 & 27.08 .98 & 0,00 \\
\hline 720 & 912827L26 & 99,19 & 20.08 .98 & 0,00 \\
\hline 721 & $912827 \times 98$ & 99,80 & 15.08 .98 & 5,88 \\
\hline 722 & $912827 \mathrm{~K} 68$ & 99,02 & 15.08 .98 & 9,25 \\
\hline 723 & $912827 \times 56$ & 99,78 & 13.08 .98 & 0,00 \\
\hline 724 & $912827 \mathrm{~K} 35$ & 99,19 & 06.08 .98 & 0,00 \\
\hline 725 & $912827 \mathrm{~J} 94$ & 99,20 & 31.07 .98 & 6,25 \\
\hline 726 & $912827 \mathrm{~J} 45$ & 99,69 & 31.07 .98 & 5,25 \\
\hline 727 & 912827 W57 & 99,22 & 30.07 .98 & 0,00 \\
\hline 728 & $912827 J 29$ & 100,08 & 23.07 .98 & 0,00 \\
\hline 729 & 912827 W32 & 99,57 & 16.07 .98 & 0,00 \\
\hline 730 & $912827 \mathrm{H} 88$ & 100,06 & 15.07 .98 & 8,25 \\
\hline 731 & $912827 \mathrm{~V} 90$ & 99,69 & 09.07 .98 & 0,00 \\
\hline 732 & 912827VS8 & 99,91 & 02.07 .98 & 0,00 \\
\hline 733 & $912827 \mathrm{H} 47$ & 99,98 & 30.06 .98 & 6,25 \\
\hline 734 & $912827 \mathrm{~V} 33$ & 100,02 & 30.06 .98 & 5,13 \\
\hline 735 & $912827 \mathrm{G} 97$ & 99,91 & 25.06 .98 & 0,00 \\
\hline 736 & $912827 \mathrm{G} 71$ & 99,94 & 18.06 .98 & 0,00 \\
\hline 737 & 912827U91 & 100,07 & 15.06 .98 & 0,00 \\
\hline 738 & $912827 \mathrm{G} 30$ & 100,01 & 11.06 .98 & 0,00 \\
\hline 739 & $912827 \cup 59$ & 100,11 & 04.06 .98 & 0,00 \\
\hline 740 & $912827 \mathrm{U34}$ & 100,05 & 31.05 .98 & 5,38 \\
\hline 741 & $9128272 \mathrm{M} 3$ & 97,84 & 26.12 .97 & 0,00 \\
\hline 742 & $912810 \mathrm{BG} 2$ & 97,20 & 18.12 .97 & 0,00 \\
\hline
\end{tabular}




\begin{tabular}{|c|c|c|c|c|}
\hline Ldf. Nummer & CUSIP & Ask-Kurs in Dollar & Falligkeit & Kupon in \% \\
\hline 743 & $912810 \mathrm{EK} 0$ & 110,39 & 20.11 .97 & 0,00 \\
\hline 744 & $912810 \mathrm{EJ3}$ & 110,38 & 15.11 .97 & 7,38 \\
\hline 745 & 912810 EG9 & 117,28 & 15.11 .97 & 8,88 \\
\hline 746 & $912810 \mathrm{EF} 1$ & 117,22 & 13.11 .97 & 0,00 \\
\hline 747 & $912810 \mathrm{EE} 4$ & 114.34 & 13.11 .97 & 0,00 \\
\hline 748 & 912810 ED6 & 110,08 & 06.11 .97 & 0,00 \\
\hline 749 & $912810 \mathrm{EC} 8$ & 118,22 & 06.11 .97 & 0,00 \\
\hline 750 & $912810 \mathrm{~EB} 0$ & 119,53 & 31.10 .97 & 5,63 \\
\hline 751 & $912810 \mathrm{EA2}$ & 120,72 & 31.10 .97 & 5,75 \\
\hline 752 & $912810 \mathrm{DZZ}$ & 117,75 & 30.10 .97 & 0,00 \\
\hline 753 & $912810 \mathrm{DY} 1$ & 116,38 & 30.10 .97 & 0,00 \\
\hline 754 & $912810 \mathrm{DV} 7$ & 121,47 & 23.10 .97 & 0,00 \\
\hline 755 & $912810 \mathrm{DT} 2$ & 127,78 & 23.10 .97 & 0,00 \\
\hline 756 & $9128100 S 4$ & 135,28 & 16.10 .97 & 0,00 \\
\hline 757 & $912810 \mathrm{DPO}$ & 141,23 & 16.10 .97 & 0,00 \\
\hline 758 & 912810DN5 & 138,41 & 15.10 .97 & 8,75 \\
\hline 759 & $912810 \mathrm{DF} 2$ & 137,83 & 09.10 .97 & 0,00 \\
\hline 760 & $912810 \mathrm{DB} 1$ & 124.53 & 09.10 .97 & 0,00 \\
\hline 761 & $912810 \mathrm{CP} 1$ & 118,29 & 02.10 .97 & 0,00 \\
\hline 762 & $912810 \mathrm{CM} 8$ & 128,63 & 02.10 .97 & 0,00 \\
\hline 763 & $912810 \mathrm{CK} 2$ & 119,61 & 30.09 .97 & 5,75 \\
\hline 764 & $912810 \mathrm{CG} 1$ & 111,81 & 30.09 .97 & 5,50 \\
\hline 765 & 912810 CE6 & 109,28 & 25.09 .97 & 0,00 \\
\hline 766 & $912810 \mathrm{CC} 0$ & 107,72 & 25.09 .97 & 0,00 \\
\hline 767 & $912810 \mathrm{DU} 9$ & 116,28 & 18.09 .97 & 0,00 \\
\hline 768 & 912810DR6 & 124,03 & 18.09 .97 & 0,00 \\
\hline 769 & $912810 \mathrm{BUI}$ & 104,16 & 15.09 .97 & 0,00 \\
\hline 770 & $912810 \mathrm{DQ} 8$ & 131,17 & 15.09 .97 & 0,00 \\
\hline 771 & $9128100 \mathrm{DM} 7$ & 127,75 & 11.09 .97 & 0,00 \\
\hline 772 & $912810 \mathrm{DG} 0$ & 126,38 & 11.09 .97 & 0.00 \\
\hline 773 & 912810 DES & 121,89 & 04.09 .97 & 0,00 \\
\hline 774 & $912810 \mathrm{DD} 7$ & 119,36 & 04.09 .97 & 0,00 \\
\hline 775 & $912810 \mathrm{DC} 9$ & 118,82 & 31.08 .97 & 5,63 \\
\hline 776 & $912810 \mathrm{DA} 3$ & 122,38 & 31.08 .97 & 6,00 \\
\hline 777 & $912810 \mathrm{CT} 3$ & 116,86 & 28.08 .97 & 0,00 \\
\hline 778 & $912827 Z N 5$ & 105,71 & 28.08 .97 & 0,00 \\
\hline 779 & $9128272 E 5$ & 106,16 & 21.08 .97 & 0,00 \\
\hline 780 & 912827 YW6 & 106,29 & 15.08 .97 & 6,50 \\
\hline 781 & $912827 \mathrm{YN} 6$ & 104,80 & 15.08 .97 & 8,63 \\
\hline 782 & $912827 \times N$ N & 105,05 & 14.08 .97 & 0,00 \\
\hline 783 & 912827 XE7 & 104,25 & 07.08 .97 & 0,00 \\
\hline 784 & $912827 \mathrm{WW} 8$ & 103,73 & 07.08 .97 & 0,00 \\
\hline 785 & 912827 WN8 & 103,75 & 31.07 .97 & 5,50 \\
\hline 786 & $912827 \mathrm{BS0}$ & 102,42 & 31.07 .97 & 5,88 \\
\hline 787 & 912827 WE8 & 102,96 & 31.07 .97 & 0,00 \\
\hline 788 & $912827 \mathrm{VW9}$ & 101,66 & 31.07 .97 & 0,00 \\
\hline 789 & $912827 \mathrm{VN} 9$ & 101,66 & 24.07 .97 & 0,00 \\
\hline
\end{tabular}

\section{Tabelle 50: US-Staatanleihen zur Herleitung der Indexkurve des Sícheren Zins}


Andreas Siemes - 978-3-631-75322-4

Downloaded from PubFactory at 01/11/2019 06:12:16AM

via free access 


\section{Literaturverzeichnis}

Adam (1996):

Adam, D.; Planung und Entscheidung, 4.A.; Wiesbaden 1996.

Adam, Best, Dehnen, Steffens (2000):

Adam, D.; Best, F.; Dehnen, K.; Steffens, Ch.; Prognosequalität neuronaler Netze; Münster 2000. Veröffentlichungen des Instituts für Industrie- und Krankenhausbetriebslehre, Universität Münster, Nr. 42.

Altman (1968):

Altman, E.I.; Financial Ratios, Discriminant Analysis and the Prediction of Corporate Bankruptcy; in: Journal of Finance, Jg. 1968, S. 589-609.

Altman (1993):

Altman, E.I.; Corporate Financial Distress and Bankruptcy - A Complete Guide to Predicting \& Avoiding Distress and Profiting from Bankruptcy, 2nd ed; New York 1993.

Altman (1996):

Altman, E.I.; Corporate Bond and Commercial Loan Portfolio Analysis; Wharton Financial Institution Center 1996.

Altman, Kishore (1996):

Altman, E.I.; Kishore, V.M.; Almost Everything You Wanted to Know about Recoveries on Defaulted Bonds; in: Financial Analyst's Journal, Vol. 52, Jg. 1996, H. 6, S. 57-64.

Altman, Saunders (1997):

Altman, E.I.; Saunders, A.; Credit Risk Measurement: Developments over the Last 20 Years; in: The Journal of Banking and Finance, Jg. 1997, H. 21, S. 1721-1742.

American Chamber of Commerce in Germany (2001):

American Chamber of Commerce in Germany e.V.; Steuern in den USA; Frankfurt a.M. 2001.

Arditti, Curran (1998):

Arditti, F.; Curran, J.; Futures Contract on the Cards; in: Credit Risk, A Risk Special Report, November 1998, S. 30-32.

Armeloh (1998):

Armeloh, K.-H.; Die Berichterstattung im Anhang; zugl. Diss. Universität Münster 1996, Düsseldorf 1998.

Arnoldi (1993):

Arnoldi, H.; Fristigkeitsstruktur des Fremdkapitals: Instrumente und Strategien der Finanzierung; Wiesbaden 1993. 
Arrow (1985):

Arrow, K.J.; The Economics of Agency; in: Zeckenhauser, R.J. (Hrsg.): Principals and Agents: The Structure of Business; Boston 1985, S. 37-51.

Backhaus (1999):

Backhaus, K.; Industriegütermarketing, 6.A; München 1999.

Baden (1992):

Baden, K.; Vergleichende Unternehmensbeurteilung und Aktienkurse; Kiel 1992.

Baden, Wilhelm (1995):

Baden, K.; Wilhelm, W.; Alle Aktien im Test; in: manager magazin, Jg. 1995, H. 11, S. 133-195.

Baestaens, van den Bergh (1995):

Baestaens, D.E.; van den Bergh, W.M.; Tracking Amsterdam Stock Index Using Neural Networks; in: Refenes, A.N. (Hrsg.): Neural Networks in the Capital Market; Chichester, England 1995, S. 149-162.

Baetge (1989):

Baetge, J.; Die Ergebnisse der empirischen Bilanzforschung als Grundlage für die Entwicklung eines kennzahlenorientierten Controlling-Konzeptes; in: Delfmann, W. u.a. (Hrsg.): Der Integrationsgedanke in der Betriebswirtschaftslehre, Festschrift zum 70. Geburtstag von Helmut Koch; Wiesbaden 1989, S. 51-71.

Baetge, (1995):

Baetge, J.; Früherkennung von Kreditrisiken; in: Rolfes, B.; Schierenbeck, H.; Schüller, S. (Hrsg.): Risikomanagement in Kreditinstituten; Frankfurt a.M. 1995, S. 191-221.

Baetge (1997):

Baetge, J.; Bilanzbonitätsbeurteilung mit modernen Verfahren der Jahresabschlussanalyse unter besonderer Berücksichtigung von Bilanzpolitik; in: Fachbereich Betriebswirtschaftslehre der European Business School Oestrich-Winkel (Hrsg.): Sonderdruck zur Akademischen Feier am 5. Februar 1997 aus Anlass der Verleihung der Ehrendoktorwürde an Prof. Dr. Jörg Baetge und Helmut Maucher; Oestrich-Winkel 1997, S. $27-52$.

Baetge (1998):

Baetge, J.; Bilanzanalyse; Düsseldorf 1998.

Baetge (1998b):

Baetge, J.; Stabilität eines Bilanzbonitätsindikators und seine Einsatzmöglichkeiten im Kreditgeschäft, 1. Teil: Künstliches Neuronales Netz als Grundlage eines solchen Indikators; in: Der Schweizer Treuhänder, Jg. 1998, H. 7, S. 605- 612. 
Baetge (2001):

Baetge, J.; Bilanzen, 5.A.; Düsseldorf 2001.

Baetge, Armeloh, Schulze (1997):

Baetge, J.; Armeloh, K.-H.; Schulze, D.; Anforderungen an die Geschäftsberichtserstattung aus betriebswirtschaftlicher und handelsrechtlicher Sicht; in: Deutsches Steuerrecht, Jg. 1997, S. 176-180.

Baetge, Armeloh, Schulze (1997b):

Baetge, J.; Armeloh, K.-H.; Schulze, D.; Empirische Befunde über die Qualität der Geschäftsberichtserstattung börsennotierter Kapitalgesellschaften; in: Deutsches Steuerrecht, Jg. 1997, S. 212-219.

\section{Baetge, Ballwieser (1978):}

Bactge, J.; Ballwieser, W.; Probleme einer rationalen Bilanzpolitik; in: Betriebswirtschaftliche Forschung und Praxis, Jg. 1978, S. 511-530.

\section{Baetge, Beuter, Feidicker (1992):}

Baetge, J.; Beuter, H.B.; Feidicker, M.; Kreditwürdigkeitsprüfung mit Diskriminanzanalysen; in: Die Wirtschaftsprüfung, Jg. 1992, S. 749-761.

\section{Baetge, Hüls, Uthoff (1995):}

Baetge, J.; Hüls, D.; Uthoff, C.; Früherkennung der Unternehmenskrise; in: Forschungsjournal der Westfälischen Wilhelms-Universität Münster, Jg. 1995, H. 2, S. 21 -29.

Baetge, Jerschensky (1996):

Baetge, J.; Jerschensky, A.; Beurteilung der wirtschaftlichen Lage von Unternehmen mit Hilfe von modernen Verfahren der Jahresabschlussanalyse; in: Der Betrieb, 49. Jg. 1996, H. 32, S. 1581-1591.

Baetge, Jerschensky (1999):

Baetge, J.; Jerschensky, A.; Frïhwarnsysteme als Instrumente eines effizienten Risikomanagements und-Controlling; in: Controlling, 11. Jg. 1999, H. 4/5, S. 171-176.

\section{Baetge, Krause (1994):}

Baetge, J.; Krause, C.; Die Berücksichtigung des Risikos bei der Unternehmensbewertung; in: Betriebswirtschaftliche Forschung und Praxis, 33. Jg. 1994, S. 433-456.

\section{Baetge, Schlösser (1993):}

Baetge, J.; Schlösser, J.; Zwischenberichterstattung in Theorie und Praxis; in: v. Fritsch, U.; Liener, G.; Schmidt, R. (Hrsg.): Die deutsche Aktie, Unternehmensfinanzierung und Vermögenspolitik vor neuen Herausforderungen; Festschrift zum vierzigjährigen Bestehen des Deutschen Aktieninstituts e.V.; Stuttgart 1993, S. 225-249. 


\section{Baetge, Sieringhaus (1996):}

Baetge, J.; Sieringhaus, I.; Bilanzbonitäts-Rating von Unternehmen; in: Büschgen, H. E.; Everling, O. (Hrsg.): Rating-Handbuch; Wiesbaden 1996, S. 221-246.

\section{Ballwieser (1982):}

Ballwieser, W.; Zur Begründbarkeit informationsorientierter Jahresabschlussverbesserung; in: Zeitschrift für betriebswirtschaftliche Forschung, Jg. 1982, S. 772-793.

\section{Ballwieser (1985):}

Ballwieser, W.; Informationsökonomie, Rechnungslegungstheorie und BilanzrichtlinienGesetz; in: Zeitschrift für betriebswirtschaftliche Forschung, Jg. 1985, S. 47-66.

\section{Ballwieser (1993):}

Ballwieser, W.; Methoden der Unternehmensbewertung; in: Gebhardt, G.; Gerke, W.; Steiner, M.(Hrsg.): Handbuch des Finanzmanagements; München 1993, S. 151-176.

Ballwieser (1993b):

Ballwieser, W.; Bilanzanalyse; in: Chmielewicz, K.; Schweitzer, M. (Hrsg.): Handwörterbuch des Rechnungswesen, 3.A.; Stuttgart 1993, Sp. 211-229.

Ballwieser (1994):

Ballwieser, W.; Adolf Moxter und der Shareholder Value-Ansatz; in: Ballwieser, W. (Hrsg.): Bilanzrecht und Kapitalmarkt; Festschrift für A. Moxter, Düsseldorf 1994, S. 1377-1405.

Balzer (1997):

Balzer, A; u.a.; Gewinnen mit Aktien; in: manager magazin, Jg. 1997, H. 11, S. 151-224.

Bamberg, Baur (2000):

Bamberg, G.; Baur, F.; Statistik, 10.A.; München u.a. 2000.

Bamberg, Coenenberg (2000):

Bamberg, G.; Coenenberg, A.; Betriebswirtschaftliche Entscheidungslehre, 10.A.; München 1994.

\section{Banken (1987):}

Banken, R.; Die Marktzinsmethode als Instrument der pretialen Lenkung in Kreditinstituten; Frankfurt a.M. 1987.

\section{Bankenfachausschuss des IdW (1978):}

Bankenfachausschuss des IdW - Stellungnahme 1/1978: Zur Abschlussprüfung bei Kreditinstituten. Einzelfragen zur Prüfung des Kreditgeschäftes und Darstellung der Prüfungsergebnisse im Prüfungsbericht; in: Die Wirtschaftsprüfung, 31. Jg. 1978, S. 486-491. 


\section{Barrett, Heuson, Kolb (1986):}

Barrett, W.B.; Heuson, A.J.; Kolb, R.W.; The Effect of Three Mile Island on Utility Bond Risk, Premium: A Note; in: The Journal of Finance, 41. Jg. 1986, H. 1, S. 255-261.

\section{Barrett, Heuson, Kolb (1986b):}

Barrett, W.B.; Heuson, A.J.; Kolb, R.W.; The Differential Effect of Sinking Funds on Bond Risk, Premium: A Note; in: The Journal of Finance Research, Vol. 9, Jg. 1986, H. 4, S. 303-312.

\section{Bartram (1991):}

Bartram, W.; Dynamische Liquiditätsanalysen mit Hilfe von Kapitalflußrechnungen; in: Buchführung, Bilanzen, Kostenrechnung, Jg. 1991, H. 29, S. 609-624.

\section{Basle Committee on Banking Supervision (1996):}

Basle Committee on Banking Supervision; Amendment to the Capital Accord Incorporate Market Risks; in: Basle 1/1996.

\section{Basler Ausschuß für Bankenaufsicht (1999):}

Basler Ausschuß für Bankenaufsicht (Hrsg.): A New Capital Framework; Basel Juni 1999.

Baun (1994):

Baun, S.; Neuronale Netze in der Aktienkursprognose; in: Rehkugler, H.; Zimmermann, H.G. (Hrsg.): Neuronale Netze in der Ökonomie; München 1994, S. 131-207.

Beaver (1981):

Beaver, W. H.; Financial Reporting. An Accounting Revolution; New Jersey 1981.

\section{Behrenwaldt (1996):}

Behrenwaldt, U.; Funktion des Rating für Anleger; in: Büschgen, H.E.; Everling, O. (Hrsg.): Rating-Handbuch; Wiesbaden 1996, S. 291-303.

\section{Benenati (1998):}

Benenati, I.; Neuronale Netze im Portfoliomanagement; Wiesbaden 1998.

\section{Bentz, Refenes (1994):}

Bentz, Y.; Refenes, A.N.; Backpropagation with weighted signs and its application to financial time series; in: Proceedings of the Neural Networks in the Capital Markets, Pasadena, California, 16.-18.11.1994.

\section{Berblinger (1996):}

Berblinger, J.; Marktakzeptanz des Rating durch Qualität; in: Büschgen, H.E.; Everling, O. (Hrsg.): Rating-Handbuch; Wiesbaden 1996, S. 21-88.

Berens (1992):

Berens, W.; Beurteilung von Heuristiken; Wiesbaden 1992. 
Berens, Delfmann (1995):

Berens, W.; Delfmann, W.; Quantitative Planung, 2.A.; Stuttgart 1995.

Berens, Strauch (1999):

Berens, W.; Strauch, J.; Herkunft und Inhalt des Begriffs Due Diligence; in: Berens, W.; Brauner, H.U. (Hrsg.): Due Diligence bei Unternehmensakquisitionen, 2.A.; Stuttgart 1999, S. 3-19.

Berens, Thonemann, Siemes, Kleinau (2001):

Berens, W.; Thonemann, U.W.; Siemes, A.; Kleinau, P.; Prognose von Optionspreisen mittels Techniken der künstlichen Intelligenz - Ein Vergleich der Bewertungsgenauigkeit von Optionen mittels Künstlichen Neuronalen Netzen, Genetischer Programmierung und dem Black \& Scholes Model; Unpublished Working Paper, Lehrstuhl für Betriebswirtschaftslehre, insb. Controlling der WWU Münster und das Institut für Supply Chain Management der WWU Münster, Münster 2001.

\section{Berger (1987):}

Berger, K.-H.; Möglichkeiten der Erfassung von Risiken im Bankbetrieb; in: Krumnow, J.; Metz, M., (Hrsg.): Rechnungswesen im Dienste der Bankpolitik; Stuttgart 1987, S. 251-265.

Berthel (1970):

Berthel, J.; Art. Modelle allgemein, in: Kosiol , E. (Hrsg.): Handwörterbuch des Rechnungswesens, Stuttgart 1970, S. 1122-1130.

Betsch, Brümmer, Hartmann, Wittberg (1997):

Betsch, O.; Brümmer, E.; Hartmann, E.; Wittberg, V.; Kreditwürdigkeitsanalyse im Firmenkundengeschäft; in: Die Bank, 3/1997, S. 150-155.

Bierman, Hass (1975):

Bierman, H.; Hass, J.; An Analytic Model of Bond Risk Differentials; in: Journal of Financial and Quantitative Analysis, Jg. 1975, H. 10, S. 757-773.

\section{Billingsley, Lamy, Marr (1985):}

Billingsley, R.S.; Lamy, R.E.; Marr, M.W.; Split Ratings and Bond Reoffering Yields; in: Financial Management, Vol. 14, 1985, S. 59-65.

Bitz (1993):

Bitz, M.; Grundlagen des finanzwirtschaftlich orientierten Risikomanagements; in: Gebhardt, G.; Gerke, W.; Steiner, M. (Hrsg.): Handbuch des Finanzmanagements; München 1993, S. 641-668.

\section{Black, Cox (1976):}

Black, F.; Cox, J.C.; Valuing Corporate Securities: Some Effects of Bond Indenture Provisions; in: Journal of Finance, Jg. 1976, Vol. 41, No. 3, S. 529-543. 


\section{Black, Scholes (1973):}

Black, F., Scholes, M.; The Pricing of Options and Corporate Liabilities; in: Journal of Political Economics, Jg. 1973, Vol. 81, S. 637-654.

Blien, Lindner (1993):

Blien, U.; Lindner, H.G.; Neuronale Netze - Werkzeuge für empirische Analysen ökonomischer Fragestellungen; in: Jahrbuch für Nationalökonomie und Statistik, Vol. 212, Jg. 1993, H. 5-6, S. 497-521.

Blume (1992):

Blume, L.; Easley, D.; Evolution and Market Behaviour; in: Journal of Economic Theory, Jg. 1992, H. 58, S. 9-40.

\section{Boardman, McEnally (1981):}

Boardman, C.M.; McEnally, R.W.; Factors Affecting Seasoned Corporate Bond Prices; in: Journal of Financial and Quantitative Analysis, Vol. 16, Jg. 1981, H. 2, S. 207-226.

Bohl, Alexander (2000):

Bohl, M.T.; Alexander, V.; Das Finanzsystem in Deutschland; in: Stein, von J.H.; Hagen, von J. (Hrsg.): Obst/Hintner - Geld-, Bank- und Börsenwesen, 40.A; Stuttgart 2000, S. 447-469.

Bondt de, Thaler (1985):

Bondt de, W.F.M.; Thaler, R.; Does the Stock Market Overreact?; in: The Journal of Finance, Vol. 40, Jg. 1985, H. 3, S. 793-808.

Bondt de, Thaler (1987):

Bondt de, W.F.M.; Thaler, R.; Further Evidence On Investor Overreaction and Stock Market Seasonality; in: The Journal of Finance, Vol. 42, Jg. 1987, H. 3, S. 557-581.

Bondt de, Thaler (1989):

Bondt de, W.F.M.; Thaler, R.; Anomalies, A Mean-Reverting Walk Down Wall Street; in: Journal of Economic Perspectives, Vol. 3, Jg. 1989, H. 1, S. 189-204.

\section{Borchert (1998):}

Borchert, M.; Geld und Kredit, 5.A.; München u.a. 1997.

Born (2001):

Born, K.; Bilanzanalyse international. Deutsche und ausländische Jahresabschlüsse lesen und beurteilen, 2.A.; Stuttgart 2001.

Börsig (1991):

Börsig, C.; Unternehmenswert und Unternehmensbewertung; in: Zeitschrift für betriebswirtschaftliche Forschung, 45. Jg. 1993, S. 79-81. 
Bös (1991):

Bös, M.; Optionsbewertung und Kapitalmarkt; zugl. Diss. Universität Köln 1990, Bergisch Gladbach u.a.1991.

Brakensiek (1991):

Brakensiek, Th.; Die Kalkulation und Steuerung von Ausfallrisiken im Kreditgeschäft der Banken; zugl. Diss. Universität Münster 1991, Frankfurt a.M. 1991.

Brause (1991):

Brause, R.; Neuronale Netze. Eine Einführung in die Neuroinformatik; Stuttgart 1991.

Bremer, Sweeney (1991):

Bremer, M.; Sweeney, R.J.; The Reversal of Large Stock-Price Decreases; in: The Journal of Finance, Vol. 46, Jg. 1991, S. 747-754.

Bridts (1990):

Bridts, Ch.; Zwischenberichtspublizität; zugl. Diss. Universität Augsburg 1990, Düsseldorf 1990.

Brister, Kennedy, Liu (1994):

Brister, B.M.; Kennedy, R.E.; Liu, P.; The Regulation Effect of Credit Ratings on Bond Interest Yield: The Case of Junk Bonds; in: Journal of Business Finance and Accounting, Vol. 21, Jg. 1994, S. 511-531.

Bröker (2000):

Bröker, F.; Quantifizierung von Kreditportfoliorisiken; Frankfurt a. M. 2000.

Brown (1993):

Brown, C.; Neural Networks with Learning Disabilities; in: Technical Analysis of Stocks \& Commodities, Jg. 1993, H. Mai, S. 50-57.

Buchner (1981):

Buchner, R.; Grundzüge der Finanzanalyse; München 1981.

Bühler (1983):

Bühler, W.; Anlagestrategien zur Begrenzung des Zinsänderungsrisikos von Portefeuilles aus festverzinslichen Titeln; in: Zeitschrift für betriebswirtschaftliche Forschung, Jg. 1983, Sonderheft 16, S. 82-137.

Bühler, Uhrig-Homburg (2000):

Bühler, W.; Uhrig-Homburg, M.; Rendite und Renditestrukturen am Rentenmarkt, in: Stein, von J.H.; Hagen, von J. (Hrsg.): Obst/Hintner - Geld-, Bank- und Börsenwesen, 40.A; Stuttgart 2000, S. 298-336. 
Bühner (1990):

Bühner, R.; Das Management-Wert-Konzept; Stuttgart 1990.

Burger (1995):

Burger, A.; Jahresabschlußanalyse; München u.a. 1995.

Büschgen (1992):

Büschgen, H.E.; Veränderungen im Wettbewerbszenario der Banken - neue Anforderungen an das bankbetriebliche Risikomanagement; in: Büschgen, H.E. (Hrsg.): Mitteilungen und Berichte des Instituts für Bankwirtschaft und Bankrecht an der Universität zu Köln, Abteilung Bankwirtschaft; Köln 1992, S. 31-79.

Campell (1997):

Campell, N.A.; Biologie; Heidelberg u.a. 1997.

Cantor, Packer, Cole (1997):

Cantor, R.; Packer, F.; Cole, K.; Split Ratings and the Pricing of Credit Risk, Research Paper; Federal Reserve Bank of New York, No. 9711, Jg. 1997.

Carpenter (1989):

Carpenter, G.A.; Neural Network Models for Pattern Recognition and Associative Memory; in: o. Hrsg.; Neural Networks; Vol. 2, o.O. 1989, S. 243-257.

\section{Cassel (1992):}

Cassel, D.; Inflation; in: Bender, D.; Berg, H; Cassel, D.; Gabisch, G.; Hartwig, K.-H.; Hübl, L.; Kath, D.; Grossekettler, H.; Siebke, J.; Thieme, H.J.; Willms, M. (Hrsg.): Vahlens Kompendium der Wirtschaftstheorie und Wirtschafspolitik, 6.A.; Bd. 1, München 1992, S. 265-323.

\section{Cassetti (1993):}

Cassetti, M.D.; A Neural Network System for Reliable Trading Signals; in: Technical Analysis of Stocks \& Commodities, Jg. 1993, H. Juni, S. 78-84.

Chaouli, Froitzheim (1992):

Chaouli, M; Froitzheim, U.J.; Das bessere Gehirn; in: WirtschaftsWoche, Jg. 1992, H. 42, S. 138-143.

\section{Chenoweth, Obradovic (1994):}

Chenoweth, T.; Obradovic, W.; Feature Selection for the Predictive Models of the Stock Market; in: Proceedings of Neural Networks in the Capital Market, Pasadena, California, 16.-18.11.1994.

Chiang, Kolb (1986):

Chiang, R.; Kolb, R.W.; An Analytical Model for the Relationship between Maturity and Bond Risk Differentials; in: Financial Review, Jg. 1986, Vol. 21, S. 191-209. 


\section{Coenenberg (1974):}

Coenenberg, A.G.; Jahresabschluß und Kapitalmarkt. Zur Diskussion empirischer Forschungsansätze und -ergebnisse zum Informationsgehalt von Jahresabschlüssen für Aktionäre; in: Zeitschrift für betriebswirtschaftliche Forschung, Jg. 1974, S. 647-657.

Coenenberg (1990):

Coenenberg, A.G.; Bilanzanalyse nach neuem Recht; Landsberg/Lech 1990.

Coenenberg (2000):

Coenenberg, A.G.; Jahresabschluß und Jahresabschlußanalyse, 17.A.; Landsberg/Lech 1997.

Coenenberg (1995):

Coenenberg, A.G.; Henes, F.; Der Informationsgehalt der Zwischenberichtspublizität nach § $44 b$ Börsengesetz; in: Zeitschrift für betriebswirtschaftliche Forschung, Jg. 1995, S. 969-995.

\section{Copeland, Koller, Murrin (1993):}

Copeland, T.E.; Koller, T.; Murrin, J.; Unternehmenswert, Methoden und Strategien für eine wertorientierte Unternehmensführung; Frankfurt a.M. u.a. 1993.

Copeland, Weston (1988):

Copeland, T.E.; Weston J.F.; Financial Theory and Corporate Policy, 3.A.; Reading, Mass u.a. 1988.

Corsten, May (1996):

Corsten, H.; May, C.; Anwendungsfelder Neuronaler Netze und ihre Umsetzung; in: Corsten, H.; May, C. (Hrsg.): Neuronale Netze in der Betriebswirtschaft - Anwendungen in Prognose, Klassifikation und Optimierung; Wiesbaden 1996, S. 1-75.

Cox, Ross, Rubinstein (1979):

Cox, J.C.; Ross, St.A.; Rubinstein, M.; Option Pricing: A Simplified Approach; in: Journal of Financial Economics, Jg. 1979, Vol. 4, S. 145-166.

Das (1996):

Das, S.R.; Pricing Credit Derivatives - Total Return Swap \& Credit Spread Products; in: Financial Products, Jg. 1996, H. 30, S. 16-20.

Das, Tufano (1996):

Das, S.R.; Tufano, P.; Pricing Credit-Sensitive Debt When Interest Ratings, Credit Ratings and Credit Spread Are Stochastic; in: Journal of Financial Engineering, Jg. 1996, Vol. 5, No. 2, S. 161-198.

Deutsche Bank (1996):

Deutsche Bank; Geschäftsbericht 1995; Frankfurt a.M. 1996. 
Deutsche Bank (1999):

Deutsche Bank; Geschäftsbericht 1998; Frankfurt a.M. 1999.

Deutsche Börse AG (1997):

Deutsche Börse AG; Leitfaden zu den Rentenindizes der Deutschen Börse AG; 0.O., Februar 1997.

Deutsche Börse AG (2001):

Deutsche Börse AG; Leitfaden zu den Rentenindizes der Deutschen Börse AG; Version 3.2, September, o.O., 2001.

Dialynas (1988):

Dialynas, C.P.; Bond Yield Spreads Revisited; in: Journal of Portfolio Management, Winter 88, Jg. 1988, S. 57-62.

Dialynas, Edington (1992):

Dialynas, C.P.; Edington, D.H.; Bond Yield Spreads: A Postmodern View; in: Journal of Portfolio Management, Jg. 1992, H. Fall, S. 68-75.

Dörner (1992):

Dörner, W.; Die Unternehmensbewertung; in: Institut der Wirtschafsprüfer in Deutschland e.V. (Hrsg.): Handbuch für Rechnungslegung, Prüfung und Beratung, Band II; Düsseldorf 1992, S. 1-136.

Dorfner (1991):

Dorfner, G.; Konnektionismus; Stuttgart 1991.

Duffee (1996):

Duffee, G.R.; Treasury Yields and Corporate Bond Yield Spreads: An Empirical Analysis Working Paper; in: Federal Reserve Board (Washington, D.C.), 1996.

Duffee (1996b):

Duffee, G.R.; Estimating the Price of Default Risk, Working Paper; in: Federal Reserve Board (Washington, D.C.), 1996.

Duffie (1996):

Duffie, D.; Dynamic Asset Pricing Theory; New Jersey 1996.

Dutta, Shekar (1988):

Dutta, S.; Shekar, S.; Bond-Rating: A Non-Conservative Application of Neural Networks; in: Trippi, R.; Turban, E.: Neural Networks in Finance and Investing, Chicago 1993, S. 257-265.

Dutta, Shekar, Wong (1994):

Dutta, S.; Shekar, S; Wong, W.Y.; Decision support in non-conservative domains: Generalization with neural networks; in: Decision Support System, Jg. 1994, H. 11, S. 527-544. 


\section{E.I. DuPont Nemours and Company (1959):}

E.I. DuPont Nemours and Company; Executive Committee, Control Charts, A Description of the DuPont Chart System for Appraising Operating Performance, 3.A.; Wilmington/Delaware 1959.

\section{Ederington (1986):}

Ederington, L.H.; Why split Ratings occur; in: Financial Management, Jg. 1986, H. Spring, S. 37-47.

\section{Ederington, Yawitz, Roberts (1987):}

Ederington, L.H.; Yawitz, J.B.; Roberts, B.E.; The Informational Content of Bond Ratings; in: Journal of Financial Research, Vol. 10, Jg. 1987, H. 3, S. 211-226.

Eller (1996):

Eller, R.; Handbuch derivativer Finanzinstrumente; Stuttgart 1996.

Endres (2001):

Endres, K.; Jahresabschlussanalyse mit Künstlichen Neuronalen Netzen und mit dem Working Capital; in: Finanz Betrieb, 6/2001, S. 371-380.

Everling (1991):

Everling, O.; Credit Rating durch internationale Agenturen: Eine Untersuchung zu den Komponenten und instrumentalen Funktionen des Ratings; Wiesbaden 1991.

Everling (1995):

Everling, O.; Rating; in: Gerke, W; Steiner, M. (Hrsg.): Handwörterbuch des Bank und Finanzwesens, 2.A.; Stuttgart 1995, Sp. 1601-1609.

Fabozzi (1995):

Fabozzi, F.J.; Bond Pricing and Return Measurement; in: Fabozzi, F.J.; Fabozzi, T.D. (Hrsg.): The Handbook of Fixed Income Securities, 4.A.; Burr Ridge, New York 1995. S. 49-82.

Fabozzi (1996):

Fabozzi, F.J.; Bond Markets, Analysis and Strategies, 3.A.; New Jersey 1996.

\section{Fabozzi, Modigliani (1992):}

Fabozzi, F.J., Modigliani, F.; Capital Markets : Institutions and Instruments; London u.a. 1992.

Fadlalla, Lin (2001):

Fadlalla, A., Lin, C.-H.; An Analysis of the Applications of Neural Networks in Finance; in: interfaces 31:4; July/August 2001, S. 112-122. 
Falter (1994):

Falter, M.; Die Praxis des Kreditgeschäfts, 14.A.; Stuttgart 1994.

Fama (1970):

Fama, E.F.; Efficient Capital Markets: A Review of Theory and Empirical Work; in: The Journal of Finance, Jg. 1970, S. 383-423.

Fama (1976):

Fama, E.F.; Foundation of Finance; New York 1976.

Fama, French (1993):

Fama, E.F.; French, K.R.; Common Risk Factors in the Returns on Stocks and Bonds; in: Journal of Financial Economics, Jg. 1993, H. 33, S. 3-56.

Fama, Miller (1972):

Fama, E.F.; Miller, M.H.; The Theory of Finance; New York u.a. 1972.

Federal Reserve System Task Force on Internal Credit Risk Models (1998):

Federal Reserve System Task Force on Internal Credit Risk Models; Credit Risk Models at the Major U.S. Banking Institutions: Current State of the Art and Implications for Assessments of Capital Adequacy; in: FED-Report, 5/1998, Jg. 1998.

Feidicker (1992):

Feidicker, M.; Kreditwürdigkeitsprüfung; Düsseldorf 1992.

Felderer, Homburg (1994):

Felderer, B.; Homburg, St.; Makroökonomie und neue Makroökonomie; Berlin u.a. 1994.

Fisher (1911):

Fisher, I.; The purchasing power of money; New York 1911.

Fisher (1937):

Fisher, I.; Income in theory and income taxation in practice; in: Econometrica, Jg. 1937, H. 5, S. 1-55.

Fisher (1959):

Fisher, L.; Determinants of the Risk Premiums on Corporate Bonds; in: Journal of Political Economy, Vol. 67, Jg. 1959, H. 3, S. 217-237.

Flesch (1994):

Flesch, J.; Die Ausfallquote ist zu hoch; in: Frankfurter Allgemeine Zeitung, Nr. 284, vom 7. Dezember 1994, S. 23. 
Flesch (1995):

Flesch, J.; Die Grundgleichung geht nicht mehr auf; in: Frankfurter Allgemeine Zeitung, Nr. 31, vom 6. Februar 1995, S. B 9.

Fons (1994):

Fons, J.S.; Using Default Rates to Model the Term Structure of Credit Risk; in: Financial Analyst's Journal, Vol. 50, Jg. 1994, H. 5 Sept./Oct., S. 25-35.

Forster (1986):

Forster, K.-H.; Ausgewählte Fragen aus dem Bereich der neuen Bewertungsbestimmungen, Sonderdruck aus dem Bericht über die Fachtagung des Institutes der Wirtschaftsprüfer am 27. Juni 1986 in Düsseldorf; Neuss 1986.

Franke, Hax (1994):

Franke, G.; Hax, H.; Finanzwirtschaft des Unternehmens und Kapitalmarkt, 3.A.; Berlin u.a. 1994.

Frantzmann (1989):

Frantzmann, H.-J.; Saisonalitäten und Bewertung am deutschen Aktien- und Rentenmarkt; zugl. Diss. Universität Karlsruhe 1989, Frankfurt a.M. 1989.

\section{Friedman (1953):}

Friedman, M.; The Case for Flexible Exchange Rates; in: Friedman, M. (Hrsg.): Essays in Positive Economics, Chicago u.a. 1953.

Friedman, Savage (1948):

Friedman, M., Savage L.J.; The utility analysis of choice involving risk; in: The Journal of Political Economy, 56, Jg. 1948, S. 279-304.

Frühwirth (1997):

Frühwirth, M.; Handbuch der Renditeberechnung; München u.a. 1997.

Fuller, Farrel (1987):

Fuller, R.J.; Farrell, J.L.; Modern Investments and Security Analysis; New York 1987.

\section{Fung, Rudd (1986):}

Fung, W.K.H.; Rudd A.; Pricing New Corporate Bond Issues: An Analysis of Issue Cost and Seasoning Effects; in: The Journal of Finance, Vol. 41, Jg. 1986, H. 3, S. 633-642.

Füser (1994):

Füser, K.; Neuronale Netze im Bereich der Finanzwirtschaft; Tagesunterlagen zum Forum Wertpapierberatung, Düsseldorf und Münster, 13. und 14.10.1994.

Füser (1995):

Füser, K.; Neuronale Netze in der Finanzwirtschaft; Wiesbaden 1995. 
Gaida (1997):

Gaida, St.; Kreditrisikokosten-Kalkulation mit Optionspreisansätzen; zugl. Diss. Universität Münster 1997, Münster u.a. 1997.

Gebhardt (1993):

Gebhardt, G.; Anleihen als Instrument der langfristigen Finanzierung; in: Gebhardt, G.; Gerke, W.; Steiner, M. (Hrsg.): Handbuch des Finanzmanagements; München 1993, S. 445-475.

\section{Gerdsmeier, Krob(1994):}

Gerdsmeier, St.; Krob, B.; Kundenindividuelle Bewertung des Ausfallrisikos mit dem Optionspreismodell; in: Die Bank, Jg. 1994, S. 469-475.

Geske (1977):

Geske, R.; The Valuation of Corporate Liabilities as Compound Options; in: Journal of Financial and Quantitative Analysis, Jg. 1977, H. 12, S. 541-552.

\section{Geske, Johnson (1984):}

Geske, R.; Johnson, H.E.; The Valuation of Corporate Liabilities as Compound Options: A Correction; in: Journal of Financial and Quantitative Analysis, Jg. 1984, H. 19, S. 231-232.

Glormann (2001):

Glormann, F.; Bilanzrating von US-GAAP Abschlüssen; zugl. Diss. Universität Münster 2000, Düsseldorf 2001 .

Goebel (1995):

Goebel, A.; Die Konzernrechnungslegung nach HGB, IAS und US-GAAP - Eine Synopse wesentlicher Unterschiede und Gemeinsamkeiten; in: Der Betrieb, 50. Jg. 1995, H. 33, S. 2489-2492.

\section{Goergen (2001):}

Goergen, A.; Unsensibler Vorstoß; in: WirtschaftsWoche, Jg. 2001, H. 11, S. 92-97.

Grabher, Klien, Stoss (1996):

Grabher, C.; Klien, W.; Stoss, K.; Neue Wege im Kreditrisikomanagement; in: Österreichisches Bankarchiv, 44. Jg. 1996, H. 1, S. 37-42.

\section{Graf (1991):}

Graf, J.; Stock Market Prediction with Neural Networks; in: Gritzmann, P. (Hrsg.): Operations Research 91, extended abstracts of the 16th Symposium on Operations Research in Trier; Trier 1991, S. 496-499.

\section{Graf (1993):}

Graf, H.P.; Die Anbieter von Neuronalen Netzen sind auf der Suche nach Märkten; in: Computerwoche, 16. Jg. 1993, S. 52-24. 
Gräfer (1992):

Gräfer, H.; Annual Report - Der US-amerikanische Jahresabschluß; Stuttgart 1992.

Granziol (1981):

Granziol, M.; Tägliche Wechselkurs-Schwankungen, Devisenmarkt-Interventionen der monetären Behörden und rationale Erwartungen; zugl. Diss. Universität Zürich 1981, Frankfurt a.M. 1981.

Green, Locke, Paul-Choudhury (1998):

Green, J.; Locke, J.; Paul-Choudhury, S.; Strength through adversity; in: Credit Risk, A Risk Special Supplement, März 1998, S. 6-9.

Grill, Perczynski (1995):

Grill, H.; Perczynski, H.; Wirtschaftslehre des Kreditwesens, 29.A.; Bad Homburg vor der Höhe 1995.

Grochla (1969):

Grochla, E.; Modelle als Instrument der Unternehmensführung; in: Zeitschrift für betriebswirtschaftliche Forschung, Jg. 1969, S. 382-397.

Grünwald (1980):

Grünwald, L.; Optionsmarkt und Kapitalmarkteffizienz: eine Analyse der Organisations- und Informationseffizienz des börsenmäßigen Optionshandels in der Bundesrepublik Deutschland und den USA; München u.a. 1980.

Grütter-Settele (1999):

Grütter-Settele, A.; Verhaltenswirkung von Informationen des externen Rechnungswesens : empirische Untersuchung zur Verhaltenswirkung quantitativer Jahresabschlußinformationen im Rahmen der Konkurrenzanalyse; zugl. Diss. Universität Düsseldorf 1998, AugsburgHaunstetten, 1999.

Heinke (1998):

Heinke, V.G.; Bonitätsrisiko und Credit Rating festverzinslicher Wertpapiere; zugl. Diss. Universität Münster 1998, Bad Soden Ts. 1998.

Hafner (1993):

Hafner, R.; Unternehmensbewertung als Instrument zur Durchsetzung von Verhandlungspositionen; in: Betriebswirtschaftliche Forschung und Praxis, Jg. 1993, S. 79-89.

Hagenmüller, Jacob (1988):

Hagenmüller, K.-F.; Jacob, A.-F. (Hrsg.): Der Bankbetrieb, Band III, Rechnungswesen und Bankpolitik, 5.A.; Wiesbaden 1988. 
Haller (1989):

Haller, A.; Behavioural Accounting; in: Die Betriebswirtschaft, Jg. 1989, S. 383-385.

Hand, Holthausen, Leftwich (1992):

Hand, J.R.M.; Holthausen, R.W.; Leftwich, R.W.; The Effect of Bond Rating Agency Announcements on Bond and Stock Pricing; in: Journal of Finance, Vol. 47, Jg. 1992, S. 733-752.

\section{Hahnenstein, Wilkens, Röder (2001):}

Hahnenstein, L.; Wilkens, S.; Röder, K.; Die Black-Scholes-Optionspreisformel - Eine Herleitung mit Hilfe des Prinzips der risikoneutralen Bewertung; Arbeitspapier der Betrieblichen Finanzwirtschaft (Nr. 2000-07), Münster 2001.

Hartmann-Wendels, Pfingsten, Weber (2000):

Hartmann-Wendels, Th.; Pfingsten, A.; Weber, M.; Bankbetriebslehre, 2.A.; Berlin u.a. 2000.

Hartung, Elpelt (1989):

Hartung, J.; Elpelt, B.; Multivariate Statistik, 3.A.; München u.a. 1989.

Hattori (1996):

Hattori, P.K.; The Chase Guide to Credit Derivatives in Europe; London 1996.

Hauschildt (1971):

Hauschildt, J.; Entwicklungslinien der Bilanzanalyse; in: Zeitschrift für betriebswirtschaftliche Forschung, Jg. 1971, S. 335-351.

Helbling (1992):

Helbling, C.; Bilanz- und Erfolgsanalyse, 8.A.; Bern u.a. 1992.

Hecht-Nilsen (1990):

Hecht-Nilsen, R.; Neurocomputing; Reading u.a. 1990.

Heinke (1998):

Heinke, V.G.; Bonitätsrisiko und Credit Rating festverzinslicher Wertpapiere; zugl. Diss. Universität Münster 1998, Bad Soden Ts. 1998.

Heri (1982):

Heri, E.W.; Bestimmungsgründe kurzfristiger Wechselkursfluktuationen: Eine empirische Analyse flexibler Wechselkurse unter besonderer Berücksichtigung der Theorie und Empirie effizienter Märkte; Hamburg 1982.

Hering (1999):

Hering, Th.; Finanzwirtschaftliche Unternehmensbewertung; zugl. Habil.-Schrift Universität Greifswald 1998, Wiesbaden 1999. 


\section{Hickman (1958):}

Hickman, W.B.; Corporate Bond Quality and Investor Experience; Princeton 1958.

Hicks (1939):

Hicks, J.R.; Value and capital; Oxford 1939.

Hiemstra (1994):

Hiemstra, Y.; Linear Regression versus Backpropagation to Predict Quarterly Excess Returns; in: Proceedings of Neural Networks in the Capital Markets, Pasadena, California, 16.-18.11.1994, o. S.

Hinton (1992):

Hinton, G.E.; Wie Neuronale Netze lernen; in: Spektrum der Wissenschaft, Jg. 1992, H. 11, S. 134-143.

\section{Hinz (1994):}

Hinz, M.; Sachverhaltsgestaltung im Rahmen der Jahresabschlußpolitik; zugl. Diss. Fernuniversität Hagen 1993/1994, Düsseldorf 1994.

\section{Hirshleifer (1973):}

Hirshleifer, J.; Where are we in the Theory of Information; in: American Economic Review, Jg. 1973, H. 63, S. 31-39.

Hodrick (1990):

Hodrick, R.J.; Volatility in the Foreign Exchange and Stock Markets: Is it Excessive?; in: American Economic Review, Vol. 80, Jg. 1990, H. 2, S. 186-191.

\section{Hoffjan (1999):}

Hoffjan, A.; Internationales Controlling; in: Berens, W.; Schmitting, W.; Hoffjan, A. (Hrsg.): Controlling in Fallstudien, Stuttgart 1999, S. 223-272.

Hoffjan, Siemes (1999):

Hoffjan, A.; Siemes, A.; Erwartungsbildung und Aktienkursentwicklung; in: Wirtschafswissenschaftliches Studium, 28. Jg. 1999, H. 4, S. 452-458.

\section{Hölscher (1987):}

Hölscher, R.; Risikokosten-Management in Kreditinstituten - Ein integratives Modell zur Messung und ertragsorientierten Steuerung der bankbetrieblichen Erfolgsrisiken; zugl. Diss. Universität Münster 1987, Frankfurt a.M. 1987.

Holzer (1990):

Holzer, Ch.S.; Anlagestrategien in festverzinslichen Wertpapieren; Wiesbaden 1990. 


\section{Hopfenbeck (1992):}

Hopfenbeck, W.; Allgemeine Betriebswirtschafts- und Managementlehre: das Unternehmen im Spannungsfeld zwischen ökonomischen, sozialen und ökologischen Interessen, 6.A.; Landsberg/Lech 1992.

\section{Hsueh, Kidwell (1988):}

Hsueh, L.P.; Kidwell, D.S.; Bond Ratings: Are Two Better Than One?; in: Financial Management, Vol. 17, Nr. 1, 1988, S. 46-53.

Hull (1997):

Hull, J.C.; Options, Futures and Other Derivatives, 3.A.; New Jersey 1997.

\section{Hüls (1995):}

Hüls, D.; Früherkennung insolvenzgefährdeter Unternehmen; zugl. Diss. Universität Münster 1995, Düsseldorf 1995.

Hürlimann (1987):

Hürlimann, W.; Künstliche Intelligenz, Expertensysteme und Problemlösungsmethoden: Wo stehen wir?; in: io, Management Zeitschrift, Jg. 1987, H. 56, S. 396-400.

Irving (1996):

Irving, R.; Credit Derivatives come good; in: Risk - Managing Risk in the Worlds Financial Markets, Jg. 1996, H. July, S. 22-26.

Jacob (1988):

Jacob, A.-F.; Gedanken zur Risikosteuerung im Bankbetrieb; in: bank und markt, Jg. 1988, S. 6-11.

\section{Jacob, Klein (1996):}

Jacob, A.-F.; Klein, S.; Investment Banking; Wiesbaden 1996.

Jaffee (1975):

Jaffee, D.; Cyclical Variations in the Risk Structure of Interest Rates; in: Journal of Monetary Economics, Vol. 1, Jg. 1975, H. July, S. 309-325.

Jansen (2001)

Jansen, S.; Ertrags- und volatilitätsgestützte Kreditwïrdigkeitsprüfung im mittelständischen Firmenkundengeschäft der Banken; (in Vorbereitung), 0.O. 2001.

Jansen (2001b):

Jansen, S.; Bankinterne Ratingansätze im Firmenkundengeschäft; in: Rolfes, B.; Schierenbeck, H. (Hrsg.): Ausfallrisiken - Quantifizierung, Bepreisung und Steuerung, Frankfurt a.M. 2001, S. 95-125. 


\section{Jarrow, Turnbull (1995):}

Jarrow, R.A.; Turnbull, St.M.; Pricing Derivatives on Financial Securities Subject to Credit Risk; in: Journal of Finance, Jg. 1995, Vol. 50, H. 1, S. 53-85.

Johnson (1967):

Johnson, R.E.; The Term Structure of Corporate Bond Yields as a Function of Risk of Default; in: Journal of Finance, Jg. 1967, Vol. 22, H. 2 May, S. 313-345.

Jonkhart (1979):

Jonkhart, M.; On the Term Structure of Interest Rates and the Risk of Default; in: Journal of Banking and Finance, Jg. 1979, Vol. 3, S. 253-261.

\section{Jurgeit (1989):}

Jurgeit, L.; Bewertung von Optionen und bonitätsrisikobehafteten Finanztiteln - Anleihen, Kredite und Fremdfinanzierungsfazilitäten; zugl. Diss. Universität Hamburg 1988, Wiesbaden 1989.

Käfer (1969):

Käfer, K; Praxis der Kapitalflußrechnung, Stuttgart, 1969.

Käfer (1984):

Käfer, K; Kapitalflußrechnung, 2.A., Stuttgart, 1984.

\section{Kaplan, Norton (1992):}

Kaplan, R.S.; Norton, D.P.; The Balanced Scorecard - Measures That Drive Performance; in: Harvard Business Review, 70. Jg. 1992, Jan./Feb., S. 71-79.

Karnin (1990):

Karnin, E.D., A Simple Procedure for Pruning Back-Propagation Trained Neural Networks, in. IEEE Transactions on Neural Networks, Vol. 1, No. 2, Juni 1990, S. 239-242.

Kath (1992):

Kath, D.; Geld und Kredit; in: Bender, D.; Berg, H; Cassel, D.; Gabisch, G.; Hartwig, K.-H.; Hübl, L.; Kath, D.; Grossekettler, H.; Siebke, J.; Thieme, H.J.; Willms, M. (Hrsg.): Vahlens Kompendium der Wirtschaftstheorie und Wirtschafspolitik, 6.A., Bd. 1, München 1992, S. $175-218$.

\section{Katz (1992):}

Katz, J.O.; Developing Neural Network Forecasters For Trading; in: Technical Analysis of Stocks \& Commodities, Jg. 1992, H. April, S. 58-70.

Kean (1992):

Kean, J.; Using Neural Nets For Intermarket Analysis; in: Technical Analysis of Stocks \& Commodities, Jg. 1992, H. November, S. 58-63. 
Kean (1993):

Kean, J.; Treasury Bond Yields: A Neural Net Analysis Approach; in: Technical Analysis of Stocks \& Commodities, Jg. 1993, H. April, S. 78-84.

Keim, Stambaugh (1986):

Keim, D.B.; Stambaugh, R.F.; Predicting returns in the Bond and Stock Markets; in: Journal of Financial Economics, Jg. 1986, H. 17, S. 357-390.

Kidwell, Koch (1982):

Kidwell, D.S.; Koch, T.W.; The Behaviour of the Interest Rate Differential between TaxExempt Revenue and General Obligation Bonds: A Test of Risk Preferences and Market Segmentation; in: The Journal of Finance, Jg. 1982, H. March, S. 73-85.

Kilhey (1987):

Kilhey, U.; Die Beurteilung des Erfolgs von Bankprodukten als Grundlage produktpolitischer Entscheidungen; Frankfurt a.M. 1987.

Kirchner (1999):

Kirchner, T.; Segmentierte Aktienmärkte; zugl. Diss. Universität Karlsruhe 1999, Wiesbaden 1999.

$\operatorname{Kjer}(1981)$ :

Kjer, von V.; Optionsanleihen: Analysen und Gestaltung einer Finanzierungs- und Anlageform; zugl. Diss. Technische Universität Berlin 1980, Berlin 1981.

Kim, Ramaswamy, Sundaresan (1993):

Kim, I. J.; Ramaswamy, K.; Sundaresan, S.; Does Default Risk in Coupons Affect the Valuation of Corporate Bonds?, A Contingent Claims Model; in: Financial Management, Vol. 22, Jg. 1993, H. 3, S. 117-131.

Kimoto, Asakawa, Yoda, Takeoka (1990):

Kimoto, T.; Asakawa, K.; Yoda, M.; Takeoka, M.; Stock Market Prediction System with Modular Neural Networks; in: Proceeding of the International Joint Conference on Neural Networks, Vol. I, San Diego, IEEE Network Council, 1990, S. 1-6.

Kirmße (1996):

Kirmße, St.; Die Bepreisung und Steuerung von Ausfallrisiken im Firmenkundengeschäft der Kreditinstitute; Frankfurt a.M. 1996.

Kirmße (2001):

Kirmße, St.; Die Anwendung des optionspreistheoretischen Ansatzes zur Bepreisung von Ausfallrisiken. Grundüberlegungen; in: Rolfes, B.; Schierenbeck, H. (Hrsg.): Ausfallrisiken Quantifizierung, Bepreisung und Steuerung, Frankfurt a.M. 2001, S. 149-172. 
Kirmße, Siemes (1999):

Kirmße, St.; Siemes, A.; Der neue Grundsatz II - eine kritische Betrachtung der Erfassung unechter Pensionsgeschäfte; in: Zeitschrift für das gesamte Kreditwesen, 52. Jg. 1999, H. 20, S. 1116-1118.

Klein, Jonas (1999):

Klein, K.-G.; Jonas, M.; Due Diligence und Unternehmensbewertung; in: Berens, W.; Brauner, H.U. (Hrsg.): Due Diligence bei Unternehmensakquisitionen, 2.A., Stuttgart 1999, S. 159-174.

Klug (1985):

Klug, M.; Zur Ableitung von Kapitalkosten aus dem diskreten Optionspreismodell; zugl. Diss. Universität Frankfurt a.M. 1984, Berlin 1985.

Knight (1921):

Knight, F.; Risk, Uncertainty and Profit; Boston u.a. 1921.

Köhle (1990):

Köhle, M.; Neuronale Netze; Wien 1990.

Kohonen (1991):

Kohonen, T. et. al.; Artificial Neural Networks; Proceedings of the 1991 International Conference on Artificial Neural Networks, ICANN-91, Espoo, Finnland, 24.-26.6.1991.

Kolb (1992):

Kolb, R.W.; Investments, 3.A.; Miami 1992.

Kosiol (1961):

Kosiol, E; Modellanalysen als Grundlage unternehmerischer Entscheidungen, in: Zeitschrift für betriebswirtschaftliche Forschung, 1961, S. 318-334.

Krause (1993):

Krause, C.; Kreditwürdigkeitsprüfung mittels Neuronalen Netzen; zugl. Diss. Universität Münster 1992, Düsseldorf 1993.

Krob (2001):

Krob, B.; Der Optionspreisansatz - Weiterentwicklungen und Anwendungsmöglichkeiten in der Praxis; in: Rolfes, B.; Schierenbeck, H. (Hrsg.): Ausfallrisiken - Quantifizierung, Bepreisung und Steuerung, Frankfurt a.M. 2001, S. 173-183.

Krüger (1983):

Krüger, R.; Der Jahresabschluß aus Sicht des Aufsichtsrates; in: Baetge, J. (Hrsg.): Der Jahresabschluß im Widerstreit der Interessen; Düsseldorf 1983, S. 269-296. 
Krumbholz (1994):

Krumbholz, M.; Die Qualität publizierter Lageberichte; zugl. Diss. Universität Münster 1993, Düsseldorf 1994.

Krumnow (1985):

Krumnow, J.; Bilanzanalyse auf Basis der neuen Rechnungslegungsvorschrift; in: Zeitschrift für betriebswirtschaftliche Forschung, Jg. 1985, S. 783-809.

\section{Kümmel (1994):}

Kümmel, A.T.; Bewertung von Kreditinstituten nach dem Shareholder Value Ansatz; Berlin u.a. 1994.

\section{Küting (1993):}

Küting, K.; Die Ertragsstarken bilanzieren eher konservativ; in: Blick durch die Wirtschaft, Jg. 1993, H. 15.12.1993, S. 7.

\section{Küting (1996):}

Küting, K.; 200 Geschäftsberichte auf dem Prüfstand. Wie Capital Unternehmen bewertet; in: Capital, Jg. 1996, H. 10, S. 61-70.

Küting, Weber (2000):

Küting, K.; Weber, C.-P.; Die Bilanzanalyse, 5.A.; Stuttgart 2000.

Lachenbruch, Sneeringer, Revo (1973):

Lachenbruch, P.A.; Sneeringer, Ch.; Revo, L.T.; Robustness of the Linear and Quadratic Discriminant Function of Certain Types of Non-Normality; in: Communications in Statistics, Jg. 1973, S. 39-56.

\section{Lachnit (1976):}

Lachnit, L.; Technik und Aussage der Bilanzanalyse; in: Wirtschafswissenschaftliches Studium, Jg. 1976, S. 13-16.

Lackes, Mack (2000):

Lackes, R.; Mack, D.; Neuronale Netze in der Unternehmensplanung; München 2000.

Lamy, Thompson (1988):

Lamy, R.; Thompson, G.R.; Risk Premia and the Pricing of Primary Issue Bonds; in: Journal of Banking and Finance, Vol. 12, Jg. 1988, H. 2, S. 585-601.

\section{Leffson (1964):}

Leffson, U.; Die Grundsätze ordnungsmäßiger Buchführung; Düsseldorf 1964.

Leffson (1984):

Leffson, U.; Bilanzanalyse, 3.A.; Stuttgart 1984. 


\section{Lerbinger, (1984):}

Lerbinger, P.; Die Leistungsfähigkeit deutscher Aktieninvestmentfonds; in: Zeitschrift für betriebswirtschaftliche Forschung, 36. Jg., Nr. 1, S. 60-73.

LeRoy (1989):

LeRoy, St.; Efficient Capital Markets and Martingales; in: Journal of Economic Literature, Vol. 27, Jg. 1989, S. 1583-1621.

\section{Lev, OhIsen (1982):}

Lev, B.; Ohlson, J.; Market-Based Empirical Research in Accounting: A Review, Interpretation and Extension; in: Journal of Accounting Research, Jg. 1982, H. Supplement, S. 249-322.

\section{Lewis (1994):}

Lewis, Th.G.; Steigerung des Unternehmenswertes; Landsberg/Lech 1994.

\section{Lintner (1965):}

Lintner, J.; The Valuation of the Risk Assets and the Selection of Risky Investment in Stock Portfolios and Capital Budgets; in: The Review of Economics and Statistics, Vol. 47, Jg. 1965, S. 13-37.

\section{Lister (1997):}

Lister, M.; Risikoadjustierte Ergebnismessung und Risikokapitalallokation; Frankfurt a.M. 1997.

\section{Litterman, Iben (1991):}

Litterman, R.; Iben, Th.; Corporate Bond Valuation and the Term Structure of Credit Spreads; in: Journal of Portfolio Management, Vol. 17, Jg. 1991, H. 3, S. 52-64.

\section{Liu, Moore (1987):}

Liu, P.; Moore, W.T.; The Impact of Split Ratings on Premia; in: Financial Review, Vol. 22, Jg. 1987, H. 1, S. 735-746.

\section{Loistl (1990):}

Loistl, O.; Zur neuern Entwicklung der Finanzierungstheorie; in: Die Betriebswirtschaft, 50. Jg., H. 1, S. 47-84.

\section{Long de, Shleifer, Summers, Waldmann (1990):}

Long, de J.B.; Shleifer, A.; Summers, L.H.; Waldmann, R.J.; Noise Trader Risk in the Financial Markets; in: Journal of Political Economy, Vol. 98, Jg. 1990, H. 4, S. 703-738.

\section{Long de, Shleifer, Summers, Waldmann (1990a):}

Long, de J.B.; Shleifer, A.; Summers, L.H.; Waldmann, R.J.; Positive Feedback Investment Strategies and Destabilizing Rational Speculation; in: The Journal of Finance, Vol. 45, Jg. 1990, No. 2, S. $379-395$. 


\section{Longstaff, Schwartz (1995):}

Longstaff, F.A.; Schwartz, E.S.; A Simple Approach to Valuing Risky Fixed and Floating Rate Debt; in: Journal of Finance, Vol. 50, Jg. 1995, H. 3, S. 789-819.

Lucas (1972):

Lucas, R.E.; Expectations and the Neutrality of Money; in: Journal of Economic Theory, Jg. 1972, S. 103-124.

\section{Lucas (1975):}

Lucas, R.E.; An Equilibrium Model of the Business Cycle; in: Journal of Political Economics, Vol. 83, Jg. 1975, H. 6, S. 1113-1144.

\section{Lucas (1981):}

Lucas, R.E.; Studies in Business-Cycle Theory; Oxford 1981.

Lynch, Cross (1995):

Lynch, R.L.; Cross, K.F.; Measure up!: Yardsticks for contimuous improvement, 2.A.; Cornwall 1995.

Ma, Rao, Peterson (1990):

Ma, Ch.K.; Rao, R.P.; Peterson, R.L.; Resiliency of the High-Yield Bond Market; in: Altman, E.I. (Hrsg.): The High-Yield Debt Market: Investment Performance and Economics Impact; Homewood 1990, S. 58-78.

Macaulay (1938):

Macaulay, F.H.; Some theoretical problems suggested by movements of interest rates, bond yield, and stock prices in the United States since 1856; New York 1938.

Makoski (1999):

Makoski, R.; Kennzahlen im Logistik-Controlling; in: Berens, W.; Hoffjan, A.; Schmitting, W. (Hrsg.); Controlling in Fallstudien, Stuttgart 1999, S. 67-94.

Malkiel (1990):

Malkiel, B.G.; A random walk down wall street, 5.A.; New York u.a. 1990.

Malliaris (1994):

Malliaris, M.E.; Modeling the Behavior of the S\&P 500 Index: A Neural Network Approach; in: Proceeding of the tenth Conference on Artificial Intelligence for Applications, San Antonio, Texas, 1.-4.3.1994.

Maltzan (2000):

Maltzan, von B.-A.; Handelsobjekt und Handelsmodalitäten, in: Stein, von J.H.; Hagen, von

J. (Hrsg.): Obst/Hintner - Geld-, Bank- und Börsenwesen, 40.A; Stuttgart 2000, S. 827-842. 
Mann (1995):

Mann, T.; Wiener-ITo-Prozesse und Zeithorizonteffekte; in: Das Wirtschaftsstudium, Jg. 1995, H. 4, S. 299-304.

Matthes (1994):

Matthes, R.; Zinsprognosen: Fehlerkorrekturmodell vs. Neuronale Netze; in: Bol, G.; Nakhaeizadeh, G.; Vollmer, K.H. (Hrsg.): Finanzmarktanwendungen Neuronaler Netze und ökonometrischer Verfahren; Heidelberg 1994, S. 41-60.

McCulloch, Pitts (1943):

McCulloch, W.; Pitts, W.; A logical calculus of the ideas immanent in nervous activity; in: Bulletin of Mathematical Biophysics, Jg. 1943, H. 5, S. 115-133.

Meffert, Bruhn (1995):

Meffert, H.; Bruhn, M.; Dienstleistungsmarketing; Wiesbaden 1995.

Menkhoff, Röckmann (1994):

Menkhoff, L.; Röckmann, Ch.; Noise Trading auf Aktienmärkten; in: Zeitschrift für betriebswirtschaftliche Forschung, 64. Jg. 1994, S. 277-295.

Merton (1974):

Merton, R.C.; On the Pricing of Corporate Debt: The Risk Structure of Interest Rates; in: Journal of Finance, Vol. 29, Jg. 1974, S. 449-470.

Meyer (1994):

Meyer, C.; Betriebswirtschaftliche Kennzahlen und Kennzahlen-Systeme; Stuttgart 1994.

Miller (1994):

Miller, M.; Das Optimieren von Neuronalen Netzen für den Einsatz zur Prognose in der Ökonomie; in: Bol, G.; Nakhaeizadeh, G.; Vollmer, K.H. (Hrsg.): Finanzmarktanwendungen Neuronaler Netze und ökonometrischer Verfahren; Heidelberg 1994, S. 125-147.

Minsky, Papert (1969):

Minsky, M.; Papert, S.; Perceptrons; Cambridge, MA u.a. 1969.

Möller (1983):

Möller, H.P.; Probleme und Ergebnisse kapitalmarktorientierter empirischer Bilanzforschung in Deutschland; in: Betriebswirtschaftliche Forschung und Praxis, Jg. 1983, S. 285-302.

Möller (1985):

Möller, H.P.; Die Informationseffizienz des deutschen Aktienmarktes - Eine Zusammenfassung; in: Zeitschrift für betriebswirtschaftliche Forschung, 37. Jg. 1985, H. 6, S. 500-518. 


\section{Möller (1986):}

Möller, H. P.; Bilanzkennzahlen und Ertragsrisiken des Kapitalmarktes; zugl. Habil.-Schrift Universität Augsburg 1985, Stuttgart 1986.

Moser, Quast (1994):

Moser, H.; Quast, W.; Organisation des Risikomanagements in einem Bankkonzern; in: Schierenbeck, H.; Moser, H. (Hrsg.): Handbuch Bankcontrolling, Wiesbaden 1994, S. 665-686.

\section{Mossin (1966):}

Mossin, J.; Equilibrium in a Capital Asset Market; in: Econometrica, Vol. 34, Jg. 1966, S. 768-783.

\section{Moxter (1983):}

Moxter, A.; Grundsätze ordnungsmäßiger Unternehmensbewertung, 2.A.; Wiesbaden 1983.

\section{Mülhaupt (1980):}

Mülhaupt, L.; Einführung in die BWL der Banken - Struktur und Grundprobleme des Bankbetriebs, 3.A.; Wiesbaden 1980.

Müller, Müller (1998):

Müller, A.; Müller, D.; Bilanzierung und Kreditvergabe; Herne u.a. 1998.

Muth (1961):

Muth, J.F.; Rational Expectations and the Theory of Price Movements; in: Economoetrica, Vol. 29, Jg. 1961, S. 315-335.

\section{Neuber (1988):}

Neuber, F.; Steuerungsinstrumente zur Verhinderung einer Krise aus Sicht einer einzelnen Bank; in: Sparkasse, 105. Jg. 1988, S. 298-303.

\section{Neumann, Morgenstern (1973):}

Neumann, J.v.; Morgenstern, O.; Theory of games and economic behavior, Princeton 1944, deutsche Übersetzung: Spieltheorie und wirtschaftliches Verhalten, 3.A.; Würzburg 1973.

Nöth (1995):

Nöth, M.; Untersuchung der Renditestruktur im Markt der DM-Euroanleihen; in: Kredit und Kapital, 28. Jg. 1995, S. 535-568.

Pellens (1989):

Pellens, B.; Der Informationswert von Konzernabschlüssen; zugl. Diss. Universität Bochum 1988, Wiesbaden 1989.

Pellens (1998):

Pellens, B.; Internationale Rechnungslegung, 2.A.; Stuttgart 1998. 
Perridon, Steiner (1997):

Perridon, L.; Steiner, M.; Finanzwirtschaft der Unternehmung, 9.A.; München 1997.

Perry, Liu, Evans (1988):

Perry, L.G.; Liu, P.; Evans, D.A.; Modified Bond Ratings: Further Evidence on the Effect of Split Ratings on Corporate Bond Yields; in: Journal of Business Finance and Accounting, Vol. 15, Jg. 1988, S. 231-241.

Potthoff (1996):

Potthoff, E.; Die Prüfung des Jahresabschlusses durch den Aufsichtsrat; in: Baetge, J. u.a. (Hrsg.): Rechnungslegung, Prüfung und Beratung, Herausforderung für den Wirtschaftsprüfer, Festschrift zum 70. Geburtstag von Rainer Ludewig; Düsseldorf 1996, S. 831-853.

Potvin (1993):

Potvin, J.-Y.; The Traveling Salesman Problem: An Neural Network Perspective; in: ORSA Journal on Computing, Vol. 5, Jg. 1993, H. 4, S. 328-348.

Priewasser (1992):

Priewasser, E.; Bankbetriebslehre, 3.A.; München u.a. 1992.

Puckler (1988):

Puckler, G.; Das Bank- und Börsenwesen in den USA, 2.A.; Frankfurt a.M. 1988.

Pytlik (1995):

Pytlik, M.; Diskriminanzanalyse und Künstliche Neuronale Netze zur Klassifizierung von Jahresabschlüssen; Frankfurt a.M. 1995.

\section{Rappaport (1995):}

Rappaport, A.; Shareholder Value; Stuttgart 1995.

Refenes (1995):

Refenes, A.N.; Neural Network Design Considerations, in: Refenes, A.N. (Hrsg.): Neural Networks in the Capital Market, Chichester, England, S. 15-32.

Rehkugler, Poddig (1990):

Rehkugler, H.; Poddig, Th.; Statistische Methoden versus Künstliche Neuronale Netzwerke zur Aktienkursprognose - Eine vergleichende Studie; in: Bamberger Betriebswirtschaftliche Beiträge, Nr. 76, Bamberg 1990.

Rehkugler, Poddig (1992):

Rehkugler, H.; Poddig, Th.; Anwendungsperspektiven und Anwendungsprobleme von Künstlichen Neuronalen Netzwerken; in: Information Management, Jg. 1992, H. 2, S. 50-58.

Rehkugler, Poddig (1998):

Rehkugler, H.; Poddig, Th.; Bilanzanalyse, 4.A.; München u.a. 1998. 
Rehkugler, Schindel (1994):

Rehkugler, H.; Schindel, V.; Finanzierung, 6.A.; München 1994.

Reichel (1995):

Reichel, R.; Wie der Firmenkredit zur Verkaufsoption wird; in: Handelsblatt, Jg. 1995, H. 185 vom 25.09 .95 , S. 23.

Reichmann (1988):

Reichmann, Th.; Controlling-Praxis: erfolgsorientierte Unternehmenssteuerung; München 1988.

Reichmann (1997):

Reichmann, Th.; Controlling mit Kennzahlen und Managementberichten, 5.A.; München 1997.

\section{Reichmann, Lachnit (1976):}

Reichmann, Th.; Lachnit, L.; Planung, Steuerung und Kontrolle mit Hilfe von Kennzahlen; in: Zeitschrift für betriebswirtschaftliche Forschung, Jg. 1976, S. 705-723.

Reinhardt, Soeder (1987):

Reinhardt, F.; Soeder, H.; dtv-Atlas zur Mathematik, Bd. 1, 6.A.; München 1987.

Reuters (1999):

Reuters AG; Reuters 3000 Fixed Income (R3FI); o.O. 1999.

Rhee (1994):

Rhee, M.J.; Forecasting Stock Market Indices with Neural Networks; in: Proceedings of the Neural Networks in the Capital Markets, Pasadena, California, 16.-18.11.1994, o. S.

Riess (1992):

Riess, M.; Ihr Netz denkt mit - Neuronale Netze im Einsatz bei Allianz Lebensversicherung; in: Versicherungsbetrieb, Jg. 1992, H. 7/8, S. 6-9.

\section{Ripper, Kempf (1998):}

Ripper, K.; Kempf, Th.; Bedeutung der Risikofaktoren am deutschen Aktienmarkt; in: Die Bank, Jg. 1998, H. 12, S. 754-758.

Ritter, Martinetz, Schulten (1991):

Ritter, H.; Martinetz, T.; Schulten, K.; Neuronale Netze - Eine Einfuihrung in die Neuroinformatik selbstorganisierender Netzwerke; Bonn u.a. 1991.

Rodriguez (1988):

Rodriguez, R.; Default Risk, Yield Spreads and Time to Maturity; in: Journal of Financial and Quantitative Analysis, Vol. 23, Jg. 1988, S. 111-117. 
Rojas (1992):

Rojas, R.; Theorie der neuronalen Netze; Berlin u.a. 1992.

Rolfes (1985):

Rolfes, B.; Die Steuerung von Zinsänderungsrisiken in Kreditinstituten; zugl. Diss. Universität Münster 1985, Frankfurt a.M. 1985.

Rolfes (1992):

Rolfes, B.; Moderne Investitionsrechnung; München u.a. 1992.

Rolfes (1999):

Rolfes, B.; Gesamtbanksteuerung; Stuttgart 1999.

Rolfes (2001):

Rolfes, B.; Steuerung des Kreditrisikos im Dualen Steuerungsmodell; in: Rolfes, B.; Schierenbeck, H. (Hrsg.): Ausfallrisiken - Quantifizierung, Bepreisung und Steuerung, Frankfurt a.M. 2001, S. 1-28.

Rometsch, Kolb (1999):

Rometsch, S.; Kolb, Ch.; Das Comeback der Industrieanleihe - verdrängen Corporate Bonds den syndizierten Kredit?; in: Zeitschrift für das gesamte Kreditwesen, Jg. 1999, H. 6, S. 296-298.

Rosenblatt (1958):

Rosenblatt, F.; The perceptron. A probabilistic model for information storage and organization in the brain.; in: Psychological review, 65. Jg. 1958, S. 386-408.

Rudolph (1981):

Rudolph, B.; Funktionen und Konditionen der Kreditinstitute im Emissionsgeschäft; in: Wirtschafswissenschaftliches Studium, Jg. 1981, H. 2, S. 60-64.

Rudolph (1994):

Rudolph, B.; Ansätze zur Kalkulation von Risikokosten für Kreditgeschäfte; in: Schierenbeck, H.; Moser, H (Hrsg.): Handbuch Bankcontrolling; Wiesbaden 1994, S. 887-904.

Ruggiero (1994):

Ruggiero, M.A.; How to build an artificial trader; in: Futures, Jg. 1994, September, S. 56-58.

Rumelhardt, Hinton, Williams (1986):

Rumelhart, D.E.; Hinton, G.E.; Williams, R.J.; Learning Internal Representations by Error Propagation; in: Rumelhart, D.E.; McClelland, J.L.; PDP Research Group (Hrsg.): Parallel distributed Processing: Explorations in the Microstructure of Cognition, Bd. 1, Foundations Cambridge u.a. 1986, S. 318-362. 
Santomero (1995):

Santomero, A.M.; Financial Risk Management: The Whys and Hows, Financial Markets, Institutions \& Instruments; Vol. 4, S. 1-14.

Sawazki (1996):

Sawazki, W.; Enwartungsbildung und Aktienkurse, Eine theoretische und empirische Betrachtung der deutschen Börse; Düsseldorf 1996.

Schäfer (1994):

Schäfer, B.; Informationsverarbeitung und Preisbildung an Aktien-und Optionsmarkt. Eine empirische Intraday-Untersuchung zur Preisanpassungsgeschwindigkeit an schweizerischen und deutschen Aktien- und Optionsmärkten; Zürich 1994.

Schaudwet (2001):

Schaudwet, Ch., Die wichtigsten Bilanzregeln: HGB, IAS und US-GAAP, in: WirtschaftsWoche, Jg. 2001, vom 8.8., o.S.

Schedelbauer (1978):

Schedelbauer, H.; Bilanzanalyse in der Praxis; in: Der Betrieb, Jg. 1978, S. 2425-2430.

Schierenbeck (1995):

Schierenbeck, H.; Konzeption eines integrierten Risikomanagements; in: Rolfes, B., Schierenbeck, H., Schüller, St. (Hrsg.): Risikomanagement in Kreditinstituten; Frankfurt a.M. 1995, S. 1-51.

Schierenbeck (1999):

Schierenbeck, H.; Ertragsorientiertes Bankmanagement, 1. Bd., 6.A.; Wiesbaden 1999.

Schierenbeck (1999b):

Schierenbeck, H.; Ertragsorientiertes Bankmanagement, 2. Bd., 6.A.; Wiesbaden 1999.

Schierenbeck (2000):

Schierenbeck, H.; Grundzüge der Betriebswirtschaftslehre, 15.A.; München u.a. 2000.

Schierenbeck, Hölscher (1998):

Schierenbeck, H.; Hölscher, R.; BankAssurance, 4.A.; Stuttgart 1998.

Schierenbeck, Rolfes (1988):

Schierenbeck, H.; Rolfes, B.; Entscheidungsorientierte Margenkalkulation; Frankfurt a.M. 1988.

Schiffmann (1999):

Schiffmann, W.; Entwurf und Optimierung mehrschichtiger feedforward Netze; zugl. Habil.-Schrift Universität Koblenz/Landau 1999, Aachen 1999. 
Schlieper (1995):

Schlieper, U.; Nobelpreis für Wirtschaftswissenschaften - die Grenzen der Konjunkturpolitik; in: Spektrum der Wissenschaft, Jg. 1995, H. 12, S. 32-35.

Schmidt (1981):

Schmidt, R.H.; Grundformen der Finanzierung, Eine Anwendung des neoinstitutionalistischen Ansatzes der Finanzierungstheorie; in: Kredit und Kapital, 14. Jg. 1981, S. 186-221.

Schmidt (1982):

Schmidt, R.H.; Rechnungslegung als Informationsproduktion auf nahezu effizienten Kapitalmärkten; in: Zeitschrift für betriebswirtschaftliche Forschung, Jg. 1982, S. 728-748.

Schmidt (1990):

Schmidt, R.; Rating börsennotierter Unternehmen; in: Gerke, W. (Hrsg.): Anleger an die Börse; Berlin u.a. 1990, S. 55-88.

Schmidt (1991):

Schmidt, R.; Handbuch Manager Magazin 500 - Unternehmenstest Deutschland 1991; Hamburg 1991.

\section{Schmidt (1995):}

Schmidt, J.G.; Die Discounted Cash-flow-Methode - nur eine kleine Abwandlung der Ertragswertmethode?; in: Zeitschrift für betriebswirtschaftliche Forschung, 47. Jg. 1995, S. 1088-1118.

Schmidt, Wilhelm (1987):

Schmidt, R.; Wilhelm, W.; Was Firmen wirklich wert sind; in: manager magazin, Jg. 1987, H. 11, S. 234-265.

Schmitting (2000):

Schmitting, W.; Das Traveling-Salesman-Problem: Anwendungen und heuristische Nutzung von Voronoi-/Delaunay-Strukturen zur Lösung euklidischer, zweidimensionaler TravelingSalesman-Probleme, zugl. Diss. Universität Düsseldorf 1999, Münster 2000.

Schmitz (1995):

Schmitz, R.H.; Emissionsgeschäft der Kreditinstitute; in: Gehrke, W., Steiner, M. (Hrsg.): Handwörterbuch des Bank- und Finanzwesens, 2.A.; Stuttgart 1995, Sp. 511-521.

Schmoll (1985):

Schmoll, A.; Interne Gefahrenquellen für das Kreditgeschäft; in: Die Bank, Jg. 1985, S. 227-236. 
Schneeweiß (1967):

Schneeweiß, H.; Entscheidungskriterien bei Risiko; zugl. Habil.-Schrift Universität Saarbrücken 1964, Berlin u.a. 1967.

Schneider (1992):

Schneider, D.; Investitionen, Finanzierung und Besteuerung, 7.A.; Wiesbaden 1992.

Schöneburg, Gantert, Reiner (1989):

Schöneburg, E.; Gantert, M.; Reiner, M.; Aktienkursprognosen mit Neuronalen Netzwerken; in: Computerwoche, Jg. 1989, H. 40, S. 121-124.

Schöneburg, Hansen, Gawelczyk (1990):

Schöneburg, E.; Hansen, N.; Gawelczyk, A.; Neuronale Netzwerke; München 1990.

Schüller (1995):

Schüller, St.; Ertragsorientierte Risikopolitik - Changemanagement des Kreditprozesses; in: Rolfes, B.; Schierenbeck, H.; Schüller, St. (Hrsg.): Risikomanagement in Kreditinstituten; Frankfurt a.M. 1995, S. 173-190.

Schulte (1996):

Schulte, R.; Kursänderungsrisiken bei festverzinslicher Wertpapieren; zugl. Diss. Fernuniversität Hagen 1995, Wiesbaden 1996.

Schulte (1996b):

Schulte, J.; Rechnungslegung und Aktienkursentwicklung; zugl. Diss. Universität Bochum 1995, Wiesbaden 1996.

Schulte-Mattler (1999):

Schulte-Mattler, H.; Basler Vorschlag zur Erfassung und Begrenzung von Kreditrisiken; in: Die Bank, Jg. 1999, H. 8, S. 530-534.

Schulte-Mattler (2001):

Schulte-Mattler, H.; Neuere Entwicklungen in der bankenaufsichtsrechtlichen Behandlung von Kreditrisiken; in: Rolfes, B.; Schierenbeck, H. (Hrsg.), Ausfallrisiken - Quantifizierung, Bepreisung und Steuerung; Frankfurt a.M. 2001, S. 53-71.

Schumann (1991):

Schumann, M.; Neuronale Netze zur Entscheidungsunterstützung in der Betriebswirtschaft; in: Biethan, J.; et. al. (Hrsg.): Wissensbasierte Systeme in der Finanzwirtschaft; Wiesbaden 1991, S. 21-50.

\section{Schumann, Lohrbach (1993):}

Schumann, M.; Lohrbach, T.; Comparing Artificial Neural Networks with Statistical Methods within the Field of Stock Market Prediction; in: International Joint Conference on System Science, Hawaii 1993, S. 597-606. 
Schuster (1989):

Schuster, L.; Neuere Entwicklungen im Risiko-Management der Banken; in: Die Unternehmung, 43. Jg. 1989, S. 204-215.

Sharpe (1964):

Sharpe, W.F.; Capital Asset Prices: A Theory of Market Equilibrium under Conditions of Risk; in: Journal of Finance, Vol. 19, Jg. 1964, S. 425-442.

Sharpe (1992):

Sharpe, W.F.; Asset Allocation: Management style and performance measurement; in: Journal of Portfolio Management, Winter 1992, S. 7-19.

Shefrin (1997):

Shefrin, H.; Behavioural Option Pricing; Department of Finance, Leavey School of Business, Santa Clara University, Working Paper 8/97.

Shefrin, Statman (1985):

Shefrin, H.; Statman, M.; The Disposition to Sell Winners too Early and Ride Losers too Long: Theory and Evidence; in: The Journal of Finance, Vol. 40, Jg. 1985, H. 3, S. 777-792.

\section{Shefrin, Statman (1994):}

Shefrin, H.; Statman, M.; Behavioural Capital Asset Pricing Theory; in: Journal of Finance and Quantitative Analysis, Vol. 29, Jg. 1994, H. 3, S. 323-349.

Shih (1991):

Shih, Y.L.; Neural Nets in Technical Analysis; in Technical Analysis of Stocks \& Commodities, Jg. 1991, H. Februar, S. 62-68.

Shiller (1981):

Shiller, R.J.; Do Stock Prices Move too Much to be Justified by Subsequent Changes in Dividends?; in: American Economic Review, Vol. 71, Jg. 1981, S. 421-438.

Shiller (1984):

Shiller, R.J.; Stock Prices and Social Dynamics; in: Brookings Papers on Economic Activity, 2. Jg. 1984, S. 457-510.

\section{Shiller, Pound (1989):}

Shiller, R.J.; Pound, J.; Survey Evidence on Diffusion of Interest and Information Among Investors; in: Journal of Economic Behaviour and Organization, Jg. 1989, H. 12, S. 47-66.

Shimko, Tejima, van Deventer (1993):

Shimko, D.C.; Tejima, N.; van Deventer, D.R.; The Pricing of Risky Debt When Interest Rates are Stochastic; in: Journal of Fixed Income, Vol. 3, Jg. 1993, S. 58-65. 
Sieben (1963):

Sieben, G.; Der Substanzwert der Unternehmung; Wiesbaden 1963.

Sieben, Schildbach (1994):

Sieben, G.; Schildbach Th.; Betriebswirtschaftliche Entscheidungstheorie, 4.A.; Düsseldorf 1994.

Siegel (1991):

Siegel, Th.; Grundlagen der Unternehmensbewertung; in: Wirtschafswissenschaftliches Studium, Jg. 1991, S. 231-237.

Siegert (1995):

Siegert, Th.; Shareholder-Value als Lenkungsinstrument; in: Zeitschrift für betriebswirtschaftliche Forschung, 47. Jg. 1995, S. 580-607.

Sinkey (1992):

Sinkey, J.F.; Commercial Bank Financial Management in the Financial Services Industry, 4th ed.; New York 1992.

Skinner (1995):

Skinner, F.S.; Bond Yield, Taxes, and the Dimensions of Default Risk; In: Financial Review, Vol. 30, Jg. 1995, No. 4, S. 738-761.

\section{Smithson (1995):}

Smithson, C., et al.; Credit Derivates; in: Risk - Managing Risk in the Worlds Financial Markets, Jg. 1995, H. December, S. 38-39.

Sommerfeld (2001):

Sommerfeld, H.; Die Steuerung von Kreditrisiken durch Hedging - Eine empirische Analyse; (in Vorbereitung), 0.0. 2001.

Spremann (1996):

Spremann, K.; Investition und Finanzierung, 5.A.; München u.a. 1996.

SPSS (1997):

SPSS Inc.; Neural Connection 2.0 - User's Guide; Chicago 1997.

Staehle (1975):

Staehle, W.H.; Das Du-Pont-System und verwandte Konzepte der Unternehmenskontrolle; in: v. Böcker, F.; Dichtl, E. (Hrsg.): Erfolgskontrolle im Marketing; Berlin 1975, S. 317-336.

Stanley, Bak (1991):

Stanley, J.; Bak, E.; Neuronale Netze. Computersimulation biologischer Intelligenz; München 1991. 
Steiner (1980):

Steiner, M.; Ertragskraftorientierte Unternehmenskredit und Insolvenzrisiko; Stuttgart 1980.

Steiner (1992):

Steiner, M.; Rating - Risikobeurteilung von Emittenten durch Rating-Agenturen; in: Wirtschafswissenschaftliches Studium, Jg. 1992, H. 10, S. 347-368.

Steiner, Bruns (1998):

Steiner, M.; Bruns, Ch.; Wertpapiermanagement, 6.A.; Stuttgart 1998.

Steiner, Heinke (1996):

Steiner, M; Heinke, V.G.; Risikobeurteilung von Lebensversicherungen durch spezialisierte Ratingagenturen; in: Versicherungswirtschaft, 51. Jg., H. 24, 1996, S. 1694-1707.

Strecker (1997):

Strecker, St.; Künstliche Neuronale Netze - Aufbau und Funktionsweise; Arbeitspapier Wirtschaftsinformatik 10/1997, Universität zu Mainz, Lehrstuhl für Allgemeine Betriebswirtschafslehre und Wirtschaftsinformatik, 1997.

Streim (1975):

Streim, H.; Heuristische Lösungsverfahren, Versuch einer Begriffserklärung; in: Zeitschrift für Operations Research, Jg. 1975, S. 143-162.

Strom, Baun (1992):

Storm, A.; Baun, S.; Neuronale Netze in der Finanzprognose; in: Sparkasse, 11/1992, S. 534-540.

\section{Süchting (1993):}

Süchting, J.; Kreditrating - Ziele und Probleme der Anwendung im Firmenkundengeschäft, in: Bankinformation, Heft 10, Jg. 1993, S. 8-10.

Tegeder (1996):

Tegeder, P.; Business Reengineering als Grundlage eines ganzheitlichen Restrukturierungsprozesses in ostdeutschen Kreditinstituten; Frankfurt a.M. 1996.

Teschner (1998):

Teschner, C.L.; Aufbau einer effektiven Portfoliosteuerung; in: Hanker, P. (Hrsg.): Management von Marktpreis- und Ausfallrisiken: Instrumente und Strategien zur Risikominimierung in Banken; Wiesbaden 1998, S. 243-247.

Thompson, Vaz (1990):

Thompson, G.R.; Vaz, P.; Dual Bond Ratings: A Test of the Certification Function of Rating Agencies; in: Financial Review, Vol. 25, Jg. 1990, H. 3 August, S. 457-471. 
Uhilg (1995):

Uhlig, H.; Finanzprognose mit Neuronalen Netzen: eine Einführung mit Anleitung; München 1995.

Uhlir (1979):

Uhlir, H.; Überprüfung der Random-Walk-Hypothese auf dem österreichischen Aktienmarkt; Wien 1979.

Uhlir, Steiner (1994):

Uhlir, H.; Steiner, P.; Wertpapieranalyse, 3.A.; Heidelberg 1994.

Utans, Moody (1991):

Utans, J.; Moody, J.; Selecting Neural Network Architectures via Prediction Risk: Application to Corporate Bond Rating Prediction; in: Proceeding of the First International Workshop on Artificial Intelligence Applications on Wall Street, S. 35-41.

Uthoff (1997):

Uthoff, C.; Erfolgsoptimale Kreditwürdigkeitsprïfung auf der Basis von Jahresabschlüssen und Wirtschaftsauskünften mit Künstlichen Neuronalen Netzen; zugl. Diss. Universität Münster 1996, Stuttgart 1997.

van Horne (1979):

van Home, J.C.; Behaviour of Default Risk Premium for Corporate Bonds and Commercial Paper; in: Journal of Business Research, Vol. 7, Jg. 1979, H. 4, S. 301-313.

van Horne (1990):

van Horne, J.C.; Financial Market Rates \& Flows; Engelwood, Cliffs 1990.

von Villiez (1989):

von Villiez, C.; Budgetkontrolle und Abweichungsanalyse in Kreditinstituten; Frankfurt a.M. 1989.

Völkel (2001):

Völkel, H.-G.; Rating als Chance; in: Initiativbanking, Februar 2001, S. 6-9.

Wansley, Clauretie (1985):

Wansley, J.W.; Clauretie, T.M.; The Impact of Credit Watch Placement on Equity Returns and Bond Prices; in: Journal of Financial Research, Vol. 8, Jg. 1985, S. 31-42.

Weber, Krahnen, Weber (1995):

Weber, M.; Krahnen, J.; Weber, A.; Scoring-Verfahren - häufige Anwendungsfehler und ihre Vermeidung; in: Der Betrieb, Jg. 1995, S. 1621-1626. 
Weinrich (1978):

Weinrich, G.; Kreditwürdigkeitsprognosen - Steuerung des Kreditgeschäfts durch Risikoklassen; Wiesbaden 1978.

Weinstein (1981):

Weinstein, M.; The Systematic Risk of Corporate Bonds; in: Journal of Financial Economics, Vol. 5, Jg. 1977, S. 257-278.

Werner (1993):

Werner, P.; Kursprognosen, Verborgene Regeln; in: WirtschaftsWoche, Jg. 1993, H. 48, S. 74-82.

West (1988):

West, K.D.; Bubbles, Fads and Stock Price Volatility Tests: A Partial Evaluation; in: The Journal of Finance, Vol. 43, Jg. 1988, H. 3, S. 639-660.

White (1988):

White, H.; Economic Prediction using Neural Networks: The Case of IBM Daily Stock Returns; in: IEEE Proceedings of the second Annual ICNN, San Diego, 24.-27.7.1988, Bd. II, S. 451-458.

Wild (1991):

Wild, K.-D.; Neuronale Netze erstellen leistungsfähiger Zinsprognosen; in: Sparkasse, Jg. 1991, H. 6/91, S. 257-260.

Wilhelm (1983):

Wilhelm, J.; Finanztitelmärkte und Unternehmensfinanzierung; Berlin u.a. 1983.

Wittkemper (1994):

Wittkemper, H.-G.; Neuronale Netze als Hilfsmittel zur Rendite- und Risikoeinschätzung von Aktien; zugl. Diss. Universität Münster 1993, Köln 1994.

Wöhe (1996):

Wöhe, G.; Einführung in die Allgemeine Betriebswirtschafslehre, 19.A.; München 1996.

Woll (2000):

Woll, A.; Allgemeine Volkswirtschaftslehre, 13.A.; München 2000.

Wu (1991):

Wu, C.; A Certainty Equivalent Approach to Municipal Bond Default Risk Estimation; in: Journal of Banking and Finance, Vol. 14, Jg. 1991, H. 3, S. 241-247.

Wu,Yu (1996):

Wu, C.; Yu, C.-H.; Risk Aversion and the Yield of Corporate Debt; in: Journal of Banking and Finance, Vol. 20, Jg. 1996, S. 267-281. 
Yawitz (1977):

Yawitz, J.B.; An Analytical Model of Interest Rate Differentials and Different Default Recoveries; in: Journal of Financial and Quantitative Analysis, Vol. 12, Jg. 1977, S. 481-490.

Yawitz, Maloney, Ederington (1985):

Yawitz, J.B.; Maloney, K.J.; Ederington, L.H.; Taxes, Default Risk and Yield Spreads in: Journal of Finance, Vol. 40, Jg. 1985, H. 4, S. 1127-1140.

Zapotocky (2000):

Zapotocky, S.; Eigengeschäfte mit Wertpapieren, in: Stein, von J.H.; Hagen, von J. (Hrsg.):

Obst/Hintner - Geld-, Bank- und Börsenwesen, 40.A; Stuttgart 2000, S. 1076-1090.

Zell (1994):

Zell, A.; Simulation Neuronaler Netze; zugl. Habil.-Schrift Universität Stuttgart 1994, Bonn u.a. 1994.

Zentralverband der Elektroindustrie (1989):

Zentralverband der Elektrotechnik-Elektroindustrie (ZVEI); ZVEI-Kennzahlensystem, 4.A.; Frankfurt a.M. 1989.

\section{Ziebart, Reiter (1992):}

Ziebart, D.A.; Reiter, S.S.; Bond Ratings, Bond Yields and Financial Information; in: Contemporary Accounting Research, Vol. 9, Jg. 1992, H. 1, S. 252-282.

\section{Zimmermann (1994):}

Zimmermann, H.G.; Neuronale Netze als Entscheidungskalkül; in: Rehkugler, H.; Zimmermann, H.G. (Hrsg.): Neuronale Netze in der Ökonomie; München 1994, S. 1-88.

\section{Zimmermann (1999):}

Zimmermann, H.-J.; Fuzzy Set Theorie; in: Wirtschafswissenschaftliches Studium, Jg. 1999, H. 1, S. 22-29. 
Andreas Siemes - 978-3-631-75322-4

Downloaded from PubFactory at 01/11/2019 06:12:16AM

via free access 


\section{Gesetzesverzeichnis}

AktG:

Aktiengesetz; in: Wirtschaftsgesetze IDW Textausgabe, 18.A., Stand: 1. Februar 2001, Düsseldorf 2001.

BösG:

Börsen Gesetzbuch; in: Wirtschaftsgesetze IDW Textausgabe, 18.A., Stand: 1. Februar 2001, Düsseldorf 2001.

\section{ESTG:}

Einkommenssteuerrecht, in: Beck-Texte im Deutschen Taschenbuchverlag, 15.A., Stand 1. Februar 2001, München.

HGB:

Handelsgesetzbuch; in: Wirtschaftsgesetze IDW Textausgabe, 18.A., Stand: 1. Februar 2001, Düsseldorf 2001.

KWG:

Kreditwesengesetz; in: Wirtschaftsgesetze IDW Textausgabe, 18.A., Stand: 1. Februar 2001, Düsseldorf 2001.

\section{Beiträge zum Controlling}

$$
\text { Herausgegeben von Wolfgang Berens }
$$

Band 1 Wolfgang Berens / Joachim Strauch: Due Diligence bei Unternehmensakquisitionen - eine empirische Untersuchung. Unter Mitarbeit von Thorsten Behrens und Julia Lescher. 2002.

Band 2 Andreas Siemes: Marktorientierte Kreditrisikobewertung. Eine empirische Untersuchung mittels Künstlicher Neuronaler Netze. 2002. 


\section{Andreas Schön}

\section{Innovationscontrolling}

Eine Controlling-Konzeption zur effektiven und effizienten Gestaltung innovativer Prozesse in Unternehmen

Frankfurt/M., Berlin, Bern, Bruxelles, New York, Oxford, Wien, 2001. XV, 272 S., zahir. Graf.

Europäische Hochschulschriften: Reihe 5, Volks- und Betriebswirtschaft. Bd. 2703

ISBN 3-631-37459-3 · br. $€ 45.50$ *

Die effektive und effiziente „Produktion" von Innovationen stellt eine der größten Herausforderungen an das Management von Unternehmen dar. Einen wesentlichen Baustein der erfolgreichen innovativen Tätigkeiten bildet dabei die Gestaltung der Innovationsprozesse. Das Management sieht sich jedoch bei der Wahrnehmung der Gestaltungsaufgabe von Innovationsprozessen mit einer zunehmenden Komplexität und Dynamik der Unternehmensum- und -innenwelt konfrontiert. Dies schlägt sich u.a. darin nieder, daß es dem Innovationsmanagement mittels des klassischen Managementinstrumentariums oftmals nicht gelingt, die richtigen Entscheidungen über Inhalte und Gestaltungsalternativen von Innovationsprozessen in der erforderlichen Zeit zu treffen.

Im Mittelpunkt des Interesses steht bei dieser Arbeit die Beratung des Innovationsmanagements bei der Wahrnehmung seiner Gestaltungsaufgabe mit einer geeigneten Controlling-Konzeption und einem ausgewählten Kennzahlensystem.

FrankfurtM - Berlin - Bern - Bruxelles - New York - Oxford - Wien

Auslieferung: Verlag Peter Lang AG

Jupiterstr. 15, $\mathrm{CH}-3000$ Bern 15

Telefax (004131) 9402131

*inklusive der in Deutschland gültigen Mehrwertsteuer

Preisänderungen vorbehalten

Homepage http://wnww.peterlang.de 
Andreas Siemes - 978-3-631-75322-4

Downloaded from PubFactory at 01/11/2019 06:12:16AM

via free access 
Andreas Siemes - 978-3-631-75322-4

Downloaded from PubFactory at 01/11/2019 06:12:16AM

via free access 